\title{
Electrospray Ionization Tandem Mass Spectrometric Determination of Ligand Binding Energies in $\mathrm{Pt}^{\mathrm{II}}$ Complexes
}

\author{
Loubna A. Hammad, Gerd Gerdes, and Peter Chen* \\ Laboratorium für Organische Chemie \\ ETH Zürich \\ Zürich, Switzerland
}

\section{Supplemental Information}

Description of the 24-pole ion guide

The difficulty in constructing high-order multipoles is that ions should not "see" insulators. While a quadrupole or octopole can be constructed from thick rods which are mounted along their outside surface, the construction of higher-order multipoles according to the same design, i.e. thick rods, leads to increasingly large inner diameters, which, in turn, leads to higher peak operating voltages. The present 24-pole was constructed from thin stainless steel plates, mounted radially in annular PEEK supports. The inner edge of the

plates were formed by electric-discharge machining to present a circular cross-section locally. The practical advantages of the radially-mounted plates is that the inner diameter of the multipole can be kept small while maintaining a mechanically stiff and rigid assembly that does not easily short due to "sagging" of thin rods. Electrical contact to the plates is also easily made. The disadvantage is that the assembly has a relatively large electrical capacitance, but this is overcome with a high-power rf sources described by Gerlich in ref 14 . 

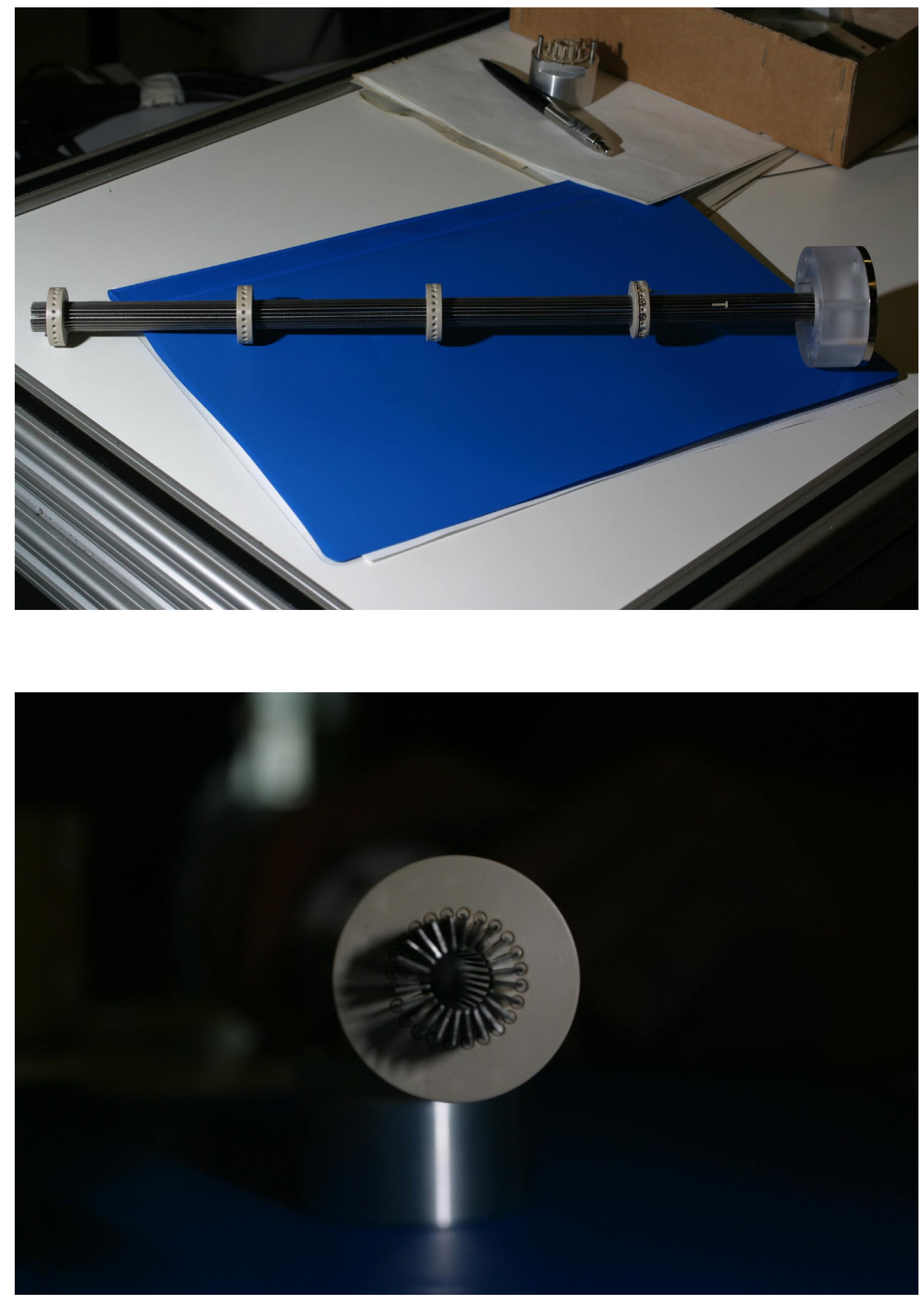


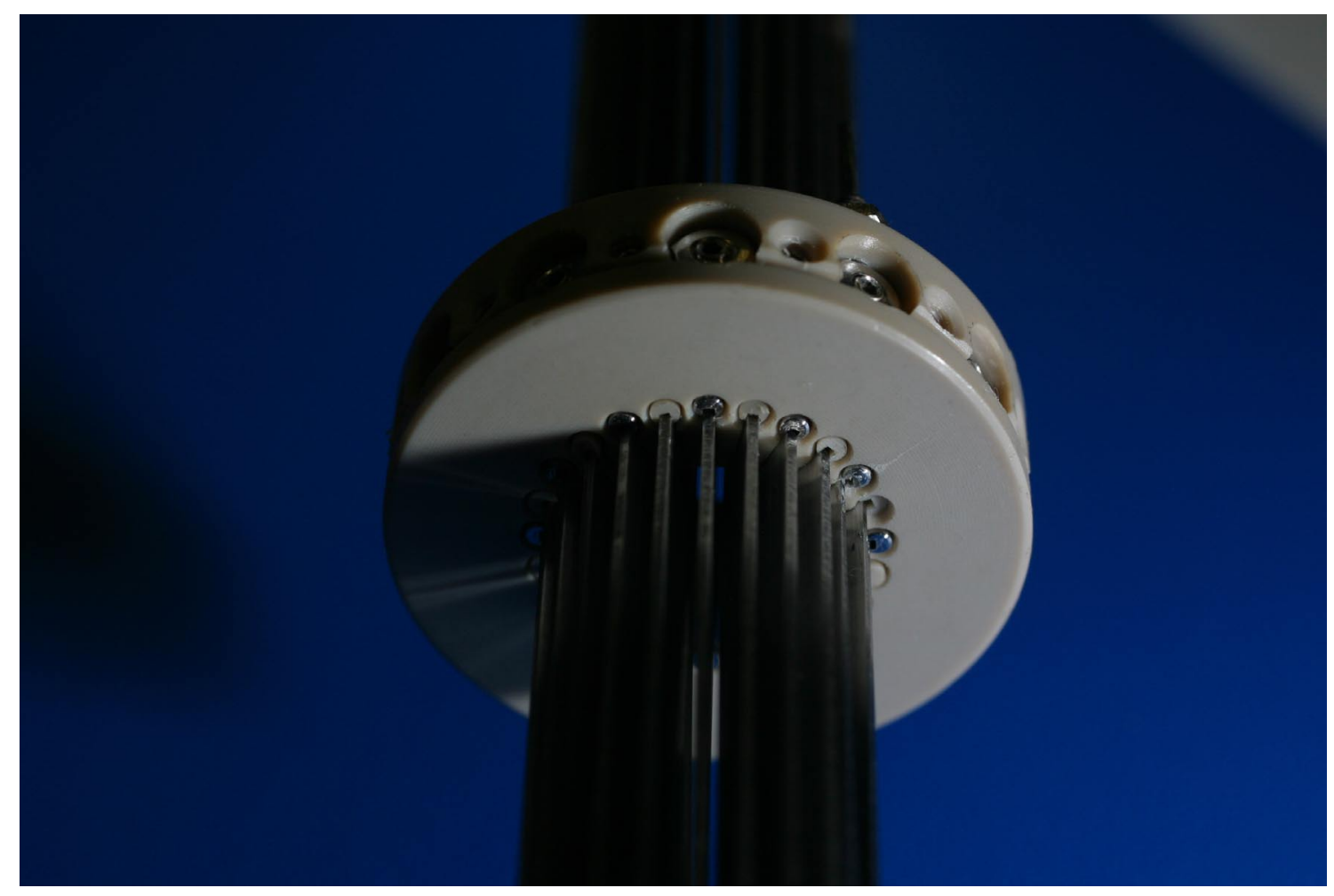




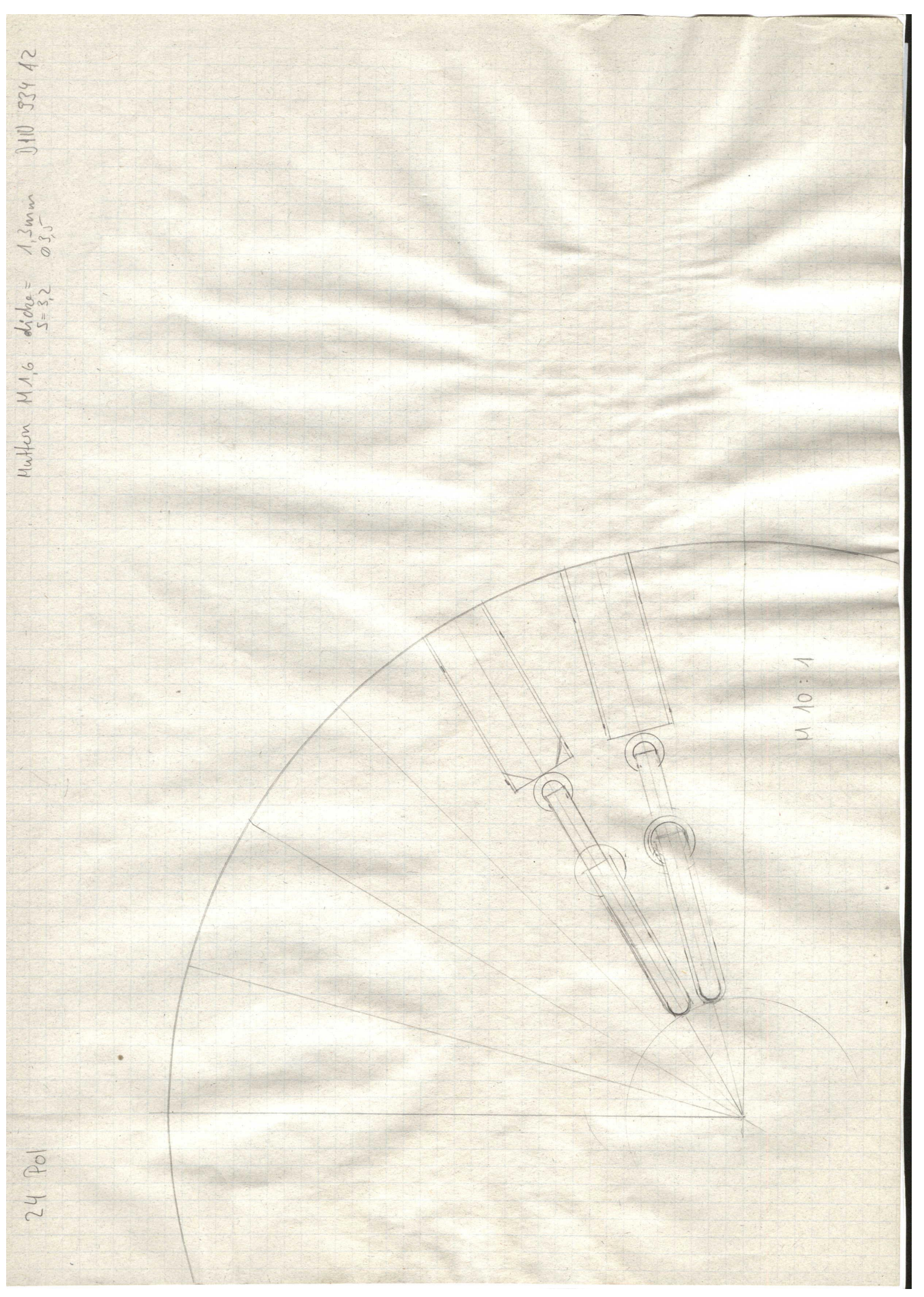




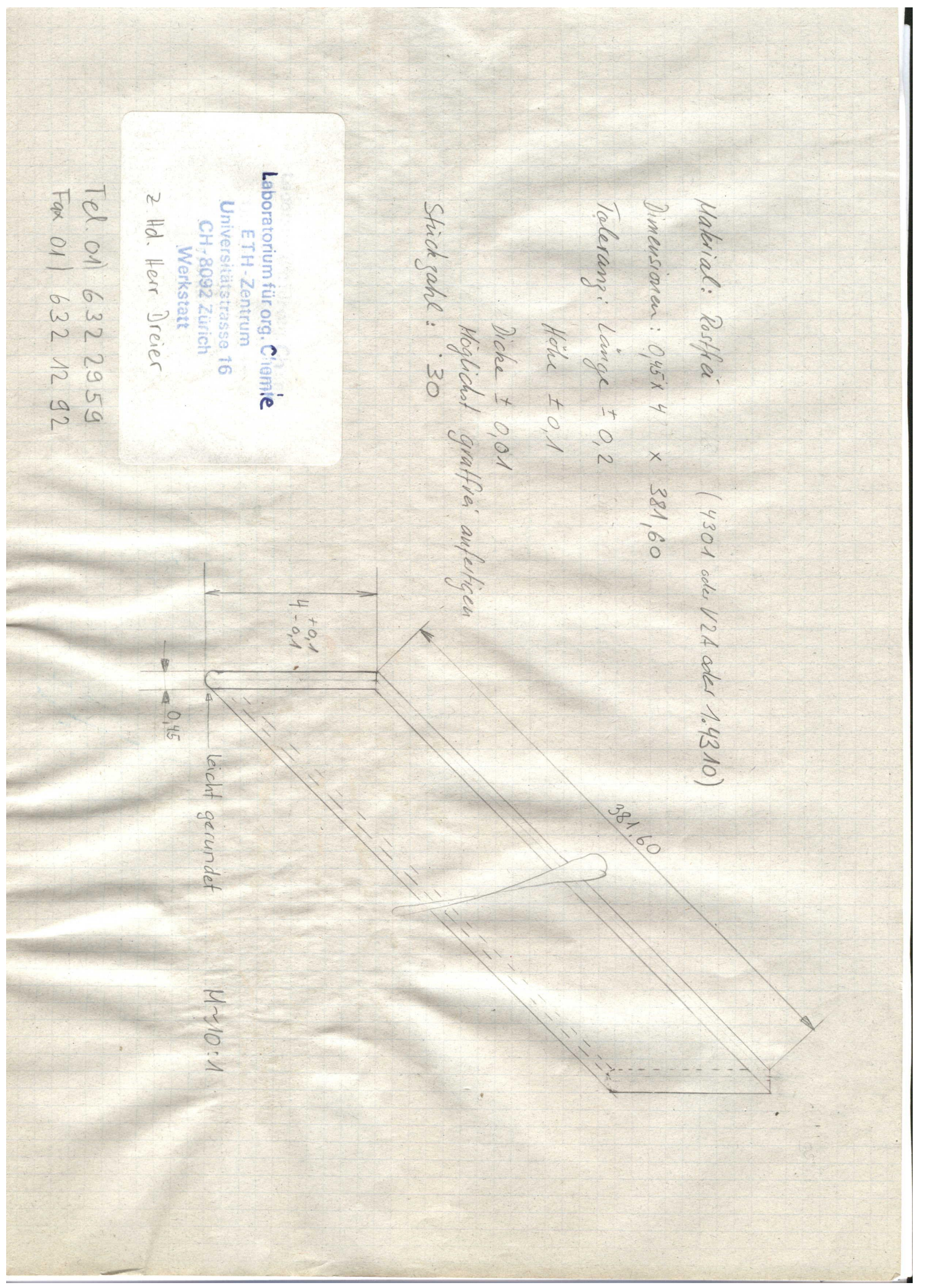




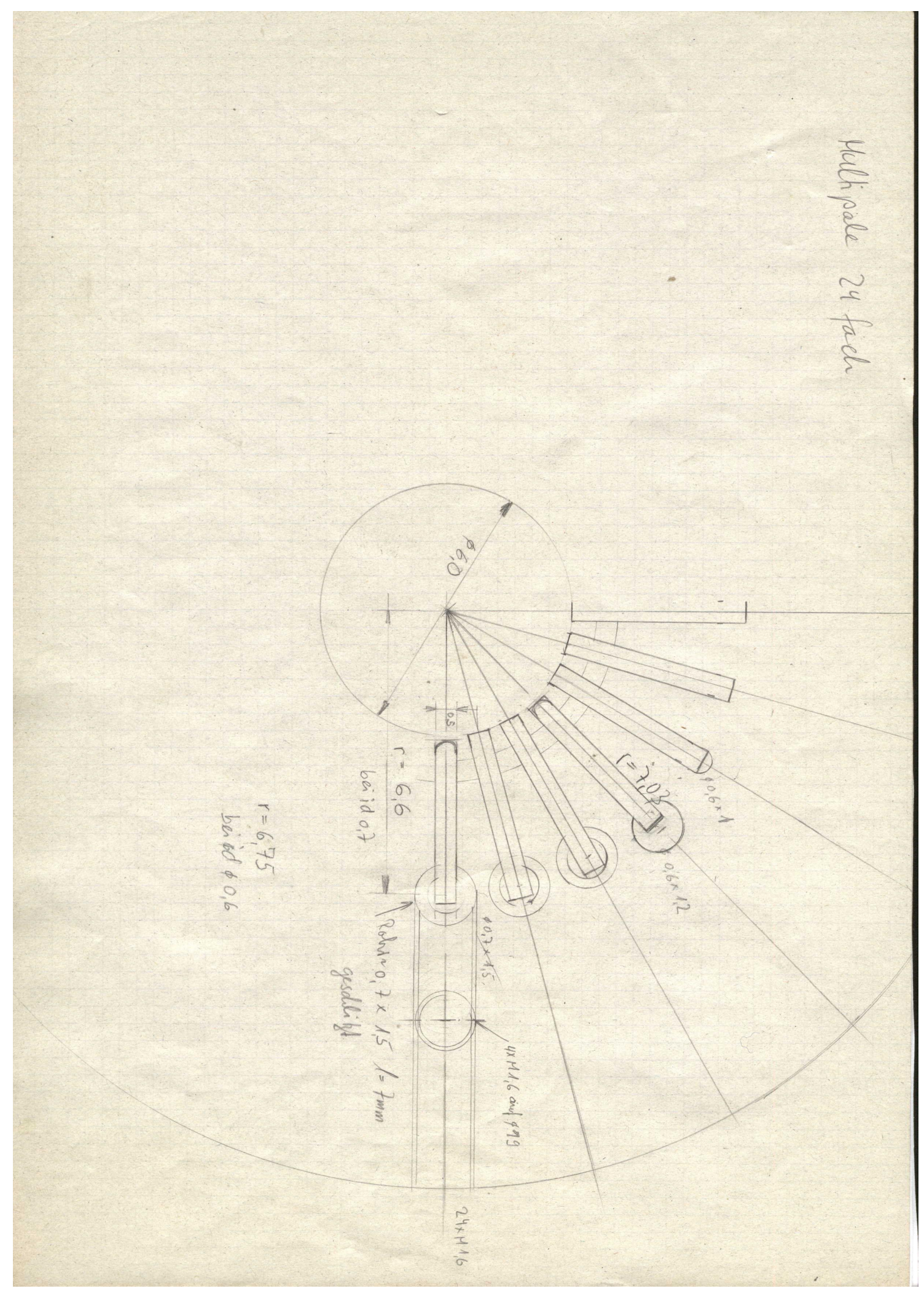




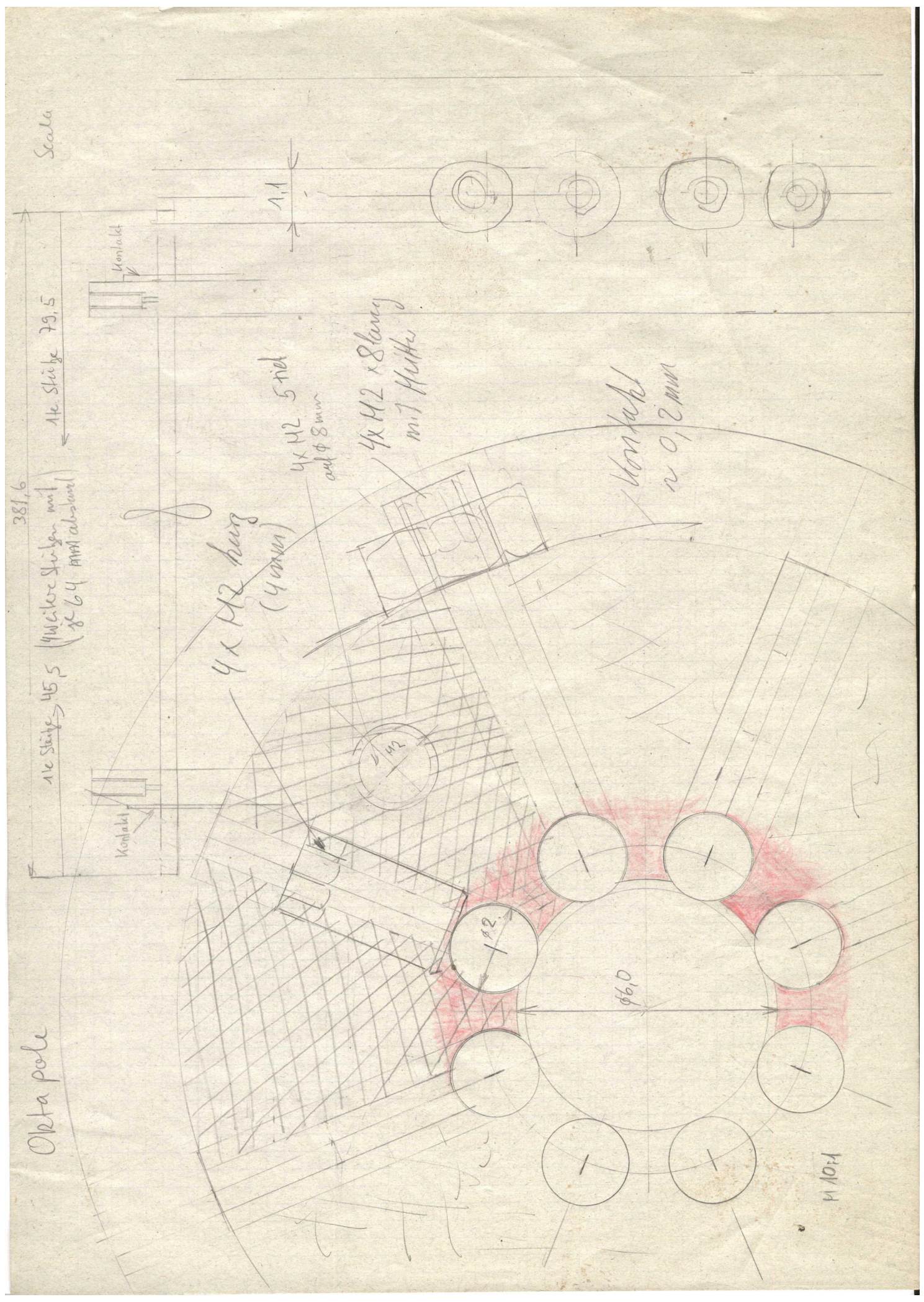


Energy-resolved Threshold Measurements with Xe instead of Ar

\begin{tabular}{cccccc}
\hline Complex & \multicolumn{2}{c}{$E_{0}($ expt, RRKM) } & $\begin{array}{c}\text { kinetic shift } \\
\mathrm{eV}\end{array}$ & $n$ & $\begin{array}{c}\Delta S^{\ddagger} \\
\text { Joules/mol K }\end{array}$ \\
\hline 1a & $1.15 \pm 0.06$ & $26.5 \pm 1.4$ & 1.00 & $1.5 \pm 0.3$ & 69 \\
1b & $1.24 \pm 0.04$ & $28.6 \pm 0.9$ & 1.14 & $1.5 \pm 0.1$ & 1 \\
1c & $1.49 \pm 0.05$ & $34.4 \pm 1.2$ & 1.85 & $1.3 \pm 0.1$ & 11 \\
\hline
\end{tabular}

Table S1. Experimental thermochemical data for the dissociation of complexes 1a-c using Xe as target gas.

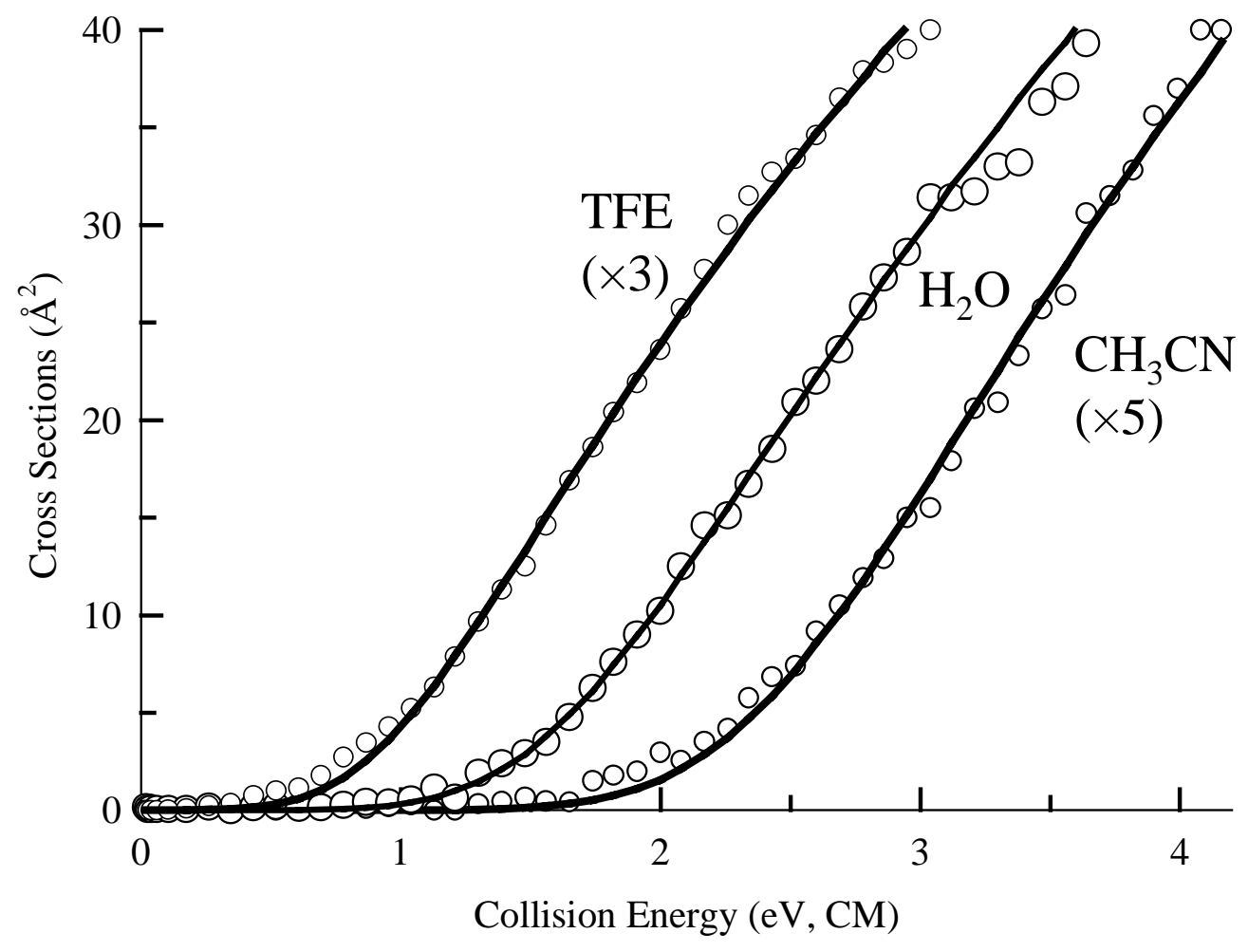

Figure S1. Relative product cross sections for collision-induced dissociation of 1a-c with Xe target as a function of translational energy in the center-of-mass frame. The experimental data are the circles, and the solid lines are the model appearance curves calculated using eq 1 convoluted over the neutral and ion kinetic energy distributions, and over RRKM dissociation probabilities. 
Calculated RRKM dissociation rates for 1a-c using the full frequency set versus a single effective frequency.

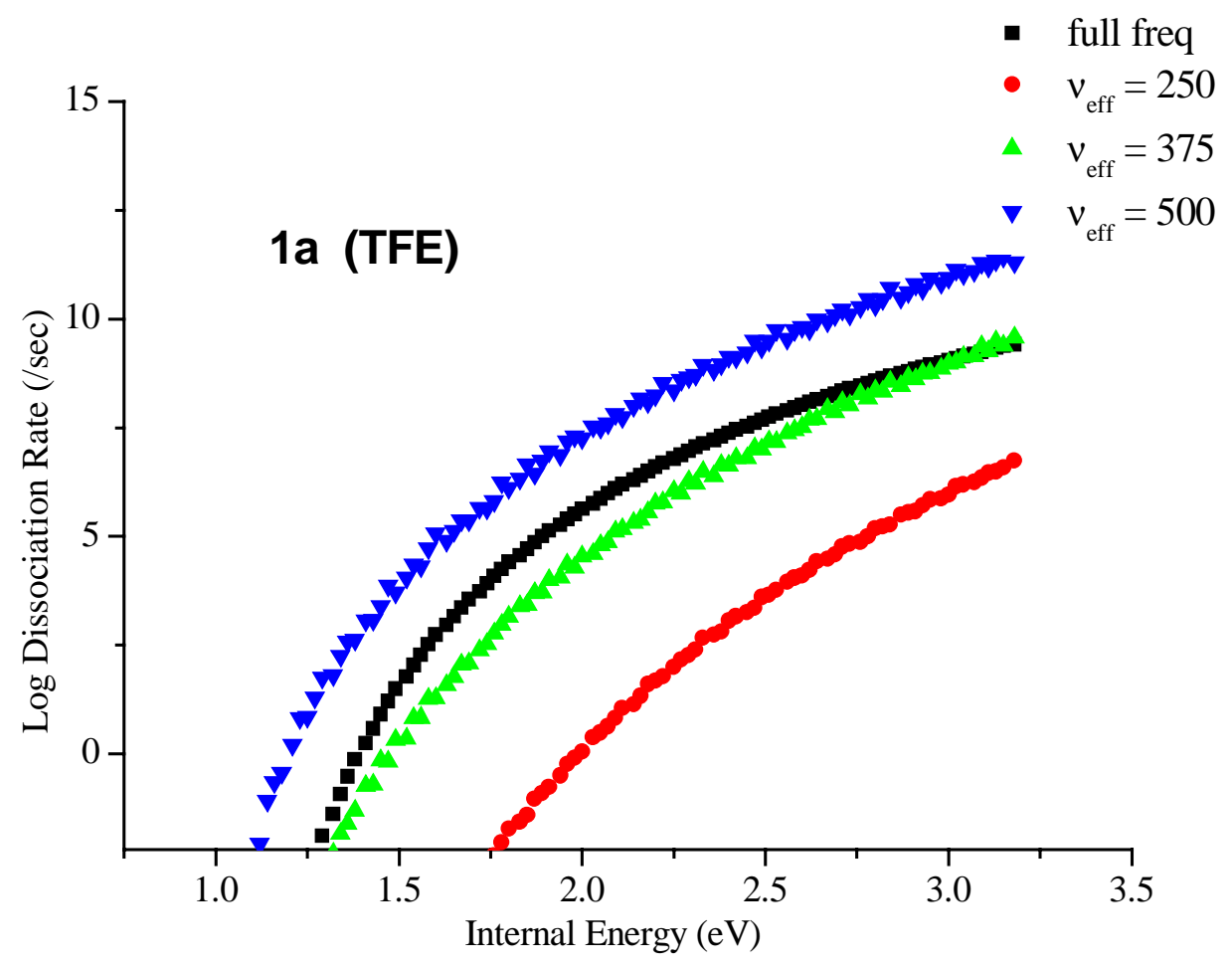



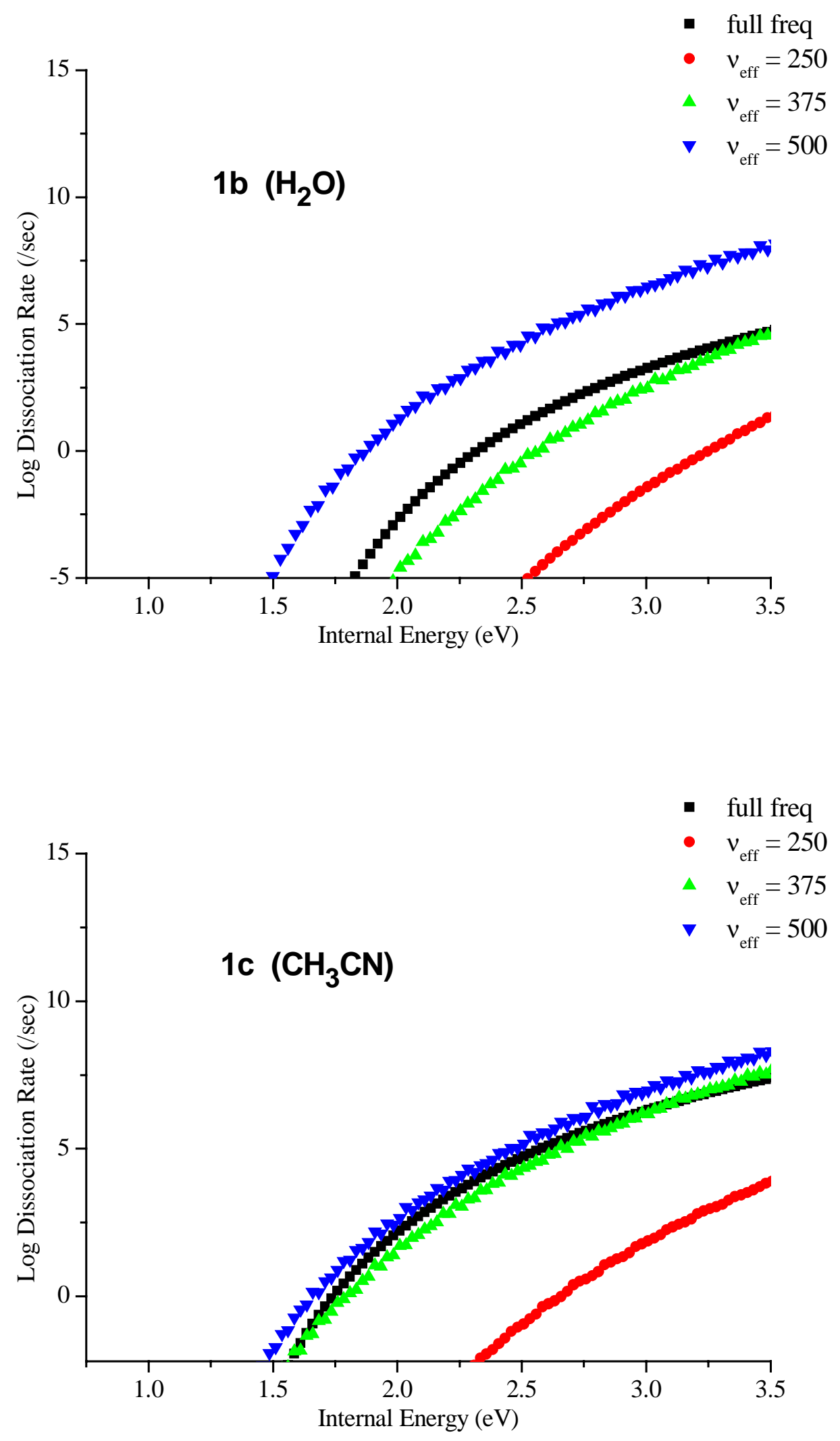
Computational Data

\begin{tabular}{|c|c|c|c|c|c|}
\hline \multicolumn{6}{|l|}{ Water } \\
\hline \multicolumn{6}{|c|}{ Standard orientation: } \\
\hline \multirow[t]{5}{*}{ Center number } & Atomic number & \multicolumn{4}{|c|}{ Coordinates $[\AA]$} \\
\hline & & & $x$ & Y & Z \\
\hline & 1 & 8 & 0 & 0.118859 & 0 \\
\hline & 2 & 1 & 0.764403 & -0.475435 & 0 \\
\hline & 3 & 1 & -0.764403 & -0.475435 & 0 \\
\hline \multicolumn{2}{|c|}{ Rotational constants [GHz]: } & \multicolumn{2}{|c|}{799.365596} & 429.09854 & \multirow[t]{2}{*}{279.215811} \\
\hline Isotopes: & & \multicolumn{2}{|c|}{$\mathrm{O}-16, \mathrm{H}-1, \mathrm{H}-1$} & & \\
\hline \multicolumn{2}{|c|}{ Electronic engergy [Hartree]: } & \multicolumn{2}{|c|}{-76.375958} & & \\
\hline \multicolumn{6}{|c|}{ 2,2,2-Trifluoroethanol (TFE) } \\
\hline \multicolumn{6}{|c|}{ Standard orientation: } \\
\hline \multirow[t]{11}{*}{ Center number } & Atomic number & \multicolumn{2}{|c|}{ Coordinates $[\AA]$} & & \\
\hline & & & $x$ & Y & Z \\
\hline & 1 & 6 & -0.416468 & -0.000106 & -0.000002 \\
\hline & 2 & 9 & -0.552274 & -0.788701 & 1.10659 \\
\hline & 3 & 6 & 0.892307 & 0.748859 & -0.000023 \\
\hline & 4 & 8 & 1.933162 & -0.204604 & 0.000016 \\
\hline & 5 & 9 & -1.4383 & 0.921415 & -0.000039 \\
\hline & 6 & 9 & -0.552265 & -0.78878 & -1.106538 \\
\hline & 7 & 1 & 0.894628 & 1.387879 & -0.892854 \\
\hline & 8 & 1 & 0.894621 & 1.387944 & 0.892762 \\
\hline & 9 & 1 & 2.775972 & 0.273086 & -0.00001 \\
\hline \multirow{2}{*}{\multicolumn{2}{|c|}{ Rotational constants [GHz]: }} & \multicolumn{2}{|c|}{5.1084036} & 2.8153932 & 2.7742136 \\
\hline & 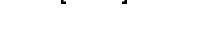 & \multicolumn{3}{|c|}{ C-12,F-19,C-12,O-16,F-19,F-19,H-1,H-1,H-1 } & \\
\hline \multicolumn{2}{|c|}{ Electronic engergy [Hartree]: } & & & & \\
\hline \multicolumn{6}{|c|}{ Acetonitrile } \\
\hline \multicolumn{6}{|c|}{ Standard orientation: } \\
\hline \multirow[t]{8}{*}{ Center number } & Atomic number & & $\operatorname{es}[\AA]$ & & \\
\hline & & & $x$ & Y & Z \\
\hline & 1 & 6 & 1.180202 & -0.000006 & -0.000043 \\
\hline & 2 & 6 & -0.275472 & 0.000027 & 0.000175 \\
\hline & 3 & 7 & -1.443405 & -0.000012 & -0.000076 \\
\hline & 4 & 1 & 1.558637 & -0.000677 & 1.028505 \\
\hline & 5 & 1 & 1.558385 & -0.890509 & -0.514956 \\
\hline & 6 & 1 & 1.558428 & 0.89114 & -0.513811 \\
\hline Rotational const & $\operatorname{tants~[GHz]:~}$ & & & 9.152611 & 9.1525885 \\
\hline Isotopes: & & & $\mathrm{N}-14, \mathrm{H}-1, \mathrm{H}-$ & & \\
\hline Electronic enger & rgy [Hartree]: & & & & \\
\hline ML3 Fragment & & & & & \\
\hline Standard orienta & & & & & \\
\hline Center number & Atomic number & & es $[\AA]$ & & \\
\hline & & & $x$ & Y & z \\
\hline & 1 & 78 & 0.001596 & -1.005199 & 0.035734 \\
\hline & 2 & 7 & 1.22082 & 0.554753 & -0.167774 \\
\hline
\end{tabular}




\begin{tabular}{|c|c|c|c|c|c|}
\hline & 3 & 7 & -1.38621 & 0.52904 & -0.291744 \\
\hline & 4 & 17 & 1.900929 & 0.905662 & 2.717562 \\
\hline & 5 & 17 & 3.021207 & 0.053789 & -2.539213 \\
\hline & 6 & 17 & -1.873091 & -2.441739 & -0.178797 \\
\hline & 7 & 17 & -3.412783 & 2.765599 & 0.651524 \\
\hline & 8 & 6 & 1.443581 & -2.470487 & 0.193969 \\
\hline & 9 & 1 & 2.090314 & -2.453954 & -0.69236 \\
\hline 1 & 10 & 1 & 2.045522 & -2.258063 & 1.088768 \\
\hline 1 & 11 & 1 & 0.993751 & -3.466652 & 0.294636 \\
\hline 1 & 12 & 6 & 2.616028 & 0.475017 & 0.125945 \\
\hline 1 & 13 & 6 & 3.549921 & 0.203948 & -0.885475 \\
\hline & 14 & 6 & 3.052609 & 0.575187 & 1.455835 \\
\hline 1 & 15 & 6 & 4.400798 & 0.41717 & 1.767925 \\
\hline 1 & 16 & 1 & 4.722499 & 0.502271 & 2.798721 \\
\hline 1 & 17 & 6 & 5.316257 & 0.149631 & 0.751215 \\
\hline 1 & 18 & 1 & 6.364756 & 0.022378 & 0.993991 \\
\hline 1 & 19 & 6 & 4.89834 & 0.042584 & -0.574081 \\
\hline 2 & 20 & 1 & 5.606428 & -0.168522 & -1.366123 \\
\hline & 21 & 6 & 0.65243 & 1.641112 & -0.640119 \\
\hline 2 & 22 & 6 & 1.400284 & 2.901195 & -0.937185 \\
\hline 2 & 23 & 1 & 2.396231 & 2.873581 & -0.488947 \\
\hline 2 & 24 & 1 & 0.857278 & 3.771673 & -0.552769 \\
\hline 2 & 25 & 1 & 1.51646 & 3.022356 & -2.023438 \\
\hline 2 & 26 & 6 & -0.824375 & 1.580856 & -0.84189 \\
\hline 2 & 27 & 6 & -1.477806 & 2.602563 & -1.718551 \\
\hline 2 & 28 & 1 & -0.889568 & 2.699208 & -2.641299 \\
\hline & 29 & 1 & -1.521473 & 3.589877 & -1.243832 \\
\hline 3 & 30 & 1 & -2.491984 & 2.298774 & -1.986433 \\
\hline 3 & 31 & 6 & -2.746161 & 0.206779 & -0.131628 \\
\hline 3 & 32 & 6 & -3.772282 & 1.12629 & 0.19948 \\
\hline & 33 & 6 & -3.121151 & -1.160496 & -0.135919 \\
\hline & 34 & 6 & -4.431828 & -1.584191 & -0.031016 \\
\hline 3 & 35 & 1 & -4.670466 & -2.640647 & -0.066099 \\
\hline 3 & 36 & 6 & -5.435433 & -0.63008 & 0.159987 \\
\hline 3 & 37 & 1 & -6.468489 & -0.945212 & 0.244191 \\
\hline 3 & 38 & 6 & -5.100429 & 0.708515 & 0.315306 \\
\hline 3 & 39 & 1 & -5.85917 & 1.442041 & 0.5593 \\
\hline Rotational consté & ants [GHz]: & \multicolumn{2}{|c|}{0.2695841} & & \\
\hline Isotopes: & & \multicolumn{4}{|c|}{$\begin{array}{l}\text { C-12,H-1, C-12,H-1, C-12,H-1,C-12,C-12,H-1,H-1,H-1,C-12,C-12,H-1, H-1, H-1, C-12,C-12, } \\
\text { C-12,C-12,H-1,C-12,H-1,C-12,H-1 }\end{array}$} \\
\hline Electronic enger & gy [Hartree]: & \multicolumn{4}{|c|}{-2722.22145} \\
\hline \multicolumn{6}{|c|}{ Pt water } \\
\hline \multicolumn{6}{|c|}{ Standard orientation: } \\
\hline \multirow[t]{5}{*}{ Center number } & Atomic number & \multicolumn{4}{|c|}{ Coordinates $[\AA]$} \\
\hline & & & $x$ & Y & Z \\
\hline & 1 & 78 & 0.036259 & -0.296959 & -0.937071 \\
\hline & 2 & 7 & 1.253506 & 0.183838 & 0.554105 \\
\hline & 3 & 7 & -1.311644 & 0.162427 & 0.6363 \\
\hline
\end{tabular}




\begin{tabular}{|c|c|c|c|c|c|}
\hline & 4 & 17 & 2.492425 & 2.727464 & -0.451604 \\
\hline & 5 & 17 & 2.540254 & -2.439701 & 1.249873 \\
\hline & 6 & 17 & -2.65369 & 2.81585 & 0.084305 \\
\hline & 7 & 17 & -2.483599 & -2.579807 & 0.81975 \\
\hline & 8 & 6 & 1.48114 & -0.665654 & -2.329646 \\
\hline & 9 & 8 & -1.364761 & -0.753326 & -2.434959 \\
\hline 1 & 0 & 6 & 0.729946 & 0.536178 & 1.706086 \\
\hline & 1 & 6 & 1.53082 & 0.913169 & 2.909887 \\
\hline 1 & 2 & 1 & 2.59784 & 0.938112 & 2.680849 \\
\hline 1 & 3 & 1 & 1.362302 & 0.186446 & 3.716323 \\
\hline & 4 & 1 & 1.222864 & 1.900528 & 3.27612 \\
\hline 1 & 5 & 6 & -0.749953 & 0.532987 & 1.752634 \\
\hline 1 & 6 & 1 & -1.12362 & 0.34611 & 3.852836 \\
\hline 1 & 7 & 1 & -1.3082 & 1.992586 & 3.214567 \\
\hline 1 & 8 & 1 & -2.554695 & 0.768799 & 2.879771 \\
\hline 1 & 9 & 6 & -1.480216 & 0.93018 & 2.995506 \\
\hline 2 & 20 & 6 & -2.714505 & 0.109526 & 0.425603 \\
\hline & 21 & 6 & -3.445773 & 1.266305 & 0.101396 \\
\hline & 2 & 6 & -4.799022 & 1.179795 & -0.221828 \\
\hline & 3 & 1 & -5.343695 & 2.082595 & -0.470301 \\
\hline 2 & 24 & 6 & -5.433971 & -0.060895 & -0.222709 \\
\hline & 25 & 1 & -6.48739 & -0.124655 & -0.469096 \\
\hline 2 & 6 & 6 & -4.727584 & -1.220065 & 0.096039 \\
\hline 2 & 27 & 1 & -5.219093 & -2.18554 & 0.112199 \\
\hline 2 & 8 & 6 & -3.372461 & -1.133957 & 0.410797 \\
\hline 2 & 29 & 6 & 2.673889 & 0.135662 & 0.368069 \\
\hline 3 & 30 & 6 & 3.358797 & 1.250786 & -0.135348 \\
\hline & 31 & 6 & 3.379917 & -1.04859 & 0.622821 \\
\hline 3 & 32 & 6 & 4.728522 & 1.182361 & -0.382687 \\
\hline 3 & 33 & 1 & 5.243559 & 2.052352 & -0.77148 \\
\hline & 34 & 6 & 5.415736 & -0.003211 & -0.12856 \\
\hline 3 & 35 & 1 & 6.48051 & -0.057671 & -0.323095 \\
\hline 3 & 36 & 6 & 4.749232 & -1.119076 & 0.37502 \\
\hline 3 & 37 & 1 & 5.280429 & -2.041424 & 0.57608 \\
\hline 3 & 38 & 1 & 2.170306 & -1.44529 & -1.976428 \\
\hline 3 & 39 & 1 & 2.052991 & 0.253431 & -2.521688 \\
\hline 4 & 10 & 1 & 1.06301 & -0.998352 & -3.296387 \\
\hline 4 & 11 & 1 & -2.141683 & -1.232688 & -2.091892 \\
\hline 4 & 12 & 1 & -0.967341 & \multirow{2}{*}{$0.1088041^{-1.287477}$} & -3.148016 \\
\hline \multicolumn{2}{|c|}{$\begin{array}{l}\text { Rotational constants [GHz]: } \\
\text { Isotopes: }\end{array}$} & \multicolumn{2}{|c|}{0.2491845} & & $\begin{array}{lrr}0.2491845 & 0.1088041 & 0.1005043 \\
\text { Pt-195,N-14,N-14,Cl-35,Cl-35,Cl-35,Cl-35,C-12,0-16,C-12,C-12,H-1,H-1,H-1, }\end{array}$ \\
\hline \multicolumn{2}{|c|}{ Isotopes: } & \multicolumn{4}{|c|}{$\begin{array}{l}\text { Pt-195,N-14,N-14,Cl-35,Cl-35,Cl-35,Cl-35,C-12,O-16,C-12,C-12,H-1,H-1,H-1, } \\
\text { C-12,H-1,H-1,H-1,C-12,C-12,C-12,C-12,H-1,C-12,H-1,C-12,H-1,C-12,C-12,C-12,C-12, } \\
\text { C-12,H-1,C-12,H-1,C-12,H-1,H-1,H-1,H-1,H-1,H-1 }\end{array}$} \\
\hline \multicolumn{2}{|c|}{ Electronic engergy [Hartree]: } & \multicolumn{4}{|c|}{-2798.64694} \\
\hline \multicolumn{6}{|c|}{ Pt TFE } \\
\hline \multirow[t]{3}{*}{ Center number } & Atomic number & \multicolumn{4}{|c|}{ Coordinates $[\AA]$} \\
\hline & & & $x$ & Y & Z \\
\hline & 1 & 6 & 5.061347 & -0.590008 & -0.761944 \\
\hline
\end{tabular}




\begin{tabular}{|c|c|c|c|c|}
\hline 2 & 6 & 3.734991 & -0.970449 & -0.569338 \\
\hline 3 & 6 & 2.921279 & -0.270753 & 0.332718 \\
\hline 4 & 6 & 3.451188 & 0.818915 & 1.037181 \\
\hline 5 & 6 & 4.777768 & 1.200702 & 0.846297 \\
\hline 6 & 6 & 5.575164 & 0.494734 & -0.052853 \\
\hline 7 & 7 & 1.539796 & -0.62179 & 0.474033 \\
\hline 8 & 6 & 1.156056 & -1.542075 & 1.327804 \\
\hline 9 & 6 & -0.295549 & -1.825264 & 1.334234 \\
\hline 10 & 6 & -0.861385 & -2.898712 & 2.20817 \\
\hline 11 & 17 & 2.445985 & 1.695997 & 2.153993 \\
\hline 12 & 17 & 3.075766 & -2.311901 & -1.463831 \\
\hline 13 & 78 & 0.169753 & 0.278951 & -0.637065 \\
\hline 14 & 8 & -1.364502 & 1.134766 & -1.809291 \\
\hline 15 & 7 & -0.984783 & -1.080271 & 0.514836 \\
\hline 16 & 6 & -2.391665 & -1.188449 & 0.35278 \\
\hline 17 & 6 & -3.279899 & -0.60309 & 1.273598 \\
\hline 18 & 6 & -4.65458 & -0.614664 & 1.037622 \\
\hline 19 & 6 & -5.151928 & -1.215763 & -0.117767 \\
\hline 20 & 6 & -4.288201 & -1.80908 & -1.03742 \\
\hline 21 & 6 & -2.914356 & -1.792197 & -0.804964 \\
\hline 22 & 17 & -2.663672 & 0.153043 & 2.717857 \\
\hline 23 & 17 & -1.835221 & -2.545769 & -1.942293 \\
\hline 24 & 6 & 1.490975 & 1.4378 & -1.71447 \\
\hline 25 & 6 & 2.08616 & -2.287663 & 2.228702 \\
\hline 26 & 1 & 3.090548 & -1.861056 & 2.195787 \\
\hline 27 & 1 & 2.144716 & -3.340698 & 1.91983 \\
\hline 28 & 1 & 1.719485 & -2.258572 & 3.261798 \\
\hline 29 & 1 & -0.308285 & -3.835875 & 2.071794 \\
\hline 30 & 1 & -0.782713 & -2.608796 & 3.264725 \\
\hline 31 & 1 & -1.91447 & -3.071784 & 1.97389 \\
\hline 32 & 1 & -5.322038 & -0.161824 & 1.760953 \\
\hline 33 & 1 & -6.220587 & -1.229924 & -0.29773 \\
\hline 34 & 1 & -4.670465 & -2.290376 & -1.929579 \\
\hline 35 & 1 & 5.173323 & 2.044306 & 1.398718 \\
\hline 36 & 1 & 6.606235 & 0.792908 & -0.203083 \\
\hline 37 & 1 & 5.677456 & -1.139248 & -1.463421 \\
\hline 38 & 1 & 2.246125 & 0.792177 & -2.180196 \\
\hline 39 & 1 & 1.986755 & 2.152993 & -1.044827 \\
\hline 40 & 1 & 0.999843 & 2.012994 & -2.517992 \\
\hline 41 & 6 & -2.357214 & 1.952182 & -1.161675 \\
\hline 42 & 1 & -0.934115 & 1.643874 & -2.525019 \\
\hline 43 & 6 & -1.726651 & 3.076518 & -0.369889 \\
\hline 44 & 1 & -3.016293 & 2.377708 & -1.923539 \\
\hline 45 & 1 & -2.923 & 1.297236 & -0.500412 \\
\hline 46 & 9 & -0.851429 & 3.778661 & -1.162452 \\
\hline 47 & 9 & -2.706151 & 3.9063 & 0.081807 \\
\hline 48 & 9 & -1.014852 & 2.612001 & 0.717485 \\
\hline Rotational constants [GHz]: & \multicolumn{2}{|c|}{0.1629126} & 0.0962928 & \\
\hline Isotopes: & \multicolumn{4}{|c|}{$\begin{array}{l}\mathrm{C}-12, \mathrm{C}-12, \mathrm{C}-12, \mathrm{C}-12, \mathrm{C}-12, \mathrm{C}-12, \mathrm{~N}-14, \mathrm{C}-12, \mathrm{C}-12, \mathrm{C}-12, \mathrm{Cl}-35, \mathrm{Cl}-35, \mathrm{Pt}-195, \mathrm{O}-16, \\
\mathrm{~N}-14, \mathrm{C}-12, \mathrm{C}-12, \mathrm{C}-12, \mathrm{C}-12, \mathrm{C}-12, \mathrm{C}-12, \mathrm{Cl}-35, \mathrm{Cl}-35, \mathrm{C}-12, \mathrm{C}-12, \mathrm{H}-1, \mathrm{H}-1, \mathrm{H}-1, \mathrm{H}-1, \mathrm{H}-1,\end{array}$} \\
\hline
\end{tabular}


Electronic engergy [Hartree]:

$\mathrm{H}-1, \mathrm{H}-1, \mathrm{H}-1, \mathrm{H}-1, \mathrm{H}-1, \mathrm{H}-1, \mathrm{H}-1, \mathrm{H}-1, \mathrm{H}-1, \mathrm{H}-1, \mathrm{C}-12, \mathrm{H}-1, \mathrm{C}-12, \mathrm{H}-1, \mathrm{H}-1, \mathrm{~F}-19, \mathrm{~F}-19, \mathrm{~F}-19$

$-3172.82797$

$\mathrm{PtCH} 3 \mathrm{CN}$

Standard orientation:

Center number Atomic number Coordinates $[\AA]$

\begin{tabular}{|c|c|c|c|c|}
\hline & & $X$ & Y & Z \\
\hline 1 & 6 & -4.877631 & 0.456901 & 1.126148 \\
\hline 2 & 6 & -3.534177 & 0.094431 & 1.201573 \\
\hline 3 & 6 & -2.840898 & -0.303245 & 0.04996 \\
\hline 4 & 6 & -3.51376 & -0.334224 & -1.179464 \\
\hline 5 & 6 & -4.857273 & 0.027232 & -1.2577 \\
\hline 6 & 6 & -5.531778 & 0.422664 & -0.103985 \\
\hline 7 & 7 & -1.442082 & -0.607052 & 0.115225 \\
\hline 8 & 6 & -1.026476 & -1.829461 & 0.339492 \\
\hline 9 & 6 & 0.446744 & -2.001379 & 0.372553 \\
\hline 10 & 6 & 1.051325 & -3.349184 & 0.609753 \\
\hline 11 & 17 & -2.663813 & -0.826949 & -2.617682 \\
\hline 12 & 17 & -2.709944 & 0.135473 & 2.735463 \\
\hline 13 & 78 & -0.065313 & 0.839753 & -0.139006 \\
\hline 14 & 7 & 1.354643 & 2.172529 & -0.372016 \\
\hline 15 & 7 & 1.117786 & -0.903046 & 0.17452 \\
\hline 16 & 6 & 2.539353 & -0.85236 & 0.148266 \\
\hline 17 & 6 & 3.235271 & -1.026109 & -1.059029 \\
\hline 18 & 6 & 4.620574 & -0.881435 & -1.10574 \\
\hline 19 & 6 & 5.319902 & -0.557574 & 0.055905 \\
\hline 20 & 6 & 4.645747 & -0.377141 & 1.262698 \\
\hline 21 & 6 & 3.26012 & -0.52042 & 1.306521 \\
\hline 22 & 17 & 2.35872 & -1.426233 & -2.510686 \\
\hline 23 & 17 & 2.412601 & -0.292281 & 2.811274 \\
\hline 24 & 6 & -1.429965 & 2.366932 & -0.4163 \\
\hline 25 & 6 & -1.929373 & -3.001406 & 0.548215 \\
\hline 26 & 1 & -2.977857 & -2.707733 & 0.471285 \\
\hline 27 & 1 & -1.757115 & -3.438684 & 1.540592 \\
\hline 28 & 1 & -1.72249 & -3.776169 & -0.201375 \\
\hline 29 & 1 & 0.697568 & -3.76535 & 1.561499 \\
\hline 30 & 1 & 0.761793 & -4.042467 & -0.190918 \\
\hline 31 & 1 & 2.14123 & -3.28237 & 0.637961 \\
\hline 32 & 1 & 5.139306 & -1.030283 & -2.045123 \\
\hline 33 & 1 & 6.398348 & -0.45425 & 0.022338 \\
\hline 34 & 1 & 5.184227 & -0.134681 & 2.170969 \\
\hline 35 & 1 & -5.361817 & -0.001524 & -2.215778 \\
\hline 36 & 1 & -6.576039 & 0.706308 & -0.163968 \\
\hline 37 & 1 & -5.398119 & 0.76267 & 2.025516 \\
\hline 38 & 1 & -2.063388 & 2.437802 & 0.477775 \\
\hline 39 & 1 & -2.052184 & 2.128015 & -1.288848 \\
\hline 40 & 1 & -0.933504 & 3.329056 & -0.581916 \\
\hline 41 & 6 & 2.19484 & 2.963158 & -0.509094 \\
\hline 42 & 6 & 3.24411 & 3.950014 & -0.679975 \\
\hline 43 & 1 & 3.148604 & 4.733714 & 0.081436 \\
\hline
\end{tabular}




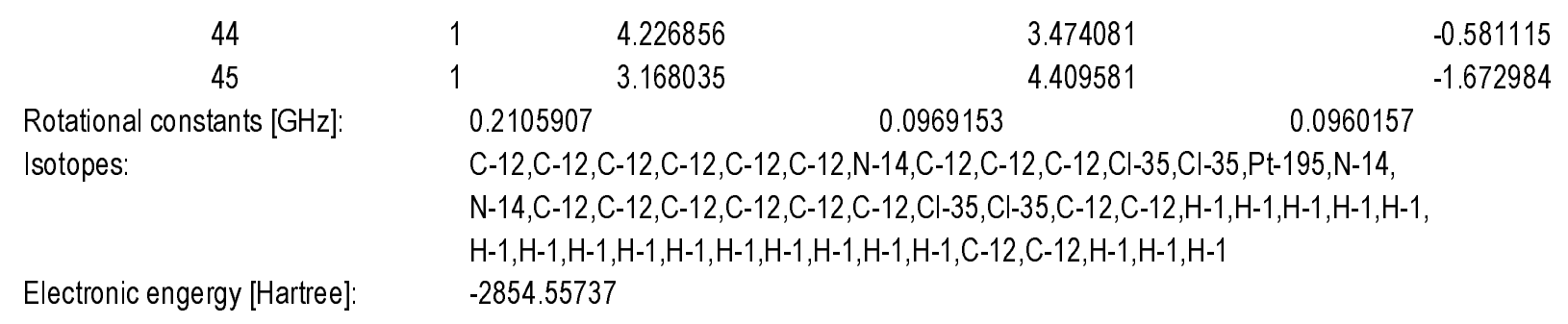




\begin{tabular}{|c|c|c|c|c|c|c|c|c|c|c|}
\hline \multicolumn{11}{|l|}{ Water } \\
\hline \multicolumn{2}{|c|}{ Frequencies } & \multicolumn{3}{|l|}{1709.58} & \multicolumn{3}{|l|}{3774.30} & \multicolumn{3}{|l|}{3896.47} \\
\hline Atom & AN & X & $\mathrm{Y}$ & Z & X & Y & Z & $x$ & $\mathrm{Y}$ & Z \\
\hline 1 & 8 & 0.00 & 0.07 & 0.00 & 0.00 & 0.05 & 0.00 & 0.07 & 0.00 & 0.00 \\
\hline 2 & 1 & -0.42 & -0.56 & 0.00 & 0.59 & -0.39 & 0.00 & -0.56 & 0.43 & 0.00 \\
\hline 3 & 1 & 0.42 & -0.56 & 0.00 & -0.5 & -0.39 & 0.00 & -0. & & 0.00 \\
\hline \multicolumn{11}{|l|}{ TFE } \\
\hline \multicolumn{2}{|c|}{ Frequencies } & 98.19 & & & 165.95 & & & 209.69 & & \\
\hline Atom & AN & X & $\mathrm{Y}$ & Z & X & $Y$ & Z & X & $\mathrm{Y}$ & Z \\
\hline 1 & 6 & 0.00 & 0.00 & -0.06 & 0.00 & 0.00 & -0.02 & 0.00 & 0.15 & 0.00 \\
\hline 2 & 9 & 0.16 & -0.03 & -0.07 & 0.04 & & -0.01 & 0.25 & & 0.02 \\
\hline 3 & 6 & 0.00 & 0.00 & -0.19 & 0.00 & 0. & -0.04 & 0. & & 0.00 \\
\hline 4 & 8 & 0.00 & 0.00 & 0.34 & 0.00 & 0.00 & -0.01 & $-0.3^{2}+3$ & -0 . & 0.00 \\
\hline 5 & 9 & 0.00 & 0.00 & 0.08 & 0.00 & 0.00 & 0.01 & -0.24 & -0.14 & 0.00 \\
\hline 6 & 9 & -0.16 & 0.03 & -0.07 & -0.04 & $-0 .(1-x-y$ & -0.01 & 0.2 & & -0.02 \\
\hline 7 & 1 & 0.11 & -0.34 & -0.43 & 0.0 & -0.1 & -0.05 & 0.20 & & -0.01 \\
\hline 8 & 1 & -0.11 & 0.34 & -0.43 & -0.02 & 0.01 & -0.05 & 0.2 & & 0.01 \\
\hline 9 & 1 & 0.00 & 0.00 & -0.39 & & 0.00 & 0.99 & & -0.65 & 0.00 \\
\hline \multicolumn{2}{|c|}{ Frequencies } & 335.73 & & & 405.80 & & & 507.91 & & \\
\hline Atom & AN & X & $\mathrm{Y}$ & Z & X & Y & Z & $X$ & $\mathrm{Y}$ & 2 \\
\hline 1 & 6 & 0.00 & 0.00 & -0.08 & -0.01 & 0.01 & 0.00 & 0.00 & 0.00 & -0.26 \\
\hline 2 & 9 & 0.19 & 0.05 & -0.01 & & 0.24 & 0.16 & -0.0 & & -0.14 \\
\hline 3 & 6 & 0.00 & 0.00 & 0.22 & 0.2 & -0.17 & 0.00 & 0.00 & & 0.03 \\
\hline 4 & 8 & 0.00 & 0.00 & 0.02 & & -0.05 & 0.00 & 0.00 & & 0.04 \\
\hline 5 & 9 & 0.00 & 0.00 & -0.13 & -0.2 & -0.32 & 0.0 & 0. & & 0.37 \\
\hline 6 & 9 & -0.19 & -0.05 & -0.01 & -0.1 & 0.24 & -0.16 & 0.1 & -0 & -0.14 \\
\hline 7 & 1 & 0.15 & 0.38 & 0.50 & 0.2 & -0.16 & 0.00 & 0.26 & & 0.29 \\
\hline 8 & 1 & -0.15 & -0.38 & 0.5 & 0.2 & -0 . & 0. & -0 . & & 0. \\
\hline 9 & 1 & 0.00 & 0.00 & -0.13 & 0.3 & 0.19 & 0.00 & 0.0 & 0.00 & -0.0 \\
\hline \multicolumn{2}{|c|}{ Frequencies } & 527.33 & & & 629.72 & & & 817.73 & & \\
\hline Atom & AN & X & $\mathrm{Y}$ & Z & $X$ & Y & Z & X & Y & Z \\
\hline 1 & 6 & 0.00 & -0.24 & 0.00 & -0.26 & 0.01 & 0.00 & -0.0 & -0 . & 0.00 \\
\hline 2 & 9 & 0. & 0.13 & 0.34 & & -0. & 0. & & & \\
\hline 3 & 6 & & -0.16 & 0.00 & & -0 . & 0. & & & 0.00 \\
\hline 4 & 8 & -0.15 & 0.06 & 0.00 & 0.2 & -0.04 & 0.00 & -0.01 & 0. & 0.00 \\
\hline 5 & 9 & 0.25 & -0.04 & 0.00 & -0. & $0.2^{2}$ & 0. & -0. & & \\
\hline 6 & 9 & & 0.13 & -0.34 & & & & -0 . & & \\
\hline 7 & 1 & -0.42 & -0.16 & 0.00 & -0 . & -0 & 0.1 & 0. & & -0.02 \\
\hline 8 & 1 & & -0.16 & 0.00 & & -0. & -0. & & & 0.0 \\
\hline 9 & 1 & -0.23 & 0.18 & 0.00 & -0.08 & 0.48 & 0.00 & & -0.47 & 0.00 \\
\hline \multicolumn{2}{|c|}{ Frequencies } & 1017.3 & & & 1117.94 & & & & & \\
\hline Atom & AN & $x$ & Y & Z & $x$ & Y & Z & $x$ & 1 & L \\
\hline 1 & 6 & 0.00 & 0.00 & 0.09 & -0.06 & -0.07 & 0.00 & -0.18 & & 0.00 \\
\hline 2 & 9 & & & -0.07 & & & & & & \\
\hline 3 & 6 & & 0.00 & 0.2 & & 0. & 0.0 & -0 & & 0. \\
\hline 4 & 8 & 0. & 0.00 & -0.04 & & -0.30 & 0.00 & 0. & & 0.00 \\
\hline 5 & 9 & & & 0.00 & & & & & & \\
\hline 6 & 9 & -0. & -0. & -0.07 & & 0. & 0. & 0. & & -0.04 \\
\hline 7 & 1 & -0.2 & -0.5 & -0.2 & & 0. & -0 . & 0. & & \\
\hline 8 & 1 & & 0.5 & -0.22 & -0 . & & & & & \\
\hline 9 & 1 & 0.00 & 0.00 & 0.02 & 0.1 & -0.01 & 0.00 & & 0.64 & 0.0 \\
\hline \multicolumn{2}{|c|}{ Frequencies } & 1210.9 & & & & & & & & \\
\hline Atom & AN & $X$ & Y & Z & $X$ & $Y$ & $Z$ & $x$ & & \\
\hline & 6 & 0.00 & 0.00 & 0.19 & & 0.08 & 0.0 & & & \\
\hline 2 & 9 & 0.01 & 0.03 & -0.06 & & -0 & 0. & & & \\
\hline 3 & 6 & 0.00 & 0.00 & -0.09 & -0.07 & 0.00 & 0.00 & -0.04 & -0. & 0.00 \\
\hline 4 & 8 & 0.00 & 0.00 & 0.05 & & -0.08 & 0.0 & & & 0.00 \\
\hline 5 & 9 & 0.00 & 0.00 & -0.01 & -0.09 & 0.05 & 0.00 & 0.01 & -0.03 & 0.00 \\
\hline 6 & 9 & -0.01 & -0.03 & -0.05 & -0.03 & -0.04 & -0.04 & -0.01 & -0.04 & -0.0 \\
\hline
\end{tabular}




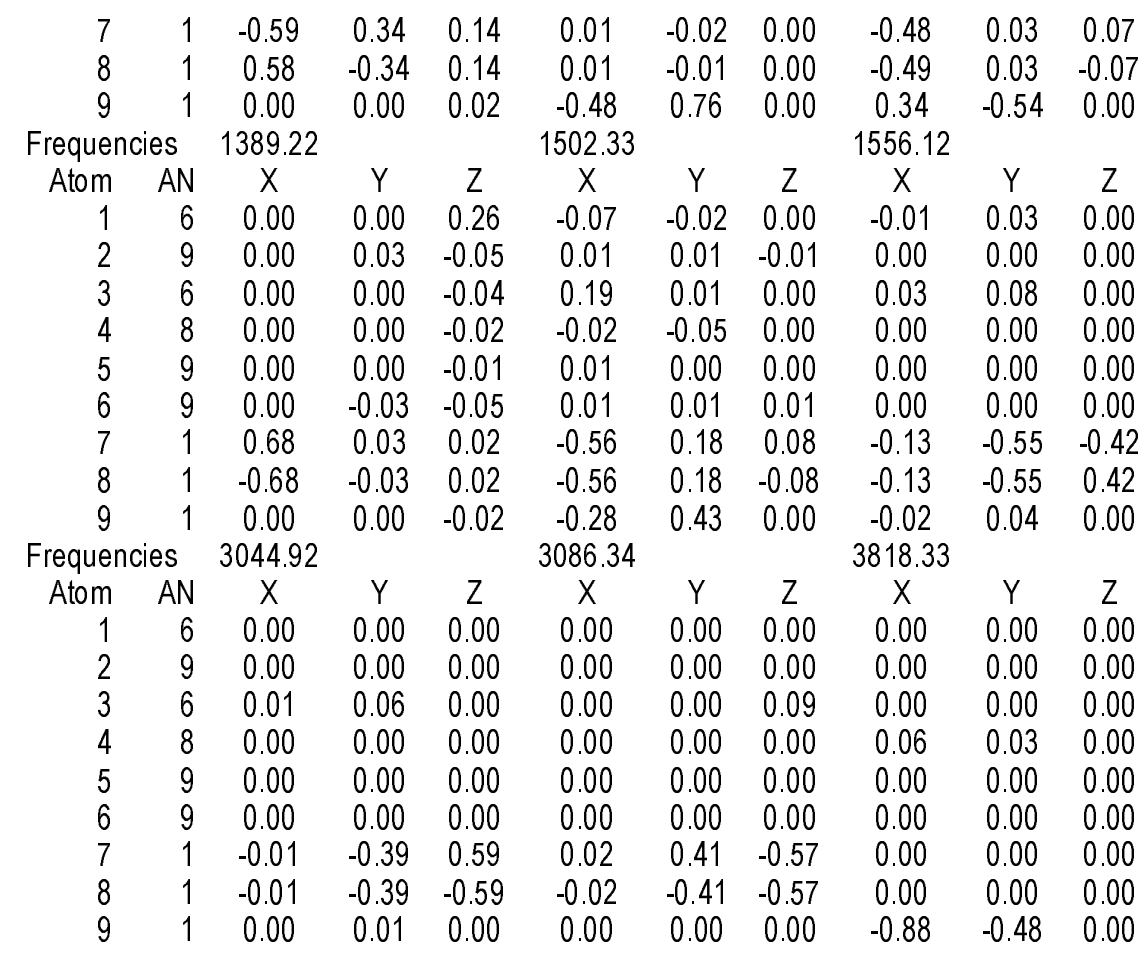

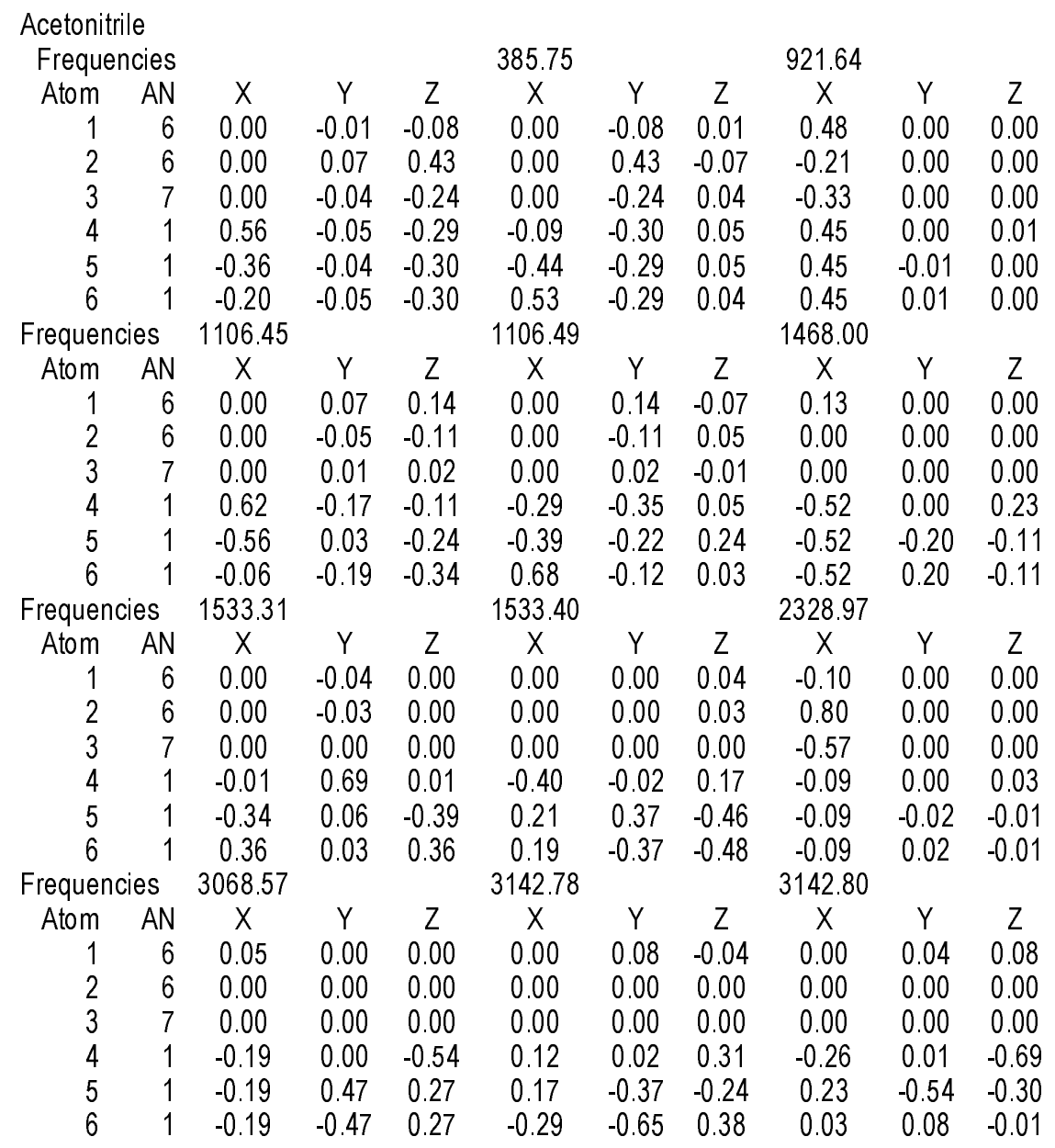




\begin{tabular}{|c|c|c|c|c|c|c|c|c|c|c|}
\hline \multicolumn{11}{|c|}{ ML3 Fragment } \\
\hline Atom & AN & $x$ & Y & 7 & $X$ & $Y$ & 7 & $x$ & Y & Z \\
\hline 1 & 78 & 0.00 & -0.02 & -0.09 & 0.00 & 0.01 & 0.03 & -0.02 & -0.03 & 0.03 \\
\hline 2 & 7 & 0.00 & -0.01 & -0.03 & 0.00 & 0.02 & 0.07 & 0.00 & -0.04 & 0.01 \\
\hline 3 & 7 & 0.00 & 0.00 & -0.01 & 0.00 & 0.02 & 0.03 & 0.01 & -0.02 & -0.02 \\
\hline 4 & 17 & -0.10 & 0.30 & -0.04 & 0.17 & 0.15 & 0.03 & 0.00 & -0.06 & 0.01 \\
\hline 5 & 17 & 0.07 & -0.24 & 0.08 & -0.15 & -0.14 & 0.01 & 0.00 & -0.03 & 0.00 \\
\hline 6 & 17 & -0.01 & 0.00 & -0.13 & 0.02 & 0.00 & -0.07 & -0. & 0.00 & -0.11 \\
\hline 7 & 17 & 0.07 & -0.03 & 0.18 & -0.08 & -0.05 & 0.05 & 0.1 & -0.02 & 0.11 \\
\hline 8 & 6 & 0.00 & -0.03 & -0.13 & 0.00 & 0.02 & 0.0 & $-0 .(1-x-1$ & -0.03 & 0.13 \\
\hline 9 & 1 & -0.08 & -0.0 & -0.19 & -0.05 & -0.06 & 0.0 & -0 & -0.08 & 0.14 \\
\hline 10 & 1 & 0.08 & 0.00 & -0.20 & 0.06 & 0.08 & 0.01 & -0.04 & 0.02 & 0.12 \\
\hline 11 & 1 & -0.01 & -0.0 & -0.0 & -0.01 & 0.0 & 0.1 & -0.04 & -0.02 & 0.17 \\
\hline 12 & 6 & -0.01 & 0.0 & 0.0 & 0.0 & 0. & 0.0 & & & 0.00 \\
\hline 13 & 6 & 0.02 & -0. & 0.0 & -0.05 & -0.08 & -0.03 & & 0. & -0.01 \\
\hline 14 & 6 & -0.06 & 0.1 & 0.0 & 0.09 & 0.05 & -0.8 & 0.1 & 0.04 & -0.01 \\
\hline 15 & 6 & -0.0 & 0.1 & 0. & & 0.02 & -0 . & & 0.19 & -0.03 \\
\hline 16 & 1 & -0.11 & 0.25 & 0.1 & 0.1 & 0.05 & -0 . & 0. & 0.23 & -0.04 \\
\hline 17 & 6 & -0.04 & 0.0 & 0.1 & 0.03 & -0.06 & -0.12 & 0.0 & 0.27 & -0.04 \\
\hline 18 & 1 & -0.0 & & 0.2 & 0.0 & -0. & -0 . & & 0.39 & -0.06 \\
\hline 19 & 6 & 0.00 & & 0. & -0.04 & -0 . & -0 . & & 0. & -0.03 \\
\hline 20 & 1 & 0.03 & -0.1 & 0.2 & -0.09 & -0.17 & -0 . & & 0.26 & -0.04 \\
\hline 21 & 6 & -0.01 & & 0.0 & 0.0 & 0. & 0.1 & & & -0.04 \\
\hline 22 & 6 & -0.0 & & 0. & & & 0. & & & -0.12 \\
\hline 23 & 1 & 0.03 & & $-0 .($ & 0.0 & 0. & 0.1 & & & \\
\hline 24 & 1 & & 0. & 0. & & & 0.1 & & & \\
\hline 25 & 1 & -0 . & 0.0 & 0.0 & 0. & 0. & 0.1 & & -0 & -0 . \\
\hline 26 & 6 & -0.01 & 0. & 0. & 0.0 & 0.0 & 0.0 & 0. & -0 . & -0.03 \\
\hline 27 & 6 & -0.0 & & 0. & & & & & & \\
\hline 28 & 1 & -0 . & 0.0 & 0. & & 0. & 0.1 & & & 0.00 \\
\hline 2 & 1 & & & 0. & & 0. & 0.1 & & -0.02 & \\
\hline 30 & 1 & -0.04 & 0.07 & 0. & 0.03 & 0. & 0. & 0. & -0.01 & 0.00 \\
\hline 31 & 6 & 0.0 & & 0.0 & & -0 & & & & -0 \\
\hline 32 & 6 & 0.0 & & 0. & -0.03 & -0. & -0 . & & 0. & 0.0 \\
\hline 33 & 6 & 0.00 & 0.0 & 0. & 0.01 & -0.01 & -0.11 & -0. & 0.02 & -0.08 \\
\hline 34 & 6 & $0 . C$ & & 0. & 0.00 & -0 & & & & -0.11 \\
\hline 3 & 1 & -0.0 & 0. & 0. & & -0. & -0.2 & -0 & 0. & -0.15 \\
\hline 36 & 6 & 0. & & 0. & -0.02 & -0. & -0 . & & & -0.07 \\
\hline 37 & 1 & 0.0 & & & -0.02 & -0 . & & & & -0.09 \\
\hline 38 & 6 & 0.0 & -0. & 0.2 & -0.04 & -0 . & -0 . & & 0.06 & -0.0 \\
\hline 39 & 1 & & -0.01 & 0.28 & -0.06 & -0.06 & -0.19 & 0.04 & 0.07 & 0.03 \\
\hline Frequer & & & & & & & & & & \\
\hline Atom & AN & $x$ & $Y$ & Z & $x$ & $Y$ & Z & $X$ & $Y$ & 2 \\
\hline & 78 & & -0.04 & -0.0 & & 0.0 & 0.0 & -0. & -0.04 & 0.02 \\
\hline 2 & 7 & & & -0. & & & -0 . & & & 0.01 \\
\hline 3 & 7 & & & 0.0 & & 0. & & & & -0.02 \\
\hline 4 & 17 & & 0.0 & -0.02 & -0.10 & 0.01 & -0.1 & 0. & 0.18 & -0.01 \\
\hline 5 & 17 & & -0.02 & 0.0 & 0.10 & 0.03 & -0.06 & 0.1 & 0.22 & -0.02 \\
\hline 6 & 17 & & & & & & & & & -0.03 \\
\hline 7 & 17 & & & & & -0 . & 0.2 & & & \\
\hline 8 & 6 & & -0. & -0.2 & 0.0 & 0.1 & 0.0 & -0. & -0 & 0.04 \\
\hline 9 & 1 & & & -0. & & & & & & 0.06 \\
\hline 10 & 1 & & -0 . & -0. & & & 0. & & & \\
\hline 11 & 1 & -0.1 & $-0 .($ & -0. & 0.0 & 0. & 0. & -0.01 & -0.05 & 0.00 \\
\hline 12 & 6 & & -0.0 & -0. & & 0.1 & -0. & & & 0.01 \\
\hline 13 & 6 & & 0.0 & 0.0 & & -0 . & -0 . & & 0. & 0.00 \\
\hline 14 & 6 & 0.0 & 0.0 & -0.0 & -0. & -0 . & -0 . & & 0.02 & \\
\hline 15 & 6 & 0.0 & 0.1 & -0.0 & -0.08 & -0.11 & 0.01 & 0.0 & -0.05 & 0.00 \\
\hline 16 & 1 & 0.02 & 0.17 & -0.01 & -0.12 & -0.14 & 0.03 & 0.01 & -0.06 & 0.00 \\
\hline
\end{tabular}




\begin{tabular}{|c|c|c|c|c|c|c|c|c|c|c|}
\hline & & & & & & & & & & \\
\hline 17 & 6 & 0.04 & 0.16 & -0.01 & -0.05 & -0.15 & 0.05 & -0.02 & -0.11 & 0.00 \\
\hline 18 & 1 & 0.05 & 0.23 & 0.00 & -0.07 & -0.21 & 0.10 & -0.03 & -0.17 & 0.00 \\
\hline 19 & 6 & 0.05 & 0.10 & 0.00 & 0.01 & -0.10 & 0.03 & -0.02 & -0.04 & -0.01 \\
\hline 2 & 1 & 0.06 & 0.14 & 0.00 & 0.03 & -0.12 & 0.06 & -0.03 & -0.03 & -0.01 \\
\hline 2 & 6 & 0.01 & -0.02 & 0.06 & -0.01 & 0.00 & -0.10 & -0.02 & -0.03 & 0.00 \\
\hline 2 & 6 & -0.01 & 0.02 & 0.15 & 0.00 & -0.03 & -0.23 & -0.07 & & 0.08 \\
\hline 23 & 1 & 0.03 & -0.05 & 0.04 & -0.02 & 0.02 & -0.17 & 0.06 & -0.11 & -0.23 \\
\hline 24 & 1 & 0.03 & -0.04 & 0.34 & -0.01 & 0.02 & -0.39 & 0.05 & -0.07 & 0.48 \\
\hline 25 & 1 & -0.13 & 0.17 & 0.16 & 0.07 & -0.19 & -0.24 & -0.42 & 0.31 & 0.08 \\
\hline 26 & 6 & 0.01 & -0.01 & 0.07 & -0.02 & 0.01 & -0.01 & -0.01 & -0.06 & -0.04 \\
\hline 27 & 6 & 0.02 & -0.01 & 0.06 & -0.09 & 0.03 & 0.07 & -0.01 & -0.12 & -0.10 \\
\hline 28 & 1 & 0.02 & -0.03 & 0.06 & -0.13 & 0.12 & 0.06 & -0.05 & -0.22 & -0.13 \\
\hline 29 & 1 & 0.02 & -0.01 & 0.05 & -0.12 & 0.00 & 0.14 & 0.04 & -0.07 & -0.19 \\
\hline 30 & 1 & 0.02 & & 0.06 & -0.09 & 0.00 & 0.09 & -0.03 & -0.11 & -0.03 \\
\hline 31 & 6 & 0.00 & 0.02 & 0.06 & 0.01 & 0.00 & 0.03 & 0.00 & -0.02 & -0.02 \\
\hline 32 & 6 & -0.01 & 0.0 & -0.02 & 0.00 & -0.01 & 0.02 & 0.04 & 0.01 & -0.01 \\
\hline 33 & 6 & -0.02 & & 0.07 & 0.01 & 0.00 & -0.02 & -0.03 & -0.01 & -0.04 \\
\hline 34 & 6 & -0.04 & 0.05 & -0.04 & 0.00 & 0.00 & -0.15 & -0.05 & 0.04 & -0.05 \\
\hline 35 & 1 & -0.06 & 0.05 & -0.04 & 0.00 & 0.00 & -0.20 & -0.09 & 0.05 & -0.06 \\
\hline 36 & 6 & -0.04 & & -0.15 & -0.01 & & -0.22 & -0.02 & .07 & -0.04 \\
\hline & 1 & -0.05 & & -0.24 & -0.02 & 0.01 & -0.34 & -0.03 & 0.11 & -0.05 \\
\hline 38 & 6 & -0.03 & & -0.14 & -0.02 & -0.01 & -0.11 & 0.03 & 0.06 & -0.01 \\
\hline 39 & 1 & -0.04 & 0.08 & -0.21 & -0.03 & -0.02 & -0.13 & 0.05 & 0.08 & 0.00 \\
\hline quer & & 87.71 & & & 93.50 & & & 99.09 & & \\
\hline tom & AN & $X$ & $Y$ & Z & $x$ & $Y$ & Z & $x$ & $Y$ & Z \\
\hline 1 & 78 & 0.00 & 0.01 & 0.00 & 0.01 & 0.01 & 0.01 & -0.02 & -0.02 & 0.03 \\
\hline 2 & 7 & & & -0.02 & 0.00 & 0.01 & -0.01 & 0.03 & -0.06 & -0.04 \\
\hline 3 & 7 & 0.00 & 0.00 & -0.03 & 0.00 & 0.00 & 0.00 & -0.01 & 0.02 & 0.01 \\
\hline 4 & 17 & -0.03 & -0.04 & -0.02 & -0.03 & -0.05 & -0.01 & 0.0 & 0.04 & -0.11 \\
\hline 5 & 17 & 0.0 & -0.05 & -0.01 & -0.01 & -0.03 & 0.00 & 0.19 & 0.04 & -0.08 \\
\hline 6 & 17 & 0.01 & 0.00 & 0.01 & 0.01 & 0.00 & -0.01 & -0.02 & 0.02 & -0.08 \\
\hline 7 & 17 & 0.02 & -0.01 & 0.02 & 0.03 & -0.01 & 0.01 & -0.14 & 0.03 & -0.04 \\
\hline 8 & 6 & 0.0 & 0.03 & 0.1 & & 0.01 & -0.01 & -0. & -0.04 & 0.15 \\
\hline$a$ & 1 & & & & -0 & 29 & -0.2 & -0 . & -0.24 & 0.07 \\
\hline 10 & 1 & & & 0. & & 0.25 & -0 . & & 0.12 & 0.03 \\
\hline 11 & 1 & 0.0 & -0.02 & -0.27 & -0.0 & 0.06 & 0.45 & -0.1 & 0.00 & 0.40 \\
\hline 12 & 6 & 0. & & -0.01 & & & -0.0 & 0. & -0.06 & -0.06 \\
\hline 13 & 6 & & & -0.01 & & & -0.0 & & -0.02 & -0.04 \\
\hline 14 & 6 & -0.0 & 0.00 & -0.0 & -0. & 0.00 & -0. & 0. & -0.02 & -0.06 \\
\hline 15 & 6 & & & -0.01 & & & 0. & 0.0 & 0.01 & -0.02 \\
\hline 16 & 1 & -0. & & -0.01 & & & 0. & 0. & 0.04 & -0.02 \\
\hline 17 & 6 & & & -0 . & & 0.0 & 0. & & 0.02 & 0.00 \\
\hline 18 & 1 & 0. & 0.04 & 0.00 & -0.01 & 0.02 & 0.0 & 0.0 & 0.04 & 0.03 \\
\hline 19 & 6 & 0.00 & & -0.01 & -0.01 & 0.00 & 0.0 & 0. & 0.01 & -0.01 \\
\hline 20 & 1 & & & 0.0 & & & & & & 0.01 \\
\hline 21 & 6 & & & & & 0. & -0 . & 0. & -0.03 & 0.02 \\
\hline 22 & 6 & -0.03 & & 0.06 & -0.1 & 0.04 & 0.0 & 0.0 & -0.02 & 0.07 \\
\hline 23 & 1 & 0.0 & & -0.20 & & & -0 . & & & -0.15 \\
\hline 24 & 1 & & & 0. & & -0. & 0. & & -0.07 & \\
\hline 25 & 1 & & & 0.06 & -0.29 & 0.22 & 0. & -0.1 & 0.18 & 0.07 \\
\hline 26 & 6 & & & -0.0 & & & -0. & & & 0.06 \\
\hline 27 & 6 & 0. & & 0. & -0. & -0.03 & -0. & 0. & 0.12 & 0.14 \\
\hline 28 & 1 & -0. & & -0.02 & -0 . & -0.07 & -0.0 & 0.06 & 0.17 & 0.15 \\
\hline 29 & 1 & & & -0. & & -0.01 & -0. & 0. & 0.08 & 0.22 \\
\hline 30 & 1 & -0. & & 0.0 & & -0.02 & 0.0 & & 0.19 & 0.12 \\
\hline 31 & 6 & 0.0 & 0.00 & -0.02 & 0. & 0.00 & 0.0 & -0.02 & 0.02 & 0.01 \\
\hline SL & 6 & 0.0 & & 0. & 0.0 & 0.00 & 0.00 & -0.05 & 0.00 & 0.00 \\
\hline 33 & 6 & 0.0 & & -0.0 & & 0.00 & -0.01 & $-0 .($ & 0.02 & 0.00 \\
\hline 34 & 6 & & 0.0 & -0.01 & 0.0 & 0.00 & -0.02 & 0.0 & -0.01 & 0.03 \\
\hline 35 & 1 & 0.00 & 0.00 & 0.00 & 0.00 & 0.00 & -0.02 & 0.02 & -0.02 & 0.04 \\
\hline
\end{tabular}




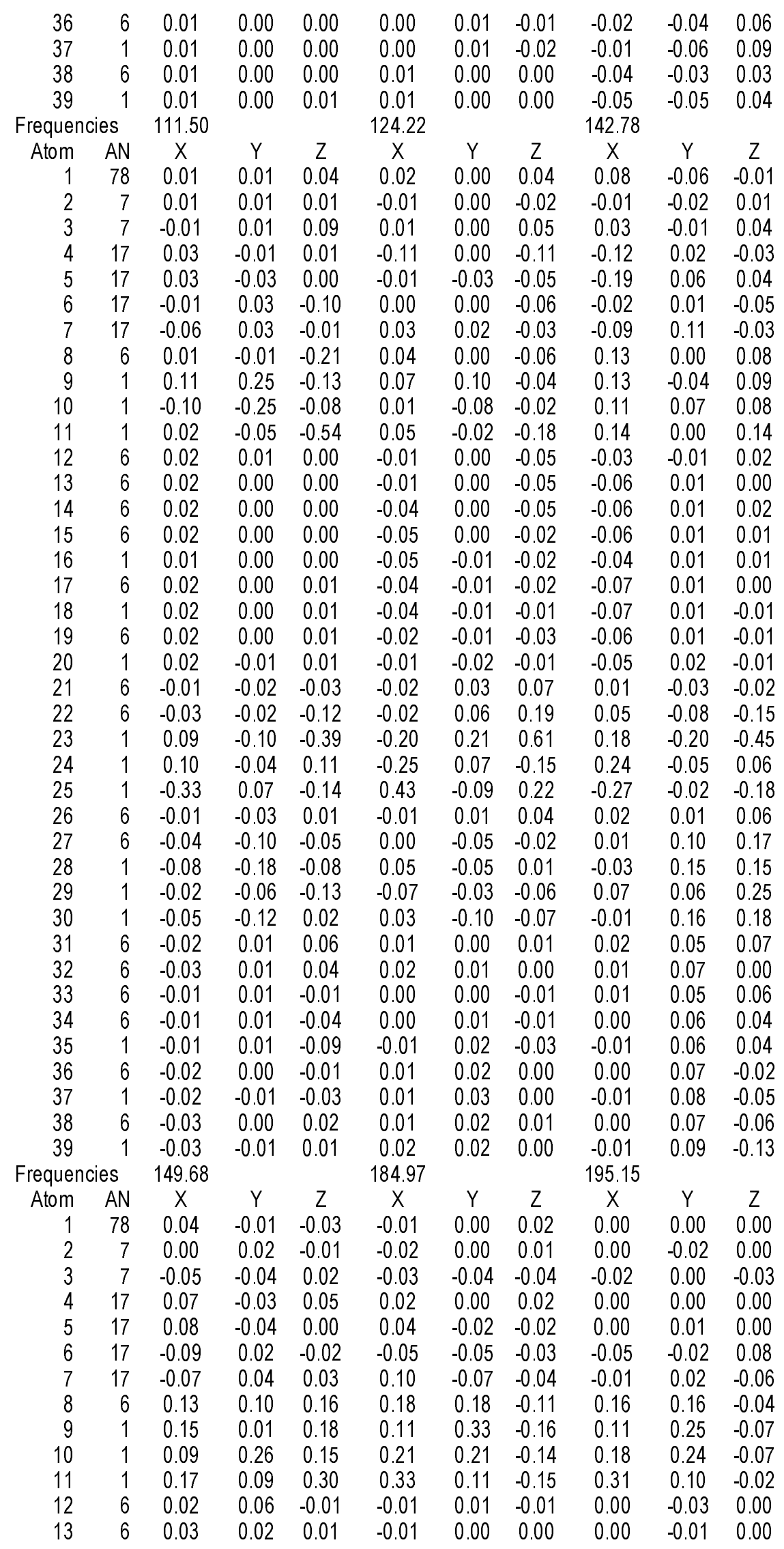




\begin{tabular}{|c|c|c|c|c|c|c|c|c|c|c|}
\hline & & & & & & & & & & \\
\hline 14 & 6 & 0.03 & 0.02 & 0.00 & 0.00 & 0.00 & -0.01 & 0.00 & -0.01 & 0.00 \\
\hline 15 & 6 & 0.02 & -0.02 & 0.00 & 0.00 & 0.00 & -0.01 & 0.00 & 0.00 & 0.00 \\
\hline 16 & 1 & 0.01 & -0.05 & 0.01 & -0.01 & 0.00 & 0.00 & 0.00 & 0.01 & 0.00 \\
\hline 17 & 6 & 0.03 & -0.04 & 0.01 & 0.00 & -0.01 & 0.00 & 0.00 & 0.00 & 0.00 \\
\hline 18 & 1 & 0.02 & -0.09 & 0.02 & 0.00 & -0.01 & 0.00 & 0.00 & 0.02 & 0.00 \\
\hline 19 & 6 & 0.02 & -0.02 & 0.02 & -0.01 & 0.00 & & & 0.00 & \\
\hline 20 & 1 & 0.01 & -0.05 & 0.02 & -0.01 & -0.01 & 0.00 & & 0.00 & .00 \\
\hline 21 & 6 & -0.06 & & 0.00 & -0.03 & 0.00 & 0.02 & 0.01 & -0.02 & 0.00 \\
\hline 22 & 6 & -0.14 & 0.06 & 0.05 & -0.02 & -0.02 & -0.04 & 0.04 & -0.04 & 0.00 \\
\hline 23 & 1 & -0.23 & & 0.2 & 0.01 & -0.04 & -0.10 & 0.05 & -0.08 & -0.02 \\
\hline 24 & 1 & -0.31 & 0.03 & -0.15 & 0.03 & 0.00 & -0.01 & 0.08 & -0.03 & 0.02 \\
\hline 25 & 1 & 0.08 & -0.03 & 0.06 & -0.09 & -0.03 & -0.05 & 0.02 & -0.03 & 0.00 \\
\hline 26 & 6 & -0.07 & -0.05 & -0.01 & -0.03 & 0.01 & 0.03 & 0.01 & 0.00 & 0.00 \\
\hline 27 & 6 & -0.12 & -0.12 & -0.05 & 0.0 & 0.13 & 0.14 & 0.0 & 0.04 & 0.00 \\
\hline 28 & 1 & -0.12 & -0.13 & -0.05 & 0.03 & 0.22 & 0.16 & 0.06 & 0.00 & 0.00 \\
\hline 29 & 1 & -0.19 & & -0.10 & 0.04 & 0.07 & 0.26 & 0.14 & 0.04 & 0.01 \\
\hline 30 & 1 & -0.10 & & -0.03 & 0.00 & 0.19 & 0.08 & 0.04 & 0.12 & 0.01 \\
\hline 31 & 6 & -0.06 & & 0.05 & -0.03 & -0.04 & -0.07 & -0.03 & 0.00 & -0.04 \\
\hline 32 & 6 & -0.05 & 0.0 & 0.03 & -0.01 & -0.01 & -0.12 & -0.01 & -0.02 & 0.10 \\
\hline 33 & 6 & -0.08 & 0. & 0.04 & -0.05 & -0.04 & 0.04 & -0.04 & 0.00 & -0.15 \\
\hline 34 & 6 & -0.10 & 0.05 & 0.01 & -0.05 & -0.01 & 0.16 & -0.05 & 0.01 & \\
\hline 35 & 1 & -0.12 & & 0.00 & -0.07 & -0.01 & 0.29 & -0.07 & 0.02 & -0.42 \\
\hline 36 & 6 & -0.09 & & -0.02 & -0.04 & 0.03 & 0.04 & -0.02 & -0.01 & 0.02 \\
\hline 37 & 1 & -0.09 & & -0.05 & -0.04 & 0.05 & 0.09 & -0.02 & -0.01 & \\
\hline 38 & 6 & -0.06 & 0.06 & -0.01 & -0.03 & 0.04 & -0.14 & 0.01 & -0.05 & 0.26 \\
\hline 39 & 1 & -0.06 & 0.08 & -0.04 & -0.02 & 0.08 & -0.22 & 0.05 & -0.08 & 0.46 \\
\hline equen & & 207.62 & & & & & & 222.05 & & \\
\hline Atom & AN & $X$ & $Y$ & Z & $x$ & $Y$ & Z & $x$ & Y & Z \\
\hline 1 & 78 & 0.00 & 0.00 & 0.00 & 0.00 & 0.01 & 0.00 & 0.06 & -0.01 & 0.03 \\
\hline 2 & 7 & & & 0.00 & & -0.07 & 0.00 & 0.00 & 0.03 & -0.04 \\
\hline 3 & 7 & 0. & 0.00 & 0.01 & -0.01 & 0.02 & -0.08 & -0.02 & -0.02 & -0.17 \\
\hline 4 & 17 & -0.01 & -0.09 & 0.02 & -0.03 & 0.01 & -0.05 & 0.02 & -0.01 & 0.03 \\
\hline 5 & 17 & & 0.09 & -0.02 & -0. & 0.03 & 0.03 & 0.0 & -0.01 & 0.00 \\
\hline 6 & 17 & & & 0.00 & -0.0 & 0.04 & 0.00 & -0.0 & & 0.04 \\
\hline 7 & 17 & & & -0.01 & & -0.03 & 0.16 & -0.0 & -0.04 & 0.03 \\
\hline 8 & 6 & -0.01 & -0.01 & 0.00 & 0.1 & 0.12 & -0.04 & -0.1 & -0.19 & -0.08 \\
\hline 9 & 1 & & & 0. & & 0.20 & -0.07 & -0 . & -0.20 & -0.10 \\
\hline 10 & 1 & & & & & & & & & -0.07 \\
\hline 11 & 1 & & & -0.0 & 0.2 & 0.08 & -0. & -0 . & -0.12 & -0.19 \\
\hline 12 & 6 & & & & & -0.09 & 0.02 & 0.0 & 0.07 & -0.01 \\
\hline 13 & 6 & & & & & -0.06 & 0.0 & & 0.04 & 0.00 \\
\hline 14 & 6 & & & -0. & & -0.04 & 0.0 & & 0.04 & 0.00 \\
\hline 15 & 6 & 0.0 & & -0.04 & 0.0 & 0.02 & 0.01 & -0.01 & 0.00 & 0.00 \\
\hline 16 & 1 & & & -0.0 & 0.0 & 0.07 & 0.0 & -0.02 & -0.03 & 0.00 \\
\hline 17 & 6 & & & & & & & & & 0.01 \\
\hline 18 & 1 & & & & & 0. & -0 . & -0 . & -0.06 & 0.01 \\
\hline 19 & 6 & -0 . & & & 0.03 & -0.02 & 0.0 & -0.01 & 0.00 & 0.00 \\
\hline 20 & 1 & & & & & & & & & 0.01 \\
\hline 21 & 6 & & & & & -0.07 & -0. & -0. & 0.02 & -0.07 \\
\hline 22 & 6 & & & & & -0.08 & 0.1 & & 0.06 & 0.00 \\
\hline 23 & 1 & & & & & -0.19 & 0.1 & & & \\
\hline 24 & 1 & & & -0 . & & -0.09 & 0.1 & -0 . & & \\
\hline 25 & 1 & & & 0. & 0.20 & 0.04 & 0.12 & 0.00 & 0.11 & 0.01 \\
\hline 26 & 6 & & & & 0.0 & -0.05 & -0 . & -0. & 0.00 & -0.13 \\
\hline 27 & 6 & & & 0.01 & 0.12 & -0.08 & -0.21 & & 0.14 & 0.00 \\
\hline 28 & 1 & 0. & & 0.01 & 0.16 & -0.12 & -0.19 & 0.04 & 0.35 & 0.05 \\
\hline 29 & 1 & & & 0.0 & 0.10 & -0.07 & -0.24 & -0.05 & 0.04 & 0.21 \\
\hline 30 & 1 & & & & & -0.09 & -0.22 & & 0.16 & -0.10 \\
\hline 31 & 6 & & & 0.0 & -0.03 & 0.04 & -0.09 & -0.05 & 0.02 & -0.20 \\
\hline 32 & 6 & 0.00 & 0.00 & 0.00 & -0.05 & 0.02 & -0.07 & -0.04 & 0.00 & -0.10 \\
\hline
\end{tabular}




\begin{tabular}{|c|c|c|c|c|c|c|c|c|c|c|}
\hline 33 & 6 & 0.00 & 0.00 & 0.00 & -0.02 & 0.04 & 0.00 & -0.07 & 0.02 & -0.14 \\
\hline & & & 0.00 & & 0.00 & & & -0.06 & 0.02 & -0.01 \\
\hline 35 & 1 & 0.00 & 1.00 & -0.01 & 0.04 & -0.02 & 0.24 & -0.05 & 01 & 06 \\
\hline 36 & 6 & 0.00 & .00 & 0.00 & -0.03 & & 0.04 & & & \\
\hline 37 & 1 & 0.00 & 0.00 & & -0.02 & -0.04 & 0.10 & -0.04 & 0.00 & 0.24 \\
\hline 38 & 6 & 0.00 & & & -0.05 & & -0.0 & & & .05 \\
\hline 39 & 1 & 0.00 & .00 & 0.0 & -0.06 & 0.00 & -0.11 & & 0.00 & .17 \\
\hline \multicolumn{2}{|c|}{ Frequencies } & \multicolumn{2}{|l|}{236.96} & & \multicolumn{2}{|l|}{260.94} & & \multicolumn{2}{|l|}{284.76} & \\
\hline Atom & AN & X & Y & Z & $x$ & Y & Z & X & $Y$ & Z \\
\hline 1 & 78 & 0.04 & 0.07 & -0.03 & 0.05 & 0.04 & -0.01 & 0.00 & -0.02 & 0.00 \\
\hline 2 & 7 & 0.09 & -0.01 & 0.06 & -0.07 & -0.04 & 0.00 & -0.01 & 0.03 & 0.03 \\
\hline 3 & 7 & -0.07 & -0.03 & 0.12 & -0.03 & 0.03 & 0.06 & -0.02 & 0.02 & 0.02 \\
\hline 4 & 17 & -0.05 & -0.02 & -0.18 & 0.0 & 0.04 & 0.15 & -0.01 & -0.01 & \\
\hline 5 & 17 & & 0.04 & 0.11 & & -0.01 & -0.14 & & -0.01 & -0.01 \\
\hline 6 & 17 & -0.1 & 0.01 & 0.01 & -0.05 & -0.01 & -0.01 & & 0.05 & 0.01 \\
\hline 7 & 17 & & -0.14 & -0.07 & & -0.05 & -0.01 & & & \\
\hline 8 & 6 & -0.1 & -0.07 & 0.06 & -0.04 & -0.05 & & & & \\
\hline 9 & 1 & -0.06 & & 0.11 & -0.01 & -0.16 & 0.05 & & 0.01 & 0.01 \\
\hline 10 & 1 & & & 0.1 & & -0.12 & 0. & & & 01 \\
\hline 11 & 1 & & & 0.0 & -0 . & 0.00 & 0.0 & 0. & -0.02 & \\
\hline 12 & 6 & 0.11 & -0.02 & 0.03 & -0 & -0.12 & 0.0 & 0.0 & 0.07 & 0.00 \\
\hline 13 & 6 & 0.1 & & 0.04 & -0 . & -0.09 & -0.0 & & 05 & -0.01 \\
\hline 1 & 6 & 0.1 & & 0.0 & & -0.08 & 0. & & & \\
\hline 15 & 6 & 0.12 & & 0.03 & -0 . & 0.01 & -0.02 & 0. & & 00 \\
\hline 16 & 1 & 0.12 & & 0.03 & & 0.06 & -0.04 & & & \\
\hline 17 & 6 & & & & & & & & & \\
\hline $1 \varepsilon$ & 1 & & & & & & & & & \\
\hline 19 & 6 & 0.11 & & 0.02 & & 0.02 & & & & \\
\hline 20 & 1 & 0.11 & & & & 0.09 & -0 & & & \\
\hline 21 & 6 & 0.02 & & & & & & & & \\
\hline 2 & 6 & 0.02 & & -0.05 & & -0.11 & & & & -0 . \\
\hline 2 & 1 & 0. & & -0 . & & -0.30 & 0. & & & \\
\hline 24 & 1 & & & -0.1 & & -0.03 & 0. & & & \\
\hline 2 & 1 & & & -0.0 & & -0.09 & 0. & & -0.09 & \\
\hline 2 & 6 & -0. & & 0. & & 0. & & & & \\
\hline 27 & 6 & 0.0 & & 0.0 & & -0.01 & -0. & & 0.02 & \\
\hline 28 & 1 & & & & & -0.27 & & & & \\
\hline 29 & 1 & & & & & & & & & \\
\hline 30 & 1 & & & & & 0. & 0. & & & \\
\hline 31 & 6 & & & & & -0.02 & & & & \\
\hline 32 & 6 & & & & & & & & & \\
\hline 33 & 6 & & & 0. & -0 & -0.02 & 0. & & 0.0 & -0.03 \\
\hline 34 & 6 & & & 0. & & -0.01 & 0. & & .01 & \\
\hline 35 & 1 & & & & & & & & & \\
\hline 36 & 6 & & & 0. & & -0. & & & & \\
\hline 37 & 1 & & & & & -0.01 & -0 . & & & \\
\hline 38 & 6 & & & & & -0.02 & & & -0.01 & -0.02 \\
\hline 39 & 1 & -0.09 & & & & -0.01 & -0.03 & & -0.02 & \\
\hline \multicolumn{2}{|c|}{ Frequencies } & & & & & & & & & \\
\hline Atom & AN & & Y & Z & & Y & Z & & Y & 2 \\
\hline & 78 & & -0.01 & -0.01 & & -0.02 & 0.00 & & & \\
\hline 2 & 7 & & & 0.06 & & -0.03 & -0.04 & & & -0.07 \\
\hline 3 & 7 & & & & & 0.04 & 0.0 & & -0.05 & -0.05 \\
\hline 4 & 17 & & & & & 0.00 & 0.0 & & & 0.00 \\
\hline 5 & 17 & & & -0.0 & & -0.01 & -0. & & & \\
\hline 6 & 17 & & & 0.0 & & 0.15 & -0.0 & & 0.08 & 0.00 \\
\hline 7 & 17 & & & $-0 .(1+x-y$ & & -0.04 & -0.0 & & & -0.02 \\
\hline 8 & 6 & & & 0.0 & & 0.00 & $0 .($ & & 0.00 & -0.01 \\
\hline 9 & 1 & & & 0.0 & 0.0 & 0.02 & 0.0 & & 0.00 & -0.03 \\
\hline 10 & 1 & 0.00 & 0.04 & 0.02 & 0.01 & 0.04 & 0.01 & 0.02 & -0.02 & -0.02 \\
\hline
\end{tabular}




\begin{tabular}{|c|c|c|c|c|c|c|c|c|c|c|}
\hline & & & & & & & & & & \\
\hline 11 & 1 & 0.04 & -0.01 & 0.04 & 0.05 & -0.01 & 0.01 & 0.00 & 0.00 & -0.02 \\
\hline 12 & 6 & -0.02 & -0.06 & 0.01 & 0.00 & 0.03 & -0.01 & -0.01 & -0.12 & 0.00 \\
\hline 13 & 6 & -0.03 & -0.05 & 0.00 & 0.01 & 0.03 & -0.01 & 0.02 & -0.11 & 0.02 \\
\hline 14 & 6 & -0.01 & -0.05 & 0.01 & -0.01 & 0.03 & -0.01 & -0.02 & -0.11 & 0.00 \\
\hline 15 & 6 & 0.00 & -0.01 & 0.00 & -0.02 & 0.01 & 0.00 & -0.01 & -0.01 & 0.00 \\
\hline 16 & 1 & 0.01 & 0.02 & -0.01 & -0.02 & -0.01 & 0.00 & -0.02 & 0.06 & 0.00 \\
\hline 17 & 6 & -0.01 & 0.04 & -0.02 & -0.02 & -0.02 & 0.01 & 0.03 & 0.07 & 0.01 \\
\hline 18 & 1 & 0.00 & 0.10 & -0.03 & -0.03 & -0.07 & 0.02 & 0.04 & 0.20 & 0.02 \\
\hline 19 & 6 & -0.02 & 0.00 & -0.01 & 0.00 & 0.00 & 0.00 & 0.03 & -0.02 & 0.02 \\
\hline 20 & 1 & -0.02 & 0.03 & -0.01 & 0.00 & -0.02 & 0.01 & 0.05 & 0.04 & 0.02 \\
\hline 21 & 6 & -0.01 & 0.02 & 0.11 & -0.01 & -0.07 & -0.07 & -0.01 & -0.07 & -0.09 \\
\hline 22 & 6 & 0.02 & -0.04 & -0.02 & -0.10 & -0.01 & 0.00 & -0.07 & -0.02 & 0.04 \\
\hline 23 & 1 & 0.08 & -0.07 & -0.15 & -0.10 & 0.05 & 0.02 & -0.14 & 0.04 & 0.20 \\
\hline 24 & 1 & 0.12 & 0.01 & -0.01 & -0.17 & -0.08 & 0.05 & -0.20 & -0.08 & \\
\hline 25 & 1 & -0.11 & -0.11 & -0.05 & -0.08 & 0.08 & 0.01 & 0.09 & 0.03 & 0.07 \\
\hline 26 & 6 & -0.01 & 0.02 & 0.11 & -0.01 & -0.04 & -0.07 & -0.03 & -0.06 & -0.07 \\
\hline 27 & 6 & -0.11 & -0.11 & 0.05 & 0.17 & 0.09 & -0.06 & -0.12 & 0.02 & 0.09 \\
\hline 28 & 1 & 0.05 & 0.15 & 0.18 & 0.44 & 0.37 & 0.15 & -0.43 & -0.19 & -0.13 \\
\hline 29 & 1 & -0.57 & -0.15 & 0.09 & -0.06 & -0.03 & 0.17 & 0.31 & 0.06 & 0.04 \\
\hline 30 & 1 & 0.04 & -0.45 & -0.18 & 0.27 & 0.08 & -0.43 & -0.28 & 0.25 & 0.47 \\
\hline 31 & 6 & -0.04 & 0.04 & -0.12 & -0.06 & 0.02 & 0.12 & -0.02 & 0.00 & 0. \\
\hline 32 & 6 & -0.04 & 0.03 & -0.12 & -0.08 & -0.02 & 0.11 & -0.01 & 0.01 & 0.00 \\
\hline 33 & 6 & -0.02 & 0.05 & -0.13 & -0.02 & 0.03 & 0.12 & -0.01 & 0.00 & 0.00 \\
\hline 34 & 6 & 0.01 & 0.01 & -0.02 & -0.02 & -0.02 & 0.00 & 0.00 & -0.01 & \\
\hline 35 & 1 & 0.04 & 0.00 & 0.04 & 0.02 & -0.03 & -0.08 & 0.00 & -0.01 & 0.00 \\
\hline 36 & 6 & 0.00 & -0.01 & 0.10 & -0.06 & -0.05 & -0.08 & 0.00 & -0.01 & -0.01 \\
\hline 37 & 1 & 0.03 & -0.05 & 0.26 & -0.06 & -0.07 & -0.21 & 0.00 & 0.00 & -0.02 \\
\hline 38 & 6 & -0.02 & 0.01 & 0.00 & -0.09 & -0.04 & 0.03 & -0.01 & 0.00 & 0.00 \\
\hline 39 & 1 & -0.02 & -0.01 & 0.07 & -0.12 & -0.06 & -0.02 & -0.01 & -0.01 & 0.00 \\
\hline Frequer & & 330.91 & & & 357.62 & & & 367.79 & & \\
\hline Atom & AN & X & Y & Z & $x$ & $Y$ & Z & $x$ & $Y$ & Z \\
\hline & 78 & -0.01 & -0.01 & 0.00 & 0.00 & 0.04 & -0.01 & 0.02 & -0.01 & 0.00 \\
\hline 2 & 7 & 0.01 & 0.00 & -0.06 & 0.03 & -0.09 & 0.15 & -0.12 & 0.09 & 0.22 \\
\hline 3 & 7 & 0.01 & 0.01 & -0.01 & -0.03 & -0.19 & -0.07 & -0.09 & 0.02 & -0.16 \\
\hline 4 & 17 & 0.01 & -0.02 & 0.01 & -0.08 & 0.00 & 0.03 & -0.01 & -0.01 & -0.11 \\
\hline 5 & 17 & -0.02 & -0.01 & 0.02 & 0.02 & -0.03 & -0.08 & 0.11 & 0.00 & 0.08 \\
\hline 6 & 17 & -0.01 & 0.05 & 0.00 & 0.12 & -0.01 & 0.01 & 0.07 & -0.02 & 0.00 \\
\hline 7 & 17 & 0.02 & -0.03 & -0.04 & -0.04 & 0.08 & 0.03 & 0.02 & 0.05 & 0.00 \\
\hline 8 & 6 & 0.00 & 0.00 & 0.00 & -0.04 & 0.00 & -0.02 & 0.01 & -0.04 & -0.02 \\
\hline 9 & 1 & 0.00 & 0.02 & 0.00 & -0.04 & -0.02 & -0.02 & -0.03 & -0.01 & -0.0 \\
\hline 10 & 1 & 0.00 & 0.01 & 0.00 & -0.02 & -0.08 & -0.01 & 0.04 & -0.12 & -0.03 \\
\hline 11 & 1 & 0.02 & -0.01 & -0.01 & -0.0 & 0.02 & -0.04 & -0.0 & -0.02 & -0.0 \\
\hline 12 & 6 & 0.02 & 0.13 & -0.03 & 0.03 & 0.09 & 0.0 & -0.08 & 0.0 & 0.11 \\
\hline 13 & 6 & 0.04 & 0.14 & -0.02 & -0.04 & 0.14 & -0.04 & -0.14 & -0.03 & 0.09 \\
\hline 14 & 6 & 0.01 & 0.14 & -0.03 & 0.0 & 0.14 & 0.06 & 0.09 & -0.07 & 0.05 \\
\hline 15 & 6 & -0.01 & 0.02 & -0.01 & & 0.03 & 0.01 & & & \\
\hline 16 & 1 & -0.04 & -0.05 & 0.01 & 0.1 & -0.04 & -0.01 & 0.20 & -0.02 & -0.06 \\
\hline 17 & 6 & -0.01 & -0.09 & 0.02 & -0.03 & -0.11 & -0.05 & 0.06 & 0.03 & -0.12 \\
\hline 18 & 1 & -0.05 & -0.28 & 0.06 & & -0.32 & -0.09 & & & \\
\hline 19 & 6 & 0.02 & 0.03 & 0.00 & & 0.03 & -0.06 & -0.1 & -0.01 & \\
\hline 20 & 1 & 0.02 & -0.04 & 0.02 & -0.08 & -0.03 & -0.07 & -0.23 & 0.01 & -0.15 \\
\hline 21 & 6 & 0.02 & 0.00 & -0.08 & & -0.19 & 0.04 & -0.10 & 0.06 & \\
\hline 22 & 6 & 0.24 & -0.08 & 0.09 & -0. & -0.21 & 0.0 & 0.0 & & \\
\hline 23 & 1 & 0.21 & -0.34 & 0.14 & -0.08 & -0.19 & 0.05 & 0.10 & -0.24 & -0.04 \\
\hline 24 & 1 & 0.39 & -0. & 0.19 & & -0.22 & 0.08 & 0.27 & & \\
\hline 25 & 1 & 0.32 & 0.06 & 0.11 & & -0.17 & 0.06 & 0.04 & -0.12 & \\
\hline 26 & 6 & -0.02 & 0.01 & -0.05 & 0.0 & -0.22 & -0.03 & -0.11 & 0.06 & -0.11 \\
\hline 27 & 6 & -0.18 & -0.02 & 0.04 & 0.1 & -0.10 & 0.13 & -0.12 & 0.13 & -0.11 \\
\hline 28 & 1 & -0.32 & 0.02 & -0.05 & & -0.01 & 0.13 & -0.10 & 0.18 & \\
\hline 29 & 1 & -0.16 & -0.02 & 0.05 & 0.21 & -0.17 & 0.28 & -0.14 & 0.10 & \\
\hline
\end{tabular}




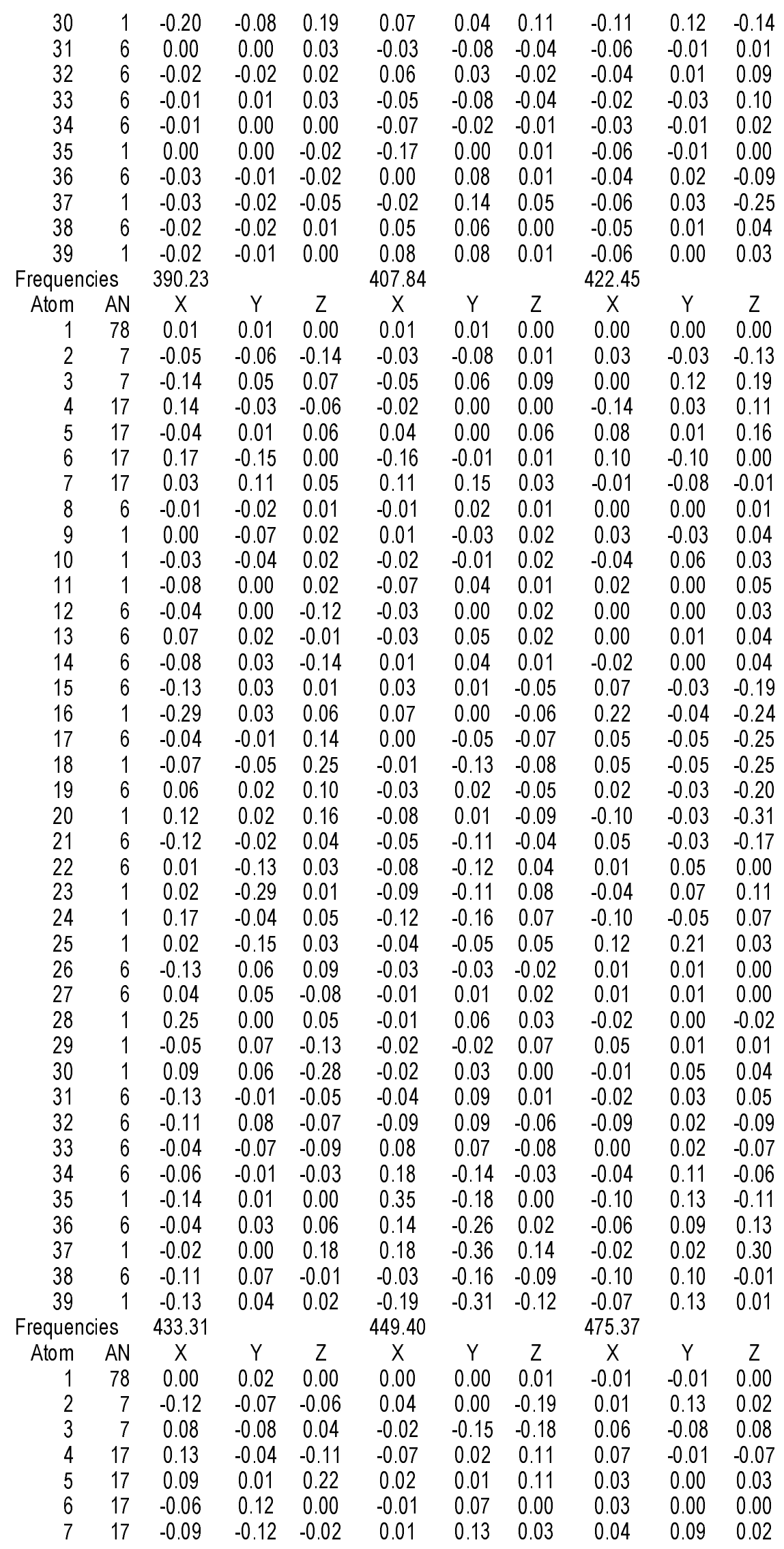




\begin{tabular}{|c|c|c|c|c|c|c|c|c|c|c|}
\hline & & & & & & & & & & \\
\hline $\begin{array}{l}8 \\
9\end{array}$ & $\begin{array}{l}6 \\
1\end{array}$ & $\begin{array}{l}0.00 \\
0.02\end{array}$ & $\begin{array}{r}0.00 \\
-0.03\end{array}$ & $\begin{array}{l}0.00 \\
0.02\end{array}$ & $\begin{array}{r}0.00 \\
-0.02\end{array}$ & $\begin{array}{l}0.00 \\
0.01\end{array}$ & $\begin{array}{l}-0.01 \\
-0.03\end{array}$ & $\begin{array}{l}0.01 \\
0.03\end{array}$ & $\begin{array}{l}-0.02 \\
-0.02\end{array}$ & $\begin{array}{l}0.00 \\
0.02\end{array}$ \\
\hline 10 & 1 & 0.00 & 0.00 & 0.01 & 0.03 & -0.02 & -0.02 & $\begin{array}{l}0.00 \\
-0.01\end{array}$ & 0.01 & 0.01 \\
\hline 11 & 1 & -0.01 & 0.01 & 0.02 & 0.00 & -0.01 & -0.02 & 0.04 & -0.03 & 0.02 \\
\hline 12 & 6 & -0.18 & 0.00 & -0.05 & 0.03 & -0.01 & -0.09 & -0.05 & 0.06 & 0.02 \\
\hline 13 & 6 & -0.13 & & 0.06 & 0.11 & -0.04 & -0.02 & -0.12 & & 0.03 \\
\hline 14 & 6 & -0.12 & 0.04 & -0.14 & -0.09 & 0.01 & -0.06 & -0.02 & -0.05 & 0.00 \\
\hline 15 & 6 & -0.15 & 0.03 & -0.14 & -0.06 & -0.03 & -0.17 & -0.05 & -0.02 & 0.02 \\
\hline 16 & 1 & -0.22 & 0.05 & -0.12 & -0.03 & -0.04 & -0.17 & -0.06 & -0.02 & 0.02 \\
\hline 17 & 6 & -0.13 & -0.04 & -0.06 & 0.03 & -0.01 & -0.12 & -0.08 & 0.08 & -0.01 \\
\hline 18 & 1 & -0.15 & -0.11 & -0.03 & 0.01 & 0.03 & 0.00 & -0.06 & 0.16 & -0.08 \\
\hline 19 & 6 & -0.15 & 0.04 & -0.02 & 0.13 & -0.04 & -0.13 & -0.15 & -0.02 & 0.03 \\
\hline 20 & 1 & -0.23 & 0.05 & -0.08 & 0.12 & -0.02 & -0.15 & -0.17 & -0.06 & 0.02 \\
\hline 21 & 6 & -0.10 & & 0.02 & -0.02 & 0.12 & 0.14 & 0.1 & 0.09 & -0.15 \\
\hline 22 & 6 & -0.08 & -0.12 & 0.02 & 0.07 & 0.06 & -0.03 & 0.15 & 0.22 & -0.05 \\
\hline 23 & 1 & -0.07 & -0.16 & 0.01 & 0.12 & 0.00 & -0.14 & 0.10 & 0.17 & 0.05 \\
\hline 24 & 1 & -0.03 & & 0.0 & 0.21 & 0.18 & -0.11 & 0.10 & 0.15 & 0.04 \\
\hline 25 & 1 & -0.08 & & 0.02 & -0.03 & -0.13 & -0.06 & 0.24 & 0.36 & -0.02 \\
\hline 26 & 6 & 0.00 & -0.03 & 0.07 & -0.02 & 0.04 & 0.15 & 0.10 & -0.15 & -0.05 \\
\hline 27 & 6 & 0.12 & & 0. & 0.04 & -0.05 & 0.01 & 0. & -0.12 & 0.11 \\
\hline 28 & 1 & 0.23 & & 0. & 0.19 & -0.13 & 0.09 & 0. & 0.01 & 0.11 \\
\hline 29 & 1 & 0.16 & -0.02 & -0.01 & -0.08 & 0.01 & -0.13 & 0. & -0.19 & 0.27 \\
\hline 30 & 1 & 0.12 & & -0 . & 0.11 & & -0.13 & 0. & -0.02 & 0.11 \\
\hline 31 & 6 & 0.13 & & 0. & -0.04 & -0.09 & -0.11 & -0.03 & -0.04 & 0.05 \\
\hline 32 & 6 & 0.18 & -0.06 & -0.02 & 0.07 & -0.02 & 0.10 & -0.04 & 0.00 & 0.01 \\
\hline 33 & 6 & 0.04 & & 0. & -0.09 & -0.09 & 0.03 & -0.11 & -0.05 & -0.08 \\
\hline 34 & 6 & 0.04 & 0.06 & -0.01 & -0.08 & -0.16 & 0.09 & -0.11 & -0.10 & -0.02 \\
\hline 35 & 1 & 0.01 & 0.07 & -0.02 & -0.08 & -0.16 & 0.21 & -0.10 & -0.11 & 0.00 \\
\hline 36 & 6 & 0.10 & & 0.0 & -0.03 & -0.06 & -0.13 & -0.09 & -0.08 & 0.07 \\
\hline 37 & 1 & 0.08 & & 0. & -0.08 & 0.06 & -0.30 & -0. & -0.05 & 0.17 \\
\hline 38 & 6 & 0.19 & & -0. & 0.07 & -0.09 & 0.02 & -0.06 & -0.05 & -0.07 \\
\hline 39 & 1 & 0.27 & & -0.0 & 0.05 & -0.10 & 0.00 & -0.09 & -0.05 & -0.15 \\
\hline Erequen & & 510.80 & & & 531.67 & & & 539.03 & & \\
\hline Atom & AN & & $\mathrm{Y}$ & Z & & $\mathrm{Y}$ & Z & $X$ & $\mathrm{Y}$ & Z \\
\hline 1 & 78 & 0.00 & -0.01 & 0.00 & 0.00 & 0.00 & 0.00 & 0.00 & 0.00 & 0.00 \\
\hline 2 & 7 & -0.02 & 0.06 & -0.01 & -0.01 & -0.05 & 0.00 & 0.0 & 0.01 & 0.01 \\
\hline 3 & 7 & & -0.02 & 0. & 0.02 & 0.07 & 0.04 & -0.01 & -0.01 & 0.00 \\
\hline 4 & 17 & & & & & & -0.01 & & & 0.00 \\
\hline 5 & 17 & & 0.00 & -0.0 & 0. & -0.01 & -0.0 & -0 . & -0.03 & 0.00 \\
\hline 6 & 17 & & & & & & 0.02 & 0. & 0.01 & 0.00 \\
\hline 7 & 17 & & & & & & -0.03 & & 0.00 & 0.01 \\
\hline 8 & 6 & & & & & -0.02 & 0. & & & 0.00 \\
\hline 9 & 1 & & & 0. & 0.0 & -0.02 & 0.00 & -0.01 & 0.00 & 0.00 \\
\hline 10 & 1 & -0. & & & 0.02 & -0.01 & 0.0 & 0. & 0.00 & -0.01 \\
\hline 11 & 1 & & & & & & 0. & & & 0.00 \\
\hline 12 & 6 & & & & -0. & & 0. & 0. & -0.01 & -0.01 \\
\hline 13 & 6 & & & & -0.01 & 0.07 & 0.0 & 0.07 & 0.40 & -0.06 \\
\hline 14 & 6 & & & & & & 0. & & & \\
\hline 15 & 6 & & & & & & 0. & & & -0.02 \\
\hline 16 & 1 & & & & & & -0.0 & 0.07 & 0.54 & -0.07 \\
\hline 17 & 6 & & & & -0.02 & -0.12 & 0. & & & 0.01 \\
\hline 18 & 1 & & & & -0.0 & -0.27 & 0. & & -0.01 & 0.02 \\
\hline 19 & 6 & & & 0. & -0.02 & 0. & 0. & 0.00 & -0.13 & 0.02 \\
\hline 20 & 1 & & & & -0.02 & & -0.0 & -0. & -0.53 & 0.07 \\
\hline 21 & 6 & & & -0.04 & 0.04 & -0.02 & -0.03 & & & 0.00 \\
\hline 22 & 6 & 0.0 & & 0.02 & -0.02 & 0.02 & -0.01 & 0.00 & & 0.00 \\
\hline 23 & 1 & & & 0.0 & -0.02 & 0.11 & 0.00 & 0.01 & -0.03 & -0.01 \\
\hline 24 & 1 & 0.1 & & & -0.12 & -0.05 & 0.00 & & 0.00 & 0.02 \\
\hline 25 & 1 & & & & -0.04 & 0.05 & -0.01 & & 0.01 & 0.00 \\
\hline 26 & 6 & -0.14 & 0.00 & -0.02 & 0.01 & 0.07 & 0.01 & -0.02 & 0.01 & 0.01 \\
\hline
\end{tabular}




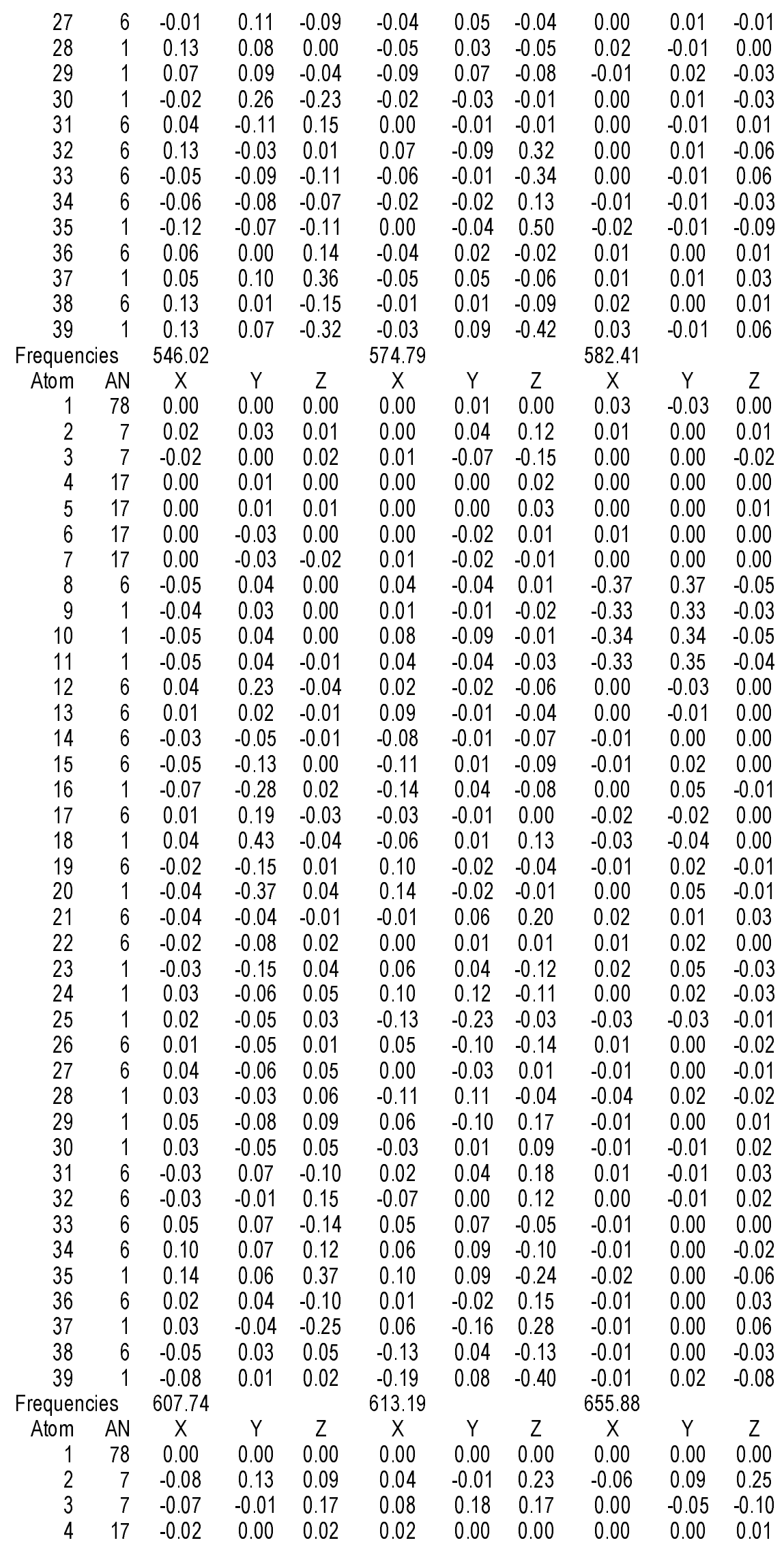




\begin{tabular}{|c|c|c|c|c|c|c|c|c|c|c|}
\hline 5 & 17 & - 01 & 000 & - & 001 & 000 & 003 & & 000 & 0,00 \\
\hline 6 & 17 & $\begin{array}{l}-0.01 \\
-0.02\end{array}$ & 0.01 & 0.00 & 0.01 & 0.00 & 0.00 & 0.00 & 0.00 & 0.00 \\
\hline 7 & 17 & -0.01 & -0.02 & -0.01 & 0.01 & 0.04 & 0.01 & 0.00 & 0.00 & 0.00 \\
\hline 8 & 6 & -0.01 & 0.02 & 0.00 & 0.01 & -0.01 & 0.00 & -0.01 & 0.02 & 0.01 \\
\hline 9 & 1 & 0.02 & -0.04 & 0.03 & 0.04 & 0.00 & 0.03 & -0.09 & 0.07 & -0.05 \\
\hline 10 & 1 & -0.05 & 0.04 & 0.02 & -0.05 & 0.08 & & & -0.13 & -0.03 \\
\hline 11 & 1 & -0.05 & 0.03 & 0.03 & 0.06 & -0.03 & 0.04 & -0.05 & 0.02 & -0.07 \\
\hline 12 & 6 & -0.04 & -0.08 & -0.03 & 0.06 & 0.02 & -0.04 & -0.01 & -0.01 & -0.02 \\
\hline 13 & 6 & 0.06 & & 0.00 & 0.08 & -0.01 & -0.06 & & -0.03 & -0.01 \\
\hline 14 & 6 & -0.02 & -0.03 & -0.05 & -0.09 & 0.03 & -0.06 & -0.05 & 0.00 & -0.05 \\
\hline 15 & 6 & 0.00 & 0.06 & -0.07 & -0.15 & -0.02 & -0.07 & -0.06 & 0.01 & -0.07 \\
\hline 16 & 1 & -0.04 & & -0.07 & -0.17 & -0.09 & -0.06 & -0.11 & 0.04 & -0.06 \\
\hline 17 & 6 & 0.07 & -0.10 & 0.03 & -0.10 & 0.05 & -0.02 & 0.02 & -0.02 & 0.01 \\
\hline 18 & 1 & 0.05 & -0.16 & 0.13 & -0.13 & 0.11 & 0.12 & -0.01 & 0.00 & 0.13 \\
\hline 19 & 6 & 0.12 & & 0.00 & 0.08 & -0.05 & -0.07 & 0.10 & & -0.02 \\
\hline 20 & 1 & 0.14 & 0.2 & -0.03 & 0.13 & -0.11 & 0.00 & 0.13 & 0.04 & -0.01 \\
\hline 21 & 6 & -0.09 & 0.0 & -0.02 & 0.09 & -0.07 & 0.0 & 0.02 & -0.08 & -0.25 \\
\hline 22 & 6 & 0.05 & & -0.01 & -0.05 & -0.06 & 0.02 & 0.00 & -0.01 & -0.03 \\
\hline 23 & 1 & 0.03 & -0.14 & 0.04 & -0.02 & 0.16 & -0.04 & -0.11 & -0.05 & \\
\hline 24 & 1 & 0.21 & & 0.01 & -0.20 & -0.14 & -0.01 & -0.17 & -0.22 & 0.20 \\
\hline 25 & 1 & 0.1 & & 0.00 & -0.16 & -0.10 & 0.0 & 0.22 & 0.42 & 0.05 \\
\hline 26 & 6 & -0.09 & & -0.12 & 0.10 & 0.02 & -0.15 & -0.02 & 0.11 & 0.18 \\
\hline 27 & 6 & 0.03 & -0.05 & 0.02 & -0.07 & 0.09 & -0.07 & 0.02 & -0.01 & 0.04 \\
\hline 28 & 1 & 0.08 & & 0.07 & -0.30 & 0.27 & -0.19 & 0.16 & -0.26 & 0.09 \\
\hline 29 & 1 & 0.26 & & 0.3 & -0.07 & 0.01 & 0. & -0.11 & 0.13 & -0.28 \\
\hline 30 & 1 & -0.04 & & -0.07 & -0.11 & 0.03 & 0.1 & 0.09 & -0.15 & -0.07 \\
\hline 31 & 6 & -0.07 & -0. & -0.07 & 0.05 & -0.02 & -0.07 & & & 0.01 \\
\hline 32 & 6 & 0.00 & & -0.03 & 0. & -0.04 & -0. & -0.01 & & 0.01 \\
\hline 3 & 6 & 0.03 & & -0.05 & -0.09 & -0.04 & 0.02 & 0.00 & 0.01 & 0.01 \\
\hline 34 & 6 & 0.07 & & 0.07 & -0.13 & -0.08 & 0.04 & 0.01 & 0.01 & -0.01 \\
\hline 35 & 1 & 0.04 & 0.0 & 0.23 & -0.12 & -0.09 & 0.10 & 0.01 & 0.01 & -0.05 \\
\hline 36 & 6 & 0.09 & & -0.10 & -0.12 & -0.03 & -0.05 & 0.00 & 0.00 & 0.02 \\
\hline 37 & 1 & 0.09 & & -0.16 & -0.16 & 0.11 & -0.07 & 0.00 & -0.02 & 0.01 \\
\hline 38 & 6 & 0.04 & & 0. & 0.0 & -0.08 & 0.03 & -0.0 & 0.00 & -0.01 \\
\hline 39 & 1 & 0.03 & & 0.2 & & -0.05 & 0.16 & -0.02 & 0.01 & -0.05 \\
\hline Frequen & & 680.83 & & & & & & 737.98 & & \\
\hline Atom & AN & X & $\mathrm{Y}$ & Z & $x$ & $\mathrm{Y}$ & Z & $X$ & $\mathrm{Y}$ & Z \\
\hline & 78 & & 0.00 & 0.00 & & 0.00 & 0.00 & 0.00 & 0.00 & 0.00 \\
\hline 2 & 7 & & & & & -0.10 & 0.0 & -0.02 & -0.05 & 0.02 \\
\hline 3 & 7 & & -0.04 & & & 0.11 & 0.0 & & -0.09 & -0.04 \\
\hline 4 & 17 & & & 0.0 & -0.02 & 0.00 & 0.0 & 0.0 & 0.00 & 0.00 \\
\hline 5 & 17 & & & & & & -0.02 & 0.0 & 0.00 & 0.00 \\
\hline 6 & 17 & & & & & & & & & \\
\hline 7 & 17 & & & 0. & & -0.02 & -0 . & 0. & 0.00 & 0.01 \\
\hline 8 & 6 & & & -0 & & 0.01 & 0.0 & -0.01 & 0.01 & 0.01 \\
\hline 9 & 1 & & & & & & & & 0.02 & -0.02 \\
\hline 10 & 1 & & & & & -0.06 & & & & -0.01 \\
\hline 11 & 1 & & & 0. & -0. & 0.05 & -0.02 & -0.02 & 0.02 & -0.02 \\
\hline 12 & 6 & & & -0 & & -0.13 & 0.00 & -0.03 & -0.14 & 0.02 \\
\hline 13 & 6 & & & & & & & & & -0.01 \\
\hline 14 & 6 & & & & & 0.12 & -0. & 0.0 & 0.1 & -0.02 \\
\hline 15 & 6 & & & -0.0 & 0.01 & -0.09 & -0.08 & -0.01 & -0.05 & -0.01 \\
\hline 16 & 1 & & & & & -0.41 & & & & 0.03 \\
\hline 17 & 6 & 0.2 & & 0. & & & 0.0 & 0.0 & 0.08 & -0.01 \\
\hline 18 & 1 & 0.2 & & 0.0 & 0.1 & -0.06 & 0.06 & 0.00 & -0.08 & 0.02 \\
\hline 19 & 6 & & & & & -0.06 & 0.0 & 0.00 & -0.05 & 0.01 \\
\hline 20 & 1 & & & 0.0 & & -0.41 & 0.0 & -0.06 & -0.33 & 0.04 \\
\hline 21 & 6 & -0.1 & & -0.0 & & -0.01 & 0.02 & 0.04 & -0.01 & 0.02 \\
\hline 22 & 6 & & & 0.0 & 0.0 & 0.07 & -0.02 & 0.03 & 0.05 & -0.01 \\
\hline 23 & 1 & & & 0.03 & 0.06 & 0.23 & -0.05 & 0.05 & 0.15 & -0.04 \\
\hline
\end{tabular}




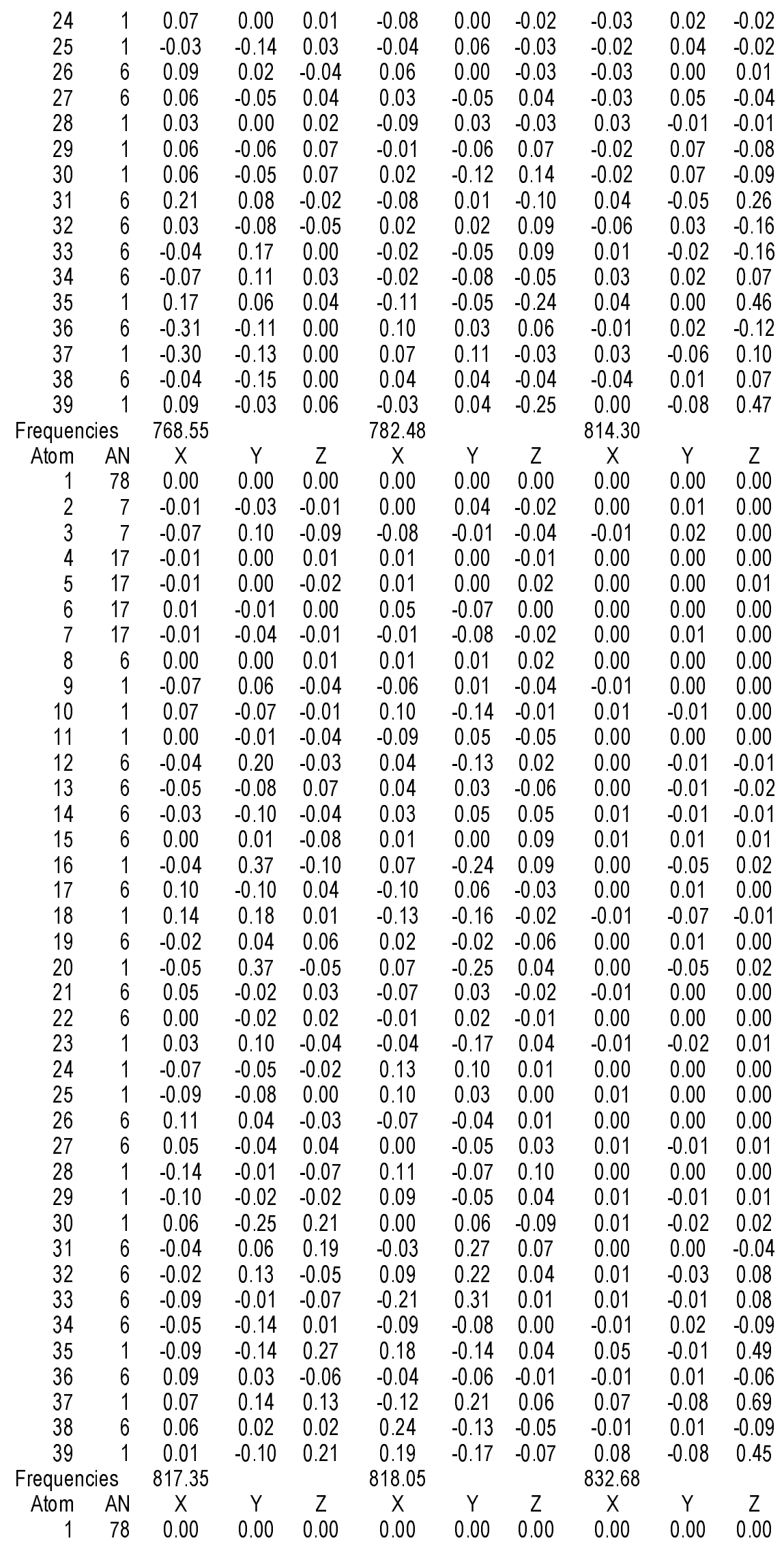




\begin{tabular}{|c|c|c|c|c|c|c|c|c|c|c|}
\hline & & & & & & & & & & \\
\hline $\begin{array}{l}2 \\
3\end{array}$ & $\begin{array}{l}7 \\
7\end{array}$ & $\begin{array}{l}-0.01 \\
-0.01\end{array}$ & $\begin{array}{l}-0.05 \\
-0.02\end{array}$ & $\begin{array}{r}-0.04 \\
0.02\end{array}$ & $\begin{array}{r}-0.01 \\
0.00\end{array}$ & $\begin{array}{l}-0.01 \\
-0.02\end{array}$ & $\begin{array}{l}0.03 \\
0.01\end{array}$ & $\begin{array}{l}0.01 \\
0.05\end{array}$ & $\begin{array}{c}0.00 \\
-0.07\end{array}$ & $\begin{array}{r}0.02 \\
-0.01\end{array}$ \\
\hline 4 & 17 & 0.04 & -0.02 & -0.06 & -0.02 & 0.00 & 0.03 & 0.00 & 0.00 & 0.00 \\
\hline 5 & 17 & -0.01 & -0.01 & -0.07 & 0.01 & 0.00 & 0.03 & 0.00 & 0.00 & -0.01 \\
\hline 6 & 17 & 0.00 & 0.00 & 0.00 & 0.00 & 0.00 & 0.00 & 0.00 & -0.01 & 0.00 \\
\hline 7 & 17 & 0.00 & 0.00 & 0.00 & 0.00 & 0.00 & 0.00 & & -0.01 & 0.00 \\
\hline 8 & 6 & 0.00 & & 0.02 & 0.00 & 0.01 & 0.0 & -0.01 & -0.03 & -0.12 \\
\hline 9 & 1 & -0.06 & 0.04 & -0.03 & 0.02 & -0.04 & 0.01 & 0.43 & -0.33 & 0.21 \\
\hline 10 & 1 & 0.09 & 0.11 & -0.01 & 0.00 & -0.02 & 0.01 & -0.48 & & 0.09 \\
\hline 11 & 1 & -0.05 & & -0.04 & -0.04 & 0.03 & 0.0 & & & 0.27 \\
\hline 12 & 6 & -0.04 & & 0.21 & 0.01 & -0.05 & -0.08 & -0.01 & 0.00 & 0.01 \\
\hline 13 & 6 & 0.05 & 0.09 & 0.21 & -0.01 & 0.08 & -0.11 & 0.00 & 0.01 & 0.02 \\
\hline 14 & 6 & -0.11 & & 0.18 & 0.07 & 0.07 & -0.0 & -0.01 & .00 & 0.00 \\
\hline 15 & 6 & -0.10 & -0.07 & -0.09 & 0.03 & -0.09 & 0. & -0.01 & 0.00 & -0.02 \\
\hline 16 & 1 & 0.09 & & -0.19 & 0.05 & 0.46 & 0. & -0.02 & 0.01 & -0.02 \\
\hline 17 & 6 & -0.01 & & -0.03 & -0.01 & -0.06 & 0.02 & 0.0 & 1.00 & 0.00 \\
\hline 18 & 1 & 0.03 & & 0.13 & 0.12 & 0.66 & -0.17 & 0.01 & 0.01 & 0.02 \\
\hline 19 & 6 & 0.12 & & -0.05 & -0.07 & -0.08 & 0.0 & 0.00 & 0.00 & 0.01 \\
\hline 20 & 1 & 0.10 & & -0.19 & 0.06 & 0.47 & 0.0 & -0. & 0.01 & -0.01 \\
\hline 21 & 6 & 0.01 & & 0.01 & 0.0 & -0.01 & 0.0 & 0.01 & -0.01 & -0.01 \\
\hline 22 & 6 & 0.02 & 0.03 & 0.00 & 0.01 & 0.02 & -0.01 & 0.00 & 0.00 & 0.00 \\
\hline 23 & 1 & 0.03 & & -0.04 & 0.01 & 0.04 & 0. & 0. & & 0.00 \\
\hline 24 & 1 & 0.02 & & -0.02 & -0.01 & 0.00 & & -0.03 & -0.02 & \\
\hline 25 & 1 & -0.01 & & -0.01 & 0.01 & 0.04 & -0.01 & 0.00 & 0.02 & 0.00 \\
\hline 26 & 6 & -0.04 & -0.02 & 0.01 & -0.02 & -0.01 & 0.0 & 0. & 0.00 & 0.02 \\
\hline 27 & 6 & -0.02 & & -0.01 & -0.01 & 0.01 & -0 & -0 & & -0.02 \\
\hline 28 & 1 & 0.05 & & 0.03 & 0.02 & 0.00 & 0. & & 0. & -0.02 \\
\hline 29 & 1 & 0.03 & & 0.0 & & & 0. & & 0.05 & -0.05 \\
\hline 30 & 1 & -0.02 & 0. & -0.07 & -0.02 & 0.05 & -0. & -0. & 0.02 & -0.04 \\
\hline 31 & 6 & 0.00 & & -0.02 & 0.00 & 0.01 & -0. & -0.02 & 0.03 & -0.01 \\
\hline 32 & 6 & 0.02 & & 0.02 & & 0.00 & 0.0 & -0.02 & 0.05 & 0.03 \\
\hline 33 & 6 & 0.00 & & 0.0 & 0.0 & 0. & & -0 & 0.02 & 0.01 \\
\hline 34 & 6 & 0.00 & & -0.0 & 0.00 & 0.01 & 0.1 & -0.01 & -0.04 & -0.01 \\
\hline 3 & 1 & 0.05 & & 0.0 & 0.03 & & 0. & -0.01 & -0.04 & 0.03 \\
\hline 36 & 6 & -0.02 & & -0 . & & & & & & -0.01 \\
\hline 37 & 1 & -0.01 & & 0.1 & -0.01 & -0.01 & & 0.04 & 0.01 & 0.04 \\
\hline 38 & 6 & 0.01 & & -0.02 & 0.01 & -0.01 & -0. & -0.0 & 0.02 & 0.00 \\
\hline 39 & 1 & 0.05 & & 0.08 & & -0.01 & & & -0.03 & \\
\hline quen & & 842.69 & & & 860 & & & & & \\
\hline Atom & AN & $X$ & $Y$ & Z & & $Y$ & Z & $x$ & $Y$ & Z \\
\hline 1 & 78 & & -0.01 & 0.00 & & 0.00 & 0.00 & & 0.00 & 0.00 \\
\hline 2 & 7 & & & -0.04 & & 0.09 & -0.0 & & 0.01 & 0.01 \\
\hline 3 & 7 & & & -0.09 & & -0.11 & 0.0 & -0. & 0.02 & -0.02 \\
\hline 4 & 17 & & & -0 & & & & & & 0.00 \\
\hline 5 & 17 & & & & & & & & & 0.00 \\
\hline 6 & 17 & & & & & 0.00 & 0. & 0. & -0.01 & 0.00 \\
\hline 7 & 17 & & & & & -0.03 & -0.01 & & 0.00 & 0.00 \\
\hline 8 & 6 & & & -0.04 & & & & & & 0.02 \\
\hline 9 & 1 & & & 0. & & 0. & & & 0.46 & -0.04 \\
\hline 10 & 1 & -0.19 & & & & -0.16 & -0 . & -0.08 & 0.37 & -0.09 \\
\hline 11 & 1 & & & & & & & & & 0.03 \\
\hline 12 & 6 & -0. & & 0.0 & & -0.07 & & & & \\
\hline 13 & 6 & 0.05 & & -0.03 & 0.00 & 0.03 & 0.0 & -0.01 & 0.01 & 0.01 \\
\hline 14 & 6 & 0.02 & & & & 0.03 & & & 0.01 & -0.02 \\
\hline 15 & 6 & 0.0 & & 0.0 & & -0.01 & -0. & & -0.01 & -0.03 \\
\hline 16 & 1 & 0.1 & & 0.0 & & 0.01 & -0. & -0. & 0.01 & -0.02 \\
\hline 17 & 6 & -0.08 & & -0.0 & & 0.00 & & & 0.00 & 0.01 \\
\hline 18 & 1 & -0. & & -0.0 & & 0.04 & & & 0.01 & 0.00 \\
\hline 19 & 6 & & & -0.07 & -0.01 & -0.01 & & & 0.00 & 0.02 \\
\hline 20 & 1 & 0.14 & 0.03 & -0.02 & -0.02 & 0.02 & 0.00 & -0.05 & 0.01 & 0.00 \\
\hline
\end{tabular}




\begin{tabular}{|c|c|c|c|c|c|c|c|c|c|c|}
\hline & & & & & & & & & & \\
\hline $\begin{array}{l}21 \\
22\end{array}$ & $\begin{array}{l}6 \\
6\end{array}$ & $\begin{array}{l}-0.11 \\
-0.10\end{array}$ & $\begin{array}{r}0.09 \\
-0.09\end{array}$ & $\begin{array}{c}-0.06 \\
0.02\end{array}$ & $\begin{array}{l}-0.02 \\
-0.06\end{array}$ & $\begin{array}{r}0.05 \\
-0.08\end{array}$ & $\begin{array}{r}-0.01 \\
0.03\end{array}$ & $\begin{array}{l}0.01 \\
0.01\end{array}$ & $\begin{array}{l}-0.01 \\
0.00\end{array}$ & $\begin{array}{l}0.00 \\
0.00\end{array}$ \\
\hline $\begin{array}{l}22 \\
23\end{array}$ & $\begin{array}{l}6 \\
1\end{array}$ & $\begin{array}{l}-0.10 \\
-0.15\end{array}$ & $\begin{array}{l}-0.09 \\
-0.42\end{array}$ & $\begin{array}{l}.02 \\
0.09\end{array}$ & $\begin{array}{l}-0.06 \\
-0.06\end{array}$ & $\begin{array}{l}-0.08 \\
-0.17\end{array}$ & $\begin{array}{l}0.03 \\
0.02\end{array}$ & $\begin{array}{l}0.01 \\
0.01\end{array}$ & 0.02 & 0.00 \\
\hline 24 & 1 & 0.14 & 0.05 & 0.03 & 0.02 & -0.02 & 0.00 & -0.01 & -0.01 & 0.00 \\
\hline 25 & 1 & 0.08 & -0.08 & 0.04 & -0.02 & -0.11 & 0.03 & 0.00 & 0.01 & 0.00 \\
\hline 26 & 6 & 0.10 & 0.09 & -0.05 & 0.07 & 0.00 & 0.00 & & -0.01 & 0.01 \\
\hline 27 & 6 & 0.11 & -0.11 & 0.09 & -0.02 & 0.08 & -0.05 & 0.00 & -0.02 & 0.01 \\
\hline 28 & 1 & -0.11 & -0.02 & -0.03 & -0.05 & 0.05 & -0.07 & 0.03 & -0.02 & 0.03 \\
\hline 29 & 1 & -0.04 & -0.10 & 0.07 & -0.06 & 0.09 & -0.09 & 0.03 & -0.02 & 0.02 \\
\hline 30 & 1 & 0.12 & -0.33 & 0.31 & -0.01 & 0.01 & -0.03 & -0.01 & 0.03 & -0.03 \\
\hline 31 & 6 & 0.03 & -0.04 & 0.07 & -0.02 & 0.04 & -0.07 & -0.01 & 0.00 & 0.01 \\
\hline 32 & 6 & -0.02 & -0.03 & -0.04 & -0.07 & 0.11 & 0.06 & 0.02 & -0.01 & -0.01 \\
\hline 33 & 6 & 0.02 & -0.10 & -0.04 & -0.06 & -0.01 & 0.04 & 0.01 & 0.01 & 0.00 \\
\hline 34 & 6 & -0.02 & & 0.01 & -0.03 & -0.15 & -0.02 & 0.01 & 0.04 & 0.00 \\
\hline 35 & 1 & -0.13 & -0.03 & 0.01 & -0.13 & -0.13 & 0.02 & 0.04 & 0.03 & -0.01 \\
\hline 36 & 6 & 0.02 & 0.03 & 0.00 & 0.13 & 0.02 & -0.01 & -0.03 & -0.01 & 0.00 \\
\hline 37 & 1 & 0.02 & & -0.04 & 0.12 & 0.04 & 0.02 & -0.03 & 0.00 & 0.00 \\
\hline 38 & 6 & -0.03 & 0.03 & 0.02 & -0.10 & 0.10 & 0.02 & 0.03 & -0.03 & -0.01 \\
\hline 39 & 1 & -0.04 & 0.04 & 0.00 & -0.23 & -0.05 & 0.06 & 0.06 & 0.00 & -0.02 \\
\hline requen & & 915.12 & & & 944.54 & & & 947.12 & & \\
\hline Atom & AN & X & $Y$ & Z & $X$ & $Y$ & Z & $x$ & $Y$ & 2 \\
\hline 1 & 78 & -0.01 & -0.01 & 0.00 & 0.00 & 0.00 & 0.00 & 0.00 & 0.00 & 0.00 \\
\hline 2 & 7 & 0.33 & 0.28 & -0.03 & & 0.00 & 0.00 & 0.00 & 0.00 & 0.00 \\
\hline 3 & 7 & & 0.02 & -0.01 & & 0.00 & 0.00 & 0.00 & 0.00 & 0.00 \\
\hline 4 & 17 & 0.00 & 0.00 & 0.01 & & 0.00 & 0.00 & 0.00 & 0.00 & 0.00 \\
\hline 5 & 17 & 0.00 & 0.00 & -0.01 & 0.0 & 0.00 & 0.0 & 0.0 & 0.00 & 0.00 \\
\hline 6 & 17 & 0.00 & 0.00 & 0.00 & & 0.00 & 0.00 & 0.00 & 0.00 & 0.00 \\
\hline 7 & 17 & 0.00 & 0.00 & 0.00 & 0.00 & 0.00 & 0.00 & 0.00 & 0.00 & 0.00 \\
\hline 8 & 6 & 0.06 & 0.05 & -0.02 & 0.0 & 0.00 & 0.0 & 0.0 & 0.00 & 0.00 \\
\hline 9 & 1 & 0.13 & -0.31 & 0.02 & & 0.00 & 0.00 & 0.0 & 0.00 & 0.00 \\
\hline 10 & 1 & 0.02 & -0.20 & 0.07 & 0 & 0.00 & 0.00 & 0.00 & 0.00 & 0.00 \\
\hline 11 & 1 & -0.39 & & 0.00 & 0.0 & 0.00 & 0.00 & 0.0 & 0.00 & 0.00 \\
\hline 12 & 6 & 0.06 & 14 & 0.04 & & 0.00 & 0.0 & 0. & 0.00 & 0.00 \\
\hline 13 & 6 & & & 0.06 & & 0.00 & 0.0 & 0. & 4 & 0.00 \\
\hline 14 & 6 & -0.04 & & -0.05 & & 0.00 & 0.0 & -0. & -0.04 & 0.01 \\
\hline 15 & 6 & -0.07 & -0.02 & -0.18 & 0.0 & 0.00 & 0.0 & 0.0 & 0.12 & -0.02 \\
\hline 16 & 1 & -0.21 & & -0.14 & & 0.00 & 0.0 & -0 . & -0.68 & 0.09 \\
\hline 17 & 6 & & & & & & 0.0 & 0. & & 0.00 \\
\hline 18 & 1 & 0. & & 0. & & 0.0 & 0.0 & 0. & -0.01 & 0.00 \\
\hline 19 & 6 & -0.14 & & 0. & & & 0.0 & -0.02 & -0.12 & 0.02 \\
\hline 20 & 1 & -0.27 & & & & & 0.8 & 0. & & -0.09 \\
\hline 21 & 6 & & & & & & 0. & 0. & & 0.00 \\
\hline 22 & 6 & -0.07 & & 0. & & 0.0 & 0.0 & 0.0 & 0.00 & 0.00 \\
\hline 23 & 1 & -0.07 & & 0. & & & 0.0 & 0. & 0.00 & 0.00 \\
\hline 24 & 1 & & & & & & & & & 0.00 \\
\hline 25 & 1 & & & & & & 0. & 0. & & 0. \\
\hline 26 & 6 & -0.0 & & 0.02 & 0.00 & 0.00 & 0.0 & 0.0 & 0.00 & 0.00 \\
\hline 27 & 6 & & & -0.01 & & & 0. & & & 0.00 \\
\hline 28 & 1 & & & -0 . & & & 0. & & & \\
\hline 29 & 1 & & & -0.0 & & & 0.0 & 0. & 0.00 & 0.00 \\
\hline 30 & 1 & & & -0.0 & & & & & & 0. \\
\hline 31 & 6 & -0 & & 0.0 & & -0. & 0. & & & \\
\hline 32 & 6 & 0.0 & & -0.01 & & -0.01 & 0.0 & 0. & 0.00 & 0.00 \\
\hline 33 & 6 & 0.0 & & -0.01 & & 0.01 & -0.0 & 0. & 0. & 0.00 \\
\hline 34 & 6 & 0.01 & 0.0 & 0.00 & & -0.01 & 0.1 & & & 0.00 \\
\hline 35 & 1 & 0.05 & 0.0 & 0.01 & -0. & 0.04 & -0.7 & 0.00 & 0.00 & 0.00 \\
\hline 36 & 6 & -0.04 & & 0.00 & 0.0 & -0.01 & -0.01 & 0.00 & 0.00 & 0.00 \\
\hline 37 & 1 & -0.04 & & 0.00 & 0.0 & -0.01 & 0.05 & 0.00 & 0.00 & 0.00 \\
\hline 38 & 6 & 0.05 & & -0.0 & 0.0 & 0.02 & -0.1 & 0.00 & 0.00 & 0.00 \\
\hline 39 & 1 & 0.08 & 0.01 & -0.04 & 0.11 & -0.12 & 0.66 & 0.00 & 0.00 & 0.00 \\
\hline
\end{tabular}




\begin{tabular}{|c|c|c|c|c|c|c|c|c|c|c|}
\hline \multicolumn{2}{|c|}{ Frequencies } & \multicolumn{3}{|l|}{1022.28} & \multicolumn{3}{|l|}{1030.30} & \multicolumn{3}{|l|}{1031.37} \\
\hline & AN & Y & $Y$ & Z & × & Y & Z & y & Y & Z \\
\hline 1 & 78 & 0.00 & 0.00 & 0.00 & 0.00 & 0.00 & 0.00 & 0.00 & 0.00 & 0.00 \\
\hline 2 & 7 & 0.02 & -0.05 & 0.02 & 0.01 & -0.01 & 0.00 & 0.00 & 0.00 & 0.00 \\
\hline 3 & 7 & -0.05 & -0.06 & 0.04 & -0.01 & 0.00 & 0.00 & 0.00 & 0.00 & 0.00 \\
\hline 4 & 17 & 0.00 & & 0.00 & 0.00 & & & & & 0.00 \\
\hline 5 & 17 & 0.00 & 0.00 & 0.00 & 0.00 & 0.00 & 0.0 & 0.00 & 0.00 & 0.00 \\
\hline 6 & 17 & 0.00 & 0.00 & 0.00 & 0.00 & 0.00 & 0.00 & 0.00 & 0.00 & 0.00 \\
\hline 7 & 17 & 0.00 & 0.00 & 0.00 & 0.00 & 0.00 & 0.00 & 0.00 & 0.00 & 0.00 \\
\hline 8 & 6 & 0.00 & 0.00 & 0.01 & 0.00 & 0.0 & & & & 0.00 \\
\hline 9 & 1 & -0.02 & 0.02 & -0.01 & 0.00 & 0.00 & 0.00 & 0.00 & 0.00 & 0.00 \\
\hline 10 & 1 & 0.02 & -0.01 & -0.01 & 0.00 & 0.00 & & 0.00 & 0.00 & 0.00 \\
\hline 11 & 1 & 0.02 & -0.01 & -0.01 & 0.00 & 0.1 & & & & 0.00 \\
\hline 12 & 6 & 0.02 & 0.00 & 0.00 & 0.01 & & & & -0.01 & 0.00 \\
\hline 13 & 6 & 0.00 & 0.00 & 0.00 & 0.00 & 0. & & & -0.01 & 0.00 \\
\hline 14 & 6 & 0.00 & & 0.00 & 0.00 & & & & & 0.00 \\
\hline 15 & 6 & -0.01 & 0.00 & -0.02 & 0.00 & 0.00 & & & & -0.01 \\
\hline 16 & 1 & -0.01 & -0.01 & -0.02 & 0.00 & & -0.01 & -0.07 & -0.44 & 0.06 \\
\hline 17 & 6 & 0.01 & 0.00 & 0.0 & 0.0 & 0. & & -0.0 & 0.14 & 0.02 \\
\hline 18 & 1 & 0.01 & & 0.0 & & & & 0.11 & 0.74 & -0.09 \\
\hline 19 & 6 & -0.01 & 0.00 & 0.02 & 0.00 & 0.00 & 0.00 & 0.01 & 0.08 & -0.01 \\
\hline 20 & 1 & -0.02 & & 0.01 & 0.00 & & & & 43 & 0.05 \\
\hline 21 & 6 & -0.07 & & 0.01 & -0.01 & & & & & \\
\hline 22 & 6 & -0.05 & 0.08 & -0.05 & -0.01 & 0.01 & -0 . & & 1.00 & 0.00 \\
\hline 23 & 1 & -0.12 & -0.37 & 0.10 & -0.01 & -0. & & & & .00 \\
\hline 24 & 1 & $0.2 \varepsilon$ & 0.2 & 0.07 & 0.0 & & & & & \\
\hline 25 & 1 & 0.2 & & -0.01 & 0.0 & & & 0. & & \\
\hline 26 & 6 & & -0.05 & 0.03 & & & & & & 0.00 \\
\hline 27 & 6 & 0.06 & & -0.05 & 0.00 & & & & & \\
\hline 28 & 1 & & & -0.28 & -0.02 & & & & & 0.00 \\
\hline 29 & 1 & & & -0.12 & -0.02 & & & 0.00 & & 0.00 \\
\hline 30 & 1 & 0.1 & & 0.3 & 0.0 & -0.02 & & & & .00 \\
\hline 31 & 6 & -0.05 & & 0. & & 0.00 & & & 0 & \\
\hline 32 & 6 & & & 0. & 0.00 & 0. & 0. & 0.0 & 0.00 & 0.00 \\
\hline 33 & 6 & & & 0. & & 0. & & & & 0.00 \\
\hline 34 & 6 & 0.0 & & 0.0 & -0.01 & 0. & & 0. & 0 & \\
\hline 35 & 1 & 0.02 & 0.06 & -0.04 & 0.06 & -0.03 & & 0. & 0.00 & 0.00 \\
\hline 36 & 6 & & & -0.01 & & & & & & \\
\hline 37 & 1 & & & 0.0 & -0.0 & 0. & & 0. & & 0.01 \\
\hline 38 & 6 & & & & -0 . & & & & & 0.00 \\
\hline 39 & 1 & & & & & -0.07 & & & & \\
\hline Frequen & & 1047.13 & & & 1061.61 & & & 1087.19 & & \\
\hline Atom & AN & X & Y & Z & & $\mathrm{Y}$ & & $x$ & Y & \\
\hline & 78 & & 0.00 & 0.00 & & 0.00 & & & & 0.00 \\
\hline 2 & 7 & & & -0.0 & & & & & & \\
\hline 3 & 7 & & -0.08 & 0.04 & & 0.02 & 0. & & 2 & 0.01 \\
\hline 4 & 17 & & & & & 0.00 & & & 0.00 & 0.00 \\
\hline 5 & 17 & & & & & & & & & \\
\hline 6 & 17 & & & & & & & & & \\
\hline 7 & 17 & & 0.00 & 0.00 & & 0.00 & & & 0.00 & 0.00 \\
\hline 8 & 6 & & & & & & & & & \\
\hline 9 & 1 & & & & & -0.01 & & & & \\
\hline 10 & 1 & & 0.00 & -0.01 & & 0.01 & & & 0.00 & 0.00 \\
\hline 11 & 1 & & -0.02 & 0.00 & & & & & & 0.00 \\
\hline 12 & 6 & & 0.00 & -0.02 & 0.0 & 0.0 & & & 0. & 0.00 \\
\hline 13 & 6 & & 0.0 & 0.0 & & 0.00 & -0. & 0. & 0.00 & 0.01 \\
\hline 14 & 6 & & & -0.0 & & 0.0 & & -0. & & 0.00 \\
\hline 15 & 6 & 0.0 & & 0.1 & 0.0 & 0.00 & & & 0 & 0.01 \\
\hline 16 & 1 & & 0.0 & 0.1 & -0.01 & 0.00 & & 0. & 0.00 & 0.01 \\
\hline 17 & 6 & -0.01 & 0.00 & 0.00 & 0.00 & 0.00 & 0.00 & 0.00 & 0.00 & 0.00 \\
\hline
\end{tabular}




\begin{tabular}{|c|c|c|c|c|c|c|c|c|c|c|}
\hline & & & & & & & & & & \\
\hline $\begin{array}{l}18 \\
19\end{array}$ & 1 & $\begin{array}{r}-0.01 \\
0.07\end{array}$ & $\begin{array}{r}0.01 \\
-0.02\end{array}$ & $\begin{array}{l}-0.01 \\
-0.08\end{array}$ & $\begin{array}{l}0.00 \\
0.00\end{array}$ & $\begin{array}{l}0.00 \\
0.00\end{array}$ & $\begin{array}{l}0.01 \\
0.00\end{array}$ & $\begin{array}{l}0.01 \\
0.00\end{array}$ & $\begin{array}{l}0.00 \\
0.00\end{array}$ & $\begin{array}{l}-0.01 \\
-0.01\end{array}$ \\
\hline 20 & 1 & 0.05 & -0.03 & $\begin{array}{l}-0.00 \\
-0.10\end{array}$ & 0.01 & 0.00 & 0.02 & -0.02 & 0.00 & -0.02 \\
\hline 21 & 6 & 0.07 & 0.06 & -0.04 & 0.00 & -0.03 & -0.06 & -0.02 & 0.05 & 0.12 \\
\hline 22 & 6 & 0.07 & -0.10 & 0.07 & -0.02 & 0.05 & 0.08 & 0.03 & -0.06 & -0.11 \\
\hline 23 & 1 & 0.15 & 0.41 & -0.12 & 0.11 & -0.05 & -0.2 & -0 . & & 0.27 \\
\hline 24 & 1 & -0.34 & -0.27 & -0.10 & 0.17 & 0.29 & -0.2 & -0.18 & -0.34 & 0.27 \\
\hline 25 & 1 & -0.35 & -0.17 & 0.01 & -0.13 & -0.39 & 0.01 & 0.17 & 0.48 & -0.02 \\
\hline 26 & 6 & 0.05 & -0.05 & 0.02 & 0.00 & -0.04 & -0.09 & 0.01 & -0.06 & -0.10 \\
\hline 27 & 6 & 0.05 & 0.08 & -0.02 & -0.02 & 0.05 & 0.11 & & & 0.09 \\
\hline 28 & 1 & -0.21 & 0.06 & -0.19 & 0.17 & -0.42 & 0.16 & 0.12 & -0.33 & 0.12 \\
\hline 29 & 1 & -0.23 & 0.10 & -0.11 & -0.12 & 0.28 & -0.41 & -0.09 & 0.23 & -0.33 \\
\hline 30 & 1 & 0.07 & -0.21 & 0.18 & 0.13 & -0.19 & -0.19 & 0.10 & 0.16 & -0.15 \\
\hline 31 & 6 & -0.10 & & 0.01 & 0.00 & 0.01 & -0.0 & 0.0 & & 0.00 \\
\hline 32 & 6 & 0.00 & 0.03 & 0.01 & 0.00 & 0.00 & 0.00 & 0.00 & 0.00 & 0.00 \\
\hline 33 & 6 & 0.01 & -0.01 & 0.00 & 0.01 & -0.01 & 0.00 & 0.01 & -0.01 & 0.00 \\
\hline 34 & 6 & 0.02 & 0.10 & 0.00 & 0.00 & 0.00 & 0.00 & 0.00 & 0.00 & \\
\hline 35 & 1 & 0.01 & & 0.04 & -0.01 & 0.00 & -0.0 & 0.00 & .00 & 0.00 \\
\hline 36 & 6 & -0.01 & -0.01 & 0.02 & -0.01 & 0.00 & 0.0 & -0.01 & 0 & .00 \\
\hline 37 & 1 & -0.02 & 0.00 & -0.07 & -0.01 & 0.01 & 0. & -0.01 & .00 & .00 \\
\hline 38 & 6 & 0.07 & & -0.03 & 0.00 & 0.00 & 0.00 & 0.00 & 0.00 & 0.00 \\
\hline 39 & 1 & 0.06 & -0.09 & 0.01 & 0.01 & 0.01 & -0.01 & 0.01 & 0.01 & 0.00 \\
\hline requer & & 1107.20 & & & 1110.13 & & & 1115.73 & & \\
\hline Atom & AN & X & $\mathrm{Y}$ & Z & X & $\mathrm{Y}$ & Z & X & $\mathrm{Y}$ & Z \\
\hline 1 & 78 & 0.00 & 0.00 & 0.00 & 0.00 & 0.00 & 0.00 & 0.00 & 0.00 & 0.00 \\
\hline 2 & 7 & 0.1 & 0.00 & 0.01 & -0.02 & 0.01 & -0.01 & 0.00 & -0.03 & 0.01 \\
\hline 3 & 7 & -0.02 & 0.01 & -0.01 & -0.01 & -0.02 & 0.01 & 0.07 & 0.02 & -0.01 \\
\hline 4 & 17 & 0.01 & 0.00 & 0.00 & 0.00 & 0.00 & 0.00 & 0.00 & 0.00 & 0.00 \\
\hline 5 & 17 & 0.01 & 0.00 & 0.01 & & 0.00 & 0.00 & 0.0 & 0.00 & 0.00 \\
\hline 6 & 17 & 0.00 & 0.00 & 0.00 & -0.02 & 0.02 & 0.00 & 0.0 & 0.01 & 0.00 \\
\hline 7 & 17 & 0. & 0.00 & 0.0 & -0.0 & -0.04 & -0.01 & 0.0 & -0.02 & -0.01 \\
\hline 8 & 6 & & 0.00 & 0.0 & & 0.00 & 0.00 & & 0.01 & 0.00 \\
\hline 9 & 1 & 0.00 & 0.00 & 0.00 & & 0.00 & 0.00 & 0.0 & -0.02 & 0.01 \\
\hline 10 & 1 & & & 0. & & 0.00 & 0.0 & 0.0 & -0.02 & 0.00 \\
\hline 11 & 1 & & & & & -0.01 & 0. & 0. & & 0.00 \\
\hline 12 & 6 & -0.02 & 0.00 & 0.00 & -0.01 & 0.00 & 0.0 & 0.0 & 0.00 & 0.00 \\
\hline 13 & 6 & -0.07 & 0. & -0.0 & & 0.00 & 0.02 & -0.0 & 0.00 & -0.01 \\
\hline 14 & 6 & -0.06 & & -0.02 & & -0.01 & 0.0 & -0. & & 0.00 \\
\hline 15 & 6 & & & 0. & & -0.01 & -0. & & & \\
\hline 16 & 1 & -0.5 & & 0. & & -0.05 & -0.12 & -0. & 0.02 & 0.04 \\
\hline 17 & 6 & 0.1 & & & -0.07 & 0.01 & -0.01 & 0.0 & 0.00 & 0.01 \\
\hline 18 & 1 & & & & & & & & & 0.01 \\
\hline 19 & 6 & & & -0 & & 0. & 0.0 & 0. & 0. & -0.02 \\
\hline 20 & 1 & & & & & & 0.2 & -0.05 & 0.00 & -0.07 \\
\hline 21 & 6 & & & & & & -0. & & & \\
\hline 22 & 6 & & & & & -0. & 0. & -0 & 0. & -0.01 \\
\hline 23 & 1 & & & & & 0. & -0.01 & -0 . & -0.14 & 0.03 \\
\hline 24 & 1 & 0. & & & & -0.04 & -0.01 & & & 0.03 \\
\hline 25 & 1 & 0.1 & & & & & & & & 0.00 \\
\hline 26 & 6 & & & & & -0. & 0.0 & 0. & 0. & \\
\hline 27 & 6 & -0.02 & & -0.01 & & & -0.02 & -0.05 & -0.04 & 0.00 \\
\hline 28 & 1 & 0.0 & & & & 0.05 & -0.0 & & & 0.13 \\
\hline 29 & 1 & & & & & -0.02 & 0. & & -0.05 & 0.05 \\
\hline 30 & 1 & -0.02 & 0. & -0.04 & -0.01 & 0.02 & 0.02 & -0.07 & 0.19 & -0.18 \\
\hline 31 & 6 & 0.02 & & & & & -0.0 & & 0.02 & 0.00 \\
\hline 32 & 6 & 0.03 & & & 0.11 & 0.27 & 0. & -0.0 & 0.18 & 0.04 \\
\hline 33 & 6 & 0.03 & -0.0 & 0.00 & 0.22 & -0.18 & -0.03 & 0.10 & -0.16 & -0.01 \\
\hline 34 & 6 & 0.0 & & & & & -0.02 & -0.06 & 0.15 & 0.01 \\
\hline 35 & 1 & 0.1 & & -0.0 & 0.4 & -0.24 & -0.09 & -0. & 0.26 & 0.08 \\
\hline 36 & 6 & -0.06 & & 0.00 & -0.30 & -0.06 & 0.01 & 0.00 & 0.00 & 0.02 \\
\hline
\end{tabular}




\begin{tabular}{|c|c|c|c|c|c|c|c|c|c|c|}
\hline $\begin{array}{r}37 \\
38 \\
39\end{array}$ & $\begin{array}{l}1 \\
6 \\
1\end{array}$ & $\begin{array}{l}-0.06 \\
-0.02 \\
0.07\end{array}$ & $\begin{array}{l}0.00 \\
0.05 \\
0.15\end{array}$ & $\begin{array}{l}0.02 \\
0.01 \\
0.02\end{array}$ & $\begin{array}{c}-0.34 \\
-0.01 \\
0.21\end{array}$ & $\begin{array}{l}0.00 \\
0.12 \\
0.37\end{array}$ & $\begin{array}{l}0.06 \\
0.02 \\
0.04\end{array}$ & $\begin{array}{c}-0.02 \\
0.06\end{array}$ & $\begin{array}{c}0.07 \\
-0.16 \\
-0.47\end{array}$ & $\begin{array}{l}-0.01 \\
-0.04 \\
-0.05\end{array}$ \\
\hline \multicolumn{2}{|c|}{ Frequencies } & 1140.57 & & & 1184.85 & & & 1211.86 & & \\
\hline om & AN & X & $Y$ & Z & X & $Y$ & Z & X & $Y$ & Z \\
\hline 1 & 78 & 0.00 & 0.00 & 0.00 & 0.00 & 0.00 & 0.00 & 0.00 & 0.00 & 0.00 \\
\hline 2 & 7 & 0.08 & -0.01 & 0.02 & -0.06 & -0.01 & -0.01 & & & 0.00 \\
\hline 3 & 7 & -0.02 & 0.02 & -0.01 & -0.05 & 0.00 & -0.01 & 0.02 & -0.03 & 0.00 \\
\hline 4 & 17 & -0.03 & 0.01 & 0.04 & 0.01 & 0.00 & -0.01 & & 0.00 & \\
\hline 5 & 17 & -0.0 & 0.00 & -0.05 & 0.00 & 0.00 & 0.0 & 0.00 & 0.00 & \\
\hline 6 & 17 & 0.00 & 0.00 & 0.00 & 0.00 & 0.00 & 0.00 & 0.00 & 0.00 & 0.00 \\
\hline 7 & 17 & 0.0 & 0.00 & 0.00 & 0.00 & 0.01 & 0.00 & 0.01 & 0.01 & \\
\hline 8 & 6 & 0.0 & 0.00 & 0.00 & -0.0 & 0.00 & 0.0 & 0.0 & 0.00 & \\
\hline 9 & 1 & -0.02 & 0.00 & -0.02 & 0.01 & 0.01 & 0.01 & 0.0 & 0.00 & 0.00 \\
\hline 10 & 1 & -0.03 & 0.01 & 0.02 & 0.0 & 0.01 & -0.01 & & 0.00 & \\
\hline 11 & 1 & -0.0 & & 0.01 & & -0.02 & 0. & & & \\
\hline 12 & 6 & -0.12 & 0.01 & -0.03 & -0.0 & 0.03 & -0.0 & & & \\
\hline 13 & 6 & 0.1 & 0.04 & 0.44 & -0.0 & -0.01 & -0. & & & \\
\hline 14 & 6 & 0.2 & & -0.36 & & & & & & \\
\hline 15 & 6 & 0.0 & & 0.08 & & -0.01 & & & & \\
\hline 1 & 1 & -0.16 & 0.04 & 0.12 & & -0.04 & -0. & & & \\
\hline 17 & 6 & & & -0.06 & & & & & & \\
\hline 18 & 1 & & & -0.07 & & & & & & \\
\hline 15 & 6 & & -0.02 & -0.05 & & 0.00 & 0.1 & & & \\
\hline 20 & 1 & -0. & -0.01 & -0.18 & 0. & -0.01 & 0. & & & \\
\hline 2 & 6 & 0.1 & -0.01 & 0.02 & & 0.09 & & & -0. & \\
\hline 22 & 6 & & 0.02 & -0.02 & -0.11 & -0.03 & -0. & 0. & 0.01 & \\
\hline 23 & 1 & -0 . & -0.31 & 0.04 & -0.1 & -0.37 & 0. & & & \\
\hline 24 & 1 & 0.2 & & 0. & & 0. & & & -0.02 & \\
\hline 25 & 1 & & 02 & 0. & & -0.08 & 0. & -0 . & & \\
\hline 26 & 6 & & -0 . & 0. & & -0.06 & & & & \\
\hline 27 & 6 & -0.0 & 0.0 & -0 . & -0. & 0.02 & -0 & & & \\
\hline 28 & 1 & & & & 0.1 & & & & & \\
\hline 29 & 1 & & & & & & & & & \\
\hline 30 & 1 & -0 . & & -0 & -0 . & & & & 01 & \\
\hline 31 & 6 & & & & -0.0 & & & & & \\
\hline 32 & 6 & & & -0 & -0.0 & -0.08 & & & & \\
\hline 33 & 6 & -0. & & 0. & 0. & 0. & & 0. & & \\
\hline 34 & 6 & & & 0. & & -0.01 & 0. & 0. & & \\
\hline 3 & 1 & & & & & & & & & \\
\hline 36 & 6 & & & 0. & & & & & & \\
\hline 37 & 1 & & & & -0. & & & & & \\
\hline 38 & 6 & & & & & & & & -0.07 & \\
\hline 39 & 1 & & & & & & & & -0.12 & \\
\hline \multicolumn{2}{|c|}{ Frequencies } & 1214.29 & & & & & & & & \\
\hline Atom & AN & & Y & Z & & Y & Z & $x$ & Y & \\
\hline 1 & 78 & & 0.00 & 0.00 & & & & & & \\
\hline 2 & 7 & & & & & & & & & \\
\hline 3 & 7 & & & & & & & & & \\
\hline 4 & 17 & & & & & & & & & \\
\hline 5 & 17 & & & & & & & & & \\
\hline 6 & 17 & & & & & 0. & & & & 0 \\
\hline 7 & 17 & & & & & & & & & \\
\hline 8 & 6 & & & & & 0. & & & & \\
\hline 9 & 1 & & & & & -0.06 & & & & \\
\hline 10 & 1 & & & & & & & & 0.01 & 0.00 \\
\hline & 1 & & & & & & & & 0.00 & \\
\hline 12 & 6 & -0.0 & 0.01 & 0.06 & & & & & -0.01 & \\
\hline 13 & 6 & -0.1 & 0.01 & -0.0 & 0.03 & 0.00 & & 0.08 & -0.01 & 0.03 \\
\hline 14 & 6 & 0.13 & -0.02 & -0.02 & 0.04 & -0.01 & -0.0 & -0.11 & 0.01 & 00 \\
\hline
\end{tabular}




\begin{tabular}{|c|c|c|c|c|c|c|c|c|c|c|}
\hline & & & & & & & & & & \\
\hline $\begin{array}{l}15 \\
16\end{array}$ & $\begin{array}{l}6 \\
1\end{array}$ & $\begin{array}{l}0.00 \\
0.08\end{array}$ & $\begin{array}{l}-0.01 \\
-0.03\end{array}$ & $\begin{array}{l}-0.07 \\
-0.10\end{array}$ & $\begin{array}{l}-0.03 \\
-0.27\end{array}$ & $\begin{array}{l}0.00 \\
0.05\end{array}$ & $\begin{array}{r}-0.03 \\
0.04\end{array}$ & $\begin{array}{l}0.05 \\
0.65\end{array}$ & $\begin{array}{r}0.00 \\
-0.12\end{array}$ & $\begin{array}{c}0.03 \\
-0.14\end{array}$ \\
\hline 17 & 6 & $\begin{array}{l}.00 \\
-0.02\end{array}$ & 0.01 & 0.09 & 0.00 & 0.00 & 0.00 & -0.01 & 0.01 & 0.04 \\
\hline 18 & 1 & -0.20 & 0.14 & 0.92 & 0.00 & 0.00 & -0.03 & -0.05 & 0.04 & 0.23 \\
\hline 19 & 6 & 0.03 & -0.01 & -0.06 & -0.04 & 0.01 & 0.01 & -0.04 & 0.01 & 0.00 \\
\hline & 1 & -0.05 & -0.01 & -0.14 & -0.16 & 0.01 & -0.10 & & 0.02 & \\
\hline 21 & 6 & 0.00 & 0.00 & 0.00 & 0.02 & 0.05 & -0.01 & 0.01 & -0.01 & 0.00 \\
\hline 22 & 6 & 0.00 & 0.00 & 0.00 & -0.02 & -0.02 & 0.00 & 0.00 & 0.01 & 0.00 \\
\hline 23 & 1 & 0.00 & 0.01 & 0.01 & -0.03 & -0.04 & 0.01 & 0.00 & -0.02 & -0.01 \\
\hline 24 & 1 & -0.01 & -0.01 & 0.00 & 0.01 & 0.00 & & & 0.02 & 0.00 \\
\hline 25 & 1 & 0.00 & 0.01 & 0.00 & 0.01 & 0.00 & 0.00 & 0.01 & -0.01 & 0.00 \\
\hline 26 & 6 & -0.01 & 0.00 & 0.00 & -0.03 & 0.13 & -0.08 & 0.00 & 0.00 & 0.00 \\
\hline 27 & 6 & 0.00 & 0.00 & 0.00 & 0.02 & -0.05 & 0.04 & 0.00 & 0.00 & 0.00 \\
\hline 28 & 1 & 0.00 & & 0.00 & 0.05 & 0.00 & 0.0 & 0.00 & -0.01 & 0.00 \\
\hline 29 & 1 & -0.01 & 0.00 & 0.00 & 0.07 & -0.04 & 0.03 & 0.00 & 0.00 & 0.00 \\
\hline 30 & 1 & 0.00 & 0.00 & 0.01 & 0.03 & -0.04 & 0.02 & 0.00 & 0.00 & 0.00 \\
\hline 31 & 6 & 0.00 & & 0.00 & -0.21 & -0.20 & 0.0 & 0.0 & -0.04 & -0.01 \\
\hline 32 & 6 & -0.01 & & 0.00 & -0.09 & 0.04 & 0.0 & 0.0 & 0.02 & 0.00 \\
\hline 33 & 6 & 0.00 & 0.00 & 0.00 & -0.04 & 0.04 & 0.0 & -0.02 & 0.00 & 0.00 \\
\hline 34 & 6 & 0.00 & & 0.00 & 0.03 & 0.06 & 0. & 0. & & \\
\hline 35 & 1 & 0.01 & & 0.00 & 0.76 & -0.09 & $-0 .($ & 0.1 & -0.03 & -0.02 \\
\hline 36 & 6 & 0.00 & & 0.00 & -0.01 & -0.03 & 0.0 & 0. & 0.01 & 0.00 \\
\hline 37 & 1 & -0.01 & & 0. & -0.09 & 0.22 & 0. & -0. & 0.11 & 0.02 \\
\hline 38 & 6 & 0.00 & & 0.00 & 0.10 & 0.03 & -0.01 & -0.01 & 0.00 & .00 \\
\hline 39 & 1 & 0.00 & -0.01 & 0.00 & 0.12 & 0.05 & -0.03 & -0.17 & -0.16 & .00 \\
\hline requen & & 1262.85 & & & 1284.27 & & & 1307.52 & & \\
\hline Atom & AN & $X$ & $Y$ & Z & $x$ & $Y$ & Z & $X$ & Y & Z \\
\hline 1 & 78 & 0.00 & 0.00 & 0.00 & 0.00 & 0.00 & 0.00 & 0.00 & 0.00 & 0.00 \\
\hline 2 & 7 & & & 0.00 & -0.17 & -0.02 & -0.02 & & 0.01 & 0.01 \\
\hline 3 & 7 & -0.04 & 02 & 0.00 & -0.09 & -0.01 & 0.01 & 0.05 & -0.01 & 0.00 \\
\hline 4 & 17 & 0.00 & 0.00 & 0.00 & 0.00 & 0.00 & 0.00 & 0.00 & 0.00 & 0.00 \\
\hline 5 & 17 & 0. & 0.00 & 0.00 & 0.00 & 0.00 & -0.01 & 0.0 & 0.00 & 0.00 \\
\hline 6 & 17 & & & 0.00 & & 0.00 & 0.0 & 0.0 & 0.00 & 0.00 \\
\hline 7 & 17 & & & & & 0.00 & 0.0 & 0. & 0.00 & 0.00 \\
\hline 8 & 6 & & & 0.00 & & 0.00 & 0.0 & & 0.07 & -0.01 \\
\hline 9 & 1 & -0.02 & 0.02 & -0.02 & 0.01 & 0.00 & 0.02 & 0.31 & -0.34 & 0.23 \\
\hline 10 & 1 & & & 0.01 & & 0.00 & -0.01 & 0.3 & -0.38 & -0.13 \\
\hline 11 & 1 & & & & & -0.03 & 0.0 & & & \\
\hline 12 & 6 & & & 0. & 0. & -0.02 & 0. & -0 . & 0.00 & -0.06 \\
\hline 13 & 6 & & & -0.02 & & -0.01 & 0.0 & -0.06 & 0.01 & 0.02 \\
\hline 14 & 6 & & & & & -0.01 & 0.0 & & 0.00 & 0.02 \\
\hline 15 & 6 & & & & & & -0 . & & & 0.03 \\
\hline 16 & 1 & -0 . & & 0.0 & -0.48 & & 0.0 & 0.1 & -0.03 & -0.02 \\
\hline 17 & 6 & & & -0.02 & -0.01 & 0.00 & 0.0 & 0.0 & -0.01 & -0.03 \\
\hline 18 & 1 & & & -0.08 & -0.02 & & & & & \\
\hline 19 & 6 & & & -0 & -0 . & & 0. & 0. & -0. & \\
\hline 20 & 1 & & & 0.21 & -0.44 & 0.03 & -0.27 & 0.09 & -0.01 & 0.05 \\
\hline 21 & 6 & & & 0.01 & & & $-0 .($ & & & 0.01 \\
\hline 22 & 6 & & & & & -0.04 & 0. & & & \\
\hline 23 & 1 & & & 0.00 & & 0.04 & 0.01 & 0. & 0.04 & 0.00 \\
\hline 24 & 1 & & & & -0 & & -0. & & & 0.00 \\
\hline 25 & 1 & & & 0.0 & -0 . & -0.02 & 0. & & 0.02 & 0.00 \\
\hline 26 & 6 & & & 0.0 & 0.13 & -0.07 & 0.05 & -0.01 & 0.06 & -0.03 \\
\hline 27 & 6 & & & 0. & -0.04 & & -0. & 0. & -0.02 & 0.01 \\
\hline 28 & 1 & & & -0.0 & 0.0 & 0.02 & 0. & & 0.01 & 0.03 \\
\hline 29 & 1 & -0. & & -0.01 & 0.05 & 0.05 & -0.05 & 0.0 & -0.02 & 0.02 \\
\hline 30 & 1 & 0.0 & & 0.01 & -0.03 & 0.09 & -0.12 & 0.00 & 0.00 & 0.02 \\
\hline 31 & 6 & & & -0.02 & 0.04 & 0.08 & 0.01 & -0.13 & 0.12 & 0.03 \\
\hline 32 & 6 & 0.0 & & 0.00 & 0.15 & -0.02 & -0.0 & 0.13 & -0.05 & -0.04 \\
\hline 33 & 6 & -0.05 & 0.01 & 0.00 & -0.07 & -0.06 & 0.00 & -0.12 & -0.10 & 0.01 \\
\hline
\end{tabular}




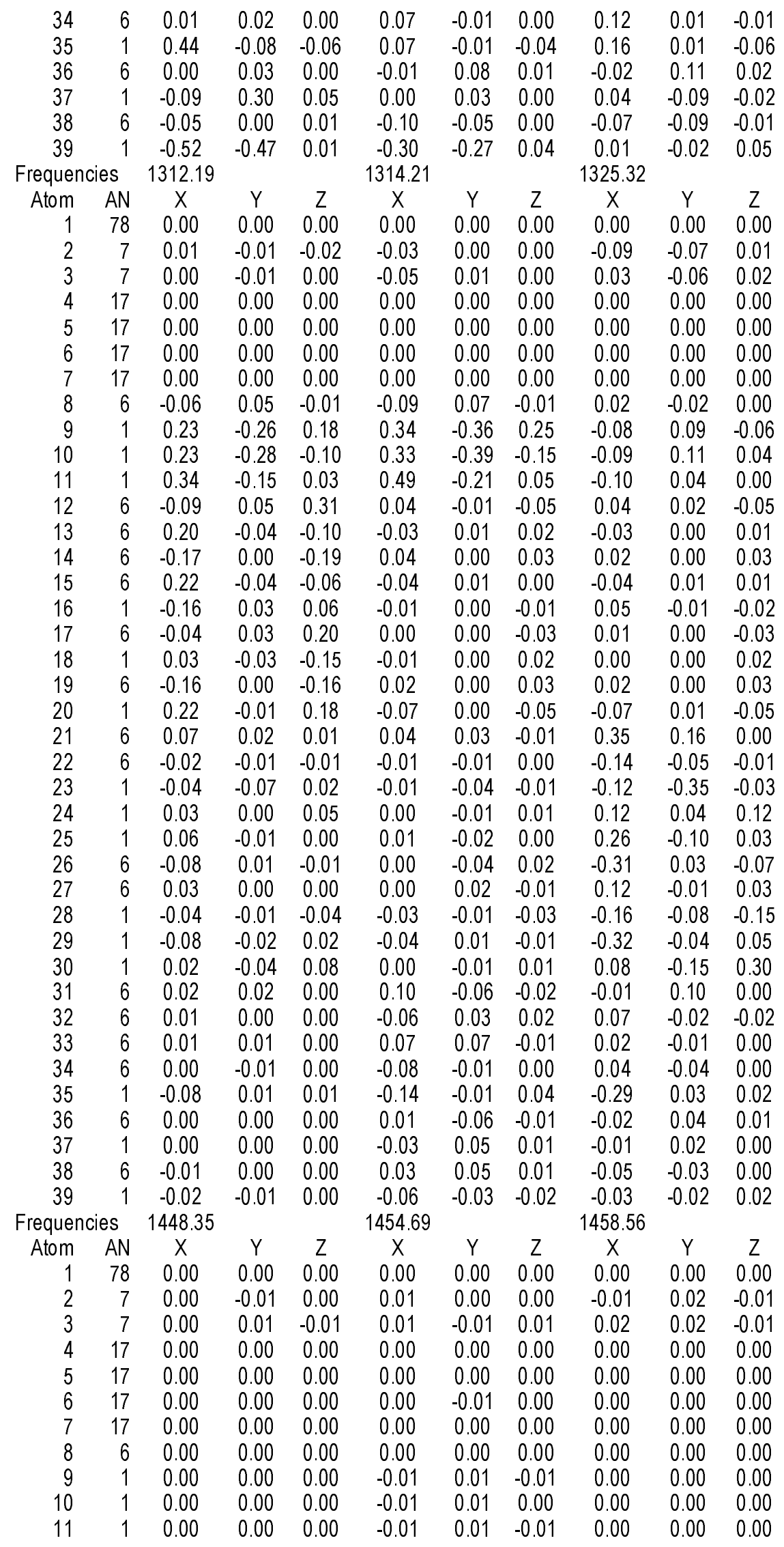




\begin{tabular}{|c|c|c|c|c|c|c|c|c|c|c|}
\hline & & & & & & & & & & \\
\hline $\begin{array}{l}12 \\
13\end{array}$ & $\begin{array}{l}6 \\
6\end{array}$ & $\begin{array}{r}0.01 \\
-0.01\end{array}$ & $\begin{array}{l}0.00 \\
0.00\end{array}$ & $\begin{array}{l}0.00 \\
0.01\end{array}$ & $\begin{array}{c}0.01 \\
-0.01\end{array}$ & $\begin{array}{l}0.00 \\
0.00\end{array}$ & $\begin{array}{l}0.00 \\
0.01\end{array}$ & $\begin{array}{r}-0.03 \\
0.03\end{array}$ & $\begin{array}{r}0.00 \\
-0.01\end{array}$ & $\begin{array}{l}-0.01 \\
-0.02\end{array}$ \\
\hline 14 & 6 & -0.01 & 0.00 & $\begin{array}{l}.0 .01 \\
-0.01\end{array}$ & -0.01 & 0.00 & -0.01 & 0.02 & 0.00 & 0.03 \\
\hline 15 & 6 & 0.00 & 0.00 & 0.00 & 0.00 & 0.00 & 0.01 & 0.01 & 0.00 & -0.01 \\
\hline 16 & 1 & 0.04 & -0.01 & -0.01 & 0.04 & -0.01 & -0.01 & -0.10 & 0.02 & 0.02 \\
\hline 17 & 6 & 0.00 & 0.00 & 0.00 & 0.01 & 0.00 & 0.00 & -0.01 & 0.00 & 0.00 \\
\hline 18 & 1 & 0.01 & 0.00 & 0.00 & 0.01 & 0.00 & 0.0 & -0.02 & 0.00 & 0.01 \\
\hline 19 & 6 & 0.00 & 0.00 & -0.01 & 0.00 & 0.00 & -0.01 & 0.00 & 0.00 & 0.01 \\
\hline 20 & 1 & 0.03 & 0.00 & 0.02 & 0.04 & 0.00 & 0.03 & -0.07 & 0.00 & -0.05 \\
\hline 21 & 6 & 0.00 & & -0.01 & -0.03 & 0.00 & 0. & 0.01 & -0.03 & 0.01 \\
\hline 22 & 6 & -0.04 & -0.08 & 0.03 & 0.00 & -0.02 & 0.01 & 0.03 & 0.07 & -0.03 \\
\hline 23 & 1 & 0.07 & 0.27 & -0.18 & 0.02 & 0.10 & -0.03 & -0.06 & -0.20 & 0.16 \\
\hline 24 & 1 & 0.22 & 0.25 & -0.32 & 0.07 & 0.07 & -0.08 & -0.17 & -0.20 & 0.27 \\
\hline 25 & 1 & 0.13 & 0.46 & 0.09 & 0.01 & 0.12 & 0. & -0.11 & -0.39 & -0.08 \\
\hline 26 & 6 & 0.00 & -0.01 & 0.01 & 0.04 & 0.05 & -0.02 & -0.01 & -0.01 & 0.01 \\
\hline 27 & 6 & -0.03 & 0.07 & -0.04 & -0.01 & -0.03 & 0.0 & -0.04 & 0.07 & -0.05 \\
\hline 28 & 1 & 0.15 & & 0.04 & -0.01 & 0.15 & 0. & 0.18 & -0.38 & \\
\hline 29 & 1 & 0.12 & & 0.38 & 0.11 & 0.05 & -0 . & 0.17 & -0.17 & 0.44 \\
\hline 30 & 1 & 0.05 & -0.30 & 0.09 & 0.00 & 0.00 & -0.05 & 0.06 & -0.35 & 0.10 \\
\hline 31 & 6 & 0.00 & & 0. & -0.17 & -0.09 & & -0.03 & -0.01 & 0.01 \\
\hline 32 & 6 & 0.00 & & & 0.16 & -0.05 & -0. & 0.02 & -0.01 & -0.01 \\
\hline 33 & 6 & 0.00 & & 0.00 & 0.10 & 0.18 & 0. & 0.01 & 0.02 & 0.00 \\
\hline 34 & 6 & 0.00 & & 0. & 0.07 & -0.07 & & 0.00 & & \\
\hline 35 & 1 & 0.02 & & 0.00 & -0.70 & 0.10 & 0.0 & -0.07 & & \\
\hline 36 & 6 & 0.00 & 0.00 & 0.00 & -0.07 & -0.05 & 0.00 & -0.01 & -0.01 & 0.00 \\
\hline 37 & 1 & 0.00 & -0 & 0. & -0.14 & 0.15 & 0. & -0.01 & 0.00 & 0.00 \\
\hline 38 & 6 & 0.00 & & 0.0 & -0.02 & 0.07 & 0.0 & 0.00 & 0.01 & \\
\hline 39 & 1 & 0.01 & 0.00 & 0.00 & -0.36 & -0.27 & 0.01 & -0.06 & -0.05 & 0.00 \\
\hline requen & & 1469.70 & & & 1484.89 & & & 1491.45 & & \\
\hline Atom & AN & $x$ & $Y$ & Z & $X$ & Y & Z & $x$ & Y & Z \\
\hline 1 & 78 & 0.00 & 0.00 & 0.00 & 0.00 & 0.00 & 0.00 & 0.00 & 0.00 & 0.00 \\
\hline 2 & 7 & & -0.03 & 0.00 & 0.00 & 0.00 & 0.00 & & 0.01 & 0.01 \\
\hline 3 & 7 & -0.01 & 0.00 & 0.00 & -0.03 & 0.00 & 0.0 & 0. & 0.00 & 0.00 \\
\hline 4 & 17 & & & 0. & 0.00 & 0.00 & 0.0 & 0. & 0.00 & 0.00 \\
\hline 5 & 17 & & 0. & 0.00 & & 0.00 & 0.0 & & 0.00 & 0.00 \\
\hline 6 & 17 & 0.00 & 0.00 & 0.00 & 0.0 & 0.00 & 0.00 & 0.0 & 0.00 & 0.00 \\
\hline 7 & 17 & & & 0. & & 0.00 & 0.0 & 0.0 & 0.00 & 0.00 \\
\hline 8 & 6 & & & & & & 0.0 & & & \\
\hline 9 & 1 & & & -0 & & 0.0 & 0. & 0. & -0.01 & 0.01 \\
\hline 10 & 1 & -0.04 & & & & 0.01 & -0.01 & 0.02 & 0.03 & -0.02 \\
\hline 11 & 1 & & & & & & -0.01 & -0. & 0.00 & -0.03 \\
\hline 12 & 6 & & & & & 0.1 & & & -0.02 & -0.12 \\
\hline 13 & 6 & -0.1 & & 0. & & 0.00 & 0.0 & 0.0 & 0.01 & 0.10 \\
\hline 14 & 6 & -0.0 & & -0 . & & 0.00 & 0.0 & -0.0 & & 0.06 \\
\hline 15 & 6 & & & & & & & & & \\
\hline 16 & 1 & & & -0 & -0.02 & 0. & 0. & -0 . & 0.08 & 0.19 \\
\hline 17 & 6 & & & 0.02 & 0.00 & 0.00 & 0.0 & 0.03 & -0.02 & -0.11 \\
\hline 18 & 1 & & & -0. & & & 0. & & 0.10 & 0.66 \\
\hline 19 & 6 & & & & & 0. & 0. & -0 . & 0.01 & \\
\hline 20 & 1 & & & & 0. & 0.00 & 0. & 0. & 0.00 & 0.38 \\
\hline 21 & 6 & & & -0. & & & & & & 0.00 \\
\hline 22 & 6 & & & & & 0. & 0. & & 0.00 & \\
\hline 23 & 1 & -0.0 & & 0. & & 0.03 & -0.01 & 0.02 & 0.02 & -0.06 \\
\hline 24 & 1 & -0.1 & & & & -0.02 & 0. & & -0.01 & 0.03 \\
\hline 25 & 1 & -0.2 & & -0.05 & 0.02 & -0.05 & 0.0 & & -0.02 & 0.01 \\
\hline 26 & 6 & 0.0 & & 0.00 & 0.01 & 0.02 & -0.0 & 0.00 & 0.00 & 0.00 \\
\hline 27 & 6 & -0.0 & & -0.1 & -0.01 & 0.00 & 0.00 & 0.00 & 0.00 & 0.00 \\
\hline 28 & 1 & 0.0 & & & 0.02 & 0.05 & 0.02 & & 0.02 & 0.00 \\
\hline 29 & 1 & 0.0 & & 0.12 & 0.15 & 0.01 & -0.0 & 0.01 & 0.01 & -0.01 \\
\hline 30 & 1 & 0.02 & -0.08 & -0.01 & 0.03 & -0.13 & -0.01 & 0.00 & -0.01 & 0.00 \\
\hline
\end{tabular}




\begin{tabular}{|c|c|c|c|c|c|c|c|c|c|c|}
\hline 31 & 6 & 0.01 & 0.00 & 0.00 & 0.07 & -0.10 & -0.02 & 0.00 & 0.00 & 0.00 \\
\hline & 6 & 0.00 & 0.00 & 0.00 & 0.01 & 0.11 & 0.02 & 0.00 & 0.00 & 0.00 \\
\hline 33 & 6 & 0.00 & 0.00 & 0.00 & -0.09 & 0.02 & 0.01 & 0.00 & 0.00 & 0.00 \\
\hline 34 & 6 & 0.00 & 0.00 & 0.00 & 0.13 & 0.02 & & & 0.00 & 0.00 \\
\hline 35 & 1 & 0.01 & 0.00 & 0.00 & -0.34 & 0.14 & 0.04 & 0.01 & 0.00 & 0.00 \\
\hline 36 & 6 & 0.00 & & & 0.04 & -0.06 & & & & .00 \\
\hline 37 & 1 & 0.00 & 1.00 & 0.0 & -0.14 & 0.56 & & 0.00 & -0.02 & .00 \\
\hline 38 & 6 & 0.00 & 0.00 & 0.00 & -0.13 & -0.10 & 0.00 & 0.00 & 0.00 & 0.00 \\
\hline 39 & 1 & 0.01 & 0.01 & 0.00 & 0.41 & 0.47 & 0.04 & -0.01 & -0.01 & 0.00 \\
\hline \multicolumn{2}{|c|}{ Frequencies } & \multicolumn{2}{|l|}{1501.24} & & \multicolumn{2}{|l|}{1502.76} & & \multicolumn{3}{|l|}{1513.37} \\
\hline Atom & AN & X & $\mathrm{Y}$ & Z & X & $\mathrm{Y}$ & Z & $x$ & $\mathrm{Y}$ & Z \\
\hline 1 & 78 & 0.00 & 0.00 & 0.00 & 0.00 & 0.00 & 0.00 & 0.00 & 0.00 & 0.00 \\
\hline 2 & 7 & 0.00 & 0.00 & 0.00 & -0.03 & 0.04 & -0.02 & 0.00 & 0.00 & 0.00 \\
\hline 3 & 7 & 0.00 & 0.00 & 0.00 & -0.01 & -0.02 & 0.01 & & 0.00 & 0.00 \\
\hline 4 & 17 & 0.00 & 0.00 & 0.00 & 0.00 & 0.00 & 0.00 & & 0.00 & 0.00 \\
\hline 5 & 17 & 0.00 & 0.00 & 0.00 & & 0.00 & 0.0 & & & .00 \\
\hline 6 & 17 & 0.00 & 0.00 & 0.00 & 0.0 & 0.00 & 0.00 & & 0.00 & 0.00 \\
\hline 7 & 17 & 0.00 & 0.00 & 0.00 & 0.00 & 0.00 & 0.00 & & 0.00 & 0.00 \\
\hline 8 & 6 & -0.04 & -0.05 & 0.04 & 0.0 & 0.00 & 0.00 & -0.02 & .03 & -0.06 \\
\hline 9 & 1 & 0.35 & 0.10 & 0.29 & -0.01 & 0.01 & -0.01 & 0.27 & 0.58 & 0.18 \\
\hline 10 & 1 & 0.44 & 0.43 & -0.40 & -0.02 & -0.02 & 0.02 & 0.12 & -0.28 & -0.06 \\
\hline 11 & 1 & -0.31 & 0.07 & -0.35 & 0.01 & 0.00 & 0.0 & -0.1 & 0.13 & 0.64 \\
\hline 12 & 6 & 0.01 & & 0.00 & 0.03 & & 0.1 & & & \\
\hline 13 & 6 & -0.01 & 0.00 & 0.00 & -0.01 & 0.00 & 0.0 & 0.1 & .00 & 0.00 \\
\hline 14 & 6 & 0.00 & 0.00 & -0.01 & 0.00 & 0.00 & -0.01 & 0. & 1.00 & 0.00 \\
\hline 15 & 6 & 0.00 & & 0.00 & -0.0 & & 0. & & & \\
\hline 16 & 1 & & & & & & $-0 .(1-x-1$ & & & \\
\hline 17 & 6 & 0.00 & 0.00 & 0.00 & & 0.00 & 0.0 & & & 00 \\
\hline 18 & 1 & 0.1 & & -0.02 & & -0.01 & -0. & & & \\
\hline 19 & 6 & & & 0. & & & $-0 .(1-x-1$ & & & \\
\hline 20 & 1 & 0.00 & & & & 0.00 & 0.0 & & & 0.00 \\
\hline 21 & 6 & 0.0 & & 0.0 & 0. & -0.04 & 0. & & & .00 \\
\hline 22 & 6 & 0.00 & & 0.1 & -0.8 & -0.01 & -0.0 & & & \\
\hline 23 & 1 & 0.00 & & & & 0. & -0.2 & & & \\
\hline 24 & 1 & 0. & & 0.0 & & -0.06 & 0.4 & & & \\
\hline 25 & 1 & & & 0.00 & 0.4 & -0.25 & 0.0 & & 0.00 & \\
\hline 26 & 6 & 0.00 & & 0. & & & -0 . & 0. & & \\
\hline 27 & 6 & & & & & & & & & \\
\hline 28 & 1 & & & 0. & 0. & 0. & 0. & & & \\
\hline 29 & 1 & & & & & & -0 . & & & \\
\hline 30 & 1 & & & & & -0.28 & & & & \\
\hline 31 & 6 & 0.00 & & 0.0 & 0.0 & 0.0 & 0.0 & 0. & 0.0 & \\
\hline 32 & 6 & & & 0. & & & 0. & 0. & & \\
\hline 33 & 6 & & & & & & & & & \\
\hline 34 & 6 & 0.00 & & 0.1 & -0. & & & & & \\
\hline 35 & 1 & & & & & & & 0. & 0.00 & \\
\hline 36 & 6 & 0.00 & & & & & & & & \\
\hline 37 & 1 & 0.00 & & & & & & & & \\
\hline 38 & 6 & & & & & & 0. & & & \\
\hline 39 & 1 & -0.01 & & 0.00 & -0.02 & -0.03 & 0.00 & & 0.00 & 0.00 \\
\hline \multicolumn{2}{|c|}{ Frequencies } & 1519.95 & & & & & & & & \\
\hline Atom & AN & X & Y & Z & $x$ & Y & Z & $x$ & Y & \\
\hline & 78 & 0.00 & 0.00 & 0.00 & 0.00 & 0.00 & 0.00 & 0. & 0.00 & 0.0 \\
\hline 2 & 7 & & -0.03 & 0.01 & & & -0.01 & & -0.01 & 0.01 \\
\hline & 7 & & & 0.02 & & 0.03 & -0.02 & & -0.02 & 0.01 \\
\hline 4 & 17 & & & 0.00 & 0.0 & 0.00 & 0.00 & 0.00 & 0.00 & 0.00 \\
\hline 5 & 17 & & & & & 0.00 & 0.0 & & 0.00 & 0. \\
\hline 6 & 17 & 0.0 & 0.0 & 0.00 & 0.0 & 0.00 & 0.0 & & 0.00 & 0.0 \\
\hline 7 & 17 & & 0.0 & 0.00 & 0.00 & 0.00 & 0.00 & 0.00 & 0.00 & 0.00 \\
\hline 8 & 6 & 0. & 0.00 & 0.00 & 0.00 & 0.00 & 0.00 & 0.00 & 0.00 & 0.00 \\
\hline
\end{tabular}




\begin{tabular}{|c|c|c|c|c|c|c|c|c|c|c|}
\hline & & & & & & & & & & \\
\hline 9 & 1 & -0.03 & -0.04 & -0.02 & 0.00 & 0.00 & 0.00 & 0.00 & -0.01 & 0.00 \\
\hline 10 & 1 & -0.02 & 0.00 & 0.01 & 0.00 & 0.00 & 0.00 & 0.00 & 0.00 & 0.00 \\
\hline 11 & 1 & 0.02 & -0.01 & -0.02 & 0.00 & 0.00 & 0.00 & 0.00 & 0.00 & -0.01 \\
\hline 12 & 6 & -0.02 & 0.00 & -0.01 & 0.01 & 0.00 & 0.00 & -0.01 & 0.00 & 0.00 \\
\hline 13 & 6 & 0.01 & 0.00 & 0.00 & 0.00 & 0.00 & 0.00 & 0.00 & 0.00 & 0.00 \\
\hline 14 & 6 & 0.00 & & 0.01 & 0.00 & 0.00 & & & .00 & 0.00 \\
\hline 15 & 6 & 0.01 & 0.00 & 0.00 & 0.00 & 0.00 & 0.0 & & 0.00 & 0.00 \\
\hline 16 & 1 & -0.04 & 0.01 & 0.01 & 0.00 & 0.00 & 0.00 & -0.01 & 0.00 & 0.01 \\
\hline 17 & 6 & 0.00 & 0.00 & -0.01 & 0.00 & 0.00 & 0.00 & 0.00 & 0.00 & -0.01 \\
\hline 18 & 1 & -0.01 & & 0.02 & 0.00 & 0.00 & 0.0 & -0.01 & 0.00 & 0.02 \\
\hline 19 & 6 & 0.00 & 0.00 & 0.01 & 0.00 & 0.00 & 0.0 & & 0.00 & 0.00 \\
\hline 20 & 1 & -0.02 & 0.00 & -0.01 & 0.00 & 0.00 & 0.00 & 0.00 & 0.00 & 0.00 \\
\hline 21 & 6 & 0.02 & 0.03 & -0.02 & 0.01 & -0.04 & -0.01 & 0.01 & 0.01 & -0.01 \\
\hline 22 & 6 & 0.02 & -0.02 & -0.01 & -0.01 & -0.03 & -0.0 & 0.0 & -0.02 & -0.02 \\
\hline 23 & 1 & -0.16 & 0.00 & 0.37 & -0.15 & 0.44 & 0.32 & -0.13 & 0.11 & 0.29 \\
\hline 24 & 1 & 0.16 & 0.13 & -0.10 & 0.47 & 0.15 & 0.28 & 0.21 & 0.11 & 0.01 \\
\hline 25 & 1 & -0.40 & & -0.04 & -0.14 & -0.15 & -0.0 & -0.28 & -0.01 & -0.04 \\
\hline 26 & 6 & -0.02 & & -0.01 & 0.00 & -0.03 & 0.03 & 0.01 & 0.01 & -0.03 \\
\hline 27 & 6 & -0.02 & 0.00 & 0.03 & 0.03 & 0.01 & 0.0 & -0.01 & -0.01 & -0.04 \\
\hline 28 & 1 & -0.19 & & -0.08 & -0.20 & -0.22 & -0 . & 0.49 & 0.11 & 0.30 \\
\hline 29 & 1 & 0.49 & & -0.06 & -0.25 & -0.07 & 0.1 & -0.14 & & -0.16 \\
\hline 30 & 1 & 0.18 & & -0.24 & & 0.23 & -0.22 & -0.18 & 0.05 & 0.57 \\
\hline 31 & 6 & 0.02 & & 0.00 & -0.02 & -0.01 & 0.0 & 0.01 & 0.00 & 0.00 \\
\hline 32 & 6 & & & 0.00 & & 0.00 & 0.00 & -0.01 & & 0.00 \\
\hline 33 & 6 & 0.01 & -0.01 & 0.00 & 0.00 & 0.00 & 0.00 & 0.00 & 0.00 & 0.00 \\
\hline 34 & 6 & -0.01 & & 0.00 & 0.00 & 0.01 & 0.0 & 0.00 & .00 & 0.00 \\
\hline 35 & 1 & 0.04 & & 0.0 & -0.01 & 0.01 & 0.0 & -0.01 & 0.00 & \\
\hline 36 & 6 & 0.00 & 0.02 & 0.00 & 0.01 & -0.01 & 0.00 & 0.00 & 0.00 & 0.00 \\
\hline 37 & 1 & 0.02 & & -0.01 & -0.01 & 0.04 & 0. & 0.00 & 0.00 & 0.00 \\
\hline 38 & 6 & 0.01 & & 0.0 & -0.01 & 0.00 & 0. & 0. & .00 & 0 \\
\hline 39 & 1 & -0.01 & & 0.00 & & 0.02 & & 0.00 & 0.00 & 0.00 \\
\hline Erequen & & 1569.29 & & & 1583.27 & & & 1595.87 & & \\
\hline Atom & AN & $x$ & $Y$ & Z & $x$ & $Y$ & Z & $x$ & $Y$ & Z \\
\hline & 78 & 0.00 & 0.00 & 0.00 & & 0.00 & 0.00 & 0.00 & 0.00 & 0.00 \\
\hline 2 & 7 & & & & & 0.15 & -0.07 & & -0.01 & 0.02 \\
\hline 3 & 7 & -0.06 & -0.05 & 0.03 & 0.1 & 0.15 & -0.08 & -0.0 & -0.01 & 0.00 \\
\hline 4 & 17 & & & 0. & & 0.00 & 0.0 & 0.0 & 0.00 & 0.00 \\
\hline 5 & 17 & & & & & 0.00 & 0. & & & 0.00 \\
\hline 6 & 17 & & & 0. & & 0.00 & 0.1 & 0. & 0.00 & 0.00 \\
\hline 7 & 17 & & & & & 0.00 & 0.0 & 0.0 & 0.00 & 0.00 \\
\hline 8 & 6 & & & & & 0.00 & 0. & 0. & 0.00 & 0.00 \\
\hline 9 & 1 & & & -0 . & & 0.01 & 0. & & & 0.00 \\
\hline 10 & 1 & -0. & & 0. & & 0.01 & -0.0 & -0.0 & -0.01 & 0.01 \\
\hline 11 & 1 & & & & & 0.00 & 0.0 & 0. & 0.00 & 0.01 \\
\hline 12 & 6 & & & & & -0.01 & & & & -0.27 \\
\hline 13 & 6 & & & & -0 . & 0.01 & 0. & -0 . & 0. & 0.15 \\
\hline 14 & 6 & & & 0. & & 0.00 & -0.01 & 0.04 & 0.02 & 0.19 \\
\hline 15 & 6 & & & & & 0.00 & & & & -0.20 \\
\hline 16 & 1 & & & & & 0.00 & -0 . & -0 . & 0.02 & -0.12 \\
\hline 17 & 6 & & & & & 0.00 & 0.02 & -0.09 & 0.06 & 0.40 \\
\hline 18 & 1 & & & & & -0.01 & -0 . & & & -0.64 \\
\hline 19 & 6 & 0. & & 0. & 0.1 & 0.00 & -0 . & -0 . & -0.03 & -0.22 \\
\hline 20 & 1 & & & & & 0.00 & 0.0 & 0.23 & -0.04 & -0.03 \\
\hline 21 & 6 & -0. & & -0 & & -0.22 & 0. & 0. & 0.02 & -0.01 \\
\hline 22 & 6 & & & & & 0.03 & 0. & & & 0.00 \\
\hline 23 & 1 & 0.0 & & -0. & -0.05 & -0.27 & 0.11 & -0.01 & 0.02 & 0.02 \\
\hline 24 & 1 & 0.0 & & 0.16 & 0.01 & 0.17 & -0.34 & 0.02 & 0.01 & 0.01 \\
\hline 25 & 1 & 0.0 & & 0.0 & -0.17 & 0.30 & 0.01 & -0.03 & -0.02 & 0.00 \\
\hline 26 & 6 & & & -0.0 & -0.09 & -0.18 & 0.10 & 0.00 & 0.01 & -0.01 \\
\hline 27 & 6 & 0.01 & 0.00 & 0.01 & -0.02 & 0.01 & -0.02 & 0.00 & 0.00 & 0.00 \\
\hline
\end{tabular}




\begin{tabular}{|c|c|c|c|c|c|c|c|c|c|c|}
\hline & & & & & & & & & & \\
\hline 28 & 1 & -0.04 & -0.25 & -0.04 & 0.02 & 0.34 & 0.04 & 0.01 & -0.01 & 0.01 \\
\hline 29 & 1 & -0.16 & -0.08 & 0.16 & 0.15 & 0.13 & -0.24 & -0.02 & 0.00 & 0.00 \\
\hline 30 & 1 & -0.01 & 0.16 & -0.11 & 0.00 & -0.17 & 0.14 & -0.01 & 0.01 & 0.01 \\
\hline 31 & 6 & 0.10 & -0.13 & -0.03 & -0.03 & -0.10 & 0.00 & 0.00 & 0.01 & 0.00 \\
\hline 32 & 6 & -0.17 & 0.05 & 0.04 & -0.03 & 0.05 & 0.01 & 0.00 & 0.00 & 0.00 \\
\hline 33 & 6 & -0.05 & 0.08 & 0.01 & 0.01 & 0.06 & 0.00 & 0.00 & 0.00 & 0.00 \\
\hline 34 & 6 & 0.20 & -0.14 & -0.03 & 0.04 & -0.07 & -0.01 & 0.00 & 0.00 & 0.00 \\
\hline 35 & 1 & -0.34 & -0.03 & 0.02 & -0.05 & -0.06 & 0.00 & 0.00 & 0.00 & 0.00 \\
\hline 36 & 6 & -0.15 & 0.27 & 0.05 & -0.04 & 0.17 & 0.02 & 0.00 & -0.01 & 0.00 \\
\hline 37 & 1 & 0.08 & -0.55 & -0.08 & 0.09 & -0.26 & -0.04 & 0.00 & 0.01 & 0.00 \\
\hline 38 & 6 & 0.11 & -0.08 & -0.03 & -0.01 & -0.10 & -0.02 & 0.00 & 0.01 & 0.00 \\
\hline 39 & 1 & 0.00 & -0.22 & -0.04 & 0.11 & 0.01 & -0.01 & -0.01 & 0.00 & 0.00 \\
\hline Frequ & ccies & 1606.31 & & & 1615.25 & & & 1641.87 & & \\
\hline Atom & AN & X & $Y$ & Z & $X$ & $Y$ & Z & $x$ & Y & Z \\
\hline 1 & 78 & 0.00 & 0.00 & 0.00 & 0.00 & 0.00 & 0.00 & 0.00 & 0.00 & 0.00 \\
\hline 2 & 7 & -0.01 & 0.00 & 0.00 & 0.07 & -0.08 & 0.04 & 0.14 & -0.19 & 0.09 \\
\hline 3 & 7 & -0.01 & -0.01 & 0.01 & -0.02 & 0.00 & 0.00 & 0.17 & 0.22 & -0.12 \\
\hline 4 & 17 & 0.00 & 0.00 & 0.00 & 0.00 & 0.00 & 0.00 & 0.00 & 0.00 & 0.00 \\
\hline 5 & 17 & 0.00 & 0.00 & 0.00 & 0.00 & 0.00 & 0.00 & 0.00 & 0.00 & 0.00 \\
\hline 6 & 17 & 0.00 & 0.00 & 0.00 & 0.00 & 0.00 & 0.00 & 0.00 & 0.00 & 0.00 \\
\hline 7 & 17 & 0.00 & 0.00 & 0.00 & 0.00 & 0.00 & 0.00 & 0.00 & 0.00 & 0.00 \\
\hline 8 & 6 & 0.00 & 0.00 & 0.00 & 0.00 & 0.00 & 0.00 & 0.00 & 0.00 & 0.00 \\
\hline 9 & 1 & -0.01 & 0.00 & -0.01 & 0.00 & 0.00 & 0.00 & 0.02 & -0.02 & 0.01 \\
\hline 10 & 1 & -0.01 & 0.00 & 0.01 & 0.00 & 0.00 & 0.00 & 0.01 & -0.01 & -0.01 \\
\hline 11 & 1 & 0.00 & 0.00 & 0.00 & -0.01 & 0.00 & 0.00 & 0.00 & 0.01 & 0.00 \\
\hline 12 & 6 & 0.14 & -0.01 & 0.04 & -0.02 & 0.00 & -0.01 & -0.05 & 0.01 & -0.01 \\
\hline 13 & 6 & -0.25 & 0.03 & -0.03 & -0.01 & 0.00 & -0.01 & 0.00 & 0.00 & -0.01 \\
\hline 14 & 6 & -0.24 & 0.02 & -0.09 & -0.01 & 0.00 & 0.00 & -0.01 & 0.00 & 0.01 \\
\hline 15 & 6 & 0.33 & -0.05 & -0.04 & 0.02 & 0.00 & 0.00 & 0.01 & 0.00 & 0.00 \\
\hline 16 & 1 & -0.47 & 0.10 & 0.21 & -0.03 & 0.01 & 0.01 & -0.0 & 0.00 & 0.01 \\
\hline 17 & 6 & -0.17 & 0.02 & -0.05 & -0.01 & 0.00 & 0.00 & -0.01 & 0.00 & 0.00 \\
\hline 18 & 1 & -0.20 & 0.03 & -0.02 & -0.01 & 0.00 & 0.00 & -0.01 & 0.00 & 0.00 \\
\hline 19 & 6 & 0.28 & -0.02 & 0.19 & 0.01 & 0.00 & 0.01 & 0.0 & 0.00 & 0.01 \\
\hline 20 & 1 & -0.35 & 0.00 & -0.39 & -0.02 & 0.00 & -0.02 & -0.0 & 0.0 & -0.02 \\
\hline 21 & 6 & -0.01 & 0.01 & 0.00 & -0.06 & 0.11 & -0.05 & -0.12 & 0.30 & -0.13 \\
\hline 22 & 6 & 0.00 & 0.00 & 0.00 & 0.00 & -0.01 & 0.00 & 0.00 & -0.04 & 0.01 \\
\hline 23 & 1 & 0.01 & -0.01 & -0.02 & 0.0 & 0.11 & -0.04 & 0.0 & 0.25 & -0.07 \\
\hline 24 & 1 & -0.0 & -0.02 & 0.01 & -0.01 & -0.08 & 0.13 & $-0 .($ & -0.16 & 0.26 \\
\hline 25 & 1 & 0.0 & -0.02 & 0.00 & 0.0 & -0.12 & -0.01 & 0.0 & -0.25 & -0.01 \\
\hline 26 & 6 & 0.0 & 0.01 & -0.01 & 0.02 & 0.00 & 0.00 & -0.12 & -0.33 & 0.18 \\
\hline 27 & 6 & 0.00 & 0.00 & 0.00 & 0.00 & 0.00 & 0.00 & 0.0 & 0.03 & -0.03 \\
\hline 28 & 1 & & -0.02 & 0.00 & & -0.04 & 0.0 & & 0. & 0.00 \\
\hline 29 & 1 & -0.01 & -0.01 & 0.02 & -0.05 & -0.01 & 0.01 & 0.1 & 0.13 & -0.25 \\
\hline 30 & 1 & 0.00 & 0.01 & -0.01 & -0.02 & 0.04 & 0.02 & 0.02 & -0.19 & 0.17 \\
\hline 31 & 6 & 0.0 & 0.01 & 0.00 & & & 0.01 & $-0 .($ & & 0.01 \\
\hline 32 & 6 & -0. & 0.00 & 0.0 & & 0.0 & -0.01 & -0. & -0.01 & 0.00 \\
\hline 33 & 6 & -0.01 & -0.01 & 0.00 & 0.24 & 0.15 & -0.02 & -0.07 & -0.02 & 0.01 \\
\hline 34 & 6 & 0.01 & 0.00 & 0.00 & & -0.06 & 0.02 & 0.0 & 0.00 & -0.01 \\
\hline 35 & 1 & -0.0 & 0.01 & 0.0 & 0.4 & -0.2 & -0.04 & -0.1 & 0.05 & 0.01 \\
\hline 36 & 6 & 0.00 & -0.01 & 0.00 & & 0.20 & 0.01 & -0.03 & -0.02 & 0.00 \\
\hline 37 & 1 & -0.0 & 0.01 & 0.00 & & -0.23 & -0.05 & -0.05 & 0.03 & 0.01 \\
\hline 38 & 6 & 0.0 & 0.01 & 0.00 & -0.2 & -0.25 & -0.01 & 0.0 & 0.04 & 0.00 \\
\hline & 1 & -0.02 & -0.01 & 0.00 & 0.35 & 0.31 & 0.03 & -0.06 & -0.07 & 0.00 \\
\hline Frequer & ies & 3025.86 & & & 3057.58 & & & 3062.17 & & \\
\hline Atom & AN & X & $Y$ & Z & X & $Y$ & Z & X & $Y$ & Z \\
\hline & 78 & 0.00 & 0.00 & 0.00 & & 0.0 & 0.00 & 0.00 & 0.00 & 0.00 \\
\hline 2 & 7 & 0.00 & 0.00 & 0.00 & 0.00 & 0.00 & 0.00 & 0.00 & 0.00 & 0.00 \\
\hline 3 & 7 & 0.00 & 0.00 & 0.00 & 0.00 & 0.00 & 0.00 & 0.00 & 0.00 & 0.00 \\
\hline 4 & 17 & 0.00 & 0.00 & 0.00 & 0.00 & 0.00 & 0.00 & 0.0 & 0.00 & 0.00 \\
\hline & 17 & 0.00 & 0.00 & 0.00 & 0.00 & 0.00 & 0.00 & 0.00 & 0.00 & 0.00 \\
\hline
\end{tabular}




\begin{tabular}{|c|c|c|c|c|c|c|c|c|c|c|}
\hline & & & & & & & & & & \\
\hline 6 & 17 & 0.00 & 0.00 & 0.00 & 0.00 & 0.00 & 0.00 & 0.00 & 0.00 & 0.00 \\
\hline 7 & 17 & 0.00 & 0.00 & 0.00 & 0.00 & 0.00 & 0.00 & 0.00 & 0.00 & 0.00 \\
\hline 8 & 6 & -0.03 & 0.03 & -0.01 & 0.00 & 0.00 & 0.00 & 0.00 & 0.00 & 0.00 \\
\hline 9 & 1 & 0.29 & 0.03 & -0.43 & 0.00 & 0.00 & 0.00 & 0.00 & 0.00 & 0.00 \\
\hline 10 & 1 & 0.34 & 0.14 & 0.53 & 0.00 & 0.00 & 0.00 & 0.00 & 0.00 & 0.00 \\
\hline 11 & 1 & -0.25 & -0.49 & 0.05 & 0.00 & 0.00 & 0.00 & 0.00 & 0.00 & 0.00 \\
\hline 12 & 6 & 0.00 & 0.00 & 0.00 & 0.00 & 0.00 & 0.0 & 0.00 & .00 & 0.00 \\
\hline 13 & 6 & 0.00 & 0.00 & 0.00 & 0.00 & 0.00 & 0.00 & 0.00 & 0.00 & 0.00 \\
\hline 14 & 6 & 0.00 & 0.00 & 0.00 & 0.00 & 0.00 & 0.00 & 0.00 & 0.00 & 0.00 \\
\hline 15 & 6 & 0.00 & 0.00 & 0.00 & 0.00 & 0.00 & 0.0 & 0.00 & 0.00 & 0.00 \\
\hline 16 & 1 & 0.00 & 0.00 & 0.00 & 0.00 & 0.00 & 0.0 & 0.00 & 0.00 & 0.00 \\
\hline 17 & 6 & 0.00 & 0.00 & 0.00 & 0.00 & 0.00 & 0.00 & 0.00 & 0.00 & 0.00 \\
\hline 18 & 1 & 0.00 & 0.00 & 0.00 & 0.00 & 0.00 & 0.0 & 0.00 & 0.00 & 0.00 \\
\hline 19 & 6 & 0.00 & 0.00 & 0.00 & 0.00 & 0.00 & 0.00 & 0.00 & 0.00 & 0.00 \\
\hline 20 & 1 & 0.00 & 0.00 & 0.00 & 0.00 & 0.00 & 0.00 & 0.00 & 0.00 & 0.00 \\
\hline 21 & 6 & 0.00 & 0.00 & 0.00 & 0.00 & 0.00 & 0.00 & 0.00 & 0.00 & 0.00 \\
\hline 22 & 6 & 0.00 & 0.00 & 0.00 & 0.01 & 0.03 & -0.03 & 0.01 & 0.02 & -0.02 \\
\hline 23 & 1 & 0.00 & 0.00 & 0.00 & -0.26 & 0.01 & -0.13 & -0.20 & 0.01 & -0.10 \\
\hline 24 & 1 & 0.00 & 0.00 & 0.00 & 0.19 & -0.29 & -0.14 & 0.14 & -0.22 & -0.11 \\
\hline 25 & 1 & 0.00 & 0.00 & 0.00 & -0.07 & -0.06 & 0.63 & -0.05 & -0.05 & 0.48 \\
\hline 26 & 6 & 0.00 & 0.00 & 0.00 & 0.00 & 0.00 & 0.0 & 0.00 & 0.00 & 0.00 \\
\hline 27 & 6 & 0.00 & 0.00 & 0.00 & 0.00 & -0.02 & 0.0 & 0.00 & 0.03 & -0.03 \\
\hline 28 & 1 & 0.00 & 0.00 & 0.00 & 0.25 & 0.03 & -0.3 & -0.32 & -0.04 & 0.50 \\
\hline 29 & 1 & 0.00 & & 0.00 & -0.02 & 0.29 & 0.15 & 0.02 & -0.39 & \\
\hline 30 & 1 & 0.00 & 0.00 & 0.00 & -0.20 & -0.06 & -0.05 & 0.28 & 0.09 & 0.07 \\
\hline 31 & 6 & 0.00 & 0.00 & 0.00 & 0.00 & 0.00 & 0.00 & 0.00 & 0.00 & 0.00 \\
\hline 32 & 6 & 0.00 & 0.00 & 0.00 & 0.00 & 0.00 & 0.00 & 0.00 & 0.00 & 0.00 \\
\hline 33 & 6 & 0.00 & 0.00 & 0.00 & 0.00 & 0.00 & 0.00 & 0.00 & 0.00 & 0.00 \\
\hline 34 & 6 & 0.00 & 0.00 & 0.00 & 0.00 & 0.00 & 0.00 & 0.00 & 0.00 & 0.00 \\
\hline 35 & 1 & 0.00 & & 0.00 & 0.00 & 0.00 & 0.0 & 0.0 & 0.00 & 0 \\
\hline 36 & 6 & 0.00 & 0. & 0.0 & 0.00 & 0.00 & 0.00 & 0.00 & 0.00 & 0.00 \\
\hline 37 & 1 & 0.00 & 0.00 & 0.00 & 0.00 & 0.00 & 0.00 & 0.00 & 0.00 & 0.00 \\
\hline 38 & 6 & 0.00 & 0. & 0.0 & 0.00 & 0.00 & 0.00 & 0.0 & 0.00 & 0.00 \\
\hline 39 & 1 & 0.00 & & 0.00 & 0.00 & 0.00 & 0.00 & 0.00 & 0.00 & \\
\hline equen & & 3114.12 & & & 3121.54 & & & 3126.67 & & \\
\hline Atom & AN & $X$ & $Y$ & Z & $X$ & $\mathrm{Y}$ & Z & $X$ & Y & Z \\
\hline 1 & 78 & 0.00 & 0.00 & 0.00 & 0.00 & 0.00 & 0.00 & 0.00 & 0.00 & 0.00 \\
\hline 2 & 7 & & & & & 0.00 & 0.0 & & 0.00 & 0.00 \\
\hline 3 & 7 & & & 0.00 & 0.00 & 0.00 & 0. & 0. & 0.00 & 0.00 \\
\hline 4 & 17 & & 0. & 0.00 & 0.00 & 0.00 & 0.00 & 0.0 & 0.00 & 0.00 \\
\hline 5 & 17 & & & 0. & & 0.00 & 0.00 & 0.00 & 0.00 & 0.00 \\
\hline 6 & 17 & & & & & 0.00 & & & & 0.00 \\
\hline 7 & 17 & & & 0.1 & & 0.00 & 0.00 & 0.00 & 0.00 & 0.00 \\
\hline 8 & 6 & & & -0.06 & 0.00 & 0.00 & 0.00 & 0.05 & 0.04 & -0.07 \\
\hline 9 & 1 & & & & & 0.00 & & & -0.01 & 0.67 \\
\hline 10 & 1 & & & & & 0.0 & -0. & 0. & 0.06 & 0.15 \\
\hline 11 & 1 & & & -0.08 & -0.01 & -0.01 & 0.00 & -0.22 & -0.47 & 0.03 \\
\hline 12 & 6 & 0.00 & & & & & & & 0.00 & 0.00 \\
\hline 13 & 6 & & & 0. & & 0. & 0. & & & \\
\hline 14 & 6 & & & 0. & & 0.00 & 0.00 & 0.00 & 0.00 & 0.00 \\
\hline 15 & 6 & 0.0 & & & & 0.00 & 0.0 & & 0.00 & 0.00 \\
\hline 16 & 1 & 0.0 & & 0.0 & 0.00 & 0.00 & 0. & & 0.00 & 0.00 \\
\hline 17 & 6 & & & & & 0.00 & 0.0 & 0.0 & 0.00 & \\
\hline 18 & 1 & 0.00 & 0. & 0.0 & 0.00 & 0.00 & 0.00 & 0.00 & 0.00 & 0.00 \\
\hline 19 & 6 & & & & & & 0.00 & & 0.00 & 0.00 \\
\hline 20 & 1 & & & 0.0 & 0.00 & & 0.0 & 0. & 0.00 & \\
\hline 21 & 6 & 0.00 & 0.0 & 0.00 & 0.00 & 0.00 & 0.00 & 0.00 & 0.00 & 0.00 \\
\hline 22 & 6 & 0.0 & & 0.0 & -0.01 & 0.02 & 0.03 & 0.00 & 0.00 & \\
\hline 23 & 1 & 0.0 & 0.0 & 0.0 & -0.09 & 0.01 & -0.0 & 0.0 & 0.00 & 0. \\
\hline 24 & 1 & 0.00 & 0.00 & 0.00 & 0.14 & -0.22 & -0.09 & 0.00 & 0.00 & 0.00 \\
\hline
\end{tabular}




\begin{tabular}{|c|c|c|c|c|c|c|c|c|c|c|}
\hline & & & & & & & & & & \\
\hline 25 & 1 & 0.00 & 0.00 & 0.00 & 0.02 & 0.03 & -0.19 & 0.00 & 0.00 & 0.00 \\
\hline 26 & 6 & 0.00 & 0.00 & 0.00 & 0.00 & 0.00 & 0.00 & 0.00 & 0.00 & 0.00 \\
\hline 27 & 6 & 0.00 & 0.00 & 0.00 & 0.04 & -0.05 & -0.06 & 0.00 & 0.00 & 0.00 \\
\hline 28 & 1 & 0.00 & 0.00 & 0.01 & -0.31 & -0.07 & 0.4 & 0.00 & 0.00 & .00 \\
\hline 29 & 1 & 0.00 & 0.01 & 0.00 & -0.02 & 0.66 & 0.3 & 0.00 & 0.00 & 0.00 \\
\hline 30 & 1 & 0.00 & 0.00 & & -0.10 & -0.05 & -0.0 & & & 0.00 \\
\hline 31 & 6 & 0.00 & & 0.0 & & & 0.0 & 0.00 & .00 & \\
\hline 32 & 6 & 0.00 & & 0.00 & & 0.00 & 0.0 & 0.00 & 0.00 & .00 \\
\hline 33 & 6 & 0.00 & 0.00 & 0.00 & & 0.00 & 0.00 & 0.00 & 0.00 & 0.00 \\
\hline 34 & 6 & 0.0 & 0.00 & 0.00 & & & & & & .00 \\
\hline 35 & 1 & 0.0 & 0.0 & 0. & & & & & & \\
\hline 36 & 6 & 0.00 & 0.00 & 0.00 & & 0.00 & 0.00 & 0.0 & & 0.00 \\
\hline 37 & 1 & 0.00 & 0.00 & 0.00 & & 0.00 & 0. & 0.0 & & \\
\hline 38 & 6 & 0.00 & & & & & & & & \\
\hline 39 & 1 & 0.00 & 0.00 & & & & & & & .00 \\
\hline reque & & 3128.19 & & & 3173.40 & & & 3175.03 & & \\
\hline Atom & AN & $x$ & Y & Z & $X$ & Y & Z & $x$ & $Y$ & Z \\
\hline 1 & 78 & 0.00 & 0.00 & 0.00 & 0.00 & 0.00 & 0.00 & & 0.00 & 0.00 \\
\hline 2 & 7 & 0.00 & 0.00 & 0.00 & & 0.00 & 0.0 & & & 0.00 \\
\hline 3 & 7 & 0.00 & 0.00 & 0.00 & 0.0 & 0.00 & 0.0 & & .00 & 0.00 \\
\hline 4 & 17 & 0.0 & 0.00 & 0.00 & 0.0 & 0.00 & 0.0 & $0 .($ & 0.00 & 0.00 \\
\hline 5 & 17 & 0.00 & 0.00 & 0.00 & & 0.00 & 0.00 & 0.0 & 0.00 & 0.00 \\
\hline 6 & 17 & 0. & 0.00 & 0.00 & & 00 & 0. & & & 0.00 \\
\hline 7 & 17 & & 0.00 & 0.00 & & 0.00 & 0.0 & & & \\
\hline 8 & 6 & 0.00 & 0.00 & 0.00 & & 0.00 & 0.00 & 0.0 & 0.00 & 0.00 \\
\hline 9 & 1 & 0.0 & 0.00 & 0.00 & & 0.00 & 0.0 & 0. & 0.00 & 0.00 \\
\hline 10 & 1 & & 0.00 & 0.00 & & & & & & \\
\hline 11 & 1 & 0. & 0.00 & 0.00 & & & 0. & & & 0.00 \\
\hline 12 & 6 & 0.0 & 0.00 & 0.00 & & 0.00 & 0.0 & & & 0.00 \\
\hline 13 & 6 & 0.0 & 0.00 & 0.0 & & 0.00 & 0. & & & \\
\hline 14 & 6 & 0. & 0.00 & 0.0 & & 0.00 & 0.0 & & & \\
\hline 15 & 6 & 0. & 0.00 & 0.0 & & & 0.0 & & & 0.00 \\
\hline 16 & 1 & 0.0 & 0.00 & 0.00 & & 0.00 & 0. & & & 0. \\
\hline 17 & 6 & 0. & 0.0 & 0. & & 0 & 0. & & & \\
\hline 18 & 1 & & 0.0 & & & & & & & \\
\hline 19 & 6 & 0. & 0.00 & 0.0 & & 0.00 & 0.0 & & 0.00 & 0. \\
\hline 20 & 1 & 0. & 0.00 & 0. & & & 0. & & & \\
\hline 21 & 6 & & 0. & & & & & & & \\
\hline 22 & 6 & 0. & -0.1 & -0 . & & & & & & 0.0 \\
\hline 23 & 1 & & -0.02 & 0.0 & & & 0.3 & -0 . & & -0.1 \\
\hline 24 & 1 & & & & & -0.32 & -0.1 & -0. & & 0.05 \\
\hline 25 & 1 & & & & & & & & & \\
\hline 26 & 6 & & 0. & & & & & & & \\
\hline 27 & 6 & & & & & & & & & -0.0 \\
\hline 28 & 1 & -0 . & -0 . & & & & & & & \\
\hline 29 & 1 & -0 . & 0.2 & & & & & & & \\
\hline 30 & 1 & -0 . & -0. & -0 . & & & & & & 0.21 \\
\hline 31 & 6 & & & 0. & & & & & & \\
\hline 32 & 6 & 0. & 0. & 0. & & & & & & \\
\hline 33 & 6 & & & 0. & & & & & & \\
\hline 34 & 6 & & & & & & & & & 0.00 \\
\hline 35 & 1 & & & 0. & & & & & & \\
\hline 36 & 6 & & & & & & & & & \\
\hline 37 & 1 & 0.0 & 0.00 & 0.00 & 0. & 0.00 & 0.00 & 0.00 & 0.00 & 0.00 \\
\hline 38 & 6 & & & 0. & & & & & & \\
\hline 39 & 1 & & 0.00 & & & & 0.00 & & & \\
\hline Freque & & 3228.08 & & & & & & 3238.18 & & \\
\hline Atom & AN & & $Y$ & Z & & $Y$ & & & & \\
\hline & 78 & & 0.00 & 0.00 & & 0.00 & 0.00 & 0.00 & 0.00 & 0.0 \\
\hline & 7 & 0.00 & 0.00 & 0.00 & 0.00 & 0.00 & 0.00 & 0.00 & 0.00 & \\
\hline
\end{tabular}




\begin{tabular}{|c|c|c|c|c|c|c|c|c|c|c|}
\hline & & & & & & & & & & \\
\hline 3 & 7 & 0.00 & 0.00 & 0.00 & 0.00 & 0.00 & 0.00 & 0.00 & 0.00 & 0.00 \\
\hline 4 & 17 & 0.00 & 0.00 & 0.00 & 0.00 & 0.00 & 0.00 & 0.00 & 0.00 & 0.00 \\
\hline 5 & 17 & 0.00 & 0.00 & 0.00 & 0.00 & 0.00 & 0.00 & 0.00 & 0.00 & 0.00 \\
\hline 6 & 17 & 0.00 & 0.00 & 0.00 & 0.00 & 0.00 & 0.00 & 0.00 & 0.00 & 0.00 \\
\hline 7 & 17 & 0.00 & 0.00 & 0.00 & 0.00 & 0.00 & 0.00 & 0.00 & 0.00 & 0.00 \\
\hline 8 & 6 & 0.00 & 0.00 & 0.00 & & 0.00 & 0.00 & 0.00 & 0.00 & 0.00 \\
\hline 9 & 1 & 0.00 & 0.00 & 0.00 & & 0.00 & 0.00 & 0.00 & 0.00 & 0.00 \\
\hline 10 & 1 & 0.00 & 0.00 & 0.00 & 0.00 & 0.00 & 0.00 & 0.00 & 0.00 & 0.00 \\
\hline 11 & 1 & 0.00 & 0.00 & 0.00 & & 0.00 & 0.00 & 0.00 & 0.00 & 0.00 \\
\hline 12 & 6 & 0.00 & 0.00 & 0.00 & & 0.00 & 0.0 & 0.00 & 0.00 & 0.00 \\
\hline 13 & 6 & 0.00 & 0.00 & 0.00 & & 0.00 & 0.00 & 0.00 & 0.00 & 0.00 \\
\hline 14 & 6 & 0.00 & 0.00 & 0.00 & & 0.00 & 0.00 & 0.00 & 0.00 & 0.00 \\
\hline 15 & 6 & 0.01 & 0.00 & 0.03 & & 0.00 & 0.00 & 0.00 & 0.00 & 0.00 \\
\hline 16 & 1 & -0.12 & -0.03 & -0.37 & & 0.00 & 0.0 & 0.0 & 0.00 & \\
\hline 17 & 6 & -0.07 & 0.01 & -0.02 & & 0.00 & 0.0 & 0.00 & 0.00 & 0.00 \\
\hline 18 & 1 & 0.79 & -0.10 & 0.18 & & 0.00 & 0.0 & 0.0 & 0.00 & 0.00 \\
\hline 19 & 6 & 0.02 & -0.01 & -0.02 & & 0.00 & 0.00 & 0.00 & & \\
\hline 20 & 1 & -0.28 & 0.08 & 0.30 & & 0.00 & 0.00 & 0.00 & 0.00 & 0.00 \\
\hline 21 & 6 & 0.00 & 0.00 & 0.00 & & 0.00 & 0.0 & 0.00 & 0.00 & 0.00 \\
\hline 22 & 6 & 0.00 & 0.00 & 0.00 & & 0.00 & 0.00 & 0.00 & 0.00 & 0.00 \\
\hline 23 & 1 & 0.00 & 0.00 & 0.00 & & 0.00 & 0.00 & 0.00 & 0.00 & 0.00 \\
\hline 24 & 1 & 0.00 & 0.00 & 0.00 & & 0.00 & 0.00 & 0.00 & 0.00 & 0.00 \\
\hline 25 & 1 & 0.00 & 0.00 & 0.00 & & 0.00 & 0.0 & 0.0 & 0.00 & 0.00 \\
\hline 26 & 6 & 0.00 & 0.00 & 0.00 & & 0.00 & 0.0 & 0.0 & 0.00 & \\
\hline 27 & 6 & 0.00 & 0.00 & 0.00 & & 0.00 & 0.00 & 0.00 & 0.00 & 0.00 \\
\hline 28 & 1 & 0.00 & 0.00 & 0.00 & & 0.00 & 0.0 & 0.00 & 0.00 & 0.00 \\
\hline 29 & 1 & 0.00 & 0.0 & 0.0 & & 0.00 & 0.0 & & & \\
\hline 30 & 1 & 0.00 & 0.00 & 0.00 & & 0.00 & 0.0 & & & 0.00 \\
\hline 31 & 6 & 0.00 & 0.00 & 0.00 & & 0.00 & 0.0 & 0.00 & 0.00 & 0.00 \\
\hline 32 & 6 & 0.00 & 0.00 & 0.00 & & 0.00 & 0.0 & 0.0 & 0.00 & \\
\hline 33 & 6 & 0.00 & 0.00 & 0.00 & & 0.00 & 0.0 & 0.0 & 0.00 & 0.00 \\
\hline 34 & 6 & 0.00 & 0.00 & 0.0 & & -0.06 & 0.0 & -0.01 & -0.05 & 0.00 \\
\hline 35 & 1 & 0.00 & 0.00 & 0.00 & 0. & 0.72 & 0.02 & 0.13 & 0.56 & 0.02 \\
\hline 36 & 6 & 0.00 & 0.00 & 0.0 & 0. & 0.02 & 0.0 & -0.03 & -0.01 & \\
\hline 37 & 1 & 0.0 & 0.0 & 0. & & -0.17 & 0.0 & 0.3 & & -0.0 \\
\hline 38 & 6 & 0.0 & 0.00 & 0.00 & -0.02 & 0.02 & 0.01 & 0.05 & -0.04 & -0.0 \\
\hline 39 & 1 & 0.00 & 0.00 & 0.00 & 0.21 & -0.20 & -0.07 & -0.51 & 0.49 & 0.1 \\
\hline treque & & 3241.01 & & & 3248.98 & & & 3250.12 & & \\
\hline Atom & AN & X & $Y$ & Z & & $Y$ & Z & $x$ & $\mathrm{Y}$ & \\
\hline 1 & 78 & & 0.00 & 0.00 & & 0.00 & 0.00 & 0.00 & 0.00 & 0.00 \\
\hline 2 & 7 & & 0.00 & 0.00 & & 0.00 & 0.00 & & 0.00 & 0.00 \\
\hline 3 & 7 & & 0.00 & 0.00 & & 0.00 & 0.0 & & 0.00 & \\
\hline 4 & 17 & & 0.00 & 0.00 & & 0.00 & 0.0 & & 0.00 & \\
\hline 5 & 17 & & 0.00 & 0.00 & & 0.00 & 0.00 & 0.00 & 0.00 & 0.00 \\
\hline 6 & 17 & & 0.00 & 0.00 & & & 0.0 & & & \\
\hline 7 & 17 & & 0.00 & 0. & & 0. & 0.0 & & & \\
\hline 8 & 6 & & 0.00 & 0.00 & & 0.00 & 0.00 & & 0.00 & \\
\hline 9 & 1 & & 0. & 0. & & 00 & 0.0 & & 0.00 & \\
\hline 10 & 1 & 0. & 0. & 0. & & & 0.0 & & & \\
\hline 11 & 1 & & 0. & 0. & & & 0. & & & \\
\hline 12 & 6 & & 0.00 & 0.00 & & 0.00 & 0.00 & 0.0 & 0.00 & 0.00 \\
\hline 13 & 6 & 0. & 0.00 & 0.0 & & 0.00 & 0.0 & & 0.00 & \\
\hline 14 & 6 & & 0.0 & 0. & & & & & & \\
\hline 15 & 6 & -0.0 & 0.00 & -0.06 & -0 & 0.00 & -0.05 & 0.00 & 0.00 & 0.00 \\
\hline 16 & 1 & & 0.05 & 0.66 & & 0.05 & 0.57 & & & \\
\hline 17 & 6 & 0. & 0.00 & 0.0 & & & -0.01 & & & \\
\hline 18 & 1 & -0.0 & 0.00 & -0.01 & 0. & -0.07 & 0.13 & 0.00 & 0.00 & 0.00 \\
\hline 19 & 6 & 0. & -0.01 & -0.05 & & 0.01 & 0.04 & 0.00 & 0.00 & \\
\hline 20 & 1 & -0. & 0.14 & 0.5 & & -0.11 & -0.40 & 0.0 & 0.00 & \\
\hline 21 & 6 & 0.00 & 0.00 & 0.00 & 0.00 & 0.00 & 0.00 & 0.00 & 0.00 & \\
\hline
\end{tabular}




$\begin{array}{ccccccccccc}22 & 6 & 0.00 & 0.00 & 0.00 & 0.00 & 0.00 & 0.00 & 0.00 & 0.00 & 0.00 \\ 23 & 1 & 0.00 & 0.00 & 0.00 & 0.00 & 0.00 & 0.00 & 0.00 & 0.00 & 0.00 \\ 24 & 1 & 0.00 & 0.00 & 0.00 & 0.00 & 0.00 & 0.00 & 0.00 & 0.00 & 0.00 \\ 25 & 1 & 0.00 & 0.00 & 0.00 & 0.00 & 0.00 & 0.00 & 0.00 & 0.00 & 0.00 \\ 26 & 6 & 0.00 & 0.00 & 0.00 & 0.00 & 0.00 & 0.00 & 0.00 & 0.00 & 0.00 \\ 27 & 6 & 0.00 & 0.00 & 0.00 & 0.00 & 0.00 & 0.00 & 0.00 & 0.00 & 0.00 \\ 28 & 1 & 0.00 & 0.00 & 0.00 & 0.00 & 0.00 & 0.00 & 0.00 & 0.00 & 0.00 \\ 29 & 1 & 0.00 & 0.00 & 0.00 & 0.00 & 0.00 & 0.00 & 0.00 & 0.00 & 0.00 \\ 30 & 1 & 0.00 & 0.00 & 0.00 & 0.00 & 0.00 & 0.00 & 0.00 & 0.00 & 0.00 \\ 31 & 6 & 0.00 & 0.00 & 0.00 & 0.00 & 0.00 & 0.00 & 0.00 & 0.00 & 0.00 \\ 32 & 6 & 0.00 & 0.00 & 0.00 & 0.00 & 0.00 & 0.00 & 0.00 & 0.00 & 0.00 \\ 33 & 6 & 0.00 & 0.00 & 0.00 & 0.00 & 0.00 & 0.00 & 0.00 & 0.00 & 0.00 \\ 34 & 6 & 0.00 & 0.00 & 0.00 & 0.00 & 0.00 & 0.00 & 0.00 & 0.03 & 0.00 \\ 35 & 1 & 0.00 & 0.01 & 0.00 & 0.00 & 0.00 & 0.00 & -0.07 & -0.32 & -0.01 \\ 36 & 6 & 0.00 & 0.00 & 0.00 & 0.00 & 0.00 & 0.00 & 0.06 & 0.02 & 0.00 \\ 37 & 1 & 0.00 & 0.00 & 0.00 & 0.00 & 0.00 & 0.00 & -0.68 & -0.21 & 0.06 \\ 38 & 6 & 0.00 & 0.00 & 0.00 & 0.00 & 0.00 & 0.00 & 0.04 & -0.04 & -0.01 \\ 39 & 1 & 0.00 & 0.00 & 0.00 & 0.00 & 0.00 & 0.00 & -0.43 & 0.42 & 0.14\end{array}$

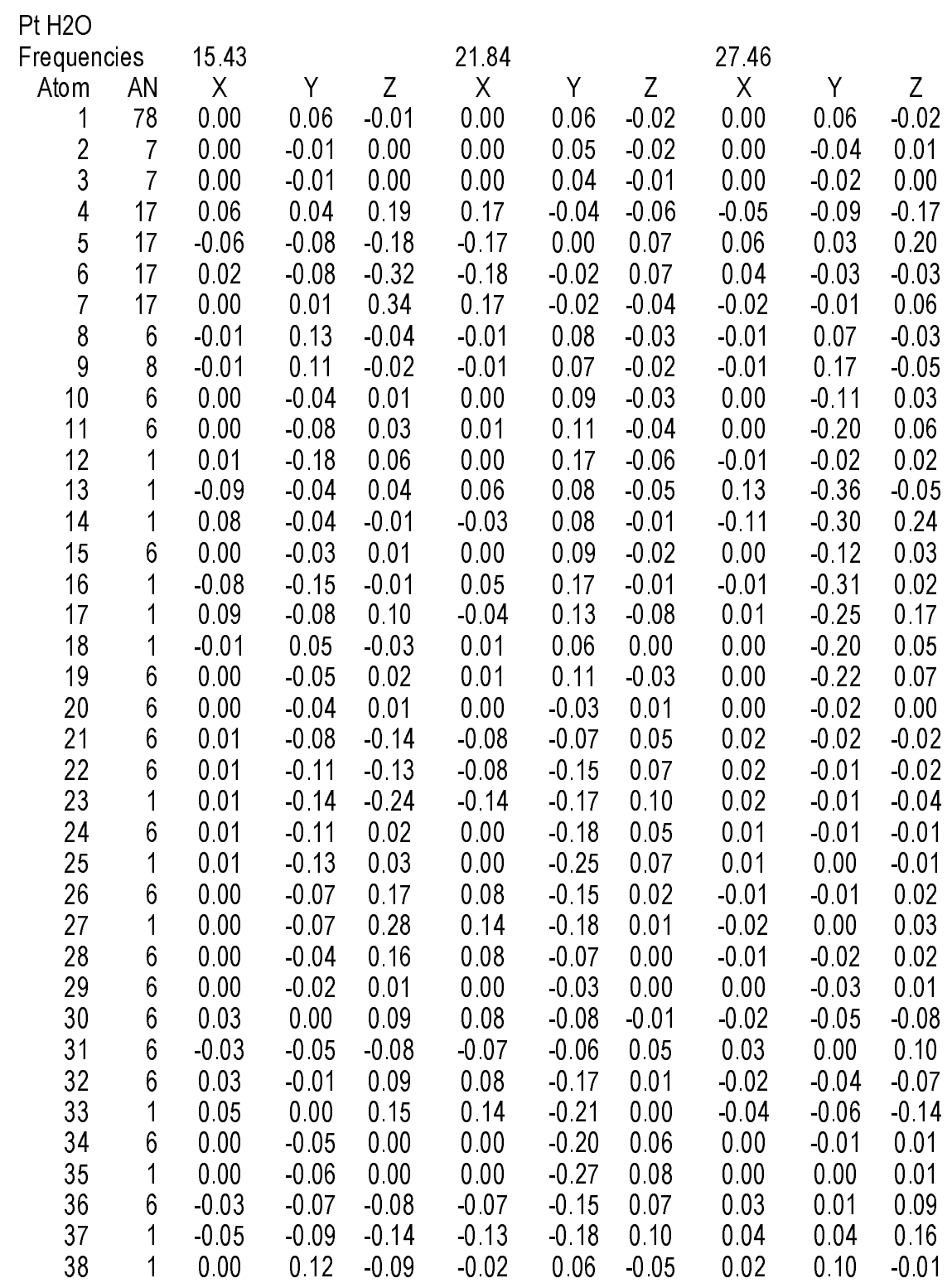




\begin{tabular}{|c|c|c|c|c|c|c|c|c|c|c|}
\hline 39 & 1 & -0.02 & 0.15 & 0.00 & 0.00 & 0.08 & -0.01 & -0.04 & 0.08 & -0.06 \\
\hline & & -0.02 & 0.19 & & -0.02 & 0.11 & -0.04 & -0.01 & 0.02 & -0.01 \\
\hline 41 & 1 & -0.04 & .14 & -0.03 & -0.04 & 0.13 & -0.01 & $-0 .($ & .21 & -0.07 \\
\hline 42 & 1 & -0.03 & 0.10 & -0.03 & -0.03 & 0.01 & 0.01 & & 0.17 & -0.06 \\
\hline \multicolumn{2}{|c|}{ Frequencies } & \multicolumn{3}{|l|}{38.96} & \multicolumn{2}{|l|}{54.27} & & \multicolumn{3}{|l|}{61.96} \\
\hline Atom & AN & X & Y & Z & X & $\mathrm{Y}$ & Z & $x$ & $Y$ & Z \\
\hline 1 & 78 & -0.02 & 0.01 & 0.04 & 0.00 & 0.00 & 0.01 & 0.03 & 0.01 & 0.02 \\
\hline 2 & 7 & 0.00 & 0.00 & 0.03 & 0.00 & 0.06 & -0.01 & -0.01 & 0.00 & 0.05 \\
\hline 3 & 7 & 0.01 & 0.02 & 0.05 & 0.00 & -0.09 & 0.04 & -0.01 & 0.00 & -0.01 \\
\hline 4 & 17 & -0.02 & -0.01 & 0.00 & 0.06 & 0.04 & -0.02 & -0. & 0.01 & 0.08 \\
\hline 5 & 17 & 0.01 & -0.01 & -0.01 & -0.07 & 0.06 & 0.02 & & 0.02 & 0.10 \\
\hline 6 & 17 & -0.01 & 0.01 & 0.04 & 0.11 & -0.06 & 0.02 & $-0 .($ & -0.01 & -0.09 \\
\hline 7 & 17 & 0.04 & 0.01 & 0.04 & -0.09 & -0.07 & 0.00 & & -0.01 & -0.09 \\
\hline 8 & 6 & -0.05 & & 0.02 & -0.02 & 0.24 & & & 0.03 & \\
\hline 9 & 8 & -0.04 & 0.03 & 0.06 & -0.01 & -0.13 & 0.0 & & 0.03 & -0.01 \\
\hline 10 & 6 & 0.0 & 0.01 & 0.03 & & 0.03 & 0.0 & & & \\
\hline 11 & 6 & 0.0 & 0.01 & 0.02 & 0.0 & 0.01 & 0.00 & & 0.01 & 0.05 \\
\hline 12 & 1 & 0.03 & -0.03 & 0.01 & -0.01 & 0.26 & -0.07 & -0.04 & -0.12 & 0.10 \\
\hline 13 & 1 & 0. & & $0 . C$ & & 16 & -0 . & & 0.10 & .11 \\
\hline 14 & 1 & 0.0 & & -0.0 & -0 . & -0.11 & 0.1 & & 0.08 & -0.05 \\
\hline 15 & 6 & 0.02 & & 0.05 & 0.00 & -0.02 & 0.0 & & 0.00 & 0.01 \\
\hline 16 & 1 & 0.00 & & 0.04 & 0. & & 0. & & -0.02 & -0.01 \\
\hline 17 & 1 & & & 0.0 & -0 . & & -0 . & & & \\
\hline 18 & 1 & 0.02 & & 0.04 & 0.02 & -0.13 & 0.0 & & & -0.05 \\
\hline 19 & 6 & 0.02 & & 0.05 & 0.00 & & 0.0 & & 0.01 & -0.01 \\
\hline 20 & 6 & 0.0 & & & & & & & & -0.0 \\
\hline 21 & 6 & 0.0 & & & & & & & & \\
\hline 22 & 6 & 0.0 & & -0.17 & & & -0.03 & & & \\
\hline 23 & 1 & 0.0 & & -0.2 & & & & & & \\
\hline 24 & 6 & 0.09 & & -0.25 & 0.02 & & -0.05 & & & 0.07 \\
\hline 25 & 1 & 0.11 & & -0.35 & 0.02 & & -0.08 & & 0.00 & 0.12 \\
\hline 26 & 6 & 0.08 & & -0.1 & & & -0 . & & & 0.05 \\
\hline 27 & 1 & 0.10 & & -0.24 & $-0 .($ & & -0. & & & \\
\hline 28 & 6 & 0.05 & & -0.06 & -0.03 & -0.04 & 0. & & & -0.01 \\
\hline 29 & 6 & & & -0 & & & -0 & & & 0.03 \\
\hline 30 & 6 & -0.02 & & -0.0 & 0. & & -0 . & & & -0.02 \\
\hline 31 & 6 & & & -0 & -0 & & 0. & & & -0.02 \\
\hline 32 & 6 & & & & & & & & & \\
\hline 33 & 1 & & & -0 & & & -0 . & & -0.08 & -0.24 \\
\hline 34 & 6 & & & & & & & & & -0.27 \\
\hline 35 & 1 & & & & & & & & & -0.41 \\
\hline 36 & 6 & & & -0 . & -0.1 & & & & -0.06 & $-0.1 \varepsilon$ \\
\hline 37 & 1 & & & -0.12 & -0 & & & & 08 & -0. \\
\hline 38 & 1 & & & & & & & & & \\
\hline 39 & 1 & & & & & & & & & \\
\hline 40 & 1 & & & & -0. & & & & & \\
\hline 41 & 1 & & & & & & 0. & & -0.26 & -0.09 \\
\hline 42 & 1 & & & & & -0.02 & 0.00 & & 0.29 & -0.15 \\
\hline Frequen & & & & & & & & & & \\
\hline Atom & AN & & $Y$ & Z & $X$ & $Y$ & Z & & $Y$ & 2 \\
\hline 1 & 78 & & 0.00 & 0.00 & -0.02 & & 0.02 & & -0.01 & -0.05 \\
\hline 2 & 7 & & & & & & & & & -0.0 \\
\hline 3 & 7 & & & 0.01 & & & & & -0.04 & -0.04 \\
\hline 4 & 17 & & -0.0 & -0.02 & & & -0. & & -0.03 & 0.11 \\
\hline 5 & 17 & & & & & & & & & 0.11 \\
\hline 6 & 17 & & & 0. & & & -0. & & & 0.19 \\
\hline 7 & 17 & & & & & & & & & 0.20 \\
\hline 8 & 6 & & & -0.0 & & & & & 0.12 & -0.05 \\
\hline 9 & 8 & & & 0.0 & -0.0 & 0.12 & & & 0.09 & -0 . \\
\hline 10 & 6 & 0.00 & 0.01 & 0.00 & 0.02 & -0.01 & 0.01 & -0.02 & 0.00 & -0.04 \\
\hline
\end{tabular}




\begin{tabular}{|c|c|c|c|c|c|c|c|c|c|c|}
\hline & & & & & & & & & & \\
\hline 11 & 6 & -0.01 & 0.09 & -0.02 & 0.03 & -0.01 & 0.00 & -0.03 & 0.05 & -0.05 \\
\hline 12 & & 0.03 & -0.34 & 0.09 & 0.03 & -0.05 & 0.00 & -0.03 & 0.04 & -0.03 \\
\hline 13 & 1 & -0.35 & 0.41 & 0.19 & 0.01 & 0.01 & 0.02 & -0.04 & 0.09 & -0.02 \\
\hline 14 & 1 & 0.29 & 0.32 & -0.38 & 0.07 & 0.01 & -0.02 & -0.03 & 0.07 & -0.09 \\
\hline 15 & 6 & 0.00 & -0.01 & 0.00 & 0.02 & 0.06 & 0.01 & -0.02 & 0.04 & -0.07 \\
\hline 16 & 1 & -0.14 & -0.21 & -0.05 & 0.10 & 0.44 & 0.06 & 0.09 & 0.44 & 0.00 \\
\hline 17 & 1 & 0.19 & -0.09 & 0.17 & -0.05 & 0.29 & -0.27 & -0.16 & 0.27 & -0.37 \\
\hline 18 & 1 & -0.01 & 0.17 & -0.06 & 0.04 & 0.13 & 0.02 & 0.00 & 0.02 & -0.04 \\
\hline 19 & 6 & 0.01 & -0.03 & 0.01 & 0.03 & 0.23 & -0.04 & -0.02 & 0.19 & -0.12 \\
\hline 20 & 6 & 0.00 & -0.02 & 0.01 & 0.01 & 0.00 & 0.02 & -0.02 & -0.02 & -0.02 \\
\hline 21 & 6 & 0.01 & & 0.01 & 0.02 & -0.01 & 0.0 & -0.03 & -0.01 & 0.02 \\
\hline 22 & 6 & 0.01 & 0.00 & 0.00 & 0.02 & -0.01 & -0.01 & -0.02 & 0.00 & -0.02 \\
\hline 23 & 1 & 0.02 & 0.01 & 0.00 & 0.02 & -0.02 & -0.03 & -0.02 & 0.00 & 0.00 \\
\hline 24 & 6 & 0.00 & 0.01 & -0.01 & 0.02 & -0.02 & 0.00 & -0.02 & 0.00 & -0.07 \\
\hline 25 & 1 & 0.00 & 0.01 & -0.01 & 0.02 & -0.02 & 0.00 & -0.01 & 0.00 & -0.11 \\
\hline 26 & 6 & -0.01 & 0.00 & -0.01 & 0.03 & -0.01 & -0.01 & -0.04 & 0.00 & -0.03 \\
\hline 27 & 1 & -0.02 & 0.00 & -0.01 & 0.03 & -0.02 & -0.02 & -0.05 & 0.00 & -0.02 \\
\hline 28 & 6 & -0.01 & -0.01 & 0.00 & 0.02 & -0.01 & 0.00 & -0.05 & -0.01 & 0.01 \\
\hline 29 & 6 & 0.00 & 0.00 & 0.00 & 0.00 & -0.05 & 0.01 & 0.01 & -0.05 & 0.01 \\
\hline 30 & 6 & 0.01 & & 0.00 & -0.02 & -0.04 & 0.01 & -0.01 & -0.03 & 0.02 \\
\hline 31 & 6 & 0.00 & 0.00 & 0.00 & 0.03 & -0.03 & 0.01 & 0.03 & -0.03 & 0.01 \\
\hline 32 & 6 & 0.01 & 0.00 & 0.01 & -0.02 & 0.01 & 0.03 & -0.0 & -0.01 & -0.03 \\
\hline 33 & 1 & 0.01 & -0.01 & 0.01 & -0.04 & 0.02 & 0.0 & -0.0 & 0.00 & -0.04 \\
\hline 34 & 6 & 0.01 & 0.00 & 0.02 & 0.02 & 0.03 & 0.0 & 0.00 & -0.01 & -0.07 \\
\hline 35 & 1 & 0.01 & 0.00 & 0.03 & 0.02 & 0.07 & 0.07 & -0.0 & 0.00 & -0.11 \\
\hline 36 & 6 & 0.00 & 0.00 & 0.02 & 0.04 & 0.01 & 0.0 & 0.0 & -0.01 & -0.04 \\
\hline 37 & 1 & 0.00 & & 0.03 & 0.07 & 0.03 & 0.04 & 0.0 & 0.00 & -0.04 \\
\hline 38 & 1 & 0.04 & 0.08 & -0.02 & 0.06 & 0.22 & -0.07 & 0.1 & 0.18 & -0.07 \\
\hline 39 & 1 & -0.05 & 0.07 & -0.04 & -0.18 & 0.19 & -0.1 & -0.02 & 0.18 & -0.03 \\
\hline 40 & 1 & 0.00 & 0.01 & 0.00 & -0.06 & 0.03 & -0.02 & 0.09 & 0.10 & -0.06 \\
\hline 41 & 1 & 0.01 & 0.00 & -0.01 & 0.09 & -0.20 & -0.02 & -0.04 & 0.25 & -0.17 \\
\hline 42 & 1 & 0.00 & 0.06 & -0.03 & -0.02 & 0.45 & -0.17 & 0.04 & -0.02 & -0.07 \\
\hline & & 87.64 & & & 90.52 & & & 93.42 & & \\
\hline & & & & & & & & & & \\
\hline Atom & AN & $X$ & $\mathrm{Y}$ & Z & $X$ & $\mathrm{Y}$ & Z & $X$ & $Y$ & Z \\
\hline 1 & 78 & -0.04 & -0.01 & -0.03 & 0.02 & 0.01 & 0.01 & 0.01 & 0.01 & 0.00 \\
\hline 2 & 7 & 0.01 & & -0.05 & -0.01 & 0.00 & 0.03 & 0. & 0.00 & 0.00 \\
\hline 3 & 7 & & & 0.00 & & -0.02 & 0.0 & & & 0.00 \\
\hline 4 & 17 & 0.07 & & 0. & -0.04 & -0.03 & -0.04 & -0.0 & -0.02 & -0.01 \\
\hline 5 & 17 & 0. & & & -0.02 & -0.02 & -0.05 & 0.0 & -0.02 & 0.01 \\
\hline 6 & 17 & & & & & -0.04 & -0.02 & & & -0.02 \\
\hline 7 & 17 & & & -0.06 & -0.04 & -0.04 & -0.02 & 0.0 & 0.01 & -0.02 \\
\hline 8 & 6 & -0.1 & & -0.0 & 0. & -0.03 & 0.0 & 0.0 & 0.10 & -0.03 \\
\hline 9 & 8 & -0.1 & & & & 0.11 & -0. & 0. & -0.13 & 0.01 \\
\hline 10 & 6 & 0.0 & & -0 . & & 0.04 & 0. & & -0.03 & 0.01 \\
\hline 11 & 6 & 0. & & -0 . & -0.02 & 0.13 & -0. & -0 . & -0.11 & 0.04 \\
\hline 12 & 1 & 0. & & -0 & -0 . & 0.28 & -0.1 & 0. & -0.29 & 0.10 \\
\hline 13 & 1 & 0. & & -0 & & 0.09 & & & & 0.06 \\
\hline 14 & 1 & 0.0 & & -0 & -0.14 & 0.08 & 0.1 & 0. & -0.03 & -0.03 \\
\hline 15 & 6 & 0.03 & & -0 & -0. & 0.00 & 0.0 & 0.0 & 0.01 & 0.00 \\
\hline 16 & 1 & 0.09 & & & & 0.16 & & & & -0.02 \\
\hline 17 & 1 & & & & -0. & 0.06 & -0. & & & 0.1 \\
\hline 18 & 1 & 0.0 & & & -0.01 & -0.17 & 0.0 & -0.0 & 0.18 & -0.06 \\
\hline 19 & 6 & 0.05 & & & -0.03 & 0.01 & -0.0 & & 0.07 & -0.02 \\
\hline 20 & 6 & 0.0 & & 0.0 & & -0.03 & 0. & & 0.01 & -0.01 \\
\hline 21 & 6 & 0.0 & & 0. & 0.0 & -0.02 & 0.01 & -0. & 0.01 & 0.00 \\
\hline 22 & 6 & 0.0 & & & & 0.00 & & & 0.01 & 0.03 \\
\hline 23 & 1 & 0.0 & & & & 0.01 & 0.0 & -0. & 0.00 & 0.05 \\
\hline 24 & 6 & 0.0 & & 0.0 & -0.01 & 0.01 & 0.05 & -0.01 & 0.00 & 0.04 \\
\hline 25 & 1 & 0.00 & 0.02 & 0.08 & -0.02 & 0.03 & 0.08 & -0.02 & 0.00 & 0.07 \\
\hline
\end{tabular}




\begin{tabular}{|c|c|c|c|c|c|c|c|c|c|c|}
\hline & & & & & & & & & & \\
\hline 26 & 6 & 0.01 & 0.00 & 0.01 & -0.02 & 0.00 & 0.02 & 0.00 & 0.00 & 0.02 \\
\hline 27 & 1 & 0.00 & 0.00 & 0.01 & -0.03 & 0.01 & 0.02 & 0.00 & 0.00 & 0.02 \\
\hline 28 & 6 & 0.01 & -0.02 & -0.01 & -0.01 & -0.03 & -0.01 & 0.00 & 0.01 & -0.01 \\
\hline 29 & 6 & 0.01 & -0.01 & -0.03 & -0.01 & 0.00 & 0.02 & 0.00 & -0.01 & 0.01 \\
\hline 30 & 6 & 0.03 & 0.01 & 0.02 & -0.02 & 0.00 & 0.0 & -0.01 & -0.01 & 0.00 \\
\hline & 6 & 0.03 & 0.01 & 0.01 & -0.01 & -0.01 & 0.0 & 0.00 & -0.01 & 0.01 \\
\hline 32 & 6 & 0.03 & 0.00 & 0.01 & -0.02 & 0.00 & 0.0 & -0.01 & 0.00 & 0.00 \\
\hline 33 & 1 & 0.03 & 0.01 & 0.04 & -0.02 & 0.00 & -0.01 & -0.02 & 0.00 & 0.00 \\
\hline 34 & 6 & 0.02 & 0.00 & -0.01 & -0.02 & 0.00 & 0.00 & 0.00 & 0.00 & 00 \\
\hline 35 & 1 & 0.02 & 0.00 & -0.02 & -0.02 & 0.00 & 0.0 & 0.00 & 0.01 & 0.00 \\
\hline 36 & 6 & 0.03 & 0.00 & 0.01 & -0.01 & 0.00 & 0.00 & 0.00 & 0.00 & 0.00 \\
\hline 37 & 1 & 0.03 & 0.01 & 0.03 & -0.01 & -0.01 & -0.02 & 0.01 & 0.00 & 0.00 \\
\hline 38 & 1 & -0.18 & -0.16 & -0.12 & -0.02 & -0.09 & 0.03 & -0.06 & 0.00 & -0.13 \\
\hline 39 & 1 & 0.00 & -0.12 & -0.01 & 0.1 & -0.06 & 0. & 0.07 & 0.09 & 0.12 \\
\hline 40 & 1 & -0.16 & 0.05 & -0.08 & 0.03 & 0.04 & 0.02 & -0.02 & 0.30 & -0.08 \\
\hline 41 & 1 & -0.35 & 0.51 & 0.12 & -0.25 & 0.63 & -0.02 & -0.19 & 0.31 & 0.12 \\
\hline 42 & 1 & -0.30 & -0.34 & 0.21 & -0.10 & -0.33 & 0.1 & -0.08 & -0.58 & 0.29 \\
\hline equen & & 120.46 & & & 121.29 & & & 128.42 & & \\
\hline Atom & AN & & $Y$ & Z & $x$ & $Y$ & Z & $X$ & $Y$ & Z \\
\hline 1 & 78 & 0.00 & 0.01 & 0.03 & 0.00 & 0.01 & -0.01 & 0.00 & 0.01 & 0.00 \\
\hline 2 & 7 & 0.04 & 0.01 & 0.01 & & 0.00 & 0.00 & 0.00 & -0.02 & 0.01 \\
\hline 3 & 7 & -0.04 & -0.01 & 0.01 & & 0.00 & 0.0 & -0.01 & 0.02 & 0.00 \\
\hline 4 & 17 & & 0.03 & -0.06 & -0.04 & -0.01 & 0. & & -0.06 & 0.02 \\
\hline 5 & 17 & & & -0.03 & -0.02 & 0.00 & 0.01 & 0.07 & -0.08 & 0.00 \\
\hline 6 & 17 & -0.12 & 0.00 & -0.04 & 0.04 & -0.01 & 0.02 & -0.05 & 0.04 & -0.02 \\
\hline 7 & 17 & -0.16 & -0.06 & -0.03 & 0.0 & 0.00 & 0.0 & 0.01 & 0.04 & -0.01 \\
\hline 8 & 6 & 0. & -0.05 & 0.05 & 0.00 & -0.03 & 0.01 & -0.01 & 0.13 & -0.03 \\
\hline 9 & 8 & -0.04 & 0.06 & 0.06 & 0.01 & 0.00 & -0.02 & 0.00 & -0.11 & 0.03 \\
\hline 10 & 6 & 0. & & 0. & 0. & 0.00 & 0.0 & 0.0 & 0.04 & -0.01 \\
\hline 11 & 6 & & -0.04 & 0.02 & & 0.01 & -0.01 & 0.0 & 0.22 & -0.07 \\
\hline 12 & 1 & & & 0.04 & 01 & -0.04 & 0.00 & -0.02 & 0.52 & -0.16 \\
\hline 13 & 1 & & & 0. & -0.03 & 0.04 & 0.0 & 0.27 & 0.15 & -0.07 \\
\hline 14 & 1 & & & 0. & 0. & 0.03 & -0.0 & -0.2 & 0.13 & -0.02 \\
\hline 15 & 6 & & & & & & 0. & 0. & -0.01 & 0.00 \\
\hline 16 & 1 & & & & & 0.04 & 0. & & -0.26 & -0.02 \\
\hline 17 & 1 & 0. & 0.04 & -0.02 & -0.02 & 0.02 & -0.0 & -0.0 & -0.18 & 0.19 \\
\hline 18 & 1 & & & -0 & & & 0. & 0. & -0.17 & 0. \\
\hline 19 & 6 & & & & & & & & -0 . & \\
\hline 20 & 6 & & & 0. & & -0.0 & 0. & -0 . & 0.03 & 0.00 \\
\hline 21 & 6 & & & -0 & & 0. & 0. & -0.02 & 0.02 & -0.01 \\
\hline 22 & 6 & & & -0 & & & -0 . & & 0.01 & -0.02 \\
\hline 23 & 1 & & & & & & & & 1 & -0.1 \\
\hline 24 & 6 & & & -0 . & & 0.00 & -0 . & -0.0 & 0.01 & -0.01 \\
\hline 25 & 1 & & & -0 . & & & -0. & -0. & 0.00 & -0.01 \\
\hline 26 & 6 & & & & & & & & & \\
\hline 27 & 1 & & & & & & 0. & 0. & 0. & \\
\hline 28 & 6 & & & -0.0 & & & 0. & -0.01 & 0.03 & 0.00 \\
\hline 29 & 6 & & & & & & & & & \\
\hline 30 & 6 & & & & & & & & & \\
\hline 31 & 6 & & & & -0.02 & & 0. & 0. & -0.04 & 0.01 \\
\hline 32 & 6 & & & -0. & & & & & & 0.01 \\
\hline 33 & 1 & 0. & & -0. & -0. & 0. & & & -0.01 & 0.01 \\
\hline 34 & 6 & & & -0.02 & -0.02 & 0.00 & 0.0 & 0. & -0.02 & 0.00 \\
\hline 35 & 1 & & & & -0.0 & & 0. & & -0.01 & 0.00 \\
\hline 36 & 6 & & & $-0 .($ & -0.1 & & 0. & & -0.02 & 0.00 \\
\hline 37 & 1 & 0. & & -0. & -0. & 0.0 & 0.0 & 0.03 & -0.02 & 0.00 \\
\hline 38 & 1 & & & -0.1 & -0.34 & -0.42 & -0.18 & -0.03 & 0.06 & -0.14 \\
\hline 39 & 1 & & & 0.3 & & -0.20 & 0.37 & & 0.14 & 0.10 \\
\hline 40 & 1 & -0. & & -0.09 & -0.0 & 0.47 & -0.1 & -0.01 & 0.28 & -0.08 \\
\hline 41 & 1 & 0.03 & -0.05 & 0.05 & 0.11 & -0.20 & -0.06 & 0.08 & -0.23 & 0.06 \\
\hline
\end{tabular}




\begin{tabular}{|c|c|c|c|c|c|c|c|c|c|c|}
\hline Frequencies & ies $^{1}$ & $\begin{array}{c}-0.03 \\
141.38\end{array}$ & 0.17 & -0.02 & $\begin{array}{c}0.08 \\
150.09\end{array}$ & 0.17 & -0.10 & $\begin{array}{c}0.05 \\
164.80\end{array}$ & -0.06 & 0.02 \\
\hline Atom & AN & $x$ & $\mathrm{Y}$ & Z & $x$ & Y & Z & X & Y & Z \\
\hline 1 & 78 & 0.00 & -0.02 & 0.00 & 0.00 & 0.07 & -0.02 & 0.06 & 0.00 & -0.01 \\
\hline 2 & 7 & 0.00 & 0.00 & 0.00 & 0.00 & 0.02 & -0.01 & 0.02 & 0.01 & 0.02 \\
\hline 3 & 7 & 0.00 & 0.00 & 0.01 & 0.00 & 0.02 & 0.00 & & -0.01 & -0.07 \\
\hline 4 & 17 & -0.01 & 0.00 & 0.00 & -0.05 & -0.05 & 0.0 & -0.05 & -0.03 & 0.01 \\
\hline 5 & 17 & -0.02 & 0.01 & 0.00 & 0.03 & -0.05 & 0.00 & -0.05 & 0.02 & -0.01 \\
\hline 6 & 17 & 0.00 & 0.00 & 0.00 & 0.05 & -0.06 & 0.03 & -0.01 & 0.01 & 0.03 \\
\hline 7 & 17 & -0.01 & -0.01 & -0.01 & -0.06 & -0.08 & -0.0 & -0.01 & & \\
\hline 8 & 6 & 0.01 & 0.04 & -0.01 & 0.01 & -0.18 & 0.06 & -0.07 & -0.03 & -0.14 \\
\hline 9 & 8 & 0.02 & 0.06 & -0.03 & 0.00 & -0.15 & 0.0 & -0.23 & 0.06 & 0.26 \\
\hline 10 & 6 & 0.01 & 0.02 & -0.01 & 0.0 & 0.05 & -0.0 & & & \\
\hline 11 & 6 & 0.02 & 0.00 & -0.01 & 0.01 & & & & & \\
\hline 12 & 1 & 0.00 & 0.23 & -0.08 & 0.01 & 0.13 & -0.0 & & 0.08 & .08 \\
\hline 13 & 1 & 0.21 & & -0.11 & & & & & & \\
\hline 14 & 1 & -0.14 & -0 . & 0.17 & -0.06 & & & & & \\
\hline 15 & 6 & 0.01 & & -0.01 & & & -0.02 & & 0.00 & -0.05 \\
\hline 16 & 1 & -0.28 & -0. & -0.10 & -0.1 & -0.1 & & & & -0.10 \\
\hline 17 & 1 & 0.45 & & 0.29 & 0.2 & 0.0 & 0.1 & -0 . & & -0.01 \\
\hline 18 & 1 & -0.02 & 0.5 & -0.13 & 0.0 & 0.37 & -0.09 & -0 . & 0.05 & -0.19 \\
\hline 15 & 6 & 0.0 & & 0.01 & & 0.10 & & & -0.02 & -0.09 \\
\hline 2 & 6 & 0.00 & & 0.01 & & -0.03 & & & -0.01 & \\
\hline 21 & 6 & 0.00 & 0.0 & 0.00 & 0.0 & -0.04 & & & 0.00 & -0.04 \\
\hline 22 & 6 & 0.00 & & 0.00 & 0.01 & -0.02 & 0.0 & & & \\
\hline 23 & 1 & 0. & & & & -0.02 & & & & \\
\hline 24 & 6 & & & & & -0.02 & & & & \\
\hline 25 & 1 & 0. & & & & -0.01 & -0.0 & & & \\
\hline 26 & 6 & 0.00 & & 0. & 0. & -0.02 & & & & \\
\hline 27 & 1 & & & & -0.01 & -0.01 & 0.00 & & & \\
\hline 28 & 6 & & & & 0.00 & -0.03 & 0.01 & & -0.01 & -0.05 \\
\hline 29 & 6 & & & -0.1 & 0. & & & & & \\
\hline 30 & 6 & -0. & & 0.0 & & -0. & 0. & & & \\
\hline 31 & 6 & & & & & -0.03 & 0. & & & \\
\hline 32 & 6 & & & & & & & & & \\
\hline 3 & 1 & -0.6 & & & -0. & & 0. & & & -0 . \\
\hline 34 & 6 & & & & & -0.01 & 0. & & & -0.01 \\
\hline 35 & 1 & & & & & & & & & \\
\hline 3 & 6 & & & & & -0 & 0.1 & -0 . & -0 . & \\
\hline 37 & 1 & & & & & & & & & -0.02 \\
\hline 38 & 1 & & & & & & & & & \\
\hline 39 & 1 & & & & -0. & -0. & -0 . & -0 . & & -0 . \\
\hline 40 & 1 & & & & & -0 & 0. & -0 & & -0 . \\
\hline 41 & 1 & & & & & & & & & \\
\hline 42 & 1 & & & -0.03 & & -0.12 & 0.06 & & & 0.07 \\
\hline \multicolumn{2}{|c|}{ Frequencies } & 167.05 & & & & & & & & \\
\hline Atom & AN & & $\mathrm{Y}$ & Z & & $\mathrm{Y}$ & Z & $x$ & Y & \\
\hline 1 & 78 & & & & & 0.00 & & & & \\
\hline 2 & 7 & & & & & & & & & \\
\hline 3 & 7 & & & & & 0.00 & 0. & & & 0.00 \\
\hline 4 & 17 & & & & & & & & & \\
\hline 5 & 17 & & & & & & & & & \\
\hline 6 & 17 & & & & & -0.01 & -0.6 & & & 0.00 \\
\hline 7 & 17 & & & & -0.0 & 0.02 & & & & \\
\hline 8 & 6 & -0.02 & & & & & & & & \\
\hline 9 & 8 & 0.00 & $-0 .(1-x-x$ & -0.0 & 0.0 & -0.01 & 0.0 & & 0.00 & 0.0 \\
\hline 10 & 6 & & & & & & & & & 0.00 \\
\hline 11 & 6 & & & & & & & & & 0.00 \\
\hline 12 & 1 & -0.1 & & & 0.0 & & & & 0.02 & -0.01 \\
\hline 13 & 1 & -0.17 & -0.06 & 0.01 & 0.00 & 0.00 & 0.00 & 0.02 & -0.02 & -0.01 \\
\hline
\end{tabular}




\begin{tabular}{|c|c|c|c|c|c|c|c|c|c|c|}
\hline & & & & & & & & & & \\
\hline $\begin{array}{l}14 \\
15\end{array}$ & 1 & $\begin{array}{l}-0.34 \\
-0.09\end{array}$ & $\begin{array}{r}-0.04 \\
0.02\end{array}$ & $\begin{array}{r}0.15 \\
-0.05\end{array}$ & $\begin{array}{l}0.00 \\
0.00\end{array}$ & $\begin{array}{l}0.00 \\
0.00\end{array}$ & $\begin{array}{l}0.00 \\
0.00\end{array}$ & $\begin{array}{l}-0.01 \\
0.00\end{array}$ & $\begin{array}{r}-0.01 \\
0.01\end{array}$ & $\begin{array}{l}0.02 \\
0.00\end{array}$ \\
\hline 16 & 1 & -0.46 & $\begin{array}{l}.02 \\
-0.17\end{array}$ & -0.13 & 0.00 & $\begin{array}{l}.00 \\
-0.02\end{array}$ & 0.00 & 0.00 & $\begin{array}{l}.0 .01 \\
-0.01\end{array}$ & 0.00 \\
\hline 17 & 1 & -0.06 & -0.05 & 0.05 & 0.03 & -0.01 & 0.02 & 0.00 & 0.00 & 0.01 \\
\hline 18 & 1 & -0.22 & 0.21 & -0.31 & 0.00 & 0.02 & 0.01 & 0.00 & 0.00 & 0.00 \\
\hline 19 & 6 & -0.21 & 0.01 & -0.11 & & 0.00 & 0.01 & & 0.00 & 0.00 \\
\hline 20 & 6 & 0.00 & -0.02 & -0.08 & 0.00 & 0.00 & 0.00 & 0.00 & 0.00 & 0.00 \\
\hline 21 & 6 & 0.02 & 0.00 & -0.03 & -0.04 & 0.02 & 0.15 & 0.00 & 0.00 & 0.00 \\
\hline 22 & 6 & 0.01 & 0.01 & 0.01 & -0.08 & 0.04 & 0.32 & 0.00 & 0.00 & 0.00 \\
\hline 23 & 1 & 0.00 & & 0.05 & -0.14 & 0.07 & & 0.00 & 0.00 & -0.01 \\
\hline 24 & 6 & 0.01 & 0.01 & 0.04 & 0.00 & 0.00 & 0.00 & 0.00 & 0.00 & 0.00 \\
\hline 25 & 1 & 0.00 & 0.01 & 0.09 & 0.00 & 0.00 & 0.00 & 0.00 & 0.00 & 0.00 \\
\hline 26 & 6 & 0.01 & -0.01 & 0.02 & 0.08 & -0.04 & -0.32 & 0.00 & 0.00 & 0.00 \\
\hline 27 & 1 & -0.01 & & 0.05 & 0.13 & -0.07 & -0.55 & 0.00 & 0.00 & 0.01 \\
\hline 28 & 6 & 0.02 & -0.02 & -0.03 & 0.04 & -0.02 & -0.16 & 0.00 & 0.00 & 0.00 \\
\hline 29 & 6 & -0.01 & 0.02 & 0.07 & 0.00 & 0.00 & 0.0 & 0.00 & 0.00 & 0.00 \\
\hline 30 & 6 & 0.01 & & 0.03 & 0.00 & 0.00 & 0.8 & 0.03 & 0.05 & 0.15 \\
\hline 31 & 6 & 0.01 & & 0.03 & 0.00 & 0.00 & 0.0 & -0.03 & -0.05 & -0.15 \\
\hline 32 & 6 & 0.01 & -0.01 & -0.01 & 0.00 & 0.00 & 0.0 & 0.06 & 0.10 & 0.32 \\
\hline 33 & 1 & -0.01 & & -0.04 & 0.00 & 0.00 & & 0.1 & 0.18 & 0.55 \\
\hline 34 & 6 & 0.01 & & -0.03 & 0.00 & 0.00 & & 0.0 & & \\
\hline 35 & 1 & 0.01 & & -0.07 & 0.00 & 0.00 & 0. & 0.0 & 0.00 & 0.00 \\
\hline 36 & 6 & 0.01 & & -0.02 & 0.00 & 0.00 & & -0. & -0.10 & \\
\hline 37 & 1 & -0.01 & & -0.04 & 0.00 & 0.00 & 0.0 & -0.11 & -0.18 & \\
\hline 38 & 1 & -0.02 & & 0.03 & 0.00 & 0.00 & 0.02 & 0.02 & 0.03 & 0.02 \\
\hline 39 & 1 & -0.01 & 0. & 0.0 & 0.02 & 0.00 & 0.0 & -0.02 & 0.01 & -0.03 \\
\hline 40 & 1 & 0.01 & & 0.01 & 0.02 & 0.00 & 0.0 & 0.01 & -0.04 & 0.01 \\
\hline 41 & 1 & -0.03 & 0.02 & -0.07 & 0.07 & -0.13 & -0.02 & 0.00 & 0.00 & 0.01 \\
\hline 42 & 1 & 0.03 & -0.03 & 0.00 & 0.05 & 0.10 & -0.06 & 0.00 & -0.01 & 0.00 \\
\hline quen & & 238.35 & & & 245.88 & & & 258.13 & & \\
\hline Atom & AN & $x$ & $Y$ & Z & $\mathrm{X}$ & $Y$ & Z & $X$ & $Y$ & Z \\
\hline 1 & 78 & -0.02 & 0.00 & 0.01 & 0.05 & -0.01 & -0.05 & 0.00 & 0.00 & -0.01 \\
\hline 2 & 7 & 0.00 & 0.02 & 0.05 & -0.08 & 0.02 & 0.06 & -0.0 & -0.01 & -0.03 \\
\hline 3 & 7 & & & -0.01 & -0.04 & 0.01 & 0.13 & 0.0 & 0.00 & -0.02 \\
\hline 4 & 17 & & & -0.01 & & 0.14 & -0.07 & & 0.10 & -0.03 \\
\hline 5 & 17 & & -0.02 & 0.00 & 0.0 & -0.16 & 0.03 & 0.03 & -0.10 & 0.04 \\
\hline 6 & 17 & -0 . & & -0.0 & & -0.02 & -0.03 & -0.02 & 0.08 & -0.01 \\
\hline 7 & 17 & & & & & & -0.03 & -0.02 & & 0.01 \\
\hline 8 & 6 & -0 . & -0.06 & -0.1 & -0.1 & -0.08 & -0 . & 0.2 & 0.08 & 0.20 \\
\hline 9 & 8 & 0. & & -0.10 & & 0.00 & 0.00 & -0.1 & 0.02 & 0.08 \\
\hline 10 & 6 & & & 0.06 & & 0.01 & 0.0 & 0. & 0.00 & -0.01 \\
\hline 11 & 6 & & & & & 0.0 & & & -0.02 & -0.07 \\
\hline 12 & 1 & & & 0. & -0.0 & 0.03 & 0.0 & 0.0 & -0.07 & -0.16 \\
\hline 13 & 1 & & & 0. & -0.0 & 0.03 & 0. & 0.1 & -0.01 & -0.06 \\
\hline 14 & 1 & & & & & & 0. & & & -0.06 \\
\hline 15 & 6 & & & 0. & & 0. & 0.1 & 0. & 0.0 & -0.01 \\
\hline 16 & 1 & & & -0.0 & & 0.09 & 0.1 & -0.06 & -0.02 & -0.03 \\
\hline 17 & 1 & & & & & 0.07 & 0. & & & -0.01 \\
\hline 18 & 1 & & & -0 & & 0. & & & -0.02 & -0.07 \\
\hline 19 & 6 & & & -0.04 & & 0.06 & 0.1 & -0.0 & -0.01 & -0.03 \\
\hline 20 & 6 & & & -0.0 & & & 0.1 & & & -0.05 \\
\hline 21 & 6 & 0. & & -0 . & -0. & 0.01 & 0.1 & 0. & & -0.03 \\
\hline 22 & 6 & 0.1 & & 0. & -0. & 0.00 & 0.00 & 0.06 & 0.00 & 0.01 \\
\hline 23 & 1 & 0.1 & & & & -0.01 & -0. & 0. & 0.00 & 0.03 \\
\hline 24 & 6 & 0.12 & & 0.07 & & -0.01 & -0.0 & & 0.01 & 0.05 \\
\hline 25 & 1 & 0.11 & 0. & 0.14 & 0.03 & -0.02 & -0.21 & 0.0 & 0.01 & 0.10 \\
\hline 26 & 6 & 0.1 & & & -0. & 0.00 & -0.01 & 0.06 & 0.00 & 0.02 \\
\hline 27 & 1 & 0.12 & & & & -0.01 & -0.09 & 0.05 & 0.01 & 0.05 \\
\hline 28 & 6 & 0.14 & -0 . & -0.03 & -0.0 & 0.02 & 0.1 & 0.07 & -0.02 & -0.03 \\
\hline 29 & 6 & -0.01 & 0.01 & 0.03 & -0.10 & 0.02 & 0.08 & -0.08 & -0.02 & -0.05 \\
\hline
\end{tabular}




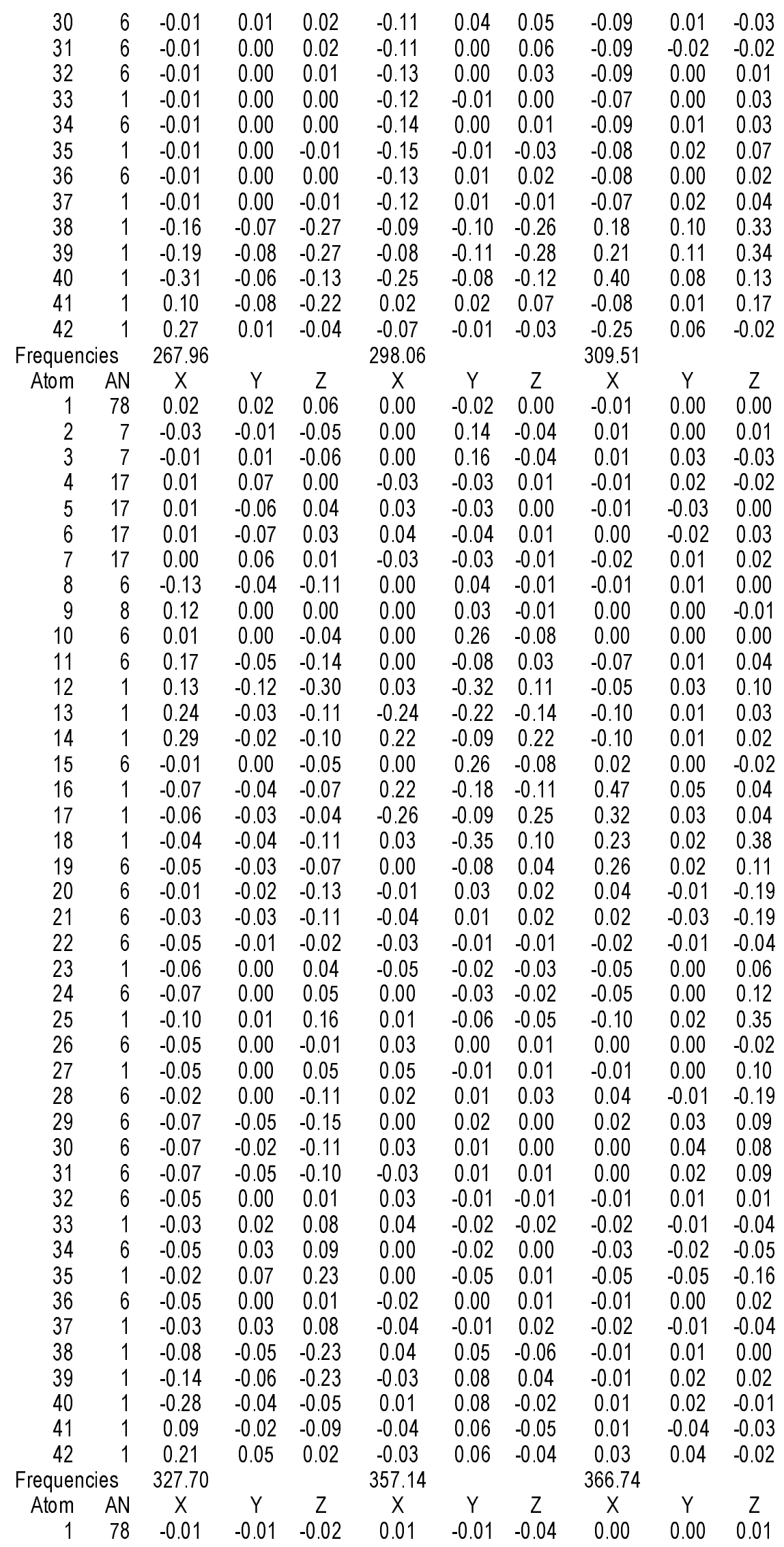




\begin{tabular}{|c|c|c|c|c|c|c|c|c|c|c|}
\hline 2 & & & & & & & & & & \\
\hline $\begin{array}{l}2 \\
3\end{array}$ & 7 & 0.00 & 0.01 & 0.05 & -0.06 & -0.02 & 0.11 & 0.02 & -0.25 & 0.06 \\
\hline 4 & $\begin{array}{r}1 \\
17\end{array}$ & $\begin{array}{l}.02 \\
0.00\end{array}$ & -0.02 & $\begin{array}{c}0.00 \\
-0.02\end{array}$ & $\begin{array}{l}0.00 \\
0.06\end{array}$ & $\begin{array}{c}0.11 \\
-0.07\end{array}$ & 0.10 & 0.07 & $\begin{array}{l}0.20 \\
0.04\end{array}$ & -0.03 \\
\hline 5 & 17 & 0.00 & 0.00 & -0.03 & 0.03 & 0.09 & $\begin{array}{l}0.00 \\
-0.01\end{array}$ & -0.09 & 0.01 & 0.01 \\
\hline 6 & 17 & -0.01 & -0.04 & 0.01 & 0.01 & -0.04 & 0.02 & 0.09 & -0.02 & 0.02 \\
\hline 7 & 17 & -0.02 & 0.04 & -0.01 & $\begin{array}{l}-0.04 \\
\end{array}$ & 0.02 & 0.00 & -0.07 & -0.04 & $\begin{array}{l}-0.02 \\
\end{array}$ \\
\hline 8 & 6 & 0.00 & 0.00 & -0.01 & 0.05 & 0.01 & & & 0.03 & -0.01 \\
\hline 9 & 8 & -0.02 & -0.01 & -0.02 & -0.06 & -0.01 & -0.02 & 0.00 & -0.02 & 0.00 \\
\hline 10 & 6 & 0.02 & 0.01 & 0.06 & -0.01 & 0.01 & 0.15 & 0.01 & -0.14 & 0.01 \\
\hline 11 & 6 & 0.27 & -0.02 & -0.07 & 0.05 & 0.05 & 0.1 & -0.02 & 0.02 & -0.03 \\
\hline 12 & 1 & 0.22 & -0.11 & -0.31 & 0.04 & 0.07 & 0.08 & -0.02 & 0.12 & -0.04 \\
\hline 13 & 1 & 0.40 & 0.01 & -0.02 & 0.10 & 0.06 & 0.1 & 0.07 & 0.09 & 0.05 \\
\hline 14 & 1 & 0.44 & & -0.03 & 0.04 & 0.05 & 0.1 & -0.13 & 0.02 & -0.13 \\
\hline 15 & 6 & -0.01 & & & & 0.07 & 0.1 & & 0.07 & -0.07 \\
\hline 16 & 1 & -0.25 & -0.01 & 0.04 & -0.16 & 0.06 & 0.2 & 0.1 & -0.05 & -0.09 \\
\hline 17 & 1 & -0.18 & & & -0.17 & 0.07 & 0.2 & -0. & -0.02 & 0.00 \\
\hline 18 & 1 & -0.13 & & -0.14 & -0.13 & 0.04 & 0.1 & & -0.07 & 0.05 \\
\hline 19 & 6 & -0.15 & & 0.00 & -0.13 & 0.07 & 0.2 & 0.0 & -0.02 & -0.05 \\
\hline 20 & 6 & 0.01 & & -0.0 & 0.03 & 0.04 & -0.0 & -0.02 & 0.14 & 0.01 \\
\hline 21 & 6 & -0.01 & & -0.03 & -0.01 & -0.01 & -0 . & -0.13 & 0.07 & 0.03 \\
\hline 22 & 6 & -0.02 & -0.01 & -0.01 & -0.03 & -0.03 & -0.04 & -0.13 & -0.04 & 0.00 \\
\hline 23 & 1 & -0.01 & & 0.0 & -0.07 & -0.04 & 0. & -0.23 & -0.11 & -0.03 \\
\hline 24 & 6 & -0.03 & & 0. & -0.04 & -0.03 & 0.1 & 0.1 & -0.14 & -0.04 \\
\hline 25 & 1 & -0.04 & 0.00 & 0.0 & -0.08 & -0.05 & 0.2 & 0.05 & $\begin{array}{l}-0.27 \\
\end{array}$ & -0.13 \\
\hline 26 & 6 & -0.01 & 0.01 & -0.01 & 0.04 & -0.01 & -0.02 & 0.12 & -0.04 & 0.05 \\
\hline 27 & 1 & 0.00 & & 0. & & -0.02 & 0. & 0. & -0.09 & \\
\hline 28 & 6 & 0.1 & & & & 0.02 & -0 . & & & \\
\hline 29 & 6 & 0.03 & 0.05 & 0.16 & -0.07 & -0.06 & -0. & 0.0 & -0.12 & 0.05 \\
\hline 30 & 6 & 0.03 & & 0. & -0.04 & -0.11 & -0 . & -0 . & -0.04 & 0.07 \\
\hline 31 & 6 & 0.04 & & & -0.01 & -0.02 & -0 . & 0.11 & -0.07 & 0.03 \\
\hline 32 & 6 & 0.01 & & & -0.02 & -0.03 & -0 . & -0.12 & 0.06 & 0.01 \\
\hline 33 & 1 & -0.0 & & -0.0 & -0. & 0.02 & 0.0 & -0.22 & 0.11 & -0.01 \\
\hline 34 & 6 & -0.01 & & -0.1 & & 0.06 & 0.1 & -0.0 & 0.12 & -0.06 \\
\hline 35 & 1 & -0.06 & & -0.3 & & & 0.3 & -0.0 & 0.22 & -0.15 \\
\hline 36 & 6 & 0. & & 0. & & 0. & -0 & 0. & 0.03 & -0.02 \\
\hline 37 & 1 & -0.01 & & -0 . & & 0.03 & 0.1 & 0.23 & 0.09 & -0.07 \\
\hline 38 & 1 & -0.0 & & 0. & & & 0. & 0.04 & 0.04 & -0.10 \\
\hline 39 & 1 & 0.00 & & & & & & & & \\
\hline 40 & 1 & 0.1 & & -0 & & 0.0 & -0 . & -0.02 & 0.10 & -0.03 \\
\hline 41 & 1 & & & & & -0.05 & 0. & 0.05 & -0.07 & 0.05 \\
\hline 42 & 1 & -0.02 & & & & & -0.0 & & -0.06 & \\
\hline quen & & 387.74 & & & & & & 420.89 & & \\
\hline Atom & AN & $x$ & $Y$ & Z & & $Y$ & Z & $X$ & $Y$ & 2 \\
\hline 1 & 78 & & 0.00 & 0.01 & & 0.01 & 0.00 & & -0.01 & -0.04 \\
\hline 2 & 7 & & & -0 . & & -0.09 & 0. & & 0.01 & 0.00 \\
\hline 3 & 7 & & & & & -0.08 & 0.0 & 0.0 & -0.01 & 0.01 \\
\hline 4 & 17 & & & & & -0.05 & -0.0 & & -0.02 & 0.00 \\
\hline 5 & 17 & & & & & & 0. & & & 0.00 \\
\hline 6 & 17 & & & & & -0.05 & -0.1 & 0.1 & -0.01 & 0.00 \\
\hline 7 & 17 & & & & & -0.02 & 0. & -0.02 & 0.02 & -0.01 \\
\hline 8 & 6 & & & -0.0 & & & & & & 0.00 \\
\hline 9 & 8 & & & 0.0 & & & & & 0.13 & \\
\hline 10 & 6 & -0.1 & & -0.0 & & 0.14 & -0.03 & 0.01 & 0.01 & 0.01 \\
\hline 11 & 6 & -0.02 & & & & -0.01 & & & 0.01 & 0.02 \\
\hline 12 & 1 & -0.0 & & -0.4 & & -0.11 & & & 0.01 & 0.04 \\
\hline 13 & 1 & 0.0 & & -0.2 & -0.0 & -0.10 & -0 . & 0. & 0.01 & 0.02 \\
\hline 14 & 1 & & & -0.1 & & -0.03 & & & 0.01 & 0.02 \\
\hline 15 & 6 & -0.18 & & & & 0.14 & -0. & 0.0 & 0.00 & 0.01 \\
\hline 16 & 1 & & & 0.07 & 0.10 & -0.10 & -0.07 & -0.02 & 0.00 & 0.00 \\
\hline 17 & 1 & 0.08 & 0.03 & 0.10 & -0.10 & -0.03 & 0.16 & -0.01 & 0.00 & 0.00 \\
\hline
\end{tabular}




\begin{tabular}{|c|c|c|c|c|c|c|c|c|c|c|}
\hline & & & & & & & & & & \\
\hline $\begin{array}{l}18 \\
10\end{array}$ & 1 & -0.04 & 0.08 & 0.34 & 0.02 & -0.12 & 0.05 & 0.00 & 0.00 & $\begin{array}{r}-0.02 \\
0.00\end{array}$ \\
\hline $\begin{array}{l}19 \\
20\end{array}$ & $\begin{array}{l}6 \\
6\end{array}$ & $\begin{array}{l}-0.01 \\
-0.14\end{array}$ & $\begin{array}{r}0.04 \\
-0.01\end{array}$ & $\begin{array}{r}0.13 \\
-0.04\end{array}$ & $\begin{array}{l}0.00 \\
0.01\end{array}$ & $\begin{array}{l}-0.02 \\
-0.11\end{array}$ & $\begin{array}{l}0.02 \\
0.01\end{array}$ & $\begin{array}{l}0.00 \\
0.02\end{array}$ & $\begin{array}{l}0.00 \\
0.01\end{array}$ & $\begin{array}{l}0.00 \\
0.01\end{array}$ \\
\hline 21 & 6 & -0.05 & 0.10 & $\begin{array}{l}-0.04 \\
-0.04\end{array}$ & 0.08 & -0.07 & 0.02 & 0.01 & 0.00 & 0.00 \\
\hline 22 & 6 & -0.06 & 0.05 & -0.03 & 0.10 & 0.09 & 0.00 & 0.01 & -0.02 & 0.00 \\
\hline 23 & 1 & -0.13 & 0.00 & -0.03 & 0.22 & 0.17 & 0.01 & 0.01 & -0.02 & -0.01 \\
\hline 24 & 6 & -0.01 & 0.00 & 0.02 & -0.01 & 0.18 & -0.01 & 0.01 & -0.01 & \\
\hline 25 & 1 & -0.02 & 0.00 & 0.05 & -0.02 & 0.27 & 0.01 & 0.00 & -0.01 & 0.02 \\
\hline 26 & 6 & -0.05 & -0.06 & -0.01 & -0.10 & 0.09 & -0.04 & 0.01 & 0.00 & 0.00 \\
\hline 27 & 1 & -0.12 & -0.02 & -0.03 & -0.22 & 0.15 & -0.06 & 0.03 & -0.01 & 0.01 \\
\hline 28 & 6 & -0.04 & -0.10 & -0.02 & -0.07 & -0.07 & -0.03 & 0.01 & 0.01 & -0.01 \\
\hline 29 & 6 & -0.08 & 0.00 & 0.04 & 0.00 & -0.11 & 0.04 & -0.01 & 0.00 & 0.00 \\
\hline 30 & 6 & -0.02 & -0.05 & 0.08 & -0.08 & -0.07 & 0.04 & -0.01 & -0.01 & 0.01 \\
\hline 31 & 6 & -0.01 & 0.07 & 0.03 & 0.08 & -0.08 & 0.00 & -0.01 & 0.01 & .00 \\
\hline 32 & 6 & -0.04 & -0.02 & 0.03 & -0.11 & 0.11 & -0.02 & -0.01 & 0.01 & .00 \\
\hline 33 & 1 & -0.10 & 0.01 & 0.02 & -0.25 & 0.18 & -0.03 & -0.03 & 0.02 & 0.00 \\
\hline 34 & 6 & -0.01 & 0.00 & -0.04 & 0.00 & 0.19 & -0.06 & -0.01 & 0.02 & -0.01 \\
\hline 35 & 1 & -0.02 & -0.02 & -0.12 & 0.00 & 0.29 & -0.09 & 0.00 & 0.02 & .00 \\
\hline 36 & 6 & -0.02 & 0.04 & 0.01 & 0.11 & 0.10 & -0.05 & -0.01 & 0.02 & \\
\hline 37 & 1 & -0.06 & 0.02 & 0.01 & 0.25 & 0.17 & -0.09 & 0.00 & 0.03 & 01 \\
\hline 38 & 1 & -0.02 & -0.03 & -0.09 & -0.02 & -0.01 & 0.0 & 0.00 & 0.02 & \\
\hline 39 & 1 & -0.02 & -0.02 & -0.08 & 0.02 & -0.02 & -0.01 & 0.02 & 0.02 & 0.07 \\
\hline 40 & 1 & -0.11 & 0.00 & 0.00 & 0.00 & -0.02 & 0.01 & 0.16 & 0.00 & -0.07 \\
\hline 41 & 1 & 0.04 & 0.01 & 0.04 & 0.02 & -0.04 & 0.02 & 0.29 & 0.25 & 38 \\
\hline 42 & 1 & 0.02 & 0.04 & 0.00 & 0.01 & -0.02 & 0.01 & 0.33 & 0.20 & 0.34 \\
\hline Frequel & & 428.40 & & & 438.30 & & & 449.93 & & \\
\hline Atom & AN & X & $\mathrm{Y}$ & Z & $X$ & $\mathrm{Y}$ & Z & $x$ & $Y$ & Z \\
\hline 1 & 78 & 0.00 & 0.00 & 0.00 & -0.01 & -0.01 & -0.02 & 0.00 & 0.01 & 0.00 \\
\hline 2 & 7 & 0.00 & 0.16 & -0.06 & 0.12 & 0.02 & 0.03 & 0.00 & -0.22 & 0.06 \\
\hline 3 & 7 & 0.00 & -0.19 & 0.06 & -0.10 & 0.02 & 0.10 & -0.01 & -0.17 & 0.07 \\
\hline 4 & 17 & 0.09 & -0.13 & 0.02 & -0.12 & 0.16 & -0.03 & -0.07 & 0.15 & -0.04 \\
\hline 5 & 17 & -0.09 & -0.12 & 0.06 & -0.13 & -0.16 & 0.08 & 0.05 & 0.12 & -0.05 \\
\hline 6 & 17 & 0.10 & 0.16 & 0.00 & 0.08 & 0.12 & 0.01 & 0.04 & 0.11 & \\
\hline 7 & 17 & -0.12 & 0.15 & -0.05 & 0.09 & -0.12 & 0.04 & -0.05 & 0.12 & -0.02 \\
\hline 8 & 6 & 0.00 & -0.01 & 0.01 & 0.00 & 0.00 & 0.01 & 0.00 & -0.01 & 0.00 \\
\hline 9 & 8 & -0.03 & -0.01 & -0.04 & 0.03 & 0.01 & 0.02 & 0.00 & -0.01 & 0.01 \\
\hline 10 & 6 & -0.01 & 0.10 & -0.04 & 0.07 & 0.02 & 0.03 & -0.01 & 0.15 & -0.06 \\
\hline 11 & 6 & -0.01 & -0.01 & -0.01 & 0.07 & 0.02 & 0.07 & & -0.02 & \\
\hline 12 & 1 & 0.00 & -0.07 & 0.02 & 0.07 & 0.02 & 0.08 & & -0.12 & \\
\hline 13 & 1 & -0.09 & -0.07 & -0.08 & 0.05 & 0.02 & 0.06 & -0.13 & -0.13 & -0.13 \\
\hline 14 & 1 & 0.05 & -0.02 & 0.06 & 0.06 & 0.02 & 0.07 & 0.08 & -0.04 & \\
\hline 15 & 6 & 0.00 & -0.07 & 0.0 & -0.04 & 0.02 & 0.07 & & & -0.1 \\
\hline 16 & 1 & -0.04 & 0.02 & 0.03 & -0.18 & 0.03 & 0.11 & 0.10 & -0.14 & \\
\hline 17 & 1 & 0.03 & 0.00 & -0.05 & -0.16 & 0.03 & 0.10 & -0.16 & -0.04 & \\
\hline 18 & 1 & -0.01 & 0.03 & -0.02 & -0.10 & 0.00 & -0.01 & 0.0 & -0.1 & 0.05 \\
\hline 19 & 6 & 0.00 & 0.00 & 0.0 & -0.11 & 0.03 & & & & \\
\hline 20 & 6 & 0.00 & -0.02 & 0.02 & -0.14 & 0.00 & 0.01 & 0.00 & -0.09 & \\
\hline 21 & 6 & 0.06 & 0.02 & 0.00 & -0.10 & 0.06 & -0.09 & 0.1 & -0.04 & 0.02 \\
\hline 22 & 6 & 0.04 & -0.2 & 0.02 & -0.13 & 0.04 & & & & \\
\hline 23 & 1 & -0.08 & -0.3 & 0.0 & -0.17 & 0.01 & -0.08 & 0.1 & -0.17 & \\
\hline 24 & 6 & 0.01 & -0.2 & 0.06 & -0.13 & 0.01 & 0.05 & 0.00 & -0.11 & 0.05 \\
\hline 25 & 1 & 0.00 & -0.2 & 0.0 & -0.16 & 0.02 & 0.1 & & & 0.09 \\
\hline 26 & 6 & 0.00 & -0.23 & 0.0 & -0.12 & -0.05 & -0.07 & & & \\
\hline 27 & 1 & 0.12 & -0.29 & 0.05 & -0.16 & -0.02 & -0.09 & -0.0 & -0.17 & 0.00 \\
\hline 28 & 6 & -0.04 & 0.01 & -0.0 & -0.08 & -0.08 & & & -0.05 & \\
\hline 29 & 6 & 0.00 & 0.01 & -0.0 & 0.19 & 0.00 & -0.0 & & & \\
\hline 30 & 6 & 0.04 & -0.01 & 0.00 & 0.14 & 0.08 & -0.10 & -0.12 & -0.03 & 0.05 \\
\hline 31 & 6 & -0.04 & -0.0 & 0.0 & 0.13 & -0.12 & & & & -0.01 \\
\hline 32 & 6 & 0.02 & 0.18 & -0.0 & 0.18 & 0.05 & & -0.11 & & 0.08 \\
\hline 33 & 1 & -0.08 & 0.24 & -0.06 & 0.25 & 0.01 & -0.09 & -0.08 & -0.20 & \\
\hline
\end{tabular}




\begin{tabular}{|c|c|c|c|c|c|c|c|c|c|c|}
\hline 34 & 6 & 0.00 & 0.20 & -0.07 & 0.16 & 0.02 & 0.01 & 0.01 & -0.15 & 0.04 \\
\hline & & 0.00 & 0.16 & -0.06 & 0.18 & 0.04 & 0.09 & 0.01 & 0.00 & -0.02 \\
\hline 36 & 6 & -0.01 & .18 & -0.05 & 0.18 & -0.06 & -0.04 & 0.14 & -0.19 & .05 \\
\hline 37 & 1 & 0.09 & 23 & -0.09 & & & & & & .08 \\
\hline 38 & 1 & -0.04 & -0.03 & 0.06 & -0.01 & 0.01 & 0.03 & -0.03 & -0.02 & 0.04 \\
\hline 39 & 1 & 0.04 & & & & & & & & .02 \\
\hline 40 & 1 & -0.02 & & & & & & & & \\
\hline 41 & 1 & -0.07 & & -0.09 & 0.0 & -0.01 & 0.0 & 0.07 & -0.07 & 0.08 \\
\hline 42 & 1 & -0.06 & 03 & -0.07 & 0.05 & -0.01 & 0.05 & 0.06 & -0.05 & .07 \\
\hline \multicolumn{2}{|c|}{ Frequencies } & \multicolumn{2}{|l|}{475.14} & & \multicolumn{2}{|l|}{521.45} & & \multicolumn{3}{|l|}{534.95} \\
\hline Atom & AN & $x$ & Y & Z & $x$ & Y & Z & $x$ & Y & Z \\
\hline 1 & 78 & -0.01 & 0.00 & -0.01 & 0.00 & 0.00 & 0.00 & 0.00 & 0.00 & 0.00 \\
\hline 2 & 7 & 0.03 & 0.04 & 0.14 & & -0.02 & -0.08 & & 0.00 & 0.00 \\
\hline 3 & 7 & 0.03 & & -0.11 & & 0.01 & 0.08 & & 0.00 & 0.00 \\
\hline 4 & 17 & 0.04 & -0.04 & 0.01 & & -0.02 & 0.0 & & 0.00 & 0.00 \\
\hline 5 & 17 & 0.04 & & & & 0.02 & -0.1 & & & 00 \\
\hline 6 & 17 & 0.06 & & 0.00 & & 0.01 & 0.0 & & & -0.03 \\
\hline 7 & 17 & & & & & -0.03 & 0.01 & & 0.00 & 0.03 \\
\hline 8 & 6 & 0.01 & & -0.0 & & 0.00 & 0.0 & & & 0.00 \\
\hline 9 & 8 & -0.02 & & -0.0 & 0.0 & -0.01 & -0.01 & 0.00 & 0.00 & -0.01 \\
\hline 10 & 6 & 0.10 & 0.07 & 0.16 & 0.1 & 0.00 & -0.04 & 0.01 & -0.02 & 0.01 \\
\hline 11 & 6 & 0.18 & & & -0.0 & 0.02 & 0.0 & & .00 & 0.01 \\
\hline 12 & 1 & 0.17 & & & & & 0.2 & & & \\
\hline 13 & 1 & 0.22 & & & & & 0.0 & & & 0.03 \\
\hline 14 & 1 & 0.25 & & & & -0.01 & 0.0 & & .01 & -0.01 \\
\hline 15 & 6 & 0.07 & & & & & & & & -0.0 \\
\hline 16 & 1 & 0.19 & & & & & & & & \\
\hline 17 & 1 & 0.17 & & & & -0.01 & -0 . & & & 0.02 \\
\hline 18 & 1 & & & & & -0 & & & -0 . & -0.03 \\
\hline 15 & 6 & & & & & & & & & \\
\hline 20 & 6 & & & & & 0.04 & & & & 0.01 \\
\hline 21 & 6 & & & & & & -0. & & & .40 \\
\hline 22 & 6 & & & & & & & & -0.02 & -0.14 \\
\hline 23 & 1 & & & & & & -0 & & -0.07 & -0.5 \\
\hline 24 & 6 & & & & & & 0. & & & \\
\hline 25 & 1 & & & & -0 . & & & & & 0.00 \\
\hline 26 & 6 & & & & & & & & & \\
\hline 27 & 1 & & & & & & & & & \\
\hline 28 & 6 & & & & & & -0 . & & 5 & -0 \\
\hline 29 & 6 & & & & & & -0 . & & & -0.01 \\
\hline 30 & 6 & & & & & & & & & \\
\hline 31 & 6 & & & & & 0. & 0. & & & \\
\hline 32 & 6 & & & & & & 0. & & & \\
\hline 33 & 1 & & & & & & & & & \\
\hline 34 & 6 & -0 . & & & & -0 . & -0 . & & & \\
\hline 35 & 1 & & & & & & -0 . & & -0.01 & -0.01 \\
\hline 36 & 6 & & & & & & & & & \\
\hline 37 & 1 & & & & & & & & & \\
\hline 38 & 1 & & & & & & -0 . & -0 . & & 0.1 \\
\hline 39 & 1 & & & & & & -0. & & -0.01 & -0.01 \\
\hline 40 & 1 & & & & & & & & & \\
\hline 41 & 1 & & & & & & & & & \\
\hline 42 & 1 & 0.00 & & 0.03 & -0.02 & 0.02 & -0.03 & 0.00 & 0.01 & -0.02 \\
\hline Frequen & & & & & & & & & & \\
\hline & AN & $X$ & $Y$ & Z & $x$ & $Y$ & & $x$ & Y & Z \\
\hline & 78 & & 0.00 & & 0.0 & 0.00 & 0.0 & & 0. & 0.00 \\
\hline 2 & 7 & & & & & 0.01 & 0.0 & & 0.04 & -0.01 \\
\hline 3 & 7 & & 0.0 & & -0. & -0.01 & 0.0 & & -0.01 & 0.00 \\
\hline 4 & 17 & & & -0.0 & & 0.00 & 0.01 & & 0.01 & 0.00 \\
\hline 5 & 17 & 0.01 & 0.01 & 0.03 & 0.01 & 0.01 & 0.00 & 0.00 & 0.01 & 0.00 \\
\hline
\end{tabular}




\begin{tabular}{|c|c|c|c|c|c|c|c|c|c|c|}
\hline & & & & & & & & & & \\
\hline 6 & 17 & 0.00 & 0.00 & 0.00 & -0.01 & -0.01 & 0.01 & 0.00 & 0.00 & 0.00 \\
\hline 7 & 17 & 0.00 & 0.00 & 0.00 & -0.01 & 0.00 & 0.00 & 0.00 & 0.00 & 0.00 \\
\hline 8 & 6 & 0.00 & 0.00 & 0.00 & -0.03 & 0.00 & 0.02 & -0.02 & 0.01 & 0.02 \\
\hline 9 & 8 & 0.00 & 0.00 & 0.00 & -0.01 & 0.01 & -0.01 & 0.04 & -0.08 & 0.05 \\
\hline 10 & 6 & 0.00 & 0.00 & 0.00 & -0.03 & 0.00 & -0.03 & 0.00 & 0.01 & 0.00 \\
\hline 11 & 6 & 0.0 & 0.00 & 0.00 & -0.02 & -0.02 & -0.07 & 0.00 & 0.00 & 0.01 \\
\hline 12 & 1 & 0.00 & 0.02 & 0.00 & -0.03 & -0.05 & -0.13 & 0.00 & 0.00 & 0.00 \\
\hline 13 & 1 & 0.01 & -0.02 & -0.02 & 0.01 & -0.02 & -0.07 & 0.01 & 0.00 & 0.00 \\
\hline 14 & 1 & -0.01 & -0.01 & 0.02 & 0.04 & -0.02 & -0.05 & 0.01 & 0.01 & 0.01 \\
\hline 15 & 6 & 0.00 & -0.02 & 0.01 & 0.01 & -0.02 & -0.04 & 0.00 & -0.02 & 0.00 \\
\hline 16 & 1 & -0.01 & 0.01 & 0.01 & 0.02 & -0.02 & -0.08 & -0.01 & 0.01 & 0.01 \\
\hline 17 & 1 & 0.02 & 0.00 & -0.03 & 0.04 & -0.03 & -0.09 & 0.02 & 0.00 & -0.03 \\
\hline 18 & 1 & 0.0 & 0.02 & -0.01 & 0.03 & -0.02 & -0.09 & 0.0 & 0.01 & -0.01 \\
\hline 19 & 6 & 0.00 & 0.00 & 0.00 & 0.03 & -0.03 & -0.08 & 0.01 & 0.00 & -0.01 \\
\hline 20 & 6 & 0.00 & 0.01 & 0.00 & -0.05 & & 0.18 & 0.00 & 0.01 & 0.00 \\
\hline 21 & 6 & -0.01 & 0.01 & 0.01 & 0.00 & 0.01 & -0.01 & -0.01 & 0.01 & 0.00 \\
\hline 22 & 6 & -0.01 & 0.00 & -0.01 & 0.03 & 0.00 & -0.12 & -0.0 & 0.01 & \\
\hline 23 & 1 & 0.00 & 0.00 & -0.03 & 0.06 & -0.03 & -0.28 & -0.02 & 0.01 & -0.01 \\
\hline 24 & 6 & 0.0 & 0.00 & 0.00 & 0.00 & 0.02 & 0.17 & 0.00 & 0.00 & 0.00 \\
\hline 25 & 1 & 0.0 & -0.01 & 0.01 & -0.04 & 0.02 & 0.37 & 0. & -0.02 & 0.01 \\
\hline 26 & 6 & $0 .($ & 0.01 & 0.00 & 0.08 & 0.00 & -0.11 & 0.01 & 0.01 & 0.00 \\
\hline 27 & 1 & 0.01 & 0.01 & 0.02 & 0.12 & -0.02 & -0.27 & 0.01 & 0.01 & 0.01 \\
\hline 28 & 6 & 0.0 & 0.00 & -0.02 & 0.04 & 0.0 & 0.0 & & & 0.00 \\
\hline 29 & 6 & 0.00 & 0.01 & 0.00 & 0.04 & & 0.21 & 0.00 & -0.02 & 0.02 \\
\hline 30 & 6 & 0.08 & 0.13 & 0.38 & -0.01 & 0.00 & -0.01 & -0.02 & -0.01 & 0.02 \\
\hline 31 & 6 & -0.08 & -0.12 & -0.39 & -0.01 & 0.00 & 0.00 & 0.03 & -0.02 & 0.00 \\
\hline 32 & 6 & -0.02 & -0.04 & -0.13 & -0.04 & -0.04 & -0.12 & -0.03 & -0.02 & 0.00 \\
\hline 33 & 1 & -0.09 & -0.17 & -0.53 & & -0.10 & -0.29 & -0.05 & -0.02 & -0.01 \\
\hline 34 & 6 & 0.0 & 0.00 & 0.01 & 0.01 & 0.06 & 0.18 & 0.01 & 0.00 & 0.01 \\
\hline 35 & 1 & 0.0 & -0.01 & 0.01 & 0.05 & 0.13 & 0.38 & 0.0 & 0.04 & 0.01 \\
\hline 36 & 6 & 0. & 0. & 0. & -0.04 & .05 & -0.13 & 0.0 & -0.02 & -0.01 \\
\hline 37 & 1 & 0. & & & & & -0.3 & 0.0 & -0.02 & -0.02 \\
\hline 38 & 1 & 0.0 & 0.01 & 0.0 & -0.03 & 0.00 & 0.01 & -0.04 & -0.01 & 0.02 \\
\hline 39 & 1 & -0.01 & 0. & -0.0 & -0.03 & 0.0 & 0.02 & 0.01 & -0.02 & -0.02 \\
\hline 40 & 1 & & & & & & 0.01 & -0.07 & -0.02 & 0.06 \\
\hline 41 & 1 & 0.0 & -0.02 & 0.0 & 0.0 & -0.05 & 0.0 & -0.39 & 0.32 & -0.40 \\
\hline 42 & 1 & 0.02 & -0.02 & 0.03 & 0.06 & -0.06 & 0.08 & -0.38 & 0.37 & -0.51 \\
\hline Frequen & & 581.88 & & & 603.66 & & & 609.79 & & \\
\hline Atom & AN & $x$ & $Y$ & Z & $X$ & $\mathrm{Y}$ & Z & $x$ & $Y$ & Z \\
\hline 1 & 78 & & 0.00 & 0.00 & -0.02 & 0.00 & 0.02 & -0.02 & 0.01 & 0.02 \\
\hline 2 & 7 & & 0.12 & -0.04 & -0 & 0.04 & 0.07 & 0.0 & -0.04 & -0.08 \\
\hline 3 & 7 & & -0.15 & 0.05 & & & -0.08 & & & 0.09 \\
\hline 4 & 17 & & 0.02 & 0.0 & & 0. & 0.0 & 0. & -0.01 & 0.00 \\
\hline 5 & 17 & & & & & & 0.01 & 0.0 & 0.01 & 0.00 \\
\hline 6 & 17 & & & & & & 0.00 & & & 0.00 \\
\hline 7 & 17 & & -0 . & & & & 0. & 0. & -0.01 & 0.00 \\
\hline 8 & 6 & & -0 . & & & -0.07 & -0.26 & 0.23 & -0.05 & -0.21 \\
\hline 9 & 8 & & 0. & & & & 0.01 & & -0.02 & 0.01 \\
\hline 10 & 6 & 0. & 0.2 & & & & 0.07 & & & -0.08 \\
\hline 11 & 6 & & & & & & 0.05 & & -0.02 & -0.07 \\
\hline 12 & 1 & & & & & & -0.14 & -0.01 & 0.05 & 0.14 \\
\hline 13 & 1 & & -0 . & & & & 0.07 & & -0.01 & -0.08 \\
\hline 14 & 1 & & & & & & 0.0 & & -0.05 & -0.13 \\
\hline 15 & 6 & & -0.21 & 0.07 & -0.08 & -0.04 & -0.05 & 0.10 & 0.05 & 0.07 \\
\hline 16 & 1 & & & & & & -0.08 & -0.1 & & 0.09 \\
\hline 17 & 1 & & & & & & -0.1 & -0.1 & 0.03 & 0.11 \\
\hline 18 & 1 & -0. & 0.12 & -0.0 & 0.00 & 0.06 & 0.15 & 0.00 & -0.07 & -0.17 \\
\hline 19 & 6 & & & & 0.0 & & -0.04 & -0.03 & 0.02 & 0.05 \\
\hline 20 & 6 & & 0. & -0. & -0. & 0.0 & 0.05 & 0.06 & -0.01 & -0.04 \\
\hline 21 & 6 & & 0.06 & & 0.01 & 0.02 & 0.03 & -0.01 & -0.02 & -0.02 \\
\hline
\end{tabular}




\begin{tabular}{|c|c|c|c|c|c|c|c|c|c|c|}
\hline & & & & & & & & & & \\
\hline 22 & 6 & -0.14 & 0.06 & -0.01 & 0.05 & 0.02 & -0.05 & -0.05 & -0.02 & 0.04 \\
\hline 23 & 1 & -0.19 & 0.04 & 0.02 & 0.05 & -0.02 & -0.16 & -0.04 & 0.01 & 0.13 \\
\hline 24 & 6 & 0.00 & -0.01 & -0.04 & 0.07 & 0.01 & 0.09 & -0.09 & -0.01 & -0.08 \\
\hline 25 & 1 & 0.02 & -0.17 & -0.06 & 0.05 & 0.02 & 0.15 & -0.08 & -0.01 & -0.13 \\
\hline 26 & 6 & 0.11 & 0.08 & 0.05 & 0.06 & -0.02 & -0.04 & -0.07 & 0.02 & 0.03 \\
\hline 27 & 1 & 0.15 & 0.07 & 0.13 & 0.05 & -0.02 & -0.15 & -0.06 & 0.02 & 0.13 \\
\hline 28 & 6 & 0.09 & 0.07 & -0.01 & 0.02 & -0.01 & 0.03 & -0.02 & 0.01 & -0.03 \\
\hline 29 & 6 & 0.00 & -0.06 & 0.02 & -0.05 & -0.02 & -0.04 & 0.05 & 0.03 & 0.07 \\
\hline 30 & 6 & -0.09 & -0.05 & 0.02 & 0.01 & -0.03 & -0.01 & 0.00 & 0.03 & 0.03 \\
\hline 31 & 6 & 0.08 & -0.06 & 0.02 & 0.03 & 0.00 & -0.03 & -0.02 & 0.00 & 0.04 \\
\hline 32 & 6 & -0.11 & -0.05 & 0.04 & 0.04 & -0.01 & 0.04 & -0.05 & 0.00 & -0.07 \\
\hline 33 & 1 & -0.15 & -0.03 & 0.05 & 0.02 & 0.03 & 0.12 & -0.04 & -0.06 & -0.21 \\
\hline 34 & 6 & -0.01 & 0.00 & 0.00 & 0.09 & -0.02 & -0.07 & -0.07 & 0.03 & 0.10 \\
\hline 35 & 1 & -0.01 & & -0.04 & 0.08 & -0.02 & -0.11 & -0.06 & 0.04 & 0.17 \\
\hline 36 & 6 & 0.10 & -0.07 & 0.00 & 0.07 & 0.02 & 0.03 & -0.06 & -0.03 & -0.06 \\
\hline 37 & 1 & 0.14 & -0.05 & -0.03 & 0.06 & 0.03 & 0.11 & -0.06 & -0.07 & -0.20 \\
\hline 38 & 1 & -0.01 & & 0.03 & 0.27 & -0.07 & -0.26 & 0.20 & -0.05 & -0.15 \\
\hline 39 & 1 & 0.03 & & -0.02 & 0.26 & -0.06 & -0.25 & 0.22 & -0.05 & -0.17 \\
\hline 40 & 1 & 0.04 & -0.02 & -0.02 & 0.25 & -0.06 & -0.25 & 0.26 & -0.06 & -0.22 \\
\hline 41 & 1 & 0.17 & & 0.17 & -0.07 & 03 & -0.07 & -0.13 & 0.05 & -0.18 \\
\hline 42 & 1 & 0.18 & & 0.24 & -0.01 & 0.03 & -0.01 & 0.03 & 0.07 & -0.03 \\
\hline equen & & 630.65 & & & 671.69 & & & 688.84 & & \\
\hline tom & AN & $x$ & $Y$ & Z & $\mathrm{x}$ & $Y$ & Z & $X$ & Y & Z \\
\hline & 78 & 0.00 & -0.01 & 0.00 & 0.00 & 0.00 & 0.00 & 0.00 & 0.00 & 0.00 \\
\hline 2 & 7 & 0.01 & 0.23 & -0.09 & & 0.28 & -0.09 & $0.1 \varepsilon$ & -0.04 & -0.02 \\
\hline 3 & 7 & 0.03 & 0.35 & -0.10 & -0.02 & -0.12 & 0.04 & -0.24 & 0.04 & 0.02 \\
\hline 4 & 17 & 0.00 & 0.01 & 0.00 & 0.00 & 0.00 & 0.00 & 0.03 & -0.03 & 0.01 \\
\hline 5 & 17 & 0.00 & 0.01 & 0.00 & 0.00 & 0.01 & 0.00 & 0.03 & 0.03 & -0.01 \\
\hline 6 & 17 & 0.00 & 0.02 & 0.00 & 0.0 & -0.01 & 0.00 & -0.03 & -0.04 & 0.00 \\
\hline 7 & 17 & & & 0.00 & & 0.00 & 0.00 & -0.0 & 0.04 & -0.01 \\
\hline 8 & 6 & -0. & 00 & 0.01 & & 0.01 & -0.01 & 0.02 & -0.01 & -0.02 \\
\hline 9 & 8 & -0.01 & 0.03 & -0.01 & -0.01 & 0.03 & -0.01 & 0.0 & -0.01 & 0.01 \\
\hline 10 & 6 & 0.0 & 01 & -0.02 & & -0.24 & 0.0 & 0.1 & 0.02 & -0.06 \\
\hline 11 & 6 & & & -0.02 & & -0.03 & 0.02 & 0. & & 0.09 \\
\hline 12 & 1 & & & 0.01 & & 0.18 & -0.07 & 0. & 0.04 & 0.21 \\
\hline 13 & 1 & -0.02 & 0.02 & -0.01 & 0.2 & 0.22 & 0.2 & -0.0 & -0.01 & 0.03 \\
\hline 14 & 1 & -0. & & -0 & -0 & 0.04 & -0.31 & 0. & 0.01 & 0.10 \\
\hline 15 & 6 & & & & & & -0.07 & & -0.05 & -0.04 \\
\hline 16 & 1 & & 0.20 & 0.2 & & -0.18 & -0.2 & -0 . & 0.04 & 0.05 \\
\hline 17 & 1 & & & -0.28 & & -0.02 & 0.30 & 0.00 & 0.01 & -0.02 \\
\hline 18 & 1 & & & -0.03 & & -0.16 & 0.0 & & & 0.07 \\
\hline 19 & 6 & & & & & & -0 . & & & 0.04 \\
\hline 20 & 6 & 0. & -0.05 & 0. & -0. & 0.01 & -0.01 & -0 . & -0.01 & -0.03 \\
\hline 21 & 6 & & & 0. & & 0.02 & -0.02 & 0.00 & 0.10 & -0.07 \\
\hline 22 & 6 & & & & & & & & & 0.04 \\
\hline 23 & 1 & & & & & & 0. & -0 . & 0.0 & 0.17 \\
\hline 24 & 6 & & & 0. & & & 0.00 & $0.3^{3}$ & 0.02 & 0.02 \\
\hline 25 & 1 & & & & & & 0.0 & & & 0.05 \\
\hline 26 & 6 & & & & & & 0. & 0. & -0.13 & 0.07 \\
\hline 27 & 1 & & & -0.0 & & 0.01 & 0. & -0.17 & -0.01 & 0.16 \\
\hline 28 & 6 & & & & & & 0. & & & -0.04 \\
\hline 29 & 6 & & & 0. & & -0.01 & 0. & 0. & -0.01 & -0.03 \\
\hline 30 & 6 & & & 0. & -0. & -0.02 & 0.02 & 0.01 & 0.09 & -0.04 \\
\hline 31 & 6 & & & & & -0.04 & -0.0 & 0. & -0.09 & 0.02 \\
\hline 32 & 6 & & & 0.0 & & -0.04 & 0.0 & & 0.11 & -0.02 \\
\hline 33 & 1 & -0 & & 0.0 & -0.1 & -0.02 & 0.03 & 0.11 & 0.03 & -0.01 \\
\hline 34 & 6 & & & 0.0 & -0.02 & 0.01 & 0.00 & -0.28 & 0.01 & 0.04 \\
\hline 35 & 1 & & & -0.0 & & 0.13 & -0.04 & & 0.00 & 0.06 \\
\hline 36 & 6 & & & 0.0 & & -0.06 & 0.01 & -0.06 & -0.10 & 0.05 \\
\hline 37 & 1 & 0.15 & -0.05 & -0.02 & 0.13 & -0.03 & 0.01 & 0.08 & -0.02 & 0.01 \\
\hline
\end{tabular}




\begin{tabular}{|c|c|c|c|c|c|c|c|c|c|c|}
\hline 38 & 1 & 0.10 & 0.04 & -0.11 & -0.10 & -0.02 & 0.13 & 0.04 & -0.01 & -0.06 \\
\hline & 1 & & & 0.11 & 0.10 & -0.07 & -0.09 & & 0.01 & $-0,02$ \\
\hline 40 & 1 & 0.02 & 09 & -0.04 & 0.01 & -0.07 & & -0.01 & 01 & -0.01 \\
\hline 41 & 1 & 0.05 & .05 & 0.03 & 0.06 & -0.05 & 0.0 & -0.07 & .02 & \\
\hline 42 & 1 & 0.10 & -0.05 & 0.10 & 0.10 & -0.08 & 0.13 & 0.04 & 0.03 & 0.00 \\
\hline \multicolumn{2}{|c|}{ Frequencies } & \multicolumn{2}{|l|}{715.81} & & \multicolumn{2}{|l|}{739.55} & & \multicolumn{3}{|l|}{765.61} \\
\hline Atom & AN & X & Y & Z & X & Y & Z & $x$ & Y & Z \\
\hline 1 & 78 & 0.00 & 0.00 & 0.00 & 0.00 & 0.00 & 0.00 & -0.01 & 0.00 & 0.00 \\
\hline 2 & 7 & 0.11 & 0.01 & 0.06 & 0.03 & 0.02 & 0.08 & 0.00 & 0.01 & 0.01 \\
\hline 3 & 7 & 0.09 & -0.06 & -0.10 & -0.01 & 0.03 & 0.0 & & 0.00 & 0.00 \\
\hline 4 & 17 & 0.02 & -0.02 & 0.00 & 0.00 & 0.00 & 0.0 & 0.00 & 0.00 & 0.00 \\
\hline 5 & 17 & 0.02 & 0.02 & -0.01 & 0.00 & 0.00 & 0.00 & 0.00 & 0.00 & 0.00 \\
\hline 6 & 17 & 0.01 & & 0.00 & & 0.00 & 0.0 & & & \\
\hline 7 & 17 & 0.01 & & 0.00 & $0 .($ & 0.00 & 0.0 & 0. & 0.00 & 0.00 \\
\hline 8 & 6 & -0. & 0.00 & 0.00 & & -0.01 & -0.0 & 0. & 0.00 & -0.01 \\
\hline 9 & 8 & $-0 .(1-x-y$ & & & & & & & & \\
\hline 10 & 6 & -0.0 & 0.00 & 0.01 & -0.06 & 0.00 & 0.0 & & & \\
\hline 11 & 6 & -0.02 & -0.01 & -0.04 & -0.05 & -0.02 & -0.08 & -0.01 & 0.00 & -0.01 \\
\hline 12 & 1 & -0. & & -0.1 & -0.0 & -0.07 & -0. & -0.0 & -0.01 & -0.03 \\
\hline 13 & 1 & & & -0.03 & & -0.02 & -0.0 & & & \\
\hline 14 & 1 & 0.05 & 0.00 & -0.02 & 0.0 & -0.01 & -0.04 & & 0.00 & 0.00 \\
\hline 15 & 6 & & & -0.0 & 0. & 0.00 & 0.0 & & .00 & .00 \\
\hline 16 & 1 & & & 0.0 & & & -0. & & & \\
\hline 17 & 1 & & & & & -0.02 & -0.0 & & & 0.00 \\
\hline 18 & 1 & & & & & -0.04 & -0.15 & & & \\
\hline 19 & 6 & & & & & & -0 & & & \\
\hline 20 & 6 & & & -0.11 & -0.0 & 0.02 & & & & \\
\hline 21 & 6 & & & 0.13 & & -0.03 & -0 . & 0.0 & & 0.00 \\
\hline 22 & 6 & & & -0.0 & & & & & & \\
\hline 23 & 1 & & & & -0.05 & & & & -0.02 & -0.02 \\
\hline 24 & 6 & & & & -0.02 & -0.01 & -0 . & $-0 .($ & & \\
\hline 25 & 1 & & & -0.0 & & 0.0 & & & & \\
\hline 26 & 6 & & & -0.0 & -0 . & & 0. & & & \\
\hline 27 & 1 & & & & -0 . & & & & -0.01 & -0.03 \\
\hline 28 & 6 & & & & & & -0 . & & & \\
\hline 29 & 6 & 0.1 & & 0. & & & 0.1 & 0. & & -0.02 \\
\hline 30 & 6 & 0.0 & & -0 . & & & -0 & 0. & & \\
\hline 31 & 6 & & & $-0 .(1$ & & & & & & \\
\hline 32 & 6 & & & & & & & 0. & & -0.01 \\
\hline 33 & 1 & & & & & & & & .01 & \\
\hline 34 & 6 & & & & & & & & & \\
\hline 35 & 1 & & & & & 0.1 & & & & -0. \\
\hline 36 & 6 & & & & & & 0. & & & \\
\hline 37 & 1 & & & & & & & & & -0. \\
\hline 38 & 1 & & & & & & & & & \\
\hline 39 & 1 & & & & & & & & & -0 \\
\hline 40 & 1 & & & & & & & & & \\
\hline 41 & 1 & & & & & & & & & \\
\hline 42 & 1 & & & & & & -0. & & -0.05 & \\
\hline Frequen & & & & & & & & 817.70 & & \\
\hline & AN & & Y & Z & & Y & & & & Z \\
\hline & 78 & & 0.00 & 0.00 & & 0.00 & & & & \\
\hline 2 & 7 & & & & & 0.01 & 0. & & & -0.03 \\
\hline 3 & 7 & & -0.01 & -0.0 & & -0.03 & & & & -0.02 \\
\hline 4 & 17 & & & 0.0 & & 0.01 & & & & -0.0 \\
\hline 5 & 17 & & & -0.01 & & 0.01 & 0. & & 0.01 & -0.01 \\
\hline 6 & 17 & & & & & & & & & 0.00 \\
\hline 7 & 17 & & & 0.0 & & -0.06 & & & -0. & 0.00 \\
\hline 8 & 6 & & 0.0 & 0.0 & & 0.02 & & & 0.01 & 0.00 \\
\hline 9 & 8 & -0.01 & 0.00 & 0.01 & -0.01 & 0.00 & 0.01 & 0.00 & 0.00 & 0.00 \\
\hline
\end{tabular}




\begin{tabular}{|c|c|c|c|c|c|c|c|c|c|c|}
\hline & & & & & & & & & & \\
\hline 10 & 6 & -0.07 & 0.01 & 0.04 & -0.03 & 0.02 & 0.01 & 0.01 & 0.00 & $\begin{array}{l}-0.01 \\
\end{array}$ \\
\hline 11 & 6 & -0.01 & 0.00 & 0.02 & -0.02 & 0.00 & -0.01 & 0.02 & 0.01 & 0.02 \\
\hline 12 & 1 & -0.05 & -0.05 & -0.16 & -0.03 & -0.03 & -0.08 & 0.02 & 0.02 & 0.05 \\
\hline 13 & 1 & 0.11 & 0.01 & 0.05 & 0.02 & -0.01 & -0.01 & 0.00 & 0.01 & 0. \\
\hline 14 & 1 & 0.11 & 0.03 & 0.05 & 0.04 & 0.01 & 0.02 & & 0.00 & 0.01 \\
\hline 15 & 6 & -0.09 & -0.01 & -0.04 & 0.01 & 0.00 & 0.01 & & -0.01 & \\
\hline 16 & 1 & 0.12 & 0.00 & -0.06 & 0.01 & -0.03 & -0.05 & 0.02 & 0.00 & 0.00 \\
\hline 17 & 1 & 0.11 & -0.01 & -0.03 & -0.01 & & 0.00 & & 0.00 & 0.02 \\
\hline 18 & 1 & -0.06 & 0.07 & 0.22 & 0.03 & -0.04 & -0.06 & -0.02 & 0.01 & 0.07 \\
\hline 19 & 6 & -0.03 & 0.00 & 0.01 & 0.02 & -0.01 & -0.04 & -0.02 & 0.01 & \\
\hline 20 & 6 & 0.02 & 0.01 & 0.19 & 0.00 & & -0.03 & 0.00 & 0.07 & -0.01 \\
\hline 21 & 6 & 0.04 & -0.08 & -0.05 & 0.09 & 0.20 & -0.04 & 0.02 & 0.07 & 0.04 \\
\hline 22 & 6 & 0.01 & -0.06 & 0.02 & 0.11 & -0.08 & 0.08 & 0.05 & -0.04 & \\
\hline 23 & 1 & 0.03 & 0.03 & 0.32 & 0.08 & & -0.21 & -0.0 & -0.02 & 0.27 \\
\hline 24 & 6 & -0.09 & -0.01 & -0.09 & -0.01 & -0.03 & 0.03 & 0.00 & -0.02 & -0.04 \\
\hline 25 & 1 & -0.16 & 0.00 & 0.19 & 0.07 & 0.17 & -0.38 & -0.10 & 0.11 & 0.36 \\
\hline 26 & 6 & 0.03 & 0.09 & 0.01 & -0.12 & -0.08 & 0.03 & -0.02 & -0.03 & \\
\hline 27 & 1 & 0.05 & 0.08 & 0.33 & 0.07 & -0.18 & -0.23 & -0.05 & -0.01 & 0.28 \\
\hline 28 & 6 & 0.05 & 0.04 & -0.07 & -0.08 & 0.20 & -0.08 & -0.04 & 0.08 & 0.02 \\
\hline 29 & 6 & 0.03 & -0.06 & -0.17 & & & 0.01 & -0.01 & -0.05 & \\
\hline 30 & 6 & 0.04 & 0.07 & 0.04 & & -0.04 & -0.03 & 0.03 & -0.02 & 0.08 \\
\hline 31 & 6 & 0.04 & -0.03 & 0.07 & -0.02 & -0.06 & -0.0 & -0.01 & -0.02 & 0.09 \\
\hline 32 & 6 & 0.02 & 0.07 & -0.03 & & & 0.03 & 0.01 & -0.01 & \\
\hline 33 & 1 & 0.04 & -0.09 & -0.34 & & & -0.27 & 0.07 & & \\
\hline 34 & 6 & -0.09 & 0.03 & 0.09 & -0.01 & & 0.03 & -0.01 & -0.01 & -0.05 \\
\hline 35 & 1 & -0.15 & -0.07 & -0.20 & -0.09 & -0.16 & -0.34 & 0.11 & 0.13 & 0.54 \\
\hline 36 & 6 & 0.01 & -0.07 & 0.01 & -0.01 & & 0.05 & -0.04 & 0.00 & -0.07 \\
\hline 37 & 1 & 0.04 & -0.13 & -0.33 & -0.03 & -0.06 & -0.26 & 0.07 & 0.15 & 0.34 \\
\hline 38 & 1 & 0.02 & 0.00 & -0.03 & -0.11 & -0 . & 0.1 & -0.02 & -0.01 & 0.01 \\
\hline 39 & 1 & -0.02 & 0.01 & 0.01 & 0.10 & -0.06 & -0.0 & 0.03 & -0.02 & -0.04 \\
\hline 40 & 1 & 0.00 & 0.01 & 0.00 & & -0.07 & 0.00 & -0.03 & -0.02 & 0.02 \\
\hline 41 & 1 & -0.08 & -0.01 & -0.16 & & & -0.16 & -0.01 & & -0.01 \\
\hline 42 & 1 & 0.14 & 0.01 & 0.10 & 0.12 & 0.02 & 0.07 & 0.01 & 0.00 & 0.0 \\
\hline (1) & & 818.35 & & & & & & 844.90 & & \\
\hline Atom & AN & $x$ & $Y$ & Z & & $Y$ & Z & $x$ & $Y$ & Z \\
\hline & 78 & 0 & 0.00 & 0.00 & 0 & 0.00 & 0.00 & 0.00 & 0.00 & 0.00 \\
\hline 2 & 7 & 0. & -0.09 & 0.02 & & & 0.00 & -0. & 0.02 & 0.02 \\
\hline 3 & 7 & & -0.01 & -0.01 & & & 0.00 & -0.1 & -0.03 & 0.07 \\
\hline 4 & 17 & & -0.09 & 0.02 & & & 0.0 & 0.1 & -0.01 & 0.00 \\
\hline 5 & 17 & $-(1$ & -0.09 & 0.04 & & & 0.00 & 0.0 & 0.00 & 0.00 \\
\hline 6 & 17 & -0 . & -0.03 & 0.00 & & & 0.00 & 0. & -0.01 & 0.00 \\
\hline 7 & 17 & & -0. & & & & 0. & & 0.00 & \\
\hline 8 & 6 & & 0.04 & -0.01 & & & -0.0 & 0.1 & -0.12 & 0.04 \\
\hline 9 & 8 & 0.00 & 0.00 & 0.01 & & & 0.00 & & 0.00 & 0.00 \\
\hline 10 & 6 & 0.0 & & & & & & & & \\
\hline 11 & 6 & & & & & & -0 . & -0 & -0.01 & -0 . \\
\hline 12 & 1 & 0.0 & -0.04 & 0.04 & & 01 & 0.02 & -0.04 & -0.02 & -0.09 \\
\hline 13 & 1 & -0. & -0.05 & -0.06 & & & -0.01 & & & 0.01 \\
\hline 14 & 1 & 0. & 0.0 & 0.0 & & & -0. & 0. & & \\
\hline 15 & 6 & -0. & 0.00 & -0.01 & & & 0.0 & 0. & 0.02 & 0.02 \\
\hline 16 & 1 & 0.0 & -0.01 & -0.0 & & & & & & -0.04 \\
\hline 17 & 1 & -0. & 0.00 & 0.0 & & 0.0 & 0.0 & -0 . & -0.01 & 0.00 \\
\hline 18 & 1 & -0. & -0.01 & 0.03 & & -0.01 & -0.02 & 0.05 & -0.04 & -0.11 \\
\hline 19 & 6 & -0. & 0.01 & 0.0 & & & 0.0 & 0. & -0.01 & -0.04 \\
\hline 20 & 6 & 0.0 & 0.08 & -0.02 & & & -0.0 & 0. & 0.01 & -0.05 \\
\hline 21 & 6 & 0.0 & 0.09 & 0.03 & & & 0.07 & 0.1 & 0.1 & 0.02 \\
\hline 22 & 6 & 0.06 & -0.04 & -0.02 & & 0.01 & -0.08 & 0.01 & 0.00 & -0.01 \\
\hline 23 & 1 & -0.0 & -0.03 & 0.23 & & 0.04 & 0.35 & -0.01 & -0.01 & 0.01 \\
\hline 24 & 6 & 0.1 & -0.02 & -0.0 & & -0.01 & -0.04 & 0.0 & 0.00 & 0.00 \\
\hline 25 & 1 & -0.08 & 0.13 & 0.31 & -0.11 & 0.08 & 0.55 & -0.01 & 0.02 & 0.04 \\
\hline
\end{tabular}




\begin{tabular}{|c|c|c|c|c|c|c|c|c|c|c|}
\hline & & & & & & & & & & \\
\hline 26 & 6 & -0.03 & -0.04 & -0.05 & 0.01 & -0.03 & -0.08 & 0.00 & -0.01 & $\begin{array}{l}-0.01 \\
\end{array}$ \\
\hline 27 & 1 & -0.05 & -0.03 & 0.24 & -0.12 & 0.04 & 0.37 & 0.00 & -0.01 & 0.01 \\
\hline & 6 & -0.04 & 0.09 & 0.02 & -0.03 & 0.00 & 0.07 & -0.01 & 0.02 & 0.02 \\
\hline 29 & 6 & 0.00 & 0.28 & -0.09 & 0.00 & -0.01 & 0.05 & 0.00 & 0.01 & -0.03 \\
\hline 30 & 6 & -0.12 & 0.30 & -0.07 & -0.01 & -0.05 & -0.05 & & 0.03 & 0.00 \\
\hline & 6 & 0.12 & 0.29 & -0.11 & -0.03 & -0.04 & -0.05 & & 0.00 & 0.01 \\
\hline 32 & 6 & -0.16 & -0.11 & 0.06 & 0.02 & 0.02 & 0.06 & 0.00 & .01 & -0.01 \\
\hline 33 & 1 & 0.01 & -0.18 & 0.12 & -0.07 & -0.07 & & 0.03 & 0.00 & 0.00 \\
\hline 34 & 6 & 0.00 & -0.05 & 0.01 & 0.02 & 0.01 & 0.02 & -0.02 & 0.00 & 0.01 \\
\hline 35 & 1 & 0.01 & & -0.02 & -0.06 & -0.15 & -0.38 & -0.02 & .03 & 0.02 \\
\hline 36 & 6 & 0.16 & -0.12 & 0.00 & -0.01 & 0.04 & 0.05 & 0.02 & -0.03 & 0.00 \\
\hline 37 & 1 & 0.00 & & 0.11 & -0.07 & -0.06 & -0.25 & 0.04 & -0.02 & 0.01 \\
\hline 38 & 1 & $\begin{array}{l}-0.14 \\
\end{array}$ & & 0.14 & -0.01 & 0.00 & 0.03 & 0.46 & 0.06 & -0.47 \\
\hline 39 & 1 & 0.15 & & -0.12 & 0.0 & 0.00 & 0.01 & -0.47 & .26 & 0.35 \\
\hline 40 & 1 & 0.00 & -0.07 & 0.03 & 0.02 & -0.01 & -0.01 & -0.02 & 0.27 & -0.08 \\
\hline 41 & 1 & -0.04 & & -0.06 & 0.03 & 0.00 & 0.05 & -0.02 & 0.00 & -0.03 \\
\hline 42 & 1 & 0.04 & & 0.02 & -0.04 & -0.01 & -0.02 & 0.00 & 0.02 & -0.01 \\
\hline Frequen & & 847.67 & & & 867.57 & & & 899.57 & & \\
\hline Atom & AN & $x$ & $Y$ & Z & $x$ & Y & Z & $x$ & Y & Z \\
\hline 1 & 78 & 0.00 & 0.00 & 0.00 & 0.00 & 0.00 & 0.00 & 0.00 & 0.00 & 0.00 \\
\hline 2 & 7 & & 0.02 & 0.10 & & 0.04 & 0.12 & 0.0 & 0.00 & 0.00 \\
\hline 3 & 7 & -0.02 & 0.08 & 0.14 & & -0.07 & -0.16 & -0.0 & 0.01 & 0.03 \\
\hline 4 & 17 & & & 0.00 & & 0.00 & 0.00 & 0.0 & 0.00 & 0.00 \\
\hline 5 & 17 & & & -0.01 & & 0.00 & 0.00 & 0.00 & 0.00 & 0.00 \\
\hline 6 & 17 & & 0.01 & 0.00 & -0.01 & -0.02 & 0.00 & 0.00 & 0.00 & 0.00 \\
\hline 7 & 17 & & 0.01 & -0.01 & 0.0 & 0.01 & 0.0 & 0.0 & 0.00 & 0.00 \\
\hline 8 & 6 & -0.01 & 0.07 & -0.03 & -0.05 & -0.03 & -0.03 & -0.08 & -0.03 & -0.09 \\
\hline 9 & 8 & -0.01 & 0.00 & 0.01 & 0.01 & -0.01 & -0.01 & 0.01 & -0.01 & -0.01 \\
\hline 10 & 6 & -0. & & 0.08 & -0.02 & 0.02 & 0.07 & 0.0 & 0.00 & -0.01 \\
\hline 11 & 6 & & -0.02 & -0.06 & -0.0 & -0.03 & -0.11 & 0. & 0.00 & 0.01 \\
\hline 12 & 1 & -0 . & & -0.33 & -0.11 & -0.08 & -0.23 & 0.0 & 0.01 & 0.02 \\
\hline 13 & 1 & & -0.03 & -0.04 & -0.01 & -0.03 & -0.09 & 0.0 & 0.00 & 0.01 \\
\hline 14 & 1 & & & 0.01 & -0.0 & -0.02 & -0.08 & 0. & 0.00 & 0.01 \\
\hline 15 & 6 & & & & & & 0.01 & -0 . & 0 & -0.01 \\
\hline 16 & 1 & & & -0.03 & & & 0.1 & & & -0.03 \\
\hline 17 & 1 & -0.0 & & -0.1 & -0.0 & 0.05 & 0.1 & 0. & -0.01 & -0.03 \\
\hline 18 & 1 & & & -0.36 & -0. & 0. & 0.0 & -0 & 0.01 & 0.03 \\
\hline 19 & 6 & & & -0.1 & & & 0.1 & & -0.01 & -0.02 \\
\hline 20 & 6 & & & -0.1 & -0. & 0.0 & 0.1 & -0 . & 0.00 & -0.01 \\
\hline 21 & 6 & & & 0. & -0 & & -0.07 & 0. & -0.01 & 0.01 \\
\hline 22 & 6 & & & -0 & & & -0.0 & & & 0.01 \\
\hline 23 & 1 & & & & & 0.04 & -0 & & & 0.02 \\
\hline 24 & 6 & & & 0. & & 0.00 & 0.0 & -0. & 0.00 & 0.00 \\
\hline 25 & 1 & & & 0. & & & 0.0 & -0. & & 0.00 \\
\hline 26 & 6 & & & -0. & & & & & & \\
\hline 27 & 1 & & & & & -0.1 & -0. & 0. & & \\
\hline 28 & 6 & & & & -0.05 & -0.06 & -0.0 & 0. & 0.01 & 0.01 \\
\hline 29 & 6 & & & -0.1 & & -0.03 & -0 . & & & -0.01 \\
\hline 30 & 6 & & & & & 0. & 0. & & & \\
\hline 31 & 6 & & & 0.0 & & 0.02 & 0.0 & & & 0.00 \\
\hline 32 & 6 & & & -0.1 & & -0.02 & -0 . & & & \\
\hline 33 & 1 & & & 0. & & 0.00 & 0. & & & \\
\hline 34 & 6 & & & & 0.0 & 0.00 & 0.0 & 0.0 & 0.00 & 0.00 \\
\hline 35 & 1 & & & & & 0.02 & 0. & & & 0.01 \\
\hline 36 & 6 & & & -0.0 & & 0.01 & -0 . & & & -0.01 \\
\hline 37 & 1 & 0. & & 0.0 & -0.0 & 0.01 & 0.0 & -0.0 & 0.01 & 0.01 \\
\hline 38 & 1 & -0.31 & & 0.34 & 0.00 & 0.10 & 0.16 & -0.12 & 0.16 & 0.40 \\
\hline 39 & 1 & & & -0.19 & -0.12 & 0.08 & 0.2 & -0.1 & 0. & 0.43 \\
\hline 40 & 1 & & & 0.0 & 0.3 & 0.00 & -0.2 & 0.62 & -0.07 & -0.37 \\
\hline 41 & 1 & -0.03 & & -0.06 & 0.02 & 0.03 & 0.07 & 0.02 & 0.03 & 0.08 \\
\hline
\end{tabular}




\begin{tabular}{|c|c|c|c|c|c|c|c|c|c|c|}
\hline $\begin{array}{c}42 \\
\text { Frequen }\end{array}$ & ies $^{1}$ & $\begin{array}{c}0.08 \\
919.10\end{array}$ & -0.01 & 0.06 & $\begin{array}{c}-0.10 \\
945.74\end{array}$ & 0.02 & -0.08 & $\begin{array}{c}-0.12 \\
949.46\end{array}$ & 0.01 & -0.09 \\
\hline Atom & AN & $x$ & $Y$ & Z & $x$ & $Y$ & Z & $x$ & Y & Z \\
\hline 1 & 78 & -0.01 & 0.00 & -0.01 & 0.00 & 0.00 & 0.00 & 0.00 & 0.00 & 0.00 \\
\hline 2 & 7 & 0.40 & 0.09 & 0.27 & 0.00 & 0.00 & 0.00 & 0.00 & 0.00 & 0.00 \\
\hline 3 & 7 & -0.13 & 0.02 & 0.05 & 0.00 & 0.00 & 0.00 & & 0.00 & 0.00 \\
\hline 4 & 17 & -0.01 & 0.01 & 0.00 & 0.00 & 0.00 & 0.00 & 0.00 & & 0.00 \\
\hline 5 & 17 & -0.01 & -0.01 & 0.00 & 0.00 & 0.00 & 0.00 & 0.00 & 0.00 & 0.00 \\
\hline 6 & 17 & 0.00 & 0.00 & 0.00 & 0.00 & 0.00 & 0.00 & 0.00 & 0.00 & 0.00 \\
\hline 7 & 17 & 0.00 & 0.00 & 0.00 & 0.00 & 0.00 & 0.00 & 0.00 & & 0.00 \\
\hline 8 & 6 & 0.05 & 0.01 & 0.03 & 0.00 & 0.00 & 0.00 & 0.00 & & 0.00 \\
\hline 9 & 8 & 0.00 & 0.00 & 0.00 & 0.00 & 0.00 & 0.00 & 0.00 & 0.00 & 0.00 \\
\hline 10 & 6 & 0.09 & 0.01 & 0.0 & 0.00 & 0.00 & 0.0 & 0.00 & & 0.00 \\
\hline 11 & 6 & -0.09 & -0.04 & -0.15 & 0.00 & 00 & 0. & 0.0 & & 0.00 \\
\hline 12 & 1 & -0.11 & -0.09 & -0.24 & 0.00 & 0.00 & 0.00 & 0.00 & 00 & 0.00 \\
\hline 13 & 1 & -0.02 & -0.05 & -0.14 & 0.00 & 0.00 & 0.0 & 0.0 & 0 & 0.00 \\
\hline 14 & 1 & 0.00 & -0.04 & -0.10 & 0.0 & & & & & \\
\hline 15 & 6 & -0.03 & -0.01 & -0.03 & 0.00 & & & 0. & & 0.00 \\
\hline 16 & 1 & -0.03 & 0.00 & 0.01 & 0.0 & 0.00 & 0. & 0. & 0 & 00 \\
\hline 17 & 1 & -0.0 & & 0. & & & & & & \\
\hline 18 & 1 & -0.0 & & -0.01 & & & & & & \\
\hline 19 & 6 & -0.0 & & 0.00 & & & & & & \\
\hline 20 & 6 & -0.03 & & -0 . & & & & & & \\
\hline 21 & 6 & 0.02 & & 0. & & 0. & & & & \\
\hline 22 & 6 & 0.05 & -0.07 & 0.0 & & & 0.00 & 0.03 & -0.01 & -0.12 \\
\hline 23 & 1 & 0.11 & -0. & 0.0 & & 0. & & -0 . & & 0.67 \\
\hline 24 & 6 & -0.05 & 0.00 & -0.0 & & 0. & 0. & 0. & & \\
\hline 25 & 1 & -0.04 & 0.00 & -0.02 & 0.0 & 0.00 & 0.0 & -0.01 & & 0.03 \\
\hline 26 & 6 & 0.04 & 0. & 0. & & 0. & & -0 . & & 0.12 \\
\hline 27 & 1 & 0.10 & & & & & 0. & 0. & & -0 . \\
\hline 28 & 6 & 0.02 & 0. & 0. & & 0. & 0. & 0.0 & & -0.03 \\
\hline 29 & 6 & 0.07 & -0.05 & -0.1 & & & & & & 0.00 \\
\hline 30 & 6 & -0.05 & & 0. & & -0.01 & -0 . & 0. & & \\
\hline 31 & 6 & & & & & & & & & \\
\hline 32 & 6 & -0. & & & & & & & & \\
\hline 33 & 1 & -0.28 & -0.09 & 0.0 & -0.13 & -0.21 & -0.6 & 0. & & \\
\hline 34 & 6 & 0.1 & & -0 . & & 0.00 & 0. & & & \\
\hline 35 & 1 & & & & & & & & & \\
\hline 36 & 6 & -0.1 & 0.1 & -0 & -0 & -0 . & & & & \\
\hline 37 & 1 & -0.28 & & & & 0.2 & & & & \\
\hline 38 & 1 & 0.04 & & & & & & & & \\
\hline 39 & 1 & 0.1 & & & & & & & & \\
\hline 40 & 1 & -0.26 & & 0. & & 0. & & 0. & & \\
\hline 41 & 1 & -0.02 & & -0 . & & 0. & & & & \\
\hline 42 & 1 & -0.01 & & -0.01 & & 0.00 & & & & \\
\hline \multicolumn{2}{|c|}{ Frequencies } & 1026.51 & & & & & & & & \\
\hline Atom & AN & $x$ & Y & Z & $\hat{n}$ & Y & Z & $x$ & Y & \\
\hline 1 & 78 & & 0.00 & 0.00 & & 0.00 & 0.0 & & & \\
\hline 2 & 7 & & & & & & & & & \\
\hline 3 & 7 & & & & & 0.00 & & & & \\
\hline 4 & 17 & & & & & & & & & \\
\hline 5 & 17 & & & 0. & & 0.00 & & & & \\
\hline 6 & 17 & & & & & 0.00 & 0. & & & \\
\hline 7 & 17 & 0.0 & & & & 0.00 & & & & 0.0 \\
\hline 8 & 6 & & & 0. & & 0.00 & & & & 0.00 \\
\hline 9 & 8 & 0.00 & 0.00 & 0. & & 0.00 & 0.0 & 0. & 0. & $0 \cap 0$ \\
\hline 10 & 6 & & & & & 0. & & & & 0.00 \\
\hline 11 & 6 & 0.0 & & -0.1 & & & & & & 0.00 \\
\hline 12 & 1 & & 0.1 & 0.3 & & 0.0 & & & & 0.0 \\
\hline 13 & 1 & -0.30 & 0.01 & -0.13 & 0.01 & 0.00 & 0.00 & -0.01 & 0.00 & 0.00 \\
\hline
\end{tabular}




\begin{tabular}{|c|c|c|c|c|c|c|c|c|c|c|}
\hline & & & & & & & & & & \\
\hline 14 & 1 & -0.32 & -0.12 & -0.15 & 0.01 & 0.00 & 0.00 & -0.01 & 0.00 & 0.00 \\
\hline 15 & 6 & -0.07 & 0.02 & 0.04 & 0.00 & 0.00 & 0.00 & 0.00 & 0.00 & 0.00 \\
\hline 16 & 1 & 0.34 & 0.01 & -0.23 & -0.02 & 0.00 & 0.01 & 0.00 & 0.00 & 0.00 \\
\hline 17 & 1 & 0.32 & -0.09 & -0.12 & -0.02 & 0.00 & 0.01 & 0.00 & 0.00 & 0.00 \\
\hline 18 & 1 & -0.13 & 0.12 & 0.42 & 0.01 & -0.01 & -0.02 & 0.00 & 0.00 & 0.00 \\
\hline 19 & 6 & -0.06 & -0.04 & -0.10 & 0.00 & 0.00 & 0.01 & 0.00 & 0.00 & 0.00 \\
\hline 20 & 6 & 0.04 & 0.00 & 0.01 & 0.00 & 0.00 & 0.0 & 0.00 & 0.00 & -0.01 \\
\hline 21 & 6 & 0.00 & 0.00 & 0.00 & 0.00 & 0.00 & 0.00 & 0.00 & 0.00 & -0.01 \\
\hline 22 & 6 & -0.02 & 0.03 & -0.01 & 0.00 & 0.00 & 0.00 & -0.02 & 0.01 & 0.08 \\
\hline 23 & 1 & -0.03 & 0.04 & 0.00 & 0.01 & 0.00 & -0.0 & 0.11 & -0.06 & -0.45 \\
\hline 24 & 6 & 0.01 & 0.00 & 0.00 & 0.00 & 0.00 & -0.01 & 0.03 & -0.02 & -0.13 \\
\hline 25 & 1 & 0.01 & 0.00 & -0.01 & -0.01 & 0.00 & 0.03 & -0.17 & 0.08 & 0.72 \\
\hline 26 & 6 & -0.02 & -0.04 & 0.00 & 0.00 & 0.00 & & -0.02 & 0.01 & 0.08 \\
\hline 27 & 1 & -0.03 & -0.04 & 0.01 & 0.01 & 0.00 & -0.02 & 0.09 & -0.05 & -0.42 \\
\hline 28 & 6 & 0.00 & 0.00 & 0.00 & 0.00 & 0.00 & 0.00 & 0.00 & 0.00 & -0.01 \\
\hline 29 & 6 & -0.02 & 0.0 & 0.00 & 0.00 & 0.00 & 0.01 & 0.00 & 0.00 & 0.00 \\
\hline 30 & 6 & 0.00 & & 0.00 & 0.00 & 0.00 & 0.01 & 0.00 & 0.00 & 0.00 \\
\hline 31 & 6 & 0.00 & 0.00 & 0.00 & 0.00 & 0.00 & 0.01 & 0.00 & 0.00 & 0.00 \\
\hline 32 & 6 & 0.01 & 0.02 & -0.01 & -0.01 & -0.02 & -0.08 & 0.00 & 0.00 & 0.00 \\
\hline 33 & 1 & 0.02 & & 0.01 & 0.08 & 0.14 & 0.41 & 0.00 & .00 & 0.02 \\
\hline 34 & 6 & 0.00 & 0.00 & 0.01 & 0.02 & 0.04 & 0.13 & 0.00 & & -0.01 \\
\hline 35 & 1 & -0.01 & -0.01 & -0.04 & -0.14 & -0.23 & -0.70 & 0.01 & 0.01 & 0.03 \\
\hline 36 & 6 & 0.01 & -0.02 & 0.00 & -0.01 & -0.03 & -0.08 & 0.00 & .00 & \\
\hline 37 & 1 & 0.02 & & 0.03 & 0.08 & 0.14 & & 0.00 & & \\
\hline 38 & 1 & 0.00 & -0.01 & -0.01 & 0.00 & 0.00 & 0.00 & 0.00 & 0.00 & 0.00 \\
\hline 39 & 1 & 0.00 & & -0.01 & 0.00 & 0.00 & 0.0 & 0.00 & .00 & .00 \\
\hline 40 & 1 & -0.01 & & 0.01 & 0.00 & 0.00 & 0.0 & 0.00 & 0.00 & \\
\hline 41 & 1 & -0.01 & 0.00 & -0.01 & 0.00 & 0.00 & 0.00 & 0.00 & 0.00 & 0.00 \\
\hline 42 & 1 & 0.00 & 0.00 & 0.00 & 0.00 & 0.00 & 0.00 & 0.00 & 0.00 & 0.00 \\
\hline requer & & 1049.93 & & & 1065.51 & & & 1093.96 & & \\
\hline Atom & AN & $X$ & $Y$ & Z & & $Y$ & Z & $X$ & $\mathrm{Y}$ & 2 \\
\hline 1 & 78 & 0.00 & 0.00 & 0.00 & 0.00 & 0.00 & 0.00 & 0.00 & 0.00 & 0.00 \\
\hline 2 & 7 & & 04 & 0.11 & & 0.01 & -0.02 & 0.0 & -0.02 & 0.01 \\
\hline 3 & 7 & & & -0.09 & & 0.02 & 0.01 & 0. & 0.03 & 0.00 \\
\hline 4 & 17 & & 0. & 0.00 & & 0.00 & 0.00 & & & 0.00 \\
\hline 5 & 17 & 0.00 & 0.00 & 0.00 & 0.0 & 0.00 & 0.00 & 0.00 & 0.00 & 0.00 \\
\hline 6 & 17 & & & 0. & & 0.00 & 0.0 & 0.0 & 0.00 & 0.00 \\
\hline 7 & 17 & & & & & 0.00 & 0. & & & 0.00 \\
\hline 8 & 6 & & & 0. & & 0.00 & 0. & 0. & 0. & 0.00 \\
\hline 9 & 8 & & & 0.00 & & 0.00 & 0.0 & 0.0 & 0.00 & 0.00 \\
\hline 10 & 6 & & & 0. & & -0.08 & 0.0 & 0.0 & 0.12 & -0.04 \\
\hline 11 & 6 & & & & & 0.11 & -0 . & & -0.11 & 0.03 \\
\hline 12 & 1 & & & 0. & & -0.27 & 0.0 & -0.03 & 0.25 & -0.10 \\
\hline 13 & 1 & & & -0.21 & & -0.24 & -0.35 & 0.17 & 0.22 & 0.34 \\
\hline 14 & 1 & & & -0.08 & & -0.01 & 0.4 & & 0.02 & \\
\hline 15 & 6 & & & & & -0. & 0. & 0. & -0.12 & \\
\hline 16 & 1 & & & 0. & & -0.24 & -0.33 & 0.14 & -0.26 & -0.33 \\
\hline 17 & 1 & & & & & & 0.4 & -0.16 & & \\
\hline 18 & 1 & & & -0.3 & & -0.24 & 0. & & -0.27 & 0.06 \\
\hline 19 & 6 & & & 0.0 & & 0.10 & -0.05 & 0.00 & 0.11 & -0.04 \\
\hline 20 & 6 & & & -0.0 & & 0.01 & 0.00 & & 0.00 & \\
\hline 21 & 6 & 0. & & 0.0 & & -0.01 & 0.0 & 0. & -0.01 & 0.00 \\
\hline 22 & 6 & & & 0.0 & & 0.01 & 0.00 & 0.00 & 0.00 & \\
\hline 23 & 1 & & & 0.0 & 0.0 & 0.02 & 0.00 & 0.01 & 0.00 & 0.00 \\
\hline 24 & 6 & & & 0.00 & & 0.00 & 0.00 & & 0.00 & \\
\hline 25 & 1 & & & -0.02 & & 0.01 & 0.0 & 0.00 & 0.01 & \\
\hline 26 & 6 & 0.0 & $0 . C$ & 0.00 & 0.00 & -0.01 & 0.00 & 0.00 & 0.00 & 0.00 \\
\hline 27 & 1 & & & 0.00 & -0.02 & -0.01 & 0.00 & -0.01 & 0.01 & \\
\hline 28 & 6 & 0.0 & -0. & 0.0 & & -0.01 & 0.0 & 0.00 & -0.01 & 0. \\
\hline 29 & 6 & & & 0.00 & 0.01 & 0.00 & 0.00 & 0.00 & 0.00 & \\
\hline
\end{tabular}




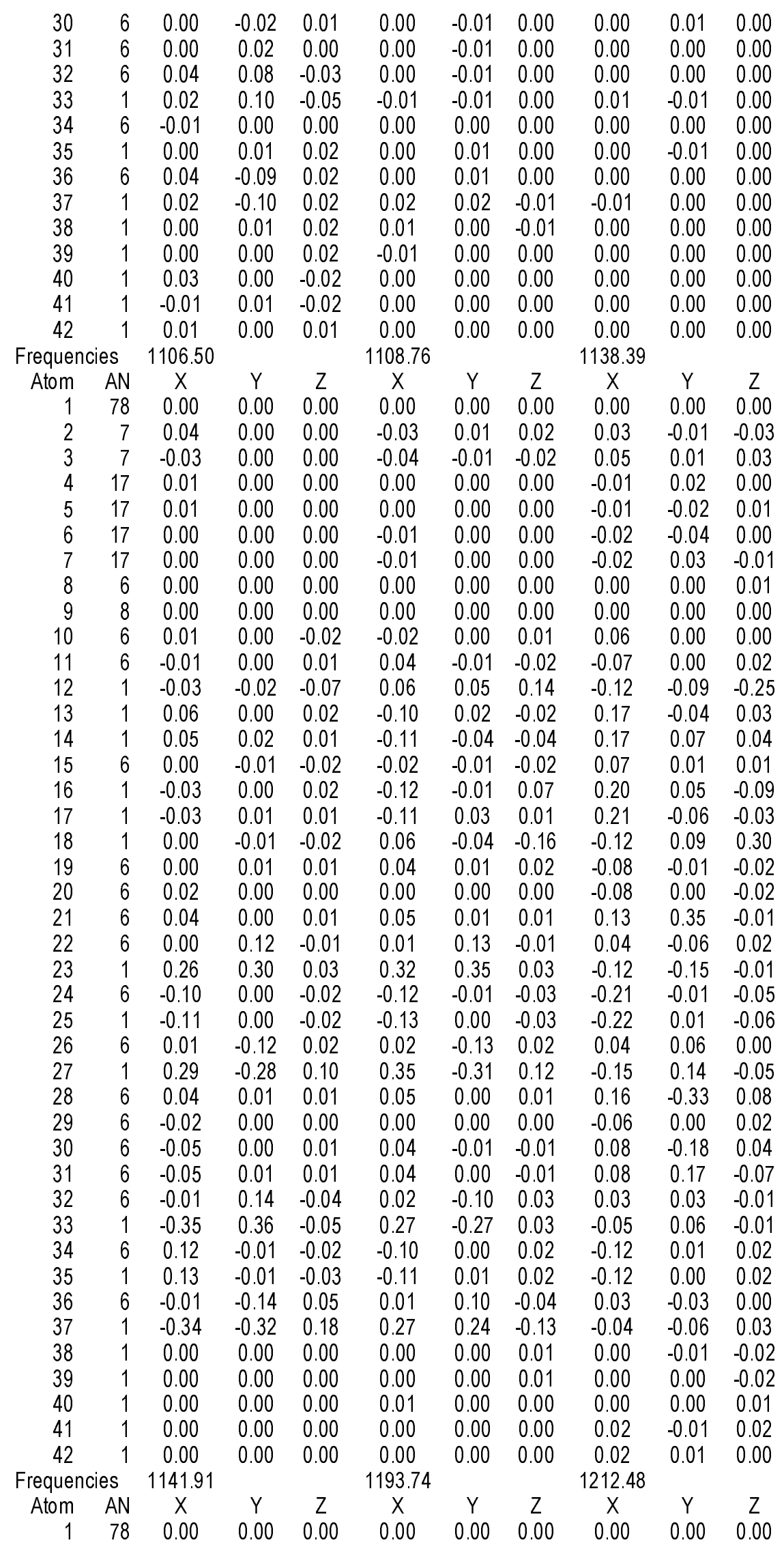




\begin{tabular}{|c|c|c|c|c|c|c|c|c|c|c|}
\hline & & & & & & & & & & \\
\hline 2 & 7 & -0.08 & 0.00 & 0.00 & -0.06 & 0.00 & 0.00 & 0.00 & 0.00 & 0.00 \\
\hline 3 & 7 & 0.05 & -0.01 & -0.01 & -0.06 & 0.00 & 0.01 & 0.00 & -0.02 & 0.00 \\
\hline 4 & 17 & 0.02 & -0.04 & 0.01 & 0.00 & -0.01 & 0.00 & 0.00 & 0.00 & 0.00 \\
\hline 5 & 17 & 0.02 & 0.04 & -0.02 & 0.00 & 0.01 & 0.00 & 0.00 & 0.00 & 0.00 \\
\hline 6 & 17 & -0.01 & -0.03 & 0.00 & 0.00 & 0.01 & 0.00 & & 0.01 & 0.00 \\
\hline 7 & 17 & -0.01 & 0.02 & -0.01 & 0.00 & -0.01 & 0.0 & -0.01 & 0.01 & .00 \\
\hline 8 & 6 & -0.01 & 0.00 & 0.01 & -0.01 & 0.00 & 0. & 0.00 & 0.00 & 0.00 \\
\hline 9 & 8 & 0.00 & 0.00 & 0.00 & 0.00 & 0.00 & 0.00 & 0.00 & 0.00 & 0.00 \\
\hline 10 & 6 & -0.05 & 0.00 & 0.03 & 0.20 & 0.03 & 0.07 & -0.01 & 0.00 & 0.00 \\
\hline 11 & 6 & 0.05 & 0.00 & -0.02 & -0.12 & -0.01 & & 0.00 & & .00 \\
\hline 12 & 1 & 0.08 & 0.06 & 0.18 & -0.18 & -0.13 & -0.3 & 0.01 & 0.01 & 0.01 \\
\hline 13 & 1 & -0.15 & 0.03 & -0.03 & 0.16 & -0.07 & -0.03 & -0.01 & 0.00 & 0.00 \\
\hline 14 & 1 & -0.15 & -0.06 & -0.02 & 0.1 & 0.07 & -0.03 & -0.01 & 0.00 & 0.00 \\
\hline 15 & 6 & -0.01 & 0.02 & 0.04 & 0.2 & -0.03 & -0.1 & 0.00 & .00 & 0.00 \\
\hline 16 & 1 & -0.01 & 0.01 & 0.01 & 0.18 & 0.08 & -0.04 & -0.01 & 0.01 & 0.01 \\
\hline 17 & 1 & 0.00 & 0.00 & -0.02 & 0.19 & -0.04 & 0.06 & -0.01 & 0.00 & -0.02 \\
\hline 18 & 1 & 0.03 & & -0.06 & -0.17 & 0.11 & & 0.0 & 0.01 & -0.01 \\
\hline 19 & 6 & 0.02 & -0.01 & -0.02 & -0.13 & 0.01 & & 0.0 & 0.00 & 0.00 \\
\hline 20 & 6 & -0.06 & 0.00 & 0.00 & -0.08 & 0.00 & -0.0 & 0.00 & 0.07 & -0.01 \\
\hline 21 & 6 & 0.09 & 0.24 & -0.01 & -0.04 & -0.06 & 0. & -0 . & -0.06 & -0.02 \\
\hline 22 & 6 & 0.03 & & 0.01 & & 0.00 & & & & 0.01 \\
\hline 23 & 1 & -0.10 & & -0.01 & & 0.11 & 0. & -0.1 & -0.13 & 0.00 \\
\hline 24 & 6 & -0.14 & -0 & -0.03 & 0. & 0.01 & 0. & 0. & 0.09 & \\
\hline 25 & 1 & -0.15 & & -0.04 & 0.01 & 0.04 & 0.0 & -0.03 & 0.94 & -0.12 \\
\hline 26 & 6 & 0.02 & 0.04 & 0.00 & 0.03 & 0.00 & 0.01 & -0.01 & -0.07 & 0.01 \\
\hline 27 & 1 & -0.11 & & -0.0 & 0.20 & -0.09 & 0.0 & 0.0 & -0.11 & 0.03 \\
\hline 28 & 6 & 0.11 & & 0.0 & -0.03 & 0.04 & -0.0 & 0.12 & -0.04 & \\
\hline 29 & 6 & 0.12 & 0.00 & -0.02 & -0.10 & 0.01 & 0.04 & 0.00 & 0.00 & 0.00 \\
\hline 30 & 6 & -0.18 & & -0.09 & -0.05 & 0.07 & -0. & -0.01 & 0.00 & 0.00 \\
\hline 31 & 6 & -0.18 & & 0.1 & -0.04 & -0.06 & 0. & 0. & 0.00 & 0 \\
\hline 32 & 6 & -0.05 & & 0. & 0.04 & 0.00 & -0.0 & 0.00 & 0.00 & 0.00 \\
\hline 33 & 1 & 0.1 & & 0. & 0.27 & -0.13 & -0. & -0.01 & 0.01 & 0.00 \\
\hline 34 & 6 & 0.2 & & -0 . & & 0.00 & 0. & 0. & -0.01 & \\
\hline 35 & 1 & 0.26 & & -0.06 & & & 0. & 0. & & \\
\hline 36 & 6 & -0.05 & & -0.0 & 0.04 & 0.00 & -0. & 0. & & 0.00 \\
\hline 37 & 1 & 0.1 & & -0.07 & 0.26 & 0.11 & -0.0 & 0.00 & 0.00 & 0.00 \\
\hline 38 & 1 & 0.03 & & -0. & & 0.01 & 0. & 0. & 0.00 & 0.00 \\
\hline 39 & 1 & & & & & & & & & \\
\hline 40 & 1 & 0.02 & & -0 & & 0.0 & & 0. & & \\
\hline 41 & 1 & 0.01 & & 0. & -0.01 & 0.00 & -0 . & 0.0 & 0.00 & 0.01 \\
\hline 42 & 1 & 0.02 & & 0.00 & & 0.00 & 0.01 & 0.00 & 0.00 & 0.00 \\
\hline quen & & 1214.11 & & & 1260.37 & & & 1261.93 & & \\
\hline Atom & AN & $X$ & $Y$ & Z & $\hat{\lambda}$ & $Y$ & Z & $x$ & $\mathrm{Y}$ & Z \\
\hline & 78 & 0.00 & 0.00 & 0.00 & 0.00 & 0.00 & 0.00 & 0.00 & 0.00 & 0.00 \\
\hline 2 & 7 & & & 0. & & & -0 . & & & -0.01 \\
\hline 3 & 7 & & & & & 0.02 & -0. & 0. & 0.00 & 0.00 \\
\hline 4 & 17 & -0.01 & & 0.00 & & 0.00 & 0.00 & 0.00 & 0.00 & 0.00 \\
\hline 5 & 17 & & & & & & & & & 0.00 \\
\hline 6 & 17 & & & & & & & & & \\
\hline 7 & 17 & & & & & 0.0 & 0. & 0. & 0. & 0.00 \\
\hline 8 & 6 & & & & & & & & & 0.00 \\
\hline 9 & 8 & 0.0 & & 0. & & 0.00 & 0. & 0. & 0.00 & 0.00 \\
\hline 10 & 6 & & & 0. & & 0.00 & 0. & 0.0 & -0.01 & 0.00 \\
\hline 11 & 6 & & & & & & 0. & & 0.00 & 0.00 \\
\hline 12 & 1 & & & 0.0 & & -0.01 & 0.0 & & -0.01 & 0.00 \\
\hline 13 & 1 & & & 0.0 & & 0.00 & 0.00 & 0.0 & -0.01 & -0.01 \\
\hline 14 & 1 & 0.0 & 0.00 & -0.1 & 0.00 & 0.00 & 0.00 & 0.00 & 0.00 & 0.01 \\
\hline 15 & 6 & 0.0 & & & & 0.00 & & & 0.00 & 0.00 \\
\hline 16 & 1 & & & 0.0 & 0.02 & 0.00 & -0.0 & 0.00 & 0.00 & 0.00 \\
\hline 17 & 1 & 0.00 & 0.00 & 0.00 & 0.01 & -0.01 & 0.01 & 0.00 & 0.00 & 0.00 \\
\hline
\end{tabular}




\begin{tabular}{|c|c|c|c|c|c|c|c|c|c|c|}
\hline & & & & & & & & & & \\
\hline $\begin{array}{l}18 \\
19\end{array}$ & $\begin{array}{l}1 \\
6\end{array}$ & $\begin{array}{l}0.00 \\
0.00\end{array}$ & $\begin{array}{l}0.00 \\
0.00\end{array}$ & $\begin{array}{l}0.00 \\
0.00\end{array}$ & $\begin{array}{l}0.00 \\
0.00\end{array}$ & $\begin{array}{r}-0.01 \\
0.00\end{array}$ & $\begin{array}{r}0.01 \\
-0.01\end{array}$ & $\begin{array}{l}0.00 \\
0.00\end{array}$ & $\begin{array}{l}0.00 \\
0.00\end{array}$ & $\begin{array}{l}0.00 \\
0.00\end{array}$ \\
\hline & 6 & 0.00 & 0.00 & 0.00 & -0.04 & -0.11 & 0.00 & 0.00 & 0.00 & 0.00 \\
\hline 21 & 6 & -0.01 & 0.00 & 0.00 & 0.07 & 0.03 & 0.01 & 0.00 & 0.00 & 0.00 \\
\hline 22 & 6 & 0.00 & 0.00 & 0.00 & -0.03 & 0.01 & -0.01 & 0.00 & 0.00 & 0.00 \\
\hline 23 & 1 & 0.00 & -0.01 & 0.0 & -0.49 & -0.29 & -0.09 & -0.01 & 0.00 & \\
\hline 24 & 6 & 0.00 & 0.01 & 0.00 & 0.00 & 0.04 & -0.01 & 0.00 & .00 & .00 \\
\hline 25 & 1 & 0.00 & 0.06 & -0.01 & -0.01 & 0.27 & -0.03 & 0.00 & 0.00 & 0.00 \\
\hline 26 & 6 & 0.00 & 0.00 & 0.00 & 0.06 & 0.03 & 0.01 & 0.00 & 0.00 & 0.00 \\
\hline 27 & 1 & 0.00 & & 0.0 & 0.67 & -0.27 & & & & 0.00 \\
\hline 28 & 6 & 0.01 & 0.00 & 0.00 & -0.10 & 0.02 & -0.03 & 0.00 & 0.00 & 0.00 \\
\hline 29 & 6 & 0.00 & 0.06 & -0.02 & 0.03 & 0.00 & 0.00 & 0.00 & -0.09 & 0.03 \\
\hline 30 & 6 & 0.12 & -0.05 & -0.01 & 0.01 & 0.00 & 0. & -0.10 & 0.02 & 0.01 \\
\hline 31 & 6 & -0.12 & -0.04 & 0.04 & 0.01 & 0.00 & 0.0 & 0.10 & 0.01 & -0.02 \\
\hline 32 & 6 & -0.01 & -0.06 & 0.02 & -0.01 & 0.00 & 0.00 & 0.06 & 0.02 & -0.02 \\
\hline 33 & 1 & 0.07 & -0.12 & 0.03 & -0.05 & 0.02 & 0.0 & 0.60 & -0.31 & -0.02 \\
\hline 34 & 6 & 0.00 & & -0.03 & 0.00 & 0.00 & 0. & 0.00 & 0.05 & -0.02 \\
\hline 35 & 1 & -0.01 & 0.90 & -0.30 & 0.00 & 0.00 & 0.0 & 0.00 & 0.24 & -0.08 \\
\hline 36 & 6 & 0.01 & -0.07 & 0.02 & -0.01 & 0.00 & 0.0 & -0.05 & 0.02 & 0.00 \\
\hline 37 & 1 & -0.07 & & 0.05 & -0.05 & -0.01 & 0. & -0.58 & -0.24 & \\
\hline 38 & 1 & 0.00 & & 0.00 & 0.00 & 0.00 & -0.0 & & & \\
\hline 39 & 1 & 0.00 & & 0.00 & 0.01 & -0.01 & -0.01 & 0. & & .00 \\
\hline 40 & 1 & 0.00 & & 0.0 & 0.01 & 0.00 & 0. & 0.0 & 0.00 & .00 \\
\hline 41 & 1 & 0.00 & & 0.00 & 0.00 & 0.00 & 0.00 & 0.00 & 0.00 & 0.00 \\
\hline 42 & 1 & 0.00 & 0.00 & 0.00 & -0.01 & 0.00 & 0.00 & 0.00 & 0.00 & 0.00 \\
\hline requen & & 1265.93 & & & 1292.56 & & & 1306.39 & & \\
\hline Atom & AN & X & $\mathrm{Y}$ & Z & X & $\mathrm{Y}$ & Z & $X$ & $Y$ & Z \\
\hline & 78 & 0.00 & 0.00 & 0.00 & 0.00 & 0.00 & 0.00 & 0.00 & 0.00 & 0.00 \\
\hline 2 & 7 & & 0. & 0.04 & 0.1 & 0.00 & -0.01 & 0.0 & 0.00 & 0.00 \\
\hline 3 & 7 & -0.11 & 02 & 0.03 & 0.16 & 0.00 & 0.00 & 0. & -0.03 & 0.00 \\
\hline 4 & 17 & 0.00 & 00 & 0.00 & & 0.00 & 0.00 & 0.00 & 0.00 & 0.00 \\
\hline 5 & 17 & 0. & 0.00 & 0.00 & & 0.00 & 0.00 & 0.0 & 0.00 & 0.00 \\
\hline 6 & 17 & 0.00 & -0 & 0. & & 0.00 & 0.0 & 0. & 0.00 & 0.00 \\
\hline 7 & 17 & & & 0.00 & & 0.00 & 0.0 & 0. & 0.00 & 0.00 \\
\hline 8 & 6 & & & -0.01 & & 0.00 & 0.0 & & 0.00 & 0.00 \\
\hline 9 & 8 & 0.00 & 0.00 & 0.00 & 0.0 & 0.00 & 0.00 & 0.00 & 0.00 & 0.00 \\
\hline 10 & 6 & 0.0 & 02 & -0.08 & -0.05 & -0.04 & -0.12 & -0.0 & 0.00 & -0.03 \\
\hline 11 & 6 & & & & & & 0.0 & & & \\
\hline 12 & 1 & & & -0 . & & 0.0 & 0.0 & & 0.03 & 0.03 \\
\hline 13 & 1 & & & & & -0.01 & 0.0 & 0. & 0.01 & 0.02 \\
\hline 14 & 1 & & & & & 0.04 & 0.0 & & & 0.00 \\
\hline 15 & 6 & & & & & & & & & 0.04 \\
\hline 16 & 1 & -0. & & 0. & & -0.02 & -0.05 & 0.0 & 0.03 & 0.01 \\
\hline 17 & 1 & -0.0 & & 0. & & -0.01 & -0.05 & 0. & -0.01 & -0.04 \\
\hline 18 & 1 & & & -0.03 & & -0.03 & -0.12 & & & -0.03 \\
\hline 19 & 6 & & & 0. & & -0.02 & -0.0 & 0. & -0.02 & -0.01 \\
\hline 20 & 6 & 0.22 & & 0.05 & -0.18 & -0.05 & -0.02 & -0.07 & 0.46 & -0.07 \\
\hline 21 & 6 & & & 0.02 & & & -0.03 & & & 0.09 \\
\hline 22 & 6 & & & -0 . & & -0.0 & 0. & -0 . & -0.18 & -0.04 \\
\hline 23 & 1 & & & -0.12 & & 0.15 & 0.07 & 0.2 & 0.15 & 0.04 \\
\hline 24 & 6 & & & 0.00 & & -0.03 & 0.0 & & 0.30 & -0.04 \\
\hline 25 & 1 & & & -0.01 & & 0.01 & 0.0 & & & 0.04 \\
\hline 26 & 6 & & & -0.0 & & 0.05 & 0.0 & 0.30 & -0.12 & 0.09 \\
\hline 27 & 1 & -0.1 & & -0.0 & & -0.08 & 0.0 & -0. & 0.07 & -0.03 \\
\hline 28 & 6 & 0.04 & & 0.01 & & 0.01 & -0.01 & & -0.23 & -0.04 \\
\hline 29 & 6 & -0.18 & & 0.03 & -0.2 & 0.01 & 0.03 & -0.04 & 0.01 & 0.00 \\
\hline 30 & 6 & -0.0 & & 0.01 & -0.09 & 0.00 & 0.02 & -0.02 & -0.01 & 0.01 \\
\hline 31 & 6 & -0.0 & & 0.02 & -0.09 & 0.01 & 0.01 & -0.01 & 0.00 & 0.00 \\
\hline 32 & 6 & 0.0 & & -0.02 & 0.07 & 0.04 & -0.0 & 0.02 & 0.01 & -0.01 \\
\hline 33 & 1 & 0.32 & -0.12 & -0.02 & 0.42 & -0.16 & -0.03 & 0.08 & -0.03 & -0.01 \\
\hline
\end{tabular}




\begin{tabular}{|c|c|c|c|c|c|c|c|c|c|c|}
\hline & & & & & & & & & & \\
\hline 34 & 6 & 0.01 & 0.00 & 0.00 & 0.01 & 0.00 & 0.00 & 0.00 & 0.00 & 0.00 \\
\hline & & 0.01 & -0.01 & 0.00 & 0.02 & 0.00 & 0.00 & 0.00 & 0.00 & 0.00 \\
\hline 6 & 6 & 0.06 & -0.03 & 0.00 & 0.07 & -0.05 & 0.00 & 0.01 & -0.01 & 0.00 \\
\hline 37 & 1 & 0.37 & .11 & -0.11 & 0.43 & 0.12 & -0.1 & 0.08 & 0.02 & -0.02 \\
\hline & 1 & -0.04 & & & & & & & & -0.02 \\
\hline & 1 & & & & 0.03 & .02 & & & & \\
\hline 40 & 1 & -0.0 & & & -0.01 & 0.00 & & & .01 & \\
\hline 41 & 1 & 0.00 & & & 0.00 & & & 0. & .00 & .00 \\
\hline 42 & 1 & 0.00 & & 0.0 & -0.02 & & -0.01 & -0.01 & 0.00 & 0.00 \\
\hline requen & & 1312.47 & & & 1341.74 & & & 1353.19 & & \\
\hline Atom & AN & X & $Y$ & Z & $x$ & Y & Z & $x$ & $Y$ & Z \\
\hline 1 & 78 & 0.00 & 0.00 & 0.00 & 0.00 & 0.00 & 0.00 & 0.00 & 0.00 & 0.00 \\
\hline 2 & 7 & 0.00 & -0.03 & 0.01 & 0.00 & 0.00 & 0.00 & & -0.02 & -0.07 \\
\hline 3 & 7 & 0.00 & 0.00 & 0.00 & -0.01 & 0.00 & 0.00 & & -0.02 & -0.06 \\
\hline 4 & 17 & 0.00 & 0.00 & 0.00 & 0.00 & 0.00 & 0.00 & 0.00 & 0.00 & 0.00 \\
\hline 5 & 17 & 0.00 & 0. & 0.00 & 0.00 & 0.00 & 0.0 & 0. & 0.00 & 0.00 \\
\hline 6 & 17 & & & 0.00 & 0.0 & & 0.0 & & 0.00 & \\
\hline 7 & 17 & & & 0.00 & & & & & 0.00 & 0.00 \\
\hline 8 & 6 & 0.00 & 0.00 & 0.00 & -0.10 & 0.02 & 0.0 & & 0.00 & -0.01 \\
\hline 9 & 8 & & & 0. & & & 0. & & & 0.00 \\
\hline 10 & 6 & & & & & & 0. & & & 0.13 \\
\hline 11 & 6 & & & & & & 0. & & -0.01 & -0.04 \\
\hline 12 & 1 & -0.01 & & -0.02 & & -0.01 & & & & -0.29 \\
\hline 13 & 1 & 0.00 & & 0.02 & & & & & & -0.07 \\
\hline 14 & 1 & & & -0.03 & & 0.01 & -0.01 & & 0.11 & -0.11 \\
\hline 15 & 6 & & & 0. & & 0.00 & & & 3 & 0.10 \\
\hline 16 & 1 & & & 0.0 & -0.0 & -0.01 & 0.0 & -0.20 & -0.15 & 0.00 \\
\hline 17 & 1 & 0.00 & 0.00 & -0.01 & -0.02 & 0.00 & 0.0 & -0.28 & 0.07 & -0.12 \\
\hline 18 & 1 & & & & & & & & -0.03 & -0.30 \\
\hline 19 & 6 & & & & & & 0. & 0. & -0.01 & -0.03 \\
\hline 20 & 6 & & & & & 0.00 & 0.0 & 0. & & 0.02 \\
\hline 21 & 6 & & & & & 0.00 & 0. & & -0.01 & 0.01 \\
\hline 22 & 6 & & & & & & & & & -0.01 \\
\hline 23 & 1 & & & & & & & & & -0.02 \\
\hline 24 & 6 & & & & & 0.00 & & & & \\
\hline 25 & 1 & & & 0. & & 0.00 & 0. & -0. & 0.01 & 0.00 \\
\hline 26 & 6 & & & & & & & & & \\
\hline 27 & 1 & & & & & & & & & \\
\hline 28 & 6 & & & & & & & & & .00 \\
\hline 29 & 6 & & & & & & & & & \\
\hline 30 & 6 & & & & & & & & & 00 \\
\hline 31 & 6 & & & & & & & & & \\
\hline 32 & 6 & & & & & & 0. & 0. & 10 & \\
\hline 33 & 1 & & & & & -0.01 & & & & 0.00 \\
\hline 34 & 6 & & & & & & & & & \\
\hline 3 & 1 & & & & & & & & & \\
\hline 36 & 6 & & & & & & 0. & 0. & & 0.00 \\
\hline 37 & 1 & & & & & & & & & \\
\hline 38 & 1 & & & & & & & & & \\
\hline 39 & 1 & & & & & & & & & 0. \\
\hline 40 & 1 & & & & & & & & & \\
\hline 41 & 1 & & & & & & & & & -0.02 \\
\hline 42 & 1 & & & 0.0 & & 0.02 & -0.02 & & 0.00 & 0.00 \\
\hline Frequen & ies & 1448.25 & & & & & & & & \\
\hline Atom & AN & & $Y$ & Z & $x$ & $Y$ & & & $Y$ & \\
\hline & 78 & & 0.0 & 0.0 & & 0.00 & 0.0 & & & \\
\hline 2 & 7 & & 0.00 & 0.01 & 0.01 & -0.01 & -0.02 & -0.01 & 0.00 & 0.00 \\
\hline 3 & 7 & & 0.00 & -0.01 & -0.01 & -0.01 & -0.02 & & 0.01 & 0.01 \\
\hline 4 & 17 & & 0.00 & 0.00 & 0.0 & 0.00 & 0.0 & & 0.00 & 0.00 \\
\hline & 17 & 0.00 & 0.00 & 0.00 & 0.00 & 0.00 & 0.00 & 0.00 & 0.00 & 0.00 \\
\hline
\end{tabular}




\begin{tabular}{|c|c|c|c|c|c|c|c|c|c|c|}
\hline & & & & & & & & & & \\
\hline 6 & 17 & 0.00 & 0.00 & 0.00 & 0.00 & 0.00 & 0.00 & 0.00 & 0.00 & 0.00 \\
\hline 7 & 17 & 0.00 & 0.00 & 0.00 & 0.00 & 0.00 & 0.00 & 0.00 & 0.01 & 0.00 \\
\hline 8 & 6 & 0.00 & 0.00 & 0.00 & 0.00 & 0.00 & 0.00 & 0.00 & 0.00 & 0.00 \\
\hline 9 & 8 & 0.00 & 0.00 & 0.00 & 0.00 & 0.00 & 0.00 & 0.00 & 0.00 & 0.00 \\
\hline 10 & 6 & 0.00 & -0.01 & -0.02 & -0.01 & 0.01 & 0.03 & 0.02 & 0.00 & 0.01 \\
\hline 11 & 6 & 0.04 & 0.02 & 0.08 & -0.03 & -0.02 & -0.08 & -0.01 & -0.01 & -0.03 \\
\hline 12 & 1 & -0.04 & -0.05 & -0.30 & 0.04 & 0.07 & 0.27 & 0.01 & 0.01 & 0.08 \\
\hline 13 & 1 & -0.18 & -0.32 & -0.29 & 0.15 & 0.29 & 0.26 & 0.06 & 0.10 & 0.10 \\
\hline 14 & 1 & -0.18 & 0.12 & -0.40 & 0.17 & -0.11 & 0.37 & 0.06 & -0.04 & 0.13 \\
\hline 15 & 6 & 0.00 & 0.00 & 0.02 & 0.01 & 0.01 & 0.03 & -0.04 & -0.02 & -0.05 \\
\hline 16 & 1 & -0.15 & 0.31 & 0.24 & -0.16 & 0.32 & 0.25 & -0.19 & 0.12 & \\
\hline 17 & 1 & -0.15 & -0.09 & 0.40 & -0.14 & -0.09 & 0.41 & -0.24 & -0.02 & 0.22 \\
\hline 18 & 1 & -0.03 & 0.13 & 0.26 & -0.03 & 0.12 & 0.27 & -0.02 & 0.15 & 0.23 \\
\hline 19 & 6 & 0.03 & -0.03 & -0.08 & 0.03 & -0.03 & -0.08 & 0.04 & -0.02 & -0.04 \\
\hline 20 & 6 & -0.01 & 0.00 & 0.00 & -0.05 & -0.01 & -0.01 & 0.20 & 0.02 & 0.05 \\
\hline 21 & 6 & 0.01 & -0.01 & 0.00 & 0.04 & -0.03 & 0.01 & -0.12 & 0.11 & -0.05 \\
\hline 22 & 6 & 0.00 & 0.00 & 0.00 & 0.00 & 0.02 & 0.00 & -0.03 & -0.07 & 0.00 \\
\hline 23 & 1 & -0.03 & -0.01 & -0.01 & -0.13 & -0.06 & -0.02 & 0.43 & 0.22 & \\
\hline 24 & 6 & 0.00 & 0.00 & 0.00 & -0.02 & 0.00 & 0.00 & 0.07 & 0.01 & 0.02 \\
\hline 25 & 1 & -0.01 & 0.00 & 0.00 & -0.02 & 0.01 & -0.01 & 0.09 & -0.04 & 0.03 \\
\hline 26 & 6 & 0.00 & -0.01 & 0.00 & 0.01 & -0.02 & 0.00 & -0.05 & 0.07 & \\
\hline 27 & 1 & -0.04 & 0.01 & -0.01 & -0.14 & 0.05 & -0.04 & 0.49 & -0.19 & 0.14 \\
\hline 28 & 6 & 0.01 & 0.01 & 0.00 & 0.03 & 0.04 & 0.00 & -0.11 & -0.14 & -0.01 \\
\hline 29 & 6 & -0.01 & 0.00 & 0.00 & 0.02 & 0.00 & 0.00 & 0.02 & 0.00 & \\
\hline 30 & 6 & 0.01 & 0.01 & 0.00 & -0.02 & -0.02 & 0.01 & -0.01 & -0.01 & \\
\hline 31 & 6 & 0.01 & -0.01 & 0.00 & -0.02 & 0.02 & 0.00 & -0.01 & 0.01 & 0.00 \\
\hline 32 & 6 & 0.00 & 0.00 & 0.00 & 0.00 & 0.01 & 0.00 & 0.00 & 0.01 & 0.00 \\
\hline 33 & 1 & -0.03 & 0.01 & 0.00 & 0.08 & -0.04 & 0.00 & 0.04 & -0.02 & 0.00 \\
\hline 34 & 6 & 0.00 & 0.00 & 0.00 & 0.01 & 0.00 & 0.00 & 0.01 & 0.00 & 0.00 \\
\hline 35 & 1 & -0.01 & 0.00 & 0.00 & 0.01 & 0.00 & 0.00 & 0.01 & 0.00 & 0.00 \\
\hline 36 & 6 & 0.00 & 0.00 & 0.00 & 0.00 & -0.01 & 0.00 & 0.00 & 0.00 & \\
\hline 37 & 1 & -0.03 & -0.01 & 0.01 & 0.08 & 0.03 & -0.02 & 0.03 & 0.01 & -0.01 \\
\hline 38 & 1 & 0.00 & 0.00 & 0.00 & 0.00 & 0.00 & 0.01 & 0.01 & 0.00 & -0.02 \\
\hline 39 & 1 & 0.00 & 0.00 & 0.00 & 0.00 & 0.00 & 0.00 & 0.01 & -0.01 & \\
\hline 40 & 1 & 0.00 & 0.00 & 0.00 & -0.01 & 0.00 & 0.00 & 0.02 & 0.00 & -0.01 \\
\hline 41 & 1 & 0.00 & 0.00 & 0.00 & 0.00 & 0.00 & 0.00 & 0.00 & 0.01 & \\
\hline 42 & 1 & 0.00 & 0.00 & 0.00 & 0.00 & 0.00 & 0.00 & 0.02 & 0.01 & 0.0 \\
\hline Frequen & & 1472.61 & & & 1488.25 & & & 1491.65 & & \\
\hline Atom & AN & $X$ & $\mathrm{Y}$ & Z & X & $\mathrm{Y}$ & Z & $X$ & $\mathrm{Y}$ & Z \\
\hline 1 & 78 & 0.00 & 0.00 & 0.00 & 0.00 & 0.00 & 0.00 & 0.00 & 0.00 & 0.00 \\
\hline 2 & 7 & -0.04 & -0.01 & -0.02 & 0. & 0.00 & 0.00 & 0.00 & -0.02 & 0.01 \\
\hline 3 & 7 & -0.01 & 0.00 & 0.00 & -0 & 0.02 & 0.00 & & 0.00 & 0.00 \\
\hline 4 & 17 & 0.00 & 0.01 & 0.00 & 0.00 & 0.00 & 0.0 & 0.0 & 0.00 & 0.00 \\
\hline 5 & 17 & 0.00 & -0.01 & 0.00 & 0.00 & 0.00 & 0.00 & 0.00 & 0.00 & 0.00 \\
\hline 6 & 17 & 0.0 & 0.00 & 0.00 & 0. & 0.00 & 0.00 & 0.0 & 0.00 & 0.00 \\
\hline 7 & 17 & 0.0 & 0.00 & 0.0 & & 0.00 & 0.0 & & 0.00 & \\
\hline 8 & 6 & 0.0 & 0.00 & 0.00 & 0. & 0.00 & 0.00 & 0.00 & 0.00 & \\
\hline 9 & 8 & 0.00 & 0.00 & 0.00 & 0. & 0.00 & 0.00 & 0.00 & 0.00 & 0.00 \\
\hline 10 & 6 & -0.0 & 0.02 & 0.06 & & 0.00 & 0.00 & & & \\
\hline 11 & 6 & & 0.01 & 0.0 & & 0.00 & 0.0 & & & \\
\hline 12 & 1 & -0.03 & -0.02 & -0.22 & 0 & -0.01 & 0.01 & 0.00 & 0.04 & -0.01 \\
\hline 13 & 1 & & -0.08 & -0.11 & & 0.00 & 0.00 & -0.02 & 0.00 & \\
\hline 14 & 1 & -0 . & 0.00 & -0.1 & & 0.00 & 0.0 & & 0.01 & \\
\hline 15 & 6 & 0.02 & 0.00 & 0.00 & 0.0 & 0.00 & 0.00 & 0.00 & 0.00 & 0.00 \\
\hline 16 & 1 & & -0.08 & -0.06 & & 0.01 & 0.01 & & 0.00 & \\
\hline 17 & 1 & 0.04 & 0.02 & -0.1 & & -0.01 & 0.02 & 0.0 & 0.00 & \\
\hline 18 & 1 & 0.0 & -0.04 & -0.0 & 0. & -0.05 & 0.00 & 0.00 & 0.01 & -0.01 \\
\hline 19 & 6 & -0.0 & 0.01 & 0.0 & 0. & 0.00 & 0.00 & 0.00 & 0.00 & 0.0 \\
\hline 20 & 6 & 0.0 & 0.00 & 0.1 & & -0.12 & 0.02 & 0.00 & 0.00 & \\
\hline 21 & 6 & 0.00 & 0.00 & 0.00 & 0.05 & 0.09 & 0.00 & 0.00 & 0.00 & \\
\hline
\end{tabular}




\begin{tabular}{|c|c|c|c|c|c|c|c|c|c|c|}
\hline & & & & & & & & & & \\
\hline 22 & 6 & 0.00 & 0.00 & 0.00 & -0.13 & -0.03 & -0.03 & 0.00 & 0.00 & 0.00 \\
\hline 23 & 1 & -0.01 & 0.00 & 0.00 & 0.39 & 0.32 & 0.05 & 0.00 & 0.00 & 0.00 \\
\hline 24 & 6 & 0.00 & 0.00 & 0.00 & 0.01 & -0.10 & 0.01 & 0.00 & 0.00 & 0.00 \\
\hline 25 & 1 & 0.00 & -0.01 & 0.00 & -0.02 & 0.66 & -0.09 & 0.00 & 0.00 & 0.00 \\
\hline 26 & 6 & 0.00 & 0.00 & 0.00 & 0.13 & -0.01 & 0.03 & 0.00 & 0.00 & 0.00 \\
\hline 27 & 1 & 0.00 & 0.00 & 0.00 & -0.35 & 0.25 & -0.12 & 0.00 & 0.00 & 0.00 \\
\hline 28 & 6 & 0.00 & 0.00 & 0.00 & -0.07 & 0.07 & -0.03 & 0.00 & 0.00 & 0.00 \\
\hline 29 & 6 & 0.22 & -0.01 & -0.04 & 0.00 & 0.00 & 0.00 & 0.00 & 0.12 & -0.04 \\
\hline 30 & 6 & -0.12 & -0.13 & 0.07 & 0.00 & 0.00 & 0.00 & 0.06 & -0.08 & 0.01 \\
\hline 31 & 6 & -0.13 & 0.14 & -0.02 & 0.00 & 0.00 & 0.0 & -0.06 & -0.07 & 0.04 \\
\hline 32 & 6 & -0.05 & 0.08 & -0.02 & 0.00 & 0.00 & 0.00 & -0.13 & 0.02 & 0.02 \\
\hline 33 & 1 & 0.52 & -0.25 & -0.02 & 0.01 & 0.00 & 0.00 & 0.36 & -0.29 & 0.02 \\
\hline 34 & 6 & 0.08 & 0.00 & -0.02 & 0.00 & 0.00 & 0.00 & 0.00 & 0.11 & -0.04 \\
\hline 35 & 1 & 0.10 & -0.01 & -0.02 & 0.00 & 0.00 & 0.00 & 0.01 & -0.65 & 0.21 \\
\hline 36 & 6 & -0.04 & -0.08 & 0.03 & 0.00 & 0.00 & 0.00 & 0.13 & 0.00 & -0.03 \\
\hline 37 & 1 & 0.51 & 0.19 & -0.17 & 0.00 & 0.00 & 0.00 & -0.36 & -0.26 & 0.16 \\
\hline 38 & 1 & -0.04 & & -0.01 & 0.00 & -0.01 & -0.02 & 0.01 & 0.02 & 0.03 \\
\hline 39 & 1 & -0.04 & 0.03 & -0.01 & 0.01 & 0.00 & 0.02 & 0.00 & -0.01 & -0.03 \\
\hline 40 & 1 & 0.02 & -0.01 & -0.01 & 0.00 & -0.03 & 0.01 & 0.00 & 0.05 & -0.01 \\
\hline 41 & 1 & 0.00 & & 0.00 & 0.00 & 0.00 & -0.01 & 0.00 & 0.00 & 0.00 \\
\hline 42 & 1 & 0.00 & & 0.00 & -0.01 & -0.01 & 0.00 & 0.00 & 0.00 & 0.00 \\
\hline requen & & 1502.35 & & & 1505.98 & & & 1517.57 & & \\
\hline Atom & AN & $x$ & $Y$ & Z & $x$ & Y & Z & $X$ & $\mathrm{Y}$ & Z \\
\hline 1 & 78 & 0.00 & 0.00 & 0.00 & 0.00 & 0.00 & 0.00 & 0.00 & 0.00 & 0.00 \\
\hline 2 & 7 & -0.03 & 0.01 & 0.04 & & 0.00 & 0.00 & 0.00 & 0.00 & 0.00 \\
\hline 3 & 7 & -0.01 & -0.01 & -0.02 & 0.00 & 0.00 & 0.00 & 0.0 & 0.00 & 0.00 \\
\hline 4 & 17 & 0.00 & 0.00 & 0.00 & 0.00 & 0.00 & 0.00 & 0.00 & 0.00 & 0.00 \\
\hline 5 & 17 & 0.00 & 0.00 & 0.00 & 0.00 & 0.00 & 0.00 & 0.00 & 0.00 & 0.00 \\
\hline 6 & 17 & 0.00 & 0.00 & 0.00 & 0.00 & 0.00 & 0.00 & 0.0 & 0.00 & 0.00 \\
\hline 7 & 17 & & & 0.00 & & 0.00 & 0.00 & 0.0 & 0.00 & 0.00 \\
\hline 8 & 6 & 0. & 0.00 & 0.00 & -0.01 & -0.07 & 0.00 & -0.03 & 0.01 & -0.06 \\
\hline 9 & 8 & 0.00 & 0.00 & 0.00 & 0.00 & 0.00 & 0.00 & 0.00 & 0.01 & 0.00 \\
\hline 10 & 6 & 0. & -0.01 & -0.04 & & 0.00 & 0.0 & 0. & 0.00 & 0.01 \\
\hline 11 & 6 & & & 0.00 & & 0.00 & 0.00 & 0. & 0.00 & 0.00 \\
\hline 12 & 1 & & & 0. & & 0.01 & 0.01 & 0. & 0.02 & -0.01 \\
\hline 13 & 1 & 0.3 & -0.26 & -0.16 & & -0.01 & -0.01 & -0.0 & 0.00 & -0.01 \\
\hline 14 & 1 & 0.32 & & -0.27 & & 0.01 & -0. & 0.0 & 0.00 & 0.00 \\
\hline 15 & 6 & & & & & & 0.0 & & & 0.00 \\
\hline 16 & 1 & 0. & & 0. & & 0.0 & 0.0 & 0. & 0. & 0.00 \\
\hline 17 & 1 & 0.24 & & 0.22 & & 0.00 & 0.01 & 0.03 & 0.00 & 0.00 \\
\hline 18 & 1 & & & -0.33 & & -0.01 & -0.02 & 0. & -0.02 & -0.01 \\
\hline 19 & 6 & & & 0. & & 0.1 & 0. & & 0.00 & 0.00 \\
\hline 20 & 6 & & & 0. & & 0.00 & 0.0 & 0.0 & 0.00 & 0.00 \\
\hline 21 & 6 & -0.01 & & 0. & & 0.00 & 0.0 & 0.0 & 0.00 & 0.00 \\
\hline 22 & 6 & & & & & & 0.0 & & & 0.00 \\
\hline 23 & 1 & & & & & & 0. & 0. & 0. & 0. \\
\hline 24 & 6 & 0.00 & & 0. & & 0.00 & 0.00 & 0.00 & 0.00 & 0.00 \\
\hline 25 & 1 & & & & & & 0.0 & & & 0.00 \\
\hline 26 & 6 & & & & & & 0. & & & \\
\hline 27 & 1 & & & 0.01 & & 0.00 & 0.0 & 0.01 & 0.00 & 0.00 \\
\hline 28 & 6 & & & & & & & & & 0.00 \\
\hline 29 & 6 & 0.0 & & -0.0 & & & 0. & & & 0.0 \\
\hline 30 & 6 & & & 0. & -0 . & 0.00 & 0.0 & 0.00 & 0. & 0.00 \\
\hline 31 & 6 & -0.0 & & & & & 0. & 0. & 0.00 & 0.00 \\
\hline 32 & 6 & 0.0 & & 0.00 & & 0.00 & 0.0 & 0. & 0.00 & 0.00 \\
\hline 33 & 1 & 0.0 & & 0.00 & & 0.01 & 0.00 & 0.01 & -0.01 & 0.00 \\
\hline 34 & 6 & $0 . C$ & & 0.0 & 0.00 & 0.00 & 0.00 & 0.00 & 0.00 & 0.00 \\
\hline 35 & 1 & 0.0 & & 0.0 & & 0.03 & -0.01 & 0.00 & -0.01 & 0.00 \\
\hline 36 & 6 & -0.0 & 0. & 0.0 & -0.0 & 0.00 & 0.00 & 0.00 & 0.00 & 0.00 \\
\hline 37 & 1 & 0.03 & 0.02 & -0.01 & 0.02 & 0.01 & -0.01 & 0.00 & 0.00 & 0.00 \\
\hline
\end{tabular}




\begin{tabular}{|c|c|c|c|c|c|c|c|c|c|c|}
\hline 38 & 1 & -0.02 & -0.02 & -0.02 & 0.23 & 0.39 & 0.46 & 0.33 & 0.32 & 0.00 \\
\hline & 1 & -0.01 & 0.01 & 0.01 & 0.11 & -0.16 & -0.27 & 0.44 & -0.16 & 0.56 \\
\hline 40 & 1 & 0.01 & .02 & 0.00 & -0.20 & 0.63 & -0.14 & -0.34 & -0.27 & 0.22 \\
\hline 41 & 1 & 0.00 & & 0.00 & 0.00 & -0.03 & & & & -0.06 \\
\hline 42 & 1 & 0.00 & & 0.00 & -0.03 & -0.02 & & & & 0.02 \\
\hline \multicolumn{2}{|c|}{ Frequencies } & \multicolumn{2}{|l|}{1521.00} & & \multicolumn{2}{|l|}{1527.20} & & \multicolumn{3}{|l|}{1534.85} \\
\hline Atom & AN & X & Y & Z & $x$ & Y & Z & $x$ & Y & 7 \\
\hline 1 & 78 & 0.00 & 0.00 & 0.00 & 0.00 & 0.00 & 0.00 & 0.00 & 0.00 & 0.00 \\
\hline 2 & 7 & -0.01 & 0.00 & 0.02 & -0.02 & 0.01 & 0.04 & & 0.00 & -0.01 \\
\hline 3 & 7 & 0.01 & 0.01 & 0.02 & 0.03 & 0.01 & 0.0 & & 0.00 & 0.00 \\
\hline 4 & 17 & 0.00 & 0.00 & 0.00 & 0.00 & 0.00 & 0.00 & 0.00 & 0.00 & 0.00 \\
\hline 5 & 17 & 0.00 & 0.00 & 0.00 & 0.00 & 0.00 & 0.0 & 0.0 & 0.00 & 0.00 \\
\hline 6 & 17 & 0.00 & 0.00 & 0.00 & 0.00 & 0.00 & 0.0 & & 0.00 & 0.00 \\
\hline 7 & 17 & 0.00 & 0.00 & 0.00 & 0.00 & 0.00 & 0.00 & 0.00 & 0.00 & 0.00 \\
\hline 8 & 6 & 0.00 & 0.00 & 0.00 & 0.00 & 0.00 & 0.0 & 0.0 & 0.00 & 0.00 \\
\hline 9 & 8 & 0.00 & 0.00 & 0.00 & 0.00 & 0.00 & & & & \\
\hline 10 & 6 & -0.01 & 0.01 & -0.03 & -0.01 & -0.02 & -0 . & & -0.01 & 0.01 \\
\hline 11 & 6 & -0.01 & 0.03 & 0.00 & -0.03 & -0.02 & 0. & & -0.03 & 0.00 \\
\hline 12 & 1 & 0.0 & -0.43 & 0.24 & 0.02 & 0.32 & 0.2 & & 0.43 & 0.14 \\
\hline 13 & 1 & 0.4 & -0.07 & 0.01 & 0.0 & -0.17 & -0.1 & -0 . & 0.02 & -0.04 \\
\hline 14 & 1 & -0.2 & -0.02 & -0.13 & & 0.17 & -0 . & & & 0.07 \\
\hline 15 & 6 & 0.02 & & -0.01 & & & & & & 0.00 \\
\hline 16 & 1 & 0.18 & & -0.14 & & & & 0.37 & & -0.12 \\
\hline 17 & 1 & -0.43 & & -0.07 & -0.19 & 0.09 & -0.27 & & 0.04 & 0.04 \\
\hline 18 & 1 & -0.0 & 0. & 0.0 & -0.0 & -0.03 & 0. & & 0.48 & 0.20 \\
\hline 19 & 6 & 0.0 & -0.02 & 0.0 & & & -0.1 & & -0. & \\
\hline 20 & 6 & -0.02 & 0.00 & 0.00 & -0.03 & 0.00 & -0.01 & 0.0 & 0.00 & .00 \\
\hline 21 & 6 & 0.00 & & 0.00 & 0.00 & -0.01 & 0. & & & .00 \\
\hline 22 & 6 & 0.0 & & 0.0 & & 0.00 & & & & \\
\hline 23 & 1 & -0.0 & & 0. & -0 . & -0.01 & 0. & 0. & & \\
\hline 24 & 6 & 0.00 & & 0.00 & -0. & 0.00 & & & & \\
\hline 25 & 1 & -0.0 & & 0.0 & -0. & 0.01 & & & & \\
\hline 26 & 6 & & & 0.0 & & & & & & \\
\hline 27 & 1 & & & -0. & & & & & & \\
\hline 28 & 6 & 0.0 & & 0.0 & 0.0 & 0. & & & & \\
\hline 29 & 6 & & & & & & & & & \\
\hline 30 & 6 & 0.00 & & & & & & & & \\
\hline 31 & 6 & & & & & & & & & \\
\hline 32 & 6 & & & & & & & & & \\
\hline 33 & 1 & & & & & & & & & \\
\hline 34 & 6 & 0. & & & & & & & & \\
\hline 35 & 1 & 0.00 & & & & & 0. & 0. & 0.02 & \\
\hline 36 & 6 & 0.00 & & & & & & 0. & & \\
\hline 37 & 1 & & & & & & & & & \\
\hline 38 & 1 & & & & & & & & & \\
\hline 39 & 1 & & & & & & & & & \\
\hline 40 & 1 & & & & & & & & & \\
\hline 41 & 1 & & & & & & & & & \\
\hline 42 & 1 & 0.00 & & 0.00 & 0.00 & 0.00 & 0.00 & 0.00 & 0.00 & 0.00 \\
\hline Frequen & & & & & & & & & & \\
\hline Atom & AN & $X$ & $Y$ & Z & 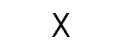 & Y & & & 1 & \\
\hline 1 & 78 & & 0.00 & 0.00 & & 0.0 & 0. & & 0. & 0.00 \\
\hline 2 & 7 & & & & & & & & & \\
\hline 3 & 7 & & & 0.01 & & & 0. & & -0.04 & -0.12 \\
\hline 4 & 17 & & & 0.00 & & & & & & $0 \cap 0$ \\
\hline 5 & 17 & & & 0.0 & 0.0 & 0.0 & 0.0 & 0.0 & 0.00 & 0.00 \\
\hline 6 & 17 & & & 0.00 & & & & & & 0.00 \\
\hline 7 & 17 & & & 0.00 & 0.0 & & & & 0.00 & 0.00 \\
\hline 8 & 6 & 0.0 & 0.0 & 0.00 & 0.00 & 0.00 & 0.00 & 0.00 & 0.00 & 0.00 \\
\hline 0 & 8 & 0.00 & 0.00 & 0.00 & 0.00 & 0.00 & 0.00 & 0.00 & 0.00 & 0.00 \\
\hline
\end{tabular}




\begin{tabular}{|c|c|c|c|c|c|c|c|c|c|c|}
\hline & & & & & & & & & & \\
\hline $\begin{array}{l}10 \\
11\end{array}$ & 6 & 0.01 & $\begin{array}{r}-0.01 \\
0.00\end{array}$ & -0.02 & $\begin{array}{l}0.01 \\
0.00\end{array}$ & $\begin{array}{r}-0.01 \\
0.00\end{array}$ & $\begin{array}{r}-0.03 \\
0.00\end{array}$ & $\begin{array}{l}-0.13 \\
-0.02\end{array}$ & $\begin{array}{r}0.10 \\
-0.01\end{array}$ & $\begin{array}{c}0.33 \\
-0.04\end{array}$ \\
\hline 12 & 1 & 000 & 0.01 & -0.02 & -0.01 & 0.03 & -0.04 & 0.06 & 010 & 0.35 \\
\hline 13 & 1 & -0.02 & 0.02 & 0.01 & -0.04 & 0.03 & 0.02 & 0.12 & -0.32 & -0.28 \\
\hline 14 & 1 & 0.01 & -0.01 & 0.03 & 0.03 & -0.01 & 0.05 & 0.07 & 0.16 & -0.42 \\
\hline 15 & 6 & 0.00 & -0.01 & -0.01 & -0.01 & -0.01 & -0.01 & 0.09 & 0.05 & 0.15 \\
\hline 16 & 1 & 0.03 & 0.00 & -0.01 & 0.02 & 0.02 & 0.01 & -0.05 & -0.19 & -0.12 \\
\hline 17 & 1 & -0.04 & & 0.02 & -0.01 & 0.00 & 0.02 & -0.07 & 0.05 & -0.20 \\
\hline 18 & 1 & -0.01 & 0.04 & -0.01 & 0.00 & 0.01 & -0.02 & -0.01 & 0.05 & 0.16 \\
\hline 19 & 6 & 0.00 & & 0.00 & 0.00 & 0.00 & 0.00 & 0.02 & 0.00 & -0.02 \\
\hline 20 & 6 & 0.02 & -0.26 & 0.03 & 0.00 & -0.01 & 0.00 & 0.07 & -0.01 & 0.02 \\
\hline 21 & 6 & -0.11 & 0.16 & -0.05 & 0.00 & 0.00 & 0.00 & -0.02 & 0.02 & -0.01 \\
\hline 22 & 6 & -0.02 & -0.20 & 0.02 & 0.00 & 0.00 & 0.00 & 0.00 & -0.01 & 0.00 \\
\hline 23 & 1 & 0.18 & & 0.05 & 0.00 & 0.00 & 0.0 & 0.01 & 0.00 & 0.00 \\
\hline 24 & 6 & -0.03 & 0.41 & -0.06 & 0.00 & 0.01 & 0.00 & 0.00 & 0.02 & 0.00 \\
\hline 25 & 1 & 0.00 & -0.67 & 0.08 & 0.00 & -0.02 & 0.00 & 0.00 & -0.03 & 0.00 \\
\hline 26 & 6 & 0.09 & & 0.05 & 0.00 & -0.01 & 0.00 & 0.01 & -0.01 & \\
\hline 27 & 1 & -0.26 & -0.06 & -0.06 & -0.01 & 0.00 & 0.0 & -0.01 & -0.01 & 0.00 \\
\hline 28 & 6 & 0.05 & 0.16 & -0.01 & 0.00 & 0.01 & 0.0 & 0.00 & 0.00 & .00 \\
\hline 29 & 6 & 0.01 & & 0.00 & 0.01 & -0.27 & 0. & -0. & 02 & \\
\hline 30 & 6 & 0.00 & & 0.0 & 0.09 & 0.16 & -0.07 & 0.02 & & \\
\hline 31 & 6 & 0.00 & & 0.00 & -0.08 & 0.17 & -0.04 & 0.01 & & 0.00 \\
\hline 32 & 6 & 0.00 & & 0.00 & 0.06 & -0.21 & 0.0 & 0.0 & & \\
\hline 33 & 1 & & & 0.00 & -0.22 & -0.06 & 0.07 & -0.03 & & \\
\hline 34 & 6 & 0.00 & -0.01 & 0.00 & 0.00 & 0.39 & -0.13 & 0.00 & 0.03 & -0.01 \\
\hline 35 & 1 & 0.00 & 0. & -0.01 & 0.01 & -0.62 & 0.20 & 0.0 & -0.04 & 0.02 \\
\hline 36 & 6 & 0.00 & & 0.00 & -0.07 & -0.21 & 0.08 & -0.01 & -0.01 & 0.00 \\
\hline 37 & 1 & 0.00 & 0.00 & 0.00 & 0.25 & -0.07 & -0.03 & 0.00 & -0.01 & 0.00 \\
\hline 38 & 1 & 0.00 & & 0.00 & 0.00 & 0.00 & 0.01 & -0.02 & -0.03 & -0.02 \\
\hline 39 & 1 & 0.00 & & -0. & 0.00 & 00 & -0.0 & -0. & 0.01 & -0.02 \\
\hline 40 & 1 & 0.01 & & -0.0 & 0.00 & 0.01 & 0.0 & 0.02 & 0.00 & 0.00 \\
\hline 41 & 1 & 0.00 & & -0.0 & 0.00 & -0.01 & -0 . & 0.01 & & \\
\hline 42 & 1 & -0.04 & -0.03 & 0.00 & -0.01 & -0.01 & 0.00 & 0.03 & 0.02 & 0.00 \\
\hline Erequen & & 1605.46 & & & 1607.03 & & & 1663.06 & & \\
\hline Atom & AN & & $Y$ & Z & $X$ & Y & Z & $X$ & $Y$ & Z \\
\hline 1 & 78 & 0. & 0.00 & 0.00 & 0.00 & 0.00 & 0.00 & 0.00 & 0.00 & 0.00 \\
\hline 2 & 7 & & 0. & 0.01 & & 0.00 & 0.01 & 0.0 & -0.01 & -0.02 \\
\hline 3 & 7 & & & & & & 0. & & & \\
\hline 4 & 17 & & & 0.0 & & 0.00 & 0.0 & 0. & 0.00 & 0.00 \\
\hline 5 & 17 & & & 0. & & 0.00 & 0.0 & 0.0 & 0.00 & 0.00 \\
\hline 6 & 17 & & & & & & 0. & 0. & 0.00 & 0.00 \\
\hline 7 & 17 & & & & & & & & & 0.00 \\
\hline 8 & 6 & & & 0. & & 0.00 & 0.0 & 0.0 & 0.00 & 0.01 \\
\hline 9 & 8 & & & & & 0.00 & 0.0 & 0.0 & 0.06 & 0.03 \\
\hline 10 & 6 & & & & & -0.01 & -0. & & & \\
\hline 11 & 6 & & & & & & 0. & & 0. & \\
\hline 12 & 1 & & & -0 & & 0.00 & -0.01 & 0. & 0.01 & 0.02 \\
\hline 13 & 1 & & & & & & & & & -0.01 \\
\hline 14 & 1 & & & & & -0. & & & & \\
\hline 15 & 6 & & & -0 & & 0.0 & -0. & -0.01 & -0.01 & -0.03 \\
\hline 16 & 1 & & & & & & & & & \\
\hline 17 & 1 & & & 0.0 & & -0.01 & 0. & & & \\
\hline 18 & 1 & & & -0.01 & & -0.01 & -0.01 & 0.00 & -0.01 & -0.02 \\
\hline 19 & 6 & & & & & 0.00 & 0. & & 0.00 & 0.00 \\
\hline 20 & 6 & & & 0.03 & & & & & 0.00 & $0 \cap 0$ \\
\hline 21 & 6 & -0.2 & & -0.06 & -0.05 & 0.00 & -0. & 0.1 & 0.00 & \\
\hline 22 & 6 & & 0.1 & 0.0 & 0.06 & 0.03 & 0.01 & 0.00 & 0.00 & 0.00 \\
\hline 23 & 1 & & & -0.0 & -0.0 & -0.07 & -0.01 & 0.00 & 0.00 & 0.00 \\
\hline 24 & 6 & & & -0.0 & -0.03 & -0.01 & -0.0 & 0.00 & 0.00 & 0. \\
\hline 25 & 1 & -0.19 & 0.05 & -0.05 & -0.04 & 0.01 & -0.01 & 0.00 & 0.00 & 0.00 \\
\hline
\end{tabular}




\begin{tabular}{|c|c|c|c|c|c|c|c|c|c|c|}
\hline 26 & 6 & 0.29 & -0.08 & 0.08 & 0.06 & -0.02 & 0.02 & 0.00 & 0.00 & 0.00 \\
\hline 27 & 1 & -0.39 & 0.28 & -0.13 & -0.08 & 0.06 & -0.03 & 0.01 & 0.00 & 0.00 \\
\hline 28 & 6 & -0.24 & -0.06 & -0.05 & -0.05 & -0.01 & -0.01 & 0.00 & 0.00 & 0.00 \\
\hline 29 & 6 & 0.03 & 0.00 & -0.01 & -0.15 & 0.01 & 0.02 & 0.00 & 0.00 & 0.00 \\
\hline 30 & 6 & -0.05 & 0.00 & 0.01 & 0.24 & 0.02 & -0.05 & 0.00 & 0.00 & 0.00 \\
\hline & 6 & -0.05 & 0.01 & 0.01 & 0.25 & -0.05 & -0.03 & 0.00 & 0.00 & 0.00 \\
\hline 32 & 6 & 0.06 & -0.03 & 0.00 & -0.30 & 0.13 & 0.0 & 0.00 & .00 & 0.00 \\
\hline 33 & 1 & -0.08 & 0.06 & 0.00 & 0.41 & -0.30 & 0.02 & 0.00 & 0.00 & 0.00 \\
\hline 34 & 6 & -0.04 & 0.00 & 0.01 & 0.17 & -0.02 & -0.03 & 0.00 & 0.00 & 0.00 \\
\hline 35 & 1 & -0.04 & 0.00 & 0.01 & 0.19 & 0.01 & -0.04 & 0.0 & .00 & 0.00 \\
\hline 36 & 6 & 0.06 & 0.02 & -0.02 & -0.30 & -0.09 & 0.09 & 0.00 & 0.00 & 0.00 \\
\hline 37 & 1 & -0.08 & -0.06 & 0.03 & 0.39 & 0.27 & -0.17 & 0.00 & 0.00 & 0.00 \\
\hline 38 & 1 & 0.00 & & -0.01 & 0.01 & 0.01 & 0.00 & -0.03 & -0.05 & -0.04 \\
\hline 39 & 1 & 0.00 & & 0.00 & 0.02 & -0.01 & 0.00 & -0.03 & 0.01 & -0.05 \\
\hline 40 & 1 & 0.01 & 0.00 & 0.00 & 0.00 & 0.00 & 0.00 & 0.08 & -0.01 & -0.02 \\
\hline 41 & 1 & 0.00 & 0.00 & -0.01 & 0.00 & 0.00 & -0.01 & 0.08 & -0.47 & -0.51 \\
\hline 42 & 1 & -0.01 & & 0.00 & -0.01 & 0.00 & 0.00 & -0.53 & -0.45 & 0.06 \\
\hline \multicolumn{2}{|c|}{ Frequencies } & 1669.92 & & & 2987.23 & & & 3058.17 & & \\
\hline Atom & AN & & $Y$ & Z & $x$ & Y & Z & $X$ & $Y$ & Z \\
\hline 1 & 78 & 0.00 & 0.00 & 0.00 & 0.00 & 0.00 & 0.00 & 0.00 & 0.00 & 0.00 \\
\hline 2 & 7 & -0.07 & 0.04 & 0.14 & 0.00 & 0.00 & 0.00 & 0.00 & 0.00 & 0.00 \\
\hline 3 & 7 & -0.20 & -0.09 & -0.26 & 0.00 & 0.00 & 0.00 & 0.00 & 0.00 & 0.00 \\
\hline 4 & 17 & 0.00 & 0.00 & 0.00 & 0. & 0.00 & 0.00 & 0.00 & 0.00 & 0.00 \\
\hline 5 & 17 & 0.00 & & 0.00 & & 0.00 & 0.00 & 0.00 & 0.00 & 0.00 \\
\hline 6 & 17 & 0.00 & 0.00 & 0.00 & 0.00 & 0.00 & 0.00 & 0.00 & 0.00 & 0.00 \\
\hline 7 & 17 & 0. & 0.00 & 0.00 & 0.00 & 0.00 & 0.00 & 0.00 & 0.00 & 0.00 \\
\hline 8 & 6 & 0.00 & 0.00 & 0.00 & 0.00 & 0.02 & 0.05 & 0.00 & 0.00 & 0.00 \\
\hline 9 & 8 & 0.01 & 0.03 & 0.02 & 0.00 & 0.00 & 0.00 & 0.00 & 0.00 & 0.00 \\
\hline 10 & 6 & 0.07 & -0.07 & -0.23 & 0.00 & 0.00 & 0.00 & 0.00 & 0.00 & 0.00 \\
\hline 11 & 6 & 0.00 & 0.01 & 0.03 & 0.00 & 0.00 & 0.0 & 0.00 & 0.00 & -0.04 \\
\hline 12 & 1 & & & -0.17 & 0.00 & 0.00 & 0.00 & 0.27 & 0.01 & -0.07 \\
\hline 13 & 1 & -0.0 & & 0.12 & 0.00 & 0.00 & 0.00 & -0.09 & -0.40 & 0.43 \\
\hline 14 & 1 & 0. & & 0. & & 0.00 & 0.0 & -0.13 & 0.42 & 0.14 \\
\hline 15 & 6 & & & 0.40 & & 0. & 0.0 & & 0.00 & 0.00 \\
\hline 16 & 1 & & & -0.19 & & 0. & 0.0 & -0 & 0.19 & -0.27 \\
\hline 17 & 1 & -0.0 & 0.05 & -0.2 & 0.00 & 0.00 & 0.0 & -0.07 & -0.40 & -0.08 \\
\hline 18 & 1 & & & 0. & & 0.00 & 0.0 & 0.22 & 0.04 & 0.03 \\
\hline 19 & 6 & & & -0 & & & 0.0 & 0. & & 0.03 \\
\hline 20 & 6 & 0. & & 0. & 0.0 & 0.0 & 0. & 0. & 0.00 & 0.00 \\
\hline 21 & 6 & & & -0. & 0.00 & 0. & 0.00 & 0.00 & 0.00 & 0.00 \\
\hline 22 & 6 & & & 0. & & 0.00 & 0.0 & 0.00 & 0.00 & 0.00 \\
\hline 23 & 1 & & & & & & & & & 0.00 \\
\hline 24 & 6 & & & & & 0.0 & 0.0 & 0.0 & 0.00 & 0.00 \\
\hline 25 & 1 & & & & 0.00 & & 0.0 & 0.00 & 0.00 & 0.00 \\
\hline 26 & 6 & & & & & & & & & \\
\hline 27 & 1 & & & & & & & 0. & & \\
\hline 28 & 6 & & & 0. & & 0. & 0.0 & 0.00 & 0.00 & 0.00 \\
\hline 29 & 6 & & & -0. & & & & & & \\
\hline 30 & 6 & 0. & & 0. & & & & & & \\
\hline 31 & 6 & & & & & 0.00 & 0.00 & 0.00 & 0.00 & 0.00 \\
\hline 32 & 6 & -0.0 & & & & & 0. & & 0.00 & 0.00 \\
\hline 33 & 1 & 0.0 & & 0.0 & & & 0. & & 0.00 & 0.00 \\
\hline 34 & 6 & & & & & & 0.0 & 0.00 & 0.00 & 0. \\
\hline 35 & 1 & 0.00 & & 0.0 & 0.0 & 0.0 & 0.00 & 0.00 & 0.00 & 0.00 \\
\hline 36 & 6 & 0.00 & & & & & 0.00 & 00 & 0.00 & 0.00 \\
\hline 37 & 1 & & & 0.0 & 0.00 & & 0.0 & 0.0 & 0.00 & 0. \\
\hline 38 & 1 & -0.03 & -0.0 & -0.01 & 0.22 & -0.24 & 0.14 & 0.00 & 0.00 & 0.00 \\
\hline 39 & 1 & & & -0.02 & 0.18 & & -0.03 & 0.00 & 0.00 & 0.00 \\
\hline 40 & 1 & & & -0. & -0.3 & -0.26 & -0.7 & 0.0 & 0.00 & 0. \\
\hline 41 & 1 & & & -0.25 & 0.00 & 0.00 & 0.00 & 0.00 & 0.00 & \\
\hline
\end{tabular}




\begin{tabular}{|c|c|c|c|c|c|c|c|c|c|c|}
\hline $\begin{array}{c}42 \\
\text { Frequen }\end{array}$ & ies $^{1}$ & $\begin{array}{c}-0.25 \\
3062.21\end{array}$ & -0.21 & 0.03 & $\begin{array}{c}0.00 \\
3071.34\end{array}$ & -0.01 & -0.01 & $\begin{array}{c}0.00 \\
3112.18\end{array}$ & 0.00 & 0.00 \\
\hline Atom & AN & $x$ & $Y$ & Z & $x$ & $Y$ & Z & $x$ & Y & Z \\
\hline 1 & 78 & 0.00 & 0.00 & 0.00 & 0.00 & 0.00 & 0.00 & 0.00 & 0.00 & 0.00 \\
\hline 2 & 7 & 0.00 & 0.00 & 0.00 & 0.00 & 0.00 & 0.00 & 0.00 & 0.00 & 0.00 \\
\hline 3 & 7 & 0.00 & 0.00 & 0.00 & 0.00 & 0.00 & 0.00 & 0.00 & 0.00 & 0.00 \\
\hline 4 & 17 & 0.00 & 0.00 & 0.00 & 0.00 & 0.00 & 0.00 & 0.00 & 0.00 & 0.00 \\
\hline 5 & 17 & 0.00 & 0.00 & 0.00 & 0.00 & 0.00 & 0.00 & 0.00 & 0.00 & 0.00 \\
\hline 6 & 17 & 0.00 & 0.00 & 0.00 & 0.00 & 0.00 & 0.00 & 0.00 & 0.00 & 0.00 \\
\hline 7 & 17 & 0.00 & 0.00 & 0.00 & 0.00 & 0.00 & 0.00 & 0.00 & 0.00 & 0.00 \\
\hline 8 & 6 & 0.00 & 0.00 & 0.00 & 0.07 & 0.02 & 0.04 & -0.01 & 0.09 & -0.03 \\
\hline 9 & 8 & 0.00 & 0.00 & 0.00 & 0.00 & 0.00 & 0.00 & 0.00 & 0.00 & 0.00 \\
\hline 10 & 6 & 0.00 & 0.00 & 0.0 & 0.00 & 0.00 & 0.00 & 0.00 & 0.00 & 0.00 \\
\hline 11 & 6 & 0.00 & 0.00 & -0.03 & 0.00 & 0.00 & 0.0 & 0.00 & 0.00 & 0.00 \\
\hline 12 & 1 & 0.21 & 0.01 & -0.0 & 0.00 & 0.00 & 0.00 & 0.00 & 0.00 & 0.00 \\
\hline 13 & 1 & -0.07 & -0.29 & 0.32 & 0.00 & 0.00 & 0.0 & 0.0 & 0.00 & 0.00 \\
\hline 14 & 1 & -0.09 & 0.30 & 0.1 & 0.00 & 0.00 & 0.0 & 0. & & \\
\hline 15 & 6 & 0.00 & 0.00 & 0.0 & 0.00 & 0.00 & 0.00 & 0.00 & & .00 \\
\hline 16 & 1 & 0.15 & -0.25 & 0.3 & 0.00 & 0.00 & 0.0 & 0.0 & 0.00 & 00 \\
\hline 17 & 1 & 0.09 & 0.57 & 0. & & 0.00 & 0. & & & \\
\hline 18 & 1 & & -0.05 & -0.04 & 0.00 & 0.00 & & & & \\
\hline 19 & 6 & 0.01 & -0. & -0.04 & 0.0 & 0.00 & 0. & & & \\
\hline 20 & 6 & 0.00 & & 0.0 & 0.00 & 0.00 & & & & \\
\hline 21 & 6 & & & 0. & 0.00 & 0.00 & & 0.00 & & \\
\hline 22 & 6 & 0.00 & 0.00 & 0. & 0.00 & 0.00 & 0.00 & 0.00 & 0.00 & .00 \\
\hline 23 & 1 & 0.00 & & 0. & 0.0 & 0.00 & 0. & 0. & & 00 \\
\hline 24 & 6 & 0.00 & & 0.0 & 0.0 & 0.00 & 0.0 & 0. & & \\
\hline 25 & 1 & 0.00 & 0.00 & 0.00 & 0.00 & 0.00 & 0.00 & 0.00 & 0.00 & .00 \\
\hline 26 & 6 & 0.00 & & 0. & 0.00 & 0.00 & 0. & 0. & & 0.00 \\
\hline 27 & 1 & 0.00 & & 0. & 0.0 & 0.00 & 0. & 0. & & \\
\hline 28 & 6 & 0. & 0. & 0. & 0.00 & 0.00 & 0.0 & 0.0 & 0. & \\
\hline 29 & 6 & 0.00 & 0.00 & 0.0 & 0.00 & 0.00 & 0.0 & & & \\
\hline 30 & 6 & 0.00 & & 0. & 0.0 & 0.0 & 0. & 0. & & \\
\hline 31 & 6 & & & & & 0.1 & 0. & & & \\
\hline 32 & 6 & & & & & 0. & 0. & & & \\
\hline 33 & 1 & 0.00 & 0.0 & 0.0 & 0.0 & 0.00 & 0.0 & 0.0 & 0.00 & \\
\hline 34 & 6 & 0. & & 0. & & 0.00 & 0. & 0. & 0. & \\
\hline 35 & 1 & & & & & & & & & \\
\hline 36 & 6 & 0. & & 0. & & 0.0 & 0. & & & \\
\hline 37 & 1 & & & & & 0. & 0. & 0. & & \\
\hline 38 & 1 & & & & $-0 .{ }^{3}$ & & & & & \\
\hline 39 & 1 & & & & $-0.2^{2}$ & -0. & & & & \\
\hline 40 & 1 & 0.00 & & 0. & -0.1 & -0.14 & -0 & 0. & 0. & \\
\hline 41 & 1 & 0.00 & & & & 0.00 & 0.0 & & & \\
\hline 42 & 1 & 0.00 & & & & -0.01 & -0.01 & & & \\
\hline Frequen & & 3118.96 & & & & & & & & \\
\hline Atom & AN & y & $Y$ & Z & n & $\mathrm{Y}$ & Z & $x$ & $\mathrm{Y}$ & $L_{L}$ \\
\hline 1 & 78 & & 0.00 & 0.00 & & 0.00 & 0.00 & & & 0.00 \\
\hline 2 & 7 & & & & & & & & & \\
\hline 3 & 7 & & & 0.00 & & 0.00 & 0. & & & 0.00 \\
\hline 4 & 17 & & & & & & & & & \\
\hline 5 & 17 & & & 0.0 & & 0.00 & & & & \\
\hline 6 & 17 & & & & & 0.00 & 0. & & & 0. \\
\hline 7 & 17 & & & & & 0.00 & 0. & & & 0.00 \\
\hline 8 & 6 & & & & & 0.0 & & & & 0.00 \\
\hline 9 & 8 & & & 0.0 & 0.00 & 0.0 & 0.0 & 0. & 0.00 & 0.00 \\
\hline 10 & 6 & 0.00 & 0.00 & 0.0 & 0.00 & 0.00 & 0.00 & 0.00 & 0.00 & 0.00 \\
\hline 11 & 6 & 0.0 & & 0.0 & 0.00 & -0.03 & 0.0 & & & 0.00 \\
\hline 12 & 1 & & -0.1 & & 0.0 & -0.0 & 0.00 & & 0.00 & -0.01 \\
\hline 13 & 1 & 0.10 & 0.37 & -0.44 & 0.04 & 0.15 & -0.18 & 0.00 & 0.00 & 0.00 \\
\hline
\end{tabular}




\begin{tabular}{|c|c|c|c|c|c|c|c|c|c|c|}
\hline & & & & & & & & & & \\
\hline 14 & 1 & -0.21 & 0.64 & 0.25 & -0.08 & 0.24 & 0.10 & 0.00 & 0.00 & 0.00 \\
\hline 15 & 6 & 0.00 & 0.00 & 0.00 & 0.00 & 0.00 & 0.00 & 0.00 & 0.00 & 0.00 \\
\hline 16 & 1 & 0.09 & -0.14 & 0.22 & -0.23 & 0.36 & -0.55 & 0.09 & -0.19 & 0.26 \\
\hline 17 & 1 & -0.04 & -0.22 & -0.05 & 0.10 & 0.58 & 0.14 & 0.01 & 0.21 & 0.03 \\
\hline 18 & 1 & -0.05 & 0.00 & -0.01 & 0.11 & -0.01 & 0.02 & 0.90 & 0.14 & 0.10 \\
\hline 19 & 6 & 0.00 & 0.03 & -0.01 & 0.00 & -0.08 & 0.03 & -0.08 & -0.01 & 0.03 \\
\hline 20 & 6 & 0.00 & 0.00 & 0.00 & 0.00 & 0.00 & 0.0 & 0.00 & 0.00 & 0.00 \\
\hline 21 & 6 & 0.00 & 0.00 & 0.00 & 0.00 & 0.00 & 0.00 & 0.00 & 0.00 & 0.00 \\
\hline 22 & 6 & 0.00 & 0.00 & 0.00 & 0.00 & 0.00 & 0.00 & 0.00 & 0.00 & 0.00 \\
\hline 23 & 1 & 0.00 & 0.00 & 0.00 & 0.00 & 0.00 & 0. & 0.00 & 0.00 & 0.00 \\
\hline 24 & 6 & 0.00 & 0.00 & 0.00 & 0.00 & 0.00 & 0.00 & 0.00 & 0.00 & 0.00 \\
\hline 25 & 1 & 0.00 & 0.00 & 0.00 & 0.00 & 0.00 & 0.00 & 0.00 & 0.00 & 0.00 \\
\hline 26 & 6 & 0.00 & 0.00 & 0.00 & 0.00 & 0.00 & 0.0 & 0.00 & 0.00 & 0.00 \\
\hline 27 & 1 & 0.00 & 0.00 & 0.00 & 0.00 & 0.00 & 0.00 & 0.00 & 0.00 & 0.00 \\
\hline 28 & 6 & 0.00 & 0.00 & 0.00 & 0.00 & 0.00 & 0.00 & 0.00 & 0.00 & 0.00 \\
\hline 29 & 6 & 0.00 & 0.00 & 0.00 & 0.00 & 0.00 & 0.00 & 0.00 & 0.00 & 0.00 \\
\hline 30 & 6 & 0.00 & 0.00 & 0.00 & 0.00 & 0.00 & 0.0 & 0.00 & 0.00 & 0.00 \\
\hline 31 & 6 & 0.00 & 0.00 & 0.00 & 0.00 & 0.00 & 0.00 & 0.00 & 0.00 & 0.00 \\
\hline 32 & 6 & 0.00 & 0.00 & 0.00 & 0.00 & 0.00 & 0.0 & 0.00 & 0.00 & 0.00 \\
\hline 33 & 1 & 0.00 & 0.00 & 0.00 & 0.00 & 0.00 & 0. & 0.00 & .00 & \\
\hline 34 & 6 & 0.00 & 0.00 & 0.00 & 0.00 & 0.00 & 0. & 0.00 & & \\
\hline 35 & 1 & 0.00 & 0.00 & 0.00 & 0.00 & 0.00 & 0.0 & 0.00 & 0.00 & 0.00 \\
\hline 36 & 6 & 0.00 & 0.00 & 0.00 & 0.00 & 0.00 & 0. & 0.0 & .00 & \\
\hline 37 & 1 & 0.00 & 0.00 & 0.00 & 0.00 & 0.00 & 0.00 & 0.00 & 0.00 & \\
\hline 38 & 1 & 0.00 & 0.00 & 0.00 & 0.00 & 0.00 & 0.00 & 0.00 & 0.00 & 0.00 \\
\hline 39 & 1 & 0.00 & 0.00 & 0.00 & 0.00 & 0.00 & 0.0 & 0.00 & 0.00 & 0.00 \\
\hline 40 & 1 & 0.00 & 0.00 & 0.00 & 0.00 & 0.00 & 0.0 & 0.00 & 0.00 & \\
\hline 41 & 1 & 0.00 & 0.00 & 0.00 & 0.00 & 0.00 & 0.00 & 0.00 & 0.00 & 0.00 \\
\hline 42 & 1 & 0.00 & 0.00 & 0.00 & 0.00 & 0.00 & 0.00 & 0.00 & 0.00 & 0.00 \\
\hline Erequer & & 3178.87 & & & 3227.48 & & & 3227.61 & & \\
\hline Atom & AN & $x$ & $Y$ & Z & $X$ & $Y$ & Z & $X$ & $\mathrm{Y}$ & Z \\
\hline 1 & 78 & 0.00 & 0.00 & 0.00 & 0.00 & 0.00 & 0.00 & 0.00 & 0.00 & 0.00 \\
\hline 2 & 7 & 0.00 & 0.00 & 0.00 & 0.00 & 0.00 & 0.00 & 0.00 & 0.00 & 0.00 \\
\hline 3 & 7 & & 0.00 & 0.00 & 0.00 & 0.00 & 0.00 & 0.0 & 0.00 & 0.00 \\
\hline 4 & 17 & & 0.00 & 0.00 & & 0.00 & 0.0 & 0.0 & & 0.00 \\
\hline 5 & 17 & 0.00 & 0.00 & 0.00 & 0.00 & 0.00 & 0.00 & 0.00 & 0.00 & 0.00 \\
\hline 6 & 17 & 0. & 0. & 0.00 & 0.00 & 0.00 & 0.00 & 0.00 & 0.00 & 0.00 \\
\hline 7 & 17 & & & 0. & & 0.00 & 0.0 & 0. & 0.00 & 0.00 \\
\hline 8 & 6 & 0. & 0. & 0.00 & 0.0 & 0.00 & 0. & 0. & 0. & 0.00 \\
\hline 9 & 8 & 0.00 & 0.00 & 0.00 & 0.00 & 0.00 & 0.00 & 0.00 & 0.00 & 0.00 \\
\hline 10 & 6 & 0.0 & 0. & 0. & 0.0 & 0.00 & 0.00 & 0.00 & 0.00 & 0.00 \\
\hline 11 & 6 & & & & & 0.00 & & & & 0.00 \\
\hline 12 & 1 & & 0.0 & -0.20 & 0. & 0.00 & 0.0 & 0.0 & 0.00 & 0.00 \\
\hline 13 & 1 & 0.0 & & -0.14 & 0.00 & 0.00 & 0.00 & 0.00 & 0.00 & 0.00 \\
\hline 14 & 1 & & & -0.09 & & 0.00 & & & & 0.00 \\
\hline 15 & 6 & & & & & 0.0 & & 0. & & 0 \\
\hline 16 & 1 & 0.00 & & -0.01 & 0.00 & 0.00 & 0.00 & 0.00 & 0.00 & 0.00 \\
\hline 17 & 1 & 0.00 & & 0.00 & & 0.00 & 0.0 & & & 0.00 \\
\hline 18 & 1 & -0. & & 0. & & 0.00 & 0. & 0. & & \\
\hline 19 & 6 & 0. & & 0. & 0. & 0.00 & 0.0 & 0.0 & 0.00 & 0.00 \\
\hline 20 & 6 & 0.0 & & & & 0.00 & 0. & & 0.00 & 0.00 \\
\hline 21 & 6 & 0.00 & 0.0 & 0.0 & 0.00 & 0.00 & 0.0 & 0. & 0.00 & 0.00 \\
\hline 22 & 6 & & & 0.00 & 0.02 & -0.02 & 0.01 & 0.0 & 0.00 & \\
\hline 23 & 1 & 0.00 & 0.0 & 0.00 & -0.19 & 0.30 & -0.08 & 0.01 & -0.01 & 0.00 \\
\hline 24 & 6 & 0.0 & 0.0 & 0.00 & -0.06 & 0.00 & -0.02 & 0.00 & 0.00 & 0.00 \\
\hline 25 & 1 & 0.0 & 0.0 & 0.00 & 0.76 & 0.05 & 0.1 & -0.02 & 0.00 & 0.00 \\
\hline 26 & 6 & 0.00 & 0.00 & 0.00 & 0.02 & 0.04 & 0.00 & 0.00 & 0.00 & 0.00 \\
\hline 27 & 1 & 0.0 & 0.0 & 0.00 & -0.23 & -0.44 & 0.01 & 0.01 & 0.01 & 0.00 \\
\hline 28 & 6 & 0.0 & 0.0 & 0.0 & 0.0 & 0.00 & 0.00 & 0.00 & 0.00 & 0. \\
\hline 29 & 6 & 0.00 & 0.00 & 0.00 & 0.00 & 0.00 & 0.00 & 0.00 & 0.00 & 0.00 \\
\hline
\end{tabular}




\begin{tabular}{|c|c|c|c|c|c|c|c|c|c|c|}
\hline & & & & & & & & & & \\
\hline $\begin{array}{l}30 \\
31\end{array}$ & $\begin{array}{l}6 \\
6\end{array}$ & $\begin{array}{l}0.00 \\
0.00\end{array}$ & 0.00 & 0.00 & $\begin{array}{l}0.00 \\
0.00\end{array}$ & $\begin{array}{l}0.00 \\
0.00\end{array}$ & $\begin{array}{l}0.00 \\
0.00\end{array}$ & $\begin{array}{l}0.00 \\
0.00\end{array}$ & $\begin{array}{l}0.00 \\
0.00\end{array}$ & $\begin{array}{l}0.00 \\
0.00\end{array}$ \\
\hline 32 & 6 & 0.00 & 0.00 & 0.00 & 0.00 & 0.00 & 0.00 & 0.02 & 0.03 & -0.01 \\
\hline 33 & 1 & 0.00 & 0.00 & 0.00 & -0.01 & & & & & 0.14 \\
\hline 34 & 6 & 0.00 & 0.00 & 0.00 & 0.00 & 0.00 & 0.00 & -0.07 & 0.00 & 0.01 \\
\hline 35 & 1 & 0.00 & 0.00 & 0.0 & 0.02 & 0.00 & 0.00 & 0.80 & -0.04 & 0.15 \\
\hline 36 & 6 & 0.00 & 0.00 & 0.0 & 0.00 & 0.00 & & & & 0.01 \\
\hline 37 & 1 & 0.00 & 0.00 & & & & & & .35 & -0.07 \\
\hline 38 & 1 & 0.00 & & & 0.00 & 0.00 & & 0.00 & & 0.00 \\
\hline 39 & 1 & 0.00 & & & 0.00 & 0.00 & & 0.00 & & 00 \\
\hline 40 & 1 & 0.00 & & & & & & & & .00 \\
\hline 41 & 1 & 0.00 & & & 0.00 & 0.00 & & 0.1 & & .00 \\
\hline 42 & 1 & 0.00 & & & 0.00 & 0.00 & & 0.00 & & .00 \\
\hline requen & & 3238.89 & & & 3240.54 & & & 3247.90 & & \\
\hline Atom & AN & $X$ & $Y$ & Z & $x$ & $Y$ & Z & $x$ & Y & Z \\
\hline 1 & 78 & 0.00 & 0.00 & 0.00 & 0.00 & 0.00 & 0.00 & 0.00 & 0.00 & 0.00 \\
\hline 2 & 7 & 0.00 & 0.00 & 0.00 & 0.00 & 0.00 & 0.00 & 0.00 & 0.00 & 0.00 \\
\hline 3 & 7 & 0.00 & 0.00 & 0.00 & 0.00 & 0.00 & 0.00 & 0.00 & 0.00 & 0.00 \\
\hline 4 & 17 & 0.00 & 0.00 & 0.00 & 0.0 & 0.00 & 0.00 & 0.0 & 0.00 & 0.00 \\
\hline 5 & 17 & & & 0.00 & & 0.00 & 0.1 & & & \\
\hline 6 & 17 & 0.00 & 0.00 & 0.00 & 0.0 & 0.00 & 0.0 & & & 0.00 \\
\hline 7 & 17 & 0.00 & 0.00 & 0.00 & 0.0 & 0.00 & 0.00 & 0.00 & .00 & 0.00 \\
\hline 8 & 6 & & & 0.0 & & 0.00 & & & & \\
\hline 9 & 8 & 0.00 & 0. & 0.00 & & 0.00 & & & & \\
\hline 10 & 6 & 0.00 & 0.00 & 0.00 & & 0.00 & 0.0 & 0. & 1.00 & .00 \\
\hline 11 & 6 & 0.00 & & 0.0 & & 0.00 & & & & 0.00 \\
\hline 12 & 1 & 0.00 & & & & 0.00 & & & & \\
\hline 13 & 1 & 0.00 & 0.00 & 0.00 & 0.0 & 0.00 & 0.0 & 0.0 & 0.00 & 0.00 \\
\hline 14 & 1 & 0.00 & 0. & 0.0 & 0.0 & 0.00 & 0.1 & 0. & 1.00 & 0.00 \\
\hline 15 & 6 & & & & & 0.00 & 0.0 & 0. & 10 & 0. \\
\hline 16 & 1 & 0.00 & 0.00 & & 0. & 0.00 & 0.0 & 0.0 & 0.00 & 0.00 \\
\hline 17 & 1 & & & 0.0 & & 0.00 & & & & 0.00 \\
\hline 18 & 1 & & & & & & 0. & & & \\
\hline 19 & 6 & & & & & 0. & 0. & 0. & & \\
\hline 20 & 6 & & & 0.0 & 0. & 0.00 & 0. & 0. & & 0.00 \\
\hline 21 & 6 & & 0. & 0.0 & 0.0 & 0.00 & 0. & 0. & & 0 \\
\hline 22 & 6 & & & 0. & & & 0. & -0 & & -0.01 \\
\hline 23 & 1 & & & & & & & & & \\
\hline 24 & 6 & & & & & 0.0 & 0. & -0 . & 10 & -0.01 \\
\hline 25 & 1 & & & & & & & & & 0.14 \\
\hline 26 & 6 & & & & & & & & & \\
\hline 27 & 1 & & & -0.0 & & 0.01 & 0. & 0.2 & 0.40 & -0.01 \\
\hline 28 & 6 & & & & & 0.00 & & 0. & 10 & 0. \\
\hline 29 & 6 & & & & & & & & & \\
\hline 30 & 6 & & & & & & & & & \\
\hline 31 & 6 & & & & & & & 0. & & \\
\hline 32 & 6 & & & & & -0.05 & & & & 0.00 \\
\hline 33 & 1 & & & & & & & & & \\
\hline 34 & 6 & & & & & & & 0. & 0. & \\
\hline 35 & 1 & 0.00 & & & & & 0. & 0. & 0.00 & 0.00 \\
\hline 36 & 6 & & & & & -0.05 & & & & \\
\hline 37 & 1 & & & & & & & & & \\
\hline 38 & 1 & 0.00 & & 0. & & 0.00 & 0.0 & 0. & 0.00 & 0.00 \\
\hline 39 & 1 & & & & & & & & & \\
\hline 40 & 1 & & & & & 0.00 & & & & \\
\hline 41 & 1 & & & 0.0 & & 0.00 & 0. & 0. & 0.00 & 0.00 \\
\hline 42 & 1 & 0.00 & & 0.00 & & 0.00 & 0.00 & & 0.00 & 0.00 \\
\hline 2 & & & & & & & & 3801.05 & & \\
\hline tom & AN & $X$ & Y & Z & $X$ & $Y$ & Z & $x$ & Y & Z \\
\hline & 78 & 0.00 & 0.00 & 0.00 & 0.00 & 0.00 & 0.00 & 0.00 & 0.00 & 0.00 \\
\hline
\end{tabular}




\begin{tabular}{|c|c|c|c|c|c|c|c|c|c|c|}
\hline 2 & 7 & 0.00 & 0.00 & 0.00 & 0.00 & 0.00 & 0.00 & 0.00 & 0.00 & 0.00 \\
\hline 3 & 7 & 0.00 & 0.00 & 0.00 & 0.00 & 0.00 & 0.00 & 0.00 & 0.00 & 0.00 \\
\hline 4 & 17 & 0.00 & 0.00 & 0.00 & 0.00 & 0.00 & & 0.00 & .00 & 0.00 \\
\hline 5 & 17 & 0.00 & & 0.00 & & 0.00 & & & .00 & 0.00 \\
\hline & 17 & 0.00 & 0.00 & 0.00 & 0.0 & 0.00 & & & .00 & .00 \\
\hline 7 & 17 & 0.00 & 0.00 & 0.0 & & 0. & & & & 1.00 \\
\hline 8 & 6 & 0.00 & 0.00 & 0.00 & & & & & & 1.00 \\
\hline 9 & 8 & 0.00 & 0.00 & 0.00 & -0.0 & -0.04 & -0.0 & & .00 & 0.05 \\
\hline & 6 & 0.00 & 0.00 & 0.0 & & 0.00 & & & .00 & .00 \\
\hline & 6 & 0.00 & 0.00 & 0.0 & & 0.0 & & & & 1.00 \\
\hline & 1 & 0.00 & 0.00 & 0.00 & & 0.00 & 0.0 & 0.0 & .00 & 0.00 \\
\hline & 1 & 0.00 & 0 & 0.0 & & 0.0 & & & 00 & .00 \\
\hline & 1 & 0.00 & 0.00 & 0.0 & & 0.0 & & & & \\
\hline & 6 & 0.00 & 0.00 & 0.00 & & & 0.0 & 0.00 & 1.00 & 0.00 \\
\hline & 1 & 0.00 & 0.00 & 0.0 & & 0. & & & .00 & 0.00 \\
\hline & 1 & 0.00 & & & & & & & & \\
\hline & 1 & 0.00 & & 0.00 & & & & & & \\
\hline & 6 & 0.00 & 0.00 & 0.00 & & 0.00 & & & .00 & 0.00 \\
\hline & 6 & 0.00 & 0.00 & 0.0 & & 0. & & & .00 & 0.00 \\
\hline & 6 & 0.00 & 0 & 0.0 & & 0. & & & & 0.00 \\
\hline & 6 & 0.00 & & 0.0 & & & & & & 0.00 \\
\hline & 1 & -0.01 & 0.01 & 0.0 & & 0.0 & 0. & 0.0 & .00 & .00 \\
\hline & 6 & 0.00 & 0.00 & 0.0 & & 0.0 & 0. & & 10 & 0.00 \\
\hline & 1 & -0.01 & & 0. & & & & & & 0.00 \\
\hline & 6 & 0.00 & 0.00 & 0.0 & & 0.0 & & 0. & 00 & 0.00 \\
\hline & 1 & 0.00 & -0.01 & 0.0 & & 0.0 & 0. & & 10 & 0.00 \\
\hline & 6 & 0.00 & 0.00 & 0.0 & & & 0. & & & 0.00 \\
\hline & 6 & 0.00 & & 0.0 & & & & & & 0.00 \\
\hline & 6 & 0.00 & & 0. & & 0. & & & .00 & 0.00 \\
\hline 3 & 6 & 0.00 & 0.00 & 0.00 & & & & & & 0.00 \\
\hline & 6 & -0.02 & -0.04 & 0.0 & & & & & & 0.00 \\
\hline & 1 & 0.28 & 0.48 & -0.2 & & & & & & 0.00 \\
\hline 3 & 6 & -0.05 & 0.00 & 0.01 & & & & & 0 & 0.00 \\
\hline & 1 & 0.56 & -0.0 & -0.1 & & & & & & 0.00 \\
\hline & 6 & -0.02 & & -0.0 & & & & & & 0.00 \\
\hline 3 & 1 & 0.28 & -0.48 & 0.10 & 0.00 & 0.0 & & & 0.00 & 0.00 \\
\hline & 1 & 0.00 & 0.00 & 0.0 & & & & & & 0.00 \\
\hline & 1 & 0.0 & 0.0 & 0.0 & & & & & & 0.00 \\
\hline & 1 & 0.0 & & 0.0 & & 0. & & 0. & 0. & \\
\hline & 1 & 0.0 & 0.0 & 0.0 & & 0.3 & & & & -0.25 \\
\hline & & 0.00 & 0.00 & 0.00 & -0.31 & 0.37 & & 0.28 & -0.38 & -0.50 \\
\hline
\end{tabular}

\begin{tabular}{|c|c|c|c|c|c|c|c|c|c|c|}
\hline $\begin{array}{l}\text { Pt TFE } \\
\text { Freque }\end{array}$ & & 18.13 & & & 21.43 & & & 30.52 & & \\
\hline Atom & AN & $X$ & Y & Z & $x$ & $Y$ & Z & $x$ & $Y$ & Z \\
\hline 1 & 6 & 0.04 & 0.15 & 0.08 & 0.05 & -0.05 & 0.12 & -0.02 & -0.08 & -0.04 \\
\hline 2 & 6 & 0.05 & 0.09 & 0.02 & 0.06 & -0.07 & 0.11 & -0.03 & -0.03 & -0.02 \\
\hline 3 & 6 & 0.00 & 0.02 & 0.03 & 0.00 & 0.01 & 0.00 & 0.0 & -0.01 & 0.02 \\
\hline 4 & 6 & -0.06 & 0.00 & 0.10 & -0.06 & 0.10 & -0.10 & 0. & -0.05 & 0.03 \\
\hline 5 & 6 & -0.07 & 0.06 & 0.16 & -0.06 & 0.11 & -0.08 & 0.1 & -0.10 & 0.00 \\
\hline 6 & 6 & -0.02 & 0.14 & 0.14 & 0.00 & 0.04 & 0.0 & 0.0 & -0.12 & -0.03 \\
\hline 7 & 7 & 0.0 & -0.04 & -0.02 & 0.0 & -0.01 & -0.0 & 0. & 0.1 & 0. \\
\hline 8 & 6 & 0.01 & -0.07 & -0.06 & 0.00 & -0.01 & -0.02 & 0.0 & 0.03 & 0.05 \\
\hline 9 & 6 & 0.01 & -0.0 & -0.0 & 0. & -0.02 & -0 . & & & \\
\hline 10 & 6 & 0.0 & -0.08 & -0.07 & 0.0 & -0.04 & -0.05 & 0.0 & 0.05 & 0.07 \\
\hline 11 & 17 & -0.13 & -0.10 & 0.12 & -0.13 & 0.20 & -0.24 & 0.15 & -0.03 & 0.07 \\
\hline 12 & 17 & 0.13 & 0.1 & -0.0 & 0.13 & -0.18 & 0.2 & -0 . & 0.02 & -0.03 \\
\hline 13 & 78 & 0.0 & -0.0 & -0.0 & $0 .($ & -0.02 & $-0 .($ & 0. & 0. & 0.02 \\
\hline 14 & 8 & 0.0 & -0.05 & -0.0 & 0.0 & 0.01 & -0.02 & 0. & -0.08 & -0.07 \\
\hline 15 & 7 & 0.01 & -0.03 & -0.02 & 0.00 & 0.00 & -0.01 & 0.02 & 0.01 & 0.01 \\
\hline
\end{tabular}




\begin{tabular}{|c|c|c|c|c|c|c|c|c|c|c|}
\hline & & & & & & & & & & \\
\hline 16 & 6 & 0.00 & 0.02 & 0.03 & 0.00 & 0.03 & 0.01 & 0.02 & 0.03 & 0.01 \\
\hline 17 & 6 & 0.06 & 0.06 & 0.07 & 0.02 & -0.04 & 0.07 & 0.03 & -0.01 & 0.04 \\
\hline 18 & 6 & 0.05 & 0.13 & 0.13 & 0.02 & 0.01 & 0.09 & 0.03 & 0.04 & 0.03 \\
\hline 19 & 6 & -0.02 & 0.16 & 0.14 & -0.01 & 0.13 & 0.04 & 0.02 & 0.12 & -0.01 \\
\hline 20 & 6 & -0.09 & 0.12 & 0.11 & -0.04 & 0.19 & -0.02 & 0.02 & 0.16 & -0.04 \\
\hline 21 & 6 & -0.08 & 0.05 & 0.05 & -0.03 & 0.14 & -0.04 & 0.02 & 0.11 & -0.03 \\
\hline 22 & 17 & 0.16 & 0.02 & 0.05 & 0.05 & -0.17 & 0.13 & 0.03 & -0.11 & 0.10 \\
\hline 23 & 17 & -0.16 & 0.00 & 0.01 & -0.07 & 0.21 & -0.12 & 0.01 & 0.15 & -0.07 \\
\hline 24 & 6 & 0.01 & -0.03 & -0.02 & 0.01 & -0.06 & -0.07 & 0.03 & 0.08 & 0.09 \\
\hline 25 & 6 & 0.01 & -0.09 & -0.08 & 0.00 & 0.01 & 0.00 & 0.01 & 0.02 & \\
\hline 26 & 1 & 0.02 & -0.12 & -0.10 & -0.02 & 0.07 & 0.07 & 0.01 & 0.02 & 0.05 \\
\hline 27 & 1 & -0.02 & -0.09 & -0.08 & 0.08 & 0.03 & -0.04 & 0.00 & 0.03 & 0.04 \\
\hline 28 & 1 & 0.03 & -0.09 & -0.07 & -0.05 & -0.06 & -0.02 & 0.01 & 0.02 & 0.05 \\
\hline 29 & 1 & 0.00 & -0.08 & -0.10 & 0.05 & -0.02 & -0.03 & 0.02 & 0.06 & \\
\hline 30 & 1 & 0.02 & -0.10 & -0.07 & -0.05 & -0.03 & -0.05 & -0.02 & 0.09 & 0.06 \\
\hline 31 & 1 & 0.00 & -0.06 & -0.06 & 0.02 & -0.07 & -0.10 & 0.01 & 0.04 & 0.05 \\
\hline 32 & 1 & 0.10 & 0.15 & 0.16 & 0.04 & -0.03 & 0.14 & 0.03 & 0.01 & 0.05 \\
\hline 33 & 1 & -0.03 & 0.21 & 0.19 & -0.01 & 0.16 & 0.06 & 0.03 & 0.16 & -0.02 \\
\hline 34 & 1 & -0.15 & 0.14 & 0.12 & -0.06 & 0.27 & -0.05 & 0.01 & 0.22 & -0.07 \\
\hline 35 & 1 & -0.12 & 0.05 & 0.21 & -0.10 & 0.18 & -0.16 & 0.15 & -0.13 & 0.01 \\
\hline 36 & 1 & -0.03 & 0.18 & 0.19 & 0.00 & 0.04 & 0.04 & 0.06 & -0.16 & -0.05 \\
\hline 37 & 1 & 0.08 & 0.21 & 0.07 & 0.10 & -0.11 & 0.21 & -0.06 & -0.09 & -0.07 \\
\hline 38 & 1 & 0.00 & -0.03 & -0.03 & 0.05 & -0.08 & 0.01 & 0.0 & 0.12 & 0.07 \\
\hline 39 & 1 & 0.02 & -0.05 & -0.01 & -0.03 & 0.00 & -0.11 & 0.00 & 0.05 & 0.14 \\
\hline 40 & 1 & 0.01 & -0.02 & -0.01 & 0.03 & -0.14 & -0.14 & 0.03 & 0.12 & 0.12 \\
\hline 41 & 6 & 0.01 & -0.04 & -0.07 & 0.01 & 0.00 & -0.03 & -0.05 & -0.13 & -0.15 \\
\hline 42 & 1 & 0.01 & -0.07 & -0.05 & 0.03 & 0.01 & -0.01 & 0.07 & -0.06 & -0.05 \\
\hline 43 & 6 & 0.01 & -0.01 & -0.10 & 0.00 & -0.05 & 0.06 & -0.17 & -0.08 & -0.12 \\
\hline 44 & 1 & 0.01 & -0.06 & -0.08 & 0.06 & 0.07 & -0.04 & -0.02 & -0.18 & -0.20 \\
\hline 45 & 1 & 0.01 & -0.02 & -0.05 & -0.05 & -0.01 & -0.09 & -0.05 & -0.15 & -0.18 \\
\hline 46 & 9 & 0.00 & -0.03 & -0.12 & 0.09 & -0.06 & 0.16 & -0.19 & -0.01 & -0.08 \\
\hline 47 & 9 & 0.00 & 0.00 & -0.12 & 0.00 & -0.03 & 0.03 & -0.26 & -0.16 & -0.17 \\
\hline 48 & 9 & 0.01 & 0.02 & -0.09 & -0.10 & -0.13 & 0.09 & -0.18 & -0.03 & -0.09 \\
\hline Frequer & & 35.93 & & & 36.91 & & & 47.24 & & \\
\hline Atom & AN & $x$ & $Y$ & Z & $x$ & $\mathrm{Y}$ & Z & $x$ & $\mathrm{Y}$ & Z \\
\hline 1 & 6 & 0.03 & -0.09 & 0.06 & 0.05 & -0.05 & 0.05 & 0.06 & -0.11 & 0.13 \\
\hline 2 & 6 & 0.02 & -0.07 & 0.05 & 0.05 & -0.05 & 0.02 & 0.02 & -0.03 & 0.04 \\
\hline 3 & 6 & 0.01 & -0.03 & 0.00 & 0.02 & -0.03 & -0.01 & 0.01 & 0.00 & 0.00 \\
\hline 4 & 6 & 0.01 & -0.01 & -0.03 & 0.01 & -0.01 & -0.02 & 0.03 & -0.03 & 0.04 \\
\hline 5 & 6 & 0.01 & -0.03 & -0.02 & 0.01 & -0.02 & 0.01 & 0.07 & -0.12 & 0.13 \\
\hline 6 & 6 & 0.02 & -0.07 & 0.03 & 0.04 & -0.04 & 0.04 & 0.08 & -0.16 & 0.18 \\
\hline 7 & 7 & 0.00 & -0.02 & 0.00 & & -0.02 & -0.03 & 0.0 & 0.03 & -0.03 \\
\hline 8 & 6 & 0.01 & -0.08 & -0.07 & 0.0 & -0.04 & -0.05 & -0.0 & 0.04 & -0.0 \\
\hline 9 & 6 & 0.02 & -0.09 & -0.08 & $0 . C$ & -0.02 & -0.05 & -0.01 & 0.05 & -0.04 \\
\hline 10 & 6 & 0.02 & -0.15 & -0.15 & -0 & -0.01 & -0.05 & -0.0 & 0.05 & -0.03 \\
\hline 11 & 17 & -0.01 & 0.05 & -0.08 & & 0.01 & -0.0 & & & \\
\hline 12 & 17 & 0.03 & -0.10 & 0.08 & 0.07 & -0.06 & 0.03 & 0.00 & 0.02 & -0.02 \\
\hline 13 & 78 & -0.01 & 0.04 & 0.06 & 0.03 & 0.01 & -0.01 & 0.01 & 0.04 & -0.05 \\
\hline 14 & 8 & -0.02 & 0.02 & 0.06 & & 0.04 & 0.00 & & & \\
\hline 15 & 7 & 0.01 & -0.04 & -0.03 & 0. & 0.00 & -0.04 & -0.0 & 0.04 & \\
\hline 16 & 6 & 0.01 & -0.04 & -0.02 & 0.02 & 0.04 & -0.03 & -0.02 & 0.00 & 0.00 \\
\hline 17 & 6 & 0.02 & -0.04 & 0.00 & & 0.16 & -0.08 & & -0.04 & \\
\hline 18 & 6 & 0.02 & -0.06 & 0.02 & 0. & 0.23 & -0.1 & -0.0 & -0.16 & \\
\hline 19 & 6 & 0.00 & -0.06 & 0.03 & 0. & 0.18 & -0.06 & -0.05 & -0.23 & 0.18 \\
\hline 20 & 6 & -0.01 & -0.05 & 0.02 & & 0.05 & 0.0 & -0.0 & -0.17 & 0.13 \\
\hline 21 & 6 & -0.01 & -0.04 & -0.01 & -0 . & -0.02 & 0.01 & -0.0 & -0.05 & \\
\hline 22 & 17 & 0.05 & -0.04 & -0.02 & 0. & 0.22 & -0.13 & 0.02 & 0.02 & 0.0 \\
\hline 23 & 17 & -0.02 & -0.03 & -0.03 & -0 & -0.17 & 0.08 & -0.06 & 0.01 & -0.01 \\
\hline 24 & 6 & -0.02 & 0.10 & 0.11 & & 0.06 & 0.03 & & 0.02 & \\
\hline 25 & 6 & 0.02 & -0.14 & -0.13 & 0.0 & -0.07 & -0.07 & -0.03 & 0.04 & \\
\hline
\end{tabular}




\begin{tabular}{|c|c|c|c|c|c|c|c|c|c|c|}
\hline & & & & & & & & & & \\
\hline 26 & 1 & 0.00 & -0.09 & -0.05 & 0.01 & -0.09 & -0.06 & -0.03 & 0.05 & 0.01 \\
\hline 27 & 1 & 0.08 & -0.10 & -0.25 & -0.01 & -0.07 & -0.10 & -0.02 & 0.04 & -0.02 \\
\hline 28 & 1 & -0.01 & -0.28 & -0.13 & 0.01 & -0.09 & -0.07 & -0.05 & 0.03 & -0.02 \\
\hline 29 & 1 & 0.05 & -0.13 & -0.19 & -0.05 & -0.03 & -0.08 & 0.00 & 0.05 & -0.02 \\
\hline 30 & 1 & 0.00 & -0.21 & -0.14 & 0.03 & -0.03 & -0.05 & -0.02 & 0.06 & -0.03 \\
\hline 31 & 1 & 0.03 & -0.16 & -0.18 & -0.02 & 0.04 & -0.03 & 0.00 & 0.03 & -0.04 \\
\hline 32 & 1 & 0.03 & -0.06 & 0.04 & 0.07 & 0.32 & -0.14 & -0.01 & -0.20 & 0.16 \\
\hline 33 & 1 & 0.00 & -0.08 & 0.05 & 0.02 & 0.23 & -0.07 & -0.06 & -0.32 & 0.25 \\
\hline 34 & 1 & -0.02 & -0.05 & 0.02 & -0.03 & 0.01 & 0.03 & -0.08 & -0.22 & 0.16 \\
\hline 35 & 1 & 0.01 & -0.01 & -0.04 & 0.00 & -0.01 & 0.00 & 0.09 & -0.15 & 0.17 \\
\hline 36 & 1 & 0.03 & -0.09 & 0.04 & 0.04 & -0.05 & 0.07 & 0.11 & -0.22 & 0.25 \\
\hline 37 & 1 & 0.03 & -0.13 & 0.09 & 0.07 & -0.07 & 0.08 & 0.07 & -0.14 & 0.16 \\
\hline 38 & 1 & -0.03 & 0.13 & 0.06 & 0.07 & 0.09 & 0.07 & 0.05 & 0.02 & 0.00 \\
\hline 39 & 1 & 0.00 & 0.06 & 0.15 & -0.04 & 0.09 & 0.05 & 0.01 & 0.03 & -0.02 \\
\hline 40 & 1 & -0.03 & 0.16 & 0.16 & 0.03 & 0.02 & 0.00 & 0.05 & 0.01 & -0.05 \\
\hline 41 & 6 & -0.03 & 0.01 & 0.06 & -0.04 & -0.07 & 0.01 & 0.01 & 0.04 & -0.07 \\
\hline 42 & 1 & -0.04 & 0.02 & 0.04 & 0.05 & 0.13 & 0.06 & 0.05 & 0.08 & -0.04 \\
\hline 43 & 6 & -0.03 & 0.11 & -0.08 & -0.16 & -0.09 & 0.12 & -0.02 & 0.01 & 0.00 \\
\hline 44 & 1 & -0.11 & -0.09 & 0.07 & -0.04 & -0.07 & 0.02 & 0.05 & 0.07 & -0.08 \\
\hline 45 & 1 & 0.06 & 0.03 & 0.15 & -0.02 & -0.16 & -0.05 & -0.02 & 0.02 & -0.12 \\
\hline 46 & 9 & -0.17 & 0.11 & -0.22 & -0.22 & 0.07 & 0.19 & 0.03 & 0.03 & 0.07 \\
\hline 47 & 9 & -0.04 & 0.07 & -0.03 & -0.24 & -0.23 & 0.19 & -0.04 & 0.00 & -0.02 \\
\hline 48 & 9 & 0.11 & 0.22 & -0.12 & -0.11 & -0.12 & 0.08 & -0.08 & -0.04 & 0.02 \\
\hline requer & & 50.01 & & & 64.40 & & & 70.65 & & \\
\hline Atom & AN & X & $Y$ & Z & $X$ & $Y$ & Z & $X$ & $Y$ & Z \\
\hline 1 & 6 & -0.03 & 0.02 & -0.06 & 0.08 & -0.11 & 0.13 & 0.06 & 0.01 & -0.02 \\
\hline 2 & 6 & -0.02 & 0.00 & -0.02 & 0.03 & 0.00 & 0.00 & 0.07 & -0.05 & -0.06 \\
\hline 3 & 6 & 0.00 & 0.00 & 0.00 & 0.01 & 0.02 & -0.03 & 0.02 & -0.08 & -0.07 \\
\hline 4 & 6 & 0.00 & 0.01 & -0.03 & 0.03 & -0.01 & 0.01 & -0.03 & -0.07 & -0.04 \\
\hline 5 & 6 & -0.01 & 0.03 & -0.07 & 0.08 & -0.11 & 0.14 & -0.0 & -0.01 & -0.01 \\
\hline 6 & 6 & -0.03 & 0.03 & -0.09 & 0.11 & -0.17 & 0.21 & 0.0 & 0.03 & 0.00 \\
\hline 7 & 7 & 0.00 & 0.00 & 0.02 & 0.01 & 0.03 & -0.04 & 0.02 & -0.08 & -0.07 \\
\hline 8 & 6 & 0.01 & -0.06 & -0.04 & 0.04 & 0.02 & -0.03 & 0.02 & -0.08 & -0.06 \\
\hline 9 & 6 & 0.01 & -0.06 & -0.05 & 0.05 & -0.01 & 0.01 & 0. & 0.03 & 0.04 \\
\hline 10 & 6 & 0.02 & -0.11 & -0.11 & 0.09 & -0.03 & 0.01 & -0.02 & 0.06 & 0.06 \\
\hline 11 & 17 & 0.02 & 0.02 & -0.02 & -0.01 & 0.08 & -0.09 & -0.10 & -0.14 & -0.05 \\
\hline 12 & 17 & -0.04 & 0.00 & 0.00 & 0.00 & 0.09 & -0.11 & 0.16 & -0.07 & -0.08 \\
\hline 13 & 78 & 0.0 & 0.04 & 0.06 & -0.04 & 0.00 & 0.00 & 0.0 & 0.01 & 0.01 \\
\hline 14 & 8 & 0.03 & -0.02 & -0.03 & -0.11 & -0.05 & 0.05 & 0.0 & 0.04 & 0.02 \\
\hline 15 & 7 & 0.01 & -0.02 & 0.00 & 0.02 & -0.01 & 0.04 & -0.01 & 0.09 & 0.10 \\
\hline 16 & 6 & 0.01 & -0.01 & 0.00 & 0.02 & -0.01 & 0.0 & -0.01 & & 0.09 \\
\hline 17 & 6 & 0.0 & 0.02 & -0.01 & 0.0 & 0.01 & 0.00 & -0.07 & 0.04 & 0.06 \\
\hline 18 & 6 & 0.01 & 0.03 & -0.02 & 0.02 & 0.10 & -0.07 & -0.0 & -0.05 & 0.01 \\
\hline 19 & 6 & 0.01 & 0.01 & 0.00 & & 0.15 & -0.11 & 0.0 & & 0.00 \\
\hline 20 & 6 & 0.00 & -0.03 & 0.02 & 0.0 & 0.10 & -0.07 & 0.0 & -0.03 & 0.02 \\
\hline 21 & 6 & 0.00 & -0.04 & 0.02 & & 0.01 & 0.0 & 0. & 0. & 0.06 \\
\hline 22 & 17 & 0.02 & 0.01 & -0.02 & & -0.07 & $0 . C$ & -0. & & 0.08 \\
\hline 23 & 17 & 0.0 & -0.10 & 0.05 & 0. & -0.06 & 0.0 & 0. & 0. & 0.09 \\
\hline 24 & 6 & 0.00 & 0.07 & 0.10 & -0.08 & -0.02 & -0.07 & 0.0 & -0.11 & -0.10 \\
\hline 25 & 6 & 0.0 & -0.13 & -0.11 & & 0.03 & -0.05 & 0. & -0.17 & -0.15 \\
\hline 26 & 1 & 0.0 & -0.10 & -0.07 & & 0.08 & -0.0 & & -0.27 & -0.25 \\
\hline 27 & 1 & 0.05 & -0.10 & -0.22 & 0.13 & 0.05 & -0.10 & -0.11 & -0.17 & -0.16 \\
\hline 28 & 1 & 0.03 & -0.24 & -0.11 & 0.06 & -0.04 & -0.05 & 0.13 & -0.13 & -0.11 \\
\hline 29 & 1 & 0.02 & -0.11 & -0.16 & & -0.02 & -0.01 & -0.11 & 0.02 & 0.00 \\
\hline 30 & 1 & 0.0 & -0.17 & -0.09 & & -0.04 & 0.0 & 0.1 & & \\
\hline 31 & 1 & 0.02 & -0.10 & -0.12 & 0.08 & -0.04 & 0.03 & -0.05 & 0.15 & 0.16 \\
\hline 32 & 1 & 0.02 & 0.05 & -0.03 & & 0.12 & -0.09 & -0.11 & -0.09 & -0.01 \\
\hline 33 & 1 & 0.0 & 0.02 & 0.00 & 0.0 & 0.22 & -0.17 & 0.02 & -0.1 & -0.02 \\
\hline 34 & 1 & 0.00 & -0.05 & 0.03 & 0.07 & 0.12 & -0.09 & 0.13 & -0.05 & 0.0 \\
\hline 35 & 1 & 0.00 & 0.04 & -0.09 & 0.09 & -0.15 & 0.18 & -0.09 & -0.01 & 0.01 \\
\hline
\end{tabular}




\begin{tabular}{|c|c|c|c|c|c|c|c|c|c|c|}
\hline & & & & & & & & & & \\
\hline 36 & 1 & -0.03 & 0.04 & -0.12 & 0.15 & -0.26 & 0.32 & -0.01 & 0.07 & 0.02 \\
\hline 3 & 1 & -0.05 & 0.02 & -0.08 & 0.10 & -0.13 & 0.17 & 0.10 & 0.04 & -0.01 \\
\hline 38 & 1 & 0.03 & 0.09 & 0.12 & -0.10 & -0.03 & -0.09 & 0.02 & -0.16 & -0.04 \\
\hline 39 & 1 & -0.03 & 0.07 & 0.12 & -0.05 & -0.01 & -0.09 & 0.03 & -0.05 & -0.17 \\
\hline 40 & 1 & 0.02 & 0.07 & 0.09 & -0.11 & -0.03 & -0.05 & 0.03 & -0.19 & -0.16 \\
\hline 41 & 6 & 0.01 & 0.07 & -0.17 & -0.07 & -0.01 & 0.06 & & 0.02 & \\
\hline 42 & 1 & 0.08 & -0.11 & -0.06 & -0.14 & -0.08 & 0.0 & 0. & 0.05 & \\
\hline 43 & 6 & 0.00 & 0.00 & -0.06 & -0.01 & 0.00 & 0.01 & -0.08 & 0.04 & -0.03 \\
\hline 44 & 1 & 0.17 & 0.14 & -0.26 & -0.08 & -0.01 & 0.07 & 0.00 & 0.00 & -0.07 \\
\hline 45 & 1 & -0.15 & 0.12 & -0.2 & -0.06 & 0.02 & 0.1 & -0 . & 0.02 & -0.05 \\
\hline 46 & 9 & 0.24 & & 0.12 & & -0.08 & -0.03 & & 0.04 & 0.00 \\
\hline 47 & 9 & 0.01 & 0.12 & -0.25 & 0.04 & 0.07 & -0.02 & -0.10 & 0.04 & -0.07 \\
\hline 48 & 9 & -0.27 & -0.09 & 0.08 & -0.03 & 0.01 & 0.02 & -0.11 & 0.06 & 0.00 \\
\hline requer & & 82.47 & & & 87.23 & & & 91.11 & & \\
\hline Atom & AN & $X$ & Y & Z & $X$ & $Y$ & Z & $x$ & $Y$ & Z \\
\hline 1 & 6 & 0.00 & -0.02 & 0.03 & 0.05 & -0.01 & 0.00 & 0.03 & -0.01 & 0.00 \\
\hline 2 & 6 & -0.01 & 0.01 & 0.01 & 0.05 & -0.01 & 0.02 & 0.03 & -0.02 & -0.03 \\
\hline 3 & 6 & 0.00 & 0.00 & 0.02 & 0.03 & 0.04 & -0.04 & 0.01 & -0.04 & -0.04 \\
\hline 4 & 6 & 0.00 & 0.01 & 0.01 & 0.07 & -0.01 & 0.02 & -0.01 & -0.03 & -0.03 \\
\hline 5 & 6 & 0.01 & -0.02 & 0.03 & 0.07 & -0.01 & 0.00 & -0.02 & -0.02 & 0.00 \\
\hline 6 & 6 & 0.02 & -0.04 & 0.06 & 0.05 & 0.02 & -0.03 & 0.01 & 0.00 & 0.01 \\
\hline 7 & 7 & 0.01 & -0.01 & 0.04 & 0.02 & 0.06 & -0.06 & 0.01 & -0.03 & -0.03 \\
\hline 8 & 6 & 0.02 & -0.04 & 0.01 & 0.04 & 0.07 & -0.04 & 0. & 0.01 & 0.02 \\
\hline 9 & 6 & 0.02 & -0.04 & 0.02 & 0.05 & 0.02 & -0.04 & 0.0 & 0.03 & 0.06 \\
\hline 10 & 6 & 0.00 & -0.01 & 0.05 & 0.09 & -0.02 & -0.06 & -0.01 & 0.17 & 0.22 \\
\hline 11 & 17 & -0.03 & 0.08 & -0.07 & 0.16 & -0.17 & 0.2 & -0. & -0.06 & -0.05 \\
\hline 12 & 17 & -0.0 & 0.08 & -0.07 & 0.14 & -0.17 & 0.20 & 0.0 & -0.04 & -0.04 \\
\hline 13 & 78 & -0.0 & -0.04 & 0.06 & -0.06 & 0.03 & -0.05 & -0.1 & -0.01 & 0.01 \\
\hline 14 & 8 & -0.11 & -0.17 & 0.09 & -0.19 & -0.03 & 0.08 & -0. & 0.02 & 0.03 \\
\hline 15 & 7 & 0.0 & -0.05 & 0.01 & 0.02 & 0.01 & -0.02 & 0. & -0.06 & -0.04 \\
\hline 16 & 6 & 0.04 & -0.01 & -0.02 & 0.02 & 0.00 & -0.01 & 0.0 & -0.05 & -0.03 \\
\hline 17 & 6 & 0.0 & & -0.04 & 0.0 & 0.01 & -0.0 & 0. & -0.03 & -0.02 \\
\hline 18 & 6 & 0.0 & -0.06 & 0.03 & 0.03 & 0.02 & -0.0 & 0. & 0.00 & -0.01 \\
\hline 19 & 6 & 0.02 & & 0. & 0.03 & 0.02 & -0.0 & 0.1 & 0.01 & -0.01 \\
\hline 20 & 6 & 0.02 & -0 . & 0.0 & 0.02 & 0.02 & -0.01 & -0. & 0.00 & -0.02 \\
\hline 21 & 6 & 0.0 & 0.0 & -0.04 & 0.02 & 0.00 & -0.02 & 0.0 & -0.03 & -0.03 \\
\hline 22 & 17 & 0.1 & & -0.20 & 0.06 & 0.04 & -0. & 0. & -0.04 & -0.03 \\
\hline 23 & 17 & & & & & & -0 . & & & -0.0 \\
\hline 24 & 6 & -0.0 & 0.0 & 0.0 & -0.13 & 0.00 & -0 . & -0 . & 0.15 & 0.15 \\
\hline 25 & 6 & 0.04 & & -0 & 0. & & -0. & 0. & 06 & 0.06 \\
\hline 26 & 1 & 0.0 & & & & & & & & -0.08 \\
\hline 27 & 1 & 0.0 & & -0 . & 0.1 & 0.1 & 0.0 & -0 . & 0.00 & 0.22 \\
\hline 28 & 1 & 0. & & & 0.02 & & -0.02 & 0. & 0.2 & 0.08 \\
\hline 29 & 1 & & & & & & -0 . & & & 0.3 \\
\hline 30 & 1 & -0. & & 0. & 0.0 & -0. & & & & \\
\hline 31 & 1 & 0.0 & & & 0.10 & -0.06 & -0. & -0 . & 0.18 & 0.28 \\
\hline 32 & 1 & 0. & & & 0.02 & & -0.01 & 0. & & 0.00 \\
\hline 33 & 1 & & & & & & & & & 0.00 \\
\hline 34 & 1 & 0.0 & & 0. & 0.0 & & -0.1 & -0 . & 0.01 & -0.02 \\
\hline 35 & 1 & 0.01 & & & 0.08 & -0.04 & 0.04 & -0 . & -0.01 & 0.01 \\
\hline 36 & 1 & 0.03 & & & & & -0.0 & & & 0.03 \\
\hline 37 & 1 & & & 0. & & -0. & & & & \\
\hline 38 & 1 & -0.0 & & -0. & -0.15 & -0.02 & -0.15 & 0.00 & 0.23 & 0.09 \\
\hline 39 & 1 & -0.0 & & 0.0 & -0.11 & & -0.21 & & 0.09 & 0.24 \\
\hline 40 & 1 & -0.1 & & 0.1 & -0.18 & -0.04 & -0 . & & 0.23 & 0.21 \\
\hline 41 & 6 & -0. & & 0.1 & -0.15 & -0.01 & 0.1 & -0.01 & 0.06 & -0.01 \\
\hline 42 & 1 & -0 . & & & -0 & -0.0 & 0. & -0. & -0.02 & 0.01 \\
\hline 43 & 6 & 0.0 & & 0.0 & -0.1 & & 0.0 & & 0.06 & 0.00 \\
\hline 44 & 1 & -0 . & -0 . & 0.14 & -0.19 & -0.03 & 0.15 & 0.02 & 0.06 & -0.03 \\
\hline 45 & 1 & -0.04 & -0.10 & 0.17 & -0.11 & 0.01 & 0.18 & -0.05 & 0.08 & -0.01 \\
\hline
\end{tabular}




\begin{tabular}{|c|c|c|c|c|c|c|c|c|c|c|}
\hline $\begin{array}{l}46 \\
47 \\
48\end{array}$ & $\begin{array}{l}9 \\
9 \\
9\end{array}$ & $\begin{array}{r}0.04 \\
0.06 \\
-0.01\end{array}$ & $\begin{array}{l}-0.24 \\
-0.02 \\
-0.07\end{array}$ & $\begin{array}{c}-0.04 \\
-0.02 \\
0.06\end{array}$ & $\begin{array}{c}-0.01 \\
0.00 \\
-0.13\end{array}$ & $\begin{array}{r}-0.15 \\
0.14 \\
0.04\end{array}$ & $\begin{array}{l}-0.02 \\
-0.05 \\
0.09\end{array}$ & $\begin{array}{c}0.02 \\
-0.01 \\
-0.05\end{array}$ & $\begin{array}{l}0.04 \\
0.08 \\
0.04\end{array}$ & $\begin{array}{c}0.02 \\
-0.03 \\
0.02\end{array}$ \\
\hline \multicolumn{2}{|c|}{ Frequencies } & 100.24 & & & 107.33 & & & 114.72 & & \\
\hline Atom & AN & X & $Y$ & Z & X & $\mathrm{Y}$ & Z & $x$ & $Y$ & Z \\
\hline 1 & 6 & 0.01 & 0.01 & 0.01 & 0.01 & 0.00 & 0.00 & 0.01 & 0.01 & -0.02 \\
\hline 2 & 6 & 0.01 & 0.01 & 0.01 & 0.01 & 0.00 & 0.00 & & -0.01 & 0.00 \\
\hline 3 & 6 & 0.00 & 0.02 & -0.01 & 0.01 & 0.00 & 0.00 & 0.02 & -0.02 & 0.01 \\
\hline 4 & 6 & 0.01 & 0.01 & 0.00 & 0.0 & & 0.00 & & -0.01 & 0.00 \\
\hline 5 & 6 & 0.01 & 0.01 & 0.00 & 0.0 & 0.00 & -0. & 0.01 & 0.01 & -0.02 \\
\hline 6 & 6 & 0.01 & 0.02 & 0.00 & 0.01 & 0.00 & 0.00 & 0.01 & 0.02 & -0.02 \\
\hline 7 & 7 & 0.00 & 0.03 & -0.01 & 0.01 & 0.00 & 0.0 & 0.01 & -0.01 & 0.01 \\
\hline 8 & 6 & 0.00 & & -0.05 & -0.01 & 0.00 & -0. & -0.02 & 0.01 & 0.01 \\
\hline 9 & 6 & -0.01 & 0.04 & 0.00 & -0.01 & 0.01 & -0.01 & -0.02 & 0.02 & -0.01 \\
\hline 10 & 6 & -0.02 & 0.13 & 0.10 & 0.00 & -0.02 & -0.04 & -0.05 & 0.04 & 00 \\
\hline 11 & 17 & & & 0.02 & & & $-0 .($ & & & -0.02 \\
\hline 12 & 17 & & & 0.04 & & & & & & -0.04 \\
\hline 13 & 78 & & & -0.01 & & & & & & \\
\hline 14 & 8 & & -0.08 & -0.05 & -0. & -0.02 & & & & \\
\hline 15 & 7 & & 0.02 & -0.01 & -0.0 & 0.01 & -0. & -0. & & -0.02 \\
\hline 16 & 6 & & & 0.01 & -0.0 & 0.01 & -0.01 & & 0.01 & -0.02 \\
\hline 17 & 6 & & & 0. & & & & & & \\
\hline 18 & 6 & & & 0.00 & -0.02 & & & & & \\
\hline 19 & 6 & -0. & & -0.01 & -0.0 & 0.00 & & & 0.00 & .00 \\
\hline 20 & 6 & & & 0.00 & -0 . & & & & & \\
\hline 21 & 6 & & & 0.0 & -0. & & & & & \\
\hline 22 & 17 & & & 0.03 & -0.03 & -0.01 & & -0. & -0.02 & 0.00 \\
\hline 23 & 17 & & & 0.03 & & & & & & \\
\hline 24 & 6 & & & 0.1 & & & & & -0.03 & \\
\hline 25 & 6 & 0. & & -0.18 & -0.04 & 0. & 0. & & & 0.03 \\
\hline 26 & 1 & & & -0.07 & & -0.28 & & & & \\
\hline 27 & 1 & & & -0.4 & -0 . & -0 & & & & \\
\hline 28 & 1 & & & & & & & & & \\
\hline 29 & 1 & & & & & -0 & & & & \\
\hline 30 & 1 & & & 0. & & -0.19 & & & & -0.02 \\
\hline 31 & 1 & & & & & & & & & \\
\hline 32 & 1 & & & & & & & & & \\
\hline 33 & 1 & & & & & & & & & \\
\hline 34 & 1 & & & & -0 . & & & & & \\
\hline 35 & 1 & & & & & & & & & \\
\hline 36 & 1 & & & & & & & & & \\
\hline 37 & 1 & & & & & & & & & \\
\hline 38 & 1 & & & & & & & & & \\
\hline 39 & 1 & & & & & & & & & \\
\hline 40 & 1 & & & & & & & & & \\
\hline 41 & 6 & & & & & & & & & \\
\hline 42 & 1 & & & & & & & & & \\
\hline 43 & 6 & & & -0. & & & & & & \\
\hline 44 & 1 & & & & & & & & & \\
\hline 45 & 1 & & & & & & & & & \\
\hline 46 & 9 & & & & & & & & & \\
\hline 47 & 9 & & & 0. & & -0 & & & & -0.06 \\
\hline 48 & 9 & & & & & 0.00 & & & 0.10 & 0.10 \\
\hline 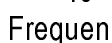 & & & & & & & & & & \\
\hline & AN & $x$ & $Y$ & Z & $x$ & $Y$ & & $x$ & Y & 2 \\
\hline 1 & 6 & & 0.03 & 0.02 & 0.08 & 0.02 & -0.02 & 0.00 & 0.00 & 0.00 \\
\hline 2 & 6 & & & 0.04 & & 0.01 & & & & \\
\hline 3 & 6 & & 0.04 & 0.04 & & -0.01 & & & -0.01 & \\
\hline 4 & 6 & & 0.05 & 0.03 & 0.08 & 0.00 & -0.03 & 0.01 & 0.00 & 0.00 \\
\hline 5 & 6 & 0. & 0.03 & 0.01 & 0.08 & 0.00 & -0.02 & 0.00 & 0.00 & 0.00 \\
\hline
\end{tabular}




\begin{tabular}{|c|c|c|c|c|c|c|c|c|c|c|}
\hline & & & & & & & & & & \\
\hline 6 & 6 & 0.04 & 0.02 & 0.01 & 0.09 & 0.02 & -0.02 & 0.01 & 0.00 & 0.00 \\
\hline 7 & 7 & 0.01 & 0.01 & 0.02 & 0.03 & -0.01 & -0.01 & 0.00 & 0.00 & 0.01 \\
\hline 8 & 6 & 0.02 & -0.04 & -0.03 & 0.01 & 0.03 & 0.02 & -0.01 & -0.01 & 0.00 \\
\hline 9 & 6 & 0.00 & -0.01 & 0.02 & 0.01 & -0.02 & -0.01 & -0.01 & 0.01 & 0.00 \\
\hline 10 & 6 & 0.01 & 0.09 & 0.14 & 0.05 & -0.10 & -0.07 & -0.06 & 0.05 & 0.02 \\
\hline 11 & 17 & 0.13 & 0.12 & & & 0.03 & -0.02 & & 0.01 & \\
\hline 12 & 17 & 0.00 & 0.05 & 0.04 & 0.18 & 00 & -0.08 & 2 & 0.00 & \\
\hline 13 & 78 & 0.00 & -0.05 & -0.01 & -0.02 & -0.02 & 0.04 & -0.01 & 0.02 & 0.03 \\
\hline 14 & 8 & 0.01 & 0.03 & 0.03 & -0.02 & -0.05 & 0.00 & -0.02 & -0.01 & 0.00 \\
\hline 15 & 7 & -0.01 & -0.04 & 0.00 & -0.03 & & 0.02 & & 0.02 & \\
\hline 16 & 6 & -0.02 & -0.03 & 0.00 & -0.05 & -0.03 & 0.02 & & 0.01 & \\
\hline 17 & 6 & -0.02 & -0.02 & -0.01 & -0.07 & 0.00 & -0.01 & 0.01 & 0.00 & -0.02 \\
\hline 18 & 6 & -0.02 & 0.00 & -0.02 & -0.07 & 0.01 & -0.02 & 0.01 & -0.01 & \\
\hline 19 & 6 & -0.03 & 0.00 & -0.02 & -0.07 & & -0.01 & & -0.01 & \\
\hline 20 & 6 & -0.03 & 0.00 & -0.01 & -0.06 & 0.00 & 0.00 & 0.01 & 0.00 & -0.01 \\
\hline 21 & 6 & -0.03 & -0.02 & -0.01 & -0.06 & -0.01 & 0.01 & 0.01 & 0.00 & \\
\hline 22 & 17 & -0.04 & 0.00 & -0.02 & -0.13 & 0.05 & -0.01 & & -0.03 & \\
\hline 23 & 17 & -0.06 & -0.01 & -0.05 & -0.11 & & -0.04 & & -0.02 & -0.02 \\
\hline 24 & 6 & 0.00 & -0.09 & -0.05 & -0.03 & -0.02 & 0.0 & -0.01 & -0.11 & .11 \\
\hline 25 & 6 & 0.04 & -0.20 & -0 . & 0.00 & & 0.1 & -0.03 & -0.05 & \\
\hline 26 & 1 & 0.09 & -0.33 & -0. & -0.06 & & 0.2 & & -0.25 & \\
\hline 27 & 1 & -0.15 & -0.19 & -0.24 & 0.22 & 0.15 & 0.0 & -0.28 & -0.11 & \\
\hline 28 & 1 & 0.1 & -0.20 & -0 . & -0.1 & & & & & \\
\hline 29 & 1 & -0.15 & 0.00 & 0.05 & & & & 0.14 & & \\
\hline 30 & 1 & 0.25 & 0.09 & 0.11 & -0.14 & -0.08 & -0.06 & -0.40 & 0.27 & -0.01 \\
\hline 31 & 1 & -0.0 & 0.23 & 0.3 & 0. & 26 & -0.2 & & -0.17 & \\
\hline 32 & 1 & -0.02 & 0.01 & -0.02 & -0.06 & 0.03 & -0.0 & & -0.02 & \\
\hline 33 & 1 & -0.03 & 0.01 & -0.02 & -0.07 & 0.01 & -0.02 & 0.01 & -0.01 & 0 \\
\hline 34 & 1 & -0.03 & 0.01 & -0. & -0.05 & 0.01 & -0.0 & 0.0 & -0.01 & -0.01 \\
\hline 35 & 1 & 0.0 & 0.03 & 0. & 0.0 & & -0.0 & & & \\
\hline 36 & 1 & 0.0 & 0.02 & 0.0 & & 0.02 & -0.0 & 0.00 & 0.00 & \\
\hline 37 & 1 & 0.00 & 0.02 & 0.1 & & 0.02 & -0.0 & 0.00 & 0.00 & \\
\hline 38 & 1 & -0.0 & -0.13 & -0. & -0.11 & -0.04 & -0.0 & 0.0 & -0.17 & \\
\hline 39 & 1 & 0.0 & -0. & -0 & 0. & & & -0.0 & & \\
\hline 40 & 1 & 0.0 & -0.12 & -0 . & -0 . & & 0. & & & \\
\hline 41 & 6 & 0.02 & 0.05 & 0.0 & 0.01 & 0.07 & -0.12 & -0.02 & 0.01 & \\
\hline 42 & 1 & 0.0 & 0.03 & 0. & 0. & -0.17 & -0.08 & -0.01 & -0.02 & \\
\hline 43 & 6 & -0. & 0.0 & & & & & -0.0 & -0.01 & \\
\hline 44 & 1 & 0.0 & 0.04 & 0. & 0. & & & & 0.02 & \\
\hline 45 & 1 & 0.0 & 0.0 & & -0.12 & & & -0.05 & 0.02 & \\
\hline 46 & 9 & -0. & 0. & & 0.01 & & & -0.0 & -0.01 & \\
\hline 47 & 9 & -0 . & & & 0. & & & & -0.01 & \\
\hline 48 & 9 & -0.04 & 0.10 & 0. & 0.02 & -0.08 & -0.09 & 0.01 & -0.05 & \\
\hline Frequer & & 152.36 & & & 156.85 & & & 168.70 & & \\
\hline Atom & AN & $x$ & $Y$ & Z & $\mathrm{Y}$ & Y & Z & & $Y$ & \\
\hline & 6 & & -0.01 & 0.00 & 0.00 & -0.01 & 0.01 & & -0.01 & \\
\hline 2 & 6 & & -0.01 & 0.00 & 0.00 & 0.00 & 0.00 & -0.01 & 0.01 & -0.0 \\
\hline 3 & 6 & & 0.00 & -0.01 & & 0.00 & & & 0.04 & \\
\hline 4 & 6 & & 0.00 & -0. & & 0.00 & & & 0.02 & \\
\hline 5 & 6 & & 0.00 & 0. & 0. & 0.00 & -0.01 & 0.0 & 0.00 & \\
\hline 6 & 6 & & 0.00 & & & & & & -0.02 & \\
\hline 7 & 7 & 0. & 0.01 & 0.0 & & & & & 0.03 & \\
\hline 8 & 6 & & 0.03 & 0.0 & & & 0.0 & 0.07 & 0.01 & \\
\hline 9 & 6 & & 0. & 0. & & -0 . & 0.0 & 0.0 & -0.03 & \\
\hline 10 & 6 & & 0.03 & & & & & & -0.05 & \\
\hline 11 & 17 & -0. & -0.03 & 0.1 & 0.0 & & & -0.07 & -0.06 & \\
\hline 12 & 17 & & 0.00 & -0.01 & 0.00 & 0.00 & 0.00 & -0.08 & -0.01 & 0.0 \\
\hline 13 & 78 & & 0.02 & & & & 0.00 & 0.01 & 0.01 & \\
\hline 14 & 8 & & 0.00 & 0.1 & & 0.03 & -0.0 & 0.05 & 0.00 & \\
\hline 15 & 7 & 0.00 & 0.00 & 0.01 & 0.00 & -0.01 & 0.00 & 0.02 & -0.05 & \\
\hline
\end{tabular}




\begin{tabular}{|c|c|c|c|c|c|c|c|c|c|c|}
\hline 16 & 6 & 0.00 & -0.01 & -0.01 & 0.00 & -0.01 & 0.01 & 0.01 & -0.07 & 0.04 \\
\hline 17 & 6 & 0.00 & -0.01 & -0.02 & 0.00 & 0.00 & 0.01 & -0.01 & -0.04 & 0.01 \\
\hline 18 & 6 & 0.00 & 0.00 & -0.01 & 0.00 & 0.00 & 0.00 & -0.01 & 0.00 & -0.02 \\
\hline 19 & 6 & 0.00 & 0.00 & -0.01 & 0.00 & 0.00 & 0.00 & -0.01 & 0.03 & -0.02 \\
\hline 20 & 6 & -0.01 & 0.00 & -0.01 & 0.00 & 0.00 & 0.00 & -0.01 & 0.01 & 0.00 \\
\hline 21 & 6 & 0.00 & -0.01 & -0.01 & 0.00 & 0.00 & 0.01 & -0.01 & -0.03 & 0.02 \\
\hline 22 & 17 & 0.02 & -0.02 & -0.02 & -0.01 & 0.01 & 0.00 & -0.10 & 0.05 & 0.01 \\
\hline 23 & 17 & -0.04 & -0.02 & -0.05 & 0.00 & 0.01 & 0.01 & -0.08 & -0.01 & -0.06 \\
\hline 24 & 6 & 0.01 & -0.08 & -0.08 & -0.01 & 0.05 & 0.05 & 0.01 & -0.02 & -0.03 \\
\hline 25 & 6 & 0.03 & 0.02 & -0.01 & 0.01 & 0.00 & 0.00 & 0.15 & 0.06 & -0.06 \\
\hline 26 & 1 & -0.04 & 0.20 & 0.19 & 0.00 & 0.01 & 0.00 & 0.19 & -0.06 & \\
\hline 27 & 1 & 0.25 & 0.09 & -0.21 & 0.02 & 0.00 & 0.00 & -0.03 & 0.00 & 0.13 \\
\hline 28 & 1 & -0.09 & -0.25 & -0.04 & 0.00 & 0.00 & 0.00 & 0.34 & 0.30 & 0.00 \\
\hline 29 & 1 & -0.22 & -0.07 & -0.27 & 0.02 & -0.01 & 0.01 & 0.42 & 0.05 & \\
\hline 30 & 1 & 0.44 & -0.15 & 0.07 & 0.00 & -0.01 & 0.00 & -0.04 & 0.11 & \\
\hline 31 & 1 & -0.08 & 0.30 & 0.36 & 0.01 & -0.03 & -0.01 & 0.26 & -0.32 & -0.09 \\
\hline 32 & 1 & 0.01 & 0.00 & -0.01 & 0.00 & 0.01 & 0.00 & 0.00 & 0.04 & -0.03 \\
\hline 33 & 1 & 0.00 & 0.00 & -0.01 & 0.00 & 0.01 & 0.00 & -0.01 & 0.07 & -0.04 \\
\hline 34 & 1 & -0.01 & 0.00 & -0.01 & 0.00 & 0.01 & 0.00 & 0.00 & 0.04 & -0. \\
\hline 35 & 1 & -0.01 & 0.00 & 0.00 & 0.00 & 0.01 & -0.01 & 0.01 & -0.02 & 0.03 \\
\hline 36 & 1 & 0.00 & 0.00 & 0.00 & 0.00 & 0.00 & 0.00 & 0.01 & -0.05 & 0.05 \\
\hline 37 & 1 & 0.00 & -0.01 & 0.01 & 0.01 & -0.01 & 0.01 & 0.01 & -0.03 & 0.02 \\
\hline 38 & 1 & 0.04 & -0.13 & 0.04 & 0.34 & 0.15 & 0.48 & 0.01 & -0.03 & -0.01 \\
\hline 39 & 1 & -0.02 & 0.02 & -0.16 & -0.44 & 0.41 & -0.02 & 0.01 & 0.01 & -0.06 \\
\hline 40 & 1 & 0.03 & -0.22 & -0.18 & 0.06 & -0.38 & -0.31 & 0.01 & -0.05 & \\
\hline 41 & 6 & 0.00 & 0.00 & -0.02 & -0. & 0.00 & -0.01 & 0.04 & -0.02 & 0.00 \\
\hline 42 & 1 & 0.02 & 0.01 & 0.01 & 0.05 & 0.08 & 0.04 & 0.05 & 0.02 & 0.00 \\
\hline 43 & 6 & 0.00 & -0.01 & -0.01 & -0.01 & 0.00 & 0.00 & 0.02 & -0.01 & \\
\hline 44 & 1 & 0.01 & 0.00 & -0.02 & -0.01 & 0.00 & 0.00 & 0.03 & -0.02 & 0.01 \\
\hline 45 & 1 & -0.01 & -0.01 & -0.03 & 0.00 & -0.01 & -0.02 & 0.06 & -0.03 & 0.00 \\
\hline 46 & 9 & 0.00 & -0.01 & -0.01 & -0.01 & -0.01 & -0.02 & 0.02 & 0.01 & 0.01 \\
\hline 47 & 9 & 0.01 & -0.01 & 0.01 & 0.02 & 0.01 & 0.01 & -0.02 & -0.03 & -0.03 \\
\hline 48 & 9 & 0.01 & -0.03 & -0.02 & 0.00 & -0.01 & -0.01 & 0.02 & 0.03 & \\
\hline Freque & & 182.29 & & & 190.48 & & & 207.75 & & \\
\hline Atom & AN & $x$ & $Y$ & Z & $x$ & $Y$ & Z & $X$ & $Y$ & Z \\
\hline & 6 & 0.01 & 0.01 & -0.01 & -0.01 & 0.01 & -0.01 & 0.09 & -0.20 & 0.24 \\
\hline 2 & 6 & 0.02 & 0.00 & 0.02 & -0.01 & 0.01 & -0.01 & 0.04 & -0.09 & 0.11 \\
\hline 3 & 6 & 0.04 & -0.01 & 0.03 & -0.02 & 0.00 & -0.01 & 0.00 & 0.00 & 0.00 \\
\hline 4 & 6 & 0.01 & 0.00 & 0.03 & -0.01 & 0.00 & 0.01 & -0.04 & 0.10 & -0.11 \\
\hline 5 & 6 & 0.01 & 0.01 & 0.01 & & -0.01 & 0.01 & -0.09 & 0.20 & -0.24 \\
\hline 6 & 6 & 0.00 & 0.02 & 0.00 & -0.01 & 0.00 & 0.00 & 0. & 0.00 & 0.00 \\
\hline 7 & 7 & 0.05 & -0.03 & 0.00 & -0.02 & -0.01 & -0.01 & 0.00 & 0.00 & 0.00 \\
\hline 8 & 6 & 0.03 & -0.02 & -0.01 & 0. & -0.01 & 0.00 & & 0.00 & -0.01 \\
\hline 9 & 6 & 0.02 & 0.00 & -0.02 & 0.01 & -0.03 & 0.0 & 0.0 & -0.01 & 0.00 \\
\hline 10 & 6 & -0.01 & 0.01 & -0.02 & 0.05 & -0.03 & 0.04 & 0.00 & 0.00 & 0.00 \\
\hline 11 & 17 & -0.05 & -0.02 & -0.01 & 0. & 0.03 & 0.01 & 0. & -0.05 & 0. \\
\hline 12 & 17 & -0.10 & 0.03 & 0.06 & & -0.01 & 0.00 & -0.0 & 0.06 & -0.0 \\
\hline 13 & 78 & 0.09 & -0.02 & -0.01 & -0.02 & 0.00 & 0.00 & 0.00 & 0.00 & 0.00 \\
\hline 14 & 8 & -0.23 & -0.04 & 0.16 & 0.11 & 0.24 & -0.06 & 0.00 & 0.01 & 0.00 \\
\hline 15 & 7 & 0.04 & 0.01 & -0.02 & & -0.04 & 0.02 & & & \\
\hline 16 & 6 & 0.03 & 0.05 & -0.02 & & -0.05 & 0.0 & & 0.00 & 0.00 \\
\hline 17 & 6 & 0.01 & 0.03 & -0.01 & 0.00 & -0.02 & 0.01 & 0.00 & 0.00 & 0.00 \\
\hline 18 & 6 & 0.01 & 0.00 & 0.00 & 0.00 & 0.01 & 0.00 & & & \\
\hline 19 & 6 & 0.00 & -0.02 & 0.0 & 0. & 0.02 & -0.0 & & 0.00 & \\
\hline 20 & 6 & 0.00 & -0.01 & 0.02 & 0.0 & 0.00 & 0.00 & 0.00 & -0.01 & 0.00 \\
\hline 21 & 6 & 0.01 & 0.02 & 0.00 & -0 & -0.03 & 0.02 & & & \\
\hline 22 & 17 & -0.08 & 0.01 & 0.04 & 0. & 0.01 & -0.0 & 0. & 0.0 & 0.0 \\
\hline 23 & 17 & -0.05 & -0.03 & -0.02 & 0.0 & 0.01 & 0.00 & 0.00 & 0.00 & 0.00 \\
\hline 24 & 6 & 0.00 & 0.06 & -0.04 & 0. & -0.07 & 0.08 & 0.0 & 0.00 & 0.01 \\
\hline 25 & 6 & 0.01 & -0.03 & 0.01 & 0.03 & 0.01 & -0.01 & 0.00 & 0.00 & 0.00 \\
\hline
\end{tabular}




\begin{tabular}{|c|c|c|c|c|c|c|c|c|c|c|}
\hline & & & & & & & & & & \\
\hline 26 & 1 & 0.01 & -0.03 & 0.04 & 0.03 & 0.01 & -0.05 & 0.01 & -0.02 & -0.02 \\
\hline 27 & 1 & 0.02 & -0.03 & -0.01 & 0.02 & 0.00 & 0.02 & -0.02 & -0.01 & 0.03 \\
\hline 28 & 1 & -0.02 & -0.05 & 0.00 & 0.06 & 0.04 & 0.00 & 0.01 & 0.03 & 0.00 \\
\hline 29 & 1 & -0.03 & 0.00 & -0.02 & 0.08 & -0.01 & 0.07 & 0.01 & 0.00 & 0.02 \\
\hline 30 & 1 & 0.00 & 0.01 & -0.02 & 0.04 & 0.01 & 0.03 & 0.00 & 0.01 & 0.00 \\
\hline 31 & 1 & -0.01 & 0.04 & -0.02 & 0.05 & -0.07 & 0.05 & 0.00 & -0.01 & 0.00 \\
\hline 32 & 1 & 0.01 & -0.02 & 0.01 & 0.00 & 0.03 & -0.02 & 0.00 & 0.01 & -0.01 \\
\hline 33 & 1 & 0.00 & -0.05 & 0.03 & 0.00 & 0.04 & -0.02 & 0.00 & 0.00 & 0.00 \\
\hline 34 & 1 & 0.01 & -0.04 & 0.03 & 0.00 & 0.02 & -0.01 & 0.00 & -0.01 & 0.01 \\
\hline 35 & 1 & 0.01 & 0.02 & 0.00 & 0.00 & -0.02 & 0.02 & -0.16 & 0.36 & -0.43 \\
\hline 36 & 1 & -0.01 & 0.03 & -0.02 & -0.01 & 0.00 & 0.00 & 0.00 & 0.00 & 0.00 \\
\hline 37 & 1 & 0.01 & 0.03 & -0.03 & -0.02 & 0.02 & -0.02 & 0.16 & -0.36 & 0.43 \\
\hline 38 & 1 & 0.02 & 0.13 & -0.10 & -0.07 & -0.16 & -0.09 & -0.05 & -0.01 & -0.07 \\
\hline 39 & 1 & -0.02 & 0.07 & -0.04 & 0.31 & -0.29 & 0.16 & 0.08 & -0.07 & 0.03 \\
\hline 40 & 1 & -0.11 & 0.05 & 0.03 & 0.18 & 0.18 & 0.22 & 0.00 & 0.08 & 0.07 \\
\hline 41 & 6 & -0.24 & 0.15 & -0.05 & -0.06 & 0.00 & -0.02 & -0.01 & 0.00 & 0.00 \\
\hline 42 & 1 & -0.36 & -0.29 & -0.08 & 0.26 & 0.51 & 0.22 & 0.00 & 0.02 & 0.00 \\
\hline 43 & 6 & -0.13 & 0.08 & -0.04 & -0.08 & -0.01 & 0.01 & -0.01 & 0.00 & 0.00 \\
\hline 44 & 1 & -0.08 & 0.16 & -0.17 & -0.10 & -0.02 & -0.01 & -0.01 & 0.00 & 0.00 \\
\hline 45 & 1 & -0.39 & 0.28 & -0.05 & 0.03 & -0.14 & -0.09 & 0.00 & 0.00 & 0.00 \\
\hline 46 & 9 & -0.10 & 0.03 & -0.04 & -0.06 & -0.12 & -0.09 & 0.00 & -0.01 & 0.00 \\
\hline 47 & 9 & 0.08 & 0.22 & 0.14 & 0.07 & 0.10 & 0.08 & 0.00 & 0.01 & 0.00 \\
\hline 48 & 9 & -0.12 & -0.20 & -0.16 & -0.05 & -0.09 & -0.05 & 0.00 & 0.00 & 0.00 \\
\hline equer & & 207.89 & & & 242.32 & & & 247.84 & & \\
\hline Atom & AN & X & $Y$ & Z & $x$ & $Y$ & Z & $x$ & $Y$ & Z \\
\hline 1 & 6 & 0.00 & 0.00 & -0.01 & 0.00 & 0.00 & 0.00 & -0.15 & -0.04 & 0.03 \\
\hline 2 & 6 & 0.00 & 0.00 & 0.00 & 0.00 & -0.01 & 0.00 & -0.13 & -0.08 & 0.03 \\
\hline 3 & 6 & 0.00 & 0.00 & 0.00 & 0.00 & -0.01 & 0.01 & -0.12 & -0.08 & 0.06 \\
\hline 4 & 6 & 0.00 & 0.00 & 0.00 & 0.00 & 0.00 & 0.00 & -0.14 & -0.04 & 0.06 \\
\hline 5 & 6 & 0.00 & 0.00 & 0.01 & 0.00 & 0.00 & 0.00 & -0.16 & -0.05 & 0.02 \\
\hline 6 & 6 & 0.00 & 0.00 & 0.00 & 0.00 & 0.00 & 0.00 & -0.17 & -0.04 & 0.01 \\
\hline 7 & 7 & 0.00 & 0.00 & 0.00 & 0.01 & -0.02 & 0.03 & -0.09 & -0.07 & 0.05 \\
\hline 8 & 6 & 0.00 & 0.00 & 0.00 & 0.03 & -0.02 & 0.05 & -0.04 & -0.09 & 0.06 \\
\hline 9 & 6 & 0.00 & 0.00 & 0.00 & 0 & 0.02 & 0.03 & -0.0 & -0.12 & 0.07 \\
\hline 10 & 6 & -0.01 & 0.00 & 0.00 & -0.14 & 0.03 & -0.08 & 0.06 & -0.13 & 0.14 \\
\hline 11 & 17 & 0.00 & 0.00 & 0.00 & 0.00 & 0.00 & 0.00 & 0.04 & 0.17 & 0.12 \\
\hline 12 & 17 & 0.00 & 0.00 & 0.00 & & 0.00 & -0.0 & 0.09 & -0.12 & -0.15 \\
\hline 13 & 78 & 0.00 & 0.00 & 0.00 & & -0.01 & 0.00 & 0.0 & & -0.03 \\
\hline 14 & 8 & 0.00 & 0.00 & 0.00 & 0.0 & 0.09 & -0.0 & -0.0 & -0.03 & 0.00 \\
\hline 15 & 7 & 0.00 & 0.00 & 0.00 & 0.12 & 0.07 & 0.00 & 0.01 & -0.12 & 0.06 \\
\hline 16 & 6 & 0.00 & 0.00 & 0.00 & & 0.12 & -0.04 & 0.02 & -0.13 & 0.08 \\
\hline 17 & 6 & 0.01 & 0.14 & -0.07 & & 0.09 & 0.0 & 0.02 & -0.09 & 0.06 \\
\hline 18 & 6 & 0.03 & 0.30 & -0.16 & 0.15 & -0.01 & 0.03 & 0.03 & 0.00 & 0.01 \\
\hline 19 & 6 & 0.00 & 0.00 & 0.00 & 0. & -0.05 & 0.05 & 0.0 & 0.05 & -0.02 \\
\hline 20 & 6 & -0.02 & -0.29 & 0.17 & & 0.01 & 0.03 & 0.0 & 0.00 & 0.01 \\
\hline 21 & 6 & -0.01 & -0.14 & 0.08 & & 0.07 & -0.0 & 0.0 & -0.09 & 0.05 \\
\hline 22 & 17 & -0.01 & -0.08 & 0.05 & -0.11 & 0.07 & 0.16 & -0.02 & 0.05 & 0.02 \\
\hline 23 & 17 & 0.01 & 0.08 & -0.05 & & -0.12 & -0.17 & 0.00 & 0.00 & -0.04 \\
\hline 24 & 6 & 0. & 0.00 & 0.00 & & 0.08 & -0.0 & -0. & & -0.15 \\
\hline 25 & 6 & 0.00 & 0.00 & 0.00 & & -0.05 & 0.0 & -0.01 & -0.06 & 0.07 \\
\hline 26 & 1 & 0.00 & 0.00 & 0.00 & & -0.07 & 0.08 & -0.02 & -0.04 & 0.06 \\
\hline 27 & 1 & 0.00 & 0.00 & 0.01 & & -0.05 & 0.03 & 0.0 & -0.06 & 0.08 \\
\hline 28 & 1 & 0.00 & 0.01 & 0.00 & & -0.07 & 0.04 & -0.01 & -0.05 & 0.06 \\
\hline 29 & 1 & 0.01 & 0.01 & 0.03 & & -0.03 & -0.11 & 0.11 & -0.10 & 0.16 \\
\hline 30 & 1 & -0.04 & 0.03 & 0.00 & & -0.06 & -0.05 & 0.10 & -0.07 & 0.12 \\
\hline 31 & 1 & 0.00 & -0.01 & -0.02 & -0.15 & 0.18 & -0.17 & 0.06 & -0.19 & 0.19 \\
\hline 32 & 1 & 0.04 & 0.52 & -0.29 & 0.14 & -0.07 & 0.05 & 0.04 & 0.06 & -0.03 \\
\hline 33 & 1 & 0.00 & 0.00 & 0.00 & & -0.14 & 0.10 & 0.04 & 0.15 & -0.07 \\
\hline 34 & 1 & -0.04 & -0.51 & 0.29 & 0.15 & -0.04 & 0.06 & 0.03 & 0.06 & -0.03 \\
\hline 35 & 1 & 0.00 & -0.01 & 0.01 & 0.00 & 0.00 & 0.00 & -0.15 & -0.04 & 0.00 \\
\hline
\end{tabular}




\begin{tabular}{|c|c|c|c|c|c|c|c|c|c|c|}
\hline & & & & & & & & & & \\
\hline 36 & 1 & 0.00 & 0.00 & 0.00 & 0.00 & 0.00 & 0.00 & -0.18 & -0.01 & $\begin{array}{l}-0.01 \\
\end{array}$ \\
\hline 3 & 1 & 0.00 & 0.01 & -0.01 & 0.00 & 0.00 & 0.00 & -0.15 & -0.02 & 0.01 \\
\hline 38 & 1 & 0.00 & 0.00 & 0.00 & -0.17 & 0.12 & -0.16 & -0.10 & 0.12 & -0.21 \\
\hline 39 & 1 & -0.01 & 0.01 & 0.00 & -0.09 & 0.04 & -0.05 & -0.06 & 0.13 & -0.21 \\
\hline 40 & 1 & -0.01 & 0.00 & -0.01 & -0.18 & 0.14 & 0.01 & -0.21 & 0.05 & -0.10 \\
\hline 41 & 6 & 0.00 & 0.00 & 0.01 & 0.03 & -0.01 & -0.01 & -0.01 & 0.00 & 0.01 \\
\hline 42 & 1 & 0.00 & 0.00 & 0.00 & 0.23 & 0.26 & & -0 . & -0.11 & 0.11 \\
\hline 43 & 6 & 0.00 & 0.00 & 0.00 & 0.00 & -0.01 & 0.0 & 0.00 & 0.01 & -0.01 \\
\hline 44 & 1 & 0.00 & -0.01 & 0.01 & 0.01 & -0.02 & 0.00 & -0.02 & 0.00 & 0.01 \\
\hline 45 & 1 & 0.01 & & 0.01 & 0.07 & -0.08 & & & .03 & .03 \\
\hline 46 & 9 & 0.00 & 0.00 & 0.00 & 0.00 & -0.03 & -0.0 & 0.0 & 0.02 & 0.01 \\
\hline 47 & 9 & 0.00 & -0.01 & 0.00 & 0.01 & 0.00 & 0.00 & 0.00 & 0.01 & 0.00 \\
\hline 48 & 9 & 0.00 & 0.01 & 0.01 & 0.01 & 0.01 & 0.01 & -0.01 & -0.01 & 0.01 \\
\hline requen & & 264.97 & & & 271.62 & & & 296.95 & & \\
\hline Atom & AN & $X$ & $Y$ & Z & $x$ & $Y$ & Z & $x$ & $Y$ & Z \\
\hline 1 & 6 & -0.08 & -0.02 & 0.01 & 0.01 & 0.00 & 0.00 & -0.03 & -0.02 & 0.01 \\
\hline 2 & 6 & -0.10 & 0.03 & -0.08 & 0.03 & -0.04 & 0.06 & -0.03 & -0.02 & 0.02 \\
\hline 3 & 6 & -0.09 & 0.07 & -0.10 & 0.03 & -0.05 & 0.07 & 0.00 & 0.00 & 0.03 \\
\hline 4 & 6 & -0.10 & 0.06 & -0.05 & 0.04 & -0.05 & 0.05 & 0.0 & 0.00 & 0.02 \\
\hline 5 & 6 & -0.08 & -0.02 & 0.01 & 0.02 & 0.00 & 0.00 & 0.02 & 0.00 & -0.01 \\
\hline 6 & 6 & -0.07 & -0.06 & 0.06 & 0.00 & 0.03 & -0.05 & -0.01 & -0.01 & -0.02 \\
\hline 7 & 7 & -0.05 & 0.04 & -0.05 & 0.00 & 0.01 & 0.03 & -0.01 & 0.10 & 0.11 \\
\hline 8 & 6 & 0.00 & 0.04 & -0.03 & -0.0 & 0.01 & 0.0 & -0 . & 0.18 & 0.19 \\
\hline 9 & 6 & -0.01 & 0.05 & -0.02 & 0.01 & 0.01 & 0.02 & -0.03 & 0.19 & 0.19 \\
\hline 10 & 6 & -0.05 & 0.05 & -0.07 & 0.04 & -0.02 & 0.01 & 0.09 & -0.07 & -0.05 \\
\hline 11 & 17 & 0.0 & 0.07 & 0.08 & -0.01 & -0.01 & -0.03 & -0.0 & -0.02 & -0.01 \\
\hline 12 & 17 & 0.03 & -0.08 & -0.05 & 0.00 & 0.02 & 0.00 & 0. & -0.02 & -0.04 \\
\hline 13 & 78 & 0.01 & -0.02 & 0.02 & -0.03 & 0.03 & -0.03 & 0.0 & -0.02 & -0.01 \\
\hline 14 & 8 & -0.05 & -0.06 & 0.04 & -0.12 & -0.07 & -0.02 & 0.0 & 0.01 & -0.02 \\
\hline 15 & 7 & 0.0 & 0.07 & -0.02 & 0.0 & -0.01 & 0.0 & -0. & 0.12 & 0.12 \\
\hline 16 & 6 & 0.02 & 0. & -0.06 & 0.03 & -0.05 & 0.0 & -0. & 0.03 & 0.02 \\
\hline 17 & 6 & 0.01 & 0.08 & -0.04 & 0.04 & -0.03 & 0.0 & & 0.01 & -0.01 \\
\hline 18 & 6 & 0.01 & 0.00 & 0.00 & 0.05 & 0.00 & 0.0 & -0.0 & 0.00 & -0.02 \\
\hline 19 & 6 & 0.01 & -0 & 0. & 0.0 & 0.03 & $-0 .($ & -0. & -0.02 & -0.03 \\
\hline 20 & 6 & 0.0 & & 0. & & 0. & 0.1 & & & \\
\hline 21 & 6 & 0.02 & 0.07 & -0.04 & 0.0 & -0.04 & 0.0 & 0.0 & 0.02 & 0.01 \\
\hline 22 & 17 & 0. & -0 & 0. & -0.02 & 0.04 & 0.0 & 0.0 & -0.03 & -0.04 \\
\hline 23 & 17 & & & -0 & & -0. & -0.0 & -0. & -0.01 & -0.02 \\
\hline 24 & 6 & 0. & & 0.13 & & & 0. & & & 0.01 \\
\hline 25 & 6 & 0.15 & & -0.12 & -0.06 & -0.05 & 0.0 & 0. & -0.08 & -0.05 \\
\hline 26 & 1 & 0. & & -0 & -0.04 & -0.11 & 0.0 & 0.0 & -0.26 & -0.18 \\
\hline 27 & 1 & & & & & -0.05 & & & & \\
\hline 28 & 1 & & & -0. & -0.09 & -0.07 & 0. & 0.2 & -0.21 & 0.02 \\
\hline 29 & 1 & -0. & & -0.0 & 0. & 0. & 0. & 0. & 0.08 & -0.23 \\
\hline 30 & 1 & & & & & & & & & \\
\hline 31 & 1 & & & & 0. & -0. & 0. & 0. & -0 . & -0.20 \\
\hline 32 & 1 & 0.1 & & 0. & 0.04 & 0. & -0.0 & -0.0 & -0.01 & -0.03 \\
\hline 33 & 1 & 0. & & & & & & & & -0.05 \\
\hline 34 & 1 & & & 0. & & 0. & & & & 0.0 \\
\hline 35 & 1 & -0. & & 0. & & 0. & -0 . & & 0. & -0 . \\
\hline 36 & 1 & -0.0 & & 0.14 & -0.03 & & -0.12 & -0.01 & -0.02 & -0.05 \\
\hline 37 & 1 & -0.1 & & 0.06 & -0.0 & 0.03 & -0.0 & & -0.03 & 0.00 \\
\hline 38 & 1 & & & 0. & & -0.24 & 0. & & & -0. \\
\hline 39 & 1 & 0.10 & -0 & 0.17 & 0.17 & -0.17 & 0.24 & -0.07 & 0.03 & 0.05 \\
\hline 40 & 1 & 0.2 & & & 0.44 & -0.12 & 0.0 & & & \\
\hline 41 & 6 & -0.0 & & 0.0 & -0.0 & 0.01 & 0.0 & & 0.00 & 0.00 \\
\hline 42 & 1 & -0.11 & -0.14 & -0.06 & -0.24 & -0.19 & -0.17 & 0.02 & 0.03 & 0.01 \\
\hline 43 & 6 & 0.0 & & & 0.0 & 0.01 & 0.00 & -0.01 & 0.01 & 0.00 \\
\hline 44 & 1 & -0.01 & & 0. & -0.05 & 0.04 & 0.0 & -0.01 & 0.00 & 0.00 \\
\hline 45 & 1 & -0.03 & 0.04 & 0.04 & -0.04 & 0.05 & 0.05 & 0.01 & -0.01 & -0.01 \\
\hline
\end{tabular}




\begin{tabular}{|c|c|c|c|c|c|c|c|c|c|c|}
\hline $\begin{array}{r}46 \\
47 \\
48\end{array}$ & $\begin{array}{l}9 \\
9 \\
9\end{array}$ & $\begin{array}{r}0.00 \\
-0.01 \\
0.00\end{array}$ & $\begin{array}{l}0.01 \\
0.00 \\
0.00\end{array}$ & $\begin{array}{l}0.01 \\
0.00 \\
0.00\end{array}$ & $\begin{array}{c}0.01 \\
-0.01 \\
0.01\end{array}$ & $\begin{array}{c}0.02 \\
-0.01 \\
0.02\end{array}$ & $\begin{array}{l}0.02 \\
0.00 \\
0.01\end{array}$ & $\begin{array}{l}-0.01 \\
0.01\end{array}$ & $\begin{array}{l}0.00 \\
0.03 \\
0.00\end{array}$ & $\begin{array}{l}0.00 \\
0.02 \\
0.00\end{array}$ \\
\hline \multicolumn{2}{|c|}{ Frequencies } & 314.35 & & & 327.97 & & & 356.83 & & \\
\hline Atom & AN & X & $Y$ & Z & $X$ & $Y$ & Z & X & Y & Z \\
\hline 1 & 6 & 0.00 & -0.01 & 0.01 & -0.01 & 0.02 & -0.02 & 0.01 & -0.01 & 0.01 \\
\hline 2 & 6 & 0.02 & -0.07 & 0.06 & -0.05 & 0.10 & -0.13 & & & 0.04 \\
\hline 3 & 6 & 0.03 & -0.06 & 0.06 & -0.04 & 0.10 & -0.13 & 0.02 & -0.03 & 0.03 \\
\hline 4 & 6 & 0.00 & -0.05 & 0.07 & -0.05 & 0.11 & -0.12 & 0.01 & -0.04 & \\
\hline 5 & 6 & -0.02 & & 0.02 & -0.01 & 0.02 & -0. & & & \\
\hline 6 & 6 & -0.04 & 0.03 & -0.04 & 0.03 & -0.06 & 0.08 & -0.03 & 0.03 & -0.03 \\
\hline 7 & 7 & 0.02 & -0.03 & -0.02 & 0.00 & 0.03 & -0.04 & 0.01 & 0.02 & -0.03 \\
\hline 8 & 6 & 0.01 & -0.04 & -0.0 & $-0 .($ & & -0.0 & & & -0.04 \\
\hline 9 & 6 & 0.02 & & -0.05 & & & & & & 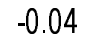 \\
\hline 10 & 6 & 0.26 & -0.02 & 0.08 & 0.1 & 0.03 & 0. & & 0.06 & -0.05 \\
\hline 11 & 17 & -0.0 & & & & & & & & \\
\hline 12 & 17 & -0.0 & -0.01 & -0.02 & & -0.02 & & & -0.01 & -0.02 \\
\hline 13 & 78 & -0.01 & & 0.00 & & -0.01 & 0.01 & -0.01 & 0.00 & 0.00 \\
\hline 14 & 8 & 0.0 & & & & 0.00 & & & & 07 \\
\hline 15 & 7 & 0.0 & 0.0 & -0.0 & -0.02 & 0.02 & -0. & & & \\
\hline 16 & 6 & 0.01 & 0.18 & -0.09 & -0.01 & -0.02 & 0.0 & -0.01 & -0.02 & 0.00 \\
\hline 17 & 6 & 0.00 & & -0.1 & & -0.02 & & & -0.03 & 02 \\
\hline 18 & 6 & -0.0 & & -0.0 & & & & & & \\
\hline 19 & 6 & -0.02 & -0.11 & 0.05 & 0.0 & 0.03 & -0 . & & & -0.01 \\
\hline 20 & 6 & 0.00 & 0.02 & -0.01 & & -0.01 & & & & 0.00 \\
\hline 21 & 6 & 0. & & & & & & & & \\
\hline 22 & 17 & & & & & & & & & \\
\hline 23 & 17 & & & & & -0.02 & & & & -0.01 \\
\hline 24 & 6 & -0. & & & & & & & & \\
\hline 25 & 6 & -0.06 & & & & & & & & \\
\hline 26 & 1 & & & & -0.2 & -0.21 & & -0. & & -0.04 \\
\hline 27 & 1 & & & & -0 & & & & & 04 \\
\hline 28 & 1 & -0 . & & & & -0.14 & -0 & & & \\
\hline 29 & 1 & & & & & & -0 & & & \\
\hline 30 & 1 & & & & & & & & & \\
\hline 31 & 1 & & & & & & & & & \\
\hline 3 & 1 & & & & & & & & & \\
\hline 3 & 1 & & & & & & & & & \\
\hline 34 & 1 & & & & & & & & & \\
\hline 35 & 1 & & & & & & & & & \\
\hline 36 & 1 & & & & & & & & & \\
\hline 37 & 1 & & & & & & & & & -0.0 \\
\hline 38 & 1 & & & & & & & & & \\
\hline 39 & 1 & & & & & & & & & \\
\hline 40 & 1 & & & & & & & & & \\
\hline 41 & 6 & & & & & & & & & 0.17 \\
\hline 42 & 1 & & & & & & & & & 0.11 \\
\hline 43 & 6 & & & & & & & & & \\
\hline 44 & 1 & & & & & & & & & \\
\hline 45 & 1 & & & & & & & & & 0.44 \\
\hline 46 & 9 & & & & & & & & & \\
\hline 47 & 9 & & & & & & & & & -0.11 \\
\hline 48 & 9 & & & & 0.00 & -0.01 & -0.0 & & -0.16 & -0.1 \\
\hline Frequen & & & & & 36557 & & & & & \\
\hline & AN & X & $Y$ & Z & $X$ & Y & & $x$ & Y & \\
\hline & 6 & -0.0 & -0.0 & 0.0 & -0.12 & -0.05 & -0.0 & -0.0 & & 0.04 \\
\hline 2 & 6 & & & & & 0.1 & 0. & & & 0.00 \\
\hline 3 & 6 & & & 0.0 & & 0.11 & & & & 0.03 \\
\hline 4 & 6 & & & 0.1 & & 0.10 & & & -0.08 & 0.00 \\
\hline 5 & 6 & 0.02 & -0.01 & 0.03 & 0.12 & 0.00 & -0.06 & -0.02 & -0.05 & -0.01 \\
\hline
\end{tabular}




\begin{tabular}{|c|c|c|c|c|c|c|c|c|c|c|}
\hline & & & & & & & & & & \\
\hline 6 & 6 & -0.06 & 0.04 & -0.09 & 0.03 & -0.12 & -0.06 & -0.01 & 0.01 & -0.04 \\
\hline$t$ & 7 & 0.04 & 0.10 & -0.02 & -0.04 & 0.17 & 0.20 & -0.12 & 0.03 & -0.04 \\
\hline 8 & 6 & -0.01 & 0.10 & -0.07 & -0.01 & 0.06 & 0.10 & -0.19 & 0.04 & -0.06 \\
\hline 9 & 6 & 0.03 & 0.11 & -0.13 & 0.01 & -0.10 & -0.05 & -0.18 & -0.04 & \\
\hline 10 & 6 & 0.08 & 0.16 & -0.12 & -0.01 & -0.03 & 0.04 & 0.01 & -0.11 & 0.10 \\
\hline 11 & 17 & $-0 .($ & 0.04 & 0.04 & -0.06 & -0.06 & -0.02 & & -0.06 & 0 \\
\hline 12 & 17 & -0.01 & -0.05 & -0.06 & 0.0 & 0.01 & -0.02 & & & \\
\hline 13 & 78 & 0.00 & -0.02 & 0.02 & 0.00 & 0.00 & 0.00 & 0.02 & -0.01 & .01 \\
\hline 14 & 8 & 0.02 & -0.01 & 0.01 & -0.02 & -0.01 & -0.06 & 0.04 & -0.02 & 0.04 \\
\hline 15 & 7 & -0.0 & & -0.14 & & -0.19 & -0.17 & & & 0.01 \\
\hline 16 & 6 & -0.01 & -0.07 & -0.01 & 0.03 & -0.03 & -0.12 & -0.14 & 0.01 & -0.03 \\
\hline 17 & 6 & 0.04 & -0.10 & 0.05 & 0.12 & 0.03 & -0.08 & -0.06 & 0.07 & 0.06 \\
\hline 18 & 6 & 0.04 & & 0.0 & 0.11 & 0.04 & 0.04 & & 0.03 & 0.03 \\
\hline 19 & 6 & 0.01 & & 0.0 & -0.03 & 0.00 & 0.13 & & -0.02 & 0.00 \\
\hline 20 & 6 & -0.05 & -0.02 & 0.01 & -0.11 & 0.03 & 0.01 & -0.04 & -0.02 & -0.06 \\
\hline 21 & 6 & -0.04 & & 0.02 & -0.08 & 0.04 & -0.10 & -0.03 & -0.02 & -0.10 \\
\hline 22 & 17 & -0.03 & & 0.02 & -0.08 & 0.00 & 0.00 & 0.0 & & \\
\hline 23 & 17 & & & -0.01 & 0.06 & & 0.05 & & -0.08 & -0.12 \\
\hline 24 & 6 & -0.05 & 0.00 & -0.02 & 0.01 & -0.03 & -0.03 & -0.03 & 0.02 & -0.03 \\
\hline 25 & 6 & & & -0.09 & & -0.03 & 0.0 & -0.05 & 0.16 & \\
\hline 26 & 1 & -0. & & -0.09 & 0.04 & -0.08 & -0.05 & & 0.27 & \\
\hline 27 & 1 & -0. & & -0.11 & -0.06 & -0.01 & -0.08 & & 0.16 & -0.15 \\
\hline 28 & 1 & -0 . & & -0.08 & 0.11 & -0.09 & 0.0 & 0.1 & 0.16 & -0.12 \\
\hline 29 & 1 & 0.08 & & -0.1 & -0.06 & -0.07 & 0.09 & 0.15 & -0.02 & 0.07 \\
\hline 30 & 1 & 0.11 & & -0.13 & & 0.05 & 0.01 & 0.09 & -0.06 & 0.08 \\
\hline 31 & 1 & 0.07 & & -0.08 & -0.03 & & 0.0 & 0.0 & -0.28 & 0.21 \\
\hline 32 & 1 & 0.07 & & 0.0 & 0.2 & & 0.11 & -0.14 & 0.00 & -0.02 \\
\hline 33 & 1 & 0.02 & 0.26 & -0.04 & -0.05 & -0.05 & 0.29 & -0.01 & -0.07 & 0.02 \\
\hline 34 & 1 & -0.09 & & 0. & -0.21 & 3 & 0.0 & -0 & -0.02 & -0.04 \\
\hline 35 & 1 & & & -0.0 & 0.2 & -0.03 & -0.09 & -0 . & -0.03 & 0.00 \\
\hline 36 & 1 & -0 . & & -0.25 & 0. & -0.25 & -0.09 & -0.03 & 0.05 & -0.09 \\
\hline 37 & 1 & -0.0 & & -0.0 & -0.22 & -0.14 & -0.0 & -0 . & & 0.01 \\
\hline 38 & 1 & & & -0. & -0.0 & -0.10 & 0. & -0. & 0. & -0.08 \\
\hline 39 & 1 & & & -0.0 & & & -0.1 & & & -0.06 \\
\hline 40 & 1 & & & -0.0 & & -0.07 & -0.0 & & -0.01 & \\
\hline 41 & 6 & 0.0 & & -0.10 & 0. & -0.04 & 0.03 & 0.02 & 0.00 & -0.02 \\
\hline 42 & 1 & 0. & & 0. & -0.0 & & -0.0 & 0. & -0.07 & -0.02 \\
\hline 43 & 6 & & & & & & & & & \\
\hline 44 & 1 & 0. & & -0. & & -0 & 0.1 & 0. & 11 & -0 \\
\hline 45 & 1 & -0.22 & & -0 . & & & 0.11 & -0. & 0.01 & -0.05 \\
\hline 46 & 9 & -0.0 & & -0. & -0.0 & & 0.02 & -0. & -0.01 & -0.02 \\
\hline 47 & 9 & & & & & & & & & \\
\hline 48 & 9 & 0.00 & & 0.0 & -0.02 & -0.02 & 0.00 & -0.01 & 0.00 & 0.01 \\
\hline equen & & & & & & & & 419.17 & & \\
\hline Atom & AN & 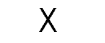 & $Y$ & Z & $x$ & $\mathrm{Y}$ & Z & $x$ & $\mathrm{Y}$ & Z \\
\hline 1 & 6 & & 0.10 & 0.06 & -0.02 & -0.04 & -0.02 & -0. & -0.08 & -0.06 \\
\hline 2 & 6 & & -0.02 & -0.06 & -0.02 & 0.01 & 0.02 & -0.02 & 0.03 & 0.02 \\
\hline 3 & 6 & & & -0.07 & & & 0.02 & & & 0.01 \\
\hline 4 & 6 & & & -0.0 & & 0. & 0.0 & & & 0.01 \\
\hline 5 & 6 & & & 0. & 0. & -0.02 & -0.03 & 0.0 & -0.07 & -0.07 \\
\hline 6 & 6 & & & & & -0.04 & -0.0 & & -0.10 & -0.08 \\
\hline 7 & 7 & 0. & & -0.0 & & 0.01 & 0.0 & & -0.04 & -0.04 \\
\hline 8 & 6 & & & 0. & -0 . & 0.01 & -0.01 & 0. & 0.0 & 0.02 \\
\hline 9 & 6 & & & 0.1 & -0. & 0.0 & -0.0 & & 0.01 & 0.04 \\
\hline 10 & 6 & & & 0.0 & & & & & -0.02 & 0.01 \\
\hline 11 & 17 & & & -0.05 & -0. & 0.00 & 0.01 & -0.05 & 0.02 & 0.04 \\
\hline 12 & 17 & & & 0.00 & 0.6 & 0.02 & 0.01 & 0.06 & 0.08 & 0.04 \\
\hline 13 & 78 & & & 0.0 & & -0.01 & 0.02 & -0.0 & 0.02 & -0.03 \\
\hline 14 & 8 & & & 0.0 & -0.2 & 0.28 & -0.04 & 0.19 & 0.00 & 0.44 \\
\hline 15 & 7 & 0.01 & -0.07 & -0.07 & -0.01 & 0.01 & 0.00 & 0.01 & 0.01 & 0.02 \\
\hline
\end{tabular}




\begin{tabular}{|c|c|c|c|c|c|c|c|c|c|c|}
\hline 16 & 6 & 0.02 & -0.05 & -0.10 & -0.01 & 0.00 & 0.01 & 0.02 & 0.00 & 0.00 \\
\hline 17 & 6 & 0.10 & -0.02 & -0.06 & -0.01 & 0.00 & 0.01 & 0.02 & -0.01 & -0.02 \\
\hline 18 & 6 & 0.09 & 0.06 & 0.09 & -0.01 & -0.01 & -0.01 & 0.02 & 0.02 & 0.03 \\
\hline 19 & 6 & -0.03 & 0.07 & 0.17 & 0.00 & 0.00 & -0.02 & 0.00 & 0.02 & 0.05 \\
\hline 20 & 6 & -0.12 & 0.05 & 0.06 & 0.00 & -0.01 & -0.01 & 0.00 & 0.02 & .04 \\
\hline 21 & 6 & -0.07 & -0.01 & -0.09 & 0.00 & -0.01 & 0.01 & 0.01 & 0.01 & 0.00 \\
\hline 22 & 17 & -0.09 & -0.02 & -0.05 & 0.01 & 0.01 & 0.02 & -0.03 & -0.02 & -0.04 \\
\hline 23 & 17 & 0.09 & -0.02 & -0.01 & 0.00 & 0.00 & 0.00 & 0.01 & 0.00 & \\
\hline 24 & 6 & 0.00 & 0.00 & -0.01 & -0.01 & 0.01 & 0.00 & 0.03 & 0.00 & \\
\hline 25 & 6 & 0.02 & -0.01 & -0.02 & -0.01 & 0.01 & -0.02 & 0.01 & -0.02 & 0.01 \\
\hline 26 & 1 & 0.04 & -0.08 & -0.10 & -0.01 & 0.01 & -0.03 & 0.01 & -0.04 & \\
\hline 27 & 1 & -0.09 & 0.02 & -0.15 & -0.01 & 0.01 & -0.02 & -0.02 & -0.01 & -0.02 \\
\hline 28 & 1 & 0.14 & -0.11 & 0.03 & 0.00 & 0.01 & -0.01 & 0.02 & -0.04 & \\
\hline 29 & 1 & 0.10 & 0.04 & -0.13 & 0.01 & 0.00 & 0.00 & 0.00 & -0.01 & -0.02 \\
\hline 30 & 1 & -0.05 & -0.19 & 0.04 & 0.01 & 0.01 & 0.00 & -0.03 & -0.06 & 0.02 \\
\hline 31 & 1 & 0.03 & -0.10 & -0.08 & 0.00 & -0.01 & 0.01 & 0.00 & -0.02 & 02 \\
\hline 32 & 1 & 0.21 & 0.10 & 0.17 & -0.03 & -0.02 & -0.02 & 0.05 & 0.03 & 06 \\
\hline 33 & 1 & -0.05 & 0.09 & 0.27 & 0.00 & 0.01 & -0.04 & 0.00 & 0.02 & \\
\hline 34 & 1 & -0.26 & 0.07 & 0.11 & 0.01 & -0.01 & -0.02 & -0.02 & 0.02 & \\
\hline 35 & 1 & -0.25 & 0.07 & 0.13 & 0.07 & -0.03 & -0.05 & 0.09 & -0.08 & \\
\hline 36 & 1 & -0.03 & 0.20 & 0.19 & 0.00 & -0.06 & -0.07 & 0.01 & .11 & 08 \\
\hline 37 & 1 & 0.19 & 0.17 & 0.09 & -0.06 & -0.06 & -0.03 & -0.09 & -0.13 & \\
\hline 38 & 1 & -0.01 & -0.03 & 0.00 & -0.02 & 0.04 & -0.05 & 0.04 & -0.02 & \\
\hline 39 & 1 & 0.03 & 0.00 & -0.03 & 0.00 & 0.02 & -0.02 & 0.01 & -0.03 & 06 \\
\hline 40 & 1 & 0.00 & -0.01 & -0.01 & -0.08 & 0.00 & $0 . c$ & 0.12 & 04 & \\
\hline 41 & 6 & -0.02 & 0.03 & 0.01 & -0.19 & 0.15 & 0.0 & 0.04 & & \\
\hline 42 & 1 & -0.02 & 0.03 & 0.04 & -0.17 & 0.48 & 0.1 & 0.14 & 25 & \\
\hline 43 & 6 & 0.00 & 0.00 & 0.00 & 0.00 & 0.00 & -0.02 & 0.00 & 01 & \\
\hline 44 & 1 & -0.01 & 0.04 & 0.01 & -0.33 & 0.22 & 0.24 & 0.39 & 09 & \\
\hline 45 & 1 & -0.04 & 0.04 & 0.01 & -0.09 & 0.14 & 0.17 & -0.28 & 0.24 & \\
\hline 46 & 9 & 0.03 & -0.03 & 0.01 & 0.18 & -0.10 & 0.09 & 0.04 & -0.08 & \\
\hline 47 & 9 & -0.04 & -0.03 & -0.03 & -0.20 & -0.15 & -0.13 & -0.08 & -0.07 & \\
\hline 48 & 9 & 0.03 & -0.01 & -0.03 & 0.18 & -0.01 & -0.14 & 0.04 & -0.05 & .06 \\
\hline Frequen & & 429.88 & & & 438.90 & & & 450.91 & & \\
\hline Atom & AN & $X$ & $Y$ & Z & $x$ & $Y$ & Z & X & $Y$ & \\
\hline 1 & 6 & 0.03 & -0.11 & -0.10 & -0.18 & -0.02 & 0.08 & -0.16 & 0.11 & 0. \\
\hline 2 & 6 & 0.03 & 0.01 & 0.01 & -0.14 & 0.01 & 0.12 & & 0.00 & 0.07 \\
\hline 3 & 6 & -0.01 & -0.02 & -0.02 & -0.19 & -0.04 & 0.01 & -0.03 & 0.09 & 0.09 \\
\hline 4 & 6 & -0.05 & -0.01 & 0.01 & -0.12 & -0.14 & -0.03 & 0.1 & 0.08 & 0.00 \\
\hline 5 & 6 & -0.01 & -0.13 & -0.09 & -0.17 & -0.11 & 0.01 & & 0.17 & 0.11 \\
\hline 6 & 6 & 0.01 & -0.12 & -0.11 & -0.16 & -0.01 & -0.01 & -0.03 & 0.10 & 0.10 \\
\hline 7 & 7 & 0.01 & -0.11 & -0.10 & -0.12 & 0.00 & -0.03 & -0.03 & 0.16 & 0.15 \\
\hline 8 & 6 & 0.01 & -0.09 & -0.09 & -0.07 & & -0.0 & & -0.11 & -0 . \\
\hline 9 & 6 & -0.01 & 0.04 & 0.03 & 0.03 & 0.05 & -0.06 & 0.03 & -0.13 & -0.14 \\
\hline 10 & 6 & 0.02 & 0.01 & 0.00 & 0.10 & 0.09 & -0.06 & 0.00 & 0.02 & 0.01 \\
\hline 11 & 17 & -0.0 & 0.05 & 0.0 & 0.14 & -0.09 & -0.1 & & & \\
\hline 12 & 17 & 0.0 & 0.11 & 0.07 & 0.10 & 0. & 0.0 & & -0.11 & \\
\hline 13 & 78 & 0.0 & -0.01 & 0.01 & 0.01 & -0.01 & 0.01 & 0.00 & -0.01 & -0.01 \\
\hline 14 & 8 & -0.08 & -0.02 & -0.21 & -0.02 & -0.01 & -0.04 & 0. & 0.02 & \\
\hline 15 & 7 & $-0 . C$ & 0.15 & 0.1 & 0.09 & & & & & \\
\hline 16 & 6 & 0.0 & 0.03 & 0.0 & 0.13 & 0.03 & 0.0 & & 0.07 & 0.07 \\
\hline 17 & 6 & -0.05 & -0.03 & -0.01 & 0.13 & -0.10 & -0.01 & -0.11 & 0.01 & 0.02 \\
\hline 18 & 6 & -0.06 & 0.09 & 0.19 & 0.15 & -0.07 & -0.03 & & 0.05 & \\
\hline 19 & 6 & -0.03 & 0.15 & 0.1 & 0.12 & 0.05 & -0.0 & & 0.09 & \\
\hline 20 & 6 & -0.01 & 0.10 & 0.20 & 0.12 & -0.04 & 0.0 & 0.08 & 0.07 & 0.15 \\
\hline 21 & 6 & 0.05 & -0.03 & 0.01 & 0.07 & -0.01 & 0.1 & & -0.01 & 0.07 \\
\hline 22 & 17 & -0.08 & -0.08 & -0.15 & -0.06 & -0.04 & -0.10 & -0.0 & -0.06 & -0.11 \\
\hline 23 & 17 & 0.12 & -0.07 & -0.11 & -0.13 & 0.08 & 0.11 & 0.0 & -0.05 & -0.09 \\
\hline 24 & 6 & -0.0 & 0.01 & 0.0 & 0.00 & 0.01 & -0.0 & & 0.01 & 0.01 \\
\hline 25 & 6 & 0.00 & 0.01 & 0.01 & -0.07 & 0.04 & -0.05 & -0.01 & 0.01 & 0.01 \\
\hline
\end{tabular}




\begin{tabular}{|c|c|c|c|c|c|c|c|c|c|c|}
\hline & & & & & & & & & & \\
\hline 26 & 1 & -0.03 & 0.08 & 0.04 & -0.08 & 0.05 & -0.06 & -0.04 & 0.09 & 0.07 \\
\hline 27 & 1 & 0.10 & -0.02 & 0.12 & -0.06 & 0.04 & -0.04 & 0.10 & -0.02 & 0.16 \\
\hline 28 & 1 & -0.08 & 0.09 & -0.02 & -0.07 & 0.05 & -0.05 & -0.13 & 0.12 & -0.03 \\
\hline 29 & 1 & 0.05 & 0.03 & -0.02 & 0.15 & 0.12 & -0.06 & -0.10 & -0.07 & 0.19 \\
\hline 30 & 1 & 0.01 & -0.02 & 0.01 & 0.15 & 0.11 & -0.07 & 0.10 & 0.25 & -0.05 \\
\hline 31 & 1 & 0.02 & -0.02 & 0.00 & 0.10 & 0.01 & 0.01 & -0.04 & 0.09 & 0.13 \\
\hline 32 & 1 & 0.05 & 0.12 & 0.27 & 0.18 & -0.06 & -0.01 & -0.10 & 0.03 & 0.15 \\
\hline 33 & 1 & -0.02 & 0.18 & 0.12 & 0.13 & 0.17 & -0.10 & 0.01 & 0.07 & -0.05 \\
\hline 34 & 1 & -0.13 & 0.13 & 0.23 & 0.19 & -0.06 & 0.03 & 0.06 & 0.08 & 0.15 \\
\hline 35 & 1 & 0.06 & -0.14 & -0.13 & -0.24 & -0.11 & 0.05 & 0.06 & 0.16 & 0.14 \\
\hline 36 & 1 & 0.00 & -0.08 & -0.09 & -0.19 & 0.04 & -0.06 & -0.01 & -0.02 & 0.00 \\
\hline 37 & 1 & -0.04 & -0.16 & -0.12 & -0.24 & -0.06 & 0.07 & -0.14 & 0.14 & 0.14 \\
\hline 38 & 1 & 0.02 & 0.08 & -0.03 & 0.01 & 0.03 & -0.03 & 0.04 & 0.05 & 0.01 \\
\hline 39 & 1 & -0.05 & 0.00 & 0.04 & 0.01 & 0.02 & -0.03 & -0.04 & 0.00 & 0.05 \\
\hline 40 & 1 & -0.05 & 0.03 & 0.04 & -0.02 & 0.00 & 0.00 & 0.02 & 0.03 & 0.02 \\
\hline 41 & 6 & -0.01 & -0.05 & -0.01 & 0.00 & -0.01 & 0.00 & -0.01 & 0.03 & 0.01 \\
\hline 42 & 1 & -0.07 & 0.07 & -0.14 & -0.02 & 0.01 & -0.03 & 0.03 & -0.01 & 0.08 \\
\hline 43 & 6 & 0.00 & -0.01 & 0.00 & 0.00 & 0.00 & 0.00 & -0.01 & 0.01 & 0.00 \\
\hline 44 & 1 & -0.17 & -0.05 & 0.12 & -0.03 & -0.01 & 0.02 & 0.07 & 0.03 & -0.05 \\
\hline 45 & 1 & 0.14 & -0.12 & 0.04 & 0.01 & -0.02 & 0.00 & -0.08 & 0.07 & -0.01 \\
\hline 46 & 9 & -0.03 & 0.04 & 0.01 & 0.00 & 0.01 & 0.00 & 0.02 & -0.02 & 0.00 \\
\hline 47 & 9 & 0.05 & 0.03 & 0.02 & 0.01 & 0.01 & 0.00 & -0.03 & -0.01 & -0.01 \\
\hline 48 & 9 & -0.03 & 0.03 & 0.04 & 0.00 & 0.01 & 0.01 & 0.02 & -0.02 & -0.03 \\
\hline requen & & 475.82 & & & 505.18 & & & 513.93 & & \\
\hline Atom & AN & X & $Y$ & Z & $X$ & $Y$ & Z & $x$ & $Y$ & Z \\
\hline 1 & 6 & -0.08 & 0.02 & -0.02 & 0.00 & 0.00 & 0.00 & 0.00 & 0.01 & 0.00 \\
\hline 2 & 6 & -0.06 & 0.02 & 0.00 & 0.00 & 0.00 & 0.00 & 0.00 & 0.00 & 0.00 \\
\hline 3 & 6 & -0.03 & -0.05 & 0.05 & 0.00 & 0.00 & 0.00 & 0.00 & 0.00 & 0.01 \\
\hline 4 & 6 & -0.06 & -0.01 & -0.04 & 0.00 & 0.00 & 0.00 & 0.01 & 0.01 & 0.00 \\
\hline 5 & 6 & -0.09 & -0.01 & -0.03 & 0.00 & 0.00 & 0.00 & 0.01 & 0.01 & 0.00 \\
\hline 6 & 6 & -0.05 & -0.07 & 0.07 & 0.00 & 0.00 & 0.00 & 0.01 & 0.00 & 0.01 \\
\hline 7 & 7 & 0.04 & -0.10 & 0.11 & 0.00 & 0.00 & 0.00 & 0.00 & -0.01 & 0.01 \\
\hline 8 & 6 & 0.12 & -0.11 & 0.11 & 0.0 & 0.00 & 0.00 & -0.01 & -0.01 & 0.00 \\
\hline 9 & 6 & 0.06 & 0.09 & -0.09 & 0.00 & 0.00 & 0.00 & -0.01 & 0.00 & 0.00 \\
\hline 10 & 6 & 0.1 & 0.15 & -0.12 & 0.00 & 0.00 & 0.00 & 0.0 & 0.00 & 0.00 \\
\hline 11 & 17 & 0.04 & -0.02 & -0.03 & 0.00 & 0.00 & 0.00 & 0.0 & 0.00 & 0.00 \\
\hline 12 & 17 & 0.0 & 0.04 & 0.03 & 0.0 & 0.00 & 0.0 & 0.0 & 0.00 & 0.00 \\
\hline 13 & 78 & & 0.00 & -0.01 & & 0.00 & 0.0 & & 0.00 & 0.00 \\
\hline 14 & 8 & -0.01 & 0.00 & 0.00 & 0.03 & 0.00 & 0.0 & -0.1 & 0.17 & 0.03 \\
\hline 15 & 7 & 0. & 0.10 & -0.08 & 0.00 & 0.00 & 0.0 & 0.0 & 0.01 & -0.01 \\
\hline 16 & 6 & -0 . & 0.03 & -0.05 & & -0.01 & 0.0 & & & -0.01 \\
\hline 17 & 6 & -0.0 & 0.00 & 0.02 & 0.00 & 0.01 & 0.0 & 0.0 & -0.01 & 0.00 \\
\hline 18 & 6 & -0.1 & -0.03 & 0.01 & 0.00 & 0.00 & 0.0 & 0.0 & -0.01 & 0.01 \\
\hline 19 & 6 & -0 . & 0.04 & -0.05 & 0.00 & -0.01 & 0. & 0. & & -0.01 \\
\hline 20 & 6 & -0.13 & -0.04 & -0.03 & 0.00 & 0.01 & 0.0 & 0.0 & -0.01 & 0.01 \\
\hline 21 & 6 & -0.09 & -0.06 & -0.03 & 0.00 & 0.00 & 0.0 & 0.0 & -0.01 & 0.00 \\
\hline 22 & 17 & 0.0 & 0.04 & 0.07 & 0.00 & 0.00 & 0.0 & & & 0.00 \\
\hline 23 & 17 & 0. & -0.02 & -0.04 & & 0.00 & 0. & & & 0.00 \\
\hline 24 & 6 & 0.0 & 0.01 & -0.01 & -0.0 & 0.00 & 0.0 & -0.0 & 0.0 & 0.01 \\
\hline 25 & 6 & 0.21 & -0.15 & 0.18 & 0.00 & 0.00 & 0.0 & 0.00 & 0.00 & 0.00 \\
\hline 26 & 1 & 0.18 & -0.08 & 0.12 & & -0.01 & 0.0 & & & -0.02 \\
\hline 27 & 1 & 0.2 & -0.15 & 0.20 & & 0.00 & 0.1 & & & 0.00 \\
\hline 28 & 1 & 0.2 & -0.12 & 0.20 & 0.00 & 0.00 & 0.0 & 0.02 & 0.00 & 0.00 \\
\hline 29 & 1 & 0.15 & 0.17 & -0.10 & -0.01 & 0.00 & 0.0 & 0.0 & & 0.00 \\
\hline 30 & 1 & 0.16 & 0.18 & -0.13 & 0.0 & 0.01 & 0.0 & & & 0.00 \\
\hline 31 & 1 & 0.11 & 0.10 & -0.05 & 0.00 & 0.01 & -0.0 & 0.00 & -0.02 & 0.02 \\
\hline 32 & 1 & -0.1 & -0.06 & 0.01 & 0.0 & 0.00 & 0.0 & & -0.02 & 0.01 \\
\hline 33 & 1 & -0.1 & 0.13 & -0.08 & 0.00 & -0.01 & 0.0 & & 0.05 & -0.03 \\
\hline 34 & 1 & -0.15 & -0.04 & -0.03 & 0.00 & 0.01 & -0.0 & 0.00 & -0.02 & 0.01 \\
\hline 35 & 1 & -0.11 & 0.01 & -0.04 & 0.00 & 0.00 & 0.00 & 0.01 & 0.01 & -0.01 \\
\hline
\end{tabular}




\begin{tabular}{|c|c|c|c|c|c|c|c|c|c|c|}
\hline & & & & & & & & & & \\
\hline 36 & 1 & -0.02 & -0.14 & 0.15 & 0.00 & 0.01 & -0.01 & 0.01 & -0.02 & 0.01 \\
\hline 3 & 1 & -0.10 & 0.03 & -0.04 & 0.00 & 0.00 & 0.01 & -0.01 & 0.01 & -0.01 \\
\hline 38 & 1 & 0.01 & 0.01 & 0.00 & 0.00 & 0.01 & 0.00 & -0.01 & 0.00 & 0.00 \\
\hline 39 & 1 & 0.01 & 0.00 & 0.00 & -0.02 & 0.00 & 0.0 & 0.01 & 0.00 & -0.01 \\
\hline 40 & 1 & 0.02 & 0.02 & -0.01 & -0.03 & 0.00 & 0.01 & -0.04 & -0.02 & 0.01 \\
\hline 41 & 6 & -0.01 & 0.01 & 0.00 & & -0.03 & 0.03 & 0.11 & 0.21 & 0.16 \\
\hline 42 & 1 & -0.01 & -0.01 & 0.00 & -0.09 & -0.09 & -0.12 & -0.22 & 0.08 & -0.09 \\
\hline 43 & 6 & 0.00 & 0.00 & 0.00 & -0.01 & 0.14 & -0.19 & 0.25 & 0.01 & 0.00 \\
\hline 44 & 1 & 0.00 & 0.01 & 0.00 & -0.34 & -0.44 & 0.12 & 0.02 & 0.34 & 0.31 \\
\hline 45 & 1 & -0.01 & & 0.0 & & -0.01 & 0.38 & & 0.31 & 0.26 \\
\hline 46 & 9 & 0.00 & 0.00 & 0.00 & 0.28 & -0.02 & -0.10 & -0.07 & 0.15 & -0.34 \\
\hline 47 & 9 & 0.00 & 0.00 & 0.00 & -0.04 & -0.14 & 0.29 & 0.07 & -0.22 & -0.11 \\
\hline 48 & 9 & 0.00 & 0.00 & 0.00 & -0.25 & 0.13 & -0.12 & -0.18 & -0.21 & 0.25 \\
\hline equer & & 520.62 & & & 538.67 & & & 539.25 & & \\
\hline Atom & AN & $x$ & Y & Z & $x$ & $\mathrm{Y}$ & Z & $x$ & $Y$ & Z \\
\hline 1 & 6 & 0.02 & 0.07 & -0.08 & 0.02 & -0.02 & 0.02 & 0.02 & -0.08 & 0.10 \\
\hline 2 & 6 & 0.02 & 0.03 & -0.04 & -0.05 & 0.13 & -0.16 & -0.11 & 0.22 & -0.25 \\
\hline 3 & 6 & 0.05 & -0.10 & 0.12 & 0.01 & -0.04 & 0.04 & -0.01 & 0.03 & -0.02 \\
\hline 4 & 6 & 0.03 & 0.03 & -0.02 & 0.06 & -0.13 & 0.15 & 0.10 & -0.22 & 0.27 \\
\hline 5 & 6 & 0.03 & 0.08 & -0.08 & -0.03 & 0.06 & -0.08 & -0.02 & 0.06 & -0.07 \\
\hline 6 & 6 & 0.11 & -0.07 & 0.09 & 0.02 & -0.03 & 0.03 & -0.01 & 0.01 & -0.02 \\
\hline 7 & 7 & -0.01 & -0.06 & 0.06 & & -0.01 & 0.01 & 0.00 & 0.00 & -0.01 \\
\hline 8 & 6 & -0.11 & -0.06 & 0.01 & -0.03 & 0.01 & 0.01 & 0.02 & 0.00 & -0.01 \\
\hline 9 & 6 & -0.12 & -0.01 & -0.01 & -0.02 & -0.01 & -0.02 & 0.01 & -0.01 & 0.00 \\
\hline 10 & 6 & 0.02 & -0.06 & 0.06 & 0.01 & 0.00 & 0.00 & 0.00 & 0.01 & 0.00 \\
\hline 11 & 17 & -0.0 & 0.01 & 0.02 & -0.0 & 0.01 & -0.0 & 0.0 & 0.01 & -0.02 \\
\hline 12 & 17 & -0.02 & -0.03 & -0.01 & 0.0 & -0.01 & 0.01 & 0.01 & -0.01 & 0.02 \\
\hline 13 & 78 & 0.00 & 0.00 & 0.00 & 0.0 & 0.00 & 0.00 & 0.00 & 0.00 & 0.00 \\
\hline 14 & 8 & 0. & -0.01 & 0. & & 0.00 & 0.0 & 0.0 & 0.00 & 0.00 \\
\hline 15 & 7 & -0.02 & 0.07 & -0.04 & & 0.01 & -0.0 & 0. & -0.01 & 0.00 \\
\hline 16 & 6 & 0.02 & 0.15 & -0.11 & 0 & -0.01 & 0.00 & 0.00 & 0.01 & 0.00 \\
\hline 17 & 6 & 0.04 & -0.09 & 0.04 & & 0.30 & -0.17 & -0.0 & -0.18 & 0.10 \\
\hline 18 & 6 & 0. & 09 & 0.04 & & -0.10 & 0.0 & -0. & 0.06 & -0.03 \\
\hline 19 & 6 & 0. & & -0 & & 0.00 & 0.0 & -0. & & 0.00 \\
\hline 20 & 6 & 0.01 & -0 . & 0. & & 0.10 & -0.0 & & & 0.04 \\
\hline 21 & 6 & 0. & 0.00 & -0.01 & -0.02 & -0.30 & 0.1 & 0.02 & 0.18 & -0.10 \\
\hline 22 & 17 & & & -0.02 & & -0.03 & 0.0 & 0.0 & 0.01 & -0.01 \\
\hline 23 & 17 & & & & & & & & & \\
\hline 24 & 6 & -0 . & & 0. & & 0.00 & 0. & 0. & 0. & 0.00 \\
\hline 25 & 6 & & & -0.0 & & 0.02 & -0.0 & 0.0 & -0.01 & 0.01 \\
\hline 26 & 1 & -0 & & -0. & -0. & 0.03 & -0.0 & 0. & & 0.05 \\
\hline 27 & 1 & & & & & & & & & 0.00 \\
\hline 28 & 1 & & & 0. & & -0.01 & 0.0 & -0.03 & -0.02 & 0.00 \\
\hline 29 & 1 & 0.1 & & & & 0. & 0. & -0. & -0.01 & 0.00 \\
\hline 30 & 1 & & & & & & & & & 0.00 \\
\hline 31 & 1 & & & & & -0. & 0. & -0 & & -0.01 \\
\hline 32 & 1 & 0. & & 0. & -0.02 & -0.39 & 0.2 & 0.00 & 0.24 & -0.13 \\
\hline 33 & 1 & & & -0. & & -0.01 & & & & -0.01 \\
\hline 34 & 1 & & & & & & -0 . & & & 0.14 \\
\hline 35 & 1 & & & -0 & & 0.2 & -0. & -0.11 & 0. & -0.32 \\
\hline 36 & 1 & & & & & -0.06 & 0. & & & -0.05 \\
\hline 37 & 1 & -0 . & & -0 . & & -0.13 & 0. & 0. & -0 . & 0.38 \\
\hline 38 & 1 & -0. & & 0. & & 0.00 & 0. & 0. & & 0.01 \\
\hline 39 & 1 & -0.0 & & & & 0.0 & 0. & 0. & 0.01 & -0.01 \\
\hline 40 & 1 & 0.0 & & 0.0 & & 0.00 & 0.0 & & -0.01 & -0.01 \\
\hline 41 & 6 & 0.0 & & 0.0 & & 0.00 & 0.0 & 0.0 & 0.00 & 0.00 \\
\hline 42 & 1 & 0.02 & & 0.02 & 0.01 & 0.00 & 0.01 & 0.00 & 0.00 & 0.00 \\
\hline 43 & 6 & -0.0 & & 0.0 & & 0.00 & 0.0 & & & 0.00 \\
\hline 44 & 1 & -0.0 & & -0.01 & 0.00 & 0.00 & 0.00 & 0.00 & 0.00 & 0.00 \\
\hline 45 & 1 & 0.00 & -0.01 & 0.00 & 0.00 & 0.00 & 0.00 & 0.00 & 0.00 & 0.00 \\
\hline
\end{tabular}




\begin{tabular}{|c|c|c|c|c|c|c|c|c|c|c|}
\hline $\begin{array}{l}46 \\
47 \\
48\end{array}$ & $\begin{array}{l}9 \\
9 \\
9\end{array}$ & $\begin{array}{l}0.01 \\
0.00 \\
0.00\end{array}$ & $\begin{array}{r}-0.01 \\
0.01 \\
0.01\end{array}$ & $\begin{array}{r}0.01 \\
0.01 \\
-0.01\end{array}$ & $\begin{array}{l}0.00 \\
0.00 \\
0.00\end{array}$ & $\begin{array}{l}0.00 \\
0.00 \\
0.00\end{array}$ & $\begin{array}{l}0.00 \\
0.00 \\
0.00\end{array}$ & $\begin{array}{l}0.00 \\
0.00\end{array}$ & $\begin{array}{l}0.00 \\
0.00 \\
0.00\end{array}$ & $\begin{array}{l}0.00 \\
0.00 \\
0.00\end{array}$ \\
\hline \multicolumn{2}{|c|}{ Frequencies } & 550.80 & & & 577.08 & & & 581.00 & & \\
\hline Atom & AN & X & $Y$ & Z & $X$ & $Y$ & Z & X & $Y$ & Z \\
\hline 1 & 6 & 0.04 & -0.07 & 0.11 & 0.01 & 0.01 & -0.02 & 0.13 & -0.03 & -0.07 \\
\hline 2 & 6 & 0.00 & 0.00 & 0.01 & 0.02 & 0.00 & 0.0 & 0.10 & -0.03 & -0.06 \\
\hline 3 & 6 & -0.07 & 0.14 & -0.16 & 0.01 & -0.02 & 0.02 & 0.01 & -0.05 & -0.06 \\
\hline 4 & 6 & 0.02 & 0.00 & 0.01 & 0.00 & -0.01 & 0.00 & -0.09 & -0.08 & -0.04 \\
\hline 5 & 6 & 0.07 & -0.07 & 0.10 & -0.01 & 0.00 & -0. & -0 . & -0.10 & $-0 \cap 3$ \\
\hline 6 & 6 & -0.02 & 0.12 & -0.14 & 0.02 & -0.01 & 0.0 & -0.01 & 0.00 & 0.00 \\
\hline 7 & 7 & -0.03 & 0.00 & -0.02 & -0.01 & 0.01 & 0.0 & -0.01 & 0.12 & 0.11 \\
\hline 8 & 6 & 0.03 & & 0.01 & -0.02 & 0.01 & & & & 0.17 \\
\hline 9 & 6 & & & 0.04 & & -0.01 & & & -0.17 & -0.17 \\
\hline 10 & 6 & -0.02 & -0.07 & 0.06 & 0.0 & 0.00 & & & -0.01 & -0.01 \\
\hline 11 & 17 & & & & & & & & & \\
\hline 12 & 17 & -0.1 & & & 0.00 & 0.00 & & 0. & & 0.02 \\
\hline 13 & 78 & 0.00 & & & -0.02 & -0.02 & & & 0.00 & 0.00 \\
\hline 14 & 8 & 0. & & 0. & -0.0 & -0.04 & & & & .01 \\
\hline 15 & 7 & 0. & & & & -0.01 & -0. & 0. & -0.12 & -0.12 \\
\hline 16 & 6 & 0.02 & 0.15 & -0.1 & -0.01 & -0.02 & 0. & 0. & & 0.03 \\
\hline 17 & 6 & 0.1 & & -0.0 & -0.01 & 0.00 & & & & 04 \\
\hline $1 \varepsilon$ & 6 & 0. & & & -0. & & & & & \\
\hline 19 & 6 & -0.03 & 0.14 & -0.1 & 0.0 & -0.02 & & 0. & 0.06 & -0.04 \\
\hline 20 & 6 & & & 0.0 & 0.0 & 0.02 & & & & 0.12 \\
\hline 21 & 6 & & & & & & & & & \\
\hline 22 & 17 & & & & & & & & & \\
\hline 23 & 17 & & & & & & & & & -0.02 \\
\hline 24 & 6 & 0. & & & 0.2 & 0.25 & & & & \\
\hline 2 & 6 & & & & -0.0 & 0.01 & & & & \\
\hline 26 & 1 & & & & -0.01 & 0.02 & & & & -0.06 \\
\hline 27 & 1 & & & & & 1 & & & & -0.24 \\
\hline 28 & 1 & & & & & & & & & \\
\hline 2 & 1 & & & & & & & & & \\
\hline 30 & 1 & & & & & & & & & \\
\hline 3 & 1 & & & & & & & & & \\
\hline 32 & 1 & & & & & & & & & \\
\hline 33 & 1 & & & & & & & & & \\
\hline 34 & 1 & & & & & & & & & \\
\hline 35 & 1 & & & & & & & & & \\
\hline 36 & 1 & & & & & & & & & \\
\hline 37 & 1 & & & & & & & & & -0 . \\
\hline 38 & 1 & & & & & & & & & \\
\hline 39 & 1 & & & & & & & & & \\
\hline 40 & 1 & & & & & & & & & \\
\hline 41 & 6 & & & & & & & & & \\
\hline 42 & 1 & & & & & & & & & 0.00 \\
\hline 43 & 6 & & & & & & & & & \\
\hline 44 & 1 & & & & & & & & & \\
\hline 45 & 1 & & & & -0.0 & & & & 01 & 0.00 \\
\hline 46 & 9 & & & & & & & & & \\
\hline 47 & 9 & & & & & & & & & \\
\hline 48 & 9 & 0.00 & & & -0.01 & 0.01 & 0.00 & 0.00 & -0.01 & 0.00 \\
\hline Frequen & & & & & & & & 628.97 & & \\
\hline & AN & X & $Y$ & Z & & Y & & $x$ & Y & \\
\hline & 6 & -0.07 & 0.0 & -0.0 & 0. & -0.01 & 0.0 & 0.1 & -0.02 & -0.06 \\
\hline 2 & 6 & & & & & 0.02 & -0. & & -0.02 & -0.06 \\
\hline 3 & 6 & & & & -0.1 & 0.02 & & & -0.0 & -0.03 \\
\hline 4 & 6 & & & & 0.0 & 0.01 & & & -0.07 & -0.02 \\
\hline 5 & 6 & -0.05 & 0.04 & -0.02 & 0.02 & -0.03 & 0.02 & -0.12 & -0.08 & -0.03 \\
\hline
\end{tabular}




\begin{tabular}{|c|c|c|c|c|c|c|c|c|c|c|}
\hline & & & & & & & & & & \\
\hline 6 & 6 & -0.07 & -0.07 & 0.06 & 0.02 & 0.04 & -0.04 & -0.02 & 0.00 & 0.01 \\
\hline 7 & 7 & 0.06 & 0.05 & -0.08 & -0.02 & -0.03 & 0.04 & -0.02 & 0.21 & 0.18 \\
\hline 8 & 6 & 0.08 & 0.06 & -0.07 & -0.04 & -0.04 & 0.03 & 0.02 & 0.00 & -0.03 \\
\hline 9 & 6 & 0.09 & -0.01 & 0.08 & -0.06 & 0.01 & -0.03 & 0.04 & -0.13 & -0.10 \\
\hline 10 & 6 & -0.03 & -0.04 & 0.04 & 0.01 & 0.01 & -0.01 & -0.01 & -0.04 & 0.02 \\
\hline 11 & 17 & 0.02 & -0.01 & -0.01 & -0.01 & 0.00 & 0.00 & & & 0.01 \\
\hline 12 & 17 & 0.01 & 0.01 & 0.01 & 0.00 & 0.00 & 0.0 & 0. & 0.01 & 0.01 \\
\hline 13 & 78 & 0.00 & & 0.00 & & & & 0.00 & -0.01 & -0.01 \\
\hline 14 & 8 & -0.04 & -0.05 & 0.01 & -0.05 & -0.06 & 0.03 & -0.01 & -0.02 & 0.00 \\
\hline 15 & 7 & 0.07 & -0.06 & 0.04 & -0.04 & 0.03 & -0.03 & -0.02 & 0.24 & 0.27 \\
\hline 16 & 6 & 0.04 & 0.05 & -0.03 & -0.03 & -0.02 & 0.0 & 0.02 & -0.05 & -0.04 \\
\hline 17 & 6 & -0.01 & 0.01 & -0.03 & 0.00 & -0.01 & 0.02 & 0.11 & -0.06 & -0.04 \\
\hline 18 & 6 & -0.04 & -0.06 & 0.00 & 0.01 & 0.03 & 0.00 & 0.15 & -0.02 & -0.07 \\
\hline 19 & 6 & -0.09 & & -0.0 & 0.05 & -0.03 & 0.0 & -0.03 & -0.03 & 0.02 \\
\hline 20 & 6 & -0.06 & -0.04 & 0.03 & 0.03 & 0.02 & -0.01 & -0.15 & -0.02 & -0.10 \\
\hline 21 & 6 & -0.02 & & 0.00 & 0.01 & -0.02 & 0.00 & -0.10 & -0.04 & -0.07 \\
\hline 22 & 17 & 0.01 & & 0.02 & -0.01 & 0.00 & -0. & 0.0 & 0.01 & 0.02 \\
\hline 23 & 17 & 0.02 & & -0.01 & -0.01 & & 0.00 & 0.0 & 0.00 & 0.01 \\
\hline 24 & 6 & -0.03 & -0.02 & 0.03 & -0.06 & -0.04 & 0.0 & -0. & -0.01 & 0.00 \\
\hline 25 & 6 & -0.05 & & -0. & 0.03 & -0.02 & 0. & -0. & 0.01 & -0.01 \\
\hline 26 & 1 & 0.01 & & 0.11 & & & -0. & -0. & 0.02 & 0.05 \\
\hline 27 & 1 & -0.1 & & -0.04 & & -0.02 & 0. & & 0.00 & 0.05 \\
\hline 28 & 1 & -0.20 & & -0.10 & & -0.02 & 0. & -0 & 0.06 & -0.04 \\
\hline 29 & 1 & -0.14 & & 0.02 & & 0.05 & -0.02 & -0.16 & -0.15 & \\
\hline 30 & 1 & -0.16 & & 0.07 & 0.07 & 0.04 & -0.02 & 0.08 & 0.26 & -0.06 \\
\hline 31 & 1 & -0.02 & & -0.13 & 0.01 & -0.08 & 0.0 & -0. & 0.08 & 0.15 \\
\hline 32 & 1 & -0.02 & & 0.07 & 0.00 & 0.07 & -0.0 & 0.22 & 0.06 & -0.06 \\
\hline 33 & 1 & -0.08 & & -0.07 & 0.05 & -0.05 & 0.0 & -0.07 & 0.06 & 0.20 \\
\hline 34 & 1 & -0.0 & & 0.07 & 0.0 & 0.07 & -0.04 & -0.19 & 0.03 & -0.11 \\
\hline 35 & 1 & -0.04 & & -0.11 & & -0.07 & 0.0 & -0 . & -0.07 & -0.02 \\
\hline 36 & 1 & -0. & & 0.09 & 0.01 & 0.06 & -0.0 & -0.04 & 0.12 & 0.12 \\
\hline 37 & 1 & -0. & & -0.11 & 0.04 & -0.07 & 0.0 & 0.17 & 0.01 & -0.05 \\
\hline 38 & 1 & & & 0. & -0.08 & 07 & 0. & 0. & 0.10 & -0.01 \\
\hline 38 & 1 & & & & -0.02 & -0.0 & 0. & -0 . & -0.05 & 0.13 \\
\hline 40 & 1 & 0. & & -0. & & -0.0 & 0. & & 0.07 & 0.03 \\
\hline 41 & 6 & -0.04 & -0.02 & 0.0 & -0.08 & -0.02 & -0.0 & 0.0 & 0.02 & 0.02 \\
\hline 42 & 1 & 0.3 & & 0. & 0.46 & & 0.6 & 0.1 & 0.06 & 0.12 \\
\hline 43 & 6 & & & & & & & & & 0.01 \\
\hline 44 & 1 & 0. & & -0.0 & 0. & & -0 . & 0. & 0.02 & 0.00 \\
\hline 45 & 1 & -0.0 & & & -0.16 & & -0.02 & -0.01 & 0.03 & 0.01 \\
\hline 46 & 9 & & & & & & & & 0.00 & -0.01 \\
\hline 47 & 9 & & & & & & & & & 0.01 \\
\hline 48 & 9 & -0.02 & 0. & 0.0 & -0.03 & 0.03 & 0.00 & 0.00 & -0.02 & 0.00 \\
\hline Frequen & & & & & & & & 689.36 & & \\
\hline Atom & AN & $X$ & $Y$ & Z & y & $\mathrm{Y}$ & Z & $x$ & $Y$ & Z \\
\hline 1 & 6 & & 0.01 & -0.0 & & -0.02 & -0.04 & -0. & -0.10 & -0.06 \\
\hline 2 & 6 & & -0.01 & 0.01 & 0.05 & 0.00 & -0.04 & 0.02 & -0.07 & -0.07 \\
\hline 3 & 6 & & & 0.01 & & & -0.01 & & 0.05 & -0.02 \\
\hline 4 & 6 & & & 0. & & -0.04 & 0.0 & & & 0.06 \\
\hline 5 & 6 & & & & $-0 .($ & -0.06 & -0.02 & -0.06 & 0.07 & 0.08 \\
\hline 6 & 6 & & & & & & 0.0 & & -0.07 & 0.04 \\
\hline 7 & 7 & & & -0.0 & & 0.2 & 0.1 & & 0.04 & -0.03 \\
\hline 8 & 6 & & & 0. & & -0.17 & -0.18 & 0.10 & 0.07 & -0.02 \\
\hline 9 & 6 & & & -0.1 & -0.0 & 0.14 & 0.1 & -0.08 & 0.01 & -0.05 \\
\hline 10 & 6 & & & -0.0 & & 0.03 & 0. & & -0.03 & 0.02 \\
\hline 11 & 17 & & & 0.00 & 0.0 & 0.00 & 0.00 & 0.03 & -0.02 & -0.02 \\
\hline 12 & 17 & & & & & & 0.0 & 0.02 & 0.03 & 0.02 \\
\hline 13 & 78 & & & & & & 0. & & 0.00 & 0.00 \\
\hline 14 & 8 & & & 0.1 & 0.01 & 0.04 & 0.04 & 0.00 & 0.01 & 0.00 \\
\hline 15 & 7 & 0.00 & 0.04 & 0.05 & 0.00 & -0.10 & -0.09 & -0.24 & -0.05 & 0.05 \\
\hline
\end{tabular}




\begin{tabular}{|c|c|c|c|c|c|c|c|c|c|c|}
\hline 16 & 6 & 0.01 & 0.00 & 0.00 & -0.01 & 0.00 & 0.01 & -0.22 & -0.02 & -0.02 \\
\hline 17 & 6 & 0.02 & 0.00 & -0.01 & -0.02 & 0.02 & 0.01 & -0.01 & 0.11 & 0.07 \\
\hline 18 & 6 & 0.02 & -0.01 & -0.01 & -0.03 & 0.00 & 0.02 & 0.07 & 0.02 & 0.14 \\
\hline 19 & 6 & -0.02 & 0.00 & 0.00 & 0.02 & 0.01 & -0.01 & 0.34 & 0.06 & 0.03 \\
\hline 20 & 6 & -0.02 & -0.01 & -0.01 & 0.03 & 0.00 & 0.02 & 0.07 & -0.11 & -0.10 \\
\hline 21 & 6 & -0.01 & 0.00 & -0.01 & 0.02 & 0.01 & 0.01 & 0.00 & -0.01 & -0.14 \\
\hline 22 & 17 & 0.00 & 0.00 & 0.00 & 0.00 & 0.00 & 0.00 & -0.03 & -0.02 & -0.04 \\
\hline 23 & 17 & 0.00 & 0.00 & 0.00 & 0.00 & 0.00 & 0.00 & -0.04 & 0.02 & 0.03 \\
\hline 24 & 6 & 0.03 & 0.02 & -0.03 & 0.01 & 0.01 & 0.01 & 0.02 & 0.01 & -0.01 \\
\hline 25 & 6 & 0.00 & 0.01 & 0.00 & 0.01 & -0.03 & -0.02 & 0.09 & -0.05 & 0.07 \\
\hline 26 & 1 & 0.02 & -0.05 & -0.01 & -0.07 & 0.15 & 0.10 & 0.13 & -0.13 & 0.15 \\
\hline 27 & 1 & -0.07 & 0.03 & -0.08 & 0.25 & -0.11 & 0.34 & 0.01 & -0.05 & 0.03 \\
\hline 28 & 1 & 0.04 & -0.06 & 0.02 & -0.23 & 0.26 & -0.11 & 0.02 & -0.06 & 0.05 \\
\hline 29 & 1 & -0.06 & -0.05 & 0.09 & 0.13 & 0.14 & -0.26 & -0.03 & -0.03 & 0.04 \\
\hline 30 & 1 & 0.03 & 0.11 & -0.04 & -0.13 & -0.32 & 0.11 & -0.01 & 0.01 & \\
\hline 31 & 1 & -0.02 & 0.05 & 0.03 & 0.05 & -0.07 & -0.16 & -0.05 & -0.06 & 0.06 \\
\hline 32 & 1 & 0.03 & -0.02 & 0.00 & -0.05 & -0.03 & 0.02 & -0.11 & -0.17 & 0.10 \\
\hline 33 & 1 & -0.02 & 0.01 & 0.02 & 0.02 & -0.01 & -0.04 & 0.34 & 0.04 & \\
\hline 34 & 1 & -0.03 & -0.03 & 0.00 & 0.03 & -0.02 & 0.03 & -0.15 & -0.19 & 0.05 \\
\hline 35 & 1 & 0.00 & 0.03 & -0.03 & -0.10 & -0.05 & -0.01 & 0.10 & 0.04 & 0.01 \\
\hline 36 & 1 & 0.01 & -0.02 & 0.01 & -0.02 & 0.09 & 0.08 & -0.27 & -0.08 & 0.04 \\
\hline 37 & 1 & -0.02 & 0.03 & -0.03 & 0.12 & 0.00 & -0.03 & 0. & 0.00 & \\
\hline 38 & 1 & 0.08 & 0.09 & -0.05 & -0.08 & -0.10 & 0.02 & 0.02 & 0.04 & -0.04 \\
\hline 39 & 1 & -0.03 & 0.02 & 0.02 & 0.11 & 0.06 & -0.13 & 0.00 & 0.03 & -0.02 \\
\hline 40 & 1 & -0.07 & 0.01 & 0.04 & -0.03 & -0.07 & -0.03 & -0 . & 0. & \\
\hline 41 & 6 & -0.18 & -0.10 & -0.09 & -0.03 & -0.01 & -0.01 & 0. & 0.00 & \\
\hline 42 & 1 & -0.53 & -0.19 & -0.46 & -0.12 & -0.04 & -0.10 & -0.04 & -0.03 & -0.06 \\
\hline 43 & 6 & 0.00 & -0.16 & -0.11 & 0.00 & -0.02 & -0.02 & 0. & 0.00 & 0.00 \\
\hline 44 & 1 & -0.19 & -0.10 & -0.09 & -0.01 & -0.01 & -0.02 & -0.01 & 0.00 & \\
\hline 45 & 1 & -0.16 & -0.13 & -0.09 & -0.04 & 0.00 & -0.01 & 0.02 & -0.01 & 0.00 \\
\hline 46 & 9 & -0.06 & 0.06 & 0.11 & -0.01 & 0.00 & 0.02 & 0.00 & 0.00 & 0.00 \\
\hline 47 & 9 & 0.25 & -0.13 & -0.06 & 0.04 & -0.02 & -0.01 & 0.00 & 0.00 & \\
\hline 48 & 9 & -0.05 & 0.14 & 0.00 & -0.01 & 0.02 & -0.01 & 0.00 & 0.00 & 0.00 \\
\hline Freque & & 717.13 & & & 739.73 & & & 781.17 & & \\
\hline Atom & AN & X & Y & Z & X & $Y$ & Z & X & $Y$ & \\
\hline & 6 & -0.01 & 0.11 & 0.02 & 0.02 & -0.05 & 0.04 & -0.02 & 0.05 & 0.06 \\
\hline 2 & 6 & 0.00 & -0.01 & 0.12 & -0.04 & 0.08 & -0.11 & -0.1 & 0.08 & 0.00 \\
\hline 3 & 6 & -0.15 & 0.02 & -0.05 & 0.06 & -0.12 & 0.15 & -0.01 & -0.12 & 0.14 \\
\hline 4 & 6 & 0.01 & -0.12 & 0.02 & -0.04 & 0.09 & -0.10 & -0.04 & -0.01 & -0.08 \\
\hline 5 & 6 & 0.01 & -0.03 & -0.10 & 0.02 & -0.03 & 0.05 & -0.0 & -0.07 & -0.05 \\
\hline 6 & 6 & 0.21 & 0.00 & 0.03 & -0.05 & 0.07 & -0.09 & 0. & 0.08 & -0.07 \\
\hline 7 & 7 & -0.12 & 0.03 & -0.05 & 0.03 & -0.06 & 0.05 & 0. & 0.03 & -0.04 \\
\hline 8 & 6 & 0.03 & 0.01 & 0.00 & & -0.03 & 0.01 & & & -0.03 \\
\hline 9 & 6 & 0.07 & 0.00 & 0.01 & 0.04 & 0.00 & 0.01 & 0. & -0.02 & 0.03 \\
\hline 10 & 6 & 0.05 & 0.09 & -0.07 & 0.04 & 0.06 & -0.05 & 0.03 & 0.01 & 0.00 \\
\hline 11 & 17 & -0.02 & 0.01 & 0.01 & & 0.00 & 0.0 & -0 & & 0.01 \\
\hline 12 & 17 & -0.01 & -0.02 & -0.01 & & 0.00 & 0.0 & & -0.01 & -0.01 \\
\hline 13 & 78 & 0.01 & 0.00 & 0.00 & 0.00 & 0.00 & 0.00 & 0.00 & 0.00 & 0.00 \\
\hline 14 & 8 & -0.01 & -0.02 & 0.00 & 0.00 & 0.00 & 0.00 & 0. & 0.00 & 0.00 \\
\hline 15 & 7 & -0.08 & -0.07 & 0.10 & & -0.03 & 0.0 & & & \\
\hline 16 & 6 & -0.07 & -0.11 & 0.05 & & -0.14 & 0.0 & & 0.17 & -0.09 \\
\hline 17 & 6 & 0.00 & 0.13 & -0.03 & 0.02 & 0.09 & -0.07 & -0.04 & -0.03 & 0.10 \\
\hline 18 & 6 & 0.02 & -0.05 & 0.07 & & -0.06 & 0.01 & -0.01 & & \\
\hline 19 & 6 & 0.11 & 0.10 & -0.04 & -0.0 & 0.08 & -0.05 & 0.1 & -0.06 & 0.05 \\
\hline 20 & 6 & -0.01 & -0.09 & -0.01 & -0.01 & -0.04 & 0.04 & -0.02 & -0.03 & -0.09 \\
\hline 21 & 6 & -0.01 & 0.09 & -0.10 & 0.01 & 0.11 & -0.05 & -0.04 & -0.09 & -0.01 \\
\hline 22 & 17 & -0.01 & -0.01 & -0.01 & 0.0 & 0.00 & 0.00 & -0. & -0.01 & -0. \\
\hline 23 & 17 & -0.01 & 0.00 & 0.01 & 0.0 & 0.00 & 0.00 & -0.1 & 0.00 & 0.00 \\
\hline 24 & 6 & 0.0 & -0.01 & 0.01 & 0.0 & 0.01 & -0.0 & 0. & 0.01 & 0.00 \\
\hline 25 & 6 & 0.03 & -0.03 & 0.03 & -0.06 & 0.05 & -0.06 & 0.01 & 0.02 & -0.01 \\
\hline
\end{tabular}




\begin{tabular}{|c|c|c|c|c|c|c|c|c|c|c|}
\hline & & & & & & & & & & \\
\hline & 1 & 0.06 & -0.09 & 0.11 & -0.10 & 0.13 & -0.15 & 0.07 & -0.12 & 0.13 \\
\hline 27 & 1 & -0.03 & -0.03 & 0.03 & 0.02 & 0.05 & -0.04 & -0.11 & 0.01 & -0.03 \\
\hline 28 & 1 & -0.05 & -0.02 & 0.01 & 0.04 & 0.04 & -0.02 & -0.13 & 0.01 & $\cap \cap 0$ \\
\hline 29 & 1 & -0.07 & 0.01 & -0.02 & -0.02 & 0.03 & -0.03 & -0.12 & -0.08 & 0.02 \\
\hline 30 & 1 & -0.04 & 0.08 & -0.07 & 0.00 & 0.06 & -0.05 & -0.11 & -0.05 & 0.02 \\
\hline 31 & 1 & 0.04 & 0.23 & -0.17 & 0.03 & 0.13 & -0.10 & 0.03 & 0.18 & -0.16 \\
\hline 32 & 1 & -0.05 & -0.34 & 0.18 & 0.01 & -0.31 & 0.16 & -0.09 & 0.27 & -0 . \\
\hline 33 & 1 & 0.09 & 0.00 & 0.05 & -0.04 & -0.06 & 0.04 & 0.13 & 0.20 & -0.06 \\
\hline 34 & 1 & -0.10 & -0.35 & 0.17 & -0.01 & -0.31 & 0.18 & -0.10 & & -0.21 \\
\hline 35 & 1 & -0.18 & 0.13 & -0.22 & 0.15 & -0.28 & 0.34 & -0.01 & -0.26 & \\
\hline 36 & 1 & 0.18 & 0.07 & -0.04 & & & 0.08 & 0.18 & -0.11 & 0.17 \\
\hline 37 & 1 & -0.18 & 0.17 & -0.19 & 0.14 & -0.28 & 0.34 & -0.01 & -0.23 & 0.28 \\
\hline 38 & 1 & 0.00 & 0.03 & -0.03 & 0.00 & & 0.01 & -0.02 & -0.03 & \\
\hline 39 & 1 & -0.01 & 0.02 & -0.01 & -0.01 & -0.01 & 0.02 & 0.01 & -0.01 & 0.00 \\
\hline 40 & 1 & -0.06 & -0.03 & 0.03 & 0.03 & 0.03 & -0.01 & 0.03 & 0.01 & -0.02 \\
\hline 41 & 6 & -0.0 & 0.00 & 0.00 & 0.00 & 0.0 & 0.00 & 0.00 & 0.00 & 0.00 \\
\hline 42 & 1 & 0.0 & 0.06 & 0.09 & 0.00 & & -0.01 & -0.01 & -0.01 & -0.02 \\
\hline 43 & 6 & 0.00 & 0.00 & 0.00 & 0.00 & 0.00 & 0.00 & 0.00 & 0.00 & 0.00 \\
\hline 44 & 1 & 0.01 & 0.01 & -0.01 & 0.00 & 0.00 & 0.00 & 0.00 & 0.00 & 0.01 \\
\hline 45 & 1 & -0.02 & 0.01 & 0.00 & 0.00 & & 0.00 & 0.01 & 0.00 & \\
\hline 46 & 9 & 0.01 & 0.00 & -0.01 & 0.00 & & 0.00 & 0.00 & & 0.00 \\
\hline 47 & 9 & 0.00 & 0.00 & 0.00 & 0.00 & 0.00 & 0.00 & 0.00 & 0.00 & 0.00 \\
\hline 48 & 9 & 0.00 & 0.00 & 0.00 & 0.00 & & 0.00 & 0.00 & & \\
\hline requen & & 815.37 & & & 817.48 & & & 818.00 & & \\
\hline Atom & AN & $X$ & $Y$ & Z & $X$ & $Y$ & Z & $x$ & $Y$ & Z \\
\hline 1 & 6 & 0.01 & -0.05 & 0.05 & -0.01 & 0.04 & -0.07 & 0.19 & -0.08 & -0.07 \\
\hline 2 & 6 & -0.02 & 0.00 & -0.07 & 0.03 & -0.03 & 0.06 & 0.07 & & 0.16 \\
\hline 3 & 6 & 0.01 & -0.03 & -0.02 & -0.01 & 0.04 & -0.02 & -0.03 & 0.21 & 0.22 \\
\hline 4 & 6 & 0.01 & 0.02 & -0.06 & 0.01 & -0.03 & 0.07 & -0.17 & 0.23 & 0.19 \\
\hline 5 & 6 & 0.04 & -0.01 & 0.07 & -0.03 & 0.04 & -0.06 & -0.13 & -0.17 & -0.02 \\
\hline 6 & 6 & -0.01 & -0.03 & 0.04 & -0.02 & 0.02 & -0.03 & 0.01 & -0.05 & -0.01 \\
\hline 7 & 7 & 0.00 & -0.02 & 0.03 & -0.01 & 0.01 & -0.02 & 0.01 & -0.06 & -0.05 \\
\hline 8 & 6 & -0.0 & -0.01 & 0.02 & 0.0 & 0.01 & 0.00 & 0.0 & 0.00 & 0.01 \\
\hline 9 & 6 & 0.0 & -0.01 & 0.01 & -0.02 & 0.00 & -0.01 & 0.0 & 0.0 & 0.00 \\
\hline 10 & 6 & 0.0 & 0.04 & -0.03 & & -0.01 & 0.01 & 0.0 & & 0.00 \\
\hline 11 & 17 & & 0.00 & 0.01 & & 0.00 & -0.01 & 0.0 & -0.06 & -0.07 \\
\hline 12 & 17 & 0. & 0.01 & 0.01 & & 0.00 & -0.0 & -0.0 & & -0.06 \\
\hline 13 & 78 & & 0.00 & 0.00 & & & 0.0 & & & \\
\hline 14 & 8 & 0.0 & 0.00 & 0.00 & & 0.0 & 0.0 & 0.0 & 0. & 0.00 \\
\hline 15 & 7 & -0. & -0.06 & 0.01 & & 0.00 & -0.0 & 0.0 & & 0.0 \\
\hline 16 & 6 & -0.0 & 0.11 & 0.15 & & & 0.0 & & & \\
\hline 17 & 6 & 0.0 & 0.11 & 0.15 & & 0.01 & 0.1 & 0.0 & 0.00 & 0.0 \\
\hline 18 & 6 & 0.1 & -0.07 & -0.04 & & 0.02 & -0.0 & 0.0 & & \\
\hline 19 & 6 & & -0.04 & -0.01 & & & & & & 0.0 \\
\hline 20 & 6 & -0.0 & -0.06 & -0.0 & & & -0.0 & & 0. & \\
\hline 21 & 6 & & 0.11 & 0.13 & & 0.02 & 0.1 & -0.0 & 0. & \\
\hline 22 & 17 & -0. & -0.03 & -0.05 & & -0.01 & -0.0 & & & \\
\hline 23 & 17 & & -0.03 & -0.0 & & & -0.1 & & & \\
\hline 24 & 6 & & 0.02 & 0.0 & & & 0.1 & 0.1 & & \\
\hline 25 & 6 & -0.0 & 0.01 & -0.01 & 0.0 & -0.01 & 0.0 & -0.01 & & 0.01 \\
\hline 26 & 1 & -0. & 0.06 & -0.08 & & & 0.0 & & & -0.04 \\
\hline 27 & 1 & & 0.02 & -0.0 & & & 0.0 & -0.1 & & \\
\hline 28 & 1 & 0.0 & 0.00 & 0.01 & 0.0 & -0.02 & 0.02 & 0.04 & & 0.02 \\
\hline 29 & 1 & 0.0 & 0.03 & -0.04 & & & -0.02 & 0.00 & & -0.01 \\
\hline 30 & 1 & -0.0 & 0.00 & -0.0 & & -0.02 & 0.0 & -0.0 & & 0.0 \\
\hline 31 & 1 & 0.0 & 0.06 & -0.0 & -0.01 & -0.06 & 0.0 & 0.01 & 0.01 & -0.02 \\
\hline 32 & 1 & & 0.12 & & & & 0.0 & 0.0 & & 0.01 \\
\hline 33 & 1 & -0.0 & 0.34 & & & & 0.2 & 0.0 & & \\
\hline 34 & 1 & 0.0 & 0.12 & -0.2 & 0.0 & & 0.08 & -0.01 & -0.03 & 0.00 \\
\hline 35 & 1 & -0.08 & 0.25 & -0.24 & 0.11 & -0.23 & 0.26 & -0.04 & -0.01 & \\
\hline
\end{tabular}




\begin{tabular}{|c|c|c|c|c|c|c|c|c|c|c|}
\hline & & & & & & & & & & \\
\hline 36 & 1 & -0.16 & 0.27 & -0.39 & 0.15 & -0.32 & 0.42 & -0.15 & 0.46 & -0.07 \\
\hline & 1 & -0.08 & 0.24 & -0.25 & 0.11 & -0.24 & 0.26 & -0.05 & 0.00 & -0.34 \\
\hline 38 & 1 & -0.09 & -0.10 & 0.04 & -0.05 & -0.04 & 0.00 & -0.09 & -0.11 & 0.04 \\
\hline 39 & 1 & 0.10 & 0.04 & -0.09 & 0.07 & 0.05 & -0.08 & 0.10 & 0.05 & -0.10 \\
\hline 40 & 1 & 0.01 & -0.05 & -0.04 & -0.05 & -0.05 & 0.00 & 0.01 & -0.04 & -0.03 \\
\hline 41 & 6 & 0.00 & 0.00 & 0.00 & -0.03 & -0.02 & -0. & -0.03 & & -0.02 \\
\hline 42 & 1 & 0.00 & 0.01 & 0.00 & -0.01 & 0.01 & & -0.01 & & -0.01 \\
\hline 43 & 6 & 0.00 & 0.00 & 0.00 & 0.00 & 0.01 & 0.0 & 0.00 & 0.01 & 0.00 \\
\hline 44 & 1 & 0.00 & 0.00 & 0.00 & -0.02 & -0.03 & -0.03 & -0.03 & -0.04 & -0.02 \\
\hline 45 & 1 & 0.00 & & 0.00 & -0.03 & -0.03 & & & & 0.02 \\
\hline 46 & 9 & 0.00 & 0.00 & 0.00 & 0.02 & 0.01 & -0.0 & 0.02 & 0.01 & -0.02 \\
\hline 47 & 9 & 0.00 & 0.00 & 0.00 & -0.01 & 0.01 & 0.00 & -0.01 & 0.01 & 0.00 \\
\hline 48 & 9 & 0.00 & 0.00 & 0.00 & 0.01 & -0.01 & 0.02 & 0.02 & -0.01 & 0.03 \\
\hline requen & & 819.33 & & & 821.78 & & & 846.59 & & \\
\hline Atom & AN & X & Y & Z & $x$ & $Y$ & Z & $x$ & $Y$ & Z \\
\hline 1 & 6 & 0.01 & 0.02 & -0.06 & 0.01 & 0.01 & -0.01 & -0.01 & 0.00 & 0.01 \\
\hline 2 & 6 & 0.03 & -0.02 & 0.05 & 0.01 & 0.02 & 0.03 & -0.01 & -0.01 & -0.01 \\
\hline 3 & 6 & -0.01 & 0.05 & -0.02 & -0.01 & 0.03 & 0.01 & 0.00 & -0.02 & 0.00 \\
\hline 4 & 6 & 0.01 & -0.01 & 0.07 & -0.01 & 0.01 & 0.03 & 0.00 & -0.01 & -0.02 \\
\hline 5 & 6 & -0.02 & 0.03 & -0.03 & -0.02 & -0.01 & -0.02 & 0.0 & 0.00 & 0.00 \\
\hline 6 & 6 & -0.02 & 0.01 & -0.02 & 0.01 & 0.00 & -0.01 & 0.0 & 0.00 & 0.00 \\
\hline 7 & 7 & -0.01 & -0.01 & 0.00 & 0.01 & -0.01 & -0.01 & 0.0 & 0.00 & -0.01 \\
\hline 8 & 6 & -0.01 & -0.01 & 0.01 & 0.00 & 0.01 & 0.0 & 0.01 & 0.01 & 0.00 \\
\hline 9 & 6 & -0.01 & 0.00 & 0.00 & -0.01 & 0.00 & -0.01 & -0.01 & -0.01 & -0.02 \\
\hline 10 & 6 & 0.00 & 0.01 & -0.01 & 0.00 & -0.01 & 0.00 & -0.01 & -0.01 & 0.00 \\
\hline 11 & 17 & 0. & 0.00 & -0.01 & 0.0 & -0.01 & -0.0 & 0.0 & 0.00 & 0.00 \\
\hline 12 & 17 & $0 .($ & 0.00 & -0.01 & 0.0 & -0.01 & -0.01 & 0.00 & 0.00 & 0.00 \\
\hline 13 & 78 & 0.00 & 0.00 & 0.00 & 0.00 & 0.00 & 0.00 & 0.00 & 0.00 & 0.00 \\
\hline 14 & 8 & 0.00 & 0.00 & 0.00 & -0.06 & -0.02 & -0.04 & 0.0 & 0.00 & 0.00 \\
\hline 15 & 7 & -0.02 & -0.01 & 0.01 & 0.0 & 0.01 & 0. & $-0 .($ & 0. & 3 \\
\hline 16 & 6 & 0.00 & -0.04 & 0.02 & 0.00 & 0.01 & 0.0 & 0.0 & -0.02 & -0.02 \\
\hline 17 & 6 & 0. & 0.06 & -0.04 & 0.01 & 0.00 & 0.0 & 0.0 & -0.01 & -0.03 \\
\hline 18 & 6 & 0. & -0.08 & 0. & & 0.02 & -0. & 0. & 0.00 & 0.00 \\
\hline 19 & 6 & & & 0.02 & & 0.02 & -0.0 & -0.01 & 0. & 0.00 \\
\hline 20 & 6 & 0.00 & & 0.06 & -0.01 & 0.02 & -0.0 & 0.01 & & \\
\hline 21 & 6 & 0.0 & 0.08 & -0.03 & -0.02 & 0.00 & 0.0 & 0.01 & -0.01 & -0.02 \\
\hline 22 & 17 & & & 0. & & 0.00 & -0. & 0. & 0.00 & 0.01 \\
\hline 23 & 17 & & & & & & & & & \\
\hline 24 & 6 & & & 0. & & 0.02 & 0. & 0. & 9 & 0.09 \\
\hline 25 & 6 & & & 0. & & 0.00 & 0.8 & 0. & 0.00 & 0.01 \\
\hline 26 & 1 & & & & & -0.01 & 0. & & -0.03 & 0.02 \\
\hline 27 & 1 & & & & & 0.1 & -0 & & & -0.01 \\
\hline 28 & 1 & & & 0. & & -0.01 & 0.0 & 0.0 & -0.01 & 0.00 \\
\hline 29 & 1 & & & -0 & & & 0. & 0. & -0.01 & 0.02 \\
\hline 30 & 1 & & & & & & & & & -0.01 \\
\hline 31 & 1 & & & & & -0 . & 0. & -0 . & & 0. \\
\hline 32 & 1 & & & -0 & 0.00 & -0.15 & 0. & 0. & 0.01 & 0.01 \\
\hline 33 & 1 & & & -0 & & & & & & -0.01 \\
\hline 34 & 1 & & & -0 . & & & & & & \\
\hline 35 & 1 & & & & & -0.05 & 0. & -0. & 01 & 0.00 \\
\hline 36 & 1 & & & & & & & & & -0.02 \\
\hline 37 & 1 & & & 0. & 0. & & 0. & & & 0.01 \\
\hline 38 & 1 & & & -0. & -0.1 & -0.12 & 0. & -0.41 & -0.43 & 0.13 \\
\hline 39 & 1 & 0.0 & & -0. & 0.1 & 0.03 & -0 . & 0. & 0.26 & -0.46 \\
\hline 40 & 1 & & & 0.0 & & -0.02 & -0 . & & -0.26 & -0.13 \\
\hline 41 & 6 & 0.0 & 0.0 & 0.0 & 0.31 & 0.22 & 0.1 & -0.01 & -0.01 & -0.01 \\
\hline 42 & 1 & 0.01 & 0.0 & 0.0 & 0.15 & -0.02 & 0.08 & -0.04 & 0.00 & -0.03 \\
\hline 43 & 6 & 0.0 & & 0.0 & 0.00 & -0.04 & 0.0 & 0.00 & 0.00 & 0.00 \\
\hline 44 & 1 & & & 0.0 & 0.27 & 0.33 & 0.2 & 0.00 & -0.01 & -0.01 \\
\hline 45 & 1 & 0.01 & 0.01 & 0.01 & 0.24 & 0.30 & 0.19 & -0.01 & -0.01 & -0.01 \\
\hline
\end{tabular}




\begin{tabular}{|c|c|c|c|c|c|c|c|c|c|c|}
\hline $\begin{array}{r}46 \\
47 \\
48\end{array}$ & $\begin{array}{l}9 \\
9 \\
9\end{array}$ & $\begin{array}{r}-0.01 \\
0.00 \\
-0.01\end{array}$ & $\begin{array}{r}-0.01 \\
0.00 \\
0.00\end{array}$ & $\begin{array}{r}0.01 \\
0.00 \\
-0.01\end{array}$ & $\begin{array}{c}-0.17 \\
0.13 \\
-0.14\end{array}$ & $\begin{array}{l}-0.15 \\
-0.07 \\
0.09\end{array}$ & $\begin{array}{c}0.16 \\
-0.04 \\
-0.24\end{array}$ & $\begin{array}{l}0.00 \\
0.00 \\
0.00\end{array}$ & $\begin{array}{l}0.00 \\
0.00 \\
0.00\end{array}$ & $\begin{array}{l}0.00 \\
0.00 \\
0.00\end{array}$ \\
\hline \multicolumn{2}{|c|}{ Frequencies } & \multicolumn{2}{|l|}{847.94} & & \multicolumn{2}{|l|}{867.65} & & \multicolumn{3}{|l|}{891.53} \\
\hline Atom & AN & X & $Y$ & Z & X & $\mathrm{Y}$ & Z & X & $Y$ & Z \\
\hline 1 & 6 & 0.05 & -0.04 & -0.09 & -0.02 & 0.02 & 0.00 & -0.01 & 0.01 & 0.01 \\
\hline 2 & 6 & 0.06 & -0.06 & 0.01 & 0.01 & -0.02 & 0.03 & & 0.00 & 0.01 \\
\hline 3 & 6 & -0.03 & 0.08 & -0.09 & -0.03 & 0.06 & -0.07 & & 0.00 & 0.00 \\
\hline 4 & 6 & 0.04 & 0.02 & 0.08 & 0.01 & -0.03 & & & & 0.00 \\
\hline 5 & 6 & 0.02 & 0.10 & 0.03 & -0.01 & 0.00 & -0.0 & 0.0 & -0.01 & -0.01 \\
\hline 6 & 6 & -0.09 & -0.03 & 0.02 & 0.01 & 0.00 & 0.00 & 0.01 & 0.00 & 0.00 \\
\hline 7 & 7 & -0.06 & -0.10 & 0.09 & 0.07 & -0.07 & 0.0 & 0.01 & 0.01 & -0.01 \\
\hline 8 & 6 & -0.10 & -0.10 & 0.08 & -0.01 & -0.05 & 0. & 0.00 & 0.01 & -0.01 \\
\hline 9 & 6 & 0.10 & -0.07 & 0.08 & 0.08 & 0.00 & 0.0 & -0.04 & 0.00 & -0.01 \\
\hline 10 & 6 & 0.12 & 0.14 & -0.12 & -0.01 & -0.07 & 0.0 & & 0.02 & -0.02 \\
\hline 11 & 17 & 0.01 & & -0.01 & 0.0 & 0.00 & & & & \\
\hline 12 & 17 & 0.00 & & 0.00 & & & & & & 0.00 \\
\hline 13 & 78 & 0.00 & & -0.0 & & 0.00 & & & & .00 \\
\hline 14 & 8 & 0.00 & & 0.0 & & 0.01 & & & & \\
\hline 15 & 7 & -0.01 & & 0.1 & 0.2 & 0.14 & & -0.07 & 34 & 0.03 \\
\hline 16 & 6 & 0.02 & & -0.1 & & -0.10 & 0. & & & -0.01 \\
\hline 17 & 6 & -0.0 & & & & & & & & \\
\hline 18 & 6 & -0.0 & & & & & & 0.03 & & \\
\hline 19 & 6 & 0.04 & & 0.02 & & 0.01 & 0.0 & & & .00 \\
\hline 20 & 6 & 0.00 & & -0.0 & -0 & -0.09 & & & & 04 \\
\hline 21 & 6 & 0.0 & & -0.0 & -0.1 & 0.02 & -0. & & & \\
\hline 22 & 17 & & 0.01 & 0.01 & -0.01 & -0.01 & -0. & 0. & & .00 \\
\hline 23 & 17 & & & & & 0.00 & & & & 00 \\
\hline 24 & 6 & 0.0 & & & & & -0 . & & & \\
\hline 25 & 6 & & 0.04 & -0.06 & -0.0 & 0.06 & -0. & & -0 . & 0.01 \\
\hline 26 & 1 & -0.2 & & & -0 . & 0.13 & & & & .03 \\
\hline 27 & 1 & 0.1 & & & -0 & & & & & \\
\hline 28 & 1 & & & & & & & & & \\
\hline 29 & 1 & & & & & -0 & & & & -0.02 \\
\hline 30 & 1 & & & & -0 & -0 & & & & -0.03 \\
\hline 31 & 1 & & & & & & & & & \\
\hline 32 & 1 & & & & & & & & & \\
\hline 33 & 1 & & & & & & & & & \\
\hline 34 & 1 & & & & -0 & -0.05 & & & & \\
\hline 35 & 1 & & & & & -0.02 & & & & \\
\hline 36 & 1 & -0 & & & & & & & & \\
\hline 37 & 1 & & & & -0 . & -0.01 & 0. & & & \\
\hline 38 & 1 & -0. & & & & & & & & \\
\hline 39 & 1 & & & & & & & & & \\
\hline 40 & 1 & & & & & & & & & -0.28 \\
\hline 41 & 6 & & & & & & -0 & & & -0.01 \\
\hline 42 & 1 & & & & & & & & & \\
\hline 43 & 6 & & & & & & & & & \\
\hline 44 & 1 & & & & & -0.02 & & & & \\
\hline 45 & 1 & & & & & & & & & \\
\hline 46 & 9 & & & & & 0.0 & & & & \\
\hline 47 & 9 & & & & & 0. & & & & 0.00 \\
\hline 48 & 9 & 0.00 & & & & 0.00 & & & 0.00 & \\
\hline Frequen & & & & & & & & & & \\
\hline Atom & AN & $X$ & $Y$ & Z & $x$ & $Y$ & Z & $x$ & Y & 2 \\
\hline 1 & 6 & -0.15 & & 0.14 & & -0.08 & 0.0 & 0.00 & 0.00 & 0.00 \\
\hline 2 & 6 & & & & & 0.02 & -0.0 & & & 00 \\
\hline 3 & 6 & 0.0 & 0.13 & -0.13 & 0.0 & 0.00 & 0.0 & & 0.00 & 0.00 \\
\hline 4 & 6 & -0.04 & -0.08 & 0.00 & 0.01 & -0.02 & 0.03 & 0.00 & 0.00 & 0.00 \\
\hline 5 & 6 & -0.10 & -0.17 & -0.12 & -0.03 & 0.08 & -0.09 & 0.00 & 0.00 & 0.00 \\
\hline
\end{tabular}




\begin{tabular}{|c|c|c|c|c|c|c|c|c|c|c|}
\hline & & & & & & & & & & \\
\hline 6 & $\begin{array}{l}6 \\
7\end{array}$ & 0.12 & & $\begin{array}{l}-0.01 \\
\end{array}$ & 0.00 & 0.00 & 0.00 & 0.00 & 0.00 & $\begin{array}{l}0.00 \\
0.00\end{array}$ \\
\hline $\begin{array}{l}7 \\
8\end{array}$ & $\begin{array}{l}7 \\
6\end{array}$ & $\begin{array}{l}0.44 \\
0.09\end{array}$ & $\begin{array}{l}-0.14 \\
-0.02\end{array}$ & $\begin{array}{l}0.21 \\
0.03\end{array}$ & $\begin{array}{l}0.00 \\
0.00\end{array}$ & $\begin{array}{l}0.00 \\
0.00\end{array}$ & $\begin{array}{l}0.00 \\
0.00\end{array}$ & $\begin{array}{l}0.00 \\
0.00\end{array}$ & $\begin{array}{l}0.00 \\
0.00\end{array}$ & \\
\hline 9 & 6 & -0.03 & 0.02 & -0.02 & 0.00 & 0.00 & 0.00 & 0.00 & 0.00 & 0.00 \\
\hline 10 & 6 & -0.01 & 0.00 & 0.00 & 0.00 & 0.00 & 0.00 & 0.00 & 0.00 & 0.00 \\
\hline 11 & 17 & -0.01 & 0.01 & 0.01 & 0.00 & 0.00 & 0.00 & 0.00 & 0.00 & 0.00 \\
\hline 12 & 17 & 0.00 & -0.01 & -0.01 & 0.00 & 0.00 & 0.00 & 0.00 & 0.00 & 0.00 \\
\hline 13 & 78 & -0.01 & 0.01 & -0.01 & 0.00 & 0.00 & 0.00 & 0.00 & 0.00 & 0.00 \\
\hline 14 & 8 & 0.00 & 0.00 & 0.00 & 0.00 & 0.00 & 0.00 & 0.00 & 0.00 & 0.00 \\
\hline 15 & 7 & -0.13 & -0.07 & 0.04 & 0.00 & 0.00 & 0.00 & 0.00 & 0.00 & 0.00 \\
\hline 16 & 6 & -0.04 & 0.04 & -0.03 & 0.00 & 0.00 & 0.00 & 0.00 & 0.00 & 0.00 \\
\hline 17 & 6 & 0.03 & -0.03 & -0.02 & 0.00 & 0.00 & 0.00 & 0.00 & 0.03 & -0.02 \\
\hline 18 & 6 & 0.06 & -0.03 & -0.06 & 0.00 & 0.00 & 0.00 & -0.01 & -0.11 & 0.06 \\
\hline 19 & 6 & -0.05 & 0.00 & 0.00 & 0.00 & 0.00 & 0.00 & 0.00 & 0.00 & 0.00 \\
\hline 20 & 6 & 0.04 & 0.04 & 0.07 & 0.00 & 0.00 & 0.00 & 0.01 & 0.11 & -0.06 \\
\hline 21 & 6 & 0.02 & 0.00 & 0.02 & 0.00 & 0.00 & 0.00 & 0.00 & -0.03 & 0.02 \\
\hline 22 & 17 & 0.00 & 0.00 & 0.01 & 0.00 & 0.00 & 0.00 & 0.00 & 0.00 & 0.00 \\
\hline 23 & 17 & 0.00 & 0.00 & 0.00 & 0.00 & 0.00 & 0.00 & 0.00 & 0.00 & \\
\hline 24 & 6 & 0.04 & -0.01 & 0.01 & 0.00 & 0.00 & 0.00 & 0.00 & 0.00 & 0.00 \\
\hline 25 & 6 & -0.11 & 0.10 & -0.11 & 0.00 & 0.00 & 0.00 & 0.00 & 0.00 & 0.00 \\
\hline 26 & 1 & -0.13 & 0.16 & -0.21 & 0.00 & 0.00 & 0.00 & 0.00 & 0.00 & \\
\hline 27 & 1 & -0.04 & 0.11 & -0.12 & 0.00 & 0.00 & 0.00 & 0.00 & 0.00 & 0.00 \\
\hline 28 & 1 & 0.00 & 0.06 & -0.07 & & 0.00 & 0.00 & 0.00 & 0.00 & 0.00 \\
\hline 29 & 1 & -0.02 & -0.01 & -0.01 & 0.00 & 0.00 & 0.00 & 0.00 & 0.00 & \\
\hline 30 & 1 & -0.03 & -0.01 & 0.01 & 0.00 & 0.00 & 0.00 & 0.00 & 0.00 & \\
\hline 31 & 1 & -0.01 & 0.00 & -0.01 & & 0.00 & 0.00 & 0.00 & 0.00 & 0.00 \\
\hline 32 & 1 & 0.13 & -0.03 & -0.01 & 0.00 & 0.00 & 0.00 & 0.05 & 0.61 & -0.34 \\
\hline 33 & 1 & -0.05 & 0.00 & -0.01 & 0.00 & 0.00 & 0.00 & 0.00 & -0.02 & 0.01 \\
\hline 34 & 1 & 0.10 & 0.04 & 0.05 & 0.00 & 0.00 & 0.00 & -0.05 & -0.60 & 0.34 \\
\hline 35 & 1 & -0.26 & -0.15 & -0.04 & 0.20 & -0.43 & 0.51 & 0.00 & 0.00 & 0.00 \\
\hline 36 & 1 & 0.11 & 0.04 & -0.03 & 0.00 & 0.00 & 0.00 & 0.00 & 0.00 & \\
\hline 37 & 1 & -0.30 & 0.00 & 0.11 & -0.20 & 0.43 & -0.51 & 0.00 & 0.00 & 0.00 \\
\hline 38 & 1 & 0.06 & 0.11 & -0.14 & 0.00 & 0.00 & 0.00 & 0.00 & 0.00 & 0.00 \\
\hline 39 & 1 & -0.01 & 0.09 & -0.0 & & 0.00 & 0.0 & 0.00 & 0.00 & \\
\hline 40 & 1 & -0.20 & -0.09 & 0.10 & & 0.00 & 0.00 & 0.00 & 0.00 & 0.00 \\
\hline 41 & 6 & 0.00 & 0.00 & 0.00 & 0.00 & 0.00 & 0.00 & 0.00 & 0.00 & 0.00 \\
\hline 42 & 1 & -0.01 & 0.01 & 0.00 & 0.00 & 0.00 & 0.00 & 0.01 & 0.00 & 0.01 \\
\hline 43 & 6 & 0.00 & 0.00 & 0.00 & 0.0 & 0.00 & 0.00 & 0.00 & 0.00 & 0.00 \\
\hline 44 & 1 & 0.00 & 0.00 & 0.00 & & 0.00 & 0.0 & -0.01 & -0.01 & \\
\hline 45 & 1 & 0.00 & -0.01 & -0.01 & 0.00 & 0.00 & 0.00 & 0.01 & 0.00 & 0. \\
\hline 46 & 9 & 0.00 & 0.00 & 0.00 & $0 . C$ & 0.00 & 0.00 & 0.00 & 0.00 & 0.00 \\
\hline 47 & 9 & 0.00 & 0.00 & 0.0 & & 0.00 & 0.00 & 0.00 & 0.00 & 0.00 \\
\hline 48 & 9 & 0.00 & 0.00 & 0.00 & 0.00 & 0.00 & 0.00 & 0.00 & 0.00 & 0.00 \\
\hline Frequen & & 984.60 & & & 1027.56 & & & 1029.89 & & \\
\hline Atom & AN & X & Y & Z & $x$ & $Y$ & Z & $X$ & $Y$ & Z \\
\hline 1 & 6 & 0.00 & 0.00 & 0.00 & -0.01 & 0.01 & 0.02 & 0.00 & 0.00 & 0.00 \\
\hline 2 & 6 & 0.00 & 0.00 & 0.00 & 0.00 & 0.00 & 0.00 & 0.00 & 0.00 & 0.00 \\
\hline 3 & 6 & 0.00 & 0.00 & 0.00 & 0.02 & 0.00 & 0.00 & 0.00 & 0.00 & 0.00 \\
\hline 4 & 6 & 0.00 & 0.00 & 0.00 & & 0.00 & 0.00 & & 0.00 & 0.00 \\
\hline 5 & 6 & 0.0 & 0.00 & 0.0 & & -0.02 & -0.0 & & 0.00 & 0.00 \\
\hline 6 & 6 & 0.0 & 0.00 & 0.00 & 0. & 0.01 & -0.01 & 0.00 & 0.00 & 0.00 \\
\hline 7 & 7 & 0.0 & 0.00 & 0.00 & & 0.04 & -0.04 & 0.00 & 0.00 & 0.00 \\
\hline 8 & 6 & 0.0 & 0.00 & 0.00 & -0 . & 0.02 & -0.02 & -0.01 & 0.00 & 0.00 \\
\hline 9 & 6 & 0.0 & 0.00 & 0.00 & $0 . C$ & 0.04 & -0.03 & 0.00 & 0.00 & 0.00 \\
\hline 10 & 6 & 0.0 & 0.00 & 0.00 & 0. & -0.07 & 0.08 & 0.00 & -0.01 & 0.01 \\
\hline 11 & 17 & 0.0 & 0.00 & 0.00 & 0. & 0.00 & 0.00 & 0.00 & 0.00 & 0.00 \\
\hline 12 & 17 & 0.0 & 0.00 & 0.00 & 0.0 & 0.00 & 0.00 & 0.00 & 0.00 & 0.00 \\
\hline 13 & 78 & 0.00 & 0.00 & 0.00 & 0.0 & 0.00 & 0.00 & 0.00 & 0.00 & 0.00 \\
\hline 14 & 8 & 0.0 & 0.01 & -0.03 & 0.0 & 0.00 & 0.00 & 0.00 & 0.00 & 0.00 \\
\hline 15 & 7 & 0.00 & 0.00 & 0.00 & -0.04 & 0.04 & -0.05 & 0.00 & 0.00 & 0.00 \\
\hline
\end{tabular}




\begin{tabular}{|c|c|c|c|c|c|c|c|c|c|c|}
\hline & & & & & & & & & & \\
\hline 16 & 6 & 0.00 & 0.00 & 0.00 & -0.04 & 0.00 & -0.01 & 0.00 & -0.01 & 0.00 \\
\hline 17 & 6 & 0.00 & 0.00 & 0.00 & 0.00 & 0.00 & 0.00 & 0.00 & -0.01 & 0.00 \\
\hline 18 & 6 & 0.00 & 0.00 & 0.00 & 0.03 & -0.02 & -0.02 & 0.01 & 0.07 & -0.04 \\
\hline 19 & 6 & 0.00 & 0.00 & 0.00 & -0.01 & 0.01 & -0.01 & -0.01 & -0.12 & 0.07 \\
\hline 20 & 6 & 0.00 & 0.00 & 0.00 & 0.02 & 0.01 & 0.04 & 0.01 & 0.07 & -0.04 \\
\hline 21 & 6 & 0.00 & 0.00 & 0.00 & 0.00 & 0.00 & 0.00 & 0.00 & -0.01 & 0.00 \\
\hline 22 & 17 & 0.00 & 0.00 & 0.00 & 0.00 & 0.00 & 0.00 & 0.00 & 0.00 & 0.00 \\
\hline 23 & 17 & 0.00 & 0.00 & 0.00 & 0.00 & 0.00 & 0.00 & 0.00 & 0.00 & 0.00 \\
\hline 24 & 6 & -0.01 & 0.01 & -0.01 & 0.00 & 0.00 & 0.00 & 0.00 & 0.00 & 0.00 \\
\hline 25 & 6 & 0.00 & 0.00 & 0.00 & -0.04 & -0.08 & 0.07 & 0.00 & -0.01 & 0.01 \\
\hline 26 & 1 & 0.01 & -0.01 & 0.01 & -0.17 & 0.24 & -0.29 & -0.02 & 0.02 & -0.03 \\
\hline 27 & 1 & -0.01 & 0.00 & 0.00 & 0.29 & -0.05 & 0.05 & 0.03 & -0.01 & 0.01 \\
\hline 28 & 1 & -0.01 & 0.00 & -0.01 & 0.32 & -0.03 & 0.19 & 0.03 & 0.00 & 0.02 \\
\hline 29 & 1 & 0.00 & 0.00 & 0.00 & -0.31 & -0.29 & 0.10 & -0.02 & -0.02 & 0.01 \\
\hline 30 & 1 & 0.00 & 0.00 & 0.00 & -0.30 & -0.12 & 0.11 & -0.02 & -0.01 & 0.01 \\
\hline 31 & 1 & 0.00 & -0.01 & 0.01 & 0.08 & 0.36 & -0.28 & 0.00 & 0.02 & -0.01 \\
\hline 32 & 1 & 0.00 & 0.01 & 0.00 & 0.03 & & -0.05 & -0.03 & & 0.21 \\
\hline 33 & 1 & 0.00 & 0.00 & 0.00 & & -0.06 & 0.03 & 0.05 & & -0.37 \\
\hline 34 & 1 & 0.00 & -0.02 & 0.01 & 0.03 & 0.06 & 0.01 & -0.03 & -0.40 & 0.23 \\
\hline 35 & 1 & 0.00 & 0.00 & 0.00 & -0.02 & 0.00 & -0.04 & -0.01 & & \\
\hline 36 & 1 & 0.00 & 0.00 & 0.00 & & -0.03 & 0.04 & & -0.02 & 0.03 \\
\hline 37 & 1 & 0.00 & 0.00 & 0.00 & -0.02 & 0.03 & -0.01 & -0.01 & 0.01 & -0.01 \\
\hline 38 & 1 & 0.00 & -0.03 & 0.04 & -0.01 & -0.01 & 0.01 & 0.0 & 0.00 & \\
\hline 39 & 1 & -0.01 & -0.04 & 0.05 & 0.01 & 0.00 & 0.00 & 0.00 & & \\
\hline 40 & 1 & 0.04 & 0.02 & -0.02 & 0.01 & 0.00 & -0.01 & 0.00 & 0.00 & 0.00 \\
\hline 41 & 6 & 0.01 & -0.12 & 0.15 & 0.00 & 0.00 & 0.00 & 0.00 & 0.00 & 0.00 \\
\hline 42 & 1 & -0.31 & 0.20 & -0.09 & & 0.00 & 0. & 0.0 & & \\
\hline 43 & 6 & 0.01 & -0.05 & 0.08 & & 0.00 & 0.00 & 0.00 & & \\
\hline 44 & 1 & 0.51 & 0.35 & -0.04 & 0.01 & 0.01 & 0.00 & 0.00 & 0.00 & 0.00 \\
\hline 45 & 1 & -0.54 & -0.09 & -0.30 & -0.01 & 0.00 & 0. & 0.0 & 00 & \\
\hline 46 & 9 & 0.06 & 0.05 & -0. & & 0.00 & & 0.0 & & \\
\hline 47 & 9 & -0.01 & 0.01 & 0.1 & & 0.00 & 0.0 & 0.00 & & 0.0 \\
\hline 48 & 9 & -0.05 & 0.01 & -0.08 & 0.00 & 0.00 & 0.00 & 0.00 & 0.00 & 0.00 \\
\hline Frequer & & 1030.48 & & & 1049.69 & & & 1052.79 & & \\
\hline Atom & AN & X & $Y$ & Z & $X$ & $\mathrm{Y}$ & Z & X & $Y$ & L \\
\hline 1 & 6 & 0.02 & -0.05 & 0.06 & 0.05 & -0.05 & -0.06 & -0.03 & 0.03 & 0.04 \\
\hline 2 & 6 & 0.00 & 0.00 & -0.01 & 0.00 & 0.02 & 0.02 & 0.00 & 0.00 & -0.01 \\
\hline 3 & 6 & 0. & 0.01 & -0.01 & -0 . & -0.02 & 0.0 & 0. & 0.01 & 0.0 \\
\hline 4 & 6 & 0. & 0.00 & 0.00 & & -0.02 & -0.0 & & 0.01 & \\
\hline 5 & 6 & 0.0 & -0.05 & 0.06 & & 0.07 & 0.05 & -0.02 & -0.05 & -0.0 \\
\hline 6 & 6 & -0.04 & 0.08 & -0.10 & & 0.00 & 0.00 & 0. & 0.00 & 0.00 \\
\hline 7 & 7 & & 0.00 & 0.00 & & -0.09 & 0.09 & & 0.05 & \\
\hline 8 & 6 & 0.1 & 0.00 & 0.0 & 0. & -0.05 & 0.04 & -0.03 & 0.04 & \\
\hline 9 & 6 & & 0.00 & 0.00 & & 0.04 & -0.05 & -0.02 & -0.02 & 0.03 \\
\hline 10 & 6 & -0 . & 0.0 & -0.01 & & & 0.0 & & 0.01 & \\
\hline 11 & 17 & & 0.00 & 0.0 & & 0.00 & 0.0 & & 0.00 & \\
\hline 12 & 17 & & 0. & 0.0 & & 0.00 & 0.00 & 0. & 0.00 & \\
\hline 13 & 78 & & 0. & 0.0 & & 0.00 & 0.0 & & 0.00 & \\
\hline 14 & 8 & 0. & 0.0 & 0.0 & & -0 . & -0.0 & 0.2 & & \\
\hline 15 & 7 & & 0. & 0.0 & -0 & & -0.06 & 0.03 & -0.03 & \\
\hline 16 & 6 & & 0.00 & 0.00 & -0.09 & -0.01 & -0.01 & 0.0 & 0.01 & 0.01 \\
\hline 17 & 6 & 0. & 0.0 & 0.00 & 0. & & 0.0 & & -0.01 & \\
\hline 18 & 6 & & 0. & 0.0 & & & -0.06 & -0.0 & 0.02 & \\
\hline 19 & 6 & 0.00 & 0.00 & 0.00 & -0.01 & 0.00 & 0.00 & 0.00 & 0.00 & 0.00 \\
\hline 20 & 6 & & 0.00 & 0.00 & & & 0.08 & -0.02 & -0.03 & \\
\hline 21 & 6 & & 0. & 0.0 & & -0.01 & -0.02 & & 0.01 & \\
\hline 22 & 17 & & 0.00 & 0.00 & 0.00 & 0.00 & 0.00 & 0.00 & 0.00 & 0.00 \\
\hline 23 & 17 & & 0.00 & 0.00 & & 0.00 & 0.00 & 0.00 & 0.00 & \\
\hline 24 & 6 & & 0. & 0.00 & -0 & 0.00 & -0.01 & 0.00 & 0.00 & \\
\hline 25 & 6 & 0.00 & 0.00 & 0.00 & 0.05 & 0.10 & -0.07 & -0.03 & -0.06 & 0.03 \\
\hline
\end{tabular}




\begin{tabular}{|c|c|c|c|c|c|c|c|c|c|c|}
\hline & & & & & & & & & & \\
\hline 26 & 1 & 0.01 & -0.01 & 0.01 & 0.20 & -0.26 & 0.26 & -0.13 & 0.17 & -0.14 \\
\hline 2 & 1 & -0.01 & 0.00 & 0.00 & -0.37 & 0.09 & -0.11 & 0.23 & -0.06 & 0.09 \\
\hline 28 & 1 & -0.02 & 0.00 & -0.01 & -0.33 & -0.02 & -0.19 & 0.18 & 0.04 & 0.10 \\
\hline 29 & 1 & 0.02 & 0.02 & -0.01 & -0.21 & -0.19 & 0.01 & 0.13 & 0.11 & 0.02 \\
\hline 30 & 1 & 0.02 & 0.01 & -0.01 & -0.25 & -0.17 & 0.13 & 0.17 & 0.12 & -0.09 \\
\hline 3 & 1 & -0.01 & -0.03 & 0.02 & 0.08 & 0.22 & -0.23 & -0.06 & -0.14 & .16 \\
\hline 32 & 1 & 0.00 & 0.02 & -0.01 & 0.04 & -0.05 & -0.08 & -0.02 & 0.06 & 04 \\
\hline 33 & 1 & 0.00 & -0.03 & 0.02 & 0.00 & 0.01 & -0.01 & 0.00 & -0.01 & 0.02 \\
\hline 3 & 1 & 0.00 & 0.02 & -0.01 & 0.01 & 0.04 & 0.09 & 0.00 & -0.03 & -0.06 \\
\hline 3 & 1 & -0.13 & & -0.33 & & & & 0.00 & -0.05 & -0.04 \\
\hline 36 & 1 & 0.22 & -0.46 & 0.55 & 0.00 & -0.01 & 0.01 & 0.00 & 0.00 & 0.00 \\
\hline 37 & 1 & -0.13 & 0.27 & -0.32 & 0.03 & -0.06 & -0.08 & -0.02 & 0.04 & 0.05 \\
\hline 38 & 1 & 0.00 & 0.00 & 0.00 & 0.00 & -0.01 & 0.02 & 0.00 & 0.00 & 0.01 \\
\hline 39 & 1 & 0.00 & & 0.0 & 0.00 & -0.02 & 0.0 & 0.00 & -0.01 & 0.00 \\
\hline 40 & 1 & 0.00 & 0.00 & 0.00 & 0.02 & 0.02 & -0.02 & 0.00 & 0.00 & 0.00 \\
\hline 41 & 6 & 0.00 & 0.00 & 0.00 & -0.11 & 0.07 & 0.0 & -0.35 & 0.22 & 0.17 \\
\hline 42 & 1 & 0.00 & & 0.00 & 0.01 & -0.01 & -0.0 & 0.02 & -0.05 & -0.21 \\
\hline 43 & 6 & 0.00 & & 0.00 & 0.02 & 0.02 & 0.01 & 0.08 & 0.05 & 0.03 \\
\hline 44 & 1 & 0.00 & 0.00 & 0.00 & -0.09 & 0.06 & 0.0 & -0.27 & 0.21 & 0.11 \\
\hline 45 & 1 & 0.00 & & 0.0 & -0.06 & 0.06 & 0. & -0.21 & 0.17 & 0.23 \\
\hline 46 & 9 & 0.00 & & 0.0 & -0.01 & -0.01 & 0.0 & -0.02 & -0.01 & 0.01 \\
\hline 47 & 9 & 0.00 & & 0.0 & 0.00 & -0.01 & -0.01 & 0.02 & -0.04 & -0.03 \\
\hline 48 & 9 & 0.00 & & 0.00 & 0.00 & 0.00 & 0.00 & -0.02 & 0.00 & -0.02 \\
\hline quen & & 1066.71 & & & 1094.48 & & & 1105.80 & & \\
\hline Atom & AN & $X$ & $Y$ & Z & $x$ & $Y$ & Z & $x$ & Y & Z \\
\hline 1 & 6 & 0.00 & 0.00 & 0.00 & 0.00 & 0.00 & 0.00 & -0.01 & 0.12 & 0.11 \\
\hline 2 & 6 & 0.00 & -0.01 & -0.01 & & 0.00 & 0.00 & 0.05 & 0.01 & -0.01 \\
\hline 3 & 6 & 0.01 & 0.00 & 0.00 & 0.00 & 0.00 & 0.00 & 0.02 & 0.00 & 0.00 \\
\hline 4 & 6 & 0.00 & -0.01 & -0.01 & 0 & 0.00 & 0.00 & 0.0 & 0.02 & 0.00 \\
\hline 5 & 6 & 0.00 & & -0.01 & & 0.00 & 0.00 & 0.0 & -0.12 & -0.11 \\
\hline 6 & 6 & 0.00 & 0. & 0.00 & 0. & 0.00 & 0.00 & -0.12 & -0.04 & 0.02 \\
\hline 7 & 7 & 0. & 0. & 0.00 & 0. & -0.02 & -0.01 & -0.05 & -0.01 & 0.00 \\
\hline 8 & 6 & 0.00 & & -0.07 & -0.02 & 0.09 & 0.0 & -0.0 & -0.02 & 0.01 \\
\hline 9 & 6 & & & -0.05 & & -0.09 & -0.0 & 0. & -0.01 & 0.01 \\
\hline 10 & 6 & & & 0.07 & -0.02 & 0.08 & 0.0 & & 0.01 & -0.01 \\
\hline 11 & 17 & 0.00 & & 0.00 & 0.00 & 0.00 & 0.00 & -0.01 & 0.00 & 0.00 \\
\hline 12 & 17 & & & 0. & & 0. & 0.0 & -0.0 & 0.00 & 0.00 \\
\hline 13 & 78 & & & & & & & & & \\
\hline 14 & 8 & & & 0. & & 0.00 & 0.0 & 0. & 0.00 & 0.00 \\
\hline 15 & 7 & & & 0. & & 0. & 0.0 & 0.1 & 0.01 & 0.00 \\
\hline 16 & 6 & & & & & & 0.0 & -0. & 0.00 & 0.00 \\
\hline 17 & 6 & & & & & & & & & 0.00 \\
\hline 18 & 6 & 0. & & 0. & & 0.0 & 0.0 & 0.0 & -0.05 & -0.09 \\
\hline 19 & 6 & & & & & & 0.0 & 0. & 0.00 & 0.01 \\
\hline 20 & 6 & & & & & & & & & 0.09 \\
\hline 21 & 6 & & & & & & -0 . & -0 . & 0. & -0.01 \\
\hline 22 & 17 & & & 0. & & & 0.0 & 0.00 & 0.00 & 0.00 \\
\hline 23 & 17 & & & & & & & & & \\
\hline 24 & 6 & & & & & & & & & \\
\hline 25 & 6 & & & & & & -0. & 0. & 0.01 & -0.01 \\
\hline 26 & 1 & & & -0 & & & 0. & & -0.05 & 0.06 \\
\hline 27 & 1 & -0.2 & & -0 & & -0.1 & 0. & & 0.01 & 0.00 \\
\hline 28 & 1 & 0. & & 0. & -0 . & & -0.1 & -0. & 0.00 & -0.04 \\
\hline 29 & 1 & 0.1 & & & & 0.20 & -0. & & 0.01 & 0.00 \\
\hline 30 & 1 & -0.0 & & 0.1 & & -0.41 & 0.2 & & 0.01 & -0.01 \\
\hline 31 & 1 & 0.0 & & -0.1 & 0.09 & -0.15 & -0.23 & 0.00 & 0.00 & 0.00 \\
\hline 32 & 1 & 0.02 & & 0.02 & 0.01 & 0.00 & 0.01 & -0.20 & -0.13 & -0.26 \\
\hline 33 & 1 & 0.0 & & 0.0 & & & 0.01 & & 0.00 & 0.01 \\
\hline 34 & 1 & -0.0 & & -0.01 & -0.01 & 0.00 & 0.00 & & 0.12 & 0.18 \\
\hline 35 & 1 & -0.01 & -0.01 & 0.00 & 0.01 & 0.00 & -0.01 & 0.41 & -0.18 & -0.31 \\
\hline
\end{tabular}




\begin{tabular}{|c|c|c|c|c|c|c|c|c|c|c|}
\hline 36 & 1 & 0.00 & 0.01 & 0.00 & 0.00 & 0.00 & 0.00 & -0.14 & -0.04 & 0.02 \\
\hline & 1 & 0.01 & 0.02 & 0.01 & -0.01 & 0.00 & 0.00 & 0.32 & 0.39 & 0.20 \\
\hline 38 & 1 & 0.01 & 0.01 & 0.00 & 0.00 & 0.00 & 0.00 & 0.00 & 0.00 & 0.00 \\
\hline 39 & 1 & -0.01 & 0.00 & 0.00 & 0.00 & 0.00 & 0.00 & 0.00 & 0.00 & 0.00 \\
\hline 40 & 1 & 0.00 & 0.00 & 0.00 & 0.00 & 0.00 & 0.00 & 0.00 & 0.00 & 0.00 \\
\hline 41 & 6 & -0.01 & 0.00 & 0.00 & 0.00 & 0.00 & 0.00 & & .00 & .00 \\
\hline 42 & 1 & 0.00 & 0.00 & 0.00 & 0.00 & 0.00 & 0.00 & 0.00 & .00 & 0.00 \\
\hline 43 & 6 & 0.00 & 0.00 & 0.00 & 0.00 & 0.00 & 0.00 & 0.00 & 0.00 & 0.00 \\
\hline 44 & 1 & 0.00 & 0.01 & 0.00 & 0.00 & 0.00 & 0.00 & 0.00 & 0.00 & 0.00 \\
\hline 45 & 1 & 0.00 & 0.00 & 0.0 & 0.00 & 0.00 & & & .00 & 0.00 \\
\hline 46 & 9 & 0.00 & 0.00 & 0.0 & 0.00 & 0.00 & 0.00 & 0.00 & .00 & 0.00 \\
\hline 47 & 9 & 0.00 & 0.00 & 0.0 & 0.00 & 0.00 & 0.00 & 0.00 & 0.00 & .00 \\
\hline 48 & 9 & 0.00 & 0.00 & 0.00 & 0.00 & 0.00 & 0.00 & & .00 & 0.00 \\
\hline \multicolumn{2}{|c|}{ Frequencies } & 1108.22 & & & 1139.36 & & & 1142.42 & & \\
\hline Atom & AN & $X$ & Y & Z & $X$ & $Y$ & Z & $x$ & $Y$ & Z \\
\hline 1 & 6 & 0.00 & 0.07 & 0.06 & 0.04 & -0.02 & -0.03 & 0.05 & -0.03 & -0.04 \\
\hline 2 & 6 & 0.04 & 0.01 & 0.00 & 0.08 & 0.21 & 0.15 & 0.11 & 0.31 & 0.22 \\
\hline 3 & 6 & 0.00 & 0.00 & 0.00 & -0.08 & -0.03 & 0.01 & -0.10 & -0.03 & 0.01 \\
\hline 4 & 6 & 0.04 & 0.01 & -0.01 & 0.14 & -0.15 & -0.18 & 0.19 & -0.22 & -0.26 \\
\hline 5 & 6 & 0.02 & -0.06 & -0.06 & 0.03 & 0.04 & 0.02 & 0.04 & 0.06 & 0.03 \\
\hline 6 & 6 & -0.08 & -0.02 & 0.01 & -0.15 & -0.04 & 0.02 & -0.21 & -0.06 & 0.03 \\
\hline 7 & 7 & -0.02 & -0.02 & 0.02 & 0.04 & 0.03 & -0.02 & 0.08 & 0.01 & 0.00 \\
\hline 8 & 6 & -0.01 & -0.01 & 0.01 & 0.07 & 0.01 & 0.00 & 0.0 & 0.03 & -0.02 \\
\hline 9 & 6 & -0.02 & 0.01 & -0.01 & 0.07 & 0.01 & 0.01 & -0.01 & 0.03 & -0.03 \\
\hline 10 & 6 & 0.04 & -0.01 & 0.02 & -0.08 & 0.00 & -0.02 & 0.00 & -0.02 & 0.02 \\
\hline 11 & 17 & & & 0.00 & -0.02 & 0.02 & 0. & -0 . & 0.02 & 0.03 \\
\hline 12 & 17 & & & & & -0.02 & -0.02 & & & -0.02 \\
\hline 13 & 78 & & 0.00 & 0.00 & 0.00 & 0.00 & 0.00 & 0.0 & 0.00 & 0.00 \\
\hline 14 & 8 & 0.00 & 0.00 & 0.00 & & 0.00 & 0.1 & 0. & 0.00 & 0.00 \\
\hline 15 & 7 & -0.04 & & -0.02 & & -0.02 & 0.02 & -0.06 & -0.02 & 0.00 \\
\hline 16 & 6 & & 0.00 & 0.00 & -0.07 & 0.00 & -0.02 & 0.07 & 0.01 & 0.01 \\
\hline 17 & 6 & 0. & 0.00 & 0.0 & 0.08 & 0.14 & 0.27 & -0.09 & -0.16 & -0.30 \\
\hline 18 & 6 & -0 . & 0.07 & 0.1 & & -0.02 & -0.03 & -0 . & 0.02 & 0.04 \\
\hline 19 & 6 & & 0.00 & -0.02 & -0.18 & 0.00 & -0.03 & 0. & & 0.03 \\
\hline 20 & 6 & 0.0 & -0.07 & -0.12 & 0.03 & 0.02 & 0.0 & -0 . & -0.03 & -0.05 \\
\hline 21 & 6 & & & 0.01 & 0.1 & -0.15 & -0.2 & -0 . & 0.15 & 0.25 \\
\hline 22 & 17 & & & 0. & & -0.02 & -0.0 & 0. & 0. & 0.03 \\
\hline 23 & 17 & & & & & 0.02 & & & & -0.03 \\
\hline 24 & 6 & & & 0. & 0.8 & 0.00 & 0. & 0. & 0. & 0.00 \\
\hline 25 & 6 & & & -0.01 & -0.08 & -0.03 & 0.01 & -0.0 & -0.02 & 0.01 \\
\hline 26 & 1 & & & 0.11 & & 0.17 & & & & -0.10 \\
\hline 27 & 1 & & & 0.0 & 0.1 & 0.00 & -0. & 0. & 0. & -0.01 \\
\hline 28 & 1 & & & -0 & & & 0.1 & 0. & & 0.05 \\
\hline 29 & 1 & & & & & & & & & -0.01 \\
\hline 30 & 1 & & & 0. & 0. & 0.03 & & & -0.02 & 0.02 \\
\hline 31 & 1 & & & -0 & -0.08 & -0.25 & & & 0. & -0.01 \\
\hline 32 & 1 & & & 0. & -0 & -0.06 & -0 . & & & 0.14 \\
\hline 33 & 1 & & & & & 0.00 & & & & 0.06 \\
\hline 34 & 1 & 0. & & -0 & -0 & & 0.1 & & -0 . & -0.10 \\
\hline 35 & 1 & 0.25 & & -0.19 & -0.07 & 0.04 & 0. & -0.13 & 0.07 & 0.11 \\
\hline 36 & 1 & & & & -0 & -0.05 & & & & 0.03 \\
\hline 37 & 1 & & & 0. & -0. & -0.08 & -0. & -0 . & & -0.07 \\
\hline 38 & 1 & 0.00 & & 0.01 & 0.00 & 0.00 & -0.02 & -0.02 & -0.03 & -0.01 \\
\hline 39 & 1 & 0.0 & & 0.0 & -0.01 & 0.01 & & & & 0.03 \\
\hline 40 & 1 & 0.0 & & 0.00 & -0.01 & -0.01 & 0.0 & -0. & -0.01 & 0.00 \\
\hline 41 & 6 & 0.0 & & 0.0 & 0.0 & 0.00 & 0.00 & 0.00 & 0.00 & -0.01 \\
\hline 42 & 1 & 0.0 & & & 0.0 & -0.01 & 0.0 & 0.0 & 0.00 & 0.01 \\
\hline 43 & 6 & 0.0 & & 0.0 & 0.0 & 0.00 & 0.00 & 0.00 & 0.00 & 0.02 \\
\hline 44 & 1 & 0.0 & -0.0 & 0.00 & 0.00 & 0.01 & 0.01 & -0.01 & -0.01 & 0.00 \\
\hline 45 & 1 & 0.00 & 0.00 & 0.00 & 0.01 & -0.03 & -0.02 & 0.01 & -0.01 & 0.00 \\
\hline
\end{tabular}




\begin{tabular}{|c|c|c|c|c|c|c|c|c|c|c|}
\hline $\begin{array}{l}46 \\
47 \\
48\end{array}$ & $\begin{array}{l}9 \\
9 \\
9\end{array}$ & $\begin{array}{l}0.00 \\
0.00 \\
0.00\end{array}$ & $\begin{array}{l}0.00 \\
0.00 \\
0.00\end{array}$ & $\begin{array}{l}0.00 \\
0.00 \\
0.00\end{array}$ & $\begin{array}{l}0.00 \\
0.00 \\
0.00\end{array}$ & $\begin{array}{l}0.00 \\
0.00 \\
0.00\end{array}$ & $\begin{array}{l}0.00 \\
0.00 \\
0.00\end{array}$ & $\begin{array}{l}0.00 \\
0.00\end{array}$ & $\begin{array}{l}0.00 \\
0.00 \\
0.00\end{array}$ & $\begin{array}{l}0.00 \\
0.00 \\
0.00\end{array}$ \\
\hline \multicolumn{2}{|c|}{ Frequencies } & \multicolumn{2}{|l|}{1175.66} & & \multicolumn{2}{|l|}{1193.78} & & \multicolumn{3}{|l|}{1212.59} \\
\hline Atom & AN & X & $Y$ & Z & $X$ & $Y$ & Z & $X$ & $Y$ & Z \\
\hline 1 & 6 & 0.00 & 0.00 & 0.00 & 0.04 & 0.01 & -0.01 & 0.00 & 0.00 & 0.01 \\
\hline 2 & 6 & 0.00 & 0.00 & 0.00 & -0.04 & -0.06 & -0.04 & & & 0.00 \\
\hline 3 & 6 & 0.00 & 0.00 & 0.00 & -0.10 & -0.04 & 0.03 & 0.00 & 0.00 & -0.01 \\
\hline 4 & 6 & 0.00 & 0.00 & 0.00 & -0.05 & 0.04 & 0.05 & & & 0.00 \\
\hline 5 & 6 & 0.00 & 0.00 & 0.00 & 0.04 & 0.01 & -0.0 & 0.00 & 0.01 & 0.00 \\
\hline 6 & 6 & 0.00 & 0.00 & 0.00 & 0.01 & 0.00 & 0.00 & 0.00 & -0.01 & -0.01 \\
\hline 7 & 7 & 0.00 & 0.00 & 0.00 & -0.06 & -0.01 & 0.00 & 0.00 & 0.00 & 0.00 \\
\hline 8 & 6 & -0.01 & 0.00 & 0.00 & 0.21 & -0.02 & 0.06 & -0.01 & 0.00 & 0.00 \\
\hline 9 & 6 & -0.01 & 0.00 & 0.00 & 0.19 & 0.11 & -0.06 & -0.01 & 0.00 & 0.00 \\
\hline 10 & 6 & 0.01 & 0.00 & 0.00 & -0.12 & -0.05 & 0.02 & & 0.00 & .00 \\
\hline 11 & 17 & & & 0. & 0.00 & -0.01 & & & & \\
\hline 12 & 17 & & & 0.00 & & 0.01 & & & & .00 \\
\hline 13 & 78 & 0.00 & 0.00 & 0.0 & & 0.00 & & & & \\
\hline 14 & 8 & -0.01 & 0.00 & 0.03 & -0.0 & & & & & \\
\hline 15 & 7 & 0.00 & 0.00 & 0.00 & -0.06 & -0.02 & 0. & 0.01 & & -0.02 \\
\hline 16 & 6 & 0.00 & 0.00 & 0.00 & -0.09 & 0.02 & -0.1 & -0.01 & & 0.06 \\
\hline 17 & 6 & 0.00 & & 0. & -0. & & & & & \\
\hline 18 & 6 & & & 0. & & & & 0.02 & & \\
\hline 19 & 6 & 0.00 & 0.00 & 0. & & 0.00 & 0.1 & & & .08 \\
\hline 20 & 6 & 0.00 & & 0. & & 0. & & & & \\
\hline 21 & 6 & & & 0. & -0.0 & 0.02 & & & -0.02 & \\
\hline 22 & 17 & & & & 0.00 & 0.00 & 0. & 0.0 & & 0.01 \\
\hline 23 & 17 & & & & & & & & & 01 \\
\hline 24 & 6 & & & & & & & & & \\
\hline 25 & 6 & & 0.00 & 0. & -0 . & & -0. & & & \\
\hline 26 & 1 & 0. & & 0. & -0. & & & & & \\
\hline 27 & 1 & -0 . & & 0. & & & -0 & & & \\
\hline 28 & 1 & & & & & & & & & \\
\hline 29 & 1 & & & & & & & & & \\
\hline 30 & 1 & & & & & -0 & & & & -0.01 \\
\hline 31 & 1 & & & & & & & & & \\
\hline 32 & 1 & & & & & & & & & \\
\hline 33 & 1 & 0. & & 0. & 0.0 & & & & & \\
\hline 34 & 1 & & & & & -0 & & 0. & & -0 . \\
\hline 35 & 1 & & & & & -0 & & & & \\
\hline 36 & 1 & & & & & & & & & \\
\hline 37 & 1 & & & & & & & & & \\
\hline 38 & 1 & & & & & & & & & \\
\hline 39 & 1 & & & & & & & & & \\
\hline 40 & 1 & & & & & & & & & \\
\hline 41 & 6 & & & & & & & & & \\
\hline 42 & 1 & & & & & & & & & \\
\hline 43 & 6 & & & & & & & & & \\
\hline 44 & 1 & & & & & 0. & & & 01 & \\
\hline 45 & 1 & & & & & & & & & \\
\hline 46 & 9 & & & -0.1 & & & & & & \\
\hline 47 & 9 & & & -0 . & & 0. & 0. & & & 0 \\
\hline 48 & 9 & & & -0.06 & 0.00 & 0.00 & 0.00 & & 0.00 & \\
\hline Frequen & & & & & & & & & & \\
\hline Atom & AN & $x$ & $Y$ & Z & $x$ & $Y$ & Z & $X$ & Y & 2 \\
\hline 1 & 6 & & -0.05 & -0.05 & 0.01 & 0.00 & 0.00 & 0.00 & 0.00 & 0.00 \\
\hline 2 & 6 & & & -0.01 & -0.01 & -0.01 & & & & 0 \\
\hline 3 & 6 & -0.0 & 0.04 & 0.04 & -0.01 & 0.00 & 0.0 & & 0.01 & \\
\hline 4 & 6 & 0.1 & 0.00 & -0.05 & 0.00 & 0.00 & 0.00 & 0.01 & 0.00 & 0.00 \\
\hline 5 & 6 & 0.00 & -0.05 & -0.04 & 0.00 & 0.00 & 0.00 & -0.01 & 0.00 & 0.00 \\
\hline
\end{tabular}




\begin{tabular}{|c|c|c|c|c|c|c|c|c|c|c|}
\hline & & & & & & & & & & \\
\hline 6 & 6 & -0.01 & 0.07 & 0.06 & 0.00 & 0.00 & 0.00 & 0.00 & 0.00 & 0.00 \\
\hline 7 & 7 & 0.00 & -0.02 & -0.01 & 0.01 & 0.00 & 0.00 & 0.00 & 0.00 & 0.00 \\
\hline 8 & 6 & 0.00 & 0.00 & 0.00 & -0.01 & 0.00 & 0.00 & 0.01 & 0.00 & 0.00 \\
\hline 9 & 6 & 0.00 & 0.00 & 0.00 & 0.00 & 0.01 & -0.01 & 0.00 & -0.01 & 0.00 \\
\hline 10 & 6 & 0.00 & 0.00 & 0.00 & 0.00 & -0.01 & 0.00 & 0.00 & 0.01 & 0.00 \\
\hline 11 & 17 & -0.01 & 0.00 & 0.01 & 0.00 & 0.00 & 0.00 & 0.00 & 0.00 & 0.00 \\
\hline 12 & 17 & 0.01 & 0.01 & 0.00 & 0.00 & 0.00 & 0.00 & 0.00 & 0.00 & 0.00 \\
\hline 13 & 78 & 0.00 & 0.00 & 0.00 & 0.00 & 0.00 & 0.00 & 0.00 & 0.00 & 0.00 \\
\hline 14 & 8 & 0.00 & 0.00 & 0.00 & -0.03 & -0.01 & -0.01 & -0.01 & 0.00 & 0.00 \\
\hline 15 & 7 & 0.00 & 0.00 & 0.00 & -0.01 & -0.01 & -0.01 & 0.00 & 0.01 & 0.02 \\
\hline 16 & 6 & 0.00 & 0.00 & 0.01 & 0.02 & 0.03 & 0.06 & 0.00 & -0.05 & -0.09 \\
\hline 17 & 6 & -0.01 & 0.00 & -0.01 & -0.03 & 0.00 & -0.01 & 0.08 & 0.01 & 0.03 \\
\hline 18 & 6 & 0.00 & 0.00 & -0.01 & 0.02 & 0.00 & 0.00 & -0.04 & 0.01 & 0.01 \\
\hline 19 & 6 & 0.00 & 0.01 & 0.01 & 0.00 & -0.01 & -0.02 & -0.01 & 0.02 & 0.04 \\
\hline 20 & 6 & 0.00 & 0.00 & -0.01 & -0.03 & -0.01 & -0.02 & 0.05 & 0.01 & 0.03 \\
\hline 21 & 6 & 0.01 & 0.00 & 0.00 & 0.06 & -0.01 & -0.01 & -0.10 & 0.01 & 0.00 \\
\hline 22 & 17 & 0.00 & 0.00 & 0.00 & 0.00 & 0.00 & 0.00 & 0.00 & 0.00 & 0.00 \\
\hline 23 & 17 & 0.00 & 0.00 & 0.00 & 0.00 & 0.00 & 0.00 & 0.00 & 0.00 & 0.00 \\
\hline 24 & 6 & 0.00 & 0.00 & 0.00 & 0.01 & 0.01 & -0.01 & 0.00 & 0.00 & 0.00 \\
\hline 25 & 6 & 0.00 & 0.00 & 0.00 & 0.01 & 0.00 & 0.00 & 0.00 & 0.00 & 0.00 \\
\hline 26 & 1 & 0.00 & 0.01 & 0.01 & 0.01 & -0.01 & 0.02 & 0.00 & 0.00 & \\
\hline 27 & 1 & 0.00 & -0.01 & 0.01 & -0.01 & 0.00 & 0.01 & 0.00 & 0.00 & 0.00 \\
\hline 28 & 1 & 0.00 & 0.01 & -0.01 & -0.01 & 0.00 & 0.00 & 0.00 & 0.00 & 0.00 \\
\hline 29 & 1 & 0.00 & 0.00 & 0.00 & -0.01 & -0.01 & 0.01 & 0.0 & 0.01 & \\
\hline 30 & 1 & 0.00 & 0.00 & 0.00 & 0.00 & 0.01 & 0.00 & 0.01 & -0.01 & 0.00 \\
\hline 31 & 1 & 0.00 & 0.01 & 0.00 & 0.00 & 0.00 & 0.01 & 0.00 & -0.01 & -0.01 \\
\hline 32 & 1 & -0.01 & -0.01 & -0.01 & 0.24 & 0.07 & 0.16 & -0.5 & -0.14 & -0.33 \\
\hline 33 & 1 & -0.02 & 0.05 & 0.09 & & -0.08 & -0.14 & -0.04 & 0.13 & 0.22 \\
\hline 34 & 1 & 0.01 & -0.01 & -0.01 & -0.40 & 0.08 & 0.09 & 0.65 & -0.14 & -0.15 \\
\hline 35 & 1 & 0.08 & -0.07 & -0.09 & -0.04 & 0.00 & 0.02 & -0.07 & 0.00 & 0.03 \\
\hline 36 & 1 & -0.11 & 0.70 & 0.63 & 0.01 & -0.02 & -0.02 & 0.00 & -0.02 & -0.02 \\
\hline 37 & 1 & -0.06 & -0.12 & -0.08 & 0.07 & 0.05 & 0.01 & 0.04 & 0.03 & 0.01 \\
\hline 38 & 1 & 0.00 & 0.00 & 0.00 & -0.02 & -0.05 & 0.03 & -0.01 & -0.01 & 0.00 \\
\hline 39 & 1 & 0.00 & 0.00 & 0.00 & -0.02 & -0.01 & 0.0 & 0.00 & 0.00 & 0.00 \\
\hline 40 & 1 & 0.00 & 0.00 & 0.00 & -0.04 & -0.01 & 0.02 & 0.00 & 0.00 & 0.00 \\
\hline 41 & 6 & 0.00 & 0.00 & 0.00 & -0.01 & -0.01 & 0.00 & 0.00 & 0.00 & 0.00 \\
\hline 42 & 1 & -0.01 & 0.00 & 0.00 & -0.04 & -0.01 & -0.02 & -0.01 & 0.00 & 0 \\
\hline 43 & 6 & 0.00 & 0.00 & 0.00 & -0.17 & 0.34 & 0.25 & -0.03 & 0.07 & 0.05 \\
\hline 44 & 1 & 0.00 & -0.01 & 0.00 & 0.07 & -0.38 & -0.28 & 0.02 & -0.07 & \\
\hline 45 & 1 & 0.00 & 0.00 & 0.00 & 0.04 & -0.34 & -0.28 & 0.01 & -0.07 & -0 \\
\hline 46 & 9 & 0.00 & 0.00 & 0.00 & 0. & -0.01 & -0.02 & 0.0 & 0.00 & 0.00 \\
\hline 47 & 9 & 0.00 & & 0.00 & & -0.13 & -0.0 & 0.0 & -0.03 & -0.02 \\
\hline 48 & 9 & 0.00 & 0.00 & 0.00 & 0.00 & -0.01 & -0.03 & 0.00 & 0.00 & -0.01 \\
\hline Frequer & & 1261.19 & & & 1265.61 & & & 1279.88 & & \\
\hline Atom & AN & $X$ & $\mathrm{Y}$ & Z & $X$ & Y & Z & $X$ & $Y$ & Z \\
\hline 1 & 6 & -0.05 & 0.00 & 0.02 & 0.07 & -0.01 & -0.03 & 0.02 & 0.00 & \\
\hline 2 & 6 & 0.09 & 0.04 & 0.00 & -0.07 & -0.03 & 0.00 & -0.02 & -0.01 & 0.0 \\
\hline 3 & 6 & 0.00 & -0.07 & -0.06 & -0.18 & -0.04 & 0.03 & -0.06 & -0.02 & 0.01 \\
\hline 4 & 6 & -0.10 & -0.02 & 0.03 & -0.06 & -0.01 & 0.02 & & -0.01 & \\
\hline 5 & 6 & 0.05 & 0.0 & 0.01 & 0. & 0.04 & 0.0 & & 0.02 & \\
\hline 6 & 6 & -0.01 & 0.04 & 0.03 & 0.01 & 0.00 & 0.00 & 0. & 0.00 & 0.00 \\
\hline 7 & 7 & 0.00 & 0.01 & 0.01 & 0.11 & -0.01 & 0.03 & & 0.00 & 0.00 \\
\hline 8 & 6 & 0.00 & 0.00 & -0.01 & 0.0 & 0.06 & -0.0 & & 0.02 & \\
\hline 9 & 6 & 0.00 & 0.00 & 0.00 & -0.04 & 0.07 & -0.08 & -0.03 & -0.01 & 0.01 \\
\hline 10 & 6 & 0.00 & 0.00 & 0.00 & 0.00 & -0.03 & 0.03 & & 0.00 & \\
\hline 11 & 17 & 0.1 & 0.00 & 0.00 & 0.0 & 0.00 & 0.0 & & 0.00 & 0.0 \\
\hline 12 & 17 & 0.00 & 0.00 & 0.00 & 0. & 0.00 & 0.00 & 0.00 & 0.00 & 0.00 \\
\hline 13 & 78 & 0.0 & 0.0 & 0.00 & 0. & 0.00 & 0.00 & & 0.00 & 0.00 \\
\hline 14 & 8 & 0.00 & 0.00 & 0.00 & 0.0 & 0.00 & 0.00 & & 0.04 & \\
\hline 15 & 7 & 0.00 & 0.00 & 0.00 & -0.11 & -0.04 & 0.02 & 0.01 & 0.00 & \\
\hline
\end{tabular}




\begin{tabular}{|c|c|c|c|c|c|c|c|c|c|c|}
\hline & & & & & & & & & & \\
\hline 16 & 6 & 0.01 & 0.00 & -0.01 & 0.24 & 0.01 & 0.04 & 0.01 & 0.00 & 0.00 \\
\hline 17 & 6 & 0.01 & 0.00 & 0.00 & 0.11 & 0.00 & 0.02 & 0.00 & 0.00 & 0.01 \\
\hline 18 & 6 & -0.01 & 0.00 & 0.00 & -0.10 & 0.02 & 0.02 & 0.00 & 0.00 & 0.00 \\
\hline 19 & 6 & 0.00 & 0.00 & 0.00 & 0.00 & 0.01 & 0.01 & 0.00 & 0.00 & 0.00 \\
\hline 20 & 6 & 0.00 & 0.00 & 0.00 & -0.05 & -0.02 & -0.05 & & 0.00 & 0.00 \\
\hline 21 & 6 & -0.01 & 0.00 & 0.00 & & -0.01 & 0.00 & & & 00 \\
\hline 22 & 17 & 0.00 & 0.00 & 0.00 & 0.00 & 0.00 & -0.01 & 0.00 & 0.00 & 0.00 \\
\hline 23 & 17 & 0.00 & 0.00 & 0.00 & 0.00 & & 0.00 & & 0.00 & 0.00 \\
\hline 24 & 6 & 0.00 & 0.00 & 0.00 & 0.02 & 0.01 & -0.01 & -0.01 & -0.01 & 0.00 \\
\hline 25 & 6 & 0.00 & 0.00 & 0.00 & 0.01 & -0.02 & 0.02 & 0.00 & -0.01 & 0.01 \\
\hline 26 & 1 & 0.00 & -0.01 & -0.01 & 0.01 & 0.01 & -0.01 & 0.00 & 0.00 & 0.00 \\
\hline 27 & 1 & 0.00 & 0.01 & -0.01 & 0.05 & -0.01 & -0.01 & 0.03 & -0.01 & 0.00 \\
\hline 28 & 1 & 0.00 & -0.01 & 0.01 & 0.04 & 0.01 & 0.02 & 0.02 & 0.00 & 0.01 \\
\hline 29 & 1 & 0.00 & & 0.00 & -0.07 & -0.06 & -0. & 0.0 & .00 & -0.01 \\
\hline 30 & 1 & 0.00 & 0.00 & 0.00 & -0.09 & 0.00 & 0.03 & -0.01 & 0.01 & 0.00 \\
\hline 31 & 1 & 0.00 & 0.00 & 0.00 & 0.00 & 0.03 & -0.02 & 0.01 & 0.02 & -0.02 \\
\hline 32 & 1 & -0.06 & & -0.03 & -0.49 & -0.10 & -0.2 & -0.03 & -0.02 & -0.01 \\
\hline 33 & 1 & 0.00 & 0.01 & 0.02 & -0.01 & 0.01 & 0.0 & 0.00 & 0.00 & 0.00 \\
\hline 34 & 1 & 0.04 & -0.01 & -0.01 & -0.37 & 0.06 & 0.0 & -0. & 0.00 & 0.00 \\
\hline 35 & 1 & 0.62 & & -0.28 & 0.31 & 0.01 & -0 . & 0. & & -0.05 \\
\hline 36 & 1 & -0.03 & & 0.16 & 0.01 & -0.01 & -0 . & 0. & & -0.01 \\
\hline 37 & 1 & -0.53 & -0.37 & -0.10 & & 0.22 & 0.1 & 0.11 & 0.07 & 0.01 \\
\hline 38 & 1 & 0.00 & & 0.00 & -0.05 & -0.08 & 0. & 0.0 & & -0.01 \\
\hline 39 & 1 & 0.00 & & 0.00 & -0.06 & -0.02 & 0.07 & 0.05 & -0.03 & -0.01 \\
\hline 40 & 1 & 0.00 & 0.00 & 0.00 & -0.08 & -0.03 & 0.03 & 0.03 & -0.01 & -0.02 \\
\hline 41 & 6 & 0.00 & & 0.00 & 0.01 & 0.01 & 0.0 & -0.10 & -0.19 & -0.19 \\
\hline 42 & 1 & 0.00 & & 0.00 & 0.01 & 0.00 & 0.00 & -0.17 & 0.23 & 0.11 \\
\hline 43 & 6 & -0.01 & 0.02 & 0.01 & -0.03 & -0.04 & -0.02 & 0.67 & 0.21 & 0.19 \\
\hline 44 & 1 & 0.00 & -0.02 & -0.01 & -0.01 & 0.03 & 0.0 & -0.05 & -0.26 & -0.27 \\
\hline 45 & 1 & 0.00 & & -0.02 & 0.01 & & 0. & 0.0 & -0.05 & 0.09 \\
\hline 46 & 9 & 0.00 & & 0.00 & 0.01 & & 0.0 & -0.14 & -0.10 & 0.07 \\
\hline 47 & 9 & 0.01 & & 0. & 0.00 & & & -0.09 & & 0.02 \\
\hline 48 & 9 & 0.00 & 0.00 & 0.00 & 0.01 & 0.00 & 0.01 & -0.10 & 0.02 & -0.12 \\
\hline Frequen & & 1292.72 & & & 1306.66 & & & 1312.69 & & \\
\hline Atom & AN & X & $Y$ & Z & $\mathrm{x}$ & Y & Z & $x$ & $Y$ & Z \\
\hline 1 & 6 & -0.07 & 0.02 & 0.04 & 0 & -0.01 & -0.01 & -0.24 & -0.19 & -0.07 \\
\hline 2 & 6 & 0.0 & 0.02 & -0.02 & -0.02 & & 0.0 & 0.28 & -0.08 & -0.17 \\
\hline 3 & 6 & & & -0.03 & -0.05 & & 0.0 & & & 0.30 \\
\hline 4 & 6 & 0.09 & 0.03 & -0.01 & -0.03 & -0.01 & 0.0 & -0 . & -0.22 & -0.10 \\
\hline 5 & 6 & -0.06 & & -0.02 & & 0. & 0.00 & 0.28 & -0.05 & -0.15 \\
\hline 6 & 6 & & & 0.00 & & & 0.0 & -0 & 0.21 & 0.19 \\
\hline 7 & 7 & & & 0. & & & 0. & & -0.02 & -0.02 \\
\hline 8 & 6 & & & 0.09 & -0.1 & 0.03 & -0.02 & 0.0 & 0.01 & 0.01 \\
\hline 9 & 6 & & & -0 . & & -0.04 & 0.0 & -0. & 0.00 & 0.01 \\
\hline 10 & 6 & & & & & & -0. & & & 0.00 \\
\hline 11 & 17 & & & & & & 0. & & 0. & 0.00 \\
\hline 12 & 17 & & & & & & 0.0 & 0.00 & 0.00 & 0.00 \\
\hline 13 & 78 & & & & & & 0.0 & & & 0.00 \\
\hline 14 & 8 & & & & & & 0. & & & \\
\hline 15 & 7 & -0.1 & & -0 & & & -0.03 & 0. & 0. & 0.00 \\
\hline 16 & 6 & & & & & & 0.37 & & & -0.01 \\
\hline 17 & 6 & & & -0.02 & & -0.10 & -0.14 & & 0.00 & 0.00 \\
\hline 18 & 6 & & & 0. & -0.22 & -0.08 & -0.19 & 0.00 & 0.00 & 0.00 \\
\hline 19 & 6 & -0.0 & & 0. & -0.0 & 0.14 & 0.2 & 0. & 0.00 & 0.00 \\
\hline 20 & 6 & & & -0.05 & & -0.06 & -0.06 & & & 0.00 \\
\hline 21 & 6 & 0. & & -0.02 & -0.27 & -0.11 & -0.23 & 0.0 & 0.00 & 0.00 \\
\hline 22 & 17 & & & 0.0 & & 0.00 & 0.00 & 0.00 & 0.00 & 0.00 \\
\hline 23 & 17 & & & 0.0 & & & 0.00 & & 0.00 & 0.00 \\
\hline 24 & 6 & & & -0.0 & -0.0 & -0.01 & 0.0 & 0.00 & 0.00 & 0.00 \\
\hline 25 & 6 & -0.02 & 0.03 & -0.03 & 0.02 & -0.01 & 0.01 & 0.00 & -0.01 & -0.01 \\
\hline
\end{tabular}




\begin{tabular}{|c|c|c|c|c|c|c|c|c|c|c|}
\hline 26 & 1 & -0.02 & 0.02 & -0.02 & 0.02 & -0.01 & 0.05 & -0.02 & 0.03 & 0.02 \\
\hline 27 & 1 & -0.09 & 0.02 & -0.02 & 0.00 & -0.02 & 0.02 & 0.00 & -0.01 & 0.02 \\
\hline 28 & 1 & -0.08 & 0.00 & -0.05 & 0.01 & 0.00 & 0.01 & 0.00 & 0.03 & -0.01 \\
\hline 29 & 1 & -0.03 & -0.04 & 0.03 & 0.03 & 0.01 & 0.02 & 0.00 & 0.00 & 0.00 \\
\hline 30 & 1 & -0.02 & -0.05 & 0.04 & 0.02 & 0.04 & -0.03 & -0.01 & 0.01 & 0.00 \\
\hline 31 & 1 & -0.03 & -0.10 & 0.07 & -0.01 & 0.04 & 0.02 & 0.00 & 0.01 & 0.00 \\
\hline 32 & 1 & -0.30 & -0.05 & -0.15 & 0.30 & 0.08 & 0.19 & -0.01 & 0.00 & -0.01 \\
\hline 33 & 1 & -0.01 & -0.01 & -0.03 & 0.04 & -0.11 & -0.19 & 0.00 & 0.00 & 0.00 \\
\hline 34 & 1 & -0.34 & 0.06 & 0.04 & -0.06 & 0.04 & 0.05 & 0.00 & 0.00 & 0.00 \\
\hline 35 & 1 & -0.43 & -0.01 & 0.16 & 0.10 & 0.00 & -0.04 & -0.32 & 0.04 & 0.15 \\
\hline 36 & 1 & -0.02 & -0.01 & 0.00 & 0.01 & 0.00 & 0.00 & 0.02 & -0.15 & -0.14 \\
\hline 37 & 1 & -0.41 & -0.24 & -0.04 & 0.10 & 0.06 & 0.01 & 0.26 & 0.20 & 0.07 \\
\hline 38 & 1 & -0.02 & -0.04 & 0.03 & 0.04 & 0.06 & -0.02 & -0.01 & 0.00 & -0.01 \\
\hline 39 & 1 & -0.03 & -0.03 & 0.04 & 0.04 & 0.02 & -0.06 & 0.01 & 0.00 & 0.00 \\
\hline 40 & 1 & 0.00 & 0.00 & -0.01 & 0.05 & 0.03 & -0.01 & 0.00 & -0.01 & 0.00 \\
\hline 41 & 6 & 0.00 & -0.01 & -0.02 & 0.00 & -0.01 & 0.01 & 0.00 & 0.00 & 0.00 \\
\hline 42 & 1 & -0.03 & 0.05 & 0.03 & 0.05 & -0.06 & -0.02 & 0.00 & 0.00 & 0.00 \\
\hline 43 & 6 & 0.05 & 0.00 & 0.02 & 0.00 & 0.01 & -0.02 & 0.00 & 0.00 & 0.00 \\
\hline 44 & 1 & -0.03 & -0.03 & -0.01 & 0.02 & 0.03 & 0.01 & 0.00 & 0.00 & 0.00 \\
\hline 45 & 1 & 0.03 & 0.01 & 0.02 & -0.02 & -0.04 & -0.03 & 0.00 & 0.00 & 0.00 \\
\hline 46 & 9 & -0.01 & 0.00 & 0.00 & 0.00 & 0.00 & 0.00 & 0.00 & 0.00 & .00 \\
\hline 47 & 9 & -0.01 & 0.01 & 0.00 & 0.00 & 0.00 & 0.00 & 0.00 & 0.00 & 0.00 \\
\hline 48 & 9 & -0.01 & 0.00 & -0.01 & 0.00 & 0.00 & 0.00 & 0.00 & 0.00 & 0.00 \\
\hline \multicolumn{2}{|c|}{ Frequencies } & 1317.11 & & & 1341.85 & & & 1355.64 & & \\
\hline Atom & AN & X & $Y$ & Z & $X$ & $Y$ & Z & $x$ & $Y$ & Z \\
\hline 1 & 6 & 0.00 & 0.00 & 0.00 & 0.00 & 0.00 & 0.00 & 0.00 & 0.00 & 0.00 \\
\hline 2 & 6 & 0.00 & 0.00 & 0.00 & 0.00 & 0.00 & 0.00 & -0.01 & 0.00 & 0.00 \\
\hline 3 & 6 & 0.00 & 0.00 & 0.00 & 0.00 & 0.00 & 0.00 & 0.02 & -0.02 & 0.02 \\
\hline 4 & 6 & 0.00 & 0.00 & 0.00 & 0.00 & 0.00 & 0.00 & -0.01 & 0.00 & 0.00 \\
\hline 5 & 6 & 0.00 & 0.00 & 0.00 & 0.00 & 0.00 & 0.00 & -0.01 & 0.00 & 0.00 \\
\hline 6 & 6 & 0.00 & 0.00 & 0.00 & 0.00 & 0.00 & 0.00 & 0.01 & 0.00 & 0.00 \\
\hline 7 & 7 & 0.00 & 0.00 & 0.00 & 0.00 & 0.00 & 0.00 & -0.09 & 0.04 & -0.05 \\
\hline 8 & 6 & -0.01 & 0.00 & 0.00 & 0.01 & 0.00 & 0.00 & 0.41 & -0.02 & 0.10 \\
\hline 9 & 6 & 0.00 & -0.01 & 0.01 & -0.01 & 0.00 & 0.00 & -0.34 & -0.14 & 0.07 \\
\hline 10 & 6 & 0.00 & 0.00 & 0.00 & 0.01 & 0.00 & 0.00 & 0.13 & 0.05 & -0.02 \\
\hline 11 & 17 & 0.00 & 0.00 & 0.00 & 0.00 & 0.00 & 0.00 & 0.00 & 0.00 & 0.00 \\
\hline 12 & 17 & 0.00 & 0.00 & 0.00 & 0.00 & 0.00 & 0.00 & 0.00 & 0.00 & 0.00 \\
\hline 13 & 78 & 0.00 & 0.00 & 0.00 & 0.00 & 0.00 & 0.00 & 0.00 & 0.00 & 0.00 \\
\hline 14 & 8 & 0.02 & -0.04 & 0.01 & 0.01 & -0.01 & 0.00 & 0.00 & 0.00 & 0.00 \\
\hline 15 & 7 & 0.02 & 0.01 & 0.00 & -0 & 0.00 & 0.00 & 0.02 & 0.05 & -0.05 \\
\hline 16 & 6 & -0.02 & 0.00 & 0.01 & 0.01 & 0.00 & 0.0 & 0.0 & -0.01 & 0.03 \\
\hline 17 & 6 & 0.00 & 0.00 & 0.00 & 0.0 & 0.00 & 0.00 & 0.04 & -0.01 & -0.01 \\
\hline 18 & 6 & 0.00 & 0.00 & -0.01 & 0.1 & 0.00 & 0.00 & -0.02 & 0.00 & 0.00 \\
\hline 19 & 6 & 0.00 & 0.00 & 0.00 & & 0.00 & 0.0 & & & \\
\hline 20 & 6 & 0.0 & 0.00 & 0.0 & & 0.00 & 0.0 & 0.00 & -0.01 & -0.02 \\
\hline 21 & 6 & -0.01 & 0.00 & -0.01 & 0. & 0.00 & 0.00 & 0.0 & 0.01 & 0.01 \\
\hline 22 & 17 & 0.0 & 0.00 & 0.00 & & 0.00 & 0.0 & & & 0.00 \\
\hline 23 & 17 & & 0.0 & 0.00 & & 0.00 & & 0.0 & 0.00 & \\
\hline 24 & 6 & 0.06 & 0.05 & -0.04 & -0.07 & -0.05 & 0.04 & 0.01 & 0.01 & -0.01 \\
\hline 25 & 6 & 0.00 & 0.00 & 0.00 & & 0.00 & 0.0 & & 0.00 & -0.03 \\
\hline 26 & 1 & 0.00 & 0.00 & 0.0 & & 0.01 & -0.0 & -0.19 & 0.12 & -0.28 \\
\hline 27 & 1 & 0.00 & 0.00 & 0.01 & 0.01 & 0.00 & -0.01 & 0.26 & 0.06 & -0.15 \\
\hline 28 & 1 & 0.00 & 0.00 & 0.00 & 0.0 & 0.01 & 0.0 & 0.14 & 0.16 & \\
\hline 29 & 1 & 0.01 & 0.00 & 0.0 & -0.0 & -0.01 & 0.0 & -0.17 & -0.10 & -0.14 \\
\hline 30 & 1 & 0.01 & 0.00 & 0.00 & -0.01 & 0.00 & 0.0 & -0.31 & 0.09 & \\
\hline 31 & 1 & 0.00 & 0.00 & 0.00 & 0.0 & 0.01 & -0.01 & 0.10 & 0.28 & -0.12 \\
\hline 32 & 1 & 0.04 & 0.01 & 0.02 & -0.03 & 0.00 & & -0.08 & -0.01 & -0.04 \\
\hline 33 & 1 & 0.00 & 0.00 & -0.01 & 0.00 & 0.00 & 0.0 & -0.02 & 0.00 & \\
\hline 34 & 1 & 0.03 & 0.00 & 0.00 & -0.03 & 0.00 & 0.00 & -0.17 & 0.03 & 0.03 \\
\hline 35 & 1 & 0.00 & 0.00 & 0.00 & 0.02 & 0.00 & -0.01 & 0.03 & 0.00 & \\
\hline
\end{tabular}




\begin{tabular}{|c|c|c|c|c|c|c|c|c|c|c|}
\hline 36 & 1 & 0.00 & 0.00 & 0.00 & 0.00 & 0.00 & 0.00 & 0.01 & 0.00 & 0.00 \\
\hline 37 & 1 & 0.00 & 0.00 & 0.00 & 0.02 & 0.01 & 0.00 & 0.01 & 0.01 & 0.00 \\
\hline 38 & 1 & -0.19 & -0.32 & 0.11 & 0.27 & 0.39 & -0.08 & -0.05 & -0.07 & 0.01 \\
\hline 39 & 1 & -0.25 & -0.12 & 0.33 & 0.33 & 0.08 & -0.35 & -0.07 & -0.02 & 0.07 \\
\hline 40 & 1 & -0.34 & -0.16 & 0.10 & 0.38 & 0.17 & -0.11 & -0.06 & -0.03 & 0.01 \\
\hline 41 & 6 & 0.02 & 0.05 & -0.07 & 0.02 & 0.03 & -0.04 & 0.00 & 0.00 & 0.00 \\
\hline 42 & 1 & -0.36 & 0.38 & 0.07 & -0.24 & 0.20 & -0.01 & 0.02 & -0.02 & 0.00 \\
\hline 43 & 6 & -0.02 & -0.09 & 0.11 & -0.03 & -0.08 & 0.10 & 0.00 & 0.01 & -0.01 \\
\hline 44 & 1 & -0.12 & -0.22 & -0.09 & -0.05 & -0.26 & -0.13 & 0.00 & 0.02 & 0.01 \\
\hline 45 & 1 & 0.11 & 0.25 & 0.21 & 0.02 & 0.27 & 0.21 & -0.01 & -0.03 & -0.03 \\
\hline 46 & 9 & 0.02 & 0.02 & -0.02 & 0.02 & 0.02 & -0.02 & 0.00 & 0.00 & 0.00 \\
\hline 47 & 9 & 0.00 & 0.01 & -0.01 & 0.00 & 0.00 & -0.01 & 0.00 & 0.00 & 0.00 \\
\hline 48 & 9 & -0.01 & 0.01 & -0.02 & 0.00 & 0.01 & -0.02 & 0.00 & 0.00 & 0.00 \\
\hline \multicolumn{2}{|c|}{ Frequencies } & 1419.83 & & & 1444.76 & & & 1448.89 & & \\
\hline Atom & AN & X & $Y$ & Z & X & $\mathrm{Y}$ & Z & $X$ & $\mathrm{Y}$ & Z \\
\hline 1 & 6 & 0.00 & 0.00 & 0.00 & 0.00 & 0.00 & 0.00 & 0.00 & 0.00 & 0.00 \\
\hline 2 & 6 & 0.00 & 0.00 & 0.00 & 0.00 & 0.00 & 0.00 & -0.01 & 0.00 & 0.01 \\
\hline 3 & 6 & 0.00 & 0.00 & 0.00 & 0.00 & 0.00 & 0.00 & 0.01 & 0.00 & 0.00 \\
\hline 4 & 6 & 0.00 & 0.00 & 0.00 & 0.00 & 0.00 & 0.00 & -0.01 & -0.01 & 0.00 \\
\hline 5 & 6 & 0.00 & 0.00 & 0.00 & 0.00 & 0.00 & 0.00 & 0.00 & 0.00 & 0.00 \\
\hline 6 & 6 & 0.00 & 0.00 & 0.00 & 0.00 & 0.00 & 0.00 & 0.00 & 0.00 & 0.00 \\
\hline 7 & 7 & 0.00 & 0.00 & 0.00 & 0.00 & 0.00 & 0.00 & 0.00 & 0.01 & -0.01 \\
\hline 8 & 6 & 0.00 & 0.00 & 0.00 & 0.00 & 0.00 & 0.00 & 0.01 & -0.01 & 0.02 \\
\hline 9 & 6 & 0.00 & 0.00 & 0.00 & 0.00 & 0.00 & 0.00 & 0.00 & 0.01 & -0.01 \\
\hline 10 & 6 & 0.00 & 0.00 & 0.00 & 0.00 & 0.00 & 0.00 & -0.02 & -0.06 & 0.06 \\
\hline 11 & 17 & 0.00 & 0.00 & 0.00 & 0.00 & 0.00 & 0.00 & 0.00 & 0.00 & 0.00 \\
\hline 12 & 17 & 0.00 & 0.00 & 0.00 & 0.00 & 0.00 & 0.00 & 0.00 & 0.00 & 0.00 \\
\hline 13 & 78 & 0.00 & 0.00 & 0.00 & 0.00 & 0.00 & 0.00 & 0.00 & 0.00 & 0.00 \\
\hline 14 & 8 & 0.03 & -0.05 & 0.04 & 0.01 & 0.03 & -0.02 & 0.00 & 0.00 & 0.00 \\
\hline 15 & 7 & 0.00 & 0.00 & 0.00 & 0.00 & 0.00 & 0.00 & 0.00 & -0.01 & 0.01 \\
\hline 16 & 6 & 0.00 & 0.00 & 0.00 & -0.02 & 0.00 & 0.00 & 0.01 & 0.00 & 0.00 \\
\hline 17 & 6 & 0.00 & 0.00 & 0.00 & 0.01 & -0.01 & -0.01 & -0.01 & 0.00 & 0.01 \\
\hline 18 & 6 & 0.00 & 0.00 & 0.00 & 0.00 & 0.00 & 0.01 & 0.00 & 0.00 & 0.00 \\
\hline 19 & 6 & 0.00 & 0.00 & 0.00 & -0.01 & 0.00 & 0.00 & 0.00 & 0.00 & 0.00 \\
\hline 20 & 6 & 0.00 & 0.00 & 0.00 & 0.00 & 0.00 & -0.01 & 0.00 & 0.00 & 0.00 \\
\hline 21 & 6 & 0.00 & 0.00 & 0.00 & & 0.01 & 0.01 & -0.01 & 0.00 & -0.01 \\
\hline 22 & 17 & 0.00 & 0.00 & 0.00 & 0. & 0.00 & 0.00 & 0.00 & 0.00 & 0.00 \\
\hline 23 & 17 & 0.00 & 0.00 & 0.00 & 0.00 & 0.00 & 0.0 & 0.00 & 0.00 & 0.00 \\
\hline 24 & 6 & -0.02 & 0.00 & 0.00 & 0.01 & -0.01 & 0.00 & 0.00 & 0.00 & 0.00 \\
\hline 25 & 6 & 0.00 & 0.00 & 0.00 & & 0.00 & 0.00 & -0.05 & 0.06 & -0.06 \\
\hline 26 & 1 & 0.00 & 0.00 & 0.00 & 0.00 & 0.01 & -0.0 & 0.09 & -0.25 & 0.18 \\
\hline 27 & 1 & 0.00 & 0.00 & 0.00 & -0.01 & 0.00 & -0.02 & 0.20 & -0.08 & 0.42 \\
\hline 28 & 1 & 0.00 & 0.00 & 0.00 & -0.0 & 0.01 & 0.00 & 0.25 & -0.36 & 0.07 \\
\hline 29 & 1 & 0.00 & 0.00 & 0.00 & & 0.00 & & 0.15 & 0.11 & -0.37 \\
\hline 30 & 1 & 0.0 & 0.00 & 0.00 & & -0.03 & 0.01 & 0.08 & 0.42 & -0.09 \\
\hline 31 & 1 & 0.00 & 0.00 & 0.00 & & -0.01 & 0.01 & 0.01 & 0.18 & -0.24 \\
\hline 32 & 1 & 0.00 & 0.00 & 0.00 & & -0.01 & -0.03 & & 0.01 & 0.02 \\
\hline 33 & 1 & 0.00 & 0.00 & 0.00 & -0.01 & 0.00 & 0.0 & 0.0 & 0.00 & 0.00 \\
\hline 34 & 1 & 0.00 & 0.00 & 0.00 & -0.05 & 0.01 & 0.01 & 0.04 & -0.01 & -0.01 \\
\hline 35 & 1 & 0.00 & 0.00 & 0.00 & & 0.00 & 0.0 & & 0.00 & -0.01 \\
\hline 36 & 1 & 0.00 & 0.00 & 0.00 & 0.00 & 0.00 & 0.0 & 0.01 & 0.00 & 0.00 \\
\hline 37 & 1 & 0.00 & 0.00 & 0.00 & 0.00 & 0.00 & 0.00 & 0.03 & 0.02 & 0.00 \\
\hline 38 & 1 & 0.07 & 0.09 & 0.01 & -0.0 & -0.07 & -0. & 0.0 & 0.00 & 0.00 \\
\hline 39 & 1 & 0.11 & -0.06 & -0.02 & -0.10 & 0.08 & -0.1 & 0.0 & 0.01 & 0.00 \\
\hline 40 & 1 & 0.04 & -0.03 & -0.04 & -0.01 & 0.05 & 0.0 & 0.00 & 0.00 & 0.00 \\
\hline 41 & 6 & 0.03 & -0.03 & -0.08 & 0.0 & -0.11 & -0.05 & 0.00 & -0.01 & 0.00 \\
\hline 42 & 1 & -0.40 & 0.41 & 0.10 & 0.30 & -0.28 & -0.07 & 0.02 & -0.02 & 0.00 \\
\hline 43 & 6 & -0.03 & 0.08 & -0.03 & -0.03 & 0.04 & 0.0 & 0.00 & 0.00 & 0.00 \\
\hline 44 & 1 & -0.25 & 0.55 & 0.46 & -0.18 & 0.21 & 0.28 & -0.01 & 0.01 & 0.02 \\
\hline 45 & 1 & 0.07 & -0.07 & -0.09 & -0.34 & 0.60 & 0.37 & -0.02 & 0.04 & 0.02 \\
\hline
\end{tabular}




\begin{tabular}{|c|c|c|c|c|c|c|c|c|c|c|}
\hline $\begin{array}{l}46 \\
47 \\
48\end{array}$ & $\begin{array}{l}9 \\
9 \\
9\end{array}$ & $\begin{array}{l}-0.01 \\
0.01 \\
0.01\end{array}$ & $\begin{array}{l}-0.01 \\
-0.01 \\
-0.01\end{array}$ & $\begin{array}{l}0.01 \\
0.00 \\
0.01\end{array}$ & $\begin{array}{l}0.00 \\
0.01 \\
0.00\end{array}$ & $\begin{array}{r}0.00 \\
-0.01 \\
0.00\end{array}$ & $\begin{array}{l}-0.01 \\
-0.01 \\
-0.01\end{array}$ & $\begin{array}{l}0.00 \\
0.00 \\
0.00\end{array}$ & $\begin{array}{l}0.00 \\
0.00 \\
0.00\end{array}$ & $\begin{array}{l}0.00 \\
0.00 \\
0.00\end{array}$ \\
\hline \multicolumn{2}{|c|}{ Frequencies } & 1458.30 & & & 1466.94 & & & 1472.41 & & \\
\hline Atom & AN & X & Y & Z & $X$ & $\mathrm{Y}$ & Z & X & Y & Z \\
\hline & 6 & 0.00 & 0.01 & 0.01 & 0.00 & -0.01 & 0.00 & -0.04 & -0.08 & -0.05 \\
\hline 2 & 6 & 0.02 & -0.01 & -0.02 & -0.01 & 0.01 & 0.01 & -0.14 & 0.07 & 0.11 \\
\hline 3 & 6 & -0.03 & -0.01 & 0.00 & 0.02 & 0.00 & 0.00 & 0.21 & 0.06 & -0.03 \\
\hline 4 & 6 & 0.02 & 0.02 & 0.01 & & -0.01 & -0.01 & -0.11 & -0.14 & -0.08 \\
\hline 5 & 6 & 0.01 & -0.01 & -0.01 & & 0.00 & 0.00 & & 0.05 & .06 \\
\hline 6 & 6 & -0.01 & 0.00 & 0.00 & & 0.00 & 0.00 & & 0.02 & -0.01 \\
\hline 7 & 7 & -0.01 & -0.02 & 0.02 & & 0.00 & 0.00 & -0.04 & 0.01 & -0.02 \\
\hline 8 & 6 & 0.01 & 0.02 & -0.02 & & 0.00 & & & .05 & \\
\hline 9 & 6 & -0.02 & 0.02 & -0.02 & -0.04 & & -0.04 & & .01 & \\
\hline 10 & 6 & -0.02 & -0.06 & 0.06 & & 0.04 & -0.03 & -0.01 & -0.01 & 0.02 \\
\hline 11 & 17 & & 0.00 & 0.0 & & 0.00 & 0.0 & & & \\
\hline 12 & 17 & & 0.00 & 0.0 & & & & & & \\
\hline 13 & 78 & 0. & 0.00 & 0.00 & & 0.00 & 0. & & & \\
\hline 14 & 8 & 0.0 & 0.00 & 0.0 & & 0.00 & 0.0 & & & \\
\hline 15 & 7 & 0.0 & -0.01 & 0.0 & & -0.01 & & & & \\
\hline 16 & 6 & 0.0 & 0.00 & 0.0 & & 0.00 & 0. & & & \\
\hline 17 & 6 & -0.04 & 0.02 & & & & & & & \\
\hline 18 & 6 & 0.0 & -0.01 & -0.02 & & & & & & \\
\hline 19 & 6 & 0.0 & 0.00 & 0.0 & 0. & 0.00 & & & & \\
\hline 20 & 6 & -0.01 & 0.01 & & & & & & & 0.00 \\
\hline 21 & 6 & -0 . & -0.02 & -0. & & & & & & \\
\hline 22 & 17 & & 0.00 & 0.1 & & & & & & \\
\hline 23 & 17 & 0.0 & 0.00 & 0.0 & & & & & & .00 \\
\hline 24 & 6 & & 0.0 & 0. & & & & & & \\
\hline 25 & 6 & 0. & -0.05 & 0.0 & & & -0. & & & \\
\hline 26 & 1 & -0 . & 0.21 & -0. & & -0.08 & 0.0 & -0.0 & 0.1 & -0 \\
\hline 27 & 1 & & 0.08 & -0. & & & & & & \\
\hline 28 & 1 & -0.2 & 0.3 & -0 . & & & 0. & -0 . & & \\
\hline 29 & 1 & & 0.1 & -0 & & & & & & \\
\hline 30 & 1 & & 0.4 & -0 & & & & & & \\
\hline 31 & 1 & 0. & 0.20 & -0.2 & & -0.16 & 0.2 & & 0.02 & \\
\hline 32 & 1 & & 0. & & & & & & & \\
\hline 33 & 1 & & & & & & & & & \\
\hline 34 & 1 & & -0. & & & & & & & \\
\hline 35 & 1 & & 0.1 & & & & -0. & & & \\
\hline 3 & 1 & & 0. & & & & & & & \\
\hline 37 & 1 & & & & & & & & & \\
\hline 38 & 1 & & 0. & & & & & -0. & -0.02 & \\
\hline 39 & 1 & & 0. & & & & & & & \\
\hline 40 & 1 & & & & & & & & & \\
\hline 4 & 6 & & -0 . & & & & & & & \\
\hline 42 & 1 & & -0 . & & & & -0.0 & & & \\
\hline 43 & 6 & & & & & & & & & \\
\hline 44 & 1 & -0 . & 0. & & & & & & & \\
\hline 45 & 1 & & 0. & & & & & & & \\
\hline 46 & 9 & & & & & & & & & \\
\hline 47 & 9 & 0. & & 0. & & & & & & \\
\hline 48 & 9 & & & & & & & & & \\
\hline Freque & & 1488.31 & & & & & & 1503.12 & & \\
\hline Atom & AN & $X$ & Y & Z & & & 2 & & & \\
\hline & 6 & & 0.00 & 0.0 & & -0.04 & & & & \\
\hline 2 & 6 & & 0.00 & 0.00 & 0.1 & 0.07 & 0.04 & 0.01 & 0.00 & -0.0 \\
\hline 3 & 6 & & 0.00 & 0.0 & & -0.09 & -0.08 & -0.0 & -0.01 & \\
\hline 4 & 6 & & 0.00 & 0.0 & & 0.04 & 0.06 & & 0.01 & \\
\hline & 6 & & 0.00 & 0.00 & & 0.03 & -0.03 & 0.01 & 0.00 & \\
\hline
\end{tabular}




\begin{tabular}{|c|c|c|c|c|c|c|c|c|c|c|}
\hline & & & & & & & & & & \\
\hline 6 & 6 & 0.00 & 0.00 & 0.00 & 0.01 & -0.09 & -0.08 & -0.01 & 0.00 & 0.00 \\
\hline 7 & 7 & 0.00 & 0.00 & 0.00 & 0.00 & 0.01 & 0.01 & 0.02 & 0.03 & -0.03 \\
\hline 8 & 6 & 0.00 & 0.00 & 0.00 & 0.00 & 0.00 & 0.00 & 0.00 & -0.02 & 0.03 \\
\hline 9 & 6 & 0.00 & 0.00 & 0.00 & 0.00 & 0.00 & 0.00 & 0.00 & 0.01 & -0.01 \\
\hline 10 & 6 & -0.01 & 0.00 & 0.00 & 0.00 & 0.00 & 0.00 & 0.03 & 0.01 & 0.00 \\
\hline 11 & 17 & 0.00 & 0.00 & 0.00 & & 0.00 & 0.00 & 0.00 & 0.00 & 0.00 \\
\hline 12 & 17 & 0.00 & 0.00 & 0.00 & 0.00 & 0.00 & 0.00 & 0.00 & 0.00 & 0.00 \\
\hline 13 & 78 & 0.00 & 0.00 & 0.00 & 0.00 & 0.00 & 0.00 & 0.00 & 0.00 & 0.00 \\
\hline 14 & 8 & 0.00 & 0.00 & 0.00 & 0.00 & 0.00 & 0.00 & 0.00 & 0.00 & 0.00 \\
\hline 15 & 7 & -0.01 & 0.01 & 0.01 & & 0.00 & 0.0 & 0.02 & -0.01 & 0.02 \\
\hline 16 & 6 & 0.02 & -0.06 & -0.11 & & 0.00 & 0.0 & -0.02 & 0.00 & -0.01 \\
\hline 17 & 6 & 0.05 & 0.04 & 0.08 & 0.00 & 0.00 & 0.00 & 0.01 & 0.00 & 0.00 \\
\hline 18 & 6 & -0.13 & -0.01 & -0.04 & 0.0 & 0.00 & 0.0 & 0.00 & 0.00 & 0.00 \\
\hline 19 & 6 & 0.02 & -0.05 & -0.09 & & 0.00 & 0. & 0.0 & 0.00 & 0.00 \\
\hline 20 & 6 & 0.13 & -0.01 & 0.01 & & 0.00 & 0.00 & 0.0 & 0.00 & 0.00 \\
\hline 21 & 6 & -0.07 & 0.04 & 0.06 & & 0.00 & 0.0 & 0.00 & 0.00 & 0.01 \\
\hline 22 & 17 & 0.00 & & & & 0.00 & & & & \\
\hline 23 & 17 & 0.00 & 0.00 & 0. & & 00 & 0. & & & \\
\hline 24 & 6 & 0.00 & 0.00 & 0.00 & & 0.00 & 0.00 & 0.00 & 0.00 & 0.00 \\
\hline 25 & 6 & 0.00 & 0.0 & 0.00 & & 0.00 & & 0.04 & & \\
\hline 26 & 1 & 0.00 & -0. & 0.00 & & -0.04 & -0.03 & -0.11 & 0.31 & \\
\hline 27 & 1 & 0.01 & 0.00 & -0.01 & 0.04 & 0.01 & 0.00 & -0.32 & -0.10 & 0.29 \\
\hline 28 & 1 & 0.00 & 0.01 & 0.00 & -0.0 & 0.00 & 0. & -0.22 & -0.39 & -0.07 \\
\hline 29 & 1 & 0.00 & 0.00 & 0.02 & -0.01 & 0.00 & 0.00 & -0.22 & -0.09 & -0.29 \\
\hline 30 & 1 & 0.07 & -0.01 & 0.00 & 0.00 & 0.00 & 0.00 & -0.30 & 0.20 & -0.03 \\
\hline 31 & 1 & 0.01 & -0.01 & -0.07 & & -0.01 & 0.00 & 0.01 & -0.26 & \\
\hline 32 & 1 & 0.34 & 0.1 & $0.2^{2}$ & & bo & 0. & -0.01 & & \\
\hline 33 & 1 & -0.10 & 0.3 & 0.5 & & & -0.0 & -0.01 & & \\
\hline 34 & 1 & -0.41 & 0.14 & 0.18 & & 0.00 & 0.0 & -0.03 & 0.01 & 0.01 \\
\hline 35 & 1 & 0.0 & 0.0 & 0.0 & -0 & 0.11 & 0. & -0.03 & 0.00 & \\
\hline 36 & 1 & 0.0 & 0.8 & 0. & & & 0.4 & -0 . & & \\
\hline 37 & 1 & 0.0 & 0.00 & 0. & & 0.31 & 0.1 & -0.03 & -0.02 & -0.01 \\
\hline 38 & 1 & -0.01 & 0.01 & -0.02 & -0.0 & 0.02 & -0.0 & 0. & 0.01 & \\
\hline 39 & 1 & 0.0 & -0.02 & & & 03 & & & -0.01 & \\
\hline 40 & 1 & 0.0 & -0. & -0 . & & -0 . & -0. & 0. & -0.02 & -0.01 \\
\hline 41 & 6 & 0.00 & 0.00 & 0.0 & 0. & 0.00 & 0.0 & 0.00 & 0.00 & \\
\hline 42 & 1 & 0.0 & 0.00 & 0.0 & & -0.01 & 0.0 & 0.0 & 0.00 & \\
\hline 43 & 6 & 0.0 & 0. & 0. & & 00 & 0. & 0. & & \\
\hline 44 & 1 & 0.0 & 0. & 0. & & & 0. & & & \\
\hline 45 & 1 & 0.0 & 0. & 0. & & 01 & 0. & 0.8 & & \\
\hline 46 & 9 & 0.0 & 0.0 & 0.0 & & 0.00 & 0.0 & 0.00 & 0.00 & 0.00 \\
\hline 47 & 9 & 0.0 & & 0. & & & & & & \\
\hline 48 & 9 & 0.0 & 0.00 & 0.00 & 0. & 0.00 & 0.0 & 0.00 & 0.00 & \\
\hline Freque & & 1505.08 & & & & & & 1523.65 & & \\
\hline Atom & AN & X & $Y$ & Z & & Y & Z & & $\mathrm{Y}$ & \\
\hline 1 & 6 & -0.01 & 0.00 & 0.00 & 0.0 & 0.00 & 0.00 & & 0.00 & \\
\hline 2 & 6 & & 0.0 & 0.00 & & 0.00 & 0.00 & & 0.00 & \\
\hline 3 & 6 & & 0.0 & 0.00 & & -0.01 & 0.0 & & 0.00 & \\
\hline 4 & 6 & 0. & 0. & 0.0 & & 0. & 0.0 & & -0.01 & \\
\hline 5 & 6 & & 0.0 & 0. & & 0. & 0. & & 0.00 & \\
\hline 6 & 6 & & 0. & 0.0 & & 0. & 0.0 & & 0.00 & 0.0 \\
\hline 7 & 7 & & 0. & 0.0 & & & -0.0 & & & \\
\hline 8 & 6 & & 0. & 0.0 & & & 0.0 & & & \\
\hline 9 & 6 & 0.0 & 0.00 & 0.00 & -0 . & -0.01 & 0.03 & 0.01 & 0.00 & 0.00 \\
\hline 10 & 6 & & 0.00 & 0.00 & & 0.02 & 0.01 & & -0.01 & \\
\hline 11 & 17 & & 0.00 & 0.0 & & 0.00 & 0.0 & & & \\
\hline 12 & 17 & & 0.00 & 0.00 & & 0.00 & 0.00 & 0.00 & 0.00 & 0.0 \\
\hline 13 & 78 & & 0.00 & 0.00 & & 0.00 & 0.0 & 0.00 & 0.00 & \\
\hline 14 & 8 & & -0.01 & 0.00 & & 0.00 & 0.0 & 0.0 & 0.01 & \\
\hline 15 & 7 & 0.00 & 0.00 & 0.00 & -0.02 & 0.01 & -0.02 & 0.00 & 0.00 & 0.00 \\
\hline
\end{tabular}




\begin{tabular}{|c|c|c|c|c|c|c|c|c|c|c|}
\hline & & & & & & & & & & \\
\hline $\begin{array}{l}16 \\
17\end{array}$ & 6 & 0.00 & 0.00 & 0.00 & 0.02 & 0.00 & 0.01 & 0.00 & 0.00 & 0.00 \\
\hline $\begin{array}{l}17 \\
18\end{array}$ & $\begin{array}{l}6 \\
6\end{array}$ & 0.00 & 0.00 & 0.00 & -0.01 & 0.00 & 0.00 & 0.00 & 0.00 & $\begin{array}{l}0.00 \\
0.00\end{array}$ \\
\hline 19 & 6 & 0.00 & 0.00 & 0.00 & 0.00 & 0.00 & 0.01 & 0.00 & 0.00 & 0.00 \\
\hline 20 & 6 & 0.00 & 0.00 & 0.00 & -0.01 & 0.00 & 0.00 & 0.00 & 0.00 & 0.00 \\
\hline 21 & 6 & 0.00 & 0.00 & 0.00 & 0.00 & 0.00 & -0.01 & 0.00 & 0.00 & 0.00 \\
\hline 22 & 17 & 0.00 & 0.00 & 0.00 & 0.00 & 0.00 & 0.00 & 0.00 & 0.00 & 0.00 \\
\hline 23 & 17 & 0.00 & 0.00 & 0.00 & 0.00 & 0.00 & 0.00 & 0.00 & 0.00 & 0.00 \\
\hline 24 & 6 & 0.01 & -0.05 & -0.04 & -0.01 & 0.01 & -0.01 & -0.04 & 0.03 & -0.05 \\
\hline 25 & 6 & 0.00 & 0.00 & 0.00 & 0.01 & -0.01 & -0.02 & 0.00 & 0.00 & 0.00 \\
\hline 26 & 1 & -0.01 & 0.01 & -0.01 & -0.14 & 0.36 & 0.19 & 0.02 & -0.05 & -0.05 \\
\hline 27 & 1 & -0.02 & 0.00 & 0.01 & -0.39 & -0.05 & 0.08 & 0.06 & 0.01 & -0.02 \\
\hline 28 & 1 & 0.00 & -0.01 & 0.00 & 0.19 & -0.11 & 0.06 & -0.05 & 0.02 & -0.02 \\
\hline 29 & 1 & 0.00 & 0.00 & 0.00 & -0.15 & -0.10 & 0.20 & 0.06 & 0.04 & -0.02 \\
\hline 30 & 1 & 0.00 & 0.01 & 0.00 & 0.49 & -0.04 & -0.02 & -0.08 & 0.00 & 0.00 \\
\hline 31 & 1 & 0.00 & 0.00 & 0.00 & 0.12 & -0.14 & -0.46 & -0.03 & 0.06 & 0.08 \\
\hline 32 & 1 & 0.00 & 0.00 & 0.01 & 0.01 & 0.00 & 0.00 & 0.00 & 0.00 & 0.00 \\
\hline 33 & 1 & 0.00 & 0.01 & 0.02 & 0.01 & -0.01 & -0.02 & 0.00 & 0.00 & 0.00 \\
\hline 34 & 1 & -0.01 & 0.00 & 0.01 & 0.04 & -0.01 & -0.01 & 0.00 & 0.00 & 0.00 \\
\hline 35 & 1 & -0.01 & 0.01 & 0.01 & -0.02 & 0.00 & 0.01 & 0.01 & 0.00 & -0.01 \\
\hline 36 & 1 & 0.00 & 0.03 & 0.02 & -0.01 & 0.01 & 0.01 & 0.00 & 0.00 & 0.00 \\
\hline 37 & 1 & 0.01 & 0.02 & 0.01 & -0.01 & -0.01 & 0.00 & 0.01 & 0.01 & 0.00 \\
\hline 38 & 1 & 0.12 & -0.22 & 0.44 & 0.07 & 0.03 & 0.08 & 0.36 & 0.12 & 0.46 \\
\hline 39 & 1 & -0.10 & 0.33 & -0.32 & 0.09 & -0.08 & 0.01 & 0.48 & -0.40 & 0.05 \\
\hline 40 & 1 & -0.12 & 0.53 & 0.46 & -0.07 & -0.04 & 0.01 & -0.37 & -0.16 & 0.09 \\
\hline 41 & 6 & 0.00 & 0.01 & 0.00 & 0.00 & 0.00 & 0.00 & 0.01 & -0.01 & 0.01 \\
\hline 42 & 1 & -0.05 & 0.07 & 0.03 & 0.02 & -0.02 & 0.00 & 0.12 & -0.08 & 0.01 \\
\hline 43 & 6 & 0.00 & 0.00 & -0.01 & 0.00 & 0.00 & 0.00 & 0.00 & 0.00 & 0.00 \\
\hline 44 & 1 & 0.01 & 0.02 & -0.01 & 0.00 & 0.00 & 0.00 & -0.04 & -0.05 & 0.03 \\
\hline 45 & 1 & 0.04 & -0.04 & -0.02 & -0.01 & 0.01 & 0.01 & -0.09 & 0.05 & -0.01 \\
\hline 46 & 9 & 0.00 & 0.00 & 0.00 & 0.00 & 0.00 & 0.00 & 0.00 & 0.00 & 0.00 \\
\hline 47 & 9 & 0.00 & 0.00 & 0.00 & 0.00 & 0.00 & 0.00 & 0.00 & 0.00 & 0.00 \\
\hline 48 & 9 & 0.00 & 0.00 & 0.00 & 0.00 & 0.00 & 0.00 & 0.00 & 0.00 & 0.00 \\
\hline Frequen & & 1528.51 & & & 1530.21 & & & 1534.96 & & \\
\hline Atom & AN & $X$ & $Y$ & Z & $\mathrm{X}$ & $Y$ & Z & $x$ & $Y$ & Z \\
\hline 1 & 6 & 0.00 & 0.00 & 0.00 & 0.00 & 0.00 & 0.00 & 0.00 & 0.00 & 0.00 \\
\hline 2 & 6 & 0.00 & 0.00 & 0.00 & 0.00 & 0.00 & 0.00 & 0.00 & 0.00 & 0.00 \\
\hline 3 & 6 & -0.02 & -0.01 & 0.00 & 0.00 & 0.00 & 0.00 & 0.00 & 0.00 & 0.00 \\
\hline 4 & 6 & 0.00 & 0.00 & 0.00 & 0.00 & 0.00 & 0.00 & 0.00 & 0.00 & 0.00 \\
\hline 5 & 6 & 0.00 & 0.00 & 0.00 & 0.00 & 0.00 & 0.00 & 0.00 & 0.00 & 0.00 \\
\hline 6 & 6 & 0.00 & 0.00 & 0.00 & 0.00 & 0.00 & 0.00 & 0.00 & -0.01 & -0.01 \\
\hline 7 & 7 & 0.02 & 0.03 & -0.03 & 0.0 & 0.00 & 0.00 & 0.0 & 0.00 & 0.00 \\
\hline 8 & 6 & 0.01 & -0.02 & 0.04 & 0.0 & 0.00 & 0.00 & 0.01 & -0.01 & -0.01 \\
\hline 9 & 6 & 0.00 & -0.03 & 0.01 & 0.00 & 0.00 & 0.00 & 0.01 & -0.01 & -0.01 \\
\hline 10 & 6 & -0.03 & -0.03 & -0.02 & 0.00 & 0.00 & 0.00 & 0.01 & -0.03 & -0.02 \\
\hline 11 & 17 & 0.00 & 0.00 & 0.00 & & & 0.00 & & & 0.00 \\
\hline 12 & 17 & 0.0 & 0.00 & 0.00 & 0.00 & 0.00 & 0.0 & 0.0 & 0.00 & 0.00 \\
\hline 13 & 78 & 0.00 & 0.00 & 0.00 & 0.00 & 0.00 & 0.00 & 0.00 & 0.00 & 0.00 \\
\hline 14 & 8 & 0.00 & 0.00 & 0.00 & -0.0 & 0.01 & 0.00 & & & \\
\hline 15 & 7 & -0.0 & 0.0 & -0.02 & & 0.00 & 0.0 & & & \\
\hline 16 & 6 & 0.02 & 0.00 & 0.00 & 0.0 & 0.00 & 0.00 & 0.00 & 0.00 & 0.00 \\
\hline 17 & 6 & 0.00 & 0.00 & 0.00 & 0.0 & 0.00 & 0.00 & & & 0.00 \\
\hline 18 & 6 & 0.00 & 0.00 & 0.00 & 0.0 & 0.00 & 0.00 & 0. & 0.0 & 0.00 \\
\hline 19 & 6 & 0.00 & 0.00 & 0.00 & 0.00 & 0.00 & 0.00 & 0.00 & 0.00 & -0.01 \\
\hline 20 & 6 & -0.01 & 0.00 & 0.00 & 0.0 & 0.00 & 0.0 & & 0.00 & 0.00 \\
\hline 21 & 6 & 0.00 & 0.00 & 0.00 & 0.0 & 0.00 & 0.00 & 0.0 & 0.00 & 0.00 \\
\hline 22 & 17 & 0.00 & 0.00 & 0.00 & 0.0 & 0.00 & 0.00 & 0.0 & 0.00 & \\
\hline 23 & 17 & 0.00 & 0.00 & 0.00 & 0.00 & 0.00 & 0.00 & 0.00 & 0.00 & 0.00 \\
\hline 24 & 6 & 0.00 & 0.00 & 0.00 & 0 & 0.00 & 0.00 & & 0.00 & 0.00 \\
\hline 25 & 6 & 0.02 & 0.02 & 0.01 & 0.00 & 0.00 & 0.00 & 0.00 & -0.02 & \\
\hline
\end{tabular}




\begin{tabular}{|c|c|c|c|c|c|c|c|c|c|c|}
\hline & & & & & & & & & & \\
\hline $\begin{array}{l}26 \\
27\end{array}$ & $\begin{array}{l}1 \\
1\end{array}$ & $\begin{array}{l}-0.02 \\
-0.05\end{array}$ & $\begin{array}{r}0.06 \\
-0.06\end{array}$ & $\begin{array}{r}-0.37 \\
0.20\end{array}$ & $\begin{array}{l}0.00 \\
0.01\end{array}$ & $\begin{array}{r}-0.01 \\
0.00\end{array}$ & $\begin{array}{r}0.00 \\
-0.01\end{array}$ & $\begin{array}{l}-0.12 \\
-0.36\end{array}$ & $\begin{array}{c}0.31 \\
-0.02\end{array}$ & $\begin{array}{c}0.41 \\
-0.03\end{array}$ \\
\hline 28 & 1 & -0.32 & -0.28 & -0.10 & 0.00 & 0.01 & 0.00 & 0.38 & 0.05 & 0.12 \\
\hline 29 & 1 & 0.42 & 0.20 & 0.28 & -0.01 & 0.00 & -0.01 & 0.29 & 0.16 & -0.04 \\
\hline 30 & 1 & 0.12 & -0.25 & 0.05 & -0.01 & 0.01 & 0.00 & -0.32 & -0.07 & 0.03 \\
\hline 31 & 1 & -0.09 & 0.47 & -0.05 & 0.00 & -0.01 & 0.01 & -0.12 & 0.31 & 0.31 \\
\hline 32 & 1 & 0.01 & 0.00 & 0.01 & -0.01 & 0.00 & 0.00 & 0.00 & 0.00 & 0.01 \\
\hline 33 & 1 & 0.01 & 0.00 & 0.00 & 0.00 & -0.01 & -0.01 & -0.01 & 0.01 & 0.02 \\
\hline 34 & 1 & 0.02 & 0.00 & -0.01 & -0.01 & 0.00 & 0.00 & -0.01 & 0.01 & 0.01 \\
\hline 35 & 1 & -0.01 & 0.00 & 0.01 & 0.00 & 0.00 & 0.00 & -0.01 & 0.00 & 0.01 \\
\hline 36 & 1 & 0.00 & 0.00 & 0.00 & 0.00 & 0.00 & 0.00 & 0.00 & 0.02 & 0.02 \\
\hline 37 & 1 & -0.01 & -0.01 & 0.00 & 0.00 & 0.00 & 0.00 & 0.00 & 0.00 & 0.00 \\
\hline 38 & 1 & -0.02 & -0.01 & -0.03 & 0.03 & 0.02 & 0.04 & -0.01 & 0.00 & \\
\hline 39 & 1 & -0.03 & 0.02 & 0.00 & 0.05 & -0.05 & 0.01 & -0.01 & 0.00 & 0.00 \\
\hline 40 & 1 & 0.03 & 0.01 & -0.01 & -0.03 & -0.03 & 0.00 & 0.01 & 0.00 & 0.00 \\
\hline 41 & 6 & 0.00 & 0.00 & 0.00 & -0.07 & -0.04 & -0.02 & 0.00 & 0.00 & 0.00 \\
\hline 42 & 1 & -0.01 & 0.01 & 0.00 & 0.02 & -0.03 & -0.01 & 0.00 & 0.00 & 0.00 \\
\hline 43 & 6 & 0.00 & 0.00 & 0.00 & -0.03 & 0.01 & 0.01 & 0.00 & 0.00 & 0.00 \\
\hline 44 & 1 & 0.02 & 0.02 & -0.01 & 0.50 & 0.42 & -0.24 & 0.01 & 0.01 & 0.00 \\
\hline 45 & 1 & 0.02 & 0.00 & 0.02 & 0.52 & -0.03 & 0.48 & 0.01 & 0.00 & 0.01 \\
\hline 46 & 9 & 0.00 & 0.00 & 0.00 & 0.00 & 0.00 & 0.00 & 0.00 & 0.00 & 0.00 \\
\hline 47 & 9 & 0.00 & 0.00 & 0.00 & 0.01 & 0.00 & 0.00 & 0.00 & 0.00 & 0.00 \\
\hline 48 & 9 & 0.00 & 0.00 & 0.00 & 0.00 & 0.00 & 0.00 & 0.00 & 0.00 & 0.00 \\
\hline requen & & 1590.59 & & & 1596.68 & & & 1600.41 & & \\
\hline Atom & AN & X & $Y$ & Z & $X$ & Y & Z & $X$ & Y & Z \\
\hline 1 & 6 & 0.00 & 0.01 & 0.00 & -0.04 & -0.18 & -0.14 & 0.01 & 0.02 & 0.01 \\
\hline 2 & 6 & 0.00 & 0.00 & 0.00 & -0.09 & 0.11 & 0.13 & -0.01 & 0.00 & 0.00 \\
\hline 3 & 6 & 0.00 & 0.01 & 0.01 & 0.04 & -0.21 & -0.19 & 0.08 & 0.05 & 0.00 \\
\hline 4 & 6 & 0.00 & -0.01 & 0.00 & 0.07 & 0.16 & 0.11 & -0.02 & -0.03 & -0.02 \\
\hline 5 & 6 & 0.00 & 0.00 & 0.00 & 0.08 & -0.14 & -0.15 & 0.00 & 0.01 & 0.01 \\
\hline 6 & 6 & 0.00 & -0.01 & -0.01 & -0.04 & 0.31 & 0.27 & 0.00 & -0.03 & -0.02 \\
\hline 7 & 7 & 0.00 & 0.00 & 0.00 & -0.02 & -0.01 & 0.03 & -0.15 & -0.19 & 0.17 \\
\hline 8 & 6 & 0.00 & -0.01 & 0.00 & & 0.03 & -0.03 & 0.0 & 0.26 & -0.24 \\
\hline 9 & 6 & 0.01 & 0.00 & 0.00 & -0.01 & 0.01 & -0.02 & -0.1 & 0.11 & -0.13 \\
\hline 10 & 6 & 0.00 & 0.00 & 0.00 & & 0.00 & 0.00 & -0.01 & -0.02 & 0.01 \\
\hline 11 & 17 & 0.00 & 0.00 & 0.00 & 0.00 & 0.00 & 0.00 & 0.00 & 0.00 & 0.00 \\
\hline 12 & 17 & 0.00 & 0.00 & 0.00 & 0.00 & 0.00 & 0.00 & 0.00 & 0.00 & 0.00 \\
\hline 13 & 78 & 0.00 & 0.00 & 0.00 & & 0.00 & 0.00 & & & 0.00 \\
\hline 14 & 8 & 0.00 & 0.00 & 0.00 & 0.00 & 0.00 & 0.00 & 0.00 & 0.00 & 0.00 \\
\hline 15 & 7 & -0.01 & 0.01 & 0.02 & 0.02 & -0.01 & 0.01 & 0.1 & -0.08 & 0.10 \\
\hline 16 & 6 & 0.04 & -0.13 & -0.23 & & 0.00 & -0.01 & -0 . & 0.00 & -0.02 \\
\hline 17 & 6 & -0.11 & 0.08 & 0.13 & 0.00 & 0.00 & 0.00 & 0.02 & -0.01 & 0.00 \\
\hline 18 & 6 & -0.02 & -0.10 & -0.19 & 0.00 & 0.00 & -0.01 & -0.02 & -0.01 & -0.01 \\
\hline 19 & 6 & -0.0 & 0.21 & 0.36 & & 0.01 & 0.01 & & & 0.01 \\
\hline 20 & 6 & 0.1 & -0.11 & -0.18 & & 0.00 & -0.01 & -0.0 & 0.1 & 0.00 \\
\hline 21 & 6 & 0.0 & 0.08 & 0.16 & 0.00 & 0.00 & 0.01 & 0.0 & 0.01 & 0.02 \\
\hline 22 & 17 & 0.00 & 0.00 & 0.00 & 0.00 & 0.00 & 0.00 & 0.0 & 0.00 & 0.00 \\
\hline 23 & 17 & 0.0 & 0.00 & 0.00 & & 0.00 & 0.00 & & & 0.00 \\
\hline 24 & 6 & 0.0 & 0.0 & 0.0 & & 0.0 & 0.0 & 0. & & \\
\hline 25 & 6 & 0.00 & 0.00 & 0.00 & 0.00 & 0.00 & 0.00 & 0.0 & -0.02 & 0.03 \\
\hline 26 & 1 & 0.00 & 0.01 & 0.02 & & 0.05 & 0.01 & -0.10 & 0.24 & -0.23 \\
\hline 27 & 1 & -0.0 & 0.00 & -0.01 & & -0.01 & 0.0 & -0.1 & -0.13 & 0.39 \\
\hline 28 & 1 & 0.02 & 0.01 & 0.00 & & -0.06 & 0.02 & 0.03 & -0.45 & 0.04 \\
\hline 29 & 1 & 0.02 & 0.02 & -0.03 & & 0.01 & 0.03 & 0.05 & -0.02 & 0.25 \\
\hline 30 & 1 & -0.0 & 0.00 & 0.01 & 0.0 & -0.03 & 0.01 & 0.1 & -0.20 & 0.05 \\
\hline 31 & 1 & -0.0 & 0.01 & 0.04 & -0.01 & 0.03 & 0.00 & -0.01 & 0.15 & -0.12 \\
\hline 32 & 1 & 0.2 & -0.04 & -0.04 & 0.01 & 0.00 & 0.00 & 0.02 & 0.01 & 0.02 \\
\hline 33 & 1 & 0.1 & -0.33 & -0.58 & 0.00 & -0.01 & -0.02 & 0.01 & 0.00 & 0.00 \\
\hline 34 & 1 & -0.2 & -0.04 & -0.10 & & 0.00 & 0.00 & 0.01 & -0.01 & -0.01 \\
\hline 35 & 1 & 0.01 & 0.00 & 0.00 & -0.21 & -0.12 & -0.02 & 0.03 & 0.01 & 0.00 \\
\hline
\end{tabular}




\begin{tabular}{|c|c|c|c|c|c|c|c|c|c|c|}
\hline & & & & & & & & & & \\
\hline 36 & 1 & 0.00 & 0.02 & 0.01 & 0.08 & -0.48 & -0.43 & -0.01 & 0.04 & 0.04 \\
\hline 3 & 1 & -0.01 & 0.00 & 0.00 & 0.25 & 0.03 & -0.07 & -0.02 & 0.00 & 0.01 \\
\hline 38 & 1 & 0.00 & 0.00 & 0.01 & 0.01 & 0.00 & 0.01 & 0.03 & 0.01 & 0.04 \\
\hline 39 & 1 & 0.00 & 0.01 & -0.01 & 0.00 & 0.00 & -0.01 & 0.03 & -0.03 & 0.00 \\
\hline 40 & 1 & 0.00 & 0.01 & 0.01 & -0.01 & 0.01 & 0.01 & -0.03 & -0.01 & 0.01 \\
\hline 41 & 6 & 0.00 & 0.00 & 0.00 & 0.00 & 0.00 & 0.0 & & 0.00 & .00 \\
\hline 42 & 1 & 0.00 & 0.00 & 0.00 & 0.00 & 0.00 & 0.0 & 0.01 & -0.01 & 0.00 \\
\hline 43 & 6 & 0.00 & 0.00 & 0.00 & 0.00 & 0.00 & 0.00 & 0.00 & 0.00 & 0.00 \\
\hline 44 & 1 & -0.01 & -0.01 & 0.01 & 0.00 & 0.00 & 0.00 & 0.01 & 0.01 & 0.00 \\
\hline 45 & 1 & -0.02 & & -0.01 & 0.00 & 0.00 & & .00 & .00 & .00 \\
\hline 46 & 9 & 0.00 & 0.00 & 0.00 & 0.00 & 0.00 & 0.00 & 0.00 & 0.00 & 0.00 \\
\hline 47 & 9 & 0.00 & 0.00 & 0.00 & 0.00 & 0.00 & 0.00 & 0.00 & 0.00 & 0.00 \\
\hline 48 & 9 & 0.00 & 0.00 & 0.00 & 0.00 & 0.00 & 0.00 & 0.00 & 0.00 & 0.00 \\
\hline equen & & 1605.10 & & & 1606.78 & & & 1666.65 & & \\
\hline Atom & AN & X & Y & Z & $X$ & Y & Z & $X$ & Y & Z \\
\hline 1 & 6 & 0.06 & 0.03 & 0.01 & 0.28 & 0.16 & 0.03 & -0.01 & 0.00 & 0.00 \\
\hline 2 & 6 & -0.05 & -0.01 & 0.01 & -0.25 & -0.04 & 0.06 & 0.00 & 0.00 & 0.00 \\
\hline 3 & 6 & 0.03 & 0.01 & -0.01 & 0.14 & 0.03 & -0.03 & 0.03 & 0.02 & -0.01 \\
\hline 4 & 6 & -0.05 & -0.02 & 0.00 & -0.23 & -0.09 & 0.01 & 0.00 & 0.00 & 0.00 \\
\hline 5 & 6 & 0.06 & 0.00 & -0.02 & 0.31 & 0.00 & -0.12 & -0.01 & 0.00 & 0.00 \\
\hline 6 & 6 & -0.03 & -0.01 & 0.01 & -0.17 & -0.04 & 0.03 & 0.00 & 0.00 & 0.00 \\
\hline 7 & 7 & -0.01 & -0.02 & 0.01 & 0.00 & 0.01 & -0.01 & -0.08 & -0.14 & 0.13 \\
\hline 8 & 6 & 0.01 & 0.02 & -0.02 & -0.02 & -0.03 & 0.0 & 0.0 & 0.22 & -0.21 \\
\hline 9 & 6 & -0.02 & 0.01 & -0.02 & 0.02 & -0.01 & 0.01 & 0.2 & -0.30 & 0.32 \\
\hline 10 & 6 & 0.00 & 0.00 & 0.00 & 0.00 & 0.00 & 0.00 & 0.00 & 0.04 & -0.03 \\
\hline 11 & 17 & 0. & 0. & 0.00 & 0.0 & 0.00 & 0.0 & 0.0 & 0.00 & 0.00 \\
\hline 12 & 17 & & & 0.00 & 0.00 & 0.00 & 0.0 & 0.00 & 0.00 & 0.00 \\
\hline 13 & 78 & 0.00 & 0.00 & 0.00 & 0.00 & 0.00 & 0.00 & 0.00 & 0.00 & 0.00 \\
\hline 14 & 8 & 0.00 & 0. & 0.00 & & 0.00 & 0.0 & 0.0 & 0.00 & 0.00 \\
\hline 15 & 7 & 0.00 & & 0.01 & -0.01 & 0.01 & -0.0 & -0. & 0.18 & -0.21 \\
\hline 16 & 6 & & 0. & 0.03 & -0.03 & 0.00 & 0.0 & 0.0 & 0.00 & 0.02 \\
\hline 17 & 6 & -0.24 & 0.01 & -0.02 & 0.05 & 0.00 & 0.0 & 0.0 & 0.01 & 0.01 \\
\hline 18 & 6 & 0.28 & 0.06 & 0.15 & -0.06 & -0.01 & -0.0 & -0.0 & 0.00 & 0.00 \\
\hline 19 & 6 & & & -0.04 & & 0.00 & 0.0 & & & 0.00 \\
\hline 20 & 6 & 0.32 & -0.05 & -0.04 & -0.07 & 0.01 & 0.0 & -0.02 & 0.00 & 0.00 \\
\hline 21 & 6 & -0.24 & -0.02 & -0.06 & 0.05 & 0.00 & 0.0 & 0.01 & -0.01 & -0.01 \\
\hline 22 & 17 & 0.0 & & 0. & & 0.00 & 0.0 & 0.0 & 0.00 & 0.00 \\
\hline 23 & 17 & & & & & & & & & \\
\hline 24 & 6 & & & 0. & & 0.00 & 0. & 0. & 0.1 & 0.00 \\
\hline 25 & 6 & & & 0. & & 0.00 & 0. & 0.1 & -0.02 & 0.02 \\
\hline 26 & 1 & -0 & & -0. & & -0.02 & 0.8 & -0. & & -0.14 \\
\hline 27 & 1 & & & & & 0.01 & & & & 0.20 \\
\hline 28 & 1 & 0. & & 0. & -0.0 & 0.04 & -0.0 & 0. & -0.23 & 0.04 \\
\hline 29 & 1 & & & & & 0.00 & -0.0 & -0.0 & 0. & -0.35 \\
\hline 30 & 1 & & & & & & & & & -0.09 \\
\hline 31 & 1 & & & & & -0 & 0. & -0 . & -0.23 & 0. \\
\hline 32 & 1 & & & -0.3 & & 0.03 & 0. & 0.0 & 0.01 & 0.01 \\
\hline 33 & 1 & & & -0.0 & & & & & & 0.00 \\
\hline 34 & 1 & & & & & -0.0 & -0. & 0. & -0.01 & -0. \\
\hline 35 & 1 & & & & -0.43 & 0.11 & 0.2 & 0.0 & 0.00 & -0.01 \\
\hline 36 & 1 & & & & & -0.07 & 0. & & & 0.00 \\
\hline 37 & 1 & & & -0.0 & -0. & -0.33 & -0 . & & & 0.00 \\
\hline 38 & 1 & & & 0. & -0.02 & -0.01 & -0. & -0.02 & -0.02 & 0.00 \\
\hline 39 & 1 & 0. & & & -0.0 & 0.01 & 0. & -0. & 0.0 & 0.02 \\
\hline 40 & 1 & 0.1 & & 0.0 & & 0.00 & 0. & & 0.00 & -0.01 \\
\hline 41 & 6 & 0. & & 0.0 & 0.0 & 0.00 & 0.0 & 0.0 & 0.00 & 0.00 \\
\hline 42 & 1 & 0.00 & 0.0 & 0.00 & 0.00 & 0.00 & 0.00 & 0.01 & 0.00 & 0.00 \\
\hline 43 & 6 & 0.0 & & 0.00 & 0.0 & 0.00 & 0.0 & 0.00 & 0.00 & 0.00 \\
\hline 44 & 1 & -0. & 0.0 & 0.0 & 0.0 & 0.00 & 0.0 & 0.00 & 0.00 & 0.01 \\
\hline 45 & 1 & -0.01 & 0.01 & 0.00 & 0.00 & 0.00 & 0.00 & 0.00 & 0.00 & 0.00 \\
\hline
\end{tabular}




\begin{tabular}{|c|c|c|c|c|c|c|c|c|c|c|}
\hline 46 & 9 & 0.00 & 0.00 & 0.00 & 0.00 & 0.00 & 0.00 & 0.00 & 0.00 & 0.00 \\
\hline 47 & 9 & 0.00 & 0.00 & 0.00 & 0.00 & 0.00 & 0.00 & 0.00 & 0.00 & 0.00 \\
\hline 48 & 9 & 0.00 & 0.00 & 0.00 & 0.00 & 0.00 & 0.00 & 0.00 & 0.00 & 0.00 \\
\hline \multicolumn{2}{|c|}{ Frequencies } & 2995.97 & & & 3057.16 & & & 3061.52 & & \\
\hline Atom & AN & $X$ & $Y$ & Z & $X$ & $Y$ & Z & $X$ & Y & Z \\
\hline 1 & 6 & 0.00 & 0.00 & 0.00 & 0.00 & 0.00 & 0.00 & 0.00 & 0.00 & 0.00 \\
\hline 2 & 6 & 0.00 & 0.00 & 0.00 & 0.00 & 0.00 & 0.00 & 0.00 & 0.00 & 0.00 \\
\hline 3 & 6 & 0.00 & 0.00 & 0.00 & 0.00 & 0.00 & 0.00 & 0.00 & 0.00 & 0.00 \\
\hline 4 & 6 & 0.00 & 0.00 & 0.00 & 0.00 & 0.00 & 0.00 & 0.00 & 0.00 & 0.00 \\
\hline 5 & 6 & 0.00 & 0.00 & 0.00 & 0.00 & 0.00 & 0.00 & 0.00 & 0.00 & 0.00 \\
\hline 6 & 6 & 0.00 & 0.00 & 0.00 & 0.00 & 0.00 & 0.00 & 0.00 & 0.00 & \\
\hline 7 & 7 & 0.00 & 0.00 & 0.00 & 0.00 & 0.00 & 0.00 & 0.00 & 0.00 & 0.00 \\
\hline 8 & 6 & 0.00 & 0.00 & 0.00 & 0.00 & 0.00 & 0.00 & 0.00 & 0.00 & 0.00 \\
\hline 9 & 6 & 0.00 & 0.00 & 0.00 & 0.00 & 0.00 & 0.00 & 0.00 & 0.00 & 0.00 \\
\hline 10 & 6 & 0.00 & 0.00 & 0.00 & 0.00 & 0.02 & -0.02 & 0.00 & 0.02 & -0.04 \\
\hline 11 & 17 & 0.00 & 0.00 & 0.00 & 0.00 & 0.00 & 0.00 & 0.00 & 0.00 & 0.00 \\
\hline 12 & 17 & 0.00 & 0.00 & 0.00 & 0.00 & 0.00 & 0.00 & 0.00 & 0.00 & 0.00 \\
\hline 13 & 78 & 0.00 & 0.00 & 0.00 & 0.00 & 0.00 & 0.00 & 0.00 & 0.00 & 0.00 \\
\hline 14 & 8 & 0.00 & 0.00 & 0.00 & 0.00 & 0.00 & 0.00 & 0.00 & 0.00 & 0.00 \\
\hline 15 & 7 & 0.00 & 0.00 & 0.00 & 0.00 & 0.00 & 0.00 & 0.00 & 0.00 & 0.00 \\
\hline 16 & 6 & 0.00 & 0.00 & 0.00 & 0.00 & 0.00 & 0.00 & 0.00 & 0.00 & 00 \\
\hline 17 & 6 & 0.00 & 0.00 & 0.00 & 0.00 & 0.00 & 0.00 & 0.00 & 0.00 & 0.00 \\
\hline 18 & 6 & 0.00 & 0.00 & 0.00 & 0.00 & 0.00 & 0.00 & 0.00 & 0.00 & \\
\hline 19 & 6 & 0.00 & 0.00 & 0.00 & 0.00 & 0.00 & 0.00 & 0.00 & 0.00 & 00 \\
\hline 20 & 6 & 0.00 & 0.00 & 0.00 & 0.00 & 0.00 & 0.00 & 0.00 & 0.00 & 0.00 \\
\hline 21 & 6 & 0.00 & 0.00 & 0.00 & 0.00 & 0.00 & 0.00 & 0.00 & 0.00 & 0.00 \\
\hline 22 & 17 & 0.00 & 0.00 & 0.00 & 0.0 & 0.00 & 0.00 & 0.00 & 0.00 & 00 \\
\hline 23 & 17 & 0.00 & 0.00 & 0.00 & 0.00 & 0.00 & 0.00 & 0.00 & 0.00 & 00 \\
\hline 24 & 6 & 0.00 & 0.04 & -0.04 & 0.00 & 0.00 & 0.00 & 0.00 & 0.00 & 0.00 \\
\hline 25 & 6 & 0.00 & 0.00 & 0.00 & 0.0 & -0.04 & 0.02 & -0.0 & 0.03 & \\
\hline 26 & 1 & 0.00 & 0.00 & 0.00 & -0.26 & -0.12 & 0.01 & 0.18 & 0.08 & -0 \\
\hline 27 & 1 & 0.00 & 0.00 & 0.00 & -0.03 & 0.62 & 0.19 & 0.02 & -0.41 & -0.13 \\
\hline 28 & 1 & 0.00 & 0.00 & 0.00 & 0.15 & -0.02 & -0.41 & -0.09 & 0.02 & \\
\hline 29 & 1 & 0.00 & 0.00 & 0.0 & 0.1 & -0.27 & -0.05 & 0. & -0.39 & \\
\hline 30 & 1 & 0.00 & 0.00 & 0.00 & 0.0 & 0.11 & 0.38 & 0.04 & 0.17 & \\
\hline 31 & 1 & 0.00 & 0.00 & 0.00 & -0.20 & -0.03 & -0.05 & -0.31 & -0.05 & -0 \\
\hline 32 & 1 & 0.00 & 0.00 & 0.00 & 0.00 & 0.00 & 0.00 & & 0.00 & \\
\hline 33 & 1 & 0.00 & 0.00 & 0.00 & 0.00 & 0.00 & 0.00 & 0.0 & 0.00 & \\
\hline 34 & 1 & 0.00 & 0.00 & 0.00 & 0.00 & 0.00 & 0.00 & 0.00 & 0.00 & \\
\hline 35 & 1 & 0.00 & 0.00 & 0.00 & 0.0 & 0.00 & 0.00 & & 0.00 & \\
\hline 36 & 1 & 0.00 & 0.00 & 0.00 & 0.00 & 0.00 & 0.00 & 0.00 & 0.00 & 00 \\
\hline 37 & 1 & 0. & 0.00 & 0.0 & 0.1 & 0.00 & 0.00 & 0.00 & 0.00 & \\
\hline 38 & 1 & -0.23 & 0.22 & & & & & & & \\
\hline 39 & 1 & -0.16 & -0.21 & -0.24 & 0.00 & 0.00 & 0.00 & 0.00 & 0.00 & 00 \\
\hline 40 & 1 & 0.42 & -0.44 & 0.62 & 0.0 & 0.00 & 0.00 & 0.00 & 0.00 & 0.00 \\
\hline 41 & 6 & 0. & 0.00 & 0. & 0. & 0.00 & 0.00 & & 0.00 & 00 \\
\hline 42 & 1 & & -0.01 & 0. & 0.1 & & 0.00 & & & 00 \\
\hline 43 & 6 & 0.00 & 0.00 & 0.00 & 0.0 & 0.00 & 0.00 & 0.00 & 0.00 & 00 \\
\hline 44 & 1 & 0.00 & 0.00 & 0.00 & 0.00 & 0.00 & 0.00 & 0.00 & 0.00 & 0.00 \\
\hline 45 & 1 & 0.00 & 0.00 & & & & & & 00 & \\
\hline 46 & 9 & 0. & 0.00 & 0.0 & 0.0 & 0.00 & 0.00 & 0.0 & 0.00 & \\
\hline 47 & 9 & 0.00 & 0.00 & 0.00 & 0.0 & 0.00 & 0.00 & 0.00 & 0.00 & 0.00 \\
\hline 48 & 9 & 0.00 & 0.00 & 0.00 & & 0.00 & 0.00 & 0.00 & 0.00 & \\
\hline Frequer & & 3085.44 & & & & & & 3120.54 & & \\
\hline Atom & AN & $X$ & Y & Z & $X$ & $Y$ & Z & $X$ & Y & \\
\hline 1 & 6 & 0.00 & 0.00 & 0.00 & 0.00 & 0.00 & 0.00 & 0.00 & 0.00 & 0.00 \\
\hline 2 & 6 & 0.00 & 0.00 & 0.00 & 0.0 & 0.00 & 0.00 & & 0.00 & \\
\hline 3 & 6 & 0.00 & 0.00 & 0.00 & 0.0 & 0.00 & 0.00 & 0.0 & 0.00 & \\
\hline 4 & 6 & 0.00 & 0.00 & 0.00 & 0.00 & 0.00 & 0.00 & 0.00 & 0.00 & 0.00 \\
\hline 5 & 6 & 0.00 & 0.00 & 0.00 & 0.00 & 0.00 & 0.00 & 0.00 & 0.00 & 0.00 \\
\hline
\end{tabular}




\begin{tabular}{|c|c|c|c|c|c|c|c|c|c|c|}
\hline & & & & & & & & & & \\
\hline 6 & 6 & 0.00 & 0.00 & 0.00 & 0.00 & 0.00 & 0.00 & 0.00 & 0.00 & 0.00 \\
\hline 7 & 7 & 0.00 & 0.00 & 0.00 & 0.00 & 0.00 & 0.00 & 0.00 & 0.00 & 0.00 \\
\hline 8 & 6 & 0.00 & 0.00 & 0.00 & 0.00 & 0.00 & 0.00 & 0.00 & 0.00 & 0.00 \\
\hline 9 & 6 & 0.00 & 0.00 & 0.00 & 0.00 & 0.00 & 0.00 & 0.00 & 0.00 & 0.00 \\
\hline 10 & 6 & 0.00 & 0.00 & 0.00 & 0.00 & 0.00 & 0.00 & -0.01 & 0.04 & 0.03 \\
\hline 11 & 17 & 0.00 & 0.00 & 0.00 & & 0.00 & 0.00 & 0.00 & 0.00 & 0.00 \\
\hline 12 & 17 & 0.00 & 0.00 & 0.00 & & 0.00 & 0.00 & 0.00 & 0.00 & .00 \\
\hline 13 & 78 & 0.00 & 0.00 & 0.00 & 0.00 & 0.00 & 0.00 & 0.00 & 0.00 & 0.00 \\
\hline 14 & 8 & 0.00 & 0.00 & 0.00 & & 0.00 & 0.00 & 0.00 & 0.00 & 0.00 \\
\hline 15 & 7 & 0.00 & 0.00 & 0.00 & & 0.00 & 0.0 & 0.00 & 0.00 & 0.00 \\
\hline 16 & 6 & 0.00 & 0.00 & 0.00 & & 0.00 & 0.00 & 0.00 & 0.00 & 0.00 \\
\hline 17 & 6 & 0.00 & 0.00 & 0.00 & & 0.00 & 0.00 & 0.00 & 0.00 & 0.00 \\
\hline 18 & 6 & 0.00 & 0.00 & 0.00 & & 0.00 & 0.00 & 0.00 & 0.00 & 0.00 \\
\hline 19 & 6 & 0.00 & 0.00 & 0.00 & & 0.00 & 0.0 & 0.0 & 0.00 & \\
\hline 20 & 6 & 0.00 & 0.00 & 0.00 & & 0.00 & 0.00 & 0.00 & 0.00 & 0.00 \\
\hline 21 & 6 & 0.00 & 0.00 & 0.00 & & 0.00 & 0.0 & 0.00 & 0.00 & 0.00 \\
\hline 22 & 17 & 0.00 & 0.00 & 0.00 & & 0.00 & 0.0 & 0.0 & & \\
\hline 23 & 17 & 0.00 & 0.00 & 0.00 & & 0.00 & 0.00 & 0.00 & 0.00 & .00 \\
\hline 24 & 6 & -0.07 & 0.01 & -0.04 & & 0.00 & 0.0 & 0.00 & 0.00 & 0.00 \\
\hline 25 & 6 & 0.00 & 0.00 & 0.00 & & 0.00 & 0.0 & 0.01 & -0.04 & \\
\hline 26 & 1 & 0.00 & 0.00 & 0.00 & & 0.00 & 0.00 & 0.13 & 0.04 & \\
\hline 27 & 1 & 0.00 & 0.00 & 0.00 & & -0.01 & 0.00 & -0.02 & 0.46 & 0.12 \\
\hline 28 & 1 & 0.00 & 0.00 & 0.00 & & 0.00 & -0.0 & -0.22 & 0.00 & 0.61 \\
\hline 29 & 1 & 0.00 & 0.00 & 0.00 & & 0.00 & 0.00 & 0.22 & -0.37 & -0.05 \\
\hline 30 & 1 & 0.00 & 0.00 & 0.00 & 0.00 & 0.00 & 0.00 & -0.03 & -0.09 & -0.34 \\
\hline 31 & 1 & 0.00 & 0.00 & 0.00 & & 0.00 & 0.00 & -0.08 & 0.00 & -0.01 \\
\hline 32 & 1 & 0.00 & 0.0 & 0. & & & 0. & 0.0 & & \\
\hline 33 & 1 & 0.00 & 0.00 & 0.0 & & 0.00 & 0.0 & & & \\
\hline 34 & 1 & 0.00 & 0.00 & 0.00 & & 0.00 & 0.0 & 0.00 & 0.00 & \\
\hline 35 & 1 & 0.00 & 0.00 & 0.00 & & 0.00 & 0.0 & 0.0 & 0. & \\
\hline 36 & 1 & 0.0 & 0.00 & 0.0 & & 0. & 0.0 & 0.0 & & \\
\hline 37 & 1 & 0.0 & 0.00 & 0.0 & & 0.00 & 0.0 & 0.0 & 0.00 & \\
\hline 38 & 1 & 0.38 & -0.35 & -0.26 & & 0.00 & 0.0 & 0.0 & 0.00 & \\
\hline 39 & 1 & 0.27 & 0.44 & 0.4 & & 0. & 0.0 & 0.0 & & \\
\hline 40 & 1 & 0.2 & -0.25 & 0. & & 0. & 0.0 & 0.0 & & \\
\hline 41 & 6 & 0.00 & 0.00 & 0.00 & -0.0 & 0.00 & -0.0 & 0.00 & 0.00 & \\
\hline 42 & 1 & 0.0 & -0.01 & 0.01 & & 0.00 & 0.0 & 0.00 & 0.00 & \\
\hline 43 & 6 & 0.0 & 0.00 & 0.0 & & 0.00 & 0.0 & 0.0 & & 0.00 \\
\hline 44 & 1 & 0.1 & 0. & 0. & & & & 0.0 & & \\
\hline 45 & 1 & 0. & 0.0 & 0. & & 0. & -0.2 & 0.00 & & \\
\hline 46 & 9 & 0.0 & 0.0 & 0.0 & & 0.00 & 0.0 & 0.00 & 0.00 & 0.00 \\
\hline 47 & 9 & 0.0 & 0. & $0 .($ & & & & & & \\
\hline 48 & 9 & 0.0 & 0.00 & 0.00 & & 0.00 & 0.0 & 0.00 & 0.00 & \\
\hline Freque & & 3123.95 & & & 312 & & & 3169.73 & & \\
\hline Atom & AN & $X$ & $Y$ & Z & & $\mathrm{Y}$ & Z & & $Y$ & Z \\
\hline & 6 & 0.0 & 0.00 & 0.00 & 0.0 & 0.00 & 0.00 & 0.00 & 0.00 & \\
\hline 2 & 6 & & 0.00 & 0.00 & & 0.00 & 0.00 & & 0.00 & 0.00 \\
\hline 3 & 6 & & 0.0 & 0.00 & & 0.00 & 0.0 & & 0.00 & \\
\hline 4 & 6 & 0. & 0.0 & 0.0 & & & 0.0 & & 0.00 & \\
\hline 5 & 6 & & 0.0 & 0.0 & & 0. & 0.0 & $0 .($ & 0.00 & \\
\hline 6 & 6 & & 0.00 & 0.00 & & 0.00 & 0.00 & 0.00 & 0.00 & 0.00 \\
\hline 7 & 7 & & 0.00 & 0.00 & & 0.00 & 0.00 & & 0.00 & \\
\hline 8 & 6 & & 0.00 & 0.0 & & & 0.0 & & & \\
\hline 9 & 6 & 0.0 & 0.00 & 0.00 & & 0.00 & 0.00 & 0.00 & 0.00 & 0.00 \\
\hline 10 & 6 & & -0.05 & -0.05 & & 0.00 & 0.00 & -0.09 & 0.01 & \\
\hline 11 & 17 & & 0.00 & 0.00 & & 0.00 & 0.0 & & & \\
\hline 12 & 17 & 0.0 & 0.00 & 0.00 & & 0.00 & 0.00 & 0.00 & 0.00 & 0.0 \\
\hline 13 & 78 & & 0.00 & 0.00 & & 0.00 & 0.0 & 0.00 & 0.00 & \\
\hline 14 & 8 & & 0.00 & 0.00 & & 0.00 & 0.0 & 0.00 & 0.00 & \\
\hline 15 & 7 & 0.00 & 0.00 & 0.00 & 0.00 & 0.00 & 0.00 & 0.00 & 0.00 & \\
\hline
\end{tabular}




\begin{tabular}{|c|c|c|c|c|c|c|c|c|c|c|}
\hline & & & & & & & & & & \\
\hline $\begin{array}{l}16 \\
17\end{array}$ & 6 & 0.00 & 0.00 & 0.00 & 0.00 & 0.00 & 0.00 & 0.00 & 0.00 & $\begin{array}{l}0.00 \\
0.00\end{array}$ \\
\hline $\begin{array}{l}17 \\
18\end{array}$ & $\begin{array}{l}6 \\
6\end{array}$ & $\begin{array}{l}0.00 \\
0.00\end{array}$ & $\begin{array}{l}0.00 \\
0.00\end{array}$ & $\begin{array}{l}0.00 \\
0.00\end{array}$ & $\begin{array}{l}0.00 \\
0.00\end{array}$ & $\begin{array}{l}0.00 \\
0.00\end{array}$ & $\begin{array}{l}0.00 \\
0.00\end{array}$ & $\begin{array}{l}0.00 \\
0.00\end{array}$ & $\begin{array}{l}0.00 \\
0.00\end{array}$ & \\
\hline 19 & 6 & 0.00 & 0.00 & 0.00 & 0.00 & 0.00 & 0.00 & 0.00 & 0.00 & 0.00 \\
\hline 20 & 6 & 0.00 & 0.00 & 0.00 & 0.00 & 0.00 & 0.00 & 0.00 & 0.00 & 0.00 \\
\hline 21 & 6 & 0.00 & 0.00 & 0.00 & 0.00 & 0.00 & 0.00 & 0.00 & 0.00 & 0.00 \\
\hline 22 & 17 & 0.00 & 0.00 & 0.00 & 0.00 & 0.00 & 0.00 & 0.00 & 0.00 & 0.00 \\
\hline 23 & 17 & 0.00 & 0.00 & 0.00 & 0.00 & 0.00 & 0.00 & 0.00 & 0.00 & 0.00 \\
\hline 24 & 6 & 0.00 & 0.00 & 0.00 & -0.02 & 0.07 & 0.06 & 0.00 & 0.00 & 0.00 \\
\hline 25 & 6 & 0.01 & -0.03 & -0.04 & 0.00 & 0.00 & 0.00 & 0.00 & 0.00 & 0.00 \\
\hline 26 & 1 & 0.08 & 0.03 & -0.01 & 0.00 & 0.00 & 0.00 & 0.03 & 0.01 & 0.00 \\
\hline 27 & 1 & -0.01 & 0.33 & 0.09 & 0.00 & 0.00 & 0.00 & 0.00 & 0.00 & 0.00 \\
\hline 28 & 1 & -0.15 & 0.00 & 0.42 & 0.00 & 0.00 & 0.00 & 0.00 & 0.00 & 0.00 \\
\hline 29 & 1 & -0.32 & 0.52 & 0.06 & 0.00 & 0.01 & 0.00 & 0.16 & -0.29 & -0.05 \\
\hline 30 & 1 & 0.05 & 0.13 & 0.51 & 0.00 & 0.00 & 0.01 & -0.01 & 0.06 & 0.20 \\
\hline 31 & 1 & 0.11 & 0.00 & 0.01 & 0.00 & 0.00 & 0.00 & 0.88 & 0.15 & 0.20 \\
\hline 32 & 1 & 0.00 & 0.00 & 0.00 & 0.00 & 0.00 & 0.00 & 0.00 & 0.00 & 0.00 \\
\hline 33 & 1 & 0.00 & 0.00 & 0.00 & 0.00 & 0.00 & 0.00 & 0.00 & 0.00 & 0.00 \\
\hline 34 & 1 & 0.00 & 0.00 & 0.00 & 0.00 & 0.00 & 0.00 & 0.00 & 0.00 & 0.00 \\
\hline 35 & 1 & 0.00 & 0.00 & 0.00 & 0.00 & 0.00 & 0.00 & 0.00 & 0.00 & 0.00 \\
\hline 36 & 1 & 0.00 & 0.00 & 0.00 & 0.0 & 0.00 & 0.00 & 0.00 & 0.00 & 0.00 \\
\hline 37 & 1 & 0.00 & 0.00 & 0.00 & 0.00 & 0.00 & 0.00 & 0.00 & 0.00 & 0.00 \\
\hline 38 & 1 & -0.01 & 0.00 & 0.00 & 0.52 & -0.44 & -0.30 & 0.00 & 0.00 & 0.00 \\
\hline 39 & 1 & 0.00 & 0.00 & 0.00 & -0.31 & -0.42 & -0.41 & 0.00 & 0.00 & 0.00 \\
\hline 40 & 1 & 0.00 & 0.00 & 0.00 & 0.00 & 0.01 & 0.03 & 0.00 & 0.00 & 0.00 \\
\hline 41 & 6 & 0.00 & 0.00 & 0.00 & 0.00 & 0.00 & 0.00 & 0.00 & 0.00 & 0.00 \\
\hline 42 & 1 & 0.00 & 0.00 & 0.00 & 0.00 & 0.00 & 0.00 & 0.00 & 0.00 & 0.00 \\
\hline 43 & 6 & 0.00 & 0.00 & 0.00 & 0.00 & 0.00 & 0.00 & 0.00 & 0.00 & 0.00 \\
\hline 44 & 1 & 0.00 & 0.00 & 0.00 & 0.00 & 0.00 & 0.00 & 0.00 & 0.00 & 0.00 \\
\hline 45 & 1 & 0.00 & 0.00 & 0.00 & 0.00 & 0.00 & 0.00 & 0.00 & 0.00 & 0.00 \\
\hline 46 & 9 & 0.00 & 0.00 & 0.00 & 0.0 & 0.00 & 0.00 & 0.00 & 0.00 & 0.00 \\
\hline 47 & 9 & 0.00 & 0.00 & 0.00 & 0.00 & 0.00 & 0.00 & 0.00 & 0.00 & 0.00 \\
\hline 48 & 9 & 0.00 & 0.00 & 0.00 & 0.00 & 0.00 & 0.00 & 0.00 & 0.00 & 0.00 \\
\hline Frequen & & 3178.33 & & & 3198.61 & & & 3227.04 & & \\
\hline Atom & AN & $X$ & $\mathrm{Y}$ & Z & $X$ & $\mathrm{Y}$ & Z & $X$ & $Y$ & Z \\
\hline 1 & 6 & 0.00 & 0.00 & 0.00 & 0.00 & 0.00 & 0.00 & 0.00 & 0.00 & 0.00 \\
\hline 2 & 6 & 0.00 & 0.00 & 0.00 & 0.00 & 0.00 & 0.00 & 0.00 & 0.00 & 0.00 \\
\hline 3 & 6 & 0.00 & 0.00 & 0.00 & 0.00 & 0.00 & 0.00 & 0.00 & 0.00 & 0.00 \\
\hline 4 & 6 & 0.00 & 0.00 & 0.00 & & 0.00 & 0.00 & 0.00 & 0.00 & 0.00 \\
\hline 5 & 6 & 0.00 & 0.00 & 0.00 & 0.00 & 0.00 & 0.00 & 0.00 & 0.00 & 0.00 \\
\hline 6 & 6 & 0.00 & 0.00 & 0.00 & 0.00 & 0.00 & 0.00 & 0.00 & 0.00 & 0.00 \\
\hline 7 & 7 & 0.00 & 0.00 & 0.00 & & 0.00 & 0.00 & 0.00 & 0.00 & 0.00 \\
\hline 8 & 6 & 0.00 & 0.00 & 0.00 & 0. & 0.00 & 0.00 & 0.00 & 0.00 & 0.00 \\
\hline 9 & 6 & 0.00 & 0.00 & 0.00 & 0.0 & 0.00 & 0.00 & 0.00 & 0.00 & 0.00 \\
\hline 10 & 6 & 0.00 & 0.00 & 0.00 & & 0.00 & 0.00 & 0.00 & 0.00 & 0.00 \\
\hline 11 & 17 & 0.0 & 0.00 & 0.00 & & 0.00 & 0.0 & & & 0.00 \\
\hline 12 & 17 & 0. & 0.00 & 0.00 & 0. & 0.00 & 0.00 & 0.00 & 0.00 & 0.00 \\
\hline 13 & 78 & 0.00 & 0.00 & 0.00 & 0. & 0.00 & 0.00 & 0.00 & 0.00 & 0.00 \\
\hline 14 & 8 & 0.00 & 0.00 & 0.00 & & 0.00 & 0.00 & 0.00 & 0.00 & 0.00 \\
\hline 15 & 7 & 0. & 0.00 & 0.0 & & 0.00 & 0.0 & & & 0.00 \\
\hline 16 & 6 & 0.0 & 0.00 & 0.00 & 0 & 0.00 & 0.00 & 0.00 & 0.00 & 0.00 \\
\hline 17 & 6 & 0.0 & 0.00 & 0.00 & & 0.00 & 0.00 & 0.00 & 0.00 & 0.00 \\
\hline 18 & 6 & 0.0 & 0.00 & 0.00 & & 0.00 & 0.00 & 0.02 & -0.01 & -0.0 \\
\hline 19 & 6 & 0.0 & 0.00 & 0.00 & 0. & 0.00 & 0.00 & -0.07 & 0.00 & -0.01 \\
\hline 20 & 6 & 0.0 & 0.00 & 0.00 & & 0.00 & 0.00 & 0.01 & 0.01 & 0.03 \\
\hline 21 & 6 & 0.00 & 0.00 & 0.00 & 0. & 0.00 & 0.00 & 0.00 & 0.00 & 0.00 \\
\hline 22 & 17 & 0.0 & 0.00 & 0.00 & & 0.00 & 0.00 & 0.00 & 0.00 & 0.00 \\
\hline 23 & 17 & 0.00 & 0.00 & 0.00 & 0.0 & 0.00 & 0.00 & 0.00 & 0.00 & 0.00 \\
\hline 24 & 6 & 0.0 & 0.00 & 0.00 & & 0.00 & 0.00 & 0.00 & 0.00 & 0.00 \\
\hline 25 & 6 & -0.08 & -0.04 & 0.02 & 0.00 & 0.00 & 0.00 & 0.00 & 0.00 & 0.00 \\
\hline
\end{tabular}




\begin{tabular}{|c|c|c|c|c|c|c|c|c|c|c|}
\hline & & & & & & & & & & \\
\hline 26 & 1 & 0.85 & 0.36 & -0.03 & 0.00 & 0.00 & 0.00 & 0.00 & 0.00 & 0.00 \\
\hline 27 & 1 & -0.03 & 0.17 & 0.06 & 0.00 & 0.00 & 0.00 & 0.00 & 0.00 & 0.00 \\
\hline 28 & 1 & 0.10 & -0.02 & -0.31 & 0.00 & 0.00 & 0.00 & 0.00 & 0.00 & 0.00 \\
\hline 29 & 1 & -0.01 & 0.02 & 0.00 & 0.00 & 0.00 & 0.00 & 0.00 & 0.00 & 0.00 \\
\hline 30 & 1 & 0.00 & 0.00 & -0.01 & 0.00 & 0.00 & 0.00 & 0.00 & 0.00 & 0.00 \\
\hline 31 & 1 & -0.03 & 0.00 & -0.01 & 0.00 & 0.00 & 0.00 & 0.00 & 0.00 & 0.00 \\
\hline 32 & 1 & 0.00 & 0.00 & 0.00 & 0.00 & 0.00 & 0.00 & -0.27 & 0.18 & 0.29 \\
\hline 33 & 1 & 0.00 & 0.00 & 0.00 & 0.00 & 0.00 & 0.00 & 0.79 & 0.01 & 0.13 \\
\hline 34 & 1 & 0.00 & 0.00 & 0.00 & 0.00 & 0.00 & 0.00 & -0.15 & -0.18 & -0.34 \\
\hline 35 & 1 & 0.00 & 0.00 & 0.00 & 0.00 & 0.00 & 0.00 & 0.00 & 0.00 & 0.00 \\
\hline 36 & 1 & 0.00 & 0.00 & 0.00 & 0.00 & 0.00 & 0.00 & 0.00 & 0.00 & 0.00 \\
\hline 37 & 1 & 0.00 & 0.00 & 0.00 & 0.00 & 0.00 & 0.00 & 0.00 & 0.00 & 0.00 \\
\hline 38 & 1 & 0.00 & 0.00 & 0.00 & 0.00 & 0.00 & 0.00 & 0.00 & .00 & 0.00 \\
\hline 39 & 1 & 0.00 & 0.00 & 0.00 & 0.00 & 0.00 & 0.00 & 0.00 & 0.00 & 0.00 \\
\hline 40 & 1 & 0.00 & 0.00 & 0.00 & 0.00 & 0.00 & 0.00 & 0.00 & 0.00 & 0.00 \\
\hline 41 & 6 & 0.00 & 0.00 & 0.00 & -0.02 & -0.06 & 0.07 & 0.00 & 0.00 & 0.00 \\
\hline 42 & 1 & 0.00 & 0.00 & 0.00 & 0.00 & -0.02 & 0.01 & 0.00 & 0.00 & 0.00 \\
\hline 43 & 6 & 0.00 & 0.00 & 0.00 & 0.00 & 0.00 & 0.00 & 0.00 & 0.00 & 0.00 \\
\hline 44 & 1 & 0.00 & 0.00 & 0.00 & -0.27 & 0.16 & -0.32 & 0.00 & 0.00 & 0.00 \\
\hline 45 & 1 & 0.00 & & 0.00 & 0.46 & 0.54 & -0.53 & 0.00 & .00 & 0.00 \\
\hline 46 & 9 & 0.00 & & 0.00 & 0.00 & 0.00 & & 0.00 & 0.00 & \\
\hline 47 & 9 & 0.00 & 0.00 & 0.00 & 0.00 & 0.00 & 0.00 & 0.00 & .00 & 0.00 \\
\hline 48 & 9 & 0.00 & & 0.00 & 0.00 & 0.00 & 0.00 & 0.00 & 0.00 & \\
\hline equen & & 3227.32 & & & 3239.01 & & & 3240.31 & & \\
\hline Atom & AN & X & $Y$ & Z & $x$ & $Y$ & Z & $x$ & Y & Z \\
\hline 1 & 6 & 0.02 & -0.02 & -0.02 & 0.00 & 0.00 & 0.00 & 0.04 & -0.03 & -0.04 \\
\hline 2 & 6 & 0.00 & 0.00 & 0.00 & 0.00 & 0.00 & 0.00 & 0.00 & 0.00 & 0.00 \\
\hline 3 & 6 & 0.00 & 0.00 & 0.00 & 0.00 & 0.00 & 0.00 & 0.00 & 0.00 & 0.00 \\
\hline 4 & 6 & 0.00 & 0.00 & 0.00 & 0.00 & 0.00 & 0.00 & 0.00 & 0.00 & 0.00 \\
\hline 5 & 6 & 0.01 & 0.03 & 0.02 & 0.00 & 0.00 & 0.00 & -0.02 & -0.05 & -0.03 \\
\hline 6 & 6 & -0.07 & -0.02 & 0.01 & 0.00 & 0.00 & 0.00 & 0.00 & 0.00 & 0.00 \\
\hline 7 & 7 & 0.00 & 0.00 & 0.00 & 0.00 & 0.00 & 0.00 & 0.00 & 0.00 & 0.00 \\
\hline 8 & 6 & 0.00 & 0.0 & 0.00 & 0.00 & 0.00 & 0.00 & 0.00 & 0.00 & 0.00 \\
\hline 9 & 6 & & & 0.00 & 0.00 & 0.00 & 0.0 & 0.00 & 0.00 & 0.00 \\
\hline 10 & 6 & & & 0.00 & & 0.00 & 0.0 & & & 0.00 \\
\hline 11 & 17 & 0.00 & 0.00 & 0.00 & 0.0 & 0.00 & 0.00 & 0.00 & 0.00 & 0.00 \\
\hline 12 & 17 & 0. & & 0. & & 0.00 & 0.00 & 0.00 & 0.00 & 0.00 \\
\hline 13 & 78 & & & & & 0.00 & 0.0 & 0.0 & 0.00 & 0.00 \\
\hline 14 & 8 & 0.00 & & 0.00 & 0.00 & 0.00 & 0.0 & 0.0 & 0.00 & 0.00 \\
\hline 15 & 7 & 0.00 & & 0. & 0.00 & 0.00 & 0.00 & 0.00 & 0.00 & 0.00 \\
\hline 16 & 6 & 0.00 & & 0. & 0.0 & 0.00 & 0.00 & 0.00 & 0.00 & 0.00 \\
\hline 17 & 6 & & & & & 0.00 & & & & 0.00 \\
\hline 18 & 6 & 0.00 & & 0. & & -0.03 & -0.04 & 0.00 & 0.00 & 0.00 \\
\hline 19 & 6 & 0.00 & & 0.00 & 0.00 & 0.00 & 0.00 & 0.00 & 0.00 & 0.00 \\
\hline 20 & 6 & 0.00 & & 0.00 & -0.02 & -0.03 & -0.05 & & & 0. \\
\hline 21 & 6 & & & & 0.0 & 0.00 & 0.0 & 0. & 0. & 0. \\
\hline 22 & 17 & 0.00 & & 0. & 0.00 & 0.00 & 0.00 & 0.00 & 0.00 & 0.00 \\
\hline 23 & 17 & 0.0 & & & & 0.00 & & & & 0.00 \\
\hline 24 & 6 & 0.0 & & 0. & & 0.00 & 0. & 0. & 0. & \\
\hline 25 & 6 & 0. & & 0. & & 0.00 & 0.00 & 0.00 & 0.00 & 0.00 \\
\hline 26 & 1 & 0.00 & & & & 0.00 & 0.0 & & 0.00 & 0.00 \\
\hline 27 & 1 & 0.00 & & 0.0 & 0.0 & 0.00 & 0. & 0. & 0.00 & 0.00 \\
\hline 28 & 1 & & & & & 0.00 & 0. & 0. & 0. & \\
\hline 29 & 1 & 0.00 & 0. & 0.0 & 0.00 & 0.00 & 0.00 & 0.00 & 0.00 & 0.00 \\
\hline 30 & 1 & 0.00 & & & & 0.00 & 0.00 & & 0.00 & 0.00 \\
\hline 31 & 1 & & & 0.0 & 0.00 & 0.00 & 0.00 & 0.0 & 0.00 & 0. \\
\hline 32 & 1 & 0.00 & 0.0 & 0.00 & -0.44 & 0.30 & 0.47 & 0.00 & 0.00 & 0.00 \\
\hline 33 & 1 & 0.0 & & 0.00 & -0.03 & 0.00 & -0.01 & 0.00 & 0.00 & 0. \\
\hline 34 & 1 & 0.0 & & 0.0 & 0.25 & 0.31 & 0.57 & 0. & 0.00 & 0. \\
\hline 35 & 1 & -0.15 & & -0.21 & 0.00 & 0.00 & 0.00 & 0.26 & 0.55 & 0.36 \\
\hline
\end{tabular}




\begin{tabular}{|c|c|c|c|c|c|c|c|c|c|c|}
\hline 36 & 1 & 0.78 & 0.22 & -0.11 & 0.00 & 0.00 & 0.00 & 0.01 & 0.00 & 0.00 \\
\hline 37 & 1 & -0.23 & 0.20 & 0.26 & 0.00 & 0.00 & 0.00 & -0.40 & 0.35 & 0.45 \\
\hline 38 & 1 & 0.00 & 0.00 & 0.00 & 0.00 & 0.00 & 0.00 & 0.00 & 0.00 & 0.00 \\
\hline 39 & 1 & 0.00 & 0.00 & 0.00 & 0.00 & 0.00 & 0.00 & 0.00 & 0.00 & 0.00 \\
\hline 40 & 1 & 0.00 & 0.00 & 0.00 & 0.00 & 0.00 & 0.00 & 0.00 & 0.00 & 0.00 \\
\hline 41 & 6 & 0.00 & 0.00 & 0.00 & 0.00 & 0.00 & 0.00 & 0.00 & 0.00 & 0.00 \\
\hline 42 & 1 & 0.00 & 0.00 & 0.00 & 0.00 & 0.00 & 0.00 & 0.00 & 0.00 & 0.00 \\
\hline 43 & 6 & 0.00 & 0.00 & 0.00 & 0.00 & 0.00 & 0.00 & 0.00 & 0.00 & 0.00 \\
\hline 44 & 1 & 0.00 & 0.00 & 0.00 & 0.00 & 0.00 & 0.00 & 0.00 & 0.00 & 0.00 \\
\hline 45 & 1 & 0.00 & 0.00 & 0.00 & 0.00 & 0.00 & 0.00 & 0.00 & 0.00 & 0.00 \\
\hline 46 & 9 & 0.00 & 0.00 & 0.00 & 0.00 & 0.00 & 0.00 & 0.00 & 0.00 & 0.00 \\
\hline 47 & 9 & 0.00 & 0.00 & 0.00 & 0.00 & 0.00 & 0.00 & 0.00 & 0.00 & 0.00 \\
\hline 48 & 9 & 0.00 & 0.00 & 0.00 & 0.00 & 0.00 & 0.00 & 0.00 & 0.00 & 0.00 \\
\hline \multicolumn{2}{|c|}{ Frequencies } & 3247.48 & & & 3248.34 & & & 3691.35 & & \\
\hline Atom & AN & $x$ & Y & Z & X & $Y$ & Z & X & $Y$ & Z \\
\hline 1 & 6 & 0.00 & 0.00 & 0.00 & -0.03 & 0.03 & 0.03 & 0.00 & 0.00 & 0.00 \\
\hline 2 & 6 & 0.00 & 0.00 & 0.00 & 0.00 & 0.00 & 0.00 & 0.00 & 0.00 & 0.00 \\
\hline 3 & 6 & 0.00 & 0.00 & 0.00 & 0.00 & 0.00 & 0.00 & 0.00 & 0.00 & 0.00 \\
\hline 4 & 6 & 0.00 & 0.00 & 0.00 & 0.00 & 0.00 & 0.00 & 0.00 & 0.00 & 0.00 \\
\hline 5 & 6 & 0.00 & 0.00 & 0.00 & -0.02 & -0.04 & -0.03 & 0.00 & 0.00 & 0.00 \\
\hline 6 & 6 & 0.00 & 0.00 & 0.00 & -0.05 & -0.01 & 0.01 & 0.00 & 0.00 & 0.00 \\
\hline 7 & 7 & 0.00 & 0.00 & 0.00 & 0.00 & 0.00 & 0.00 & 0.00 & 0.00 & 0.00 \\
\hline 8 & 6 & 0.00 & 0.00 & 0.00 & 0.00 & 0.00 & 0.00 & 0.00 & 0.00 & 0.00 \\
\hline 9 & 6 & 0.00 & 0.00 & 0.00 & 0.00 & 0.00 & 0.00 & 0.00 & 0.00 & 0.00 \\
\hline 10 & 6 & 0.00 & 0.00 & 0.00 & 0.00 & 0.00 & 0.00 & 0.00 & 0.00 & 0.00 \\
\hline 11 & 17 & 0.00 & 0.00 & 0.00 & 0.00 & 0.00 & 0.00 & 0.00 & 0.00 & 0.00 \\
\hline 12 & 17 & 0.00 & 0.00 & 0.00 & 0.00 & 0.00 & 0.00 & 0.00 & 0.00 & 0.00 \\
\hline 13 & 78 & 0.00 & 0.00 & 0.00 & 0.00 & 0.00 & 0.00 & 0.00 & 0.00 & 0.00 \\
\hline 14 & 8 & 0.00 & 0.00 & 0.00 & 0.00 & 0.00 & 0.00 & 0.03 & 0.03 & -0.05 \\
\hline 15 & 7 & 0.00 & 0.00 & 0.00 & 0.00 & 0.00 & 0.00 & 0.00 & 0.00 & 0.00 \\
\hline 16 & 6 & 0.00 & 0.00 & 0.00 & 0.00 & 0.00 & 0.00 & 0.00 & 0.00 & 0.00 \\
\hline 17 & 6 & 0.00 & 0.00 & 0.00 & 0.00 & 0.00 & 0.00 & 0.00 & 0.00 & 0.00 \\
\hline 18 & 6 & -0.03 & 0.02 & 0.03 & 0.00 & 0.00 & 0.00 & 0.00 & 0.00 & 0.00 \\
\hline 19 & 6 & -0.05 & 0.00 & -0.01 & 0.00 & 0.00 & 0.00 & 0.00 & 0.00 & 0.00 \\
\hline 20 & 6 & -0.02 & -0.02 & -0.04 & 0.00 & 0.00 & 0.00 & 0.00 & 0.00 & 0.00 \\
\hline 21 & 6 & 0.00 & 0.00 & 0.00 & 0.00 & 0.00 & 0.00 & 0.00 & 0.00 & 0.00 \\
\hline 22 & 17 & 0.00 & 0.00 & 0.00 & 0.00 & 0.00 & 0.00 & 0.00 & 0.00 & 0.00 \\
\hline 23 & 17 & 0.00 & & 0.0 & 0.00 & 0.00 & 0.0 & 0.00 & 0.00 & 0.00 \\
\hline 24 & 6 & 0.00 & 0.00 & 0.00 & 0.00 & 0.00 & 0.00 & 0.00 & 0.00 & 0.00 \\
\hline 25 & 6 & 0.00 & 0.00 & 0.00 & 0.00 & 0.00 & 0.00 & 0.00 & 0.00 & 0.00 \\
\hline 26 & 1 & 0.00 & 0. & 0.00 & 0.00 & 0.00 & 0.00 & 0.00 & 0.00 & 0.00 \\
\hline 27 & 1 & 0.00 & 0.00 & 0.00 & 0.00 & 0.00 & 0.00 & 0.00 & 0.00 & 0.00 \\
\hline 28 & 1 & 0.00 & 0.00 & 0.00 & 0.00 & 0.00 & 0.00 & 0.00 & 0.00 & 0.00 \\
\hline 29 & 1 & 0.00 & 0. & 0.00 & 0.00 & 0.00 & 0.00 & 0.00 & 0.00 & 0.00 \\
\hline 30 & 1 & 0.00 & 0. & 0.00 & 0.00 & 0.00 & 0.0 & 0.0 & 0.00 & 0.00 \\
\hline 31 & 1 & 0.00 & & 0.00 & 0.00 & 0.00 & 0.00 & 0.00 & 0.00 & 0.00 \\
\hline 32 & 1 & 0.34 & -0.23 & -0.36 & 0.00 & 0.00 & 0.00 & 0.00 & 0.00 & 0.00 \\
\hline 33 & 1 & 0.59 & 0.01 & 0.10 & 0.00 & 0.00 & 0.00 & 0.00 & 0.00 & 0.00 \\
\hline 34 & 1 & 0.21 & & 0.48 & 0.0 & 0.00 & 0.0 & 0.00 & 0.00 & 0.0 \\
\hline 35 & 1 & 0.00 & 0.00 & 0.00 & 0.21 & 0.44 & 0.29 & 0.00 & 0.00 & 0.00 \\
\hline 36 & 1 & 0.00 & & 0.00 & 0.54 & 0.16 & -0.08 & 0.00 & 0.00 & 0.00 \\
\hline 37 & 1 & 0.00 & 0.0 & 0.00 & 0.34 & -0.30 & -0.38 & 0.00 & 0.00 & 0.00 \\
\hline 38 & 1 & 0.00 & 0.0 & 0.00 & 0.00 & 0.00 & 0.00 & 0.00 & 0.00 & 0.00 \\
\hline 39 & 1 & 0.00 & 0.0 & 0.00 & 0.00 & 0.00 & 0.00 & 0.00 & 0.00 & 0.00 \\
\hline 40 & 1 & 0.00 & 0.00 & 0.00 & 0.00 & 0.00 & 0.00 & -0.01 & 0.01 & -0.02 \\
\hline 41 & 6 & 0.00 & 0.00 & 0.00 & 0.00 & 0.00 & 0.00 & 0.00 & 0.00 & 0.00 \\
\hline 42 & 1 & 0.00 & 0.0 & 0.00 & 0.00 & 0.00 & 0.00 & -0.43 & -0.51 & 0.74 \\
\hline 43 & 6 & 0.00 & 0.0 & 0.0 & 0.00 & 0.00 & 0.00 & 0.00 & 0.00 & 0.00 \\
\hline 44 & 1 & 0.00 & 0.00 & 0.0 & 0.00 & 0.00 & 0.00 & 0.00 & 0.00 & 0.00 \\
\hline 45 & 1 & 0.00 & 0.00 & 0.00 & 0.00 & 0.00 & 0.00 & -0.01 & -0.01 & 0.01 \\
\hline
\end{tabular}




$\begin{array}{lllllllllll}46 & 9 & 0.00 & 0.00 & 0.00 & 0.00 & 0.00 & 0.00 & 0.00 & 0.00 & 0.00 \\ 47 & 9 & 0.00 & 0.00 & 0.00 & 0.00 & 0.00 & 0.00 & 0.00 & 0.00 & 0.00 \\ 48 & 9 & 0.00 & 0.00 & 0.00 & 0.00 & 0.00 & 0.00 & 0.00 & 0.00 & 0.00\end{array}$

\section{$\mathrm{PtCH} 3 \mathrm{CN}$}

Frequencies 8.51

Atom AN $X$

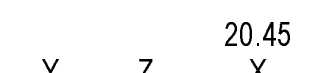

$\begin{array}{ccccccccccc}\text { m } & \text { AN } & X & Y & Z & X & Y & Z & X & Y & Z \\ 1 & 6 & 0.01 & 0.05 & 0.00 & 0.03 & -0.06 & 0.13 & 0.08 & 0.09 & 0.08\end{array}$

$\begin{array}{lllllllllll}2 & 6 & 0.01 & 0.05 & 0.01 & 0.03 & -0.07 & 0.08 & 0.07 & 0.07 & 0.02\end{array}$

$\begin{array}{lllllllllll}3 & 6 & 0.00 & 0.00 & 0.02 & 0.00 & 0.01 & 0.03 & 0.00 & 0.00 & 0.00\end{array}$

$\begin{array}{lllllllllll}4 & 6 & -0.01 & -0.05 & 0.02 & -0.03 & 0.10 & 0.05 & -0.07 & -0.05 & 0.04\end{array}$

$\begin{array}{lllllllllll}5 & 6 & -0.01 & -0.05 & 0.02 & -0.03 & 0.11 & 0.10 & -0.07 & -0.04 & 0.10\end{array}$

$\begin{array}{lllllllllll}6 & 6 & 0.00 & 0.00 & 0.00 & 0.00 & 0.03 & 0.14 & 0.00 & 0.03 & 0.12\end{array}$

$\begin{array}{lllllllllll}7 & 7 & 0.00 & 0.00 & 0.02 & 0.00 & 0.00 & -0.01 & 0.00 & -0.01 & -0.06\end{array}$

$\begin{array}{lllllllllll}8 & 6 & 0.00 & 0.01 & 0.06 & 0.00 & -0.01 & -0.03 & 0.00 & -0.02 & -0.11\end{array}$

$\begin{array}{lllllllllll}9 & 6 & 0.00 & 0.01 & 0.06 & 0.00 & -0.01 & -0.02 & 0.00 & -0.02 & -0.11\end{array}$

$\begin{array}{lllllllllll}10 & 6 & 0.00 & 0.02 & 0.12 & 0.00 & 0.00 & 0.00 & 0.00 & -0.03 & -0.18\end{array}$

$\begin{array}{lllllllllll}11 & 17 & -0.02 & -0.11 & 0.04 & -0.06 & 0.20 & -0.01 & -0.17 & -0.14 & 0.01\end{array}$

$\begin{array}{lllllllllll}12 & 17 & 0.02 & 0.12 & 0.00 & 0.06 & -0.19 & 0.06 & 0.17 & 0.14 & -0.03\end{array}$

$\begin{array}{rrrrrrrrrrr}13 & 78 & 0.00 & -0.01 & -0.04 & 0.00 & -0.01 & -0.05 & 0.00 & -0.01 & -0.02 \\ 14 & 7 & 0.00 & -0.02 & -0.10 & 0.00 & -0.02 & -0.12 & 0.00 & 0.01 & 0.05\end{array}$

$\begin{array}{ccccccccccc}14 & 7 & 0.00 & -0.02 & -0.10 & 0.00 & -0.02 & -0.12 & 0.00 & 0.01 & 0.05 \\ 15 & 7 & 0.00 & 0.00 & 0.01 & 0.00 & 0.00 & -0.02 & 0.00 & -0.01 & -0.04\end{array}$

$\begin{array}{lllllllllll}16 & 6 & 0.00 & 0.01 & 0.04 & 0.00 & 0.01 & 0.04 & 0.00 & 0.00 & 0.00\end{array}$

$\begin{array}{lllllllllll}17 & 6 & 0.00 & 0.16 & 0.02 & 0.06 & 0.00 & 0.07 & 0.05 & 0.00 & 0.03\end{array}$

$\begin{array}{lllllllllll}18 & 6 & 0.00 & 0.16 & 0.04 & 0.06 & 0.02 & 0.13 & 0.05 & 0.01 & 0.08\end{array}$

$\begin{array}{lllllllllll}19 & 6 & 0.00 & 0.02 & 0.08 & 0.00 & 0.03 & 0.16 & 0.00 & 0.03 & 0.10\end{array}$

$\begin{array}{lllllllllll}20 & 6 & 0.00 & -0.14 & 0.10 & -0.05 & 0.03 & 0.13 & -0.05 & 0.03 & 0.08 \\ 21 & 6 & 0.00 & -0.14 & 0.08 & -0.05 & 0.02 & 0.07 & -0.05 & 0.02 & 0.03\end{array}$

$\begin{array}{lllllllllll}21 & 6 & 0.00 & -0.14 & 0.08 & -0.05 & 0.02 & 0.07 & -0.05 & 0.02 & 0.03\end{array}$

$\begin{array}{lllllllllll}22 & 17 & 0.00 & 0.34 & -0.03 & 0.12 & -0.01 & 0.04 & 0.11 & -0.02 & 0.00\end{array}$

$\begin{array}{lllllllllll}23 & 17 & 0.00 & -0.33 & 0.11 & -0.12 & 0.02 & 0.03 & -0.11 & 0.01 & -0.01\end{array}$

$\begin{array}{lllllllllll}24 & 6 & 0.00 & -0.01 & -0.06 & 0.00 & -0.01 & -0.05 & 0.00 & -0.01 & -0.05 \\ 25 & 6 & 0.00 & 0.02 & 0.10 & 0.00 & -0.01 & -0.03 & 0.00 & -0.02 & -0.13\end{array}$

$\begin{array}{lllllllllll}25 & 6 & 0.00 & 0.02 & 0.10 & 0.00 & -0.01 & -0.03 & 0.00 & -0.02 & -0.13 \\ 26 & 1 & 0.00 & 0.03 & 0.15 & 0.00 & -0.02 & -0.11 & 0.00 & -0.02 & -0.08\end{array}$

$\begin{array}{lllllllllll}27 & 1 & 0.05 & 0.01 & 0.09 & -0.06 & 0.04 & 0.00 & 0.03 & -0.07 & -0.16\end{array}$

$\begin{array}{lllllllllll}28 & 1 & -0.04 & 0.02 & 0.09 & 0.05 & -0.04 & 0.01 & -0.03 & 0.01 & -0.17\end{array}$

$\begin{array}{lllllllllll}29 & 1 & 0.08 & 0.10 & 0.18 & -0.03 & -0.01 & -0.02 & 0.00 & -0.08 & -0.20\end{array}$

$\begin{array}{lllllllllll}30 & 1 & -0.08 & -0.04 & 0.20 & 0.03 & 0.00 & -0.02 & 0.00 & 0.00 & -0.21 \\ 31 & 1 & 0.01 & 0.01 & 0.02 & 0.00 & 0.01 & 0.04 & 0.00 & -0.03 & -0.17\end{array}$

$\begin{array}{lllllllllll}31 & 1 & 0.01 & 0.01 & 0.02 & 0.00 & 0.01 & 0.04 & 0.00 & -0.03 & -0.17 \\ 32 & 1 & 0.01 & 0.28 & 0.02 & 0.10 & 0.01 & 0.16 & 0.08 & 0.01 & 0.10\end{array}$

$\begin{array}{lllllllllll}33 & 1 & 0.00 & 0.02 & 0.09 & 0.00 & 0.04 & 0.21 & 0.00 & 0.04 & 0.14\end{array}$

$\begin{array}{lllllllllll}34 & 1 & 0.00 & -0.25 & 0.13 & -0.10 & 0.05 & 0.15 & -0.08 & 0.04 & 0.10\end{array}$

$\begin{array}{lllllllllll}35 & 1 & -0.01 & -0.09 & 0.02 & -0.05 & 0.18 & 0.11 & -0.13 & -0.08 & 0.13 \\ 36 & 1 & 0.00 & 0.00 & 0.00 & 0.00 & 0.04 & 0.19 & 0.00 & 0.04 & 0.17\end{array}$

$\begin{array}{lllllllllll}36 & 1 & 0.00 & 0.00 & 0.00 & 0.00 & 0.04 & 0.19 & 0.00 & 0.04 & 0.17 \\ 37 & 1 & 0.01 & 0.09 & -0.01 & 0.05 & -0.12 & 0.17 & 0.13 & 0.14 & 0.10\end{array}$

$\begin{array}{lllllllllll}38 & 1 & 0.06 & 0.06 & -0.02 & -0.08 & -0.09 & -0.10 & 0.13 & 0.12 & 0.03\end{array}$

$\begin{array}{lllllllllll}39 & 1 & -0.06 & -0.06 & 0.00 & 0.09 & 0.05 & -0.12 & -0.13 & -0.11 & 0.07\end{array}$

$\begin{array}{lllllllllll}40 & 1 & 0.00 & -0.03 & -0.16 & 0.00 & 0.01 & 0.07 & 0.00 & -0.04 & -0.24\end{array}$

$\begin{array}{lllllllllll}41 & 6 & 0.00 & -0.02 & -0.14 & 0.00 & -0.03 & -0.18 & 0.00 & 0.02 & 0.11\end{array}$

$\begin{array}{lllllllllll}42 & 6 & 0.00 & -0.03 & -0.19 & 0.00 & -0.04 & -0.26 & 0.00 & 0.03 & 0.19\end{array}$

$\begin{array}{lllllllllll}43 & 1 & 0.03 & -0.02 & -0.20 & 0.03 & -0.01 & -0.29 & -0.06 & 0.02 & 0.20\end{array}$

$\begin{array}{lllllllllll}44 & 1 & 0.00 & -0.04 & -0.22 & 0.00 & -0.05 & -0.27 & 0.00 & 0.04 & 0.24\end{array}$

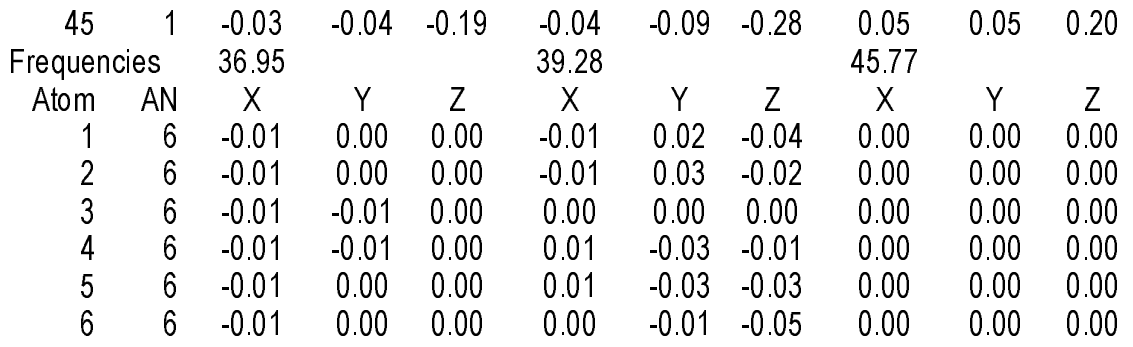




\begin{tabular}{|c|c|c|c|c|c|c|c|c|c|c|}
\hline & & & & & & & & & & \\
\hline 7 & 7 & -0.01 & -0.01 & 0.00 & 0.00 & 0.00 & 0.02 & 0.00 & 0.00 & 0.00 \\
\hline 8 & 6 & -0.01 & -0.01 & 0.00 & 0.00 & -0.01 & -0.06 & 0.00 & 0.00 & 0.00 \\
\hline 9 & 6 & -0.01 & -0.01 & 0.00 & 0.00 & -0.01 & -0.08 & 0.00 & 0.00 & 0.00 \\
\hline 10 & 6 & 0.00 & 0.00 & 0.00 & 0.00 & -0.03 & -0.15 & 0.00 & 0.00 & -0.01 \\
\hline 11 & 17 & -0.01 & -0.01 & 0.00 & 0.03 & -0.06 & 0.01 & 0.00 & 0.00 & 0.00 \\
\hline 12 & 17 & -0.01 & -0.01 & 0.00 & -0.03 & 0.06 & -0.01 & 0.00 & 0.00 & 0.00 \\
\hline 13 & 78 & -0.01 & -0.01 & 0.01 & 0.00 & 0.01 & 0.07 & 0.00 & 0.00 & \\
\hline 14 & 7 & 0.04 & -0.06 & 0.01 & 0.00 & 0.00 & -0.01 & 0.00 & 0.00 & 0.00 \\
\hline 15 & 7 & -0.01 & 0.00 & 0.00 & 0.00 & 0.00 & -0.03 & 0.00 & 0.00 & 0.00 \\
\hline 16 & 6 & -0.02 & 0.03 & -0.01 & 0.00 & -0.01 & -0.02 & 0.00 & 0.00 & 0.00 \\
\hline 17 & 6 & -0.02 & 0.07 & -0.01 & 0.02 & -0.01 & -0.01 & 0.00 & 0.00 & 0.00 \\
\hline 18 & 6 & -0.03 & 0.14 & -0.03 & 0.02 & -0.01 & 0.01 & 0.00 & 0.00 & 0.00 \\
\hline 19 & 6 & -0.03 & 0.17 & -0.03 & 0.00 & -0.01 & 0.02 & 0.00 & 0.00 & 0.00 \\
\hline 20 & 6 & -0.03 & 0.14 & -0.02 & -0.02 & 0.00 & 0.01 & 0.00 & 0.00 & 0.00 \\
\hline 21 & 6 & -0.02 & 0.07 & -0.01 & -0.02 & 0.00 & -0.01 & 0.00 & 0.00 & 0.00 \\
\hline 22 & 17 & -0.01 & 0.03 & -0.01 & 0.04 & -0.01 & -0.02 & 0.00 & 0.00 & 0.00 \\
\hline 23 & 17 & -0.02 & 0.03 & -0.01 & -0.04 & 0.00 & -0.02 & 0.00 & 0.00 & 0.00 \\
\hline 24 & 6 & 0.01 & 0.01 & 0.01 & 0.00 & 0.03 & 0.18 & 0.00 & 0.00 & 0.00 \\
\hline 25 & 6 & -0.01 & -0.01 & 0.00 & 0.00 & -0.03 & -0.15 & 0.00 & 0.00 & 0.00 \\
\hline 26 & 1 & -0.01 & -0.01 & 0.00 & 0.00 & -0.01 & -0.06 & 0.0 & 0.00 & 0.00 \\
\hline 27 & 1 & 0.00 & -0.02 & -0.01 & 0.05 & -0.15 & -0.22 & 0.0 & 0.00 & -0.01 \\
\hline 28 & 1 & -0.01 & 0.00 & -0.01 & -0.05 & 0.06 & -0.26 & 0.0 & 0.00 & -0.01 \\
\hline 29 & 1 & 0.00 & -0.01 & 0.00 & 0.02 & -0.06 & -0.16 & 0.0 & 0.00 & -0.01 \\
\hline 30 & 1 & 0.00 & -0.01 & 0.00 & -0.02 & 0.01 & -0.17 & 0.0 & 0.00 & -0.01 \\
\hline 31 & 1 & 0.00 & 0.00 & -0.01 & 0.00 & -0.03 & -0.16 & 0.00 & 0.00 & -0.01 \\
\hline 32 & 1 & -0.03 & 0.16 & -0.03 & 0.04 & -0.02 & 0.02 & 0.00 & 0.00 & 0.00 \\
\hline 33 & 1 & -0.04 & 0.23 & -0.04 & 0.00 & -0.01 & 0.0 & 0.0 & 0.00 & 0.00 \\
\hline 34 & 1 & -0.03 & 0.16 & -0.03 & -0.03 & 0.00 & 0.02 & 0.00 & 0.00 & 0.00 \\
\hline 35 & 1 & -0.01 & 0.00 & 0.00 & 0.02 & -0.06 & -0.04 & 0.00 & 0.00 & 0.00 \\
\hline 36 & 1 & -0.01 & 0.00 & 0.00 & 0.00 & -0.01 & -0.06 & 0.00 & 0.00 & 0.00 \\
\hline 37 & 1 & -0.01 & 0.00 & 0.00 & -0.02 & 0.04 & -0.05 & 0.00 & 0.00 & 0.00 \\
\hline 38 & 1 & 0.02 & 0.02 & 0.01 & 0.02 & -0.02 & 0.19 & 0.01 & 0.00 & 0.01 \\
\hline 39 & 1 & 0.01 & 0.02 & 0.01 & -0.02 & 0.09 & 0.18 & -0.01 & 0.00 & 0.01 \\
\hline 40 & 1 & 0.03 & 0.00 & 0.01 & 0.00 & 0.04 & 0.23 & 0.00 & 0.00 & 0.00 \\
\hline 41 & 6 & 0.12 & -0.15 & 0.02 & -0.01 & -0.01 & -0.13 & 0.00 & 0.00 & 0.00 \\
\hline 42 & 6 & 0.26 & -0.29 & 0.03 & -0.01 & -0.04 & -0.30 & 0.01 & -0.01 & -0.01 \\
\hline 43 & 1 & 0.38 & -0.30 & 0.06 & 0.10 & -0.01 & -0.31 & -0.33 & 0.30 & -0 \\
\hline 44 & 1 & 0.19 & -0.43 & 0.01 & -0.01 & -0.05 & -0.40 & 0.00 & 0.09 & 0.56 \\
\hline 45 & 1 & 0.31 & -0.24 & 0.05 & -0.13 & -0.07 & -0.30 & 0.35 & -0.42 & \\
\hline Frequer & & 50.30 & & & 64.31 & & & 67.06 & & \\
\hline Atom & AN & $x$ & $Y$ & Z & $x$ & $Y$ & Z & $x$ & $Y$ & Z \\
\hline 1 & 6 & 0.07 & 0.19 & -0.04 & -0.02 & 0.01 & -0.01 & 0.07 & 0.16 & -0.03 \\
\hline 2 & 6 & 0.03 & 0.06 & -0.01 & -0.02 & 0.01 & 0.02 & 0.02 & 0.00 & -0.01 \\
\hline 3 & 6 & 0.02 & 0.00 & 0.00 & 0.00 & 0.01 & 0.03 & 0.01 & -0.04 & 0.00 \\
\hline 4 & 6 & 0.04 & 0.06 & -0.01 & 0. & 0.00 & 0.0 & 0.0 & 0.01 & -0.01 \\
\hline 5 & 6 & 0.07 & 0.19 & -0.04 & 0. & 0.01 & -0.01 & 0.0 & 0.16 & \\
\hline 6 & 6 & 0.09 & 0.26 & -0.05 & 0.01 & 0.02 & -0.02 & 0.09 & 0.26 & -0.04 \\
\hline 7 & 7 & 0.00 & -0.05 & 0.01 & 0.00 & 0.00 & 0.04 & 0.00 & -0.04 & 0.00 \\
\hline 8 & 6 & -0.01 & -0.05 & 0.01 & 0.0 & 0.01 & 0.05 & 0.04 & -0.03 & -0.01 \\
\hline 9 & 6 & 0.00 & -0.06 & 0.01 & 0.1 & 0.00 & 0.0 & 0. & 0.02 & 0.00 \\
\hline 10 & 6 & 0.00 & -0.06 & 0.01 & 0.00 & 0.02 & 0.07 & 0.08 & 0.03 & -0.01 \\
\hline 11 & 17 & 0.02 & -0.01 & 0.00 & 0.05 & -0.01 & 0.04 & -0.03 & -0.14 & \\
\hline 12 & 17 & 0.01 & -0. & 0.00 & -0 . & 0.00 & 0.04 & -0.01 & -0.15 & \\
\hline 13 & 78 & 0.01 & -0.05 & 0.01 & 0.00 & 0.00 & -0.02 & -0.04 & 0.00 & 0.00 \\
\hline 14 & 7 & -0.01 & -0.03 & 0.01 & 0.00 & 0.00 & -0.02 & -0.05 & 0.01 & \\
\hline 15 & 7 & -0.01 & -0.06 & 0.01 & 0.0 & -0.01 & -0.0 & 0.0 & 0.04 & \\
\hline 16 & 6 & -0.01 & -0.01 & 0.00 & 0.00 & -0.01 & -0.07 & 0.02 & 0.04 & 0.01 \\
\hline 17 & 6 & -0.02 & 0.04 & -0.01 & 0.0 & -0.01 & -0.04 & 0.01 & 0.01 & 0.01 \\
\hline 18 & 6 & -0.03 & 0.16 & -0.03 & 0.04 & -0.01 & 0.00 & 0.03 & -0.14 & 0.0 \\
\hline 19 & 6 & -0.04 & 0.22 & -0.05 & 0.00 & -0.01 & 0.03 & 0.05 & -0.23 & 0.0 \\
\hline
\end{tabular}




\begin{tabular}{|c|c|c|c|c|c|c|c|c|c|c|}
\hline & & & & & & & & & & \\
\hline $\begin{array}{l}20 \\
21\end{array}$ & 6 & $\begin{array}{l}-0.03 \\
-0.02\end{array}$ & $\begin{array}{l}0.16 \\
0.04\end{array}$ & $\begin{array}{l}-0.03 \\
-0.01\end{array}$ & $\begin{array}{l}-0.03 \\
-0.04\end{array}$ & $\begin{array}{l}-0.01 \\
-0.01\end{array}$ & $\begin{array}{r}0.00 \\
-0.04\end{array}$ & $\begin{array}{l}0.05 \\
0.03\end{array}$ & $\begin{array}{c}-0.14 \\
0.01\end{array}$ & $\begin{array}{l}0.03 \\
0.01\end{array}$ \\
\hline $\begin{array}{l}21 \\
22\end{array}$ & $\begin{array}{r}6 \\
17\end{array}$ & $\begin{array}{l}-0.02 \\
-0.01\end{array}$ & $\begin{array}{r}0.04 \\
-0.04\end{array}$ & $\begin{array}{c}-0.01 \\
0.01\end{array}$ & $\begin{array}{c}-0.04 \\
0.09\end{array}$ & $\begin{array}{l}-0.01 \\
-0.01\end{array}$ & $\begin{array}{l}-0.04 \\
-0.08\end{array}$ & $\begin{array}{r}0.03 \\
-0.02\end{array}$ & $\begin{array}{l}0.01 \\
0.18\end{array}$ & $\begin{array}{c}0.01 \\
-0.02\end{array}$ \\
\hline 23 & 17 & -0.01 & -0.04 & 0.01 & -0.09 & 0.00 & -0.08 & 0.03 & 0.18 & -0.01 \\
\hline 24 & 6 & 0.01 & -0.05 & 0.01 & 0.00 & 0.03 & 0.16 & -0.06 & -0.03 & -0.02 \\
\hline 25 & 6 & -0.02 & -0.04 & 0.01 & 0.00 & 0.02 & 0.12 & 0.07 & -0.05 & -0.02 \\
\hline 26 & 1 & -0.02 & -0.03 & 0.00 & 0.00 & 0.02 & 0.15 & 0.06 & -0.08 & -0.04 \\
\hline 27 & 1 & -0.03 & -0.04 & 0.01 & 0.03 & 0.06 & 0.13 & 0.06 & -0.04 & -0.01 \\
\hline 28 & 1 & -0.03 & -0.04 & 0.01 & -0.03 & -0.01 & 0.14 & 0.10 & -0.05 & -0.01 \\
\hline 29 & 1 & 0.00 & -0.06 & 0.01 & 0.07 & 0.10 & 0.13 & 0.05 & -0.01 & -0.03 \\
\hline 30 & 1 & 0.00 & -0.06 & 0.01 & -0.06 & -0.05 & 0.15 & 0.13 & 0.04 & -0.04 \\
\hline 31 & 1 & 0.00 & -0.05 & 0.01 & 0.01 & 0.01 & -0.02 & 0.07 & 0.06 & 0.03 \\
\hline 32 & 1 & -0.04 & 0.20 & -0.04 & 0.07 & -0.01 & 0.02 & 0.02 & -0.17 & 0.03 \\
\hline 33 & 1 & -0.05 & 0.32 & -0.07 & 0.01 & -0.02 & 0.06 & 0.06 & -0.35 & 0.06 \\
\hline 34 & 1 & -0.03 & 0.20 & -0.04 & -0.06 & -0.01 & 0.02 & 0.06 & -0.17 & 0.03 \\
\hline 35 & 1 & 0.09 & 0.24 & -0.05 & 0.04 & 0.01 & -0.01 & 0.06 & 0.21 & -0.03 \\
\hline 36 & 1 & 0.12 & 0.36 & -0.07 & 0.01 & 0.02 & -0.04 & 0.13 & 0.39 & -0.06 \\
\hline 37 & 1 & 0.08 & 0.24 & -0.05 & -0.03 & 0.02 & -0.02 & 0.09 & 0.20 & -0.03 \\
\hline 38 & 1 & 0.03 & -0.02 & 0.02 & 0.34 & 0.24 & 0.39 & -0.03 & 0.00 & 0.00 \\
\hline 39 & 1 & -0.01 & -0.06 & 0.03 & -0.35 & -0.09 & 0.45 & -0.09 & -0.07 & 0.01 \\
\hline 40 & 1 & 0.02 & -0.05 & -0.02 & 0.00 & -0.04 & -0.21 & -0.07 & -0.03 & -0.08 \\
\hline 41 & 6 & -0.07 & 0.02 & -0.01 & 0.00 & 0.00 & 0.01 & -0.01 & -0.03 & 0.00 \\
\hline 42 & 6 & -0.15 & 0.10 & -0.03 & 0.00 & 0.01 & 0.07 & 0.04 & -0.09 & 0.00 \\
\hline 43 & 1 & -0.21 & 0.11 & -0.04 & -0.02 & -0.02 & 0.09 & 0.09 & -0.08 & -0.01 \\
\hline 44 & 1 & -0.11 & 0.19 & -0.04 & 0.00 & 0.01 & 0.08 & 0.02 & -0.15 & 0.00 \\
\hline 45 & 1 & -0.20 & 0.09 & -0.04 & 0.03 & 0.04 & 0.08 & 0.07 & -0.09 & -0.01 \\
\hline Frequer & & 70.73 & & & 81.52 & & & 83.44 & & \\
\hline Atom & AN & X & $Y$ & Z & $X$ & $Y$ & Z & X & $Y$ & Z \\
\hline 1 & 6 & 0.00 & -0.01 & 0.00 & -0.01 & -0.06 & 0.01 & 0.03 & -0.01 & 0.00 \\
\hline 2 & 6 & 0.00 & -0.01 & -0.01 & 0.01 & 0.02 & 0.02 & 0.03 & -0.01 & -0.04 \\
\hline 3 & 6 & 0.00 & 0.00 & -0.02 & 0.02 & -0.01 & 0.03 & 0.00 & -0.02 & -0.06 \\
\hline 4 & 6 & -0.01 & 0.00 & -0.01 & 0.04 & 0.01 & 0.02 & -0.02 & 0.00 & -0.04 \\
\hline 5 & 6 & -0.01 & -0.01 & 0.00 & 0.02 & -0.07 & 0.01 & -0.03 & 0.00 & 0.00 \\
\hline 6 & 6 & 0.00 & -0.01 & 0.00 & -0.01 & -0.14 & 0.01 & 0.00 & -0.02 & 0.02 \\
\hline 7 & 7 & 0.00 & 0.00 & -0.02 & 0.00 & -0.06 & 0.03 & 0.00 & -0.02 & -0.05 \\
\hline 8 & 6 & 0.00 & 0.00 & -0.01 & -0.02 & -0.07 & 0.03 & 0.00 & -0.01 & -0.02 \\
\hline 9 & 6 & 0.00 & 0.00 & 0.00 & -0.02 & -0.09 & -0.01 & 0.00 & 0.00 & 0.07 \\
\hline 10 & 6 & 0.00 & 0.00 & -0.01 & -0.03 & -0.11 & -0.13 & 0.00 & 0.04 & 0.29 \\
\hline 11 & 17 & -0.01 & 0.02 & -0.02 & 0.13 & 0.23 & 0.00 & -0.06 & 0.03 & -0.08 \\
\hline 12 & 17 & 0.0 & -0.02 & -0.01 & 0.05 & 0.24 & -0.01 & 0.10 & 0.01 & -0.07 \\
\hline 13 & 78 & 0.0 & 0.00 & 0.00 & 0.02 & -0.10 & 0.02 & 0.00 & -0.01 & 0.01 \\
\hline 14 & 7 & & 0.00 & 0.01 & 0.03 & -0.10 & 0.01 & 0.00 & -0.01 & 0.02 \\
\hline 15 & 7 & 0.0 & 0.00 & 0.03 & -0.03 & -0.08 & 0.03 & 0. & -0.01 & -0.02 \\
\hline 16 & 6 & 0.0 & 0.00 & 0.02 & -0.04 & -0.04 & 0.01 & 0.00 & 0.00 & 0.00 \\
\hline 17 & 6 & -0.0 & 0.00 & 0.02 & -0.04 & 0.02 & 0.00 & -0.01 & 0.00 & -0.01 \\
\hline 18 & 6 & -0.1 & 0.0 & 0.00 & & & 0.01 & -0.01 & & -0.01 \\
\hline 19 & 6 & 0.0 & 0.00 & -0.01 & -0.04 & -0.10 & 0.03 & 0.00 & -0.01 & -0.01 \\
\hline 20 & 6 & 0. & 0.01 & 0.00 & -0.04 & -0.04 & 0.02 & 0.00 & 0.00 & -0.01 \\
\hline 21 & 6 & & 0.00 & 0.01 & & & 0.00 & 0.00 & 0.00 & -0.01 \\
\hline 22 & 17 & -0.0 & & 0.0 & -0.08 & 0.27 & -0.05 & -0.02 & 0.02 & -0.01 \\
\hline 23 & 17 & & 0.00 & 0.03 & -0.09 & 0.26 & -0.06 & 0.00 & 0.03 & -0.01 \\
\hline 24 & 6 & & 0.00 & -0.01 & 0.05 & -0.09 & -0.08 & 0.00 & 0.02 & 0.19 \\
\hline 25 & 6 & & 0.0 & -0.02 & -0.05 & & 0.07 & 0.00 & -0.03 & -0.08 \\
\hline 26 & 1 & 0. & -0.01 & -0.09 & -0.04 & -0.04 & -0.02 & 0.00 & -0.01 & -0.02 \\
\hline 27 & 1 & & 0.04 & 0.00 & -0.12 & & 0.13 & 0.04 & -0.13 & -0.14 \\
\hline 28 & 1 & 0. & -0.03 & 0.02 & 0.00 & -0.12 & 0.17 & -0.04 & 0.05 & -0.17 \\
\hline 29 & 1 & -0. & $-0 .($ & -0.05 & -0.12 & -0.26 & -0.23 & 0.10 & 0.27 & 0.43 \\
\hline 30 & 1 & 0.0 & 0.03 & -0.06 & 0.07 & 0.00 & -0.27 & -0.10 & -0.14 & 0.49 \\
\hline 31 & 1 & & 0.0 & 0.0 & -0.03 & -0.10 & -0.01 & 0.01 & 0.03 & 0.16 \\
\hline 32 & 1 & & 0.00 & -0.0 & -0.04 & -0.01 & 0.01 & -0.01 & -0.01 & -0.01 \\
\hline
\end{tabular}




\begin{tabular}{|c|c|c|c|c|c|c|c|c|c|c|}
\hline 33 & & & & & & & & & 0 & $\cap 01$ \\
\hline & 1 & 0.02 & 0.01 & $\begin{array}{l}-0.02 \\
-0.01\end{array}$ & -0.05 & $\begin{array}{l}-0.10 \\
-0.02\end{array}$ & 0.01 & 0.00 & $\begin{array}{l}-0.02 \\
0.00\end{array}$ & -0.01 \\
\hline 35 & 1 & -0.02 & 0.00 & 0.00 & 0.04 & -0.06 & 0.00 & -0.05 & 0.00 & 0.01 \\
\hline 36 & 1 & -0.01 & -0.02 & 0.01 & -0.03 & -0.21 & 0.01 & 0.00 & -0.02 & 0.04 \\
\hline 37 & 1 & 0.01 & -0.02 & 0.00 & -0.02 & -0.05 & 0.00 & 0.06 & -0.01 & 0.01 \\
\hline 38 & 1 & 0.38 & 0.35 & 0.24 & 0.06 & -0.01 & -0.08 & 0.01 & -0.07 & 0.20 \\
\hline 39 & 1 & -0.38 & -0.24 & 0.34 & 0.04 & -0.13 & -0.06 & -0.01 & 0.12 & 0.17 \\
\hline 40 & 1 & 0.01 & -0.09 & -0.54 & 0.07 & -0.11 & -0.14 & 0.00 & 0.04 & 0.29 \\
\hline 41 & 6 & 0.00 & 0.00 & -0.01 & -0.02 & -0.06 & 0.01 & 0.00 & -0.01 & 0.01 \\
\hline 42 & 6 & 0.00 & 0.00 & -0.04 & -0.10 & 0.03 & -0.01 & -0.01 & 0.00 & -0.02 \\
\hline 43 & 1 & 0.02 & 0.00 & -0.05 & -0.16 & 0.02 & 0.00 & -0.01 & 0.01 & -0.03 \\
\hline 44 & 1 & 0.00 & 0.00 & -0.06 & -0.06 & 0.11 & -0.02 & -0.01 & 0.01 & -0.01 \\
\hline 45 & 1 & -0.02 & -0.01 & -0.04 & -0.16 & 0.03 & 0.00 & -0.02 & -0.02 & -0.02 \\
\hline quen & & 92.82 & & & 94.47 & & & 115.19 & & \\
\hline Atom & AN & $x$ & $Y$ & Z & $X$ & $Y$ & Z & $x$ & $Y$ & Z \\
\hline 1 & 6 & -0.05 & -0.05 & 0.00 & 0.01 & 0.00 & -0.01 & 0.00 & 0.01 & 0.01 \\
\hline 2 & 6 & -0.04 & -0.01 & -0.01 & 0.01 & 0.00 & -0.02 & 0.00 & 0.01 & 0.03 \\
\hline 3 & 6 & -0.01 & 0.07 & -0.02 & 0.00 & -0.01 & -0.02 & 0.00 & 0.01 & 0.04 \\
\hline 4 & 6 & -0.04 & 0.00 & -0.01 & -0.01 & 0.00 & -0.02 & 0.01 & 0.00 & 0.03 \\
\hline 5 & 6 & -0.06 & -0.04 & 0.01 & -0.01 & 0.00 & -0.01 & 0.01 & 0.00 & 0.02 \\
\hline 6 & 6 & -0.05 & -0.04 & 0.01 & 0.00 & 0.00 & -0.01 & 0.01 & 0.00 & 0.01 \\
\hline 7 & 7 & -0.01 & 0.07 & -0.02 & 0.00 & -0.01 & -0.02 & 0.00 & 0.00 & 0.03 \\
\hline 8 & 6 & -0.06 & 0.05 & -0.01 & 0.00 & 0.00 & 0.01 & 0.00 & -0.01 & -0.05 \\
\hline 9 & 6 & -0.08 & -0.02 & 0.01 & 0.00 & 0.00 & 0.01 & 0.00 & 0.00 & 0.01 \\
\hline 10 & 6 & -0.13 & -0.04 & 0.03 & 0.01 & 0.00 & -0.01 & 0.00 & 0.01 & 0.07 \\
\hline 11 & 17 & -0.11 & -0.17 & 0.01 & -0.02 & 0.00 & -0.03 & 0.04 & -0.01 & 0.05 \\
\hline 12 & 17 & -0.08 & -0.16 & 0.02 & 0.03 & 0.00 & -0.03 & -0.03 & 0.02 & 0.04 \\
\hline 13 & 78 & 0.11 & 0.00 & 0.00 & -0.01 & 0.00 & 0.01 & 0.00 & 0.00 & -0.03 \\
\hline 14 & 7 & 0.16 & -0.04 & 0.02 & -0.01 & 0.01 & 0.03 & 0.00 & -0.01 & -0.08 \\
\hline 15 & 7 & -0.04 & -0.05 & 0.01 & 0.00 & 0.00 & -0.01 & 0.00 & 0.00 & -0.01 \\
\hline 16 & 6 & -0.04 & -0.05 & 0.01 & 0.00 & 0.00 & -0.01 & 0.00 & 0.00 & 0.01 \\
\hline 17 & 6 & -0.06 & 0.00 & 0.00 & 0.01 & 0.00 & -0.01 & -0.01 & 0.00 & 0.01 \\
\hline 18 & 6 & -0.06 & 0.03 & -0.01 & 0.01 & 0.00 & 0.00 & -0.01 & 0.00 & 0.00 \\
\hline 19 & 6 & -0.07 & 0.03 & -0.01 & 0.00 & 0.00 & 0.00 & -0.01 & 0.00 & 0.00 \\
\hline 20 & 6 & -0.06 & 0.03 & 0.00 & 0.00 & 0.00 & 0.00 & 0.00 & 0.00 & 0.00 \\
\hline 21 & 6 & -0.06 & 0.00 & 0.00 & 0.00 & 0.00 & -0.01 & 0.00 & 0.00 & 0.01 \\
\hline 22 & 17 & -0.09 & 0.09 & -0.01 & 0.02 & -0.01 & -0.01 & -0.03 & 0.00 & 0.02 \\
\hline 23 & 17 & & 0.08 & -0.03 & & -0.02 & -0.01 & & & 0.02 \\
\hline 24 & 6 & 0.2 & 0.09 & 0.01 & -0.01 & 0.01 & 0.05 & 0.00 & 0.02 & 0.08 \\
\hline 25 & 6 & -0.12 & 0.10 & 0.01 & 0. & 0.02 & 0.11 & 0.00 & -0.04 & -0.20 \\
\hline 26 & 1 & -0. & 0.12 & -0.11 & & -0.06 & -0.26 & & -0.09 & -0.48 \\
\hline 27 & 1 & -0.2 & 0.18 & 0.07 & -0.25 & 0.35 & 0.30 & -0.24 & 0.03 & -0.12 \\
\hline 28 & 1 & -0.07 & 0.02 & 0.10 & & -0.21 & 0.42 & 0.23 & -0.07 & -0.09 \\
\hline 29 & 1 & -0.2 & -0.06 & -0.01 & -0.2 & -0.16 & -0.17 & -0. & -0.13 & -0.11 \\
\hline 30 & 1 & -0.1 & -0.01 & -0.01 & 0. & 0. & -0.1 & & & -0.13 \\
\hline 31 & 1 & -0.1 & -0.07 & 0.11 & 0.0 & 0.05 & 0.29 & -0.01 & 0.08 & 0.45 \\
\hline 32 & 1 & -0.06 & 0.07 & -0.01 & 0.0 & 0.00 & 0.00 & -0.01 & 0.00 & 0.00 \\
\hline 33 & 1 & -0. & 0.06 & -0.01 & & & 0.0 & & 0.0 & -0.01 \\
\hline 34 & 1 & -0.0 & 0.0 & -0.01 & 0. & -0. & 0.0 & 0.0 & 0.0 & 0.00 \\
\hline 35 & 1 & -0.07 & -0.10 & 0.02 & -0.01 & 0.00 & -0.01 & 0.02 & -0.01 & 0.01 \\
\hline 36 & 1 & -0.0 & -0.07 & 0.02 & & & 0.00 & & & 0.00 \\
\hline 37 & 1 & -0.0 & -0.10 & 0.02 & 0. & 0.0 & -0.01 & -0.0 & & 0.01 \\
\hline 38 & 1 & 0.1 & 0.06 & -0.02 & -0.06 & -0.07 & 0.02 & 0.02 & -0.04 & 0.10 \\
\hline 39 & 1 & 0.2 & 0.16 & -0.04 & & 0.06 & 0.00 & -0.02 & 0.08 & 0.08 \\
\hline 40 & 1 & 0.2 & 0.08 & 0.10 & -0.01 & 0.02 & 0.14 & 0.00 & 0.03 & 0.14 \\
\hline 41 & 6 & 0.0 & 0.03 & 0.00 & 0.00 & 0.00 & 0.01 & 0.00 & 0.00 & -0.04 \\
\hline 42 & 6 & -0.03 & 0.16 & -0.04 & 0. & -0.01 & -0.02 & 0.00 & 0.01 & 0.06 \\
\hline 43 & 1 & & 0.15 & -0.04 & 0.0 & 0.00 & -0.0 & -0.05 & -0.03 & 0.10 \\
\hline 44 & 1 & 0.0 & 0.29 & -0.06 & 0.00 & -0.02 & -0.03 & 0.00 & 0.01 & 0.09 \\
\hline 45 & 1 & -0.13 & 0.14 & -0.04 & -0.01 & -0.03 & -0.03 & 0.05 & 0.06 & 0.08 \\
\hline
\end{tabular}




\begin{tabular}{|c|c|c|c|c|c|c|c|c|c|c|}
\hline \multicolumn{2}{|c|}{ Frequencies } & \multicolumn{2}{|l|}{120.65} & \multicolumn{3}{|c|}{138.31} & \multicolumn{3}{|c|}{164.26} & \multirow[b]{2}{*}{ Z } \\
\hline & AN & $x$ & $\mathrm{Y}$ & Z & X & Y & Z & X & $\mathrm{Y}$ & \\
\hline 1 & 6 & 0.16 & -0.05 & 0.02 & 0.00 & 0.00 & 0.01 & 0.01 & -0.02 & 0.02 \\
\hline 2 & 6 & 0.16 & -0.04 & 0.01 & 0.00 & 0.00 & 0.01 & 0.02 & 0.03 & 0.00 \\
\hline 3 & 6 & 0.12 & -0.01 & -0.01 & & 0.00 & 0.01 & & & -0.01 \\
\hline 4 & 6 & 0.16 & -0.04 & -0.01 & 0.0 & 0.00 & 0.01 & 0.02 & & -0.01 \\
\hline 5 & 6 & 0.16 & -0.05 & -0.01 & 0.00 & 0.00 & 0.01 & 0.01 & -0.02 & -0.01 \\
\hline 6 & 6 & 0.18 & -0.05 & 0.01 & 0.00 & 0.00 & 0.01 & 0.01 & -0.04 & 0.01 \\
\hline 7 & 7 & 0.08 & -0.01 & 0.00 & 0.0 & 0.00 & 0.0 & -0.02 & & -0.01 \\
\hline 8 & 6 & 0.03 & -0.02 & 0.01 & & & & & & -0.02 \\
\hline 9 & 6 & -0.01 & -0.01 & 0.00 & 0.0 & 0.01 & 0.04 & -0.1 & 0.04 & 0.00 \\
\hline 10 & 6 & 0.05 & 0.02 & -0.03 & & 0.03 & 0. & -0.2 & & 0.02 \\
\hline 11 & 17 & 0.28 & -0.08 & 0.07 & & & & & & 0.07 \\
\hline 12 & 17 & & -0.1 & -0.05 & & & & & & -0.04 \\
\hline 13 & 78 & & & -0.01 & & 0. & & $-0 .(1-x)$ & & 0.00 \\
\hline 14 & 7 & -0.04 & 0.10 & 0.00 & 0.0 & 0.02 & 0.0 & -0.03 & 0.00 & 0.00 \\
\hline 15 & 7 & -0.08 & & 0.00 & & 0.0 & 0.0 & & -0.08 & 0.01 \\
\hline 16 & 6 & -0.11 & & -0.01 & & -0.01 & -0 . & & & 0.02 \\
\hline 17 & 6 & -0.15 & -0.01 & -0.01 & & -0.01 & -0.0 & 0.02 & & 0.02 \\
\hline 18 & 6 & -0.15 & -0.04 & -0.01 & & 0.00 & -0.0 & & & 0.01 \\
\hline 19 & 6 & -0.17 & $-0 .(1-x-1$ & 0.01 & & 0. & & 0. & & -0.01 \\
\hline 20 & 6 & -0 . & & 0.02 & & $-0 .(1-x-y$ & -0 . & 0. & & -0.0 \\
\hline 21 & 6 & & 0.1 & 0.01 & & -0.01 & & & & 0.00 \\
\hline 22 & 17 & & & 0.07 & & 0.0 & & & & -0.07 \\
\hline 23 & 17 & -0 . & -0 & -0.05 & & -0 . & & & & \\
\hline 2 & 6 & -0 . & 0. & -0.04 & 0. & -0. & -0. & -0. & & \\
\hline 25 & 6 & & & 0.05 & & -0. & & & & -0.03 \\
\hline 26 & 1 & 0.0 & 0. & 0.1 & & -0.06 & -0 . & -0 & & -0.1 \\
\hline 27 & 1 & & & 0. & -0 . & 0. & -0 . & & & \\
\hline 28 & 1 & & & 0.02 & & -0. & & & & 0.04 \\
\hline 29 & 1 & & & 0.0 & & 0. & & -0.2 & & 0.07 \\
\hline 30 & 1 & -0.0 & & 0.01 & & -0.02 & 0. & -0.4 & & \\
\hline 31 & 1 & & 0. & -0.13 & & 0.06 & 0. & -0 . & & -0.06 \\
\hline 32 & 1 & & & 0.00 & & 0. & & & & -0.0 \\
\hline 33 & 1 & & & 0. & & 0. & -0 . & & & \\
\hline 34 & 1 & & & 0. & & -0. & -0 . & & & -0.02 \\
\hline 35 & 1 & & & 0. & & 0.1 & & & & .00 \\
\hline 36 & 1 & & -0. & 0. & & 0. & 0. & & & 0. \\
\hline 37 & 1 & & & & & & & & & \\
\hline 38 & 1 & & & -0 . & & & & & & \\
\hline 39 & 1 & & 0.0 & -0.0 & & -0.2 & -0 . & -0. & & -0.02 \\
\hline 40 & 1 & & & -0.0 & & -0. & -0 . & & & \\
\hline 41 & 6 & & & & & & & & & \\
\hline 42 & 6 & & & -0. & & & & & & \\
\hline 43 & 1 & & & -0.0 & & 0. & & 0. & & 0.00 \\
\hline 44 & 1 & & & & & & & & & 0.00 \\
\hline 45 & 1 & & & -0.02 & & -0.06 & -0.07 & & & \\
\hline Frequer & & 171.90 & & & & & & & & \\
\hline tom & AN & & $Y$ & Z & & $Y$ & Z & $x$ & Y & \\
\hline 1 & 6 & & 0.00 & 0.00 & & -0.02 & 0.02 & & -0.01 & \\
\hline 2 & 6 & & 0.00 & -0.01 & & -0.01 & 0.0 & & & \\
\hline 3 & 6 & & 0.00 & -0.01 & & 0.0 & 0. & 0. & 0. & 0.00 \\
\hline 4 & 6 & & 0.00 & -0.01 & & & & & & \\
\hline 5 & 6 & & 0.00 & 0.00 & & 0.0 & & 0.0 & & 0.01 \\
\hline 6 & 6 & & 0.00 & 0.00 & & 0. & 0. & 0.0 & & 0.00 \\
\hline 7 & 7 & & 0.0 & $0.0^{\prime}$ & & -0.01 & -0. & 0. & 0.00 & 0.00 \\
\hline 8 & 6 & & 0.01 & 0.04 & & -0.01 & & & & \\
\hline 9 & 6 & -0.0 & 0.01 & 0.04 & & 0.00 & -0. & 0.0 & -0.05 & 0.01 \\
\hline 10 & 6 & -0.0 & 0.0 & 0.02 & 0.0 & 0.01 & 0.02 & -0.08 & -0.09 & 0.02 \\
\hline 11 & 17 & & 0.00 & -0.01 & 0.04 & -0.01 & 0.06 & -0.04 & 0.00 & -0.04 \\
\hline
\end{tabular}




\begin{tabular}{|c|c|c|c|c|c|c|c|c|c|c|}
\hline & & & & & & & & & & \\
\hline 12 & 17 & 0.01 & -0.01 & -0.01 & -0.03 & 0.03 & 0.04 & -0.05 & 0.02 & 0.04 \\
\hline 13 & 78 & 0.00 & 0.00 & 0.00 & 0.00 & -0.01 & -0.06 & 0.03 & 0.01 & 0.00 \\
\hline 14 & 7 & 0.00 & 0.00 & 0.00 & 0.01 & 0.05 & 0.35 & -0.12 & 0.20 & -0.02 \\
\hline 15 & 7 & 0.00 & 0.00 & 0.01 & 0.00 & 0.00 & -0.04 & 0.03 & -0.07 & 0.01 \\
\hline 16 & 6 & 0.00 & -0.01 & 0.00 & 0.00 & 0.01 & 0.00 & & -0.10 & 0.02 \\
\hline 17 & 6 & 0.00 & -0.01 & 0.00 & 0.00 & 0.00 & & & & 0.01 \\
\hline 18 & 6 & 0.00 & 0.00 & 0.00 & 0.00 & 0.00 & 0.0 & 0.01 & 0.02 & -0.01 \\
\hline 19 & 6 & 0.00 & & 0.00 & 0.00 & 0.00 & 0.01 & 0.01 & 0.04 & -0.01 \\
\hline 20 & 6 & 0.00 & 0.00 & 0.00 & 0.00 & 0.00 & 0.00 & 0.02 & 0.00 & 0.00 \\
\hline 21 & 6 & 0.00 & 0.00 & 0.00 & -0.01 & 0.01 & 0.0 & 0.02 & -0.06 & 0.01 \\
\hline 22 & 17 & 0.01 & 0.00 & -0.01 & -0.02 & 0.00 & 0.0 & -0.03 & 0.02 & 0.03 \\
\hline 23 & 17 & 0.00 & 0.00 & -0.01 & 0.02 & 0.01 & 0.02 & -0.03 & 0.01 & -0.03 \\
\hline 24 & 6 & 0.00 & 0.00 & 0.00 & 0.01 & 0.02 & 0.0 & -0.19 & -0.19 & 0.04 \\
\hline 25 & 6 & -0.01 & & 0.03 & 0.00 & 0.00 & 0.0 & -0.04 & 0.02 & -0.01 \\
\hline 26 & 1 & -0.02 & 0.09 & 0.39 & 0.00 & 0.01 & 0.09 & -0.03 & 0.07 & 0.00 \\
\hline 27 & 1 & 0.25 & -0.25 & -0.14 & 0.08 & -0.04 & -0.0 & -0.06 & 0.01 & -0.01 \\
\hline 28 & 1 & -0.27 & & -0.23 & -0.07 & 0.02 & -0. & -0.08 & 0.02 & -0.01 \\
\hline 29 & 1 & -0.32 & -0.21 & -0.20 & -0.07 & 0.00 & -0.0 & -0.13 & -0.07 & 0.01 \\
\hline 30 & 1 & 0.31 & 0.14 & -0.23 & 0.09 & 0.00 & -0.0 & -0.11 & -0.07 & 0.01 \\
\hline 31 & 1 & -0.02 & & 0.41 & 0.00 & 0.03 & 0. & -0.08 & -0.16 & 0.04 \\
\hline 32 & 1 & 0.00 & & 0.00 & 0.00 & -0.01 & 0.0 & & 0.07 & -0.02 \\
\hline 33 & 1 & 0.00 & & 0.00 & 0.00 & 0.00 & 0.0 & 0.01 & 0.10 & -0.02 \\
\hline 34 & 1 & 0.00 & & 0.1 & -0.01 & & 0. & & 0.03 & 0.00 \\
\hline 35 & 1 & 0.00 & & 0.00 & 0.00 & 0.04 & 0.0 & 0.03 & -0.01 & 0.01 \\
\hline 36 & 1 & 0.00 & -0.01 & 0.00 & 0.00 & 0.01 & 0.0 & 0.02 & -0.01 & 0.00 \\
\hline 37 & 1 & 0.00 & 0. & 0.00 & 0.00 & -0.03 & 0.0 & 0.03 & -0.01 & 0.00 \\
\hline 38 & 1 & 0.01 & & 0.00 & 0.00 & -0.05 & 0.0 & -0.16 & -0.31 & 0.07 \\
\hline 39 & 1 & -0.01 & 0.00 & 0.00 & 0.01 & 0.11 & 0.04 & -0.17 & -0.30 & 0.06 \\
\hline 40 & 1 & 0.00 & & -0.01 & 0.02 & 0.04 & 0.1 & -0.35 & -0.11 & 0.03 \\
\hline 41 & 6 & 0.00 & & 0.00 & 0.01 & 0.05 & 0.3 & -0.12 & 0.19 & -0.02 \\
\hline 42 & 6 & 0.00 & & 0.00 & -0.01 & -0.03 & -0.1 & 0.11 & -0.04 & 0.00 \\
\hline 43 & 1 & 0.00 & & 0.0 & 0.25 & 0.19 & -0 . & 0.29 & -0.01 & -0.01 \\
\hline 44 & 1 & 0.00 & & 0.0 & 0.01 & -0.04 & -0.3 & -0.02 & -0.30 & 0.04 \\
\hline 45 & 1 & 0.00 & & & -0.28 & -0.31 & -0.28 & 0.26 & -0.03 & -0.01 \\
\hline equer & & 207.59 & & & 208.85 & & & 241.40 & & \\
\hline Atom & AN & $X$ & $Y$ & Z & $X$ & $\mathrm{Y}$ & Z & $X$ & $Y$ & Z \\
\hline 1 & 6 & 0. & 0.00 & 0.00 & 0.09 & 0.32 & -0.06 & -0.09 & 0.03 & 0.00 \\
\hline 2 & 6 & & & & & 0.15 & -0.02 & & 0.07 & -0.03 \\
\hline 3 & 6 & 0.00 & 0. & 0.00 & 0.0 & 0.00 & 0.0 & -0. & 0.10 & -0.02 \\
\hline 4 & 6 & & & 0. & -0.04 & -0.15 & 0.03 & -0.07 & 0.07 & 0.00 \\
\hline 5 & 6 & & & & -0.09 & -0.32 & 0.0 & -0.08 & 0.02 & -0.01 \\
\hline 6 & 6 & & & 0.0 & & 0.00 & 0. & & 0.00 & 0.00 \\
\hline 7 & 7 & 0. & & 0.00 & 0. & 0.00 & 0.00 & -0.04 & 0.11 & -0.02 \\
\hline 8 & 6 & & & & 0. & 0.00 & -0.0 & 0.00 & 0.13 & -0.02 \\
\hline 9 & 6 & & & & & & -0. & & 0. & -0.02 \\
\hline 10 & 6 & & & & & & 0.0 & -0.0 & 0.09 & -0.02 \\
\hline 11 & 17 & & & 0.00 & 0.02 & 0.08 & -0.01 & 0.04 & 0.00 & 0.11 \\
\hline 12 & 17 & & & & -0.03 & -0.08 & 0.02 & & -0.04 & -0.10 \\
\hline 13 & 78 & & & & & & 0. & & -0.03 & 0.01 \\
\hline 14 & 7 & & & 0. & & & 0.02 & 0.06 & -0.09 & 0.02 \\
\hline 15 & 7 & & & & & & -0. & 0. & 0.07 & -0.01 \\
\hline 16 & 6 & & & 0. & & 0.00 & 0. & 0. & 0.04 & -0.01 \\
\hline 17 & 6 & & & -0.03 & & 0.00 & 0.00 & 0.12 & 0.03 & 0.01 \\
\hline 18 & 6 & -0.0 & & -0.07 & & & 0. & 0.13 & 0.02 & -0.01 \\
\hline 19 & 6 & 0.00 & & 0.0 & 0.0 & & 0.0 & 0.1 & 0.01 & 0.00 \\
\hline 20 & 6 & 0.04 & & 0.07 & 0.0 & 0.00 & 0.00 & 0.13 & 0.02 & 0.00 \\
\hline 21 & 6 & & & 0. & & 0.6 & 0.0 & 0.12 & 0.02 & -0.03 \\
\hline 22 & 17 & & & 0.02 & & & 0.0 & -0.06 & 0.02 & 0.16 \\
\hline 23 & 17 & -0.1 & & -0.02 & 0.0 & 0.00 & 0.00 & -0.07 & -0.05 & -0.15 \\
\hline 24 & 6 & 0.01 & 0.01 & 0.00 & 0.00 & 0.00 & 0.01 & -0.17 & -0.19 & 0.03 \\
\hline
\end{tabular}




\begin{tabular}{|c|c|c|c|c|c|c|c|c|c|c|}
\hline & & & & & & & & & & \\
\hline 25 & 6 & 0.00 & 0.00 & 0.00 & 0.00 & 0.00 & 0.00 & -0.01 & 0.15 & -0.03 \\
\hline & 1 & 0.00 & 0.00 & 0.00 & 0.00 & 0.00 & -0.02 & 0.00 & 0.18 & -0.03 \\
\hline 27 & 1 & 0.00 & -0.01 & -0.01 & -0.02 & 0.03 & 0.01 & -0.04 & 0.14 & -0.03 \\
\hline 28 & 1 & 0.00 & 0.00 & -0.01 & 0.01 & -0.02 & 0.02 & -0.04 & 0.14 & -0.03 \\
\hline 29 & 1 & -0.01 & -0.01 & -0.01 & 0.00 & 0.01 & 0.01 & -0.09 & 0.10 & -0.03 \\
\hline 30 & 1 & 0.02 & 0.01 & -0.02 & 0.00 & -0.01 & 0.01 & -0.07 & 0.11 & \\
\hline 31 & 1 & 0.00 & 0.01 & 0.01 & 0.00 & 0.00 & 0.0 & -0.05 & 0.04 & \\
\hline 32 & 1 & -0.06 & 0.57 & -0.12 & 0.00 & 0.00 & 0.00 & 0.12 & 0.00 & -0.01 \\
\hline 33 & 1 & 0.00 & 0.00 & 0.00 & 0.00 & 0.00 & 0.00 & 0.14 & 0.00 & 0.00 \\
\hline 34 & 1 & 0.06 & & 0. & 0.00 & & 0. & 0.12 & 0. & \\
\hline 35 & 1 & 0.00 & & 0.00 & -0.16 & -0.56 & 0.10 & -0.09 & -0.01 & -0.01 \\
\hline 36 & 1 & 0.00 & & 0.00 & 0.00 & 0.00 & 0.00 & -0.11 & -0.04 & 0.01 \\
\hline 37 & 1 & 0.00 & & 0.00 & & & -0.1 & -0.09 & -0.01 & 0.01 \\
\hline 38 & 1 & 0.01 & & 0.00 & -0.02 & -0.03 & 0.0 & -0.16 & -0.26 & 0.04 \\
\hline 39 & 1 & 0.01 & 0.01 & 0.00 & 0.02 & 0.02 & -0.01 & -0.16 & -0.26 & 0.04 \\
\hline 40 & 1 & 0.01 & & 0.00 & 0.00 & 0.01 & 0.0 & -0.29 & -0.13 & 0.02 \\
\hline 41 & 6 & 0.00 & & 0.00 & 0.00 & 0.0 & 0.0 & 0.08 & -0.11 & 0.02 \\
\hline 42 & 6 & 0.00 & 0.00 & 0.00 & 0.00 & 0.00 & -0.01 & -0.05 & 0.01 & 0.00 \\
\hline 43 & 1 & -0.01 & & 0.0 & 0.01 & 0.01 & -0.02 & -0.14 & 0.00 & 0.00 \\
\hline 44 & 1 & 0.00 & & 0.00 & 0.00 & 0.00 & -0.02 & 0.03 & 0.16 & -0.03 \\
\hline 45 & 1 & -0.01 & 0.00 & 0.00 & -0.01 & -0.02 & -0.01 & -0.14 & 0.00 & 0.00 \\
\hline requen & & 245.43 & & & 260.15 & & & 267.56 & & \\
\hline Atom & AN & $X$ & $Y$ & Z & $x$ & $\mathrm{Y}$ & Z & $X$ & $Y$ & Z \\
\hline 1 & 6 & -0.07 & 0.02 & 0.00 & -0.12 & 0.03 & -0.01 & 0.01 & 0.00 & 0.00 \\
\hline 2 & 6 & -0.06 & 0.03 & -0.02 & -0.14 & -0.09 & -0.01 & -0.0 & -0.08 & 0.02 \\
\hline 3 & 6 & -0.06 & 0.04 & -0.01 & -0.14 & -0.14 & 0.0 & -0.02 & -0.10 & 0.02 \\
\hline 4 & 6 & -0.06 & 0.03 & 0.01 & -0.15 & -0.08 & 0.04 & -0.01 & -0.08 & 0.01 \\
\hline 5 & 6 & -0.07 & 0.02 & -0.01 & -0.12 & 0.03 & 0.00 & 0.01 & 0.00 & 0.00 \\
\hline 6 & 6 & -0.08 & 0.02 & 0.00 & -0.11 & 0.10 & -0.02 & 0.02 & 0.05 & -0.01 \\
\hline 7 & 7 & -0.04 & 0.03 & -0.01 & -0.08 & -0.07 & 0.01 & 0.00 & -0.03 & 0.01 \\
\hline 8 & 6 & -0.04 & 0.03 & -0.01 & -0.01 & -0.05 & 0.01 & 0.01 & -0 . & 0.01 \\
\hline 9 & 6 & -0.0 & 0.07 & -0.02 & -0.01 & -0.04 & 0.0 & -0.0 & -0.03 & 0.01 \\
\hline 10 & 6 & 0.1 & 0.15 & -0.03 & -0.0 & -0.07 & 0.0 & -0.05 & -0 . & 0.01 \\
\hline 11 & 17 & & & 0.0 & & 0.04 & 0.1 & 0.0 & 0. & -0.01 \\
\hline 12 & 17 & & & -0. & 0. & & -0 . & 0.0 & & 0.01 \\
\hline 13 & 78 & & & 0. & 0.0 & 0.02 & 0.0 & 0.0 & 0.05 & -0.01 \\
\hline 14 & 7 & & & -0 & -0.04 & 0.08 & -0. & 0.1 & -0 . & 0.01 \\
\hline 15 & 7 & & & & & & & & & 0.01 \\
\hline 16 & 6 & & & -0 & & & 0.0 & -0. & -0. & 0.02 \\
\hline 17 & 6 & & & & & & 0. & -0 . & -0 . & 0.01 \\
\hline 18 & 6 & & & & & & 0.8 & & & 0.00 \\
\hline 19 & 6 & & & 0. & 0.0 & 0.0 & -0. & -0.0 & 0. & -0.01 \\
\hline 20 & 6 & -0 . & & 0. & 0.0 & 0. & 0. & -0.03 & 0. & 0.00 \\
\hline 21 & 6 & & & & & & 0. & & & \\
\hline 22 & 17 & & & & & & 0. & & & -0 . \\
\hline 23 & 17 & & & 0. & & & -0.02 & 0.0 & 0. & 0.03 \\
\hline 24 & 6 & & & & & & -0 . & -0 & & 0.03 \\
\hline 25 & 6 & & & & & & & & & 0.01 \\
\hline 26 & 1 & & & & & & 0.0 & 0. & & 0.02 \\
\hline 27 & 1 & & & & & & 0. & 0.09 & -0.06 & 0.01 \\
\hline 28 & 1 & & & & & & 0. & & & 0.01 \\
\hline 29 & 1 & & & & & & & & & 0.01 \\
\hline 30 & 1 & 0.1 & & & -0.0 & -0. & 0.0 & -0.07 & -0. & 0.00 \\
\hline 31 & 1 & 0.1 & & -0.0 & -0.0 & & 0. & -0.05 & -0 . & 0.01 \\
\hline 32 & 1 & -0. & & & 0.0 & 0. & -0 . & -0.0 & & -0.01 \\
\hline 33 & 1 & -0 . & & 0.0 & 0.0 & 0. & -0 . & -0.05 & 0.10 & -0.02 \\
\hline 34 & 1 & & & & & & -0 . & -0. & 0. & -0.01 \\
\hline 35 & 1 & -0.0 & & -0.0 & -0.0 & & -0. & 0.0 & 0.05 & -0.01 \\
\hline 36 & 1 & -0 . & & 0.0 & -0.08 & 0.23 & -0.02 & 0.05 & 0.14 & -0.03 \\
\hline 37 & 1 & -0.07 & 0.01 & 0.00 & -0.08 & 0.10 & -0.01 & 0.02 & 0.05 & -0.01 \\
\hline
\end{tabular}




\begin{tabular}{|c|c|c|c|c|c|c|c|c|c|c|}
\hline & & & & & & & & & & \\
\hline 38 & 1 & -0.12 & -0.30 & 0.05 & 0.11 & 0.12 & -0.01 & -0.18 & -0.26 & 0.05 \\
\hline 3 & 1 & -0.12 & -0.30 & 0.06 & 0.10 & 0.11 & -0.02 & -0.18 & -0.26 & 0.05 \\
\hline 40 & 1 & -0.30 & -0.12 & 0.02 & 0.16 & 0.06 & -0.01 & -0.36 & -0.06 & \\
\hline 41 & 6 & -0.03 & 0.08 & -0.02 & -0.08 & .12 & -0.0 & 0. & -0.15 & \\
\hline 42 & 6 & 0.05 & 0.0 & 0.00 & 0.05 & -0.01 & 0. & & 0.11 & \\
\hline 43 & 1 & 0. & & & & & & & .08 & -0.02 \\
\hline 44 & 1 & 0.01 & & & -0.03 & & & & & \\
\hline 45 & 1 & 0.12 & 0.02 & 0.00 & 0.15 & 0.01 & 0.00 & & 0.08 & -0.0 \\
\hline Frequen & & 302.25 & & & 315.45 & & & 328.83 & & \\
\hline Atom & AN & $X$ & $\mathrm{Y}$ & Z & $X$ & $Y$ & Z & $x$ & $Y$ & Z \\
\hline 1 & 6 & -0.02 & 0.01 & -0.01 & -0.01 & 0.02 & -0.01 & -0.01 & -0.02 & 0.00 \\
\hline 2 & 6 & -0.03 & 0.01 & 0.01 & 0.02 & 0.11 & -0.03 & -0.0 & -0.11 & 0.01 \\
\hline 3 & 6 & 0.00 & 0.01 & 0.02 & 0.03 & 0.12 & -0.02 & -0. & -0.12 & 0.02 \\
\hline 4 & 6 & 0.0 & 0.00 & 0.01 & 0.02 & 0.12 & -0.01 & & -0.11 & \\
\hline 5 & 6 & 0.03 & & 0.00 & & 0.02 & 0.00 & & -0.02 & 0.01 \\
\hline 6 & 6 & 0.0 & -0.01 & -0.02 & -0.0 & -0.07 & 0.01 & & & -0.0 \\
\hline 7 & 7 & 0.00 & 0.03 & 0.14 & 0.02 & 0.01 & 0.00 & & -0.05 & \\
\hline 8 & 6 & 0.0 & 0.05 & 0.25 & 0.00 & 0.00 & 0.01 & -0 . & -0.06 & 0.02 \\
\hline 9 & 6 & & 0.05 & 0.26 & & -0.02 & 0.01 & & -0.07 & \\
\hline 10 & 6 & -0.0 & -0.01 & -0.09 & 0.2 & & -0.0 & & -0.01 & \\
\hline 11 & 17 & -0.0 & 0.00 & -0.03 & -0.01 & -0.01 & 0.03 & & 0.02 & \\
\hline 12 & 17 & 0.0 & & -0.02 & -0.01 & -0.02 & -0.0 & & 0.01 & -0.0 \\
\hline 13 & 78 & & -0.01 & -0.0 & & & 0. & & & \\
\hline 14 & 7 & & 0.00 & 0.0 & -0.0 & & 0.0 & & 0.05 & \\
\hline 15 & 7 & & & 0.17 & & & 0.02 & & -0.04 & 0.02 \\
\hline 16 & 6 & 0. & & 0.03 & & & 0.0 & & & \\
\hline 17 & 6 & -0. & & & & & 0.0 & & & \\
\hline 18 & 6 & -0. & & & & & 0.0 & & & 0.0 \\
\hline 19 & 6 & 0.1 & -0.0 & -0. & & & -0.0 & & -0.02 & \\
\hline 20 & 6 & & 0.0 & -0.0 & -0.0 & & 0.0 & & 0.00 & -0.0 \\
\hline 2 & 6 & & & & & & 0. & & & -0 \\
\hline 22 & 17 & & & & & & -0 . & & & \\
\hline 23 & 17 & -0 . & & & & & 0. & & -0.01 & -0.0 \\
\hline 24 & 6 & & & & & & 0. & & & 0.0 \\
\hline 2 & 6 & & & & & & & & & \\
\hline 2 & 1 & & -0.08 & & & & -0. & -0 . & 0.33 & -0.0 \\
\hline 27 & 1 & -0. & & & & & 0. & & 0.04 & -0.0 \\
\hline 2 & 1 & & & & & & & & & -0.0 \\
\hline 2 & 1 & & & & & & & & & \\
\hline 3 & 1 & & & & & & 0. & & -0.04 & \\
\hline 31 & 1 & & & & & & -0.0 & & & -0.0 \\
\hline 32 & 1 & & & & & & & & & \\
\hline 3 & 1 & & & & & & -0 . & & & \\
\hline 3 & 1 & & & & & & & & & \\
\hline 3 & 1 & & & & & & & & & \\
\hline 3 & 1 & & & & & & 0. & & & \\
\hline 3 & 1 & & & & & & 0. & & & -0.01 \\
\hline 3 & 1 & & & & & & & & & \\
\hline 3 & 1 & & & & & & & & & \\
\hline 4 & 1 & & & & & & & & & \\
\hline 41 & 6 & & & & & & & & & \\
\hline 42 & 6 & & & & & & & & & \\
\hline 43 & 1 & & & & & & & & & \\
\hline 44 & 1 & & -0. & & & & 0.00 & & 0.07 & -0.01 \\
\hline 45 & 1 & & & & & & 0.00 & & 0.06 & \\
\hline Oaun & & & & & & & & & & \\
\hline Atom & AN & $X$ & $Y$ & Z & $X$ & $Y$ & Z & $x$ & $Y$ & Z \\
\hline 1 & 6 & 0.00 & -0.02 & 0.01 & -0.01 & & -0.03 & -0.12 & 0.02 & -0.05 \\
\hline 2 & 6 & & -0.12 & 0.03 & & & -0.07 & -0.1 & 0.03 & 0.0 \\
\hline & 6 & & -0.10 & 0.02 & 0.07 & 0.10 & -0.01 & -0.01 & 0.02 & \\
\hline
\end{tabular}




\begin{tabular}{|c|c|c|c|c|c|c|c|c|c|c|}
\hline & & & & & & & & & & \\
\hline $\begin{array}{l}4 \\
5\end{array}$ & $\begin{array}{l}6 \\
6\end{array}$ & $\begin{array}{c}-0.03 \\
0.00\end{array}$ & $\begin{array}{r}-0.12 \\
-0.03\end{array}$ & $\begin{array}{l}0.02 \\
0.00\end{array}$ & $\begin{array}{l}0.04 \\
0.01\end{array}$ & $\begin{array}{l}0.17 \\
0.04\end{array}$ & $\begin{array}{l}0.02 \\
0.01\end{array}$ & $\begin{array}{l}0.10 \\
0.12\end{array}$ & $\begin{array}{l}-0.03 \\
-0.04\end{array}$ & $\begin{array}{c}0.07 \\
-0.04\end{array}$ \\
\hline 6 & 6 & 0.03 & 0.08 & -0.01 & -0.07 & -0.12 & 0.01 & 0.01 & -0.01 & -0.13 \\
\hline 7 & 7 & 0.00 & 0.02 & 0.00 & 0.05 & -0.10 & 0.04 & -0.01 & 0.05 & 0.26 \\
\hline 8 & 6 & -0.01 & 0.02 & -0.01 & -0.01 & -0.14 & 0.04 & 0.00 & 0.03 & 0.13 \\
\hline 9 & 6 & 0.00 & 0.03 & 0.00 & 0.04 & -0.19 & 0.03 & & 0.00 & -0.09 \\
\hline 10 & 6 & 0.03 & 0.05 & -0.01 & 0.09 & -0.22 & 0.0 & 0.0 & 0.02 & 0.00 \\
\hline 11 & 17 & 0.01 & 0.01 & -0.02 & -0.05 & 0.01 & 0.08 & -0.08 & 0.02 & -0.04 \\
\hline 12 & 17 & 0.01 & 0.02 & 0.01 & -0.03 & -0.03 & -0.0 & 0.0 & -0.03 & -0.02 \\
\hline 13 & 78 & 0.06 & & -0.01 & 0.01 & 0.05 & -0.1 & & 0.00 & 0.00 \\
\hline 14 & 7 & -0.20 & -0.19 & 0.03 & -0.07 & -0.02 & 0.00 & 0.0 & -0.01 & -0.05 \\
\hline 15 & 7 & 0.01 & 0.03 & 0.00 & -0.02 & -0.15 & 0.0 & 0.0 & -0.04 & -0.27 \\
\hline 16 & 6 & 0.01 & & 0.01 & -0.02 & 0.08 & -0.0 & & -0.04 & -0.13 \\
\hline 17 & 6 & 0.00 & & 0.00 & 0.00 & & -0.02 & 0.12 & -0.02 & -0.07 \\
\hline 18 & 6 & 0.00 & & 0.0 & & 0.04 & 0. & 0.1 & 0.01 & 0.04 \\
\hline 19 & 6 & -0.01 & & -0.01 & 0.0 & -0.12 & & 0.0 & .04 & 0.13 \\
\hline 20 & 6 & 0.00 & & 0.00 & -0.01 & 0.03 & -0.01 & -0 . & 0.00 & 0.05 \\
\hline 21 & 6 & 0.00 & & 0.01 & -0.02 & 0.14 & -0.05 & -0.11 & -0.05 & -0.06 \\
\hline 22 & 17 & & & -0.01 & 0.0 & -0.01 & & -0. & 0.00 & 0.03 \\
\hline 23 & 17 & $\begin{array}{l}-0.01 \\
\end{array}$ & & 0.01 & 0.02 & -0.02 & -0.0 & 0. & 0.02 & 0.03 \\
\hline 24 & 6 & -0.02 & -0.02 & 0.00 & -0.05 & -0.01 & 0.00 & 0.0 & -0.01 & -0.04 \\
\hline 25 & 6 & -0.08 & & -0.02 & -0.06 & -0.14 & 0.0 & 0.0 & 0.00 & -0.02 \\
\hline 26 & 1 & -0.06 & & -0.03 & -0.05 & -0.09 & 0.1 & 0. & -0.02 & -0.12 \\
\hline 27 & 1 & -0.14 & 0.07 & -0.01 & -0.09 & -0.15 & 0.0 & -0 . & -0.08 & -0.04 \\
\hline 28 & 1 & -0.1 & & -0.01 & -0.08 & -0.1 & 0.0 & 0. & 0.07 & -0.06 \\
\hline 29 & 1 & 0.0 & 0. & -0.0 & 0.1 & -0.2 & 0. & -0 . & 0. & \\
\hline 30 & 1 & 0. & & -0.01 & 0. & -0.23 & 0.0 & 0. & -0.03 & 0.03 \\
\hline 31 & 1 & 0.0 & & -0.02 & & -0.1 & 0. & & 0.04 & 0.05 \\
\hline 32 & 1 & 0.00 & & 0. & & -0.02 & 0.1 & 0.2 & 0.04 & 0.10 \\
\hline 33 & 1 & -0.01 & & -0.02 & 0.05 & -0.32 & 0.0 & 0. & 0.10 & 0.25 \\
\hline 34 & 1 & 0.00 & & 0.00 & -0.02 & -0.03 & 0.0 & -0 . & 0.01 & 0.11 \\
\hline 35 & 1 & 0.0 & & -0.0 & & -0 & 0. & 0. & -0.06 & -0.09 \\
\hline 36 & 1 & 0.08 & & -0.04 & -0.14 & -0.3 & 0.1 & 0.1 & -0.02 & -0.25 \\
\hline 37 & 1 & & & -0.01 & 0.00 & -0.04 & 0. & -0 . & 0.03 & -0.11 \\
\hline 38 & 1 & & & 0. & & & 0. & -0 . & & -0.08 \\
\hline 39 & 1 & -0.01 & & 0.1 & -0.03 & -0.07 & 0. & 0. & -0.09 & -0.05 \\
\hline 40 & 1 & -0 . & & 0. & -0.12 & 0. & -0. & 0. & -0.02 & -0.11 \\
\hline 41 & 6 & & & & & & & & & 0.12 \\
\hline 42 & 6 & -0.2 & & 0. & -0.0 & & & 0. & 10 & -0.02 \\
\hline 43 & 1 & & & & -0.1 & & 0. & 0.1 & 0.08 & -0.09 \\
\hline 44 & 1 & & & & & & & & & -0.07 \\
\hline 45 & 1 & -0.27 & & 0. & -0.1 & -0.07 & 0.0 & -0 . & -0.10 & -0.06 \\
\hline equer & & & & & & & & 423.21 & & \\
\hline tom & AN & & $Y$ & Z & & $Y$ & Z & $X$ & $Y$ & Z \\
\hline 1 & 6 & -0.02 & 0.04 & 0.03 & 0. & 0.00 & 0.10 & -0 . & -0.02 & -0.16 \\
\hline 2 & 6 & & & 0. & & -0.04 & -0.0 & 0. & 0. & 0.0 \\
\hline 3 & 6 & & 0. & -0 & & -0.02 & -0.1 & & & 0.02 \\
\hline 4 & 6 & & & -0 . & & & -0. & & & 0.03 \\
\hline 5 & 6 & -0. & & -0.0 & -0.1 & & 0.0 & 0. & -0.04 & -0.16 \\
\hline 6 & 6 & & & 0.01 & & & 0.18 & 0. & -0.04 & -0.21 \\
\hline 7 & 7 & & & & & & -0.09 & & -0.02 & -0.11 \\
\hline 8 & 6 & & & & & & 0.1 & & & 0.00 \\
\hline 9 & 6 & & & 0.00 & 0.00 & 0.03 & 0.15 & 0. & 0.01 & 0.07 \\
\hline 10 & 6 & & & -0.03 & & & -0.03 & & 0.00 & -0.01 \\
\hline 11 & 17 & & & -0.11 & & -0.03 & -0. & & 0.04 & 0.10 \\
\hline 12 & 17 & 0. & & 0.12 & -0.09 & 0.02 & -0.04 & 0. & 0.00 & 0.11 \\
\hline 13 & 78 & & & & & & 0. & & 0.00 & -0.01 \\
\hline 14 & 7 & & & 0.0 & 0.0 & & 0.0 & & 0.06 & 0.31 \\
\hline 15 & 7 & & & -0.01 & 0.0 & -0.02 & -0.08 & & 0.01 & 0.07 \\
\hline 16 & 6 & -0.14 & -0.02 & 0.01 & 0.00 & -0.02 & -0.11 & 0.00 & 0.00 & -0.01 \\
\hline
\end{tabular}




\begin{tabular}{|c|c|c|c|c|c|c|c|c|c|c|}
\hline 17 & & & & & & & & & & \\
\hline $\begin{array}{l}71 \\
18\end{array}$ & $\begin{array}{l}6 \\
6\end{array}$ & $\begin{array}{l}-0.04 \\
-0.05\end{array}$ & $\begin{array}{r}-0.01 \\
0.00\end{array}$ & $\begin{array}{l}0.10 \\
0.05\end{array}$ & $\begin{array}{l}0.08 \\
0.10\end{array}$ & $\begin{array}{l}0.00 \\
0.02\end{array}$ & $\begin{array}{l}-0.07 \\
0.09\end{array}$ & $\begin{array}{c}-0.01 \\
0.01\end{array}$ & $\begin{array}{l}0.00 \\
0.02\end{array}$ & $\begin{array}{l}-0.02 \\
0.09\end{array}$ \\
\hline 19 & 6 & 0.00 & 0.02 & -0.01 & 0.00 & 0.04 & 0.18 & 0.00 & 0.02 & 0.11 \\
\hline 2 & 6 & -0.05 & -0.02 & -0.05 & -0.10 & 0.01 & 0.10 & -0.01 & 0.02 & 0.09 \\
\hline 2 & 6 & -0.04 & -0.05 & -0.09 & -0.08 & -0.03 & -0.06 & 0.01 & 0.00 & -0.02 \\
\hline 22 & 17 & 0.10 & & 0.16 & -0.09 & -0.02 & -0.03 & & -0.02 & -0.06 \\
\hline 23 & 17 & 0.09 & -0.02 & -0.16 & 0.09 & 0.00 & -0.04 & 0.05 & -0.01 & -0.06 \\
\hline 24 & 6 & -0.02 & -0.03 & 0.01 & 0.00 & 0.00 & -0.01 & 0.00 & 0.00 & 0.01 \\
\hline 25 & 6 & -0.05 & & 0.05 & 0.00 & 0.00 & -0.02 & 0.00 & 0.00 & -0.01 \\
\hline 26 & 1 & -0.11 & -0.4 & 0.08 & 0.0 & -0.02 & -0.14 & 0.00 & 0.00 & -0.03 \\
\hline 27 & 1 & 0.08 & -0.21 & 0.04 & -0.12 & -0.12 & -0.06 & -0.02 & -0.02 & -0.02 \\
\hline 28 & 1 & 0.09 & -0.2 & 0.04 & 0.11 & 0.09 & -0.09 & 0.02 & 0.01 & -0.02 \\
\hline 29 & 1 & 0.12 & & -0.01 & 0.09 & -0.15 & -0.06 & 0.04 & -0.06 & -0.02 \\
\hline 30 & 1 & 0.10 & & -0.02 & -0.09 & 0.13 & -0.11 & -0.04 & 0.05 & -0.04 \\
\hline 31 & 1 & -0.01 & & -0.07 & 0.01 & -0.03 & -0.14 & 0.00 & -0.01 & -0.05 \\
\hline 32 & 1 & -0.12 & & 0.0 & 0. & 0.04 & 0.16 & 0.07 & 0.03 & 0.12 \\
\hline 33 & 1 & -0.01 & & -0.01 & 0.00 & 0.07 & 0.27 & 0.00 & 0.02 & 0.11 \\
\hline 34 & 1 & -0.12 & -0.01 & -0.01 & -0.23 & 0.03 & 0.17 & -0.07 & 0.03 & 0.13 \\
\hline 35 & 1 & -0.09 & & -0.01 & -0.23 & 0.07 & 0.15 & 0.12 & -0.08 & -0.21 \\
\hline 36 & 1 & -0.04 & & 0.03 & 0.00 & 0.05 & 0.27 & 0.00 & -0.04 & -0.20 \\
\hline 37 & 1 & -0.08 & & 0.01 & 0.23 & -0.01 & 0.17 & -0.12 & 0.00 & -0.23 \\
\hline 38 & 1 & -0.02 & & 0.01 & -0.02 & 0.01 & -0.02 & 0.01 & -0.03 & 0.02 \\
\hline 39 & 1 & -0.02 & & 0.0 & 0.02 & -0.02 & -0.01 & -0.01 & & \\
\hline 40 & 1 & -0.11 & & 0.00 & 0.00 & 0.00 & -0.02 & 0.00 & 0.01 & 0.05 \\
\hline 41 & 6 & -0.01 & & -0.01 & 0.00 & -0.01 & -0.08 & 0.00 & -0.06 & -0.32 \\
\hline 42 & 6 & 0.0 & & 0. & 0. & 0.00 & 0.01 & 0.00 & & 0.02 \\
\hline 43 & 1 & 0.0 & & 0. & -0.06 & -0.05 & 0.0 & -0.25 & -0.20 & 0.21 \\
\hline 44 & 1 & -0.01 & & 0. & & 0.01 & 0.0 & 0.00 & & 0.18 \\
\hline 45 & 1 & 0.01 & 0.00 & 0.00 & 0.06 & 0.06 & 0.03 & 0.25 & 0.27 & 0.13 \\
\hline equen & & 433.88 & & & & & & 449.80 & & \\
\hline Atom & AN & X & $Y$ & Z & $x$ & $Y$ & Z & $X$ & Y & 2 \\
\hline 1 & 6 & -0.01 & 0.01 & 0.05 & 0.17 & -0.08 & -0.04 & 0.02 & 0.00 & 0.00 \\
\hline 2 & 6 & -0.02 & & 0.00 & & -0.10 & -0.09 & 0.02 & 0.00 & -0.01 \\
\hline 3 & 6 & 0.0 & 0. & 0.01 & & -0.03 & 0.01 & 0. & 0.00 & 0.00 \\
\hline 4 & 6 & & & 0.00 & & -0.06 & 0.12 & 0. & 0.00 & 0.01 \\
\hline 5 & 6 & 0.0 & & 0.06 & 0.1 & -0.06 & 0.0 & 0.0 & 0.00 & 0.01 \\
\hline 6 & 6 & 0.00 & 0. & 0. & 0.1 & 0.01 & 0.0 & 0.0 & -0.01 & 0.00 \\
\hline 7 & 7 & & & & & & 0.0 & & & \\
\hline 8 & 6 & 0.1 & & 0. & & 0.0 & -0.0 & 0.0 & 0.02 & 0.00 \\
\hline 9 & 6 & & & 0. & & & -0.02 & 0.0 & -0.04 & 0.01 \\
\hline 10 & 6 & & & & & & -0.02 & & & 0.01 \\
\hline 11 & 17 & & & -0 . & & 0.07 & 0.1 & -0. & 0.01 & 0.02 \\
\hline 12 & 17 & & & -0.0 & & 0.01 & -0.1 & -0.0 & 0.00 & -0.02 \\
\hline 13 & 78 & & & & & & 0.0 & & & 0.00 \\
\hline 14 & 7 & & & & & 0.05 & & & & 0.08 \\
\hline 15 & 7 & & & -0. & & & -0.0 & $-0 .($ & -0. & 0.01 \\
\hline 16 & 6 & & & -0.0 & & & 0.0 & -0.02 & -0.01 & 0.00 \\
\hline 17 & 6 & & & & & & & & & 0.01 \\
\hline 18 & 6 & & & & & & 0.0 & -0. & & \\
\hline 19 & 6 & 0. & & -0.20 & -0 & 0.06 & -0.01 & -0.0 & -0.02 & 0.00 \\
\hline 20 & 6 & & & -0.19 & & & -0.0 & & & -0.01 \\
\hline 21 & 6 & & & & & & -0. & & & -0.02 \\
\hline 22 & 17 & 0.0 & & 0.1 & & 0.04 & 0.12 & 0.02 & 0.00 & 0.02 \\
\hline 23 & 17 & & & & & -0.01 & -0.13 & 0.0 & 0.00 & -0.02 \\
\hline 24 & 6 & 0.1 & & -0.0 & & 0.02 & 0.00 & -0.0 & -0.05 & 0.01 \\
\hline 25 & 6 & 0. & & -0.0 & 0.0 & 0.06 & -0.01 & 0.0 & 0.03 & -0.01 \\
\hline 26 & 1 & & & -0. & & 0. & -0.01 & 0.04 & 0.01 & 0.00 \\
\hline 27 & 1 & -0 . & & -0.0 & 0.0 & 0.06 & -0.01 & 0.05 & 0.04 & -0.01 \\
\hline 28 & 1 & 0.1 & & -0.1 & 0.06 & 0.05 & -0.0 & 0.05 & 0.04 & 0.00 \\
\hline 29 & 1 & 0.02 & -0.04 & -0.02 & -0.16 & 0.12 & -0.02 & 0.04 & -0.06 & 0.01 \\
\hline
\end{tabular}




\begin{tabular}{|c|c|c|c|c|c|c|c|c|c|c|}
\hline & & & & & & & & & & \\
\hline 30 & 1 & -0.02 & 0.03 & -0.03 & -0.15 & 0.11 & -0.02 & 0.05 & -0.07 & 0.01 \\
\hline 31 & 1 & 0.00 & -0.01 & -0.04 & -0.10 & 0.01 & 0.00 & 0.03 & -0.04 & 0.01 \\
\hline 32 & 1 & -0.04 & -0.07 & -0.24 & -0.19 & -0.05 & 0.03 & -0.04 & 0.01 & 0.00 \\
\hline 33 & 1 & 0.00 & -0.01 & -0.13 & -0.14 & 0.18 & -0.04 & -0.02 & -0.05 & 01 \\
\hline 34 & 1 & 0.04 & -0.04 & -0.24 & -0.19 & -0.06 & 0.00 & -0.04 & 0.00 & 0.00 \\
\hline 35 & 1 & -0.01 & 0.01 & 0.07 & & -0.09 & 0.04 & 0.03 & -0.01 & 0.01 \\
\hline 36 & 1 & 0.00 & 0.01 & 0.04 & 0.19 & & -0.02 & 0.02 & -0.02 & 0.00 \\
\hline 37 & 1 & 0.01 & 0.02 & 0.07 & 0.24 & -0.10 & 0.00 & 0.03 & & 0.00 \\
\hline 38 & 1 & -0.04 & 0.03 & -0.04 & 0.00 & & -0.01 & -0.06 & -0.09 & 0.01 \\
\hline 39 & 1 & 0.04 & -0.05 & -0.03 & 0.00 & & 0.00 & -0.06 & -0.09 & 0.02 \\
\hline 40 & 1 & 0.00 & 0.00 & -0.03 & 0.02 & & 0.00 & -0.11 & & 0.01 \\
\hline 41 & 6 & 0.00 & -0.05 & -0.29 & 0.04 & -0.05 & 0.01 & -0.27 & 0.27 & -0.05 \\
\hline 42 & 6 & 0.00 & 0.00 & 0.02 & -0.01 & & 0.00 & 0.00 & -0.02 & 0.00 \\
\hline 43 & 1 & -0.23 & -0.19 & 0.19 & -0.06 & -0.01 & 0.00 & 0.30 & 0.01 & 0.00 \\
\hline 44 & 1 & 0.00 & 0.03 & 0.15 & 0.03 & 0.07 & -0.01 & -0.21 & -0.47 & 0.09 \\
\hline 45 & 1 & 0.23 & 0.24 & 0.12 & -0.06 & -0.01 & 0.00 & 0.31 & 0.02 & 0.00 \\
\hline equen & & 454.66 & & & 473.67 & & & 522.71 & & \\
\hline Atom & AN & $X$ & $Y$ & Z & $X$ & $\mathrm{Y}$ & Z & $x$ & Y & Z \\
\hline 1 & 6 & -0.14 & 0.06 & 0.18 & -0.09 & -0.02 & 0.02 & -0.02 & 0.11 & -0.02 \\
\hline 2 & 6 & -0.14 & 0.06 & 0.05 & -0.07 & -0.02 & 0.03 & -0.02 & 0.04 & 0.00 \\
\hline 3 & 6 & 0.00 & 0.03 & 0.13 & -0.03 & 0.07 & -0.01 & -0.0 & -0.16 & 0.03 \\
\hline 4 & 6 & 0.13 & -0.04 & 0.07 & -0.07 & -0.03 & -0.02 & -0.0 & 0.04 & -0.01 \\
\hline 5 & 6 & 0.13 & 0.00 & 0.19 & & -0.03 & -0.01 & -0. & 0.11 & -0.02 \\
\hline 6 & 6 & 0.00 & 0.03 & 0.13 & & & -0.02 & -0.11 & -0.12 & 0.02 \\
\hline 7 & 7 & 0.00 & 0.04 & 0.22 & & 0.15 & -0.03 & 0.01 & -0.08 & 0.01 \\
\hline 8 & 6 & 0.00 & -0.02 & -0.12 & & 0.16 & -0.0 & 0.1 & -0.04 & 0.01 \\
\hline 9 & 6 & 0.00 & -0.03 & -0.18 & & -0.12 & 0.02 & 0.1 & 0.01 & 0.00 \\
\hline 10 & 6 & 0.00 & 0.00 & 0.02 & & -0.19 & 0.03 & -0.02 & -0.08 & 0.01 \\
\hline 11 & 17 & 0.05 & -0.04 & -0.14 & 04 & -0.01 & -0.05 & 0.0 & -0.02 & -0.02 \\
\hline 12 & 17 & -0.05 & -0.01 & -0.14 & & & 0.0 & 0.1 & -0.01 & 0.02 \\
\hline 13 & 78 & 0.00 & 0.00 & -0.02 & & & 0.0 & 0.0 & 0.00 & 0.00 \\
\hline 14 & 7 & -0.01 & 0.05 & 0.23 & -0 & & -0.0 & 0.0 & 0.00 & 0.00 \\
\hline 15 & 7 & 0. & 0.02 & 0.14 & & -0.13 & 0.0 & 0.0 & 0. & -0.0 \\
\hline 16 & 6 & 0.1 & 0.02 & 0.09 & & & 0.0 & -0 . & & -0.03 \\
\hline 17 & 6 & -0 . & -0.01 & 0.05 & & & 0. & -0 . & -0.05 & \\
\hline 18 & 6 & -0.0 & 0.03 & 0.12 & -0.11 & 0. & 0.01 & -0.0 & -0.11 & 0.03 \\
\hline 19 & 6 & 0. & 0.01 & 0.09 & & & 0.01 & -0. & 0.15 & -0.03 \\
\hline 20 & 6 & & 0.03 & & & & -0 . & -0 . & -0.11 & 0.02 \\
\hline 21 & 6 & & 0.03 & 0.04 & & & -0.0 & -0. & -0.04 & 0.01 \\
\hline 22 & 17 & -0.03 & -0.02 & -0.09 & & 0.02 & 0.0 & 0. & 0.01 & 0.02 \\
\hline 23 & 17 & 0.0 & -0.02 & -0.09 & & -0.01 & -0.0 & 0. & & -0.02 \\
\hline 24 & 6 & & 0.00 & 0.01 & & & & & & 0.00 \\
\hline 25 & 6 & & 0.0 & 0.0 & & 0. & -0.0 & -0 . & 0.07 & -0.01 \\
\hline 26 & 1 & 0. & 0.02 & 0.10 & & 11 & -0.0 & 0. & 0. & -0.05 \\
\hline 27 & 1 & 0. & 0.10 & 0.04 & & & & & & \\
\hline 2 & 1 & & -0.0 & 0.0 & & & -0. & -0. & & -0 \\
\hline 29 & 1 & -0 . & 0.19 & 0.07 & & -0.21 & 0.0 & -0.16 & 0.00 & -0.01 \\
\hline 30 & 1 & 0.1 & -0.17 & 0.13 & & & 0. & & & \\
\hline 31 & 1 & & 0.0 & 0.1 & & & 0. & & & \\
\hline 32 & 1 & -0.08 & 0.0 & 0.12 & & & 0. & -0.02 & -0.21 & 0.05 \\
\hline 33 & 1 & $0 .($ & -0.02 & -0.02 & & & 0. & -0 . & & -0.08 \\
\hline 34 & 1 & 0. & 0.02 & 0.13 & & & -0 . & -0. & -0.2 & 0.0 \\
\hline 35 & 1 & 0. & 0.03 & 0.20 & & & 0. & 0.0 & 0.22 & -0.04 \\
\hline 36 & 1 & 0. & 0.00 & -0.04 & -0 . & 0.21 & -0.04 & -0.16 & -0.31 & 0.05 \\
\hline 37 & 1 & -0. & 0.04 & 0.1 & & -0.04 & 0.01 & 0.0 & 0.22 & -0.04 \\
\hline 38 & 1 & 0. & -0.02 & 0.0 & & & 0.0 & 0.0 & -0.02 & 0.00 \\
\hline 39 & 1 & $-0 . C$ & 0.04 & 0.02 & 0.01 & 0.01 & 0.00 & 0.02 & -0.02 & 0.01 \\
\hline 40 & 1 & 0.0 & 0.01 & 0.04 & & -0.02 & 0.00 & 0.00 & 0.00 & 0.00 \\
\hline 41 & 6 & 0.0 & -0.04 & -0.18 & 0.03 & -0.04 & 0.01 & 0.00 & 0.00 & 0.00 \\
\hline 42 & 6 & 0.00 & 0.00 & 0.01 & -0.01 & -0.01 & 0.00 & 0.00 & 0.00 & 0.00 \\
\hline
\end{tabular}




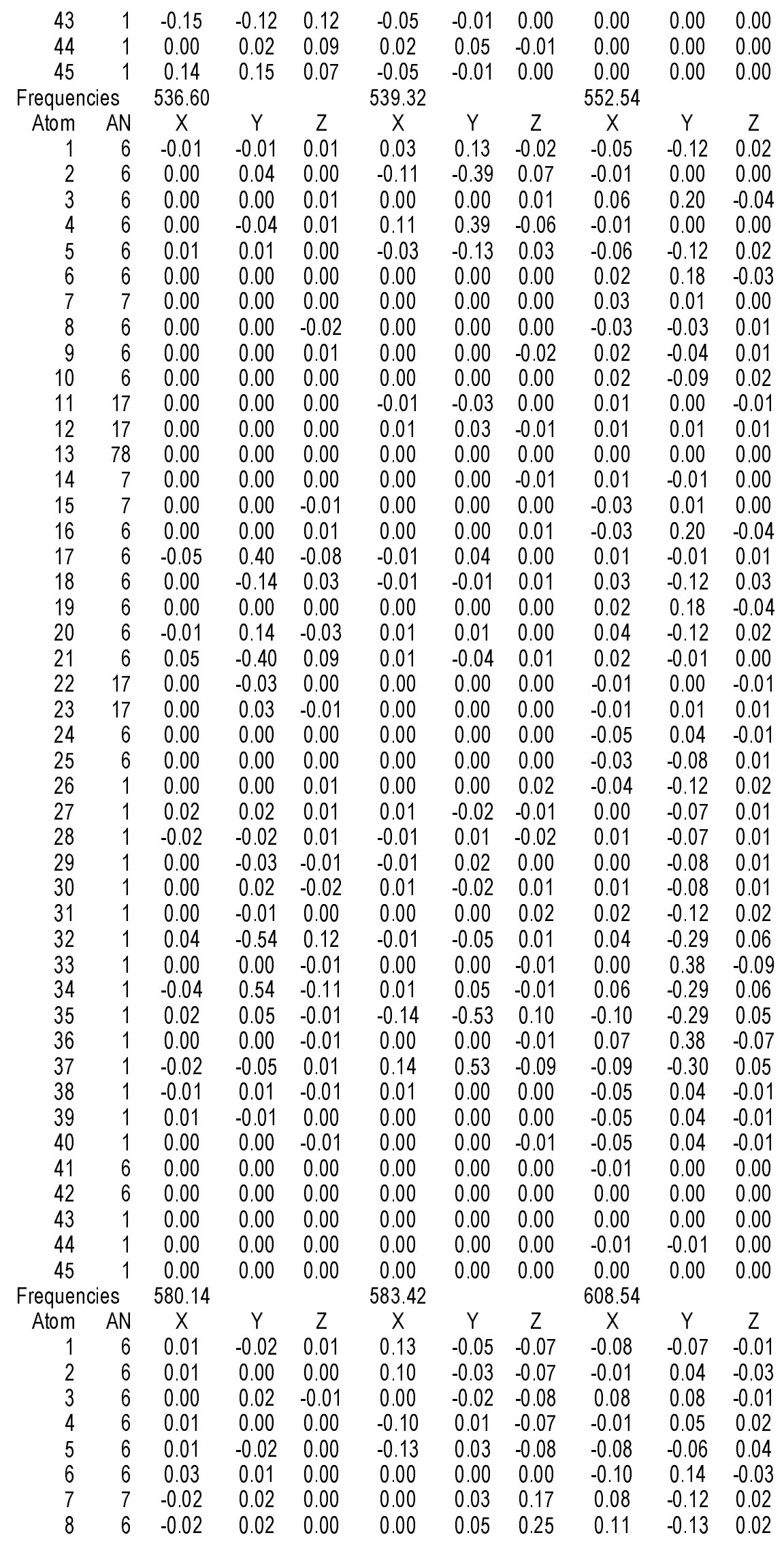




\begin{tabular}{|c|c|c|c|c|c|c|c|c|c|c|}
\hline & & & & & & & & & & \\
\hline 9 & 6 & -0.01 & -0.01 & 0.00 & 0.00 & -0.04 & -0.25 & 0.14 & 0.09 & $\begin{array}{l}-0.01 \\
\end{array}$ \\
\hline 10 & 6 & 0.01 & -0.01 & 0.00 & 0.00 & 0.00 & -0.02 & -0.04 & 0.07 & -0.01 \\
\hline 11 & 17 & 0.00 & 0.00 & 0.01 & 0.00 & 0.00 & 0.02 & 0.02 & -0.01 & -0.02 \\
\hline 12 & 17 & 0.00 & 0.00 & 0.00 & 0.00 & 0.00 & 0.02 & 0.02 & 0.00 & 0.02 \\
\hline 13 & 78 & -0.03 & 0.03 & 0.00 & & 0.00 & 0.00 & -0.01 & 0.01 & 0.00 \\
\hline 14 & 7 & -0.02 & 0.02 & 0.00 & & 0.00 & 0.02 & & -0.02 & \\
\hline 15 & 7 & -0.01 & -0.01 & 0.00 & & -0.03 & -0.17 & 0.10 & 0.10 & -0.03 \\
\hline 16 & 6 & -0.01 & 0.04 & 0.00 & 0.00 & 0.01 & 0.08 & 0.07 & -0.08 & \\
\hline 17 & 6 & 0.00 & 0.01 & 0.01 & -0.11 & 0.01 & 0.08 & -0.02 & -0.04 & -0.02 \\
\hline 18 & 6 & 0.01 & -0.02 & 0.01 & -0.14 & 0.01 & 0.08 & -0.07 & 0.06 & -0.04 \\
\hline 19 & 6 & 0.02 & 0.04 & -0.01 & 0.00 & -0.01 & 0.00 & -0.12 & -0.11 & 0.02 \\
\hline 20 & 6 & 0.02 & -0.02 & 0.00 & 0.14 & 0.04 & 0.07 & -0.07 & 0.08 & 0.01 \\
\hline 21 & 6 & 0.01 & 0.00 & 0.00 & 0.11 & 0.02 & 0.07 & -0.02 & -0.03 & 0.03 \\
\hline 22 & 17 & 0.00 & 0.00 & 0.00 & 0.00 & -0.01 & -0.02 & 0.02 & 00 & 0.02 \\
\hline 23 & 17 & 0.00 & 0.00 & 0.00 & 0.00 & -0.01 & -0.03 & 0.02 & 0.00 & -0.02 \\
\hline 24 & 6 & 0.36 & -0.37 & 0.07 & -0.0 & 0.01 & 0.00 & 0.04 & -0.04 & 0.01 \\
\hline 25 & 6 & 0.00 & 0.00 & 0.00 & 0.00 & 0.00 & 0.02 & -0.06 & -0.08 & 0.01 \\
\hline 26 & 1 & -0.01 & -0.05 & 0.01 & & -0.02 & -0.16 & 0.02 & 0.21 & -0.04 \\
\hline 27 & 1 & 0.03 & 0.00 & 0.00 & -0.19 & -0.23 & -0.06 & -0.2 & -0.11 & 0.03 \\
\hline 28 & 1 & 0.04 & 0.01 & 0.00 & & .19 & -0.13 & -0.2 & & \\
\hline 29 & 1 & 0.03 & -0.02 & 0.01 & -0.1 & 0.24 & 0.05 & -0.2 & & -0.04 \\
\hline 30 & 1 & 0.04 & -0.03 & 0.01 & 0.1 & -0.23 & 0.13 & -0.2 & 15 & -0.02 \\
\hline 31 & 1 & 0.01 & 0.03 & 0.00 & & & 0.1 & & & \\
\hline 32 & 1 & 0.01 & -0.07 & 0.02 & -0.20 & & 0.06 & & & \\
\hline 33 & 1 & 0.01 & 0.07 & -0.02 & 0.00 & -0.06 & -0.18 & -0.12 & -0.18 & 0.04 \\
\hline 34 & 1 & 0.02 & -0.06 & 0.01 & 0. & 0.06 & 0.04 & -0.04 & 0.21 & -0.04 \\
\hline 35 & 1 & -0.01 & -0.04 & 0.01 & -0.18 & 0.05 & -0.06 & -0.08 & -0.24 & \\
\hline 36 & 1 & 0.04 & 0.03 & 0.00 & 0.00 & 0.03 & 0.17 & -0.08 & 0.21 & -0.04 \\
\hline 37 & 1 & 0.00 & -0.05 & 0.01 & 0. & -0.06 & -0.04 & -0.09 & -0.24 & 0.04 \\
\hline 38 & 1 & 0.3 & -0.35 & 0.05 & -0. & & -0.0 & & -0.01 & \\
\hline 39 & 1 & 0.34 & -0.35 & 0.07 & 0. & -0.03 & -0.02 & 0. & -0.01 & 0.00 \\
\hline 40 & 1 & 0.29 & -0.34 & 0.06 & -0.0 & 0.01 & -0.0 & 0.07 & -0.05 & 0.0 \\
\hline 41 & 6 & 0.01 & -0.01 & 0.00 & & 0.00 & -0.0 & 0. & & \\
\hline 42 & 6 & 0.0 & 0.00 & 0.0 & & & & & & \\
\hline 43 & 1 & -0. & 0.00 & 0.0 & & & & & & \\
\hline 44 & 1 & 0.00 & 0.01 & 0.00 & 0.0 & 0.00 & 0.0 & 0.0 & -0.01 & \\
\hline 45 & 1 & -0.01 & 0.00 & 0.00 & 0.01 & 0.01 & 0.00 & 0.01 & 0.00 & 0.00 \\
\hline O & & 632.71 & & & 670.79 & & & 687.63 & & \\
\hline Atom & AN & $X$ & $Y$ & Z & $\gamma$ & $Y$ & Z & $x$ & & Z \\
\hline 1 & 6 & & -0.04 & -0.06 & & -0.03 & -0.05 & -0. & 0.00 & -0.11 \\
\hline 2 & 6 & & -0.04 & -0.05 & & -0.03 & -0.03 & & -0.03 & -0.09 \\
\hline 3 & 6 & & -0.01 & -0.04 & & 0.00 & -0.0 & & & \\
\hline 4 & 6 & -0. & 0.02 & -0.06 & -0.05 & 0.02 & -0.04 & 0.0 & 0.00 & 0.10 \\
\hline 5 & 6 & -0.12 & 0.02 & -0.07 & -0.09 & 0.01 & -0.05 & -0. & 0.04 & 0.11 \\
\hline 6 & 6 & & 0.00 & 0.01 & & 0.00 & & & & \\
\hline 7 & 7 & & 0.04 & 0.2 & & 0. & 0. & & -0.04 & \\
\hline 8 & 6 & & -0.01 & -0.01 & & -0.05 & -0.25 & & -0.06 & 0.02 \\
\hline 9 & 6 & & -0.03 & -0.18 & & 0.03 & 0.20 & & & \\
\hline 10 & 6 & & 0.00 & -0.01 & & 0.00 & 0.0 & & & \\
\hline 11 & 17 & & 0.00 & 0.0 & 0 & 0.00 & 0.0 & & -0.01 & \\
\hline 12 & 17 & & & 0.0 & & & 0.0 & & & \\
\hline 13 & 78 & & 0.00 & -0.1 & & 0.00 & 0.0 & & & \\
\hline 14 & 7 & & 0.0 & 0. & & 0. & 0.04 & & -0.01 & \\
\hline 15 & 7 & 0.0 & 0.07 & 0.3 & -0 & -0.02 & -0.12 & -0.2 & & \\
\hline 16 & 6 & 0. & -0.01 & -0.06 & & 0.00 & 0.0 & & & \\
\hline 17 & 6 & 0.1 & 0.02 & -0.08 & -0.02 & -0.01 & 0.0 & 0.00 & -0.04 & \\
\hline 18 & 6 & 0.16 & -0.01 & -0. & -0.03 & 0.00 & 0.02 & 0.07 & 0.08 & 0.12 \\
\hline 19 & 6 & 0.00 & 0.01 & 0.0 & & 0.00 & 0.00 & & & \\
\hline 20 & 6 & -0.1 & -0.03 & -0.0 & & 0.01 & 0.0 & & 0.03 & \\
\hline 21 & 6 & -0.11 & -0.04 & -0.06 & 0.02 & 0.01 & 0.01 & -0.01 & -0.09 & -0.10 \\
\hline
\end{tabular}




\begin{tabular}{|c|c|c|c|c|c|c|c|c|c|c|}
\hline & & & & & & & & & & \\
\hline 22 & 17 & 0.00 & 0.00 & 0.02 & 0.00 & 0.00 & 0.00 & -0.03 & -0.01 & -0.04 \\
\hline 2 & 17 & 0.00 & 0.00 & 0.02 & 0.00 & 0.00 & 0.00 & -0.03 & 0.01 & 0.04 \\
\hline & 6 & 0.00 & 0.00 & 0.00 & 0.00 & 0.00 & 0.02 & 0.02 & -0.01 & 0.00 \\
\hline 25 & 6 & 0.00 & 0.00 & 0.00 & 0.00 & 0.00 & -0.03 & 0.08 & 0.08 & -0.01 \\
\hline 26 & 1 & 0.00 & 0.01 & 0.04 & 0.00 & 0.03 & 0.20 & 0.12 & 0.19 & -0.04 \\
\hline 27 & 1 & 0.02 & 0.04 & 0.0 & 0.25 & 0.33 & 0.0 & 0.01 & 0.05 & -0.01 \\
\hline 28 & 1 & -0.04 & -0.04 & 0.03 & -0.24 & -0.27 & 0.19 & 0.02 & 0.07 & -0.02 \\
\hline 29 & 1 & -0.15 & 0.30 & 0.07 & 0.13 & -0.30 & -0.06 & -0.02 & 0.03 & 0.00 \\
\hline 30 & 1 & 0.14 & -0.26 & 0.17 & -0.14 & 0.27 & -0.16 & -0.01 & 0.01 & 0.00 \\
\hline 31 & 1 & -0.01 & 0.04 & 0.19 & 0.01 & -0.04 & -0.1 & & 0.08 & -0.01 \\
\hline 32 & 1 & 0.22 & -0.02 & -0.06 & -0.04 & 0.01 & 0.01 & -0.13 & 0.20 & -0.02 \\
\hline 3 & 1 & 0.00 & 0.05 & 0.22 & 0.01 & -0.01 & -0.04 & 0.35 & 0.01 & 0.00 \\
\hline 34 & 1 & -0.22 & -0.02 & -0.05 & 0.04 & 0.00 & 0.01 & -0.14 & 0.18 & -0.07 \\
\hline 35 & 1 & -0.16 & 0.02 & -0.05 & -0.12 & 0.01 & -0. & 0.11 & 0.02 & 0.02 \\
\hline 36 & 1 & -0.01 & 0.03 & 0.16 & -0.01 & 0.02 & 0.13 & -0.28 & 0.07 & -0.02 \\
\hline 37 & 1 & 0.16 & -0.05 & -0.03 & 0.12 & -0.02 & -0.03 & 0.10 & 0.01 & -0.02 \\
\hline 38 & 1 & 0.10 & & 0.08 & -0.10 & 0.11 & -0. & 0.02 & -0.04 & \\
\hline 39 & 1 & -0.10 & & 0.04 & 0.10 & -0.13 & $-0 .($ & 0.01 & -0.03 & 0.01 \\
\hline 40 & 1 & 0.00 & 0.01 & 0.09 & 0.00 & -0.01 & -0.08 & -0.02 & 0.01 & 0.00 \\
\hline 41 & 6 & 0.00 & & -0.01 & 0.00 & 0.00 & -0.01 & 0.00 & 0.00 & \\
\hline 42 & 6 & 0.00 & & 0.00 & 0.00 & 0.00 & & -0.01 & -0.01 & .00 \\
\hline 43 & 1 & -0.01 & & 0.01 & -0.01 & -0.01 & & -0.01 & -0.01 & 0.00 \\
\hline 44 & 1 & 0.00 & 0. & 0.01 & 0.00 & 0.00 & 0.0 & -0.01 & -0.01 & 0.00 \\
\hline 45 & 1 & 0.01 & & 0.00 & 0.01 & 0.01 & 0.00 & -0.01 & -0.01 & 0.00 \\
\hline Frequen & & 715.58 & & & 739.22 & & & 782.94 & & \\
\hline Atom & AN & $X$ & $Y$ & Z & $X$ & $\mathrm{Y}$ & Z & $x$ & $Y$ & Z \\
\hline 1 & 6 & 0.00 & -0.04 & 0.10 & 0.02 & 0.06 & -0.01 & 0.02 & -0.02 & -0.07 \\
\hline 2 & 6 & 0.00 & 0.11 & 0.06 & -0.03 & -0.13 & 0.02 & 0.05 & 0.04 & -0.07 \\
\hline 3 & 6 & -0.15 & -0.06 & 0.01 & 0.05 & 0.19 & -0.03 & $0.0^{\prime}$ & -0.18 & 0.03 \\
\hline 4 & 6 & 0.01 & 0.08 & -0.09 & -0.03 & -0.13 & 0.03 & 0.05 & 0.06 & 0.05 \\
\hline 5 & 6 & 0.00 & -0.07 & -0.08 & 0.02 & 0.06 & -0.01 & 0.02 & 0.01 & 0.08 \\
\hline 6 & 6 & 0.21 & 0.03 & 0.00 & -0.04 & -0.11 & 0.02 & -0.09 & 0.09 & -0.02 \\
\hline 7 & 7 & -0.12 & -0.06 & 0.01 & 0. & 0.08 & -0.0 & 0.0 & 0.05 & -0.01 \\
\hline 8 & 6 & & & 0.00 & -0.05 & 0.03 & -0.0 & -0.0 & 0.05 & -0.01 \\
\hline 9 & 6 & & & 0.00 & & 0.01 & 0.0 & -0.0 & -0.03 & 0.01 \\
\hline 10 & 6 & & -0.11 & 0.02 & 0.02 & -0.09 & 0.02 & -0.02 & 0.00 & 0.00 \\
\hline 11 & 17 & & & 0. & & 0.00 & 0.0 & 0.0 & 0.00 & -0.01 \\
\hline 12 & 17 & & & & & & & & & \\
\hline 13 & 78 & & & 0. & & 0.00 & 0. & 0.0 & 0.00 & 0.00 \\
\hline 14 & 7 & & & 0. & & 0.00 & 0.0 & 0.0 & 0.00 & 0.00 \\
\hline 15 & 7 & & & -0 & & 0. & -0 . & 0. & -0.06 & 0.01 \\
\hline 16 & 6 & & & -0.02 & & & & & & -0.04 \\
\hline 17 & 6 & 0. & -0.11 & 0. & 0.0 & -0.11 & 0.0 & 0.0 & -0.08 & -0.05 \\
\hline 18 & 6 & & & 0. & 0.00 & 0.05 & -0. & 0.0 & -0.01 & -0.08 \\
\hline 19 & 6 & & & & -0.02 & -0.10 & & & & \\
\hline 20 & 6 & & & -0. & -0.0 & 0. & 0. & 0. & 0. & 0.07 \\
\hline 21 & 6 & & & -0.01 & 0.02 & -0.10 & 0.0 & 0.04 & -0.05 & 0.07 \\
\hline 22 & 17 & & & -0.01 & & & & & & 0.01 \\
\hline 23 & 17 & & & & & & & & & -0.01 \\
\hline 24 & 6 & & & 0. & 0.00 & -0.01 & 0.0 & 0.00 & 0.01 & 0.00 \\
\hline 25 & 6 & & & & & & & & 0.01 & 0.00 \\
\hline 26 & 1 & 0. & & -0.0 & -0 & -0.20 & 0. & -0 & -0.18 & 0.03 \\
\hline 27 & 1 & & & -0 & 0. & -0.06 & 0.8 & 0.12 & 0.04 & -0.02 \\
\hline 28 & 1 & & & -0. & & -0.05 & 0. & 0. & 0.04 & 0.00 \\
\hline 29 & 1 & & & 0.01 & -0.0 & -0.05 & & 0.1 & -0.06 & 0.02 \\
\hline 30 & 1 & -0 . & -0 . & 0.02 & -0.02 & -0.06 & 0.0 & 0.11 & -0.05 & 0.00 \\
\hline 31 & 1 & & -0.2 & 0.05 & 0.05 & -0.18 & 0.03 & -0.03 & 0.21 & -0.04 \\
\hline 32 & 1 & & & -0.07 & -0.01 & & -0.08 & 0.08 & 0.34 & -0.10 \\
\hline 33 & 1 & & & 0.00 & -0.0 & 0.08 & -0.0 & -0.14 & 0.24 & -0.05 \\
\hline 34 & 1 & -0.10 & 0.37 & -0.09 & -0.02 & 0.35 & -0.07 & 0.08 & 0.35 & -0.05 \\
\hline
\end{tabular}




\begin{tabular}{|c|c|c|c|c|c|c|c|c|c|c|}
\hline & & & & & & & & & & \\
\hline 35 & 1 & -0.18 & -0.27 & 0.03 & 0.13 & 0.43 & -0.08 & 0.02 & -0.34 & 0.09 \\
\hline & 1 & 0.18 & -0.07 & 0.01 & 0.02 & 0.11 & -0.02 & & -0.21 & 0.04 \\
\hline 37 & 1 & -0.18 & -0.26 & 0.07 & 0.13 & 0.43 & -0.08 & 0.02 & -0.35 & In \\
\hline 38 & 1 & 0.00 & -0.03 & 0.00 & 0.00 & .01 & 0.00 & 0. & & \\
\hline 39 & 1 & 0.00 & -0.03 & 0.01 & 0.00 & & 0. & 0. & & .00 \\
\hline 40 & 1 & -0.07 & & -0.01 & & -0.02 & 0. & & & \\
\hline 41 & 6 & 0.00 & 0.00 & 0.0 & 0.00 & & & & & \\
\hline 42 & 6 & 0.00 & 0.00 & 0.00 & 0.00 & & 0. & & & \\
\hline 43 & 1 & 0.00 & 0.00 & 0.00 & 0.00 & & 0.00 & 0. & 00 & 0.00 \\
\hline 44 & 1 & 0.01 & .01 & 0.00 & 0.00 & & & & & \\
\hline 45 & 1 & 0.00 & 1.00 & 0.00 & & & 0.00 & & & 0.00 \\
\hline Frequen & & 815.86 & & & 817.01 & & & 818.52 & & \\
\hline Atom & AN & $X$ & $Y$ & Z & $X$ & $Y$ & Z & $X$ & Y & Z \\
\hline 1 & 6 & -0.02 & -0.06 & 0.02 & -0.06 & 0.01 & 0.04 & 0.18 & -0.09 & -0.13 \\
\hline 2 & 6 & 0.01 & 0.06 & 0.00 & -0.04 & 0.01 & -0.11 & & & 0.34 \\
\hline 3 & 6 & -0.01 & -0.01 & 0.01 & & -0.02 & -0.10 & & & 0.34 \\
\hline 4 & 6 & 0.01 & 0.06 & -0.01 & & -0.02 & -0.11 & & & \\
\hline 5 & 6 & -0. & -0.06 & 0.00 & 0.0 & -0.02 & 0.05 & & & -0.14 \\
\hline 6 & 6 & 0.0 & -0.04 & 0.01 & 0.00 & -0.01 & 0.02 & & -0.02 & -0.05 \\
\hline 7 & 7 & 0.0 & -0.03 & 0.00 & & 0.00 & 0.02 & & & -0.10 \\
\hline 8 & 6 & 0.0 & -0.02 & 0.00 & 0.00 & 0. & 0.02 & & & 0.01 \\
\hline 9 & 6 & -0.02 & -0.01 & 0.00 & & 0.00 & -0.01 & & 0.00 & 0.00 \\
\hline 10 & 6 & & 0.03 & -0.0 & & & 0. & & & 0.01 \\
\hline 11 & 17 & & 0.00 & 0.00 & & & 0.03 & & & -0.10 \\
\hline 12 & 17 & 0.0 & 0.00 & 0.00 & 0.0 & 0.00 & 0.03 & -0 . & -0.01 & -0.11 \\
\hline 13 & 78 & & 0.00 & 0.00 & & 0.00 & 0.00 & & & 0.00 \\
\hline 14 & 7 & & 0.00 & 0.00 & & & 0.00 & & & -0.01 \\
\hline 15 & 7 & & -0.04 & 0.01 & & & -0.06 & & & -0.01 \\
\hline 16 & 6 & 0.0 & -0.01 & -0.01 & & & 0. & & & 0.10 \\
\hline 17 & 6 & $-0 . C$ & 0. & -0.0 & & & & & & \\
\hline 18 & 6 & 0.0 & -0.06 & 0.02 & & & -0.1 & & & \\
\hline 19 & 6 & 0.0 & -0.04 & 0.01 & & -0.02 & -0.0 & & & -0.0 \\
\hline 20 & 6 & 0.02 & -0.06 & 0.02 & & -0.07 & -0 . & & & -0.04 \\
\hline 2 & 6 & 0.00 & 0.05 & -0.02 & & & 0.3 & -0 & & 0.11 \\
\hline 22 & 17 & & 0.00 & 0.01 & & & -0 . & & & -0.0 \\
\hline 23 & 17 & & 0.00 & 0.0 & & & -0. & & & -0.0 \\
\hline 2 & 6 & & 0. & 0.0 & & & 0. & 0 & & \\
\hline 2 & 6 & 0. & 0.02 & 0.0 & & & & & & \\
\hline 26 & 1 & & 0.07 & $-0 . C$ & & & & & & -0.0 \\
\hline 2 & 1 & & 0.01 & 0.0 & & -0 . & 0. & & & -0.0 \\
\hline $2 \varepsilon$ & 1 & -0. & 0.01 & 0.00 & & & & & & -0.04 \\
\hline 2 & 1 & & & 0.00 & & & & & & \\
\hline 3 & 1 & & 0. & 0. & & & -0 . & & & -0 . \\
\hline 3 & 1 & & & & & & & & & \\
\hline 3 & 1 & & & & & & & & & \\
\hline 3 & 1 & -0 . & 0.4 & -0 . & & 0. & 0. & & & \\
\hline 3 & 1 & & & & & & & & & \\
\hline 3 & 1 & & & -0 . & & & & & & -0.25 \\
\hline 3 & 1 & & & & & & -0 . & & & \\
\hline 3 & 1 & & & & & & 0. & & & -0 . \\
\hline 38 & 1 & & -0. & 0.0 & & & & & & -0.06 \\
\hline 39 & 1 & 0.0 & -0. & 0.0 & & & & & & -0.02 \\
\hline 40 & 1 & & & 0. & & -0 . & -0 . & -0 . & & -0 . \\
\hline 41 & 6 & 0.0 & 0.0 & 0.00 & & 0.00 & 0.0 & 0. & & 0.00 \\
\hline 42 & 6 & & 0.0 & 0.00 & & & & & & \\
\hline 43 & 1 & & 0. & 0.0 & & & 0. & & & \\
\hline 44 & 1 & 0.0 & 0.00 & 0.00 & & 0.00 & 0.0 & & & 0.00 \\
\hline 45 & 1 & 0.00 & 0.00 & 0.00 & 0.00 & 0.00 & 0.00 & 0.00 & 0.00 & 0.00 \\
\hline & & & & & & & & & & \\
\hline & $\Delta \mathrm{N}$ & $x$ & $Y$ & Z & & $\mathrm{Y}$ & Z & & & \\
\hline
\end{tabular}




\begin{tabular}{|c|c|c|c|c|c|c|c|c|c|c|}
\hline & & & & & & & & & & \\
\hline 2 & $\begin{array}{l}6 \\
6\end{array}$ & $\begin{array}{c}0.02 \\
-0.02\end{array}$ & $\begin{array}{c}0.07 \\
-0.07\end{array}$ & $\begin{array}{l}0.00 \\
0.04\end{array}$ & $\begin{array}{l}0.04 \\
0.05\end{array}$ & $\begin{array}{c}-0.06 \\
0.04\end{array}$ & $\begin{array}{l}-0.08 \\
-0.06\end{array}$ & $\begin{array}{c}-0.01 \\
0.00\end{array}$ & $\begin{array}{l}0.00 \\
0.00\end{array}$ & $\begin{array}{r}0.00 \\
-0.01\end{array}$ \\
\hline 3 & 6 & 0.01 & 0.06 & 0.01 & -0.02 & -0.13 & 0.02 & 0.00 & 0.00 & -0.01 \\
\hline 4 & 6 & -0.03 & -0.07 & 0.02 & 0.05 & 0.06 & 0.04 & 0.00 & 0.00 & -0.01 \\
\hline 5 & 6 & 0.00 & 0.07 & -0.04 & 0.03 & -0.02 & 0.09 & & .00 & 0.01 \\
\hline 6 & 6 & 0.03 & 0.03 & -0.01 & -0.09 & 0.03 & -0 . & 0.00 & 00 & 0.00 \\
\hline 7 & 7 & 0.01 & 0.00 & 0.00 & -0.05 & 0.14 & -0.02 & 0.00 & 0.00 & -0.01 \\
\hline 8 & 6 & 0.02 & -0.01 & 0.00 & -0.10 & 0.12 & -0.02 & 0.00 & 0.00 & 0.01 \\
\hline 9 & 6 & 0.02 & 0.00 & 0.0 & 0.10 & 0.11 & & 0.00 & 0.00 & -0.02 \\
\hline 10 & 6 & 0.00 & 0.02 & 0.0 & 0.12 & -0.18 & 0.0 & 0.00 & 0.00 & -0.01 \\
\hline 11 & 17 & 0.00 & 0.00 & 0.00 & 0.00 & -0.01 & -0.01 & 0.00 & 0.00 & 0.00 \\
\hline 12 & 17 & 0.00 & & -0.01 & 0.00 & 0.00 & 0.0 & 0.00 & 0.00 & 0.00 \\
\hline 13 & 78 & 0.00 & & 0.00 & 0.0 & -0.01 & 0.0 & 0.0 & & -0.01 \\
\hline 14 & 7 & 0.00 & 0.00 & 0.00 & 0.00 & 0.01 & 0.0 & 0.00 & 0.00 & 0.00 \\
\hline 15 & 7 & 0.02 & -0.01 & 0.0 & -0.01 & 0.22 & -0.0 & 0.0 & 0.01 & 0.04 \\
\hline 16 & 6 & 0.00 & & 0. & 0.02 & -0.19 & & 0.0 & & -0.02 \\
\hline 17 & 6 & -0.02 & & 0.00 & -0.03 & 0.09 & & & -0.01 & -0.02 \\
\hline 18 & 6 & 0.00 & -0.07 & 0.04 & -0.01 & -0.04 & 0. & -0.01 & 0.00 & 0.01 \\
\hline 15 & 6 & 0.03 & -0.04 & 0.01 & 0.04 & 0.01 & 0. & 0.00 & & .00 \\
\hline 2 & 6 & 0.00 & -0.08 & -0.01 & -0.01 & -0.05 & -0.02 & 0.00 & 0.00 & 0.00 \\
\hline 21 & 6 & -0.02 & 0.07 & -0.03 & -0.02 & 0.08 & -0.05 & 0.01 & 0.00 & -0.03 \\
\hline 22 & 17 & 0.00 & & 0.00 & 0.00 & 0.00 & 0. & 0.0 & .00 & 0.01 \\
\hline 23 & 17 & 0.00 & & 0. & & 0.00 & 0.01 & 0.00 & & 0.01 \\
\hline 24 & 6 & 0.00 & 0.00 & 0.00 & 0.00 & 0.00 & -0.01 & 0.00 & 0.02 & 0.12 \\
\hline 25 & 6 & 0.00 & & 0. & -0.12 & -0.07 & 0.0 & 0.0 & 0.00 & 0.00 \\
\hline 26 & 1 & 0.01 & & & -0.22 & -0.42 & 0. & & -0.01 & -0.01 \\
\hline 2 & 1 & -0.03 & & 0.00 & 0.11 & -0.04 & -0. & -0.01 & -0.02 & 0.00 \\
\hline 28 & 1 & -0.03 & -0.01 & 0. & 0.12 & -0.02 & 0. & 0.01 & 0.01 & -0.01 \\
\hline 29 & 1 & -0.02 & & & -0.08 & -0.08 & 0. & -0 . & & 0.00 \\
\hline 30 & 1 & -0.02 & & 0. & -0.08 & -0.10 & 0. & & -0.03 & 0.01 \\
\hline 31 & 1 & & & & & -0.51 & & & & 0.01 \\
\hline 32 & 1 & -0.07 & & -0.07 & -0.06 & 0.05 & 0. & 0. & 0.00 & 0.01 \\
\hline 3 & 1 & -0.03 & & -0. & 0. & 0.16 & -0. & 0. & 0.00 & -0.01 \\
\hline 3 & 1 & & & -0. & & & & & & 0.01 \\
\hline 35 & 1 & & & 0. & & 0 & & 0. & & 0.01 \\
\hline 3 & 1 & -0.1 & & & -0.0 & & -0.02 & 0. & 0.00 & -0.02 \\
\hline 37 & 1 & -0.1 & & 0. & & -0.01 & -0.03 & 0.0 & 0.00 & 0.01 \\
\hline 38 & 1 & & & & & & & & & -0.24 \\
\hline 3 & 1 & 0.0 & & & -0.0 & & 0. & 0. & & -0.07 \\
\hline & 1 & 0.03 & & 0. & 0.0 & -0.01 & 0. & -0.01 & -0.04 & -0.28 \\
\hline 41 & 6 & & & 0. & & 0.00 & & & & 0.00 \\
\hline 42 & 6 & 0. & & & 0. & 0. & 0. & 0. & 0.00 & 0.00 \\
\hline 43 & 1 & 0.00 & & & & & 0. & 0. & 00 & 0.00 \\
\hline 44 & 1 & & & & & & & & & \\
\hline 45 & 1 & 0. & & 0. & 0. & 0.00 & 0.0 & & 0.00 & 0.00 \\
\hline riequet & & & & & & & & 916.32 & & \\
\hline & AN & & $\mathrm{Y}$ & Z & & $\mathrm{Y}$ & Z & & $Y$ & Z \\
\hline 1 & 6 & -0.0 & -0.01 & 0.02 & -0.0 & 0.02 & 0.0 & & -0.02 & -0.06 \\
\hline 2 & 6 & 0.1 & & 0.00 & -0. & 0.04 & 0.0 & 0. & -0.02 & -0.02 \\
\hline 3 & 6 & -0.03 & & & & -0.08 & 0.01 & -0. & & -0.01 \\
\hline 4 & 6 & & & -0.02 & & & & & & 0.02 \\
\hline 5 & 6 & -0.02 & -0.02 & -0.02 & $-0 .($ & -0.01 & -0.09 & 0. & 0.00 & 0.06 \\
\hline 6 & 6 & & & 0.00 & 0.0 & -0.01 & 0.00 & -0.04 & 0.01 & 0.00 \\
\hline 7 & 7 & 0.09 & & -0.02 & & 0.09 & -0.01 & & & 0.02 \\
\hline 8 & 6 & & & & & & & & -0.02 & \\
\hline 9 & 6 & 0.10 & 0.0 & 0.00 & -0.04 & -0.02 & 0.00 & 0.00 & 0.01 & 0.00 \\
\hline 10 & 6 & & & & & -0.02 & & & & 0.00 \\
\hline 11 & 17 & & & & 0.0 & & & & 0.00 & 0.00 \\
\hline 12 & 17 & 0.0 & 0.0 & 0.00 & 0.0 & 0.00 & -0.01 & 0.00 & 0.00 & 0.00 \\
\hline & 78 & & 0.0 & 0.00 & 0.0 & 0.00 & 0.00 & 0.01 & 0.01 & 0.00 \\
\hline
\end{tabular}




\begin{tabular}{|c|c|c|c|c|c|c|c|c|c|c|}
\hline 14 & 7 & & & & 002 & & & & 001 & \\
\hline $\begin{array}{l}4 \\
15\end{array}$ & $\begin{array}{l}7 \\
7\end{array}$ & $\begin{array}{l}0.01 \\
0.28\end{array}$ & $\begin{array}{r}0.00 \\
-0.20\end{array}$ & $\begin{array}{l}0.00 \\
0.03\end{array}$ & $\begin{array}{c}0.03 \\
-0.10\end{array}$ & $\begin{array}{l}0.02 \\
0.06\end{array}$ & $\begin{array}{r}0.00 \\
-0.01\end{array}$ & $\begin{array}{r}-0.01 \\
0.02\end{array}$ & $\begin{array}{l}-0.01 \\
-0.01\end{array}$ & $\begin{array}{l}0.00 \\
0.00\end{array}$ \\
\hline 16 & 6 & -0.01 & 0.14 & -0.03 & -0.02 & -0.03 & 0.01 & 0.01 & 0.01 & 0.00 \\
\hline 17 & 6 & -0.06 & -0.04 & 0.10 & 0.02 & 0.01 & -0.02 & 0.00 & 0.00 & 0.00 \\
\hline 18 & 6 & -0.10 & 0.05 & 0.17 & 0.04 & -0.01 & -0.06 & -0.01 & 0.00 & 0.01 \\
\hline 19 & 6 & 0.16 & 0.01 & 0.00 & -0.04 & 0.00 & 0.00 & 0.01 & 0.00 & 0.00 \\
\hline 20 & 6 & -0.10 & -0.03 & -0.17 & 0.04 & 0.01 & 0.05 & -0.01 & 0.00 & -0.01 \\
\hline 21 & 6 & -0.06 & -0.08 & -0.07 & 0.02 & 0.01 & 0.02 & 0.00 & 0.00 & 0.00 \\
\hline 22 & 17 & -0.01 & 0.00 & -0.01 & 0.00 & 0.00 & 0. & 0.00 & 0.00 & 0.00 \\
\hline 23 & 17 & -0.01 & 0.0 & 0.01 & 0.00 & 0.00 & 0.0 & 0.00 & 0.00 & 0.00 \\
\hline 24 & 6 & -0.04 & -0.03 & 0.00 & -0.07 & -0.08 & 0.02 & -0.08 & -0.08 & 0.02 \\
\hline 25 & 6 & -0.11 & -0.12 & 0.02 & -0.03 & -0.05 & 0.01 & 0.04 & 0.06 & -0.01 \\
\hline 26 & 1 & -0.15 & -0.26 & 0.05 & -0.03 & -0.06 & 0.0 & 0.05 & 0.10 & -0.02 \\
\hline 27 & 1 & -0.02 & -0.10 & 0.02 & -0.01 & -0.05 & 0.01 & 0.02 & 0.05 & -0.01 \\
\hline 28 & 1 & -0.02 & -0.09 & 0.02 & -0.01 & -0.04 & 0.01 & 0.01 & 0.05 & -0.01 \\
\hline 29 & 1 & -0.08 & 0.14 & -0.04 & 0.02 & -0.03 & 0.0 & 0.02 & -0.02 & 0.01 \\
\hline 30 & 1 & -0.08 & 0.15 & -0.02 & 0.02 & -0.03 & 0.0 & 0.02 & -0.02 & 0.00 \\
\hline 31 & 1 & -0.01 & 0.01 & 0.00 & -0.01 & 0.03 & -0.01 & 0.00 & 0.01 & 0.00 \\
\hline 32 & 1 & -0.28 & -0.01 & 0.08 & 0.09 & 0.00 & -0.03 & -0.02 & 0.00 & \\
\hline 33 & 1 & 0.16 & -0.03 & 0.00 & -0.04 & 0.00 & 0.0 & 0.01 & 0.00 & 0.00 \\
\hline 34 & 1 & -0.28 & -0.04 & -0.07 & 0.09 & 0.02 & 0.0 & -0.02 & 0.00 & -0.01 \\
\hline 35 & 1 & -0.03 & 0.02 & -0.01 & -0.13 & 0.03 & -0 . & 0.10 & -0.01 & 0.04 \\
\hline 36 & 1 & 0.0 & 0.05 & -0.01 & 0.06 & -0.01 & 0.0 & -0.04 & 0.02 & 0.00 \\
\hline 37 & 1 & -0.03 & 0.02 & 0.01 & -0.13 & 0.04 & 0.04 & 0.10 & -0.03 & -0.03 \\
\hline 38 & 1 & -0.0 & 0.20 & -0.01 & -0.10 & 0.40 & -0.05 & -0.10 & 0.42 & -0.04 \\
\hline 39 & 1 & -0.04 & 0.20 & -0.06 & -0.09 & 0.38 & -0.0 & -0.08 & 0.40 & -0.11 \\
\hline 40 & 1 & 0.31 & -0.21 & 0.04 & 0.55 & -0.39 & 0.07 & 0.58 & -0.41 & 0.07 \\
\hline 41 & 6 & 0.00 & 0.00 & 0.00 & 0.02 & 0.02 & 0.00 & 0.00 & 0.00 & 0.00 \\
\hline 42 & 6 & -0.01 & -0.0 & 0.0 & -0.04 & -0.04 & 0.0 & 0.01 & 0.01 & \\
\hline 43 & 1 & -0.01 & & 0.0 & -0.04 & -0.03 & 0.00 & 0.01 & 0.01 & .00 \\
\hline 44 & 1 & -0.01 & & 0.0 & -0.04 & -0.03 & 0.01 & 0.01 & 0.01 & 0.00 \\
\hline 45 & 1 & -0.01 & -0.01 & 0.00 & -0.04 & -0.03 & 0.01 & 0.01 & 0.01 & \\
\hline Frequer & & 945. & & & 945.84 & & & 966.89 & & \\
\hline Atom & AN & $x$ & $Y$ & Z & $x$ & $Y$ & Z & $X$ & $\mathrm{Y}$ & Z \\
\hline & 6 & $\hat{0.03}$ & 0.12 & -0.02 & 0.00 & 0.00 & 0.00 & 0.02 & -0.01 & -0.03 \\
\hline 2 & 6 & -0. & -0.04 & 0.01 & & 0.00 & 0.00 & 0.01 & -0.01 & 0.00 \\
\hline 3 & 6 & 0.0 & & 0.00 & & 0.00 & 0.00 & -0.02 & 0.02 & 0.00 \\
\hline 4 & 6 & 0. & 0.0 & -0.0 & 0. & 0.00 & 0.0 & 0.0 & -0.01 & 0.00 \\
\hline 5 & 6 & -0.03 & -0.12 & 0.02 & 0.00 & 0.00 & 0.0 & 0.0 & 0.00 & 0.03 \\
\hline 6 & 6 & & & 0. & & 0. & 0. & -0. & 0.00 & 0.00 \\
\hline 7 & 7 & 0.1 & 0. & 0.0 & 0. & 0. & 0. & -0. & -0.03 & 0.00 \\
\hline 8 & 6 & 0. & & 0.00 & & 0.0 & 0.0 & 0.0 & -0.01 & 0.00 \\
\hline 9 & 6 & & 0. & 0. & & & 0.0 & 0.1 & 0.00 & 0.00 \\
\hline 10 & 6 & & & & & & & & & \\
\hline 11 & 17 & & & & & 0.0 & 0.0 & 0.0 & 0.00 & 0.00 \\
\hline 12 & 17 & & & & & & 0.0 & 0.0 & 0.00 & 0.00 \\
\hline 13 & 78 & & & & & & 0. & & & 0.00 \\
\hline 14 & 7 & & & & & & 0.1 & 0.2 & 0.26 & 0 \\
\hline 15 & 7 & & & 0. & & 0. & 0. & 0. & -0.01 & 0.00 \\
\hline 16 & 6 & & & & & & & & & \\
\hline 17 & 6 & & & 0. & & 0.0 & -0.1 & 0. & 0.00 & 0. \\
\hline 18 & 6 & 0. & & 0. & 0. & -0.12 & 0.0 & -0.01 & 0.00 & 0.01 \\
\hline 19 & 6 & 0.0 & & & 0.0 & & 0. & & 0.00 & 0.00 \\
\hline 20 & 6 & 0.0 & 0.0 & 0.0 & -0.1 & & -0.0 & & 0.00 & -0.01 \\
\hline 21 & 6 & 0.0 & & 0. & 0.0 & -0.04 & 0.01 & 0.00 & 0.00 & 0.00 \\
\hline 22 & 17 & & & 0. & & 0.0 & 0.00 & 0.00 & 0.00 & 0.00 \\
\hline 23 & 17 & & & 0.00 & 0.0 & 0.00 & 0.0 & & 0.00 & 0.00 \\
\hline 24 & 6 & 0.0 & 0.0 & 0.00 & 0.0 & 0.00 & 0.0 & & 0.02 & 0.00 \\
\hline 25 & 6 & 0.0 & 0.0 & 0.00 & 0.00 & 0.00 & 0.00 & 0.02 & 0.01 & 0.00 \\
\hline 26 & & 0.00 & 0.00 & 0.00 & 0.00 & 0.00 & 0.00 & 0.04 & 0.08 & \\
\hline
\end{tabular}




\begin{tabular}{|c|c|c|c|c|c|c|c|c|c|c|}
\hline 27 & 1 & & & & & & & & & \\
\hline 28 & $\begin{array}{l}1 \\
1\end{array}$ & 0.00 & 0.00 & 0.00 & 0.00 & 0.00 & 0.00 & -0.04 & 0.00 & $\begin{array}{l}0.01 \\
\end{array}$ \\
\hline $\begin{array}{l}\text { ZO } \\
29\end{array}$ & 1 & 0.00 & 0.00 & 0.00 & 0.00 & $\begin{array}{l}0.00 \\
0.00\end{array}$ & 0.00 & $\begin{array}{r}-0.04 \\
0.02\end{array}$ & $\begin{array}{l}0.00 \\
-0.01\end{array}$ & $\begin{array}{l}-0.01 \\
0.01\end{array}$ \\
\hline 30 & 1 & 0.00 & 0.00 & 0.00 & 0.00 & 0.00 & 0.00 & 0.02 & -0.01 & 0.00 \\
\hline 31 & 1 & 0.00 & 0.00 & 0.00 & 0.00 & 0.00 & 0.00 & -0.01 & 0.03 & -0.01 \\
\hline 32 & 1 & 0.00 & -0.01 & 0.00 & -0.08 & 0.67 & & & 0.00 & 0.01 \\
\hline 33 & 1 & 0.00 & 0.00 & 0.00 & 0.00 & 0.00 & 0.0 & 0.00 & 0.01 & 0.00 \\
\hline 34 & 1 & 0.00 & 0.01 & 0.00 & 0.07 & -0.68 & 0.14 & -0.02 & 0.00 & -0.01 \\
\hline 35 & 1 & 0.19 & 0.66 & -0.12 & 0.00 & 0.01 & 0.00 & 0.04 & -0.01 & 0.02 \\
\hline 36 & 1 & 0.00 & 0.00 & 0.00 & 0.00 & 0.00 & 0.0 & -0.01 & 0.01 & 0.00 \\
\hline 37 & 1 & -0.19 & -0.66 & 0.12 & 0.00 & -0.01 & 0.00 & 0.04 & -0.02 & -0.02 \\
\hline 38 & 1 & 0.00 & 0.00 & 0.00 & 0.00 & 0.00 & 0.00 & 0.01 & -0.07 & 0.00 \\
\hline 39 & 1 & 0.00 & 0.00 & 0.00 & 0.00 & 0.00 & & 0.01 & -0.07 & 0.02 \\
\hline 40 & 1 & 0.00 & 0.00 & 0.00 & 0.00 & 0.00 & & -0.11 & 0.08 & -0.01 \\
\hline 41 & 6 & 0.00 & 0.00 & 0.00 & 0.00 & 0.00 & 0.0 & 0.20 & 0.19 & -0.03 \\
\hline 42 & 6 & 0.00 & & 0.00 & 0.00 & 0.00 & & -0.34 & -0.32 & 0.05 \\
\hline 43 & 1 & 0.00 & 0.00 & 0.00 & 0.00 & 0.00 & & -0.29 & -0.29 & 0.03 \\
\hline 44 & 1 & 0.00 & 0.00 & 0.00 & 0.00 & 0.00 & 0.00 & -0.31 & -0.27 & 0.05 \\
\hline 45 & 1 & 0.00 & 1.00 & 0.00 & 0.00 & 0.00 & 0.00 & -0.29 & -0.28 & 0.06 \\
\hline equer & & 1025.91 & & & 1027.96 & & & 1029.92 & & \\
\hline Atom & AN & $x$ & $Y$ & Z & $X$ & $Y$ & Z & $x$ & Y & Z \\
\hline 1 & 6 & 0.01 & -0.01 & -0.02 & 0.00 & 0.00 & 0.00 & 0.02 & 0.08 & -0.01 \\
\hline 2 & 6 & 0.00 & 0.00 & 0.00 & 0.00 & 0.00 & 0.00 & 0.00 & -0.01 & 0.00 \\
\hline 3 & 6 & -0.02 & 0.00 & 0.00 & 0.00 & 0.00 & 0.00 & 0.00 & -0.01 & 0.00 \\
\hline 4 & 6 & 0.00 & 0.00 & 0.00 & 0.00 & 0.00 & 0.00 & 0.00 & -0.01 & 0.00 \\
\hline 5 & 6 & 0.01 & 0.00 & 0.02 & 0.00 & 0.00 & 0.00 & 0.02 & 0.08 & -0.01 \\
\hline 6 & 6 & & & 0.00 & & 0.00 & 0.00 & -0.04 & -0.13 & 0.02 \\
\hline 7 & 7 & -0.01 & 0.06 & -0.01 & 0.00 & 0.00 & 0.00 & 0.00 & 0.00 & 0.00 \\
\hline 8 & 6 & 0.07 & 03 & 0.00 & 0. & 0.00 & 0.0 & 0.0 & 0.00 & 0.00 \\
\hline 9 & 6 & -0.07 & & -0.01 & & 0.00 & 0.00 & 0.00 & 0.00 & 0.00 \\
\hline 10 & 6 & -0.07 & & 0.02 & 0.00 & -0.01 & 0.00 & 0.00 & -0.01 & 0.00 \\
\hline 11 & 17 & 0.00 & 0. & 0.00 & 0.0 & 0.00 & 0.0 & 0.0 & 0.00 & 0.00 \\
\hline 12 & 17 & & & 0.1 & & 0.00 & 0.0 & 0.0 & 0.00 & 0.00 \\
\hline 13 & 78 & & & 0. & & 0.00 & 0.0 & 0.0 & 0.00 & 0.00 \\
\hline 14 & 7 & -0.0 & -0 & 0.00 & 0.0 & 0.00 & 0. & 0. & 0.00 & 0.00 \\
\hline 15 & 7 & & & -0.01 & & 0.00 & 0.0 & 0.0 & 0.00 & 0.00 \\
\hline 16 & 6 & & & 0.00 & & -0.01 & 0.0 & 0.0 & 0.00 & 0.00 \\
\hline 17 & 6 & & & 0.00 & & -0.01 & 0.0 & & & \\
\hline 18 & 6 & -0.02 & & 0.0 & -0.0 & 0.08 & -0.02 & 0. & 0.00 & 0.1 \\
\hline 19 & 6 & & & 0.00 & & -0.13 & 0.0 & 0. & 0.00 & 0.00 \\
\hline 20 & 6 & & & -0.03 & & 0.08 & -0.0 & & & \\
\hline 21 & 6 & 0.0 & & 0.00 & 0.0 & -0.01 & 0.0 & 0.0 & 0.00 & 0.00 \\
\hline 22 & 17 & & & 0. & & 0.00 & 0.0 & 0.0 & 0.00 & 0.00 \\
\hline 23 & 17 & & & & & & & & & 0. \\
\hline 24 & 6 & & & 0. & & 0. & 0. & & & 0. \\
\hline 25 & 6 & & & 0. & & -0.01 & 0. & 0. & 0.0 & 0. \\
\hline 26 & 1 & & & -0 . & & 0.02 & 0. & & & \\
\hline 27 & 1 & & & 0. & & & & & & 0.00 \\
\hline 28 & 1 & & & -0.0 & -0.02 & -0. & 0. & -0 . & -0.01 & \\
\hline 29 & 1 & & & 0.11 & & -0.01 & 0. & 0.02 & -0.01 & 0.01 \\
\hline 30 & 1 & & & -0.02 & & -0.01 & & & & 0.00 \\
\hline 31 & 1 & & & -0.0 & 0.0 & & & & & \\
\hline 32 & 1 & -0.0 & & 0.03 & 0.05 & -0.44 & 0.10 & 0.00 & 0.00 & 0.00 \\
\hline 33 & 1 & & & 0.01 & -0.07 & 0.73 & -0.15 & & 0.01 & 0.00 \\
\hline 34 & 1 & -0. & & -0.04 & 0.05 & -0.44 & 0.0 & & 0.00 & 0.00 \\
\hline 35 & 1 & 0.0 & & 0.02 & 0.00 & 0.00 & 0.00 & -0.12 & -0.42 & 0.08 \\
\hline 36 & 1 & -0.0 & & 0.0 & 0.0 & -0.01 & 0.0 & 0.20 & 0.71 & -0.13 \\
\hline 37 & 1 & 0.0 & & -0.02 & 0.0 & 0.00 & 0.0 & -0.1 & -0.42 & 0.07 \\
\hline 38 & 1 & & & 0.00 & 0.00 & 0.00 & 0.0 & 0.00 & 0.00 & 0.00 \\
\hline 39 & 1 & 0.00 & -0.01 & 0.00 & 0.00 & 0.00 & 0.00 & 0.00 & 0.00 & 0.00 \\
\hline
\end{tabular}




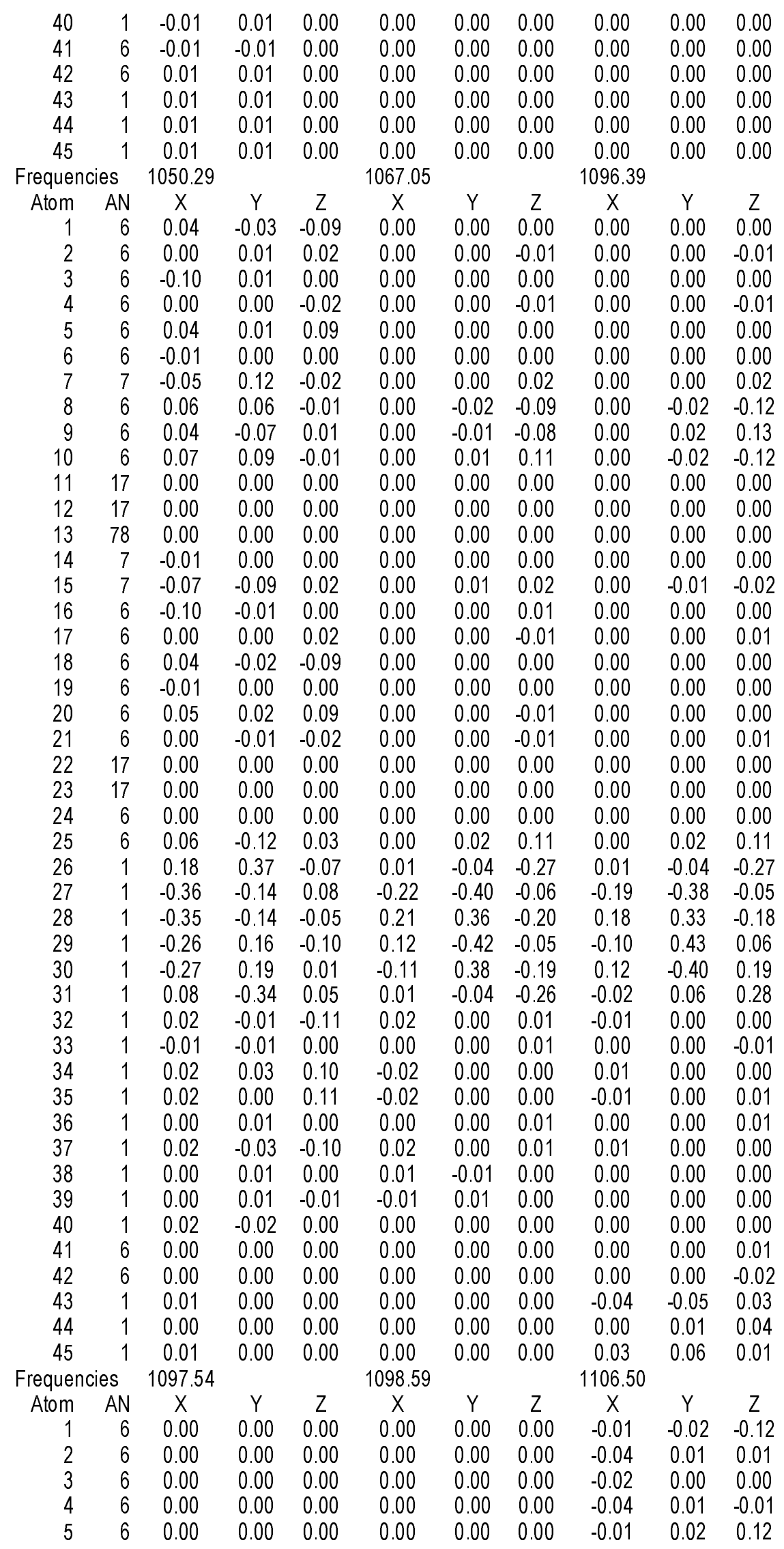




\begin{tabular}{|c|c|c|c|c|c|c|c|c|c|c|}
\hline & & & & & & & & & & \\
\hline 6 & 6 & 0.00 & 0.00 & 0.00 & 0.00 & 0.00 & 0.00 & 0.09 & -0.02 & 0.01 \\
\hline 7 & 7 & 0.00 & 0.00 & 0.00 & 0.00 & 0.00 & 0.00 & 0.04 & 0.01 & 0.00 \\
\hline 8 & 6 & 0.00 & 0.00 & 0.01 & 0.00 & 0.00 & 0.00 & 0.00 & -0.02 & 0.00 \\
\hline 9 & 6 & 0.00 & 0.00 & -0.01 & 0.00 & 0.00 & 0.00 & -0.01 & -0.03 & 0.00 \\
\hline 10 & 6 & 0.00 & 0.00 & 0.01 & 0.00 & 0.00 & 0.00 & 0.01 & 0.02 & 0.00 \\
\hline 11 & 17 & 0.00 & 0.00 & 0.00 & 0.00 & 0.00 & 0.0 & 0. & & 0.00 \\
\hline 12 & 17 & 0.00 & 0.00 & 0.00 & 0.00 & 0.00 & 0. & & 00 & 0.00 \\
\hline 13 & 78 & 0.00 & 0.00 & 0.00 & 0.00 & 0.00 & 0.0 & 0. & 0.00 & 0.00 \\
\hline 14 & 7 & 0.00 & & -0.01 & 0.01 & -0.01 & & & & 0.00 \\
\hline 15 & 7 & 0.00 & 0.00 & 0.00 & 0.00 & 0.00 & 0. & & 30 & 0.00 \\
\hline 16 & 6 & 0.00 & 0.00 & 0.00 & 0.00 & 0.00 & 0.0 & 0. & 0.00 & 0.00 \\
\hline 17 & 6 & 0.00 & 0.00 & 0.00 & 0.00 & 0.00 & 0.00 & & & -0.01 \\
\hline 18 & 6 & 0.00 & 0.00 & 0.00 & 0.00 & 0.00 & 0.01 & 0.01 & 0.03 & 0.15 \\
\hline 19 & 6 & 0.00 & 0.00 & 0.00 & -0.01 & 0.00 & 0.00 & -0.12 & & \\
\hline 20 & 6 & 0.00 & 0.00 & 0.00 & 0.00 & 0.00 & -0.01 & 0.01 & -0.03 & -0.15 \\
\hline 21 & 6 & 0.00 & 0.00 & 0.00 & 0.00 & 0.00 & 0.00 & 0.05 & 0.01 & 0.00 \\
\hline 22 & 17 & 0.00 & 0.00 & 0.00 & 0.00 & 0.00 & 0. & -0.01 & 0 & 0.00 \\
\hline 23 & 17 & 0.0 & & 0.00 & 0.00 & 0.00 & 0. & -0.01 & & \\
\hline 24 & 6 & 0.00 & 0.00 & 0.00 & 0.00 & 0.00 & 0.00 & 0.00 & 0.00 & 0.00 \\
\hline 25 & 6 & 0.00 & 0.00 & -0.01 & 0.00 & 0.00 & 0. & 0. & 0.01 & 0.00 \\
\hline 26 & 1 & 0.00 & & 0.03 & 0.00 & 0.00 & 0. & & & 0.01 \\
\hline 27 & 1 & 0.02 & 0. & 0.01 & & 0.00 & 0. & & & -0.01 \\
\hline 28 & 1 & -0.02 & -0.03 & 0.02 & 0.00 & 0.00 & 0. & & 0.01 & 0.01 \\
\hline 29 & 1 & 0.01 & -0.04 & -0.01 & -0.01 & 00 & 0. & & & \\
\hline 30 & 1 & -0.01 & & -0.02 & & & 0. & & & 0.01 \\
\hline 31 & 1 & 0.00 & -0.01 & -0.02 & 0.00 & -0.01 & 0.0 & 0.01 & -0.06 & 0.01 \\
\hline 32 & 1 & 0.00 & 0.0 & 0.0 & & 0.00 & 0.0 & & & \\
\hline 33 & 1 & 0.00 & & 0.00 & -0.01 & 0.00 & 0.00 & -0.1 & -0.01 & 0.00 \\
\hline 34 & 1 & 0.00 & 0.00 & 0.00 & 0.02 & 0.00 & -0.02 & 0.3 & -0.04 & -0.36 \\
\hline 35 & 1 & 0.00 & 0.00 & 0.00 & -0.01 & 0.00 & 0.0 & -0.27 & 0.12 & 0.27 \\
\hline 36 & 1 & 0.00 & & 0.00 & & 0.00 & 0.00 & 0. & & 0.01 \\
\hline 37 & 1 & 0.00 & 0.0 & 0.00 & -0.01 & 0.00 & -0.01 & -0.2 & 0.02 & -0.30 \\
\hline 38 & 1 & 0.00 & 0.0 & 0.00 & 0.00 & 0.00 & 0.0 & 0.0 & 0. & 0.00 \\
\hline 39 & 1 & 0.0 & & 0.00 & 0. & & 0. & & & \\
\hline 40 & 1 & 0.0 & & 0.00 & 0.0 & & 0.00 & & & 0.00 \\
\hline 41 & 6 & -0.01 & 0.0 & 0.11 & -0.08 & 0.08 & -0.02 & & -0.01 & 0.00 \\
\hline 42 & 6 & 0.01 & -0.04 & -0.15 & 0.11 & -0.11 & 0.0 & -0. & 0.01 & 0. \\
\hline 43 & 1 & -0.3 & & 0.26 & -0.45 & 02 & -0.1 & & & 0.01 \\
\hline 44 & 1 & & & 0.40 & 0.41 & & -0.1 & & & \\
\hline 45 & 1 & 0.30 & 0. & 0.11 & -0.5 & 0.00 & 0.1 & & 0.00 & \\
\hline equen & & 1108.89 & & & 1139.53 & & & 1142.16 & & \\
\hline tom & AN & $X$ & $\mathrm{Y}$ & Z & $X$ & $\mathrm{Y}$ & Z & $x$ & $Y$ & Z \\
\hline 1 & 6 & 0.02 & 0.02 & 0.14 & 0.03 & -0.02 & -0.04 & 0.05 & -0.02 & -0.05 \\
\hline 2 & 6 & 0.0 & -0.01 & 0.01 & & 0.01 & 0.25 & & 0.0 & 0.36 \\
\hline 3 & 6 & 0.00 & 0.00 & 0.00 & -0.07 & 0.03 & -0.01 & & 0.02 & -0.01 \\
\hline 4 & 6 & 0.0 & $-0 .($ & 0.00 & & & -0.24 & & & \\
\hline 5 & 6 & 0.0 & -0.0 & -0.13 & & 0.00 & 0.04 & & 0.00 & 0.06 \\
\hline 6 & 6 & -0.1 & 0.0 & -0.01 & -0.15 & 0.04 & -0.01 & & 0.06 & -0.01 \\
\hline 7 & 7 & $-0 . C$ & 0.0 & 0.00 & & & 0.01 & & & \\
\hline 8 & 6 & $-0 .($ & & 0.00 & & -0.01 & 0.0 & & & \\
\hline 9 & 6 & -0.0 & -0. & 0.00 & & 0.00 & 0.00 & & -0.04 & 0.01 \\
\hline 10 & 6 & 0.0 & 0.0 & 0.00 & & -0.02 & 0.00 & & 0.02 & 0.00 \\
\hline 11 & 17 & $-0 . C$ & 0.0 & 0.00 & -0. & 0.01 & 0.0 & & 0.0 & \\
\hline 12 & 17 & $-0 . C$ & 0.0 & 0.00 & -0. & 0.00 & -0.03 & -0.0 & 0.00 & -0.04 \\
\hline 13 & 78 & & & 0.0 & & & & & & 0.00 \\
\hline 14 & 7 & 0.00 & 0.0 & 0.00 & & 0.00 & 0.00 & & 0.00 & \\
\hline 15 & 7 & -0.03 & -0.0 & 0.00 & & 0.03 & -0.01 & & & \\
\hline 16 & 6 & 0.0 & 0.00 & 0.0 & -0. & -0.01 & 0.00 & 0.08 & 0.00 & 0.00 \\
\hline 17 & 6 & & & 0.0 & & 0.07 & & & -0.08 & \\
\hline 18 & 6 & 0.02 & 0.02 & 0.10 & 0.04 & -0.01 & -0.05 & -0.04 & 0.01 & \\
\hline
\end{tabular}




\begin{tabular}{|c|c|c|c|c|c|c|c|c|c|c|}
\hline & & & & & & & & & & \\
\hline $\begin{array}{l}19 \\
20\end{array}$ & $\begin{array}{l}6 \\
6\end{array}$ & $\begin{array}{r}-0.10 \\
0.01\end{array}$ & $\begin{array}{l}-0.01 \\
-0.02\end{array}$ & $\begin{array}{r}0.00 \\
-0.10\end{array}$ & $\begin{array}{r}-0.18 \\
0.04\end{array}$ & $\begin{array}{r}-0.02 \\
0.01\end{array}$ & $\begin{array}{l}0.01 \\
0.05\end{array}$ & $\begin{array}{r}0.20 \\
-0.04\end{array}$ & $\begin{array}{r}0.02 \\
-0.01\end{array}$ & $\begin{array}{l}-0.01 \\
-0.05\end{array}$ \\
\hline 21 & 6 & 0.04 & 0.00 & -0.01 & 0.13 & -0.05 & -0.29 & -0.14 & 0.05 & 0.32 \\
\hline 22 & 17 & 0.00 & 0.00 & 0.00 & -0.02 & -0.01 & -0.03 & 0.02 & 0.01 & 0.03 \\
\hline 23 & 17 & 0.00 & 0.00 & 0.00 & -0.02 & 0.00 & 0.03 & 0.02 & -0.01 & -0.03 \\
\hline 24 & 6 & 0.00 & 0.00 & 0.0 & 0.00 & 0.00 & 0.0 & 0.01 & -0.01 & 0.00 \\
\hline 25 & 6 & 0.04 & -0.03 & 0.00 & -0.08 & 0.03 & -0.01 & -0.02 & 0.02 & -0.01 \\
\hline 26 & 1 & 0.08 & 0.15 & -0.03 & -0.16 & -0.27 & 0.05 & -0.06 & -0.11 & 0.02 \\
\hline 27 & 1 & -0.12 & -0.02 & 0.03 & 0.19 & 0.01 & -0.06 & 0.11 & 0.01 & -0.03 \\
\hline 28 & 1 & -0.12 & & & & 0.04 & & 0.10 & .02 & 0.03 \\
\hline 29 & 1 & -0.10 & 0.05 & -0.04 & 0.2 & -0.07 & 0.08 & -0.05 & 0.02 & -0.02 \\
\hline 30 & 1 & -0.10 & 0.05 & 0.02 & 0.20 & -0.07 & -0.04 & -0.05 & 0.03 & 0.01 \\
\hline 31 & 1 & 0.04 & -0.15 & 0. & -0.09 & 0.31 & -0.06 & 0.00 & -0.02 & 0.00 \\
\hline 32 & 1 & 0.27 & & 0.2 & -0.11 & -0.03 & -0.1 & 0.13 & 0.04 & 0.13 \\
\hline 33 & 1 & -0.11 & -0.01 & 0.0 & -0.19 & -0.03 & 0.01 & 0.21 & 0.03 & -0.01 \\
\hline 34 & 1 & 0.27 & -0.03 & -0.27 & -0.11 & 0.02 & 0.12 & 0.13 & -0.02 & -0.13 \\
\hline 35 & 1 & 0.34 & & -0.31 & -0.08 & 0.04 & 0.0 & -0.12 & 0.06 & 0.13 \\
\hline 36 & 1 & -0.13 & & -0.01 & -0.16 & 0.05 & -0.01 & -0.23 & 0.07 & -0.01 \\
\hline 37 & 1 & 0.33 & -0.03 & 0.35 & -0.07 & 0.00 & -0.1 & -0.12 & 0.01 & -0.15 \\
\hline 38 & 1 & 0.00 & & 0. & 0.0 & -0.01 & 0. & -0.03 & .01 & -0.03 \\
\hline 39 & 1 & 0.00 & & 0. & $-0 .($ & -0.01 & & -0.03 & & \\
\hline 40 & 1 & 0.00 & & 0.00 & 0.00 & 0.01 & 0. & -0.02 & 0.01 & 0.00 \\
\hline 41 & 6 & 0.00 & & & 0. & 0.00 & 0. & 0.00 & 0.00 & 0.00 \\
\hline 42 & 6 & 0.00 & & & 0.0 & 0.00 & 0.00 & 0.00 & 0.00 & 0.00 \\
\hline 43 & 1 & 0.01 & & 0.00 & 0.01 & 0.00 & 0.00 & 0.00 & 0.00 & 0.00 \\
\hline 44 & 1 & -0.01 & -0.01 & 0.00 & 0.00 & -0.01 & 0.0 & 0.00 & .00 & 0.00 \\
\hline 45 & 1 & 0.01 & & 0.0 & & 0.00 & 0.00 & 0.00 & 0.00 & \\
\hline equen & & 1194.17 & & & 1211.91 & & & 1213.58 & & \\
\hline Atom & AN & $X$ & $Y$ & Z & $x$ & $Y$ & Z & $X$ & $Y$ & Z \\
\hline 1 & 6 & 0.04 & -0.01 & 0.00 & & 0.00 & 0.00 & 0.01 & -0.02 & -0.07 \\
\hline 2 & 6 & -0.04 & 0.00 & -0.06 & & 0.00 & 0.00 & -0.12 & 0.02 & -0.05 \\
\hline 3 & 6 & -0.09 & 0.05 & -0.01 & & 0.00 & 0.00 & 0.00 & 0.01 & 0.06 \\
\hline 4 & 6 & -0.04 & 0.02 & 0.06 & -0 & 0.00 & 0.0 & 0.12 & -0.04 & -0.04 \\
\hline 5 & 6 & & & 0.00 & & 0.00 & 0.0 & -0.01 & -0.01 & -0.07 \\
\hline 6 & 6 & & 0.00 & 0.00 & & 0.00 & -0.0 & 0.00 & 0.02 & 0.09 \\
\hline 7 & 7 & -0.06 & 0.01 & 0.00 & 0. & 0.00 & 0.0 & 0.00 & 0.00 & -0.02 \\
\hline 8 & 6 & 0.2 & & -0.01 & & 0.00 & 0.0 & 0.0 & 0.00 & 0.00 \\
\hline 9 & 6 & & & & & & 0.0 & & & \\
\hline 10 & 6 & & 0. & -0.01 & & 0.00 & -0. & 0. & 0.00 & 0.00 \\
\hline 11 & 17 & & & -0.01 & & 0.00 & 0.0 & -0.01 & 0.00 & 0.01 \\
\hline 12 & 17 & & & & & 0.00 & 0.8 & & & 0.01 \\
\hline 13 & 78 & & & & & & & & & 0.00 \\
\hline 14 & 7 & & & & & 0.00 & 0.0 & 0.0 & 0.00 & 0.00 \\
\hline 15 & 7 & & & & & 0.00 & -0.02 & 0.0 & 0.00 & 0.00 \\
\hline 16 & 6 & & & & & & & & & \\
\hline 17 & 6 & & & & & -0.02 & -0 . & -0 . & 0. & 0. \\
\hline 18 & 6 & & & 0. & & -0.01 & -0.07 & 0.0 & 0.00 & 0.00 \\
\hline 19 & 6 & & & & & & & & & 0.01 \\
\hline 20 & 6 & & & & & -0.02 & -0. & & & \\
\hline 21 & 6 & & & & & 0.00 & -0.05 & & 0 & 0.00 \\
\hline 22 & 17 & & & & & & & & & 0.00 \\
\hline 23 & 17 & & & -0 . & & 0.0 & & & & \\
\hline 24 & 6 & & & 0. & & 0.00 & 0.00 & 0.0 & 0.00 & 0.00 \\
\hline 25 & 6 & -0.1 & & & & 0.00 & 0. & & 0. & -0.01 \\
\hline 26 & 1 & & & 0.06 & & 0.00 & 0.0 & & & 0.01 \\
\hline 27 & 1 & 0.1 & & -0.06 & 0.00 & 0.00 & 0.0 & 0.00 & 0.01 & 0.00 \\
\hline 28 & 1 & 0.16 & & 0.08 & 0.00 & 0.00 & 0.00 & 0.00 & -0.01 & 0.01 \\
\hline 29 & 1 & & & & & 0.02 & 0.0 & & & 0.00 \\
\hline 30 & 1 & & & -0.0 & 0.00 & -0.01 & 0.0 & 0.00 & 0.00 & 0.00 \\
\hline 31 & 1 & -0.14 & 0.44 & -0.09 & 0.00 & 0.00 & 0.02 & 0.00 & 0.00 & 0.00 \\
\hline
\end{tabular}




\begin{tabular}{|c|c|c|c|c|c|c|c|c|c|c|}
\hline 32 & 1 & & & & 70 & & & & & \\
\hline $\begin{array}{l}32 \\
33 \\
\end{array}$ & $\begin{array}{l}1 \\
1\end{array}$ & 0.21 & 0.04 & 0.09 & -0.07 & $\begin{array}{l}-0.03 \\
0.03\end{array}$ & $\begin{array}{r}-0.12 \\
0.93\end{array}$ & -0.01 & 0.00 & $\begin{array}{r}-0.01 \\
0.07\end{array}$ \\
\hline 34 & 1 & 0.20 & 0.00 & $\begin{array}{l}.01 \\
-0.10\end{array}$ & 0.07 & $\begin{array}{l}0.20 \\
-0.02\end{array}$ & $\begin{array}{l}-0.12 \\
\end{array}$ & 0.01 & 0.00 & -0.01 \\
\hline 35 & 1 & 0.25 & -0.09 & -0.10 & -0.01 & 0.00 & 0.01 & 0.07 & -0.04 & -0.12 \\
\hline 36 & 1 & 0.01 & -0.01 & 0.00 & 0.00 & -0.01 & -0.07 & -0.01 & 0.17 & 0.93 \\
\hline 37 & 1 & 0.25 & -0.04 & 0.13 & 0.00 & 0.00 & & -0.07 & 0.00 & -0.13 \\
\hline 38 & 1 & 0.00 & 0.01 & 0.00 & 0.00 & 0.00 & & & 0.00 & 0.00 \\
\hline 39 & 1 & 0.00 & 0.01 & -0.01 & 0.00 & 0.00 & 0.00 & 0.00 & 0.00 & .00 \\
\hline 40 & 1 & 0.02 & -0.01 & 0.00 & 0.00 & 0.00 & & 0.00 & 0.00 & .00 \\
\hline 41 & 6 & 0.00 & 0.00 & 0.00 & 0.00 & 0.00 & 0.0 & 0.00 & 0.00 & 0.00 \\
\hline 42 & 6 & 0.00 & 0.00 & 0.00 & 0.00 & 0.00 & 0.00 & 0.00 & 0.00 & 0.00 \\
\hline 43 & 1 & 0.00 & 0.00 & 0.00 & 0.00 & 0.00 & 0.00 & 0.00 & 0.00 & 0.00 \\
\hline 44 & 1 & 0.00 & 0.00 & 0.00 & 0.00 & 0.00 & 0.0 & 0.00 & 0.00 & 0.00 \\
\hline 45 & 1 & 0.00 & 0.00 & 0.00 & 0.00 & 0.00 & 0.00 & 0.00 & 0.00 & 0.00 \\
\hline reque & & 1260.62 & & & 1261.70 & & & 1264.99 & & \\
\hline Atom & AN & $X$ & $Y$ & Z & $X$ & $\mathrm{Y}$ & Z & $x$ & $\mathrm{Y}$ & Z \\
\hline 1 & 6 & 0.00 & 0.00 & 0.00 & -0.05 & 0.02 & 0.02 & 0.06 & -0.02 & -0.03 \\
\hline 2 & 6 & 0.00 & 0.00 & 0.00 & 0.10 & -0.02 & 0.02 & -0.07 & 0.02 & -0.02 \\
\hline 3 & 6 & 0.00 & 0.00 & 0.00 & 0.00 & -0.02 & -0.09 & -0.18 & 0.04 & -0.01 \\
\hline 4 & 6 & 0.00 & 0.00 & 0.00 & -0.10 & 0.03 & 0.01 & -0.06 & 0.02 & 0.01 \\
\hline 5 & 6 & 0.00 & 0.00 & 0.00 & 0.05 & -0.01 & 0.02 & 0.06 & -0.01 & 0.04 \\
\hline 6 & 6 & 0.00 & 0.00 & 0.00 & 0.00 & 0.01 & 0.05 & 0.01 & 0.00 & 0.00 \\
\hline 7 & 7 & 0.00 & 0.00 & 0.00 & 0.00 & 0.00 & 0.02 & 0.10 & 0.03 & -0.01 \\
\hline 8 & 6 & 0.00 & 0.00 & 0.00 & 0.00 & 0.00 & 0.00 & 0.00 & -0.08 & 0.01 \\
\hline 9 & 6 & 0.00 & 0.00 & 0.01 & 0.00 & 0.00 & 0.00 & -0.03 & -0.11 & 0.02 \\
\hline 10 & 6 & 0.00 & 0.00 & -0.01 & 0.00 & 0.00 & 0.00 & 0.00 & 0.04 & -0.01 \\
\hline 11 & 17 & 0.00 & 0.00 & 0.00 & 0.00 & 0.00 & 0.00 & 0.00 & 0.00 & 0.00 \\
\hline 12 & 17 & 0. & 0.00 & 0.0 & 0.00 & 0.00 & 0.00 & 0.00 & 0.00 & 0.01 \\
\hline 13 & 78 & & & 0.00 & & 0.00 & 0.0 & 0.0 & 0.00 & 0.00 \\
\hline 14 & 7 & & 0. & 0.00 & 0.00 & 0.00 & 0.00 & 0.00 & 0.00 & 0.00 \\
\hline 15 & 7 & 0.00 & 0.00 & -0.02 & 0.00 & 0.00 & 0.00 & -0.11 & 0.04 & 0.00 \\
\hline 16 & 6 & 0.00 & 0.02 & 0.10 & 0.00 & 0.00 & 0.0 & 0.2 & 0.02 & -0.01 \\
\hline 17 & 6 & -0.10 & & -0.02 & & 0.00 & 0.0 & 0.0 & 0.01 & 0.01 \\
\hline 18 & 6 & 0.05 & & -0.02 & & 0.00 & 0. & -0. & 0.00 & 0.04 \\
\hline 19 & 6 & 0.00 & -0.01 & -0.04 & 0.00 & 0.00 & 0.0 & 0.0 & 0.00 & 0.00 \\
\hline 20 & 6 & & & -0.02 & & 0.00 & 0.0 & -0.08 & -0.02 & -0.04 \\
\hline 21 & 6 & & & -0.02 & & 0.00 & 0.0 & 0.08 & 0.01 & -0.01 \\
\hline 22 & 17 & 0. & & 0.1 & 0. & 0.00 & 0.1 & 0.1 & 0.00 & -0.01 \\
\hline 23 & 17 & & & 0. & & 0.0 & 0.0 & 0.0 & 0.00 & 0.01 \\
\hline 24 & 6 & & & 0. & & 0. & 0. & 0. & -0.02 & 0.00 \\
\hline 25 & 6 & 0. & & 0. & 0.0 & 0. & 0. & 0. & 0.03 & 0.00 \\
\hline 26 & 1 & 0.00 & & 0.00 & 0.00 & 0.00 & -0.1 & 0.0 & -0.01 & 0.00 \\
\hline 27 & 1 & 0. & & 0. & 0. & -0.01 & 0.0 & 0.04 & 0.00 & -0.02 \\
\hline 28 & 1 & & & & & & & & & \\
\hline 29 & 1 & & & 0. & & 0.0 & 0.1 & -0.07 & 0.02 & -0.04 \\
\hline 30 & 1 & & & 0. & & 0.00 & 0. & -0. & 0.03 & 0.03 \\
\hline 31 & 1 & & & & & & & & & \\
\hline 32 & 1 & & & 0. & & & & -0 . & -0.09 & -0.15 \\
\hline 33 & 1 & & & -0 . & & 0.00 & 0. & -0.01 & & 0.01 \\
\hline 34 & 1 & & & & & & & & & 0.16 \\
\hline 35 & 1 & & & 0. & & -0.21 & -0 . & 0.3 & -0.11 & -0.10 \\
\hline 36 & 1 & & & 0. & 0. & 0. & 0.2 & 0. & 0.00 & -0.01 \\
\hline 37 & 1 & & & & -0.5 & 0.1 & -0. & & -0.08 & 0.15 \\
\hline 38 & 1 & 0.0 & 0.00 & 0.0 & 0.0 & 0.0 & 0.1 & & 0.08 & -0.06 \\
\hline 39 & 1 & & 0.00 & 0.0 & 0.0 & 0.00 & 0.8 & -0.07 & 0.09 & 0.03 \\
\hline 40 & 1 & & & 0.0 & 0.0 & 0.0 & 0.00 & -0.11 & 0.05 & -0.01 \\
\hline 41 & 6 & & & 0.00 & & 0.00 & 0.0 & & 0.00 & 0.00 \\
\hline 42 & 6 & 0.0 & 0.0 & 0.00 & 0.00 & 0.00 & 0.1 & 0.0 & 0.00 & 0.00 \\
\hline 43 & 1 & 0.0 & 0.0 & 0.00 & 0.00 & 0.00 & 0.00 & 0.01 & 0.00 & 0.00 \\
\hline 44 & 1 & 0.00 & 0.00 & 0.00 & 0.00 & 0.00 & 0.00 & 0.00 & 0.00 & 0.00 \\
\hline
\end{tabular}




\begin{tabular}{|c|c|c|c|c|c|c|c|c|c|c|}
\hline \multicolumn{2}{|c|}{ Frequencies } & $\begin{array}{c}0.00 \\
1290.57\end{array}$ & 0.00 & 0.00 & $\begin{array}{c}0.00 \\
1307.98\end{array}$ & 0.00 & 0.00 & $\begin{array}{c}0.01 \\
1312.69\end{array}$ & 0.00 & 0.00 \\
\hline & AN & $x$ & Y & Z & $x$ & Y & Z & $x$ & Y & Z \\
\hline 1 & 6 & -0.07 & 0.03 & 0.04 & -0.01 & 0.00 & 0.00 & -0.26 & 0.04 & -0.18 \\
\hline 2 & 6 & 0.09 & -0.03 & 0.00 & 0.01 & 0.00 & 0.00 & 0.26 & -0.10 & -0.19 \\
\hline 3 & 6 & 0.22 & -0.05 & 0.01 & 0.00 & 0.00 & 0.01 & -0.01 & & 0.45 \\
\hline 4 & 6 & 0.09 & -0.02 & 0.01 & -0.01 & 0.00 & -0.01 & -0.26 & & -0.22 \\
\hline 5 & 6 & -0.07 & 0.01 & -0.05 & 0.01 & 0.00 & 0.00 & 0.27 & -0.10 & -0.14 \\
\hline 6 & 6 & -0.01 & 0.00 & 0.00 & 0.00 & 0.00 & & 0.00 & & 0.29 \\
\hline 7 & 7 & -0.19 & 0.02 & -0.0 & 0.00 & 0.00 & & 0.00 & & 0.03 \\
\hline 8 & 6 & 0.06 & 0.12 & -0.02 & -0.01 & & & & & \\
\hline 9 & 6 & 0.10 & -0.16 & 0.03 & 0.00 & 0.00 & 0.0 & & & .01 \\
\hline 10 & 6 & -0.03 & 0.05 & -0.01 & 0.00 & 0.00 & & 0.00 & & .00 \\
\hline 11 & 17 & 0.00 & 0.00 & 0.00 & 0.00 & 0.00 & 0.00 & 0.00 & .00 & .00 \\
\hline 12 & 17 & 0.00 & 0.00 & 0.00 & 0.00 & 0.00 & 0.0 & 0.00 & 0.00 & .00 \\
\hline 13 & 78 & 0.00 & & 0. & 0.00 & 0.00 & & 0.0 & & .00 \\
\hline 14 & 7 & 0.00 & & 0.0 & 0.00 & 0.00 & 0.0 & 0.00 & & \\
\hline 15 & 7 & -0.15 & 0.01 & 0.00 & 0.00 & -0.01 & -0.03 & 0.00 & 0.00 & 0.00 \\
\hline 16 & 6 & 0.1 & 0.01 & 0.00 & 0.00 & 0.10 & 0.4 & 0. & & \\
\hline 17 & 6 & 0.07 & & -0.01 & & -0.02 & -0.22 & -0. & & \\
\hline 18 & 6 & -0.05 & 0.00 & 0.04 & -0.29 & -0.06 & -0.15 & 0.0 & & .00 \\
\hline 19 & 6 & -0.01 & & 0.00 & 0.00 & 0.06 & 0.3 & & & \\
\hline 20 & 6 & -0.05 & & -0.0 & 0.28 & 0.00 & & & & \\
\hline 21 & 6 & 0.07 & & 0.00 & -0.29 & -0.07 & & & & \\
\hline 22 & 17 & 0.00 & & & 0.00 & 0.00 & & & & \\
\hline 23 & 17 & 0. & & & & & & & & \\
\hline 24 & 6 & 0. & & 0. & & 0.00 & & & & \\
\hline 25 & 6 & & & 0. & 0.00 & 0.00 & 0.0 & 0. & & \\
\hline 26 & 1 & -0 . & & 0.1 & 0.0 & 0.1 & & & & \\
\hline 27 & 1 & & & 0.0 & & 0.01 & & 0. & & \\
\hline 28 & 1 & -0.09 & & -0.02 & 0.00 & -0.01 & 0.0 & 0.0 & -0.03 & .02 \\
\hline 2 & 1 & & & -0 . & & & & & & \\
\hline 30 & 1 & & & -0 . & & & & & & \\
\hline 3 & 1 & & & -0 . & & & & & & \\
\hline 32 & 1 & & & -0 . & & & & & & \\
\hline 33 & 1 & & & & & -0 & & & & \\
\hline 34 & 1 & & & & & 01 & 0. & & & \\
\hline 35 & 1 & & & & & & & & & \\
\hline 3 & 1 & & & & & 0. & & & & -0 \\
\hline 37 & 1 & & & -0 . & & & & & & \\
\hline 38 & 1 & & & & & & & & & \\
\hline $3 s$ & 1 & & & 0. & -0. & 0.01 & & & & \\
\hline 40 & 1 & & & & & 0.0 & & -0 & & \\
\hline 41 & 6 & & & & & & & & & \\
\hline 42 & 6 & & & & & & & & & \\
\hline 43 & 1 & & & & & & & & & \\
\hline 44 & 1 & & & & & & & & & \\
\hline 45 & 1 & 0.00 & & & & 0.00 & & & & \\
\hline requen & & 1316.46 & & & & & & & & \\
\hline Atom & AN & & Y & Z & V & $Y$ & Z & $x$ & Y & 2 \\
\hline 1 & 6 & & 0.00 & 0.00 & & 0.00 & & & & -0.01 \\
\hline 2 & 6 & & & & & & & & & \\
\hline 3 & 6 & -0.0 & 0.00 & 0.00 & & 0.03 & -0.01 & & 0.00 & 0.00 \\
\hline 4 & 6 & & 0.00 & -0.0 & & 0.00 & & & & -0.01 \\
\hline 5 & 6 & & & & & & & & & \\
\hline 6 & 6 & 0. & & 0.0 & & 0.0 & 0. & & & 0.00 \\
\hline 7 & 7 & & & & -0.0 & -0.0 & & & & 0.00 \\
\hline 8 & 6 & 0.0 & & 0.0 & & 0.1 & & & 02 & 0.00 \\
\hline 9 & 6 & -0. & 0.0 & 0.0 & -0.35 & 0.14 & & & -0.02 & 0.00 \\
\hline 10 & 6 & 0.00 & 0.00 & 0.00 & 0.13 & -0.04 & 0.01 & -0.02 & 0.08 & -0.01 \\
\hline
\end{tabular}




\begin{tabular}{|c|c|c|c|c|c|c|c|c|c|c|}
\hline 11 & 17 & & & & & & & & & \\
\hline 11 & $\begin{array}{l}17 \\
17\end{array}$ & 0.00 & 0.00 & 0.00 & 0.00 & 0.00 & 0.00 & 0.00 & 0.00 & 0.00 \\
\hline & 78 & & & & 0.00 & & 0.00 & 0.00 & & \\
\hline 14 & 7 & 0.00 & 0.00 & 0.00 & 0.00 & 0.00 & 0.00 & 0.00 & 0.00 & 0.00 \\
\hline 15 & 7 & -0.02 & 0.01 & 0.00 & 0.02 & -0.07 & 0.01 & 0.00 & 0.01 & 0.00 \\
\hline 16 & 6 & 0.02 & 0.00 & 0.00 & 0.01 & 0.03 & 0.00 & 0.01 & .00 & .00 \\
\hline 17 & 6 & 0.01 & 0.00 & 0.00 & 0.04 & 0.00 & -0.01 & -0.01 & 0.00 & 0.01 \\
\hline 18 & 6 & -0.01 & 0.00 & 0.01 & -0.01 & 0.00 & 0.01 & 0.00 & 0.00 & 0.00 \\
\hline 19 & 6 & 0.00 & 0.00 & 0.00 & -0.01 & 0.00 & 0.00 & 0.00 & 0.00 & 0.00 \\
\hline 20 & 6 & -0.01 & 0.00 & -0.01 & -0.01 & 0.00 & -0. & 0.00 & 0.00 & 0.00 \\
\hline 21 & 6 & 0.01 & 0.00 & 0.00 & 0.03 & 0.00 & 0.0 & -0.01 & 0.00 & -0.01 \\
\hline 22 & 17 & 0.00 & 0.00 & 0.00 & 0.00 & 0.00 & 0.00 & 0.00 & 0.00 & 0.00 \\
\hline 23 & 17 & 0.00 & 0.00 & 0.00 & 0.00 & 0.00 & & 0.00 & 0.00 & 0.00 \\
\hline 24 & 6 & -0.09 & 0.08 & -0.01 & 0.01 & -0.01 & 0. & 0.0 & 0. & 0.00 \\
\hline 25 & 6 & 0.00 & 0.00 & 0.00 & -0.15 & -0.02 & 0.0 & -0.05 & -0.09 & 0.02 \\
\hline 26 & 1 & 0.00 & -0.01 & 0.00 & -0.22 & -0.31 & 0. & 0.07 & 0.32 & 0.06 \\
\hline 27 & 1 & 0.00 & -0.01 & 0.00 & 0.21 & -0.13 & -0.1 & 0.22 & 0.40 & 0.17 \\
\hline 28 & 1 & 0.00 & 0.00 & 0.01 & 0.20 & -0.08 & 0.15 & 0.23 & 0.31 & -0.30 \\
\hline 29 & 1 & -0.01 & & -0.01 & -0.23 & -0.06 & -0.1 & 0.11 & -0.35 & 0.14 \\
\hline 30 & 1 & -0.01 & & 0.00 & -0.26 & -0.03 & 0.1 & 0.11 & -0.29 & 0.24 \\
\hline 31 & 1 & 0.00 & -0.01 & 0.00 & 0.13 & -0.31 & 0.08 & 0.00 & -0.26 & 0.03 \\
\hline 32 & 1 & -0.05 & -0.01 & -0.01 & -0.11 & -0.02 & -0.0 & 0.03 & 0.01 & 0.01 \\
\hline 33 & 1 & 0.00 & & 0.00 & -0.01 & 0.00 & 0. & 0.01 & 0.00 & \\
\hline 34 & 1 & -0.05 & 0.00 & 0.02 & -0.13 & 0.00 & 0.0 & 0.03 & 0.00 & -0.01 \\
\hline 35 & 1 & 0.02 & -0.01 & 0.00 & 0.04 & -0.01 & -0.02 & 0.03 & -0.01 & -0.01 \\
\hline 36 & 1 & 0.00 & & 0. & 0.01 & 0.00 & & 0.01 & 0.00 & 0.00 \\
\hline 37 & 1 & 0.02 & & & & & & 0.03 & & \\
\hline 38 & 1 & 0.34 & -0.37 & 0.29 & -0.04 & 0.03 & -0.0 & 0.00 & 0.00 & .00 \\
\hline 39 & 1 & 0.34 & -0.4 & -0.14 & -0.04 & 0.04 & 0.0 & 0.0 & .00 & .00 \\
\hline 40 & 1 & 0.49 & -0.2 & 0.05 & -0.03 & 0.01 & 0.0 & 0.00 & 0.00 & 0.00 \\
\hline 41 & 6 & 0.00 & & 0.0 & 0.00 & 0.00 & 0.0 & 0.00 & 0.00 & 0.00 \\
\hline 42 & 6 & 0.00 & & 0.0 & 0.00 & 0.00 & 0. & 0.00 & 0.00 & 0.00 \\
\hline 43 & 1 & 0.00 & & 0. & 0.00 & 0.00 & 0. & 0.0 & 0.00 & 0.00 \\
\hline 44 & 1 & 0.00 & & & 0.00 & 0.00 & 0.0 & 0.0 & 0.00 & 0.00 \\
\hline 45 & 1 & 0.00 & 0.00 & 0.00 & 0.00 & 0.00 & 0.00 & 0.00 & 0.00 & 0.00 \\
\hline quen & & 1459.39 & & & 1464.23 & & & 1470.56 & & \\
\hline Atom & AN & $X$ & $Y$ & Z & $X$ & $\mathrm{Y}$ & Z & $X$ & Y & Z \\
\hline 1 & 6 & 0.00 & 0.00 & -0.01 & 0.00 & 0.00 & 0.00 & & 0.00 & -0.01 \\
\hline 2 & 6 & -0.02 & 0.01 & 0.02 & & 0.00 & 0.0 & $-0 .($ & 0.01 & 0.01 \\
\hline 3 & 6 & 0.02 & & 0.00 & & 0.00 & 0.0 & 0. & 0.00 & 0.00 \\
\hline 4 & 6 & -0.02 & & -0.02 & & & & & & -0.02 \\
\hline 5 & 6 & 0.00 & & 0.0 & 0.0 & 0.00 & 0.0 & 0.0 & 0.00 & 0.01 \\
\hline 6 & 6 & & & 0. & & 0.00 & 0.0 & 0.0 & 0.00 & 0.00 \\
\hline 7 & 7 & & & & & & 0. & & & 0.00 \\
\hline 8 & 6 & -0. & & 0. & & & 0. & & & 0.00 \\
\hline 9 & 6 & & & -0.01 & & 0. & 0. & -0.0 & -0.05 & 0.01 \\
\hline 10 & 6 & & & 0. & & -0.01 & 0.0 & 0. & -0.04 & 0.01 \\
\hline 11 & 17 & & & & & & & & & 0.00 \\
\hline 12 & 17 & & & & & & & & & \\
\hline 13 & 78 & & & & & & 0.0 & 0.00 & 0.00 & 0.00 \\
\hline 14 & 7 & & & & & & 0. & & & 0.00 \\
\hline 15 & 7 & & & & & & & & & \\
\hline 16 & 6 & -0.0 & & 0.00 & 0.00 & 0.00 & 0.00 & 0.21 & 0.02 & -0.01 \\
\hline 17 & 6 & 0.0 & & -0.03 & & & 0.0 & -0.12 & 0.02 & 0.13 \\
\hline 18 & 6 & 0.01 & & 0.0 & 0.00 & 0.00 & 0.0 & -0.0 & -0.02 & -0.07 \\
\hline 19 & 6 & -0.02 & & 0.0 & 0.00 & 0.00 & 0.00 & 0.08 & 0.01 & 0.00 \\
\hline 20 & 6 & & & -0.0 & & 0.0 & 0. & -0.04 & 0.01 & 0.08 \\
\hline 21 & 6 & & & 0.0 & 0.00 & 0.00 & 0. & -0.1 & -0.04 & -0.12 \\
\hline 22 & 17 & & & 0.0 & 0.00 & 0.00 & 0.0 & 0.00 & 0.00 & -0.01 \\
\hline 23 & 17 & 0.00 & 0.00 & 0.00 & 0.00 & 0.00 & 0.00 & 0.00 & 0.00 & 0.01 \\
\hline
\end{tabular}




\begin{tabular}{|c|c|c|c|c|c|c|c|c|c|c|}
\hline 24 & 6 & 0.00 & 0.00 & 0.00 & 0.00 & 0.00 & 0.00 & 0.00 & 0.00 & 0.00 \\
\hline & 6 & -0.04 & -0.07 & 0.01 & 0.00 & 0.00 & 0.00 & -0.01 & -0.02 & 0.00 \\
\hline 26 & 1 & 0.06 & 0.25 & -0.04 & 0.00 & 0.02 & 0.00 & 0.01 & 0.05 & -0.01 \\
\hline 27 & 1 & 0.16 & 0.32 & 0.13 & 0.01 & 0.02 & 0.01 & 0.05 & 0.10 & 0.05 \\
\hline 28 & 1 & 0.18 & 0.25 & -0.24 & 0.01 & 0.02 & -0.02 & 0.05 & 0.08 & -0.08 \\
\hline 29 & 1 & -0.13 & 0.42 & 0.17 & -0.01 & 0.03 & 0.01 & -0.19 & 0.21 & 0.03 \\
\hline 30 & 1 & -0.13 & 0.35 & -0.30 & -0.01 & 0.02 & -0.02 & -0.21 & 0.19 & -0.10 \\
\hline 31 & 1 & 0.00 & 0.32 & -0.04 & 0.00 & 0.02 & 0.00 & 0.01 & 0.27 & -0.02 \\
\hline 32 & 1 & -0.13 & -0.03 & -0.05 & -0.01 & 0.00 & 0.00 & 0.49 & 0.10 & 0.20 \\
\hline 33 & 1 & -0.02 & 0.00 & 0.00 & 0.00 & 0.00 & 0.0 & 0.09 & 0.01 & 0.00 \\
\hline 34 & 1 & -0.13 & 0.00 & 0.06 & -0.01 & 0.00 & 0.00 & 0.47 & 0.01 & -0.22 \\
\hline 35 & 1 & 0.08 & -0.03 & -0.03 & 0.00 & 0.00 & 0.00 & 0.05 & -0.02 & -0.02 \\
\hline 36 & 1 & 0.01 & 0.00 & 0.00 & 0.00 & 0.00 & 0.0 & 0.01 & 0.00 & 0.00 \\
\hline 37 & 1 & 0.07 & -0.01 & 0.04 & 0.00 & 0.00 & 0.00 & 0.05 & -0.01 & 0.03 \\
\hline 38 & 1 & 0.00 & 0.01 & 0.00 & 0.00 & 0.00 & 0.00 & 0.01 & -0.01 & 0.01 \\
\hline 39 & 1 & 0.00 & & 0.00 & 0.00 & 0.00 & 0.0 & 0.01 & -0.01 & 0.00 \\
\hline 40 & 1 & -0.01 & 0.00 & 0.00 & 0.00 & 0.00 & 0.0 & 0.02 & -0.01 & 0.00 \\
\hline 41 & 6 & 0.00 & 0.00 & 0.00 & 0.00 & 0.00 & 0.00 & 0.00 & 0.00 & 0.00 \\
\hline 42 & 6 & -0.01 & & 0.0 & 0.09 & & -0.01 & 0.00 & 0.00 & \\
\hline 43 & 1 & 0.03 & & -0.02 & -0.46 & -0.25 & 0.24 & 0.00 & 0.00 & 0.00 \\
\hline 44 & 1 & 0.02 & 0.04 & -0.01 & -0.22 & -0.51 & 0.09 & 0.00 & 0.00 & 0.00 \\
\hline 45 & 1 & 0.03 & 0.02 & 0.01 & -0.46 & -0.32 & -0.15 & 0.00 & 0.00 & 0.00 \\
\hline \multicolumn{2}{|c|}{ Frequencies } & 1474.24 & & & 1489.46 & & & 1491.51 & & \\
\hline Atom & AN & $X$ & $Y$ & Z & $x$ & $Y$ & Z & $x$ & $Y$ & Z \\
\hline 1 & 6 & -0.04 & 0.00 & -0.08 & 0.00 & 0.00 & 0.00 & 0.13 & -0.03 & 0.02 \\
\hline 2 & 6 & -0.12 & 0.06 & 0.13 & 0.00 & 0.00 & 0.00 & -0.06 & 0.00 & -0.08 \\
\hline 3 & 6 & 0.21 & -0.06 & 0.01 & 0.00 & 0.00 & 0.00 & 0.00 & 0.02 & 0.12 \\
\hline 4 & 6 & -0.12 & 0.01 & -0.15 & 0.00 & 0.00 & 0.00 & 0.06 & -0.03 & -0.07 \\
\hline 5 & 6 & -0.05 & 0.03 & 0.08 & 0. & 0.00 & 0.00 & -0.1 & 0.04 & 0.00 \\
\hline 6 & 6 & 0.08 & -0.02 & 0.01 & 0.00 & 0.00 & 0.00 & 0.00 & 0.02 & 0.12 \\
\hline 7 & 7 & -0.04 & -0.02 & 0.00 & 0.00 & 0.00 & 0.00 & 0.00 & 0.00 & -0.02 \\
\hline 8 & 6 & -0.03 & 0.06 & -0.01 & 0.0 & 0.00 & 0.0 & 0.0 & 0.00 & 0.00 \\
\hline & 6 & & & 0. & & 0.00 & 0.0 & 0. & 0.00 & 0.00 \\
\hline 10 & 6 & & 0. & 0. & & 0.00 & 0.0 & & 0.00 & 0.00 \\
\hline 11 & 17 & 0. & 0.00 & 0.0 & & 0.00 & 0.0 & 0.0 & 0.00 & 0.00 \\
\hline 12 & 17 & & & -0 & & 0.00 & 0.0 & 0.00 & 0.00 & 0.00 \\
\hline 13 & 78 & & & 0. & & 0.00 & 0.0 & 0.0 & 0.00 & 0.00 \\
\hline 14 & 7 & & & 0. & & 0.00 & 0.8 & 0. & 0.00 & 0.00 \\
\hline 15 & 7 & -0.01 & & 0. & & 0.00 & 0.0 & 0.0 & 0.00 & 0.00 \\
\hline 16 & 6 & -0 . & & & & -0.03 & -0 . & & & 0. \\
\hline 17 & 6 & 0.0 & 0. & -0.01 & & 0.02 & 0.0 & & & 0. \\
\hline 18 & 6 & 0.00 & & 0.00 & -0.13 & -0.02 & -0.01 & 0.1 & 0.00 & 0. \\
\hline 19 & 6 & 0.00 & 0. & 0. & & -0.02 & -0 . & 0.0 & 0.00 & 0.00 \\
\hline 20 & 6 & 0.00 & & 0. & & 0.01 & -0 . & & & \\
\hline 21 & 6 & & & 0. & -0.06 & 0.0 & 0.0 & 0.1 & 0.00 & 0. \\
\hline 22 & 17 & & & & & 0.00 & 0.0 & 0. & 0.00 & 0.00 \\
\hline 23 & 17 & & & & & & & & & \\
\hline 24 & 6 & & & & & & 0. & & & \\
\hline 25 & 6 & 0. & & 0. & & 0.00 & 0.0 & 0. & 0.00 & -0.01 \\
\hline 26 & 1 & & & & & & & & & \\
\hline 27 & 1 & -0 & & -0.0 & & 0.00 & & -0 . & 0.00 & ח \\
\hline 28 & 1 & -0 . & & 0. & -0.01 & 0.0 & 0.0 & 0. & 0.00 & 0.01 \\
\hline 29 & 1 & 0.0 & & -0.0 & -0.0 & & 0. & & & 0.00 \\
\hline 30 & 1 & 0.0 & & 0.0 & & 0.00 & 0. & 0. & 0.00 & 0.00 \\
\hline 31 & 1 & 0.0 & & 0.00 & 0.0 & -0.01 & -0.04 & 0.00 & 0.00 & 0.01 \\
\hline 32 & 1 & -0.0 & & -0 . & 0.3 & 0.10 & 0.2 & 0. & 0.00 & 0.00 \\
\hline 33 & 1 & 0.0 & & & & & 0.6 & & 0.00 & 001 \\
\hline 34 & 1 & -0.0 & & 0.0 & -0.37 & 0.02 & 0.3 & 0.00 & 0.00 & 0.00 \\
\hline 35 & 1 & 0.5 & -0.1 & -0.20 & 0.00 & 0.00 & 0.00 & 0.35 & -0.15 & -0.26 \\
\hline 36 & 1 & 0.10 & -0.03 & 0.00 & 0.00 & 0.00 & -0.01 & 0.01 & -0.12 & 0 \\
\hline
\end{tabular}




\begin{tabular}{|c|c|c|c|c|c|c|c|c|c|c|}
\hline 37 & 1 & 0.49 & -0.10 & 0.25 & 0.00 & 0.00 & 0.00 & -0.35 & 0.05 & -0.31 \\
\hline & 1 & -0.06 & -0.03 & -0.04 & 0.00 & -0.01 & 0.00 & 0.00 & 0.02 & 0.00 \\
\hline 39 & 1 & -0.06 & -0.01 & 0.05 & 0.00 & 0.01 & 0.00 & .00 & -0.02 & 0.00 \\
\hline 40 & 1 & 0.03 & & 0.00 & 0.00 & 0.00 & & & & 0.03 \\
\hline 41 & 6 & 0.00 & & & 0.00 & & & & & .00 \\
\hline 42 & 6 & 0.00 & & 0.0 & 0.00 & 0. & & & & 00 \\
\hline 43 & 1 & 0.00 & & 0.0 & 0.00 & & & & & 0.00 \\
\hline 44 & 1 & 0.00 & & 0.0 & 0.00 & & & 0 & & 0.00 \\
\hline 45 & 1 & 0.00 & & 0.00 & 0.00 & 0.00 & 00 & 0.00 & .00 & .00 \\
\hline \multicolumn{2}{|c|}{ Frequencies } & \multicolumn{2}{|l|}{1503.12} & & \multicolumn{2}{|l|}{1503.54} & & \multicolumn{2}{|l|}{1516.72} & \multirow[b]{2}{*}{ Z } \\
\hline \multicolumn{2}{|c|}{ Atom AN } & X & Y & Z & $x$ & & Z & $X$ & Y & \\
\hline 1 & 6 & 0.00 & 0.00 & 0.00 & -0.01 & 0.00 & -0.01 & 0.00 & 0.00 & 0.00 \\
\hline 2 & 6 & 0.00 & 0.00 & 0.00 & -0.01 & 0.00 & 0.01 & 0.00 & & 0.00 \\
\hline 3 & 6 & -0.01 & 0.00 & 0.00 & 0.03 & -0.01 & 0.00 & 0.00 & 0.00 & 0.00 \\
\hline 4 & 6 & 0.00 & 0.00 & 0.00 & -0.01 & 0.00 & -0.01 & 0.00 & .00 & 0.00 \\
\hline 5 & 6 & 0.00 & & 0.00 & 0.0 & 0.00 & 0.0 & 0.00 & & 0.00 \\
\hline 6 & 6 & 0.00 & & 0.00 & & 0.00 & & & .00 & 0.00 \\
\hline 7 & 7 & 0.02 & -0.03 & 0.00 & -0.02 & 0.03 & -0. & & .00 & 0.00 \\
\hline 8 & 6 & 0.00 & & 0.00 & & -0.02 & & & & 0.00 \\
\hline 9 & 6 & 0.00 & & 0.00 & & 0.02 & & 0.00 & & 0.00 \\
\hline 10 & 6 & 0.02 & & 0.00 & -0.03 & 0.00 & 0. & 0.00 & 0.00 & 0.00 \\
\hline 11 & 17 & 0. & & 0.00 & 0. & & & & 0 & 0.00 \\
\hline 12 & 17 & 0. & & 0.00 & 0.0 & & & & & \\
\hline 13 & 78 & & & & & 0.00 & & & 0.00 & 0.00 \\
\hline 14 & 7 & 0. & & 0.0 & & 0.00 & & & & 0.00 \\
\hline 15 & 7 & & & 0.1 & -0. & -0.02 & & & & \\
\hline 16 & 6 & & & 0.0 & & 0.00 & 0.0 & & & 0.00 \\
\hline 17 & 6 & 0.00 & & 0.0 & & 0.00 & & & .00 & 0.00 \\
\hline 18 & 6 & & & 0.0 & & 0.00 & 0. & & & 0.00 \\
\hline 19 & 6 & 0.00 & & 0.0 & 0. & 0.00 & 0. & 0.0 & & 0.00 \\
\hline 20 & 6 & 0. & & 0.0 & & 0.00 & & & 0 & 0.00 \\
\hline 21 & 6 & 0. & & 0.0 & 0. & 0.00 & & 0. & & 0. \\
\hline 22 & 17 & & & & & & & & & \\
\hline 23 & 17 & & & & & & & & & 0.00 \\
\hline 24 & 6 & & & 0. & -0. & -0.03 & 0. & & & 0. \\
\hline 25 & 6 & & & & & & & & & 0.00 \\
\hline 26 & 1 & & & & & & & & & 0.00 \\
\hline 27 & 1 & & & 0. & & -0.23 & & & & \\
\hline 28 & 1 & & & -0.16 & & -0.16 & & & & 0.00 \\
\hline 29 & 1 & & & & & & & & & 0.00 \\
\hline 30 & 1 & & & 0.12 & 0.22 & 0.10 & & & & \\
\hline 31 & 1 & & & -0.03 & & -0.28 & 0. & & & 0. \\
\hline 32 & 1 & & & & & & & & & 0.00 \\
\hline 33 & 1 & & & & & & & & & \\
\hline 34 & 1 & & & & & 0. & & & & 0.00 \\
\hline 35 & 1 & & & & & & & & & 0.00 \\
\hline 36 & 1 & & & & & & & & & \\
\hline 37 & 1 & & & & 0.0 & & & & & \\
\hline 38 & 1 & & & & & & & & & 0.01 \\
\hline 39 & 1 & & & & & & & & & \\
\hline 40 & 1 & & & 0.0 & -0.22 & 0.0 & & & & 0.00 \\
\hline 41 & 6 & & & 0. & 0. & 0. & & -0.02 & & 0.00 \\
\hline 42 & 6 & & & & & & & & & 0.00 \\
\hline 43 & 1 & 0.00 & & -0.0 & 0.0 & & & & & 0.45 \\
\hline 44 & 1 & & & 0.0 & 0.0 & -0.01 & 0.1 & 0. & 0.41 & -0.09 \\
\hline 45 & 1 & 0.00 & & 0.00 & 0.00 & 0.01 & 0.00 & & -0.54 & -0.27 \\
\hline- & & & & & & & & & & \\
\hline Atom & AN & $X$ & Y & Z & $X$ & $\mathrm{Y}$ & Z & & $\mathrm{Y}$ & Z \\
\hline & 6 & 0.00 & 0.00 & 0.00 & 0.00 & 0.00 & 0.00 & 0.00 & 0.00 & 0.00 \\
\hline & 6 & 0.00 & 0.00 & 0.00 & 0.00 & 0.00 & 0.00 & 0.00 & 0.00 & 0.00 \\
\hline
\end{tabular}




\begin{tabular}{|c|c|c|c|c|c|c|c|c|c|c|}
\hline & & & & & & & & & & \\
\hline 3 & 6 & 0.00 & 0.00 & 0.00 & 0.00 & 0.00 & 0.00 & 0.00 & 0.00 & 0.00 \\
\hline 4 & 6 & 0.00 & 0.00 & 0.00 & 0.00 & 0.00 & 0.00 & 0.00 & 0.00 & 0.00 \\
\hline 5 & 6 & 0.00 & 0.00 & 0.00 & 0.00 & 0.00 & 0.00 & 0.00 & 0.00 & 0.00 \\
\hline 6 & 6 & 0.00 & 0.00 & 0.00 & 0.00 & 0.00 & 0.00 & 0.00 & 0.00 & 0.00 \\
\hline 7 & 7 & 0.00 & 0.00 & 0.00 & 0.00 & -0.01 & 0.00 & 0.00 & 0.00 & 0.00 \\
\hline 8 & 6 & 0.00 & 0.00 & 0.00 & & 0.00 & -0.02 & 0.00 & 0.00 & 0.00 \\
\hline 9 & 6 & 0.00 & 0.00 & 0.00 & & 0.01 & 0.02 & 0.00 & 0.00 & 0.00 \\
\hline 10 & 6 & 0.00 & 0.00 & 0.00 & .00 & 0.00 & 0.03 & 0.00 & 0.00 & -0.01 \\
\hline 11 & 17 & 0.00 & 0.00 & 0.00 & & 0.00 & 0.00 & 0.00 & 0.00 & 0.00 \\
\hline 12 & 17 & 0.00 & 0.00 & 0.00 & & 0.00 & 0.0 & 0.00 & 0.00 & 0.00 \\
\hline 13 & 78 & 0.00 & 0.00 & 0.00 & & 0.00 & 0.0 & 0.00 & 0.00 & \\
\hline 14 & 7 & 0.00 & 0.00 & 0.00 & & 0.00 & 0.00 & 0.00 & 0.00 & 0.00 \\
\hline 15 & 7 & 0.00 & 0.00 & 0.00 & & -0.01 & 0.00 & 0.00 & 0.00 & 0.00 \\
\hline 16 & 6 & 0.00 & 0.00 & 0.00 & & 0.00 & 0.0 & & & \\
\hline 17 & 6 & 0.00 & 0.00 & 0.00 & & 0.00 & 0.0 & & & 0.00 \\
\hline 18 & 6 & 0.00 & 0.00 & 0.00 & & 0.00 & 0.0 & 0.0 & 0.00 & 0.00 \\
\hline 19 & 6 & 0.00 & 0.00 & 0.00 & & 0.00 & 0.0 & 0.0 & & \\
\hline 20 & 6 & 0.00 & 0.00 & 0.00 & & 0.00 & 0.00 & 0.0 & 0.00 & \\
\hline 21 & 6 & 0.00 & 0.00 & 0.00 & & 0.00 & 0.00 & 0.0 & 0.00 & 0.00 \\
\hline 22 & 17 & 0.00 & 0.00 & 0.00 & & 0.00 & 0.0 & 0.0 & .00 & \\
\hline 23 & 17 & 0.00 & 0.00 & 0.00 & & 0.00 & 0.00 & 0.00 & 0.00 & \\
\hline 24 & 6 & 0.00 & 0.00 & 0.01 & & 0.00 & -0.01 & 0.00 & -0.01 & -0.07 \\
\hline 25 & 6 & 0.00 & 0.00 & 0.00 & & -0.01 & -0.03 & 0.0 & 0.00 & 0.00 \\
\hline 26 & 1 & 0.00 & 0.00 & 0.00 & -0.02 & 0.05 & 0.49 & 0.00 & -0.01 & -0.07 \\
\hline 27 & 1 & 0.00 & 0.00 & 0.00 & -0.38 & 0.00 & 0.05 & 0.05 & 0.00 & -0.01 \\
\hline 28 & 1 & 0.00 & 0.00 & 0.00 & & 0.06 & 0.01 & -0.04 & -0.01 & 0.00 \\
\hline 29 & 1 & 0.0 & 0.00 & 0.00 & & & -0.03 & 0.0 & -0.02 & \\
\hline 30 & 1 & 0.00 & 0.00 & 0.00 & & -0.09 & -0.04 & -0.06 & 0.02 & 0. \\
\hline 31 & 1 & 0.00 & 0.00 & 0.00 & & -0.13 & -0.47 & 0.00 & 0.02 & \\
\hline 32 & 1 & 0.00 & 0.00 & 0.00 & & 0.00 & 0. & & & \\
\hline 33 & 1 & 0.0 & 0.00 & 0.00 & & 0. & & & & \\
\hline 34 & 1 & 0.0 & 0.00 & 0.0 & & 0. & -0.0 & 0. & & 0.0 \\
\hline 35 & 1 & 0.00 & 0.00 & 0.00 & & 0.00 & 0. & & 0. & \\
\hline 36 & 1 & 0.0 & 0.00 & 0.0 & & 50 & & & & \\
\hline 37 & 1 & 0.1 & 0.00 & 0.0 & & & 0.0 & & & 0.01 \\
\hline 38 & 1 & -0.01 & -0.05 & 0.00 & & 0.06 & 0.0 & 0.08 & 0.44 & -0.01 \\
\hline 39 & 1 & 0.0 & 0.05 & -0.02 & -0.02 & -0.07 & 0.03 & -0.12 & -0.44 & 0.17 \\
\hline 40 & 1 & 0.0 & -0.01 & -0.07 & & 0.02 & 0.1 & 0.0 & 0.12 & 0.72 \\
\hline 41 & 6 & 0. & 0. & 0. & & & 0.0 & & & \\
\hline 42 & 6 & 0.0 & 0.0 & 0.0 & & 0 & 0.0 & 0.0 & & \\
\hline 43 & 1 & -0.52 & 0.0 & -0.06 & & 0.00 & 0.00 & -0.05 & 0.00 & 0.00 \\
\hline 44 & 1 & 0.00 & -0 . & -0. & & & -0.01 & & -0.01 & \\
\hline 45 & 1 & 0 & -0.01 & -0.03 & & 0.00 & 0.00 & 0.0 & 0.00 & \\
\hline Frequer & & 1526.70 & & & 153 & & & 1593.47 & & \\
\hline Atom & AN & X & $\mathrm{Y}$ & Z & , & $\mathrm{Y}$ & Z & & $Y$ & Z \\
\hline & 6 & -0.01 & 0.00 & 0.00 & 0.00 & 0.00 & 0.00 & & 0.00 & 0.01 \\
\hline 2 & 6 & 0. & 0.0 & 0.01 & & 0.00 & 0.00 & & 0.00 & -0.01 \\
\hline 3 & 6 & 0. & -0.01 & 0.00 & & 0.00 & 0.00 & & 0.00 & \\
\hline 4 & 6 & 0. & 0.00 & -0.01 & & & 0.0 & & & \\
\hline 5 & 6 & 0. & 0.0 & 0.0 & & & 0.00 & & 0.00 & \\
\hline 6 & 6 & 0. & 0.0 & 0.0 & & 0.00 & -0.01 & 0. & 0.00 & -0.0 \\
\hline 7 & 7 & $-0 .($ & 0.04 & $-0 .($ & & & 0.00 & & 0.00 & \\
\hline 8 & 6 & -0. & -0.04 & 0. & & & -0.0 & & 0.00 & \\
\hline 9 & 6 & 0.0 & -0.04 & 0.01 & & 0.00 & -0.01 & 0.00 & 0.00 & 0.00 \\
\hline 10 & 6 & & 0.00 & 0.01 & & & -0.03 & & & \\
\hline 11 & 17 & & 0.00 & 0.00 & & & 0.0 & & & \\
\hline 12 & 17 & & 0.00 & 0.00 & & 0.00 & 0.00 & 0.00 & 0.00 & 0.00 \\
\hline 13 & 78 & & 0.00 & 0.00 & & 0.00 & 0.00 & 0.00 & 0.00 & \\
\hline 14 & 7 & & 0.00 & 0.00 & & 0.00 & 0.00 & 0.0 & 0.00 & \\
\hline 15 & 7 & 0.04 & 0.03 & -0.01 & 0.00 & 0.00 & 0.00 & 0.00 & 0.00 & \\
\hline
\end{tabular}




\begin{tabular}{|c|c|c|c|c|c|c|c|c|c|c|}
\hline 16 & 6 & -0.03 & -0.01 & 0.00 & 0.00 & 0.00 & 0.00 & 0.00 & -0.06 & -0.27 \\
\hline 17 & 6 & 0.00 & 0.00 & -0.01 & 0.00 & 0.00 & 0.00 & -0.08 & 0.03 & 0.17 \\
\hline 18 & 6 & 0.01 & 0.00 & 0.00 & 0.00 & 0.00 & 0.00 & -0.06 & -0.05 & -0.21 \\
\hline 19 & 6 & -0.01 & 0.00 & 0.00 & 0.00 & 0.00 & -0.01 & 0.00 & 0.09 & 0.41 \\
\hline & 6 & 0.01 & 0.00 & -0.01 & 0.00 & 0.00 & 0.00 & 0.06 & -0.04 & -0.22 \\
\hline 21 & 6 & 0.00 & 0.00 & 0.01 & 0.00 & 0.00 & 0.0 & 0.08 & 0.05 & 0.17 \\
\hline 22 & 17 & 0.00 & 0.00 & 0.00 & 0.00 & 0.00 & 0.00 & 0.00 & 0.00 & 0.00 \\
\hline 23 & 17 & 0.00 & 0.00 & 0.00 & 0.00 & 0.00 & 0.00 & 0.00 & 0.00 & 0.00 \\
\hline 24 & 6 & 0.00 & 0.00 & 0.00 & 0.00 & 0.00 & 0.00 & 0.00 & 0.00 & 0.00 \\
\hline 25 & 6 & -0.03 & 0.00 & -0.0 & 0.00 & -0.01 & -0.03 & 0.00 & 0.00 & 0.00 \\
\hline 26 & 1 & 0.08 & 0.34 & 0.03 & -0.01 & 0.08 & 0.49 & 0.00 & 0.00 & 0.02 \\
\hline 27 & 1 & 0.17 & -0.22 & -0.13 & -0.37 & -0.02 & 0.04 & -0.02 & 0.00 & 0.00 \\
\hline 28 & 1 & 0.29 & -0.14 & 0.22 & 0.34 & 0.04 & 0.0 & 0.02 & 0.00 & 0.00 \\
\hline 29 & 1 & -0.37 & -0.21 & -0.23 & 0.35 & -0.11 & 0.07 & 0.04 & -0.02 & 0.01 \\
\hline 30 & 1 & -0.31 & -0.16 & 0.25 & -0.32 & 0.11 & 0.00 & -0.04 & 0.02 & 0.00 \\
\hline 31 & 1 & 0.00 & 0.43 & -0.12 & -0.02 & 0.06 & & 0.0 & 0.01 & 0.04 \\
\hline 32 & 1 & -0.03 & & -0.01 & 0.00 & 0.00 & 0.0 & 0.2 & 0.01 & -0.09 \\
\hline 33 & 1 & -0.01 & 0.00 & 0.00 & 0.00 & 0.01 & 0.0 & -0.01 & -0.14 & -0.65 \\
\hline 34 & 1 & -0.03 & & 0.02 & 0.00 & 0.00 & 0. & -0.24 & -0.04 & -0.06 \\
\hline 35 & 1 & 0.02 & & -0.01 & 0.00 & 0.00 & 0. & 0.01 & 0.00 & 0.01 \\
\hline 36 & 1 & 0.01 & & 0.00 & 0.00 & 0.00 & 0.0 & 0.00 & 0.01 & 0.04 \\
\hline 37 & 1 & 0.02 & & 0. & 0.00 & 0.00 & 0. & -0.01 & .00 & 0.00 \\
\hline 38 & 1 & & & 0.01 & 0.00 & -0.01 & 0.0 & 0.00 & & \\
\hline 39 & 1 & 0.01 & 0.00 & -0.01 & 0.00 & 0.01 & 0.0 & 0.00 & -0.01 & 0.00 \\
\hline 40 & 1 & -0.01 & & 0.0 & 0.00 & 0.00 & -0.0 & 0.00 & 0.00 & 0.01 \\
\hline 41 & 6 & 0.00 & & 0. & 0.00 & 0.00 & 0.1 & 0.00 & .00 & 0.00 \\
\hline 42 & 6 & 0.00 & & 0. & 0.00 & 0.00 & 0. & 0.00 & 0.00 & 0.00 \\
\hline 43 & 1 & 0.00 & & 0.0 & 0.00 & 0.00 & 0.0 & 0.00 & 0.00 & 0.00 \\
\hline 44 & 1 & 0.00 & & 0. & 0.00 & 0.00 & 0.1 & 0.1 & 0.00 & 0.00 \\
\hline 45 & 1 & 0.00 & & 0.00 & 0.00 & 0.00 & 0.00 & 0.00 & 0.00 & 0.00 \\
\hline \multicolumn{2}{|c|}{ Frequencies } & 1597.85 & & & 1607.31 & & & 1608.27 & & \\
\hline Atom & AN & $X$ & $Y$ & Z & $x$ & Y & Z & $X$ & Y & Z \\
\hline 1 & 6 & -0.06 & -0.02 & -0.22 & 0.14 & -0.03 & 0.06 & 0.26 & -0.05 & 0.12 \\
\hline 2 & 6 & -0.08 & & 0.17 & -0.12 & 0.04 & 0.02 & -0. & & 0.02 \\
\hline 3 & 6 & 0.00 & -0.05 & -0.28 & 0.10 & -0.03 & 0.01 & 0.13 & -0.04 & 0.01 \\
\hline 4 & 6 & 0.08 & & 0.18 & -0.12 & 0.03 & -0.03 & -0.22 & 0.05 & -0.04 \\
\hline 5 & 6 & 0.06 & & -0.21 & 0.14 & -0.05 & -0.04 & 0.27 & -0.09 & -0.09 \\
\hline 6 & 6 & 0.00 & & 0.41 & -0.08 & 0.02 & 0.00 & -0.15 & 0.04 & -0.01 \\
\hline 7 & 7 & 0.00 & 0.00 & 0.02 & -0.06 & 0.09 & -0.02 & -0.01 & -0.01 & 0.00 \\
\hline 8 & 6 & & & -0 . & & -0.12 & 0.02 & -0.0 & & 0.00 \\
\hline 9 & 6 & 0. & & 0. & -0.05 & -0.07 & 0.0 & 0. & 0. & 0.00 \\
\hline 10 & 6 & & & 0. & 0.00 & 0.01 & 0.00 & 0.0 & 0.00 & 0.00 \\
\hline 11 & 17 & & & 0. & & 0.00 & 0.00 & 0.00 & 0.00 & 0.00 \\
\hline 12 & 17 & & & & & & & & & 0.00 \\
\hline 13 & 78 & & & & & 0.0 & 0.0 & 0.0 & 0.1 & 0.00 \\
\hline 14 & 7 & & & & & 0.00 & 0.00 & 0.0 & 0.00 & 0.00 \\
\hline 15 & 7 & & & & & 0.04 & & & & 0.00 \\
\hline 16 & 6 & & & & & 0.0 & 0.0 & -0. & -0.01 & 0.00 \\
\hline 17 & 6 & & & 0. & & -0.02 & 0. & 0.12 & & -0.02 \\
\hline 18 & 6 & & & & & & & -0 . & & -0.05 \\
\hline 19 & 6 & & & 0.0 & -0.14 & -0.02 & 0.0 & & 0.01 & 0.00 \\
\hline 20 & 6 & 0. & & -0.0 & 0.25 & 0.01 & -0.10 & -0.15 & 0.00 & 0.06 \\
\hline 21 & 6 & & & & -0.21 & -0.03 & -0. & & 0.02 & 0.01 \\
\hline 22 & 17 & 0.0 & & 0.0 & 0.00 & 0.00 & 0.0 & 0.0 & 0.00 & 0.00 \\
\hline 23 & 17 & & & 0. & & 0.00 & 0.0 & 0.00 & 0.00 & 0.00 \\
\hline 24 & 6 & & 0. & 0.0 & 0.0 & 0. & 0.00 & 0.00 & 0.00 & 0.00 \\
\hline 25 & 6 & & & & & 0.01 & & & 0.00 & 0.00 \\
\hline 26 & 1 & & & 0.0 & -0.03 & -0.12 & 0.0 & 0.0 & 0.02 & 0.00 \\
\hline 27 & 1 & -0.0 & -0.0 & 0.00 & -0.03 & 0.13 & 0.06 & 0.00 & -0.04 & -0.01 \\
\hline 28 & 1 & 0.04 & 0.01 & 0.00 & -0.02 & 0.10 & -0.10 & 0.00 & -0.03 & 0.03 \\
\hline
\end{tabular}




\begin{tabular}{|c|c|c|c|c|c|c|c|c|c|c|}
\hline 29 & 1 & 0.02 & -0.01 & 0.00 & 0.02 & 0.09 & 0.04 & 0.00 & -0.03 & -0.01 \\
\hline & 1 & -0.02 & 0.01 & 0.00 & 0.03 & 0.07 & -0.07 & -0.01 & -0.02 & 0.02 \\
\hline 31 & 1 & 0.00 & 0.00 & 0.02 & 0.00 & -0.07 & 0.01 & 0.00 & 0.02 & 0.00 \\
\hline 32 & 1 & 0.01 & 0.00 & 0.00 & -0.35 & -0.09 & -0.23 & 0.21 & 0.05 & 0.14 \\
\hline 33 & 1 & 0.00 & -0.01 & -0.04 & -0.16 & -0.02 & 0.01 & 0.10 & 0.01 & -0.01 \\
\hline 34 & 1 & -0.01 & 0.00 & 0.00 & -0.34 & 0.02 & 0.26 & 0.20 & -0.01 & 015 \\
\hline 35 & 1 & -0.23 & & -0.08 & -0.18 & 0.07 & 0.13 & -0.35 & 0.14 & 0.24 \\
\hline 36 & 1 & 0.01 & -0.12 & -0.64 & -0.09 & 0.02 & -0.01 & -0.17 & 0.05 & -0.01 \\
\hline 37 & 1 & 0.23 & -0.08 & -0.05 & -0.18 & 0.02 & -0.15 & -0.35 & .05 & -0.28 \\
\hline 38 & 1 & 0.00 & 0.0 & 0.00 & 0.01 & 0.00 & 0.01 & -0.02 & 0.00 & -0.01 \\
\hline 39 & 1 & 0.00 & -0.01 & 0.00 & 0.01 & 0.00 & -0.01 & -0.02 & 0.00 & 0.01 \\
\hline 40 & 1 & 0.00 & & 0.02 & 0.00 & 0.00 & 0.00 & 0.00 & 0.00 & 0.00 \\
\hline 41 & 6 & 0.00 & & 0.00 & 0.00 & 0.00 & 0.0 & 0.00 & 0.00 & 0.00 \\
\hline 42 & 6 & 0.00 & 0.00 & 0.00 & 0.00 & 0.00 & 0.00 & 0.00 & 0.00 & 0.00 \\
\hline 43 & 1 & 0.00 & 0.00 & 0.00 & 0.00 & 0.00 & 0.00 & 0.00 & 0.00 & 0.00 \\
\hline 44 & 1 & 0.00 & & 0.00 & 0.00 & 0.00 & 0.00 & 0.00 & 0.00 & \\
\hline 45 & 1 & 0.00 & 0.00 & 0.00 & 0.00 & 0.00 & 0.00 & 0.00 & 0.00 & 0.00 \\
\hline \multicolumn{2}{|c|}{ Frequencies } & 1609.04 & & & 1674.09 & & & 2392.22 & & \\
\hline Atom & AN & $x$ & Y & Z & $x$ & Y & Z & $x$ & Y & Z \\
\hline 1 & 6 & 0.04 & -0.01 & 0.02 & -0.01 & 0.00 & 0.00 & 0.00 & 0.00 & 0.00 \\
\hline 2 & 6 & -0.03 & 0.00 & -0.01 & 0.00 & 0.00 & 0.01 & 0.00 & 0.00 & 0.00 \\
\hline 3 & 6 & -0.06 & 0.02 & 0.00 & 0.03 & -0.02 & 0.00 & 0.00 & 0.00 & 0.00 \\
\hline 4 & 6 & -0.03 & & 0.01 & & 0.00 & -0.01 & 0.00 & 0.00 & \\
\hline 5 & 6 & 0.04 & -0.02 & -0.01 & -0.01 & 0.00 & 0.00 & 0.00 & 0.00 & 0.00 \\
\hline 6 & 6 & -0.03 & 0.01 & 0.00 & 0.00 & 0.00 & 0.00 & 0.00 & 0.00 & 0.00 \\
\hline 7 & 7 & 0.15 & -0.24 & 0.04 & -0.08 & 0.19 & -0.04 & 0.0 & 0.00 & \\
\hline 8 & 6 & -0.10 & 0.34 & -0.06 & 0.06 & -0.30 & 0.05 & 0.00 & 0.00 & 0.00 \\
\hline 9 & 6 & 0.11 & 0.17 & -0.03 & 0.21 & 0.44 & -0.08 & 0.00 & 0.00 & 0.00 \\
\hline 10 & 6 & 0.01 & -0.02 & 0.00 & 0.00 & -0.05 & 0.0 & 0.0 & 0.00 & 0. \\
\hline 11 & 17 & 0.00 & 0.00 & 0.00 & 0.00 & 0.00 & 0.00 & 0.00 & 0.00 & 0.00 \\
\hline 12 & 17 & 0.00 & & 0.00 & & 0.00 & 0.00 & 0.00 & 0.00 & 0.00 \\
\hline 13 & 78 & & & 0.00 & & 0.00 & 0.0 & 0.0 & 0.00 & 0 \\
\hline 14 & 7 & & & 0.00 & & 0.00 & 0.0 & -0.43 & -0.41 & 0.07 \\
\hline 15 & 7 & & & 0.02 & & -0.28 & 0. & 0.00 & 0.00 & 0.00 \\
\hline 16 & 6 & 0.11 & & 0.00 & 0.10 & 0.01 & 0.0 & 0.0 & 0.00 & 10 \\
\hline 17 & 6 & & & 0.02 & & 0.00 & 0.01 & 0.00 & 0.00 & 0.00 \\
\hline 18 & 6 & & & 0.03 & & 0.00 & 0.00 & 0.00 & & \\
\hline 19 & 6 & -0.05 & & 0.0 & 0. & 0.00 & 0.0 & 0.0 & 0. & 0. \\
\hline 20 & 6 & 0.08 & & -0.03 & -0.01 & 0.00 & 0.00 & 0.00 & 0.00 & 0. \\
\hline 21 & 6 & & & -0.02 & & -0.01 & -0.01 & 0.0 & & 0. \\
\hline 22 & 17 & & & 0. & & 0.00 & 0. & & & \\
\hline 23 & 17 & & & 0. & & 0.00 & 0.0 & 0. & 0.00 & \\
\hline 24 & 6 & 0.00 & & & & 0. & 0.0 & 0.0 & 0.00 & 0.00 \\
\hline 25 & 6 & & & & & & & & & \\
\hline 26 & 1 & 0. & & -0.0 & & -0.20 & 0.0 & 0.1 & 0.1 & \\
\hline 27 & 1 & 0. & & -0 . & -0. & 0.21 & 0. & 0. & 0.00 & \\
\hline 28 & 1 & & & & & & & & & \\
\hline 29 & 1 & & & -0.10 & -0. & -0.31 & -0 . & 0. & & \\
\hline 30 & 1 & & & 0. & & -0.24 & 0. & & 10 & \\
\hline 31 & 1 & & & & & & & & & \\
\hline 32 & 1 & & & -0.0 & & 0.00 & & & & \\
\hline 33 & 1 & & & 0.0 & & 0.00 & 0. & 0. & 0.00 & 0. \\
\hline 34 & 1 & & & & & 0.00 & & & & \\
\hline 35 & 1 & & & 0.05 & & -0.01 & -0 . & 0. & 0. & \\
\hline 36 & 1 & -0. & & 0.0 & & 0.00 & 0.0 & 0.00 & 0.00 & 0.00 \\
\hline 37 & 1 & -0. & & -0.0 & & 0.00 & 0. & 0. & 0.00 & 0.00 \\
\hline 38 & 1 & & & -0.02 & & 0.01 & & & 0.01 & \\
\hline 39 & 1 & -0. & & 0.02 & -0.02 & 0.01 & & 0.0 & 0.01 & 0. \\
\hline 40 & 1 & 0.0 & $-0 .($ & 0.00 & 0.00 & 0.00 & 0.00 & -0.02 & 0.00 & 0.00 \\
\hline 41 & 6 & 0.00 & 0.00 & 0.00 & 0.00 & 0.00 & 0.00 & 0.56 & 0.53 & \\
\hline
\end{tabular}




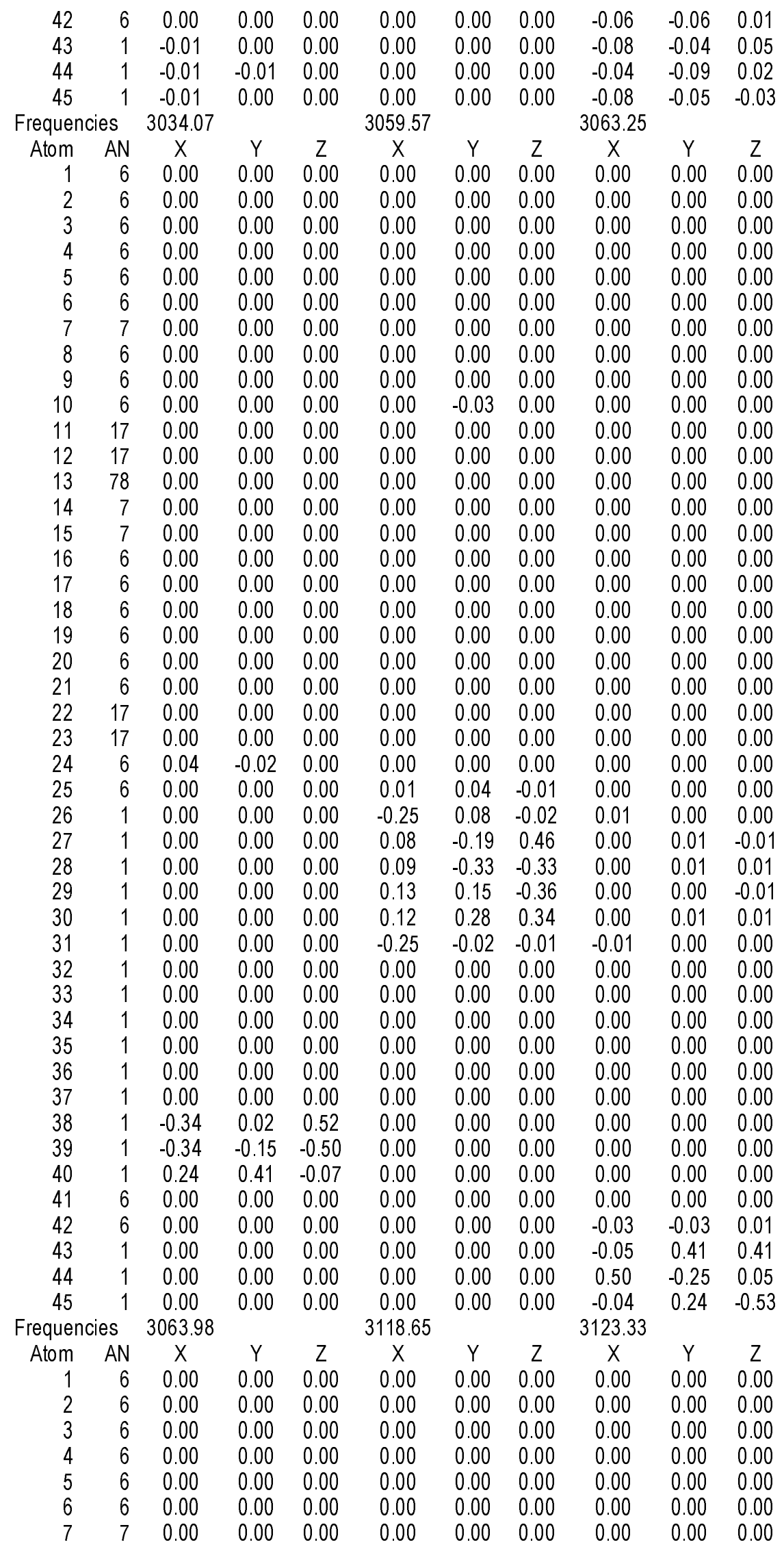




\begin{tabular}{|c|c|c|c|c|c|c|c|c|c|c|}
\hline & & & & & & & & & & \\
\hline $\begin{array}{l}8 \\
9\end{array}$ & $\begin{array}{l}6 \\
6\end{array}$ & $\begin{array}{l}0.00 \\
0.00\end{array}$ & $\begin{array}{l}0.00 \\
0.00\end{array}$ & $\begin{array}{l}0.00 \\
0.00\end{array}$ & $\begin{array}{l}0.00 \\
0.00\end{array}$ & $\begin{array}{l}0.00 \\
0.00\end{array}$ & $\begin{array}{l}0.00 \\
0.00\end{array}$ & $\begin{array}{l}0.00 \\
0.00\end{array}$ & $\begin{array}{l}0.00 \\
0.00\end{array}$ & $\begin{array}{l}0.00 \\
0.00\end{array}$ \\
\hline 10 & 6 & 0.00 & 0.04 & 0.00 & 0.00 & 0.01 & 0.04 & 0.00 & 0.00 & 0.02 \\
\hline 11 & 17 & 0.00 & 0.00 & 0.00 & 0.00 & 0.00 & 0.00 & 0.00 & 0.00 & 0.00 \\
\hline 12 & 17 & 0.00 & 0.00 & 0.00 & 0.00 & 0.00 & 0.00 & 0.00 & 0.00 & 0.00 \\
\hline 13 & 78 & 0.00 & 0.00 & 0.00 & 0.00 & 0.00 & 0.00 & 0.00 & 0.00 & 0.00 \\
\hline 14 & 7 & 0.00 & 0.00 & 0.00 & 0.00 & 0.00 & 0.0 & 0.00 & 00 & 0.00 \\
\hline 15 & 7 & 0.00 & 0.00 & 0.00 & 0.00 & 0.00 & 0.00 & 0.00 & 0.00 & 0.00 \\
\hline 16 & 6 & 0.00 & 0.00 & 0.00 & 0.00 & 0.00 & 0.00 & 0.00 & .00 & 0.00 \\
\hline 17 & 6 & 0.00 & 0.00 & 0.00 & 0.00 & 0.00 & 0.0 & 0.00 & 0.00 & 0.00 \\
\hline 18 & 6 & 0.00 & 0.00 & 0.00 & 0.00 & 0.00 & 0.00 & 0.00 & 0.00 & 0.00 \\
\hline 19 & 6 & 0.00 & 0.00 & 0.00 & 0.00 & 0.00 & 0.00 & 0.00 & 0.00 & 0.00 \\
\hline 20 & 6 & 0.00 & 0.00 & 0.00 & 0.00 & 0.00 & 0.0 & 0.00 & 0.00 & 0.00 \\
\hline 21 & 6 & 0.00 & 0.00 & 0.00 & 0.00 & 0.00 & 0.00 & 0.00 & 0.00 & 0.00 \\
\hline 22 & 17 & 0.00 & 0.00 & 0.00 & 0.00 & 0.00 & 0.00 & 0.00 & 0.00 & 0.00 \\
\hline 23 & 17 & 0.00 & 0.00 & 0.00 & 0.00 & 0.00 & 0.00 & 0.00 & 0.00 & 0.00 \\
\hline 24 & 6 & 0.00 & 0.00 & 0.00 & 0.00 & 0.00 & 0.0 & 0.00 & 0.02 & 0.09 \\
\hline 25 & 6 & 0.01 & 0.03 & -0.01 & 0.00 & -0.02 & -0.08 & 0.00 & 0.00 & 0.01 \\
\hline 26 & 1 & -0.23 & 0.07 & -0.02 & 0.02 & -0.01 & -0.02 & 0.00 & 0.00 & 0.00 \\
\hline 27 & 1 & 0.07 & -0.17 & 0.41 & 0.10 & -0.26 & 0.55 & -0.02 & 0.04 & -0.08 \\
\hline 28 & 1 & 0.08 & -0.29 & -0.29 & -0.13 & 0.46 & 0.43 & 0.02 & -0.07 & -0.06 \\
\hline 29 & 1 & -0.15 & -0.16 & 0.40 & 0.11 & 0.13 & -0.28 & 0.06 & 0.07 & -0.15 \\
\hline 30 & 1 & -0.13 & -0.32 & -0.38 & -0.09 & -0.20 & -0.22 & -0.04 & -0.10 & -0.11 \\
\hline 31 & 1 & 0.29 & 0.02 & 0.01 & -0.02 & 0.00 & 0.01 & -0.01 & 0.00 & \\
\hline 32 & 1 & 0.00 & 0.00 & 0.00 & 0.00 & 0.00 & 0.00 & 0.00 & 0.00 & 0.00 \\
\hline 33 & 1 & 0.00 & 0.00 & 0.00 & 0.00 & 0.00 & 0.0 & 0.00 & 0.00 & 0.00 \\
\hline 34 & 1 & 0.00 & & 0.00 & 0.00 & 0.00 & 0.0 & 0.00 & 0.00 & 0.00 \\
\hline 35 & 1 & 0.00 & 0.00 & 0.00 & 0.00 & 0.00 & 0.00 & 0.00 & 0.00 & 0.00 \\
\hline 36 & 1 & 0.00 & 0.00 & 0.00 & 0.00 & 0.00 & 0.00 & 0.00 & 0.00 & 0.00 \\
\hline 37 & 1 & 0.00 & & 0.00 & 0.00 & 0.00 & 0.00 & 0.0 & 0.00 & 0. \\
\hline 38 & 1 & 0.00 & 0. & 0.00 & 0.01 & 0.00 & -0.01 & 0.40 & -0.03 & -0.54 \\
\hline 39 & 1 & 0.00 & 0.00 & 0.00 & -0.01 & 0.00 & -0.01 & -0.39 & -0.16 & -0.53 \\
\hline 40 & 1 & 0.00 & & 0. & 0.00 & 0.00 & 0. & 0.00 & 0.00 & 0.03 \\
\hline 41 & 6 & & & 0.00 & 0.00 & 0.00 & 0. & 0.00 & 0.00 & \\
\hline 42 & 6 & 0.00 & & 0.00 & & 0.00 & & 0.00 & & \\
\hline 43 & 1 & 0.00 & 0.01 & 0.01 & 0.00 & 0.00 & 0.00 & 0.00 & 0.00 & 0.00 \\
\hline 44 & 1 & 0.01 & & 0.00 & 0.00 & 0.00 & 0.00 & 0.00 & 0.00 & 0.00 \\
\hline 45 & 1 & 0.00 & & -0.02 & 0.00 & 0.00 & 0.00 & 0.00 & 0.00 & 0.00 \\
\hline requen & & 3123.60 & & & 3138.67 & & & 3139.86 & & \\
\hline Atom & AN & $x$ & $Y$ & Z & $X$ & $Y$ & Z & $X$ & Y & Z \\
\hline 1 & 6 & 0.00 & 0.00 & 0.00 & 0.00 & 0.00 & 0.00 & 0.00 & 0.00 & 0.00 \\
\hline 2 & 6 & & & & & 0.00 & 0.00 & & & 0.00 \\
\hline 3 & 6 & & & 0.00 & & 0.00 & 0.00 & 0.0 & 0.00 & 0.00 \\
\hline 4 & 6 & 0.00 & 0. & 0.00 & 0.00 & 0.00 & 0.00 & 0.00 & 0.00 & 0.00 \\
\hline 5 & 6 & & & 0.00 & & 0.00 & 0.0 & & 0.00 & 0.00 \\
\hline 6 & 6 & & & & & 0.00 & 0.0 & 0. & 0.00 & 0 \\
\hline 7 & 7 & & & 0.00 & & 0.00 & 0.00 & 0.00 & 0.00 & 0.00 \\
\hline 8 & 6 & & & & & & & & 0.00 & 0.00 \\
\hline 9 & 6 & & & 0. & & 0.00 & 0. & 0. & & \\
\hline 10 & 6 & & & 0.0 & & 0.00 & 0.00 & 0.00 & 0.00 & 0.00 \\
\hline 11 & 17 & & & & & 0.00 & 0.0 & 0.0 & 0.00 & 0.00 \\
\hline 12 & 17 & & 0. & 0.0 & 0.00 & 0.00 & 0. & 0. & 0.00 & 0.00 \\
\hline 13 & 78 & & & & & 0.00 & 0.0 & 0.0 & 0.00 & \\
\hline 14 & 7 & 0.00 & 0.00 & 0.00 & 0.00 & 0.00 & 0.00 & 0.00 & 0.00 & 0.00 \\
\hline 15 & 7 & & & 0.00 & & 0.00 & 0.00 & & 0.00 & 0.00 \\
\hline 16 & 6 & & & 0.00 & 0.0 & 0.00 & 0.0 & 0.0 & 0.00 & 0.0 \\
\hline 17 & 6 & 0.00 & 0.00 & 0.00 & 0.00 & 0.00 & 0.00 & 0.00 & 0.00 & 0.00 \\
\hline 18 & 6 & & & 0.0 & & 0.00 & 0.00 & 0.00 & 0.00 & 0.00 \\
\hline 19 & 6 & 0.0 & 0.0 & 0.0 & 0.0 & 0.00 & 0.00 & 0.0 & 0.00 & 0.0 \\
\hline 20 & 6 & & 0.00 & 0.00 & 0.00 & 0.00 & 0.00 & 0.00 & 0.00 & 0.00 \\
\hline
\end{tabular}




\begin{tabular}{|c|c|c|c|c|c|c|c|c|c|c|}
\hline & & & & & & & & & & \\
\hline 21 & 6 & 0.00 & 0.00 & 0.00 & 0.00 & 0.00 & 0.00 & 0.00 & 0.00 & 0.00 \\
\hline 22 & 17 & 0.00 & 0.00 & 0.00 & 0.00 & 0.00 & 0.00 & 0.00 & 0.00 & 0.00 \\
\hline 23 & 17 & 0.00 & 0.00 & 0.00 & 0.00 & 0.00 & 0.00 & 0.00 & 0.00 & 0.00 \\
\hline 24 & 6 & 0.00 & 0.00 & -0.02 & -0.06 & -0.07 & 0.01 & 0.00 & 0.00 & 0.00 \\
\hline 25 & 6 & 0.00 & 0.01 & 0.04 & 0.00 & 0.00 & 0.00 & 0.00 & 0.00 & 0.00 \\
\hline 26 & 1 & -0.01 & 0.00 & 0.01 & 0.00 & 0.00 & 0.00 & 0.00 & 0.00 & 0.00 \\
\hline 27 & 1 & -0.05 & 0.13 & -0.27 & 0.00 & 0.00 & 0.00 & 0.00 & 0.00 & 0.00 \\
\hline 28 & 1 & 0.06 & -0.22 & -0.20 & 0.00 & 0.00 & 0.00 & 0.00 & 0.00 & 0.00 \\
\hline 29 & 1 & 0.21 & 0.25 & -0.53 & 0.00 & 0.00 & 0.00 & 0.00 & 0.00 & 0.00 \\
\hline 30 & 1 & -0.16 & -0.38 & -0.41 & 0.00 & 0.00 & 0.00 & 0.00 & .00 & 0.00 \\
\hline 31 & 1 & -0.04 & 0.00 & 0.02 & 0.00 & 0.00 & 0.00 & 0.00 & 0.00 & 0.00 \\
\hline 32 & 1 & 0.00 & 0.00 & 0.00 & 0.00 & 0.00 & 0.00 & 0.00 & 0.00 & 0.00 \\
\hline 33 & 1 & 0.00 & 0.00 & 0.00 & 0.00 & 0.00 & 0.00 & 0.00 & 0.00 & 0.00 \\
\hline 34 & 1 & 0.00 & 0.00 & 0.00 & 0.00 & 0.00 & 0.0 & 0.00 & 0.00 & 0.00 \\
\hline 35 & 1 & 0.00 & 0.00 & 0.00 & 0.00 & 0.00 & 0.00 & 0.00 & 0.00 & 0.00 \\
\hline 36 & 1 & 0.00 & 0.00 & 0.00 & 0.00 & 0.00 & 0.00 & 0.00 & 0.00 & 0.00 \\
\hline 37 & 1 & 0.00 & 0.00 & 0.00 & 0.00 & 0.00 & & 0.00 & 0.00 & 0.00 \\
\hline 38 & 1 & -0.11 & 0.01 & 0.15 & 0.18 & -0.04 & & 0.00 & 00 & 0.00 \\
\hline 39 & 1 & 0.11 & 0.04 & 0.15 & 0.17 & 0.06 & 0.2 & 0.00 & 0.00 & 0.00 \\
\hline 40 & 1 & 0.00 & 0.00 & -0.01 & 0.41 & 0.76 & -0 . & 0.00 & 0.00 & \\
\hline 41 & 6 & 0.00 & 0.00 & 0.00 & 0.00 & 0.00 & & 0.00 & & 0.00 \\
\hline 42 & 6 & 0.00 & 0.00 & 0.00 & 0.00 & 0.00 & 0.00 & 0.00 & 0.02 & 0.09 \\
\hline 43 & 1 & 0.00 & 0.00 & 0.00 & 0.00 & 0.00 & 0.0 & 0.06 & -0.51 & -0.47 \\
\hline 44 & 1 & 0.00 & 0.00 & 0.00 & 0.00 & 0.00 & 0.00 & -0.01 & 0.01 & 0.02 \\
\hline 45 & 1 & 0.00 & 0.00 & 0.00 & 0.00 & 0.00 & 0.00 & -0.05 & 0.31 & -0.64 \\
\hline Frequen & & 3143.11 & & & 3172.12 & & & 3179.68 & & \\
\hline Atom & AN & $X$ & $\mathrm{Y}$ & Z & X & $\mathrm{Y}$ & Z & $X$ & $\mathrm{Y}$ & Z \\
\hline 1 & 6 & 0.00 & 0.00 & 0.00 & 0.00 & 0.00 & 0.00 & 0.00 & 0.00 & 0.00 \\
\hline 2 & 6 & 0.00 & 0.00 & 0.00 & 0.00 & 0.00 & 0.00 & 0.00 & 0.00 & 0.00 \\
\hline 3 & 6 & 0.00 & 0.00 & 0.00 & 0.00 & 0.00 & 0.00 & 0.0 & 0.00 & 0.00 \\
\hline 4 & 6 & 0.00 & 0.00 & 0.00 & 0.00 & 0.00 & 0.00 & 0. & 0.00 & 0.00 \\
\hline 5 & 6 & 0.00 & 0.00 & 0.00 & 0.00 & 0.00 & 0.00 & 0. & 0.00 & 0.00 \\
\hline 6 & 6 & 0.00 & 0.00 & 0.00 & 0.00 & 0.00 & 0.00 & 0.00 & 0.00 & 0.00 \\
\hline 7 & 7 & 0.00 & 0.00 & 0.00 & 0.00 & 0.00 & 0.00 & 0.0 & 0.00 & 0.00 \\
\hline 8 & 6 & & & 0.00 & & 0.00 & 0.0 & 0. & 0.00 & 0.00 \\
\hline 9 & 6 & 0.00 & 0.00 & 0.00 & 0.00 & 0.00 & 0.00 & 0.00 & 0.00 & 0.00 \\
\hline 10 & 6 & 0. & 0. & 0.00 & -0.09 & -0.02 & 0.00 & 0.00 & 0.00 & 0.00 \\
\hline 11 & 17 & & & 0. & 0.00 & 0.00 & 0.00 & 0.0 & 0.00 & 0.00 \\
\hline 12 & 17 & & & 0.00 & 0.0 & 0.00 & 0. & & 0. & 0.00 \\
\hline 13 & 78 & & & 0.00 & 0.00 & 0.00 & 0.0 & 0.00 & 0.00 & 0.00 \\
\hline 14 & 7 & 0.00 & 0. & 0.00 & 0.00 & 0.00 & 0.00 & 0.0 & 0.00 & 0.00 \\
\hline 15 & 7 & & & 0.00 & & 0.00 & 0. & & & 0.00 \\
\hline 16 & 6 & & & 0. & & 0.00 & 0.0 & 0. & 0.00 & 0.00 \\
\hline 17 & 6 & & & 0.00 & 0.00 & 0.00 & 0.00 & 0.0 & 0.00 & 0.00 \\
\hline 18 & 6 & & & & & 0.00 & & & & \\
\hline 19 & 6 & & & 0. & 0. & 0.00 & 0. & 0. & 0. & \\
\hline 20 & 6 & & & 0. & 0.00 & 0.00 & 0.00 & 0. & 0.00 & 0.00 \\
\hline 21 & 6 & & & & & 0.00 & 0.0 & & & 0.00 \\
\hline 22 & 17 & & & 0. & & 0.00 & 0. & & 0. & 0. \\
\hline 23 & 17 & & & 0. & & 0.00 & 0. & 0. & 0. & 0. \\
\hline 24 & 6 & $0 . C$ & & 0.0 & 0.00 & 0.00 & 0.0 & 0.00 & 0.00 & 0.00 \\
\hline 25 & 6 & 0.0 & & 0.0 & 0.00 & 0.00 & 0.0 & $-0 .($ & 0.04 & -0.01 \\
\hline 26 & 1 & & & 0.0 & & -0.01 & 0.0 & 0.9 & -0.25 & 0.07 \\
\hline 27 & 1 & 0.00 & 0.0 & 0.00 & 0.00 & 0.00 & 0.00 & 0.02 & -0.08 & 0.22 \\
\hline 28 & 1 & 0.00 & & & & 0.00 & 0.00 & & -0.17 & -0.18 \\
\hline 29 & 1 & 0.00 & & 0.0 & & 0.10 & -0.2 & 0.0 & -0.01 & 0.01 \\
\hline 30 & 1 & 0.00 & 0.0 & 0.00 & 0.05 & 0.15 & 0.19 & 0.00 & -0.01 & -0.01 \\
\hline 31 & 1 & 0.0 & & 0.00 & & 0.06 & 0.0 & -0.03 & 0.00 & 0.00 \\
\hline 32 & 1 & 0.0 & & 0.0 & 0.00 & 0.00 & 0.00 & 0.00 & 0.00 & 0.00 \\
\hline 33 & 1 & 0.00 & 0.00 & 0.00 & 0.00 & 0.00 & 0.00 & 0.00 & 0.00 & 0.00 \\
\hline
\end{tabular}




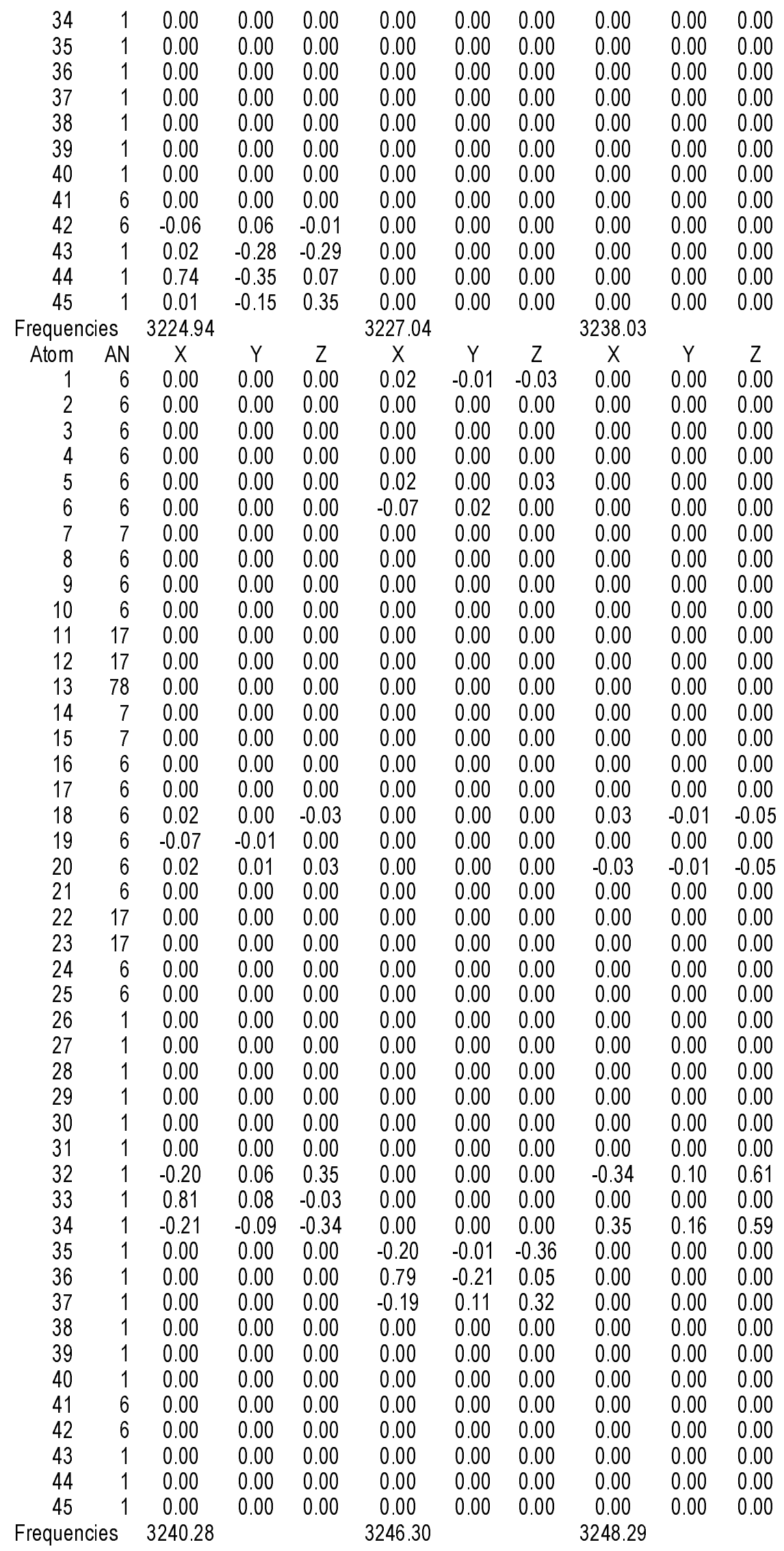




\begin{tabular}{|c|c|c|c|c|c|c|c|c|c|c|}
\hline Atom & AN & $X$ & Y & Z & $X$ & $Y$ & Z & $X$ & Y & , \\
\hline 1 & 6 & 0.03 & -0.02 & -0.05 & 0.00 & 0.00 & 0.00 & -0.02 & 0.02 & 0. \\
\hline 2 & 6 & 0.00 & 0.00 & 0.00 & 0.00 & 0.00 & 0.00 & 0.00 & 0.00 & \\
\hline 3 & 6 & 0.00 & 0.00 & 0.00 & 0.00 & 0.00 & 0.00 & 0.00 & 0.00 & \\
\hline 4 & 6 & 0.00 & 0.00 & 0.00 & 0.00 & 0.00 & 0.00 & 0.00 & 0.00 & \\
\hline 5 & 6 & -0.03 & 0.00 & -0.06 & 0.00 & 0.00 & 0.00 & -0.02 & 0.00 & \\
\hline 6 & 6 & 0.00 & 0.00 & 0.00 & 0.00 & 0.00 & 0.00 & -0.05 & 0.01 & \\
\hline 7 & 7 & 0.00 & 0.00 & 0.00 & 0.00 & 0.00 & 0.00 & 0.00 & 0.00 & \\
\hline 8 & 6 & 0.00 & 0.00 & 0.00 & 0.00 & 0.00 & 0.00 & 0.00 & 0.00 & \\
\hline 9 & 6 & 0.00 & 0.00 & 0.00 & 0.00 & 0.00 & 0.00 & 0.00 & 0.00 & \\
\hline 10 & 6 & 0.00 & 0.00 & 0.00 & 0.00 & 0.00 & 0.00 & 0.00 & 0.00 & \\
\hline 11 & 17 & 0.00 & 0.00 & 0.00 & 0.00 & 0.00 & & & 0.00 & \\
\hline 12 & 17 & 0.00 & 0.00 & 0.00 & 0.00 & 0.00 & 0.00 & 0.00 & 0.00 & \\
\hline 13 & 78 & 0.00 & 0.00 & 0.00 & 0.00 & 0.00 & 0.0 & 0.00 & 0.00 & \\
\hline 14 & 7 & 0.00 & 0.00 & 0.00 & 0.00 & 0.00 & 0.00 & 0.00 & 0.00 & \\
\hline 15 & 7 & 0.00 & 0.00 & 0.00 & 0.00 & 0.00 & 0.0 & 0.00 & 0.00 & \\
\hline 16 & 6 & 0.00 & 0.00 & 0.00 & 0.00 & 0.00 & 0.0 & 0.00 & 0.00 & \\
\hline 17 & 6 & 0.00 & 0.00 & 0.00 & 0.00 & 0.00 & 0.00 & 0.00 & 0.00 & \\
\hline 18 & 6 & 0.00 & 0.00 & 0.00 & 0.02 & -0.01 & -0.05 & 0.00 & 0.00 & \\
\hline 19 & 6 & 0.00 & 0.00 & 0.00 & 0.05 & 0.01 & 0.00 & 0.00 & 0.00 & \\
\hline 20 & 6 & 0.00 & 0.00 & 0.00 & 0.02 & 0.01 & 0.04 & 0.00 & 0.00 & \\
\hline 21 & 6 & 0.00 & 0.00 & 0.00 & 0.00 & 0.00 & 0.00 & 0.00 & 0.00 & \\
\hline 22 & 17 & 0.00 & 0.00 & 0.00 & 0.00 & 0.00 & 0.0 & 0.0 & & \\
\hline 23 & 17 & 0.0 & 0.00 & 0.00 & 0.00 & 0.00 & 0. & & 0.00 & \\
\hline 24 & 6 & 0.00 & 0.00 & 0.00 & 0.00 & 0.00 & 0.00 & 0.00 & 0.00 & \\
\hline 25 & 6 & 0.00 & 0.00 & 0.00 & 0.00 & 0.00 & 0.00 & 0.00 & 0.00 & \\
\hline 26 & 1 & 0.00 & 0.00 & 0.0 & 0.00 & & & & 00 & \\
\hline 27 & 1 & 0.00 & 0.00 & 0.00 & 0.00 & 0.00 & 0.00 & 0.00 & 0.00 & \\
\hline 28 & 1 & 0.00 & 0.00 & 0.0 & 0.00 & 0.00 & 0.00 & 0.00 & 0.00 & \\
\hline & 1 & 0.0 & 0.00 & 0. & & & & & & \\
\hline 30 & 1 & 0.00 & 0.00 & 0.00 & 0.00 & 0.00 & 0.0 & 0.00 & 0.00 & \\
\hline 31 & 1 & 0.00 & 0.00 & 0.00 & 0.00 & 0.00 & 0.00 & 0.00 & 0.00 & \\
\hline 32 & 1 & 0.00 & 0.00 & 0.0 & -0.27 & 0.08 & & & & \\
\hline 33 & 1 & 0.00 & 0.00 & 0.0 & -0.57 & -0.05 & & & 0.00 & \\
\hline 34 & 1 & 0.00 & 0.00 & 0.00 & -0.29 & -0.13 & -0.49 & 0.0 & 0.00 & \\
\hline 35 & 1 & 0.34 & 0.02 & 0.6 & 0.00 & 0.00 & 0.0 & & & \\
\hline 36 & 1 & 0.0 & -0.0 & 0.0 & 0.0 & 0.00 & & & -0.15 & \\
\hline 37 & 1 & -0.33 & 0.19 & 0.5 & 0.00 & 0.00 & & & -0.17 & \\
\hline 38 & 1 & 0.00 & 0.00 & 0.0 & 0.00 & 0.00 & & 0.0 & 0.00 & \\
\hline 39 & 1 & 0.0 & 0.00 & 0.0 & 0.0 & & & & & \\
\hline 40 & 1 & 0.00 & 0.00 & 0.0 & 0.00 & & & & & \\
\hline 41 & 6 & 0.00 & 0.00 & 0.00 & 0.00 & 0.00 & 0. & 0.00 & 0.00 & \\
\hline 42 & 6 & 0.0 & 0.0 & 0.0 & 0.00 & & & & & \\
\hline 43 & 1 & 0.0 & 0.0 & 0.0 & 0.0 & & & & & \\
\hline 44 & 1 & 0.0 & 0.0 & 0.0 & 0.00 & 0. & & 0. & 0.00 & \\
\hline 4 & 1 & 0.0 & 0.0 & 0.0 & 0.00 & 0.00 & & 0.00 & 0.00 & \\
\hline
\end{tabular}

